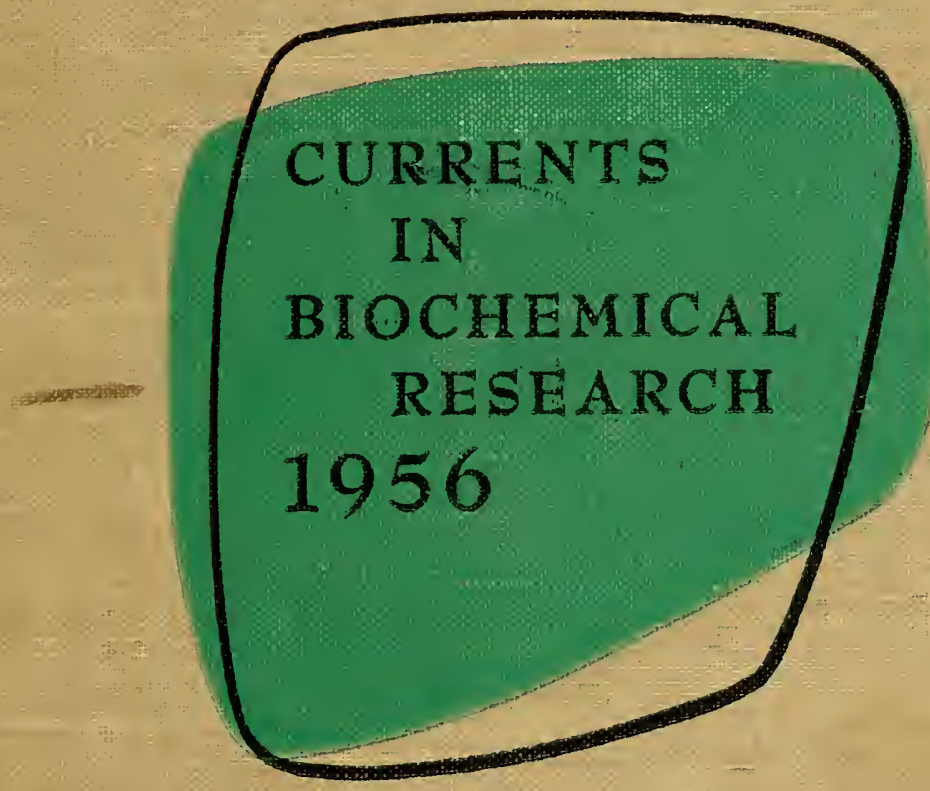




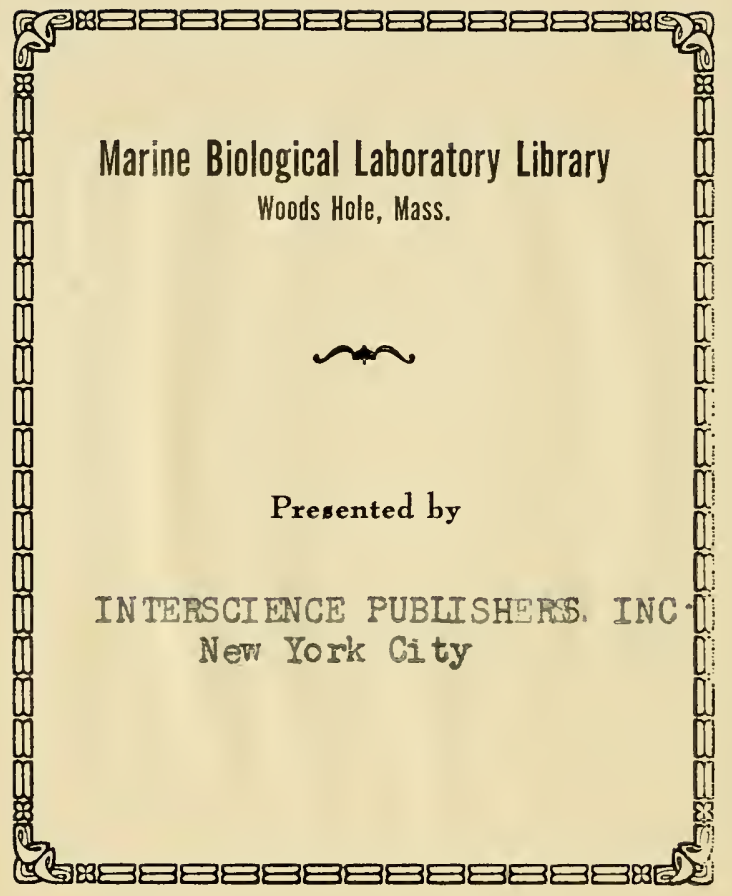







\section{CURRENTS IN \\ BIOCHEMICAL RESEARCH \\ 1956}





\section{CURRENTS IN BIOCHEMICAL RESEARCH 1956}

\section{Edited by DAVID E. GREEN}

Twenty-seven essays charting the present course of biochemical research and considering the intimate relationship of biochemistry to medicine, physiology, and biology

INTERSCIENCE PUBLISHERS, INC., NEW YORK Interscience Publishers, Ltd., London 
(C) 1956 by Interscience Publishers, Inc.

Library of Congress Catalog Card Number 56-9503

Interscience Publishers, Inc.

250 Fifth Ave., New York 1, N. Y.

For Great Britain and Northern Ireland:

Interscience Publishers Ltd.

88/90 Chancery Lane, London, W.C. 2, England

PRINTED IN THE UNITED STATES OF AMERIGA

BY MACK PRINTING COMPANY, EASTON, PENNSYLVANIA 


\section{P R E F A C E}

Ten years ago the first volume of Currents in Biochemical Research made its debut. The cordial reception which was given this volume by biologists, chemists, biochemists, and clinicians indicated clearly that at appropriate intervals there exists a real need to pause and reflect on what has been accomplished, to distinguish between tree and forest, to learn the lessons of the years which have passed, and to prepare intellectually for the years to come. Once again a distinguished group of twenty-seven contributors has been invited to make a decennial survey of progress in most, although by no means all, important fields of biochemical research. They have been asked to write as simply and as lucidly as the requirements of scholarship permit. The objectives of these essays have been to communicate to nonspecialists an overall impression of the present status of the significant problems in each field, to point up the broad strategy of current research, and finally, to speculate on the likely paths of future research. Above all, the essays were intended to bring light without overwhelming the reader with tedious detail. It would be too much to expect that each of the twenty-seven essays has met all these exacting stipulations, but at least I have high hopes that enough of them come sufficiently close to justify the aims of this second volume.

The past decade has witnessed a rate of progress vastly greater than in any comparable period since the early beginnings of biochemistry as a science more than 100 years ago. There is little doubt that this phenomenal rate of development has been sparked by a revolution in methodology. The emergence of filter paper chromatography as a tool for separating minute 
amounts of material rapidly, selectively, and even quantitatively, gave wings to biochemistry. In turn, this led to the intensive exploitation and development of a large variety of adsorption and partition procedures which almost overnight made it not only possible, but even relatively simple to separate and isolate constituents of any mixture, regardless of how similar the constituents may happen to be. Isolation ceased to be a roadblock or a major difficulty in biochemical investigation.

The spectrophotometer occupies a preeminent position among the instruments which have facilitated the dramatic advances in methodology. This was of course not a new physical tool, but until some ten years ago it was a highly expensive, custom-built apparatus available only to relatively few laboratories with considerable resources. With the advent of the mass-produced spectrophotometer, the universal application of spectrophotometric techniques to biochemical problems became possible. It is no exaggeration to say that spectrophotometry has increased manyfold the forward rate of progress of biochemistry.

Other tools and techniques have played an equally important role in the revolution of biochemical methodology during the past decade. The isotope technique with its countless ramifications and variations and the mass-produced ultracentrifuge and electrophoresis apparatus were also very dominant factors in the over-all picture.

With such a plethora of powerful tools available, structural problems that had long resisted solution were dusted off the shelf and liquidated in record time. Nucleotides, nucleic acid, polysaccharides, lipides, polypeptides, and even some proteins of low molecular weight were no match for the many and incisive methods which were brought to bear in the elucidation of stucture. Even efforts at synthesis took a new lease of life now that effective methods were available for separation of similar reaction products, or isomers, as in the case of nucleotides. It was a triumph of methodology to ferret out the exact structural formula of insulin and to accomplish the synthesis of flavin 
adenine dinucleotide and vasopressin to mention but two outstanding examples.

Methodology was not the principal limiting factor in all structural problems. The solution of the problem of the molecular architecture and stereochemistry underlying protein structure required new ideas and concepts as well as more powerful tools for analysis. The helix principle which has been shown to apply successfully to a variety of proteins and to nucleic acid has provided one of the keys for deciphering the hitherto unintelligible $\mathrm{x}$-ray diagrams of a variety of macromolecules.

A direct consequence of the advances in methodology was the growth of enzyme chemistry from a toddler to a giant dominating the whole of biochemistry. During the past ten years practically all the enzymatic and chemical details of the citric, pentose, fatty acid, and urea cycles have been elucidated. The enzymatic synthesis of nucleic acid, lecithin, cephalin, fatty acids, sucrose, lactose, purines, pyrimidines, and dinucleotides has been accomplished by means of isolated enzymes or cellfree enzyme systems. The chemical details of the reactions which intervene in the enzymatic synthesis of hemin and cholesterol are now almost fully documented. Hundreds of enzymes which implement not only the above sequences but many others too numerous to be listed have been isolated, characterized, and studied in detail. Indeed few of the classical problems of metabolism remain unsolved or are not already close to solution.

The list of coenzymes has more than doubled during the past ten years. Lipoic acid, coenzyme A, uridine diphosphoglucose, uridine diphosphogalactose, uridine diphosphoglucuronic acid, glucose-1,6-diphosphate, 1,3-diphosphoglyceric acid, guanosine diphosphate, molybdenum, and cytosine diphosphocholine are some of the more prominent recent additions to the roster of known cofactors.

The era of each investigator preparing his own cofactors, special chemicals, and even enzymes is now all but over. A 
whole new industry has sprung up which provides practically every important biochemical compound relatively inexpensively and of high purity. The importance of this development to the rapid progress of biochemical research cannot be underestimated.

Enzymology has indeed prospered by application of new methodological tools but the traffic has gone both ways. In turn, the enzymes have proved to be tools of such breath-taking elegance and simplicity as to revolutionize the tactics of determining structure or of analyzing for minute amounts of material. The elucidation of the structure of nucleic acid, of nucleotides, and of various nucleotide cofactors has been accomplished principally, if not exclusively, by enzymatic procedures and with relatively small amounts of compound.

The current procedures for isolating enzymes are still largely classical, but signs are multiplying that big changes in methodology are imminent. Chromatography on columns of cellulose derivatives or of calcium phosphate, electroconvection, starch and filter paper electrophoresis, ion exchange resin chromatography, and partition methods are portents of the things to come.

In recent years there has been a growing recognition of the role of integrated enzyme systems in cellular processes. The concept of the mitochondrion as an organized mosaic of several hundreds of enzymes linked together structurally as well as functionally and with unique operational principles is now well established in biochemical thinking. It is only in terms of large molecular aggregates and of enzymes bonded together in a precise pattern that it is at all possible to approach problems such as oxidative phosphorylation, electron transfer, and muscular contraction. The organized enzyme systems have provided the bridge between classical enzyme chemistry and cellular processes. In addition to the mitochondrion, other functional particles have been described such as the chloroplast in photosynthesis, and microsomes and nuclei in protein synthesis.

Progress in the field of the physical chemistry of biological 
systems and materials has been slower but still impressive. Kinetic analysis of enzymes has graduated to a very sophisticated and exact science which has been taken over by specialists well grounded in physical chemical theory and practice. The concepts of energy transformations and of the high-energy bond have lost their earlier primitive character and are now couched in the more rigorous language of quantum mechanics and valency theory. The interpretation of the properties and behavior of proteins and other macromolecules has now attained a high degree of precision. Certainly the molecular picture of how myosin contracts is constructed in terms which would have been inconceivable even ten years ago.

It now appears that biochemistry has reached a stage in development where fragmentation cannot be avoided. The choice now lies between chemistry and physiology since the meso area has all but disappeared. The classical areas of biochemistry such as nutrition, vitamins, minerals, enzymology, metabolism, and the chemical structure of biologically important compounds are becoming leaner and leaner, and the focus is shifting inexorably either to more chemical and molecular aspects or to the broader and as yet unsolved physiological problems.

It is at present quite clear which chemical problems will dominate the scene in the next few years. Certainly efforts will be redoubled to determine the exact stereochemistry of protein molecules after the spectacular success of the $\alpha$ helix concept. Such efforts will be paralleled by companion efforts to elucidate the structure of the active groups of enzymes. Once an understanding of the architecture of the active groups of enzymes has been attained then the old problem of the mechanism of enzyme action will be in line for renewed assault. The nature of the forces and bonds which lead to enzyme-substrate interactions is an area of investigation which badly needs sparking by new leads.

The real frontier areas of biochemistry now reach to the borderlines of physiology, genetics, cytology, medicine, and 
theoretical chemistry. The biochemical description of muscular contraction, nerve conduction, glomerular filtration, and secretion and membrane phenomena is still relatively virgin territory. The structural analysis and interpretation of the way in which mitochondria, nuclei, membranes, myelin sheath, and other complex cellular structures are constructed in a chemical sense have yet to reach even the blueprint stage. A fabulous area of exploitation awaits the investigators who can hurdle the conceptual barriers in the way of the biochemical study of hormone action at the molecular level. If current progress on the reconstruction of in vitro systems for synthesis of protein is to serve as a guide, then we must surely anticipate the polygamous marriage, in the not too distant future, of biochemistry with genetics, immunology, hematology, and virology. The older problems of biochemistry are clearly moribund but the newer problems have the fascination and challenge of youth.

David E. Green

February 1956 


\section{CONTR I B U T OR S}

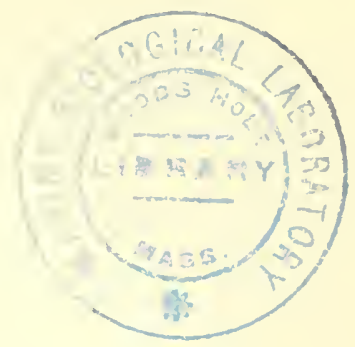

Robert A. Alberty, Ph.D. 1ssociate Professor of Chemistry, Lniversity of Wisconsin, Madison, Wis. Eli Lilly Award, 1956.

H. A. Barker, Ph.D. Professor of Plant Biochemistry, University of California, Berkeley, Calif.

James A. Bassham, Ph.D. Assistant Director, Photosynthesis Laboratory, Bio-Organic Chemistry Group, Radiation Laboratory, University of California, Berkeley, Calif.

Konrad E. Bloch, Ph.D. Higgins Professor of Biochemistry, Harrard University, Cambridge, Mass.

Iean Botts, Ph.D. Physiologist, Division of Physical Biochemistry, Naval Medical Research Institute, National Naval Medical Center, Bethesda, Md.

Melvin Calvin, Ph.D. Professor of Chemistry, and Director, Bio-Organic Chemistry Group, Radiation Laboratory, University of California, Berkeley, Calif.

Allan M. Campbell, Ph.D. Instructor in Bacteriology, University of Michigan, Ann Arbor, Mich.

Britton Chance, Ph.D., D.Sc. Professor of Biophysics, and Director of the Johnson Research Foundation, University of Pennsylvania Medical School, Philadelphia, Pa. President's Certificate of Merit, 1950; Paul Lewis Award, 1950.

Waldo E. Cohn, Ph.D. Principal Biochemist, Oak Ridge National Laboratory, Oak Ridge, Tenn.

Carl F. Cori, M.D. Professor of Biological Chemistry, Washington University School of Medicine, St. Louis, Mo. Nobel Prize, 1947.

John T. Edsall, M.D. Professor of Biological Chemistry, Harvard University, Cambridge, Mass.

Philip George, Ph.D. Research Professor of Biophysical Chemistry, The University of Pennsylvania, Philadelphia, Pa.

G. Robert Greenberg, Ph.D. Associate Professor of Biochemistry, School of Medicine, Western Reserve University, Cleveland, Ohio. 
A. D. Hershey, Ph.D. Staff Member, Department of Genetics, Carnegie Institution of Washington, Cold Spring Harbor, N. Y.

Frank M. Huennekens, Ph.D. Associate Professor of Biochemistry, School of Medicine, University of IWashington, Seattle, Wash.

Luis F. Leloir, M.D. Director of the Instituto de Investigaciones Bioquímicos, Buenos Aires, Argentina.

Fritz Lipmann, M.D., Ph.D. Head, Biochemical Research Laboratory, Massachusetts General Hospital, and Professor of Biological Chemistry, Harvard Medical School, Boston, Mass. Carl Neuberg Medal, 1948; Mead Johnson \& Co. Award, 1948; Nobel Prize, 1953.

Barbara Low, Ph.D. Assistant Professor of Physical Chemistry, Department of Biological Chemistry, Harvard Medical School, Boston, Mass.

Henry R. Mahler, Ph.D. Associate Professor of Chemistry, Indiana University, Bloomington, Ind.

Manuel F. Morales, Ph.D. Chief of the Division of Physical Biochemistry, Naval Medical Research Institute, National Naval Medical Center, Bethesda, Md. American Physiological Society Travel Award, 1950.

David Nachmansohn, M.D. Professor of Biochemistry, College of Physicians and Surgeons, Columbia University, New York, N. Y. Pasteur Medal, 1952; Carl Neuberg Medal, 1954.

Gregory Pivcus, D.Sc. Director of Laboratories, The Worcester Foundation for Experimental Biology, Shrewsbury, and Research Professor of Biology, Boston University, Boston, Mass.

Efraim Racker, M.D. Chief, Division of Nutrition and Physiology, The Public Health Research Institute of the City of New York, Inc., and Adjunct Professor, Department of Microbiology, New York L'niversity College of Medicine, New York, N.Y.

Harold P. Rusch, M.D. Professor of Oncology, and Director of the McArdle Memorial Laboratory for Cancer Research, University of IVisconsin, Madison, Wis.

Frederick Sanger, Ph.D., F.R.S. Member of the External Staff of the Medical Research Council, Department of Biochemistry, University of Cambridge, Cambridge, England.

David Shemin, Ph.D. Professor of Biochemistry, College of Physicians and Surgeons, Columbia Lniversity, New York, N. I. 
Esmond E. Snell, Ph.D. Professor of Chemistry, and Associate Director of the Biochemical Institute, University of Texas, Austin, Texas. Eli Lilly Award, 1945; Mead Johnson \& Co. Award, 1946; OsborneMendel Award, 1951.

S. Spiegelman, Ph.D. Professor of Bacteriology, University of Illinois, Urbana, Ill.

DeWitt Stetten, JR., M.D., Ph.D. Associate Director in Charge of Research, National Institute of Arthritis and Metabolic Diseases, National Institute of Health, Bethesda, and Lecturer in Medicine and Physiological Chemistry, The Johns Hopkins University, Baltimore, Md.

D. M. Surgenor, Ph.D. Assistant Professor of Biological Chemistry, Harvard Medical School, Boston, Mass.

Hugo Theorell, M.D., Professor. Head of the Medical Nobel Institute, Biochemical Department, Stockholm, Sweden. Nobel Prize, 1955.

Irwin B. Wilson, Ph.D. Assistant Professor of Biochemistry, College of Physicians and Surgeons, Columbia University, New York, I. Y. 



\section{CONTENTS}

Chemistry and Viral Growth

by A. D. Hershey. . . . . . . . . . . . . . . . 1

Photosynthesis

by J. A. Bassham and M. Calvin................. 29

Bacterial Fermentations

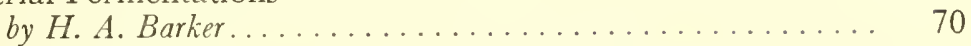

Some Aspects of Vitamin and Growth Factor Research by Esmond E. Snell. . . . . . . . . . . . . . . . . . . . 87

The Significance of Induced Enzyme Formation by S. Spiegelman and A. M. Campbell............... 115

Certain Problems in the Biochemical Study of Disease by DeWitt Stetten, Jr......................... 162

The Hormones, Their Present Significance, Their Future by Gregory Pincus............................ 176

Problems of Cellular Biochemistry by Carl F. Cori............................. 198

Enzymes as Reagents by Efraim Racker.

Attempts at the Formulation of Some Basic Biochemical Questions by Fritz Lipmann. . . . . . . . . . . . . . . . . . . . . 241

Enzyme Complexes and Complex Enzymes

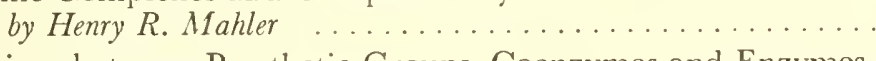

Relations between Prosthetic Groups, Coenzymes and Enzymes by Hugo Theorell........................... 275

Enzyme-Substrate Compounds and Electron Transfer by Britton Chance. . . . . . . . . . . . . . . . . . . . 308

On the Nature of Hemoprotein Reactions

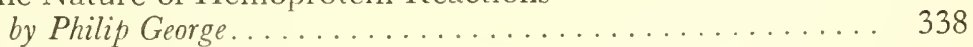

Aspects of Protein Structure by Barbara W. Low and John T. Edsall.............. 378

The Structure of Insulin by F. Sanger............................ 434

A New Concept of Ribonucleic Acids by Waldo E. Cohn. . . 
Chemical Structure as a Guide to the Study of Biochemical Syntheses by Konrad Bloch.... . . . . . . . . . . . . . . . . . . . . . 474

The Role of Nucleotides and Coenzymes in Enzymatic Processes by Frank M. Huennekens. . . . . . . . . . . . . . . . . . . .

The Biosynthesis of Porphyrins; The Succinate-Glycine Cycle by David Shemin........................ 518

Problems in the Study of Multiple Enzyme Systems by G. Robert Greenberg...................... 537

Enzyme Kinetics by Robert A. Alberty....................... . 560

The Interconversion of Sugars in Nature by Luis F. Leloir. . . . . . . . . . . . . . . . . . . . . . . . 585

A Theory of the Primary Event in Muscle Action by Manuel F. Morales and Jean Botts................. 609

Trends in the Biochemistry of Nerve Activity by David Nachmansohn and Irwin B. Wilson.............. 628

Blood: Some Functional Considerations by Douglas M. Surgenor.... . . . . . . . . . . . . . . . . . . 653

An Integrated Concept of Carcinogenesis by Harold P. Rusch............................ 675 


\title{
CHEMISTRY AND VIRAL GROWTH
}

\author{
A. D. HERSHEY, Department of Genetics, Carnegie Institution of \\ Washington, Cold Spring Harbor, New York
}

\section{Introduction}

The points of contact between biochemistry and the central mysteries of biology-meaning here the reproduction and functioning of hypothetical determinants of heredity-are necessarily few (3). In recent years the study of viruses has emerged as one of them, important if only because of its relative isolation.

Work by many investigators with many different viruses has contributed to this development, not always in tangible ways. It would be difficult, and perhaps not at present very rewarding, to trace this genealogy. Even the work with bacterial viruses has recently split into two parts calling for separate review $(5,45)$. In this discussion I shall focus attention on what happens when bacteriophage $\mathrm{T} 2$ infects the common intestinal bacterium, Escherichia coli.

\section{The Reproductive Cycle}

Morphological, biochemical, and genetic studies have revealed a developmental cycle that can be represented very simply. Figure 1 names the recognized stages and processes. The basic information from which this cycle has been deduced may be summarized as follows. 


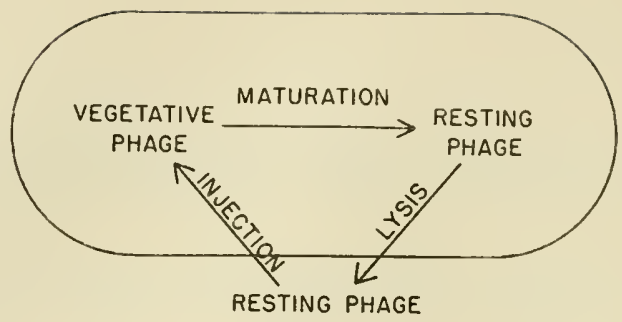

Figure 1.

\section{RESTING PHAGE}

Extracellular virus is called resting because it is stable and metabolically inert. Purified preparations of T2 consist of uniform tadpole-shaped particles with a polyhedral head measuring about 0.1 micron across, and a tail about 0.1 micron long and perhaps one-fourth as broad $(2,67)$. The surfaces of the head and tail contain distinct antigenic proteins (36). One particle contains about $2 \times 10^{-10} \gamma$ of deoxypentose nucleic acid (DNA) $(6,28)$, which can be expelled by osmotic shock leaving a proteinaceous ghost that retains the shape and some of the biological properties of the intact particle $(1,21)$. The ratio of protein to DNA in the whole particle is about one to one. The DNA is variously reported to be released in the free state (23), or combined with protein (62).

The proteins of $\mathrm{T} 2$ differ antigenically from the proteins of the host. The DNA of T2 contains 5-hydroxymethyl cytosine but no cytosine (69), in which respects it differs from all known examples of DNA from other organisms. Both facts suggest that virus and bacterium have pursued separate, though not independent, evolutionary paths for a long time. Otherwise these facts can be regarded as analytical aids pure and simple.

\section{INJECTION}

When a particle of T2 makes its specific, irreversible attachment to a bacterium (living or dead), the viral DNA passes into the cell, leaving the empty protein shell outside (27). This husk may now be seen adhering to the cell by the end of its tail 
(2), and can be stripped off in a Waring blendor without affecting the subsequent course of the infection (27). A spurious injection, simulating osmotic shock, can be observed when virus particles react with specific substances of bacterial origin (32), or even with an ion-exchange resin (52). The material released in this way consists of fibers $20 \mathrm{~A}$ or more in diameter and several microns per phage particle in aggregate length $(32,67)$. The visible fibers are destroyed by deoxyribonuclease (32). Their number has not been counted.

Several of the facts mentioned seem to show that injection occurs independently of bacterial metabolism. Benzer (4) finds, however, that an external food supply is required. The ability of the virus to inject resists multiple lethal doses of ultraviolet light, but is nearly as sensitive as the infective property to formaldehyde and ionizing radiations $(27,29)$. At present a satisfactory mechanism of injection cannot even be imagined.

Whatever the details of injection, it seems permissible to conclude that a resting particle of $\mathrm{T} 2$ consists of a protein syringe that functions chiefly to get the viral DNA into the cell, and that only the injected materials, and possibly the nonstrippable stub of the tail (amounting to 20 per cent of the total protein of the virus particle), participate directly in viral growth.

\section{VEGETATIVE PHAGE}

The events that follow injection are strictly dependent on a source of nutrients external to the cell, except that starved, infected cells tend to lose their potentiality to yield virus. Given adequate food and a favorable temperature (usually $30^{\circ}$ to $37^{\circ} \mathrm{C}$.), the normal course of events leads to cellular lysis with the liberation of 20 to 2000 new viral particles per bacterium, the yield depending chiefly on nutrient conditions and the length of time ( 20 minutes to about 2 hours) during which cellular lysis can be delayed.

Beginning with the injection, marked changes occur in the biosynthetic pattern of the cell. These changes will be described presently, but do not yet help to define vegetative phage, which 
is a genetic concept. This concept emerges from the following facts.

Doermann (10) showed that infected cells broken open during the first 10 minutes following infection do not yield any infective virus. This result has since been accounted for by the act of injection: what goes into the cell is not virus. Doermann's experiments went much further than this, however. He proved that genetic determinants, that is, enumerable structures possessing viral specificity, multiplied during the period in which the cell remained free of infective virus. This proof is summarized in a recent review (11). The term vegetative phage refers to these noninfective structures that multiply and produce new genetic combinations, and must be thought of as the connecting links between parent and offspring virus. The central problem of phage growth, therefore, is the problem of structure and function of vegetative phage.

\section{MATURATION}

The process by which vegetative phage is converted into resting phage is called maturation. From the end result we know that it involves the reformation of envelope and tail, but we do not know where it starts; to answer this it is necessary to know what vegetative phage is like. It seems possible, as will be discussed below, that vegetative phage may consist of replicas of the injected DNA-containing fibrils. If so, maturation calls for the complete synthesis of envelopes.

Probable intermediate stages in maturation have been identified. These consist of empty, tailless envelopes (40), accompanied by detached tails, or of tailed, but probably DNA-less envelopes (46). They are found only 2 or 3 minutes before the phage particles of which they are the presumed precursors, and so are probably not themselves vegetative phage particles, which must be formed appreciably earlier. The absence of DNA is puzzling, and it seems preferable to assume that the observed structures lose their DNA during isolation, rather than to imagine that they receive their charge of DNA as a final act 
of maturation. The fact that nearly all the DNA in infected cells, excepting that contained in resting phage particles, is sensitive to deoxyribonuclease may be significant in this connection. It is perhaps not quite established that the DNA-less structures are intermediates at all. In view of these uncertainties, little more can be said about them at present.

The time course of maturation is linear, beginning about 10 minutes after infection and continuing until the cells burst. During this time vegetative phage probably continues to multiply, as indicated by progressive increase in proportion of recombinants (41), and continued synthesis of viral protein and DNA $(46,58)$.

Since maturation is irreversible (22), there is no need to distinguish between mature intracellular virus and resting extracellular virus. The individual particles probably play a passive role, if any, in the release of virus from the cell.

\section{The Priming Substance}

We have seen that the interesting period in the life of a phage particle begins when its charge of DNA enters a sensitive bacterial cell to form vegetative phage. It is a question of considerable ideological importance (64) whether this charge is exclusively DNA or contains protein as well. Agreement about it has not yet been reached at the level of experimental fact (62) and, what is worse, it is doubtful whether direct analytical methods can answer it satisfactorily (23). I wish to make the point that the main biological implications of the injection phenomenon are largely independent of this specific question. Let us assume almost the worst, namely, that the charge that enters the cell consists of nucleoprotein fibers and that these, furthermore, are functionless until they have made specific attachments to complicated structures in the bacterium. What remains of our reproductive cycle that is biologically unique?

In my opinion three very important features. First, these hypothetical fibers would have to carry all the intelligence that 
T2 has acquired during its long evolutionary history (excepting what it has delegated to the bacterium). In a sense this is the kind of demonstration that has been the goal of experimental genetics since its beginning, and one that remains a will-o'-thewisp $(16,55)$ if it is not provided by T2. Second, these fibers must be linear molecules, or very nearly, in which respect they meet the theoretical requirements of a genetic material (68), as the visible chromosomes of cells do not. Third, they can be observed to multiply in a cell that is phylogenetically remote from their proper origin, as the chromosomes of cells cannot. The experimental meaning of this feature is brought out by the fact that both nucleic acids and proteins of virus and host are distinguishable by qualitative analytical methods. In this last respect, the uniqueness is not a matter of opinion.

Needless to say, the blendor experiment alone does not quite establish these claims (23). A genetic function of the nonstrippable tip of the tail has first to be excluded. In itself this may be a trivial point; it is not likely that even the staunchest biological holist would insist that the fertilized egg contain a representative molecule of every specific substance the organism is capable of producing. The question becomes important in connection with the thesis that viral lineage (defined somewhat abstractly in terms of viral properties that are invariant with respect to variations in the host) is determined exclusively by a structurally homogeneous class of linearly differentiated molecules. It is interesting also because it can be scrutinized by both genetic and chemical methods. Both tend to exclude the tail-tip as the primordium from which new membrane protein comes. I shall give an example of the genetic evidence first.

The genetic control of the specificity of attachment of virus to bacterium, which means control of the chemical structure of the tail protein, has been studied in a particularly instructive way by several investigators, most thoroughly by Streisinger (59). $\mathrm{T} 2$ and $\mathrm{T} 4$ are two closely related viruses differing, among other ways, in their tail proteins. Genetic experiments show that this difference is determined by a single gene. We may ask 
whether the determinant of this character is the organ of attachment itself or a different organ, that is, whether the gene and the agent of its expression are the same or different substances. The following circumstances show that they are different.

A single bacterium infected with both $\mathrm{T} 2$ and $\mathrm{T} 4$ yields a mixed viral progeny among which the individual particles can be classified in two ways. First, one can determine whether their attachment to bacteria shows the specificity of $\mathrm{T} 2$ or the specificity of T4. This test shows that some particles resemble T2 and some T4. Second, one can classify the particles in terms of the progenies they yield when they multiply individually. By this test, too, some prove to resemble T2 and some T4. The remarkable thing about the two tests is that they do not agree: many particles adsorb like T2 and yield progenies like T4. The best interpretation of this result is that, owing to the special conditions prevailing in the mixedly infected bacterium, some of the particles receive tail protein manufactured for a T2 particle, together with a tail-protein-determining gene derived from the T4 lineage, and vice versa. If so the two are different structures. The genetic results just described show that tail specificity, as such, is not conserved during reproduction. Analogous radiochemical results show that tail substance is not conserved during reproduction. Evidently tail protein is not the seed from which new tail protein comes. These two results seem to show that the injected materials direct the complete resynthesis of tail protein.

I have cited one item of a considerable body of genetic information (24) leading to the conclusion that the genetic material of $\mathrm{T} 2$ consists of linear structures. All the chemical evidence implicates DNA as the chief constituent of these structures. If one wishes to assume that DNA is the prime hereditary material, justification can be borrowed from the beautiful work of the past decade with bacterial transforming systems (31), or it can be sought directly, as will be discussed below. 


\section{Metabolic Effects of Infection}

Soon after infection, bacteria stop making respiratory and other enzymes $(9,51)$ and fail to respond to inductors of adaptive enzymes (49), as if the metabolic equipment of the cell were frozen at the time of infection. Protein synthesis as a whole continues unabated, however. By contrast, the synthesis of bacterial RNA and DNA stops abruptly, being replaced by the synthesis of viral DNA (6). The latter point has been confirmed by transferring infected cells into $\mathrm{C}^{14}$-glucose, when it is found that no $\mathrm{C}^{14}$ enters DNA-cytosine (29). The bacterial DNA that was formed before infection disappears afterward, in contrast to bacterial RNA and protein which remain comparatively inert. Cytological changes suggest that the bacterial nuclei are quickly dispersed (44).

These facts have led to the following generalizations. Infection causes a shift from the synthesis of characteristic bacterial constituents to the synthesis of characteristic viral constituents, without major substitutions in enzymatic equipment (9). (2) Viral genes replace bacterial genes: infection is parasitism at the genetic level (44). For the present these two ideas are no more than colorful, rather clouded, statements of fact but, as Luria (44) has pointed out, it may be useful to look for connections between them.

\section{Structure of Vegetative Phage}

I have summarized reasons for believing that the substance derived from a single viral particle and directly initiating viral growth consists of one or more linear molecules. If this is so we want to know their number and function, and whether they multiply before or after forming more complex structures. Structure can be got at in several ways besides the attempt to isolate vegetative phage, and some of these are surveyed below. About function only one question can be asked in advance of information about structure. It is concerned with 
the conservation of materials during multiplication, which in turn leads back to questions about structure and function.

THE NUMBER OF LINEAR MOLECULES

The number of structures injected into the bacterium is not known beyond the fact that one phage particle contains about $2 \times 10^{-10} \gamma$ of DNA, which corresponds to about 20 molecules in the isolated form (19). Presumably the number could be determined either by physical or genetic methods. The following genetic experiment illustrates a principle of wide application to questions of this kind.

T2 contains at least three regions of genetic material that assort independently during genetic recombination (24). If a bacterial cell is infected with two phage particles genetically marked in one of these regions, and if the infections are spaced by an interval of 2 minutes or more, the second particle will generally not contribute its genetic marker to the progeny (15). At least some of the DNA of the second particle enters the cell, because about half of the DNA of the superinfecting particles is quickly split into acid-soluble materials (38). The mechanism of exclusion is otherwise unknown, but one can ask whether the unit of exclusion is the genetically intact phage particle, or something smaller. To answer this, one infects bacteria in succession with two phage particles marked in two of the independently assorting regions of genetic material, and examines yields from single bacteria to see whether one of the two markers can contribute while the other is excluded. A single test of this kind (26) showed that the two markers were always excluded together. For what it is worth, this result suggests that the genetic unit called vegetative phage (or its precursor) is also a structural unit.

\section{PHYSICAL STRUGTURE}

The composition of vegetative phage is difficult to investigate, first because there is no unambiguous way to identify interesting structures if they could be isolated; second because 
any method used to break open the infected cell might alter important structural relations.

Direct microscopy of infected cells (50) has not revealed any characteristic intracellular structures that might be vegetative phage particles. Similarly, examination of extracts of infected bacteria (40) brought to light only normal cell components, resting phage particles, and empty, tailless membranes that have been interpreted as early products of the maturation cycle. Watanabe, Stent, and Schachman (63) labeled viral precursor DNA by infecting cells with $\mathrm{P}^{32}$-containing phage, and then subjected extracts prepared at various times to centrifugal analysis. Citrate was used to inhibit bacterial deoxyribonculease, but otherwise the conditions of lysis may have been favorable for enzymatic degradation. They found that most of the viral precursor DNA in the lysates sedimented like free nucleate.

None of these results either contradicts or justifies the assumption that vegetative T2 consists of free molecules of DNA.

\section{VIRAL PRECURSOR DNA}

Early tracer experiments showed that the phosphorus used to make viral progeny comes from three distinct sources: materials assimilated by the bacterium before infection (8), materials assimilated after infection (8), and parental materials (53). Weed and Cohen (66), Stent and Maalфe (58), and French et al. (18) found that atoms derived from any of these three sources entered unequally into the viral progeny formed at successive times, suggesting the existence of a precursor pool containing a limited amount of phosphorus. These facts prompted a search for viral precursor DNA, which was greatly facilitated by the interim discovery that the DNA of T2 contains the distinctive constituent 5-hydroxymethyl cytosine (69). This search brought to light the following information.

Cytosineless DNA, that is, DNA having the composition of the DNA of viral particles, begins to form in infected bacteria promptly after infection, and already measures roughly 50 phage 
equivalents per bacterium 10 minutes after infection, at which time infective virus particles begin to form. Also at later times, infected cultures contain more cytosineless DNA than can be accounted for by infective particles, and the amount of surplus DNA remains approximately constant at 50 to 100 phage equivalents per bacterium (28). Tracer experiments (22) show that this surplus DNA is the principal immediate precursor of the particles. First, because phosphorus incorporated early into the surplus DNA disappears from it later on, as if it were being transferred to infective viral particles by an efficient, irreversible process. Second, because the kinetics of the transfer, whatever the source of labeled phosphorus, calls for a precursor pool containing 50 to 100 phage equivalents of phosphorus per bacterium, in agreement with direct measurements of the amount of surplus DNA. This agreement shows that the precursor pool is sampled more or less at random when a phage particle is formed. In this sense it must contain chiefly a single precursor, as opposed to a sequential series of precursors. This important distinction, based on analytical methods admittedly not very exact, is recalled below by the term unitary pool.

\section{IDENTITY OF PREGURSOR DNA AND VEGETATIVE PHAGE}

The idea of vegetative phage is based on genetic experiments pointing to the existence of a mating pool or, more generally, a genetic precursor pool. The chemical experiments reveal a pool of viral precursor DNA. These two pools can be compared with respect to unity, size, and time of formation, and should resemble each other in all these respects if vegetative phage is or contains the viral precursor DNA.

Strictly speaking, the pool of precursor DNA cannot be a unitary pool, because at intermediate stages during maturation, any DNA contained in the immature particles would belong neither functionally to a randomly sampled pool, nor analytically to mature phage particles. The effect of this complication would be to cause the pool of precursor phosphorus, assayed 
kinetically, to be smaller than the pool of precursor DNA measured directly. The fact that no such discrepancy is found means that the time precursor DNA spends in the maturation cycle is short compared to the washout time of the pool (about 8 minutes). However, since the methods are far from precise, and viral growth in different cells is not synchronized, only extreme alternatives can be excluded; for example, linear multiplication such that each offspring particle enters its maturation cycle directly at birth. To this extent the chemical findings suggest a geometric mechanism of synthesis of DNA. More to the point, however, is the likelihood that the amount of precursor DNA determined analytically should exceed appreciably the amount contained in the genetically defined vegetative structures.

The chemical facts show that the infected cell contains 50 to 100 phage equivalents of viral precursor DNA, much of which belongs to a unitary pool in the sense that it is sampled at random for the maturation of infective particles. This pool is largely filled 10 minutes after infection, at the time maturation begins.

What are the characteristics of the genetic pool? Luria's (43) analysis of viral mutation showed that genetic determinants increase geometrically; this is probably equivalent to saying that they share a pool from which samples are drawn at random during maturation. This was shown more directly by Visconti and Garen (61), who found that genetic markers contributed by superinfecting phage, entering the bacterium 7 or 8 minutes after primary infection, were sampled from the same pool with markers derived from the primary infection. Moreover, the kinetics of genetic recombination suggest that the mating pool has already reached its maximal size, estimated at 30 vegetative particles per bacterium, at the time maturation begins (41). Thus the genetic pool and the viral precursor DNA pool are similar with respect to unity, size, and time of formation. It is a reasonable though not quite necessary inference that the precursor DNA is contained in the genetic pool. 
VIRAL PRECURSOR PROTEIN

Cohen (7) found that there was no interruption of protein synthesis at the time of infection, and that protein continued to accumulate until viral growth ceased. His findings have been confirmed by measuring the assimilation of $\mathrm{S}^{35}$ into acid-insoluble materials after infection (29). These measurements show that bacteria synthesize protein at nearly identical rates before, during, and for some time after infection. When does the synthesis of viral protein start?

This question has been attacked in two ways. Luria (44) and his collaborators studied the formation of viral antigens in infected cells. Their results led to the conclusion that viral antigens are formed relatively late during the process of viral growth, as though they were products of the maturation cycle. If this is so, the protein synthesized immediately after infection is not virus specific.

Hershey et al. (29) analyzed viral precursor protein by tracer methods. They fed $S^{35}$ to infected bacteria for intervals of 5 minutes, and measured the amount of $\mathrm{S}^{35}$ subsequently incorporated into virus and total intrabacterial protein, respectively. The amount incorporated into protein of all kinds was virtually independent of the period of assimilation tested, but the proportion of this incorporated into virus varied greatly. For the period 0 to 5 minutes after infection, only 13 per cent went into virus; for 5 to 10 minutes, 25 per cent; for later times, 50 to 60 per cent. This means that infected bacteria synthesize two classes of protein, one viral precursor and one not, and that the maximal rate of precursor protein synthesis is not reached until shortly before the beginning of maturation (15 minutes after infection under the conditions of these experiments). Kinetic analysis of sulfur assimilation during the period in which virus was accumulating at a linear rate showed that the interval between assimilation of sulfur atoms and their incorporation into unspecified protein averaged about 2 minutes. The interval between assimilation and incorporation into mature virus averaged about 10 minutes. Hence labeled viral precursor protein 
persists in the infected cell for an average of 8 minutes. Labeled viral precursor DNA, under the same conditions, persists about 14 minutes. This difference seems to show that the cell contains more precursor DNA than precursor protein, and therefore that the DNA destined for a given phage particle is formed somewhat earlier than the protein destined for that particle.

Both the serological and tracer methods are consistent with the idea that protein synthesis is a terminal phase in the formation of the phage particle, but neither has yielded satisfactory proof of this idea. A more promising approach might be to look for conditions under which maturation is inhibited while DNA synthesis proceeds, or conditions permitting DNA synthesis but not protein synthesis. Cultures containing proflavine furnish an example of the first class. This substance appears to block only a late step in maturation and permits both protein and DNA synthesis (44). One inhibitor of protein synthesis has been tested. Cohen (7) finds that 5-methyl tryptophan blocks both protein and DNA synthesis in infected bacteria. This suggests either that vegetative phage contains protein or that DNA synthesis is dependent on the synthesis of protein for other reasons. The attempt to confirm or refute this conclusion by studying the effects of specific metabolic blockade seems at present to offer the most general method of defining the composition of vegetative phage.

\section{RNA EGONOMY IN INFECTED BAGTERIA}

Cohen (8) showed that the amount of RNA in infected bacteria remains constant from the moment of infection, and that not more than 2 to 9 per cent of its phosphorus is replaced by newly assimilated phosphorus during 60 minutes of viral growth. Manson (48) confirmed the latter result by a more rigorous method; in his experiments replacement up to 5 per cent of the total RNA could not be excluded because of the presence of uninfected bacteria in the cultures. In order to see what these results mean it is necessary to make some quantitative comparisons. 
The amount of DNA in an uninfected bacterium growing in glucose-ammonia medium averages about 40 phage equivalents $\left(8 \times 10^{-9} \gamma\right)$. After infection, viral precursor DNA is synthesized at the rate of about three phage equivalents per bacterium per minute and accumulates to roughly 40 equivalents per bacterium. The amount of bacterial phosphorus (assimilated before infection) that is used to make viral DNA measures about 40 phage equivalents. The total amount of preassimilated RNA phosphorus per bacterium is nearly 400 phage equivalents.

If 5 per cent of the total RNA were undergoing moderately rapid turnover, RNA synthesis could easily keep pace with DNA synthesis, without having been detected in the experiments cited above. It has been suggested that this happens (22).

If 10 per cent of the total preassimilated RNA were converted into viral DNA, this source would account for an appreciable part of the observed use of preassimilated materials by the virus, and would not readily be detected as a significant decrease in RNA content of the culture. As a matter of fact, one does observe a significant decrease in acid-insoluble RNA when measured in terms of preassimilated isotope, and indirect evidence suggests that some of the purine and pyrimidine carbon enters DNA (29).

These remarks are intended to show that an active role of RNA in the growth of T2 has not been entirely excluded. Since the T2-infected bacterium is the only known biological system in which synthesis of RNA during growth may not occur, more penetrating experiments designed to test this possibility are called for.

\section{MATERIALS CONSERVED DURING REPRODUCTION}

A special method of defining the composition of vegetative phage-perhaps a minimum composition, perhaps an irrelevant one-consists in tracing those structures that pass intact from parental to offspring phage. It is now generally agreed that only the constituents of the parental DNA are transferred (17. 
27,35). It is also agreed that the efficiency of transfer never exceeds 40 or 50 per cent $(18,22,65)$. The mechanism of transfer, and therefore its significance, remains undecided. The question of mechanism is conveniently discussed in terms of alternative explanations for the low efficiency of transfer.

1. The low efficiency of transfer may result from losses occurring in some but not all the infected bacteria, either because preparations of phage always contain inviable particles or because for other reasons some of the bacteria yield few or no viral progeny. Although losses from both causes undoubtedly occur and cannot at present be measured satisfactorily, most observers have considered this explanation inadequate. Losses of this type, of course, have nothing to do with the mechanism of transfer.

2. The loss may be an essential feature of viral growth, but occur before multiplication starts. This would imply that about half the parental DNA goes into vegetative phage, and the other half serves a different, presumably nongenetic, function. This possibility has been tested in two ways. Maalфe and Watson (47) measured the efficiency of transfer of $\mathrm{P}^{32}$ during two successive cycles of growth, and found only the usual transfer of 40 per cent during the second cycle. This showed that the transferred atoms from the parental DNA are incorporated into both transferred and nontransferred parts of the DNA of the progeny. Hershey et al. (29) found that the four bases of the DNA of $\mathrm{C}^{14}$-labeled $\mathrm{T} 2$ are transferred with equal efficiency. These two results show that the loss is random, that is, if the parental DNA splits into two parts that perform different functions, and if only one part is conserved among the progeny, the choice is probably made between identical parts. In this form the idea of functional differentiation within the viral DNA is unattractive but cannot be considered disproved.

3. All the parental DNA may enter vegetative phage where it is subject to a succession of small losses during replication. The losses would have to amount to 5 or 10 per cent per generation to account for an observed transfer of 50 per cent. 
Losses of this magnitude might well be expected on general grounds. An attempt was made to test this idea by feeding $\mathrm{P}^{32}$ to infected cells during the first 4 minutes after infection (22). It was found that the labeled DNA content of the infected cells rose to a maximum during the first 25 minutes, and that 70 to 90 per cent of this was eventually incorporated into virus. This high efficiency fails to exclude the hypothesis under consideration because of the slow entry of $\mathrm{P}^{32}$ into viral precursor DNA. It does show, however, that maturation is not the step that limits the efficiency of transfer from parents to offspring.

4. Finally, perhaps only a small part or none of the parental DNA goes into vegetative phage directly. Half of it, after playing some unspecified role, could then be broken down and fed into the general pool of DNA precursors. This idea was at first supported by Kozloff's finding that parental DNA phosphorus is transferred more efficiently than parental DNA nitrogen $(34,35)$. However, subsequent and technically superior experiments showed equal transfer of phosphorus and purine carbon (65), and equal transfer of the four bases (29). Moreover, the intermediates must be nucleotides or larger fragments, because free bases or nucleosides, which compete effectively with $\mathrm{CO}_{2}$ (33) or glucose (20) as a source of viral DNA carbon, fail to compete during the transfer from $\mathrm{C}^{14}$-labeled virus (29). In view of the seeming contradictions, Kozloff's result ought to be reinvestigated by the isolation of doubly labeled nucleotides. In the meantime, the bulk of evidence is opposed to, but does not disprove, the idea of indirect use of parental materials.

At the moment, enumeration of these alternatives (the list is not exhaustive) is of value chiefly to show that the relatively low efficiency of transfer does not permit any conclusions about the mechanism of transfer. In addition, it may be pointed out that the transfer of purine and pyrimidine carbon (except cytosine) from bacterial DNA to viral DNA is nearly or quite perfectly efficient (29). Since this may surely be taken as an example of "indirect" transfer, the assumption that the transfer 
from parents to progeny involves breakdown and resynthesis of DNA leaves the low efficiency of the process unexplained.

All the evidence short of isolation and identification leads to the conclusion that vegetative phage contains large amounts of DNA. The possibility that DNA is its principal functional component is suggested by the analysis of resting phage particles, by examination of the injection mechanism, and by the composition of the material conserved during viral growth. A more general confirmation of this idea has not yet proved possible but promises to emerge from considerations to which we now turn.

\section{CORRELATION OF MATERIAL AND GENETIC CONTINUITY}

A direct correlation between the transfer of genetic markers and of labeled DNA from parental to offspring virus would not only answer the question about biochemical pathways but also rule out the unpleasant possibility that most of the viral DNA resembles more closely in function the white of an egg than it does the nuclear apparatus of an ovum. Such a demonstration would, in fact, reduce genetic questions about T2 to purely biochemical questions about structure and function of DNA. Kozloff (34) recognized this very early but was unable to find any correlation between genetic and biochemical results.

Kozloff's basic experiment is the following. Bacteria were simultaneously infected with isotopically labeled phage, previously exposed to ultraviolet light or x-rays, and with unlabeled, unirradiated phage. Previous experiments of this type with genetically marked phage had shown that the genetic contribution from the irradiated phage to the mixed yield of virus could be suppressed by a sufficient dose of radiation. Kozloff reasoned that the contribution of isotope ought also to be suppressed if the material transfer had any direct connection with genetic function. Since he did not find any appreciable effect of irradiation on isotope transfer, Kozloff $(34,35)$ concluded that the material transfer from parents to progeny probably had nothing to do with genetic function, and that it might very well involve breakdown to nonspecific fragments en route. Watson 
and Maalфe (65) and French et al. (18) described similar results without, however, subscribing to Kozloff's conclusion.

Subsequent experiments (29) have shown that the earlier results can be questioned on strictly technical grounds. For this purpose it is necessary to distinguish between the effects of ultraviolet light and ionizing radiations.

Phage inactivated by ionizing radiations are found to be largely incapable of injecting their DNA into bacterial cells, although their ability to attach to the cells is not affected. Following mixed infection with irradiated and unirradiated virus, the viral yield after cellular lysis is heavily contaminated with the original particles of irradiated virus. For this reason it is not possible to measure isotopic contribution from the irradiated virus to the progeny of the mixed infection (29).

The early experiments with phage inactivated by ultraviolet light are questionable for two reasons. First, the genetic potency of ultraviolet-killed phage in mixed infections is only now being investigated in a quantitative fashion (12) and proves to be much greater than previously supposed. Second, the isotopic contribution is not independent of the radiation dosage, as Kozloff believed, but falls slowly and continuously with increasing doses (29). This effect cannot be attributed solely to loss of the ability to inject; the latter property is extremely resistant to ultraviolet light. The careful experiments that will be required to assess the significance of the correlation between the behavior of genetic and isotopic tracers remain to be done, but it is clear that a correlation exists.

A very clear qualitative correlation can be demonstrated for the following special situation. As previously mentioned, phage particles attaching to bacteria that have already been infected 2 minutes or so earlier fail to contribute genetic markers to the progeny, and half the DNA of the superinfecting particles is quickly split into acid-soluble material. At first it was thought that the chemical breakdown might be the cause of the genetic exclusion. However, French et al. (18) found that no chemical breakdown occurs when the bacterial deoxyribonuclease is 
inhibited by streptomycin, and that even under these conditions there is no transfer of isotope from superinfecting phage to the offspring of the primary infection.

We have recently studied superinfection a little further, inhibiting bacterial deoxyribonuclease when desired by reducing the concentration of magnesium in the cultures to $10^{-5} M$ (29). At this low magnesium concentration, injection and growth of the primary infecting phage, and the transfer of its phosphorus to viral offspring, are normal. Independently of magnesium concentration, superinfecting phage injects only half its DNA (or half the particles inject), and both genetic and isotopic markers derived from it are excluded from the viral yield. The half that is injected may or may not be broken down, depending on the magnesium concentration. When the bacterial deoxyribonuclease is inhibited, superinfecting phage is excluded from genetic participation in growth and half its DNA enters the cells and remains intact without metabolic participation in viral growth. Unfortunately, the significance of this parallel is doubtful because the mechanism of exclusion is unknown.

\section{RADIOGENETIC STRUCTURE ANALYSIS}

Viral radiogenetics began some years ago when Luria (42) proposed that phage particles inactivated by ultraviolet light could be revived by a process of genetic substitution. His idea was appealing, first, because it promised a test of the much older idea that radiations produce localized genetic damages to biological materials and, second, because it led to rather special assumptions about the mechanism of viral growth. His idea subsequently lost favor without really being disproved, partly because Dulbecco (13) showed that the inactive particles could also be revived by something resembling repair, partly because the quantitative predictions of the substitution hypothesis failed at high radiation doses (14), partly because the early radiogenetic experiments suffered from what is best described as lack of faith.

Stent (57) and Doermann (12) have recently revived the 
idea of reactivation by genetic substitution. Their experiments are of the following type. Bacteria are infected with two kinds of phage differing by several genetic markers, one kind carrying radiation damage and one not. In Stent's experiments, the damages result from decay of $\mathrm{P}^{32}$ incorporated into the virus during prior growth. In Doermann's experiments they are produced by irradiating phage with ultraviolet light. In both instances the damage can be ascribed plausibly to localized effects on the viral DNA, and both types of damage show the same behavior in radiogenetic experiments.

The following description refers specifically to Doermann's experiments. He uses T4 particles irradiated with ultraviolet light until they are unable individually to infect a bacterium in the usual way. Such particles may be called dead, and the radiochemical process producing them may be called primary killing. The dead particles can be revived in several ways. In what follows we are concerned with only one of them, called cross-reactivation. It shows the following characteristics.

1. Yields from individual bacteria infected with one or more live plus one dead phage particle frequently show some but not others of the genetic markers introduced with the dead particle. The response of the individual markers to varying radiation dosage corresponds to a target volume that is 4 per cent of the target volume for primary killing.

2. Markers known from genetic tests to be unlinked are inactivated independently of each other.

3. For moderate radiation dosages, markers derived from the irradiated phage particles are either absent entirely, or appear in numerous copies in yields from individual bacteria.

4. Inactivation of genetically linked markers occurs in a correlated manner.

Doermann interprets these facts in the following way. Most of the radiation damage results from localized photochemical reactions in genetic material; otherwise correlated inactivation of unlinked markers would be observed. Undamaged pieces of genetic material are rescued by recombina- 
tion away from damaged pieces, since both the rescue and authentic recombination are subject to the same linkage rules. The photosensitive target measured in these experiments is the average size of pieces that can be rescued by recombination. It measures one twenty-fifth of the total genetic material and is large compared to the size of the individual markers, since linked markers tend to be rescued together.

Stent's conclusions are consistent with these and lead further in one direction. Since the primary lethal damage produced by assimilated $\mathrm{P}^{32}$ results from one out of twelve atomic disintegrations (30), at least this fraction of the DNA must be associated with genetic material. The fraction must, in fact, be larger, because the radiochemical efficiency is dependent on temperature (56).

These methods are evidently capable of yielding rather precise descriptions of genetic material, possibly in physical terms, certainly in radiochemical terms that can be compared with genetic descriptions. Moreover, like the earlier results of Luria, they call for interesting assumptions about the mechanism of viral growth. The problem is to explain how rescue by recombination is achieved without greatly setting back the multiplication of the rescued piece relative to comparable genetic material that does not require rescue. Such a setback does, in fact, appear when the rescue is made more difficult by high radiation dosages. In this situation we come face to face with the problem of structure and function of vegetative phage. Since both the facts and ideas bearing on the interpretation of the radiogenetic experiments are changing rapidly, it would be rash to predict how they are to be reconciled with each other.

\section{Relation between Virus and Cell Nucleus}

There are a number of indications from other phagebacterium systems that some bacteriophages engage directly with components of the bacterial nucleus $(5,29,37,45,70)$. These are only beginning to be worked out and cannot be dis- 
cussed here. For T2 none of the biological tests of this idea are applicable, but chemical tests are.

As already mentioned, the first cytochemical effect of the infection is a rapid dispersal of the bacterial chromatin (44). By chemical analysis, one observes a more or less complete disappearance of cytosine-containing DNA, and the atoms of this DNA are rapidly incorporated into viral DNA. The conversion appears to be highly efficient. The direct analytical results indicate a phosphorus transfer of 50 per cent $(22,54)$, subject to the assumption that all the transferred phosphorus comes from bacterial DNA. This becomes nearly 100 per cent when corrected for the fact that only half the phosphorus of the bacterial DNA fraction, but nearly all the phosphorus in isolated phage, is really DNA phosphorus (28). Similar conclusions follow when the transfer of $\mathrm{C}^{14}$-labeled bacterial precursors is measured. All the DNA purine and pyrimidine carbon (except cytosine), and all or most of the carbon of specifically labeled bacterial DNA-thymine, appears either in viral DNA or in residual bacterial DNA in infected bacteria (29). The conversion is more or less complete, and occupies the first 30 minutes of viral growth.

One would like to interpret these facts as an illustration of Luria's idea of parasitism at the genetic level. Before doing so, two questions have to be answered. First, does the virus cause the breakdown of bacterial DNA, or merely block its resynthesis? Second, is bacterial DNA the only characteristic bacterial substance that is used to make virus?

The first question has been answered by showing that bacterial DNA, and indeed characteristic bacterial substances in general, do not turn over at rates comparable to rates of synthesis in growing bacteria (25). Moreover, bacterial RNA does not turn over appreciably in infected bacteria $(8,22,48)$. Statements made about turnover are, of course, intelligible only in terms of actual experiments. Two examples will suffice here. The presence of thymidine in unlabeled culture medium during viral growth will specifically suppress the conversion of labeled 
bacterial DNA-thymine into viral DNA-thymine (29). The presence of thymidine in unlabeled culture medium during bacterial growth will not suppress the conservation of labeled bacterial DNA-thymine (25). In fact, the amounts of radiocarbon in all the DNA-bases remain constant during 6 hours of growth at one generation per hour in media containing any of a number of competitive substrates. One concludes that infection stimulates a decomposition of bacterial DNA that is different in rate or kind from any that may occur during bacterial growth. It may be added that the decomposition in infected bacteria is not prevented at concentrations of magnesium too low to permit the action of intracellular deoxyribonuclease as tested in another way (29), so that the effect may not be due merely to the activation of this enzyme (35).

Bacterial DNA is not the only source of preassimilated material available for the synthesis of viral substance. There is another source of purines and pyrimidines, probably RNA (29). There is also a small amount of preassimilated sulfur, which may or may not be derived from protein, that can be used to make viral protein (29). However, these materials are used at rates altogether negligible compared to the rate of conversion of bacterial to viral DNA.

It is reasonable to conclude that the preferential breakdown and re-use of bacterial DNA indicates some close relationship, structural or spatial or both, between the two kinds of DNA in infected bacteria.

\section{T2 and Other Organisms}

At the present time, if one wished to invent a genetic material, one would almost certainly choose a DNA constructed along the lines proposed by Watson and Crick (64). A few organisms have actually chosen DNA, the evidence being very good for several bacteria (31), and what I have related above for $\mathrm{T} 2$.

These facts immediately raise two questions. The first is 
whether all organisms have chosen DNA for a genetic material, which might imply that the choice had been made only once. At the present time this seems unlikely, because a number of plant viruses probably lack DNA. Evidently one of the tasks of the plant virologists is to make very sure of this.

The second question is the following. In an organism that does make use of DNA, is DNA the sole agent of genetic continuity, or is genetic specificity passed from substance to substance like the token in a relay race? The answer to this question can only be guessed, but one can hope to get it from T2, as I have tried to show.

More generally, one wonders what connection there can be between the linear molecules that pass from pneumococcus to pneumococcus, or from T2 to its host, and the relatively enormous chromosomes of most cells. A similar question puzzled geneticists, of course, long before anything was known about inheritance in bacteria or viruses (68). I think it is doubtful whether T2 can throw any light on this question except, perhaps, by renewing interest in it.

More specifically, what connection can there be between genetic recombination in $\mathrm{T} 2$ and reciprocal crossing-over between chromosomes? Here the answer may be none (60). Not only because the discrepancy in size forbids analogy; also because recombination in $\mathrm{T} 2$ does not seem to be reciprocal (39).

One suspects that in $\mathrm{T} 2$ the mechanical and biochemical bases of inheritance are reduced to their simplest terms; indeed, this is what one hopes. If this proves to be true, it will be unfair to complain that information about this organism cannot be applied directly to more complicated ones.

\section{Conclusion}

In studying viral growth, one necessarily studies many biochemical problems simultaneously. The main ones involve interrelationships among deoxyribonucleic acids, ribonucleic 
acids, and proteins, during the synthesis of at least two of these classes of substance. But to state them in this way is to ignore the unique problems presented by T2. These depend on the fact that the material thread connecting parental and offspring virus seems to be an analytically recognizable deoxyribonucleic acid and nothing else. The real biochemical problems for the present are the relation of this substance to viral inheritance, on the one hand, and the degree of autonomy of nucleic acid replication, on the other. No outstanding solution to these problems has come from the work with T2. Yet many experiments that might have yielded very depressing results have failed to do so. On the contrary, their net effect is to increase the hope one attaches to the many penetrating experiments that remain to be done.

\section{References}

1. Anderson, T. F., Botan. Rev., 15, 464 (1949).

2. Anderson, T. F., Cold Spring Harbor Symposia Quant. Biol., 18, 197 (1953).

3. Beadle, G. W., in D. E. Green, ed., Currents in Biochemical Research. Interscience, New York-London, 1946.

4. Benzer, S., personal communication.

5. Bertani, G., Cold Spring Harbor Symposia Quant. Biol., 18, 65 (1953).

6. Cohen, S. S., Cold Spring Harbor Symposia Quant. Biol., 12, 35 (1947).

7. Cohen, S. S., J. Biol. Chem., 174, 281 (1948).

8. Cohen, S. S., J. Biol. Chem., 174, 295 (1948).

9. Cohen, S. S., Bacteriol. Revs., 13, 1 (1949).

10. Doermann, A. H., Carnegie Inst. Wash. Yr. Bk., 47, 176 (1948).

11. Doermann, A. H., Cold Spring Harbor Symposia Quant. Biol., 18, 3 (1953).

12. Doermann, A. H., Symposium on Mechanisms of Genetic Recombination, Oak Ridge Natl. Lab., April, 1954, J. Cellular Comp. Physiol, 45, Suppl. 2, 51 (1955).

13. Dulbecco, R., J. Bacteriol., 59, 329 (1950).

14. Dulbecco, R., J. Bacteriol., 63, 199 (1952).

15. Dulbecco, R., J. Bacteriol., 63, 209 (1952).

16. Ephrussi, B., Nucleo-cytoplasmic Relations in Microorganisms. Clarendon Press, Oxford, 1953.

17. French, R. C., J. Bacteriol., 67, 45 (1954).

18. French, R. C., A. F. Graham, S. M. Lesley, and C. E. van Rooyen, J. Bacteriol., 64, 597 (1952). 
19. Garen, A., and E. Reichmann, personal communication.

20. Graham, A. F., and L. Siminovitch, Can. J. Microbiol., in press.

21. Herriott, R. M., J. Bacteriol., 61, 752 (1951).

22. Hershey, A. D., J. Gen. Physiol., 37, 1 (1953).

23. Hershey, A. D., Cold Spring Harbor Symposia Quant. Biol., 18, 135 (1953).

24. Hershey, A. D., Advances in Genet., 5, 89 (1953).

25. Hershey, A. D., J. Gen. Physiol., 38, 145 (1954).

26. Hershey, A. D., unpublished.

27. Hershey, A. D., and M. Chase, J. Gen. Physiol., 36, 39 (1952).

28. Hershey, A. D., J. Dixon, and M. Chase, J. Gen. Physiol., 36, 777 (1953).

29. Hershey, A. D., A. Garen, D. Fraser, and J. D. Hudis, Carnegie Inst. Wash. Yr. Bk., 53, 210 (1954).

30. Hershey, A. D., M. Kamen, J. Kennedy, and H. Gest, J. Gen. Physiol., 34, 305 (1951).

31. Hotchkiss, R. D., in Dynamics of Virus and Rickettsial Infections. Blakiston, New York, 1954.

32. Jesaitis, M. A., and W. F. Goebel, Cold Spring Harbor Symposia Quant. Biol., 18, 205 (1953).

33. Koch, A. L., F. W. Putnam, and E. A. Evans, Jr., J. Biol. Chem., 197, 105 (1952).

34. Kozloff, L. M., J. Biol. Chem., 194, 95 (1952).

35. Kozloff, L. M., Cold Spring Harbor Symposia Quant. Biol., 18, 207 (1953).

36. Lanni, F., and Y. T. Lanni, Cold Spring Harbor Symposia Quant. Biol., 18, 159 (1953).

37. Lederberg, E. M., and J. Lederberg, Genetics, 38, 51 (1953).

38. Lesley, S. M., R. C. French, A. F. Graham, and C. E. van Rooyen, Can. J. Med. Sci., 29, 128 (1951).

39. Levinthal, C., Genetics, 39, 169 (1954).

40. Levinthal, C., and H. W. Fisher, Cold Spring Harbor Symposia Quant. Biol., 18, 29 (1953).

41. Levinthal, C., and N. Visconti, Genetics, 38, 500 (1953).

42. Luria, S. E., Proc. Natl. Acad. Sci. U. S., 35, 253 (1947).

43. Luria, S. E., Cold Spring Harbor Symposia Quant. Biol., 16, 463 (1951).

44. Luria, S. E., in Dynamics of Virus and Rickettsial Infections. Blakiston, New York, 1954.

45. Lwoff, A., Bacteriol. Revs., 17, 270 (1953).

46. Maalфe, O., and N. Symonds, J. Bacteriol., 65, 177 (1953).

47. Maalфe, O., and J. D. Watson, Proc. Natl. Acad. Sci. U. S., 37, 507 (1951).

48. Manson, L. A., J. Bacteriol., 66, 703 (1953).

49. Monod, J., and E. Wollman, Ann. inst. Pasteur, 73, 937 (1947).

50. Mudd, S., J. Hillier, E. H. Beutner, and P. Hartman, Biochim. et Biophys. Acta, 10, 153 (1953). 
51. Pardee, A. B., and I. Williams, Ann. inst. Pasteur, 84, 117 (1953).

52. Puck, T. T., Cold Spring Harbor Symposia Quant. Biol., 18, 149 (1953).

53. Putnam, F. W., and L. M. Kozloff, J. Biol. Chem., 182, 243 (1950).

54. Siddiqi, M., L. M. Kozloff, F. W. Putnam, and E. A. Evans, Jr., J. Biol. Chem., 199, 165 (1952).

55. Sonneborn, T. M., Proc. Intern. Congr. genet. 9th Congr. Bellagio, Italy, 1953.

56. Stent, G. S., Cold Spring Harbor Symposia Quant. Biol., 18, 255 (1953).

57. Stent, G. S., Proc. Natl. Acad. Sci. U. S., 39, 1234 (1953).

58. Stent, G. S., and O. Maalde, Biochim. et Biophys. Acta, 10, 55 (1953).

59. Streisinger, G., Thesis, University of Illinois, Urbana, 1954.

60. Sturtevant, A. H., Symposium on Mechanisms of Genetic Recombination, Oak Ridge Natl. Lab., April, 1954, J. Cellular Comp. Physiol., 45, Suppl. 2, 237 (1955).

61. Visconti, N., and A. Garen, Proc. Natl. Acad. Sci. U. S., 39, 620 (1953).

62. Volkin, E., Federation Proc., 13, 315 (1954).

63. Watanabe, I., G. S. Stent, and H. K. Schachman, Biochim. et Biophys. Acta, 15, 38 (1954).

64. Watson, J., and F. H. C. Crick, Nature, 171, 737 (1953).

65. Watson, J., and O. Maaløe, Biochim. et Biophys. Acta, 10, 432 (1953).

66. Weed, L. L., and S. S. Cohen, J. Biol. Chtm., 192, 693 (1951).

67. Williams, R. C., Cold Spring Harbor Symposia Quant. Biol., 18, 185 (1953).

68. Wright, S., Physiol. Revs., 27, 487 (1941).

69. Wyatt, G. R., and S. S. Cohen, Biochem. J. (London), 55, 774 (1953).

70. Zinder, N., Cold Spring Harbor Symposia Quant. Biol., 18, 261 (1953). 


\section{PHOTOSYNTHESIS*}

J. A. BASSHAM and M. CALVIN, Radiation Laboratory and Department of Chemistry, University of California, Berkeley, California

During the past decade, considerable advance in the understanding of the complex process of photosynthesis has been realized. This achievement has resulted both from the use of new methods of investigation and from the stimulation of interest, partly as a result of these new techniques, which has led to very widespread participation in the study of this problem throughout the scientific world. Fortunately, compilation and discussion of this immense amount of work $(17,26,27,34,46,47,65)$-together with review articles of the most recent work $(30,36,41)$-have generally kept pace with the work itself. In the present instance, therefore, no responsibility for a complete inclusion of published work will be assumed, but rather, an attempt will be made to present some current opinions regarding selected aspects of photosynthesis, together with some speculations in areas that may be expected to prove fruitful in the near future.

From the discoveries of recent years, it has become increasingly apparent that photosynthesis includes a number of cyclic processes which are coupled to one another in such a way

* The preparation of this paper and the original work described were sponsored by the U. S. Atomic Energy Commission. 
that there is a continuous flow of energy from one cycle to another resulting in the conversion of light energy into the potential energy of new chemical bonds. These cyclic processes, although similar to better known cyclic processes occurring during respiration in plants and animals, do not seem to be in any instance simple reversals of respiratory cycles but do appear to have many points of contact with respiratory reactions, including common intermediates and enzymes. These points of contact may well lead to interaction between the two processes which could alter the course of each (18). It appears that the organization of the green plant, both structural and enzymatic, may provide mechanisms for partially preventing such interactions where they would prove deleterious to the efficiency of the photosynthetic reaction, and possibly for permitting or even inducing them where interaction is beneficial.

\section{Function of the Chloroplast}

One such device for isolating photosynthesis from respiration is the chloroplast itself. It appears likely that the entire reaction of photosynthesis, from the absorption of light, carbon dioxide, and water to the formation of various end products, may occur in the chloroplasts. This fact was indicated long ago by the observed formation of starch granules in chloroplasts. (However, starch and other high molecular weight compounds are also formed outside the chloroplasts from simple sugars, amino acids, and other low molecular weight compounds which diffuse out of the chloroplast.) It has long been known that isolated chloroplasts can, under suitable conditions, retain the ability to evolve oxygen at rates comparable to those of photosynthesis, and at the same time, transfer reducing power to a suitable oxidizing agent. Efforts to demonstrate $\mathrm{CO}_{2}$ reduction with isolated chloroplasts were generally unsuccessful in the past. However, Gerretsen (32) found a decrease in the oxidation-reduction potential in illuminated chloroplast suspensions when they were supplied with carbon dioxide and concluded that there might be an uptake 
of carbon dioxide, though to only about $3 \%$ of the rate of photosynthesis. Boychenko and Baranov (7) have demonstrated the incorporation of carbon dioxide into reduced organic compounds by isolated chloroplasts under illumination. This incorporation was determined through use of carbon-fourteen labeled $\mathrm{CO}_{2}$, providing a very sensitive method for detecting reduced carbon. This result recently has been confirmed by Arnon et al. (2). The rate of carbon reduction compared with the rate of carbon reduction in a corresponding amount of photosynthesis in intact leaves was not more than $0.5 \%$, and many of the intermediates of the carbon reduction cycle have not yet been found. In any event, if it turns out that the rate is small compared with photosynthesis and that only some steps involved in the complete cyclic reduction during photosynthesis are present in the isolated chloroplasts, these facts may be taken merely as an indication that some of the factors involved in the rather complex reduction cycle have been lost from the chloroplasts during their isolation, or that only limited amounts of the necessary enzymes are carried down with them.

We could think of the chloroplast as a container of a complex arrangement of enzymes and cofactors, some of which are lost when the chloroplast is removed from the cytoplasmic environment of the cell. Possibly such loss could be prevented if the chloroplasts could be preserved throughout their isolation in a solution exactly duplicating that contained in the cell. Perhaps it would be worth while to prepare such a solution by disrupting cells and removing chloroplasts and cell-wall fragments by centrifugation. This solution could then be used during the preparation of chloroplasts from fresh cells.

The difference in susceptibility to inactivation during chloroplast isolation displayed by the carbon-reducing apparatus as compared to that found with the system for the photolysis of water can be considered as a difference in susceptibility to loss of factors or to the disruption of the organization of various systems. It appears that the enzymatic apparatus for the absorption of light, the conversion of light energy to chemical energy, the decom- 
position of water by this energy, and the formation of oxygen and reducing power are all rather resistant to inactivation, provided relatively simple buffer solutions are employed in the isolation of the chloroplasts. This seems to indicate the presence of an organized enzymatic apparatus, perhaps with microscopically large and intricate structure and with all necessary cofactors and prosthetic groups firmly attached. Such a view is consistent with the apparent structure of the grana and lamellae as revealed by electron microscope studies $(40,57)$.

The carbon reduction cycle, however, must be easily inactivated or separated during preparation of isolated chloroplasts. This may indicate that the enzymes involved in this cycle are not part of an organized structure, except insofar as the chloroplast structure tends to enclose these enzymes and retain within itself a space with high reducing potential.

It seems likely that this reducing power does not diffuse out of the chloroplast. If it did, one might expect greater inhibition of respiration in the light than is actually observed in cells which contain chloroplasts $(12,31)$. It is significant that inhibition of respiration during photosynthesis is most pronounced in some organisms which do not contain organized chloroplasts $(11,13)$ and this fact again indicates that one role of the chloroplast structure may be to retain reducing power at a high level for photosynthesis. Another bit of evidence relating to this possibility is the observation that the conversion of photosynthetic intermediates to respiratory intermediates is inhibited in the light (18). This might be attribtued to the reducing condition within the chloroplast. In this case, the inhibition is believed to be due to the reduction of a specific cofactor rather than to a general reduction of metabolic intermediates which might otherwise enter the oxidative pathway.

The mechanism by which the chloroplast might retain the reducing power is not entirely clear, since such carriers of reducing power as TPNH might be expected to diffuse out of the chloroplast rather freely. One possibility is that the primary carrier of the reducing power is itself a protein complex resistant 
to dialysis. Thus some type of metalloprotein might carry the reducing power. Another possibility is that reduced thioctic acid, a dithiol, might be a carrier of reducing power and might be attached by its carboxyl group to form a part of a lipid. It may be simply that the enzymes which reduce $\mathrm{CO}_{2}$ in the chloroplast are so active that the reducing power is largely used before it can diffuse out of the chloroplast.

The other reagent requirement for the reduction of $\mathrm{CO}_{2}$ at photosynthetic rates seems to be a high level of ATP, as will be seen later in this discussion. Bradley (D. F. Bradley, private communication) finds that the level of ATP is higher in the dark than in the light if the plant is in an atmosphere of $4 \% \mathrm{CO}_{2}$ in air, lower in the dark than in the light if the atmosphere is nitrogen. Strehler (54) finds that there is an increase in the level of ATP in the cell upon turning on the light after a period of darkness. This increase occurred during the induction period when the rate of carbon reduction had not yet reached its maximum value. These facts might be explained in the following way. In the dark with $\mathrm{O}_{2}$, respiration and the production of ATP by some of the energy available from respiration proceed in both the chloroplast and the space outside. When the light is turned on the rate of production outside the chloroplast is not immediately affected, but inside the chloroplast there is a combination of several effects. Normal dark respiration ceases owing to the production of reducing power and in particular reduced thioctic acid (18), while at the same time the production of ATP through energetic coupling of the recombination of some photochemically produced oxidizing and reducing agents begins (4). The result of these various rate changes is an initial increase in the level of ATP. As the rate of $\mathrm{CO}_{2}$ reduction increases, the demand for ATP increases. This results in a decrease in the level of ATP in the chloroplast, perhaps to a value much less than during the dark time, and ATP will begin to flow into the chloroplast from the cellular space outside. In an atmosphere of $\mathrm{N}_{2}$, on the other hand, respiration will cease in the dark, leading to a lower level of ATP in both chloroplast and cytoplasm, 
but upon turning on the light some ATP will be formed in the chloroplast, owing to the oxidation (back reaction) of some of the photochemically generated reducing power by $\mathrm{O}_{2}$ liberated from photosynthesis, or by an intermediate oxidant. The formation of ATP by the oxidation of an intermediate reductant (i.e., TPNH) is known from studies of oxidative phosphorylation. That some of the ATP was formed during the transfer of the electrons between intermediate reductant (TPNH) and intermediate oxidant (i.e., metalloflavin and/or cytochromes) is also an intergral part of this process, although ultimately the electrons $([\mathrm{H}])$ are transferred to molecular $\mathrm{O}_{2}$. The experimental observation of the formation of ATP by the recombination of photo-produced intermediate reductants and oxidants without the possibility of the intervention of molecular oxygen has been made by Frenkel (28) using the plastids isolated from purple bacteria which are incapable of making molecular $\mathrm{O}_{2}$. A similar observation has been made by Arnon and co-workers (2) using specially prepared whole chloroplasts from spinach. In the latter case it was necessary to add other redox systems to replace molecular $\mathrm{O}_{2}$, such as the naphthoquinone related to vitamin $\mathrm{K}$, or ascorbic acid. In addition some participation of added FMN was demonstrated.

In regard to the location of the processes of photosynthesis, then, the chloroplast or its immediate environs seems to be the unique location of all important parts and may provide a container or matrix for the complex system of enzymes and cofactors involved in the carbon reduction cycle as well as a support for the pigment and enzyme structure which carries out energy conversion and decomposition of water.

\section{The Carbon-Reducing Enzymes}

Two powerful tools have been brought to bear on the problem of the path of carbon dioxide reduction during photosynthesis during the past decade: tracer elements and paper chromatography. It is unlikely that our present degree of knowledge of 
carbon reduction would be anywhere near as far advanced had either of these tools been missing. In any event, it is now possible to trace the entire path of carbon reduction from the entry of carbon dioxide into the plant cell to the formation of sugars and other end products (4). The essential steps in this process are (7) the carboxylation of a sugar phosphate, ribulose diphosphate, to give two three-carbon molecules, both of which are probably phosphoglyceric acid (see discussion later in this section); (2) the reduction (with the aid of ATP) of phosphoglyceric acid by reducing agents formed from water by the photochemical reactions; and (3) the rearrangement of most of the molecules of reduction product, phosphoglyceraldehyde, to give (with the aid of ATP) more ribulose diphosphate for continued carboxylation, with a smaller part of the sugar phosphates being drained off as end products. The individual steps in this process are shown in Figure 1, together with the enzymes believed to be involved. The carbon balance of this system is indicated in the following scheme.

$$
\begin{gathered}
3 \mathrm{C}_{5}+3 \mathrm{CO}_{2} \stackrel{3 \mathrm{ATP}}{\longrightarrow} 6 \mathrm{PGA} \text { (phosphoglycerate) } \\
6 \mathrm{PGA} \stackrel{12[\mathrm{H}]}{\longrightarrow} 6 \mathrm{C}_{3} \\
2 \mathrm{C}_{3} \longrightarrow \mathrm{C}_{6} \\
\mathrm{C}_{6}+2 \mathrm{C}_{3} \longrightarrow \mathrm{C}_{5}+\mathrm{C}_{7} \\
\mathrm{C}_{7}+\mathrm{C}_{3} \longrightarrow 2 \mathrm{C}_{5} \\
\stackrel{\longrightarrow}{\longrightarrow} \mathrm{C}_{3}+3 \mathrm{H}_{2} \mathrm{O}
\end{gathered}
$$

A slightly different version of the sugar rearrangement might be proposed in which different reactions replace those between fructose-6-phosphate and glyceraldehyde phosphate by transketolase to give ribulose-5-phosphate and a four-carbon aldose which then combines with dihydroxyacetone phosphate (aldolase?) to give sedoheptulose-1,7-diphosphate. The modified version would postulate that two molecules of fructose-6phosphate are split and recombined by transketolase and transaldolase to give directly sedoheptulose-7-phosphate and ribulose- 
J. A. BASSHAM AND M. GALVIN

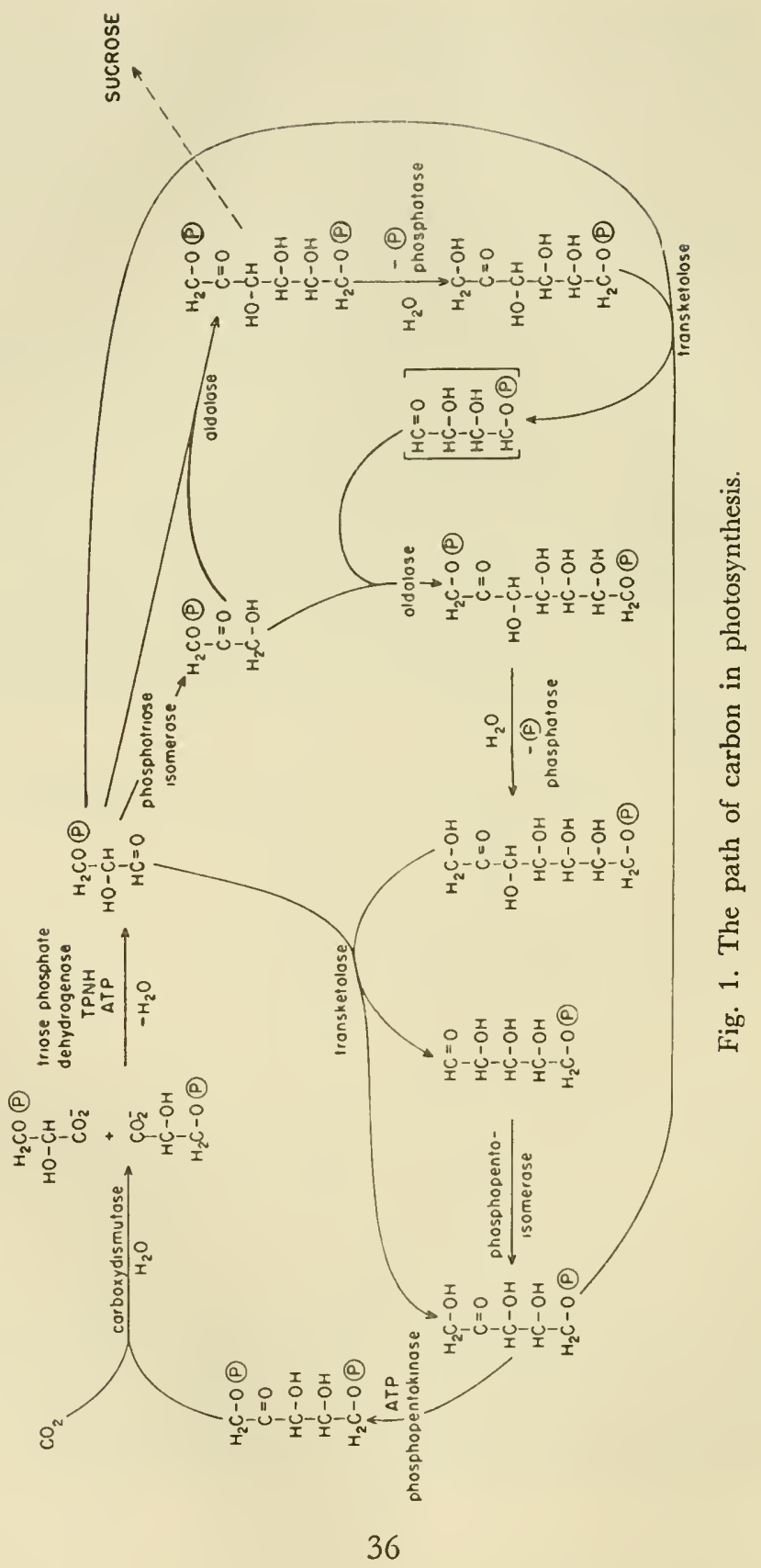


5-phosphate. Although it is not possible to choose unequivocally between these two possibilities at present, evidence obtained from degradation of the various radioactive sugar phosphates obtained from soy bean leaves which were exposed to $\mathrm{C}^{14} \mathrm{O}_{2}$ for a very short time, indicates that the original proposal (as shown in Figure 1) is correct. These degradation results are shown in Table I, which is derived from Table I of reference 4 by assuming that the carbon atoms numbers 1,2 , and 6 have the same $\mathrm{C}^{14}$ level as that found in carbon 7.

TABLE I

Distribution of $\mathrm{G}^{14}$ in Sedoheptulose

Isolated from Soy Bean Leaf

\begin{tabular}{ccc}
\hline & \multicolumn{2}{c}{ Time of exposure to $\mathrm{C}^{14} \mathrm{O}_{2}$, sec. } \\
\cline { 2 - 3 } & 0.4 & 0.8 \\
\hline $\mathrm{H}_{2} \mathrm{C}-\mathrm{OH}$ & 0 & 2 \\
$\mid \mathrm{C}=\mathrm{O}$ & 0 & 2 \\
$\mathrm{I}-\mathrm{O}$ & 33 & 39 \\
$\mathrm{HO}-\mathrm{C}-\mathrm{H}$ & 8 & 18 \\
$\mathrm{H}-\mathrm{OH}$ & 49 & 38 \\
$\mathrm{H}-\mathrm{O}-\mathrm{OH}$ & 0 & 2 \\
$\mathrm{HC}-\mathrm{OH}$ & 0 & 2 \\
$\mathrm{H}_{2} \mathrm{C}-\mathrm{OPO}_{3} \mathrm{H}^{-}$ & 0 & 2 \\
\hline
\end{tabular}

In either version for sugar rearrangement carbons numbers 4 and 5 of sedoheptulose are derived from carbons 3 and 4 of fructose, respectively. However, in the original version (Figure 1) carbon 3 of sedoheptulose is derived from the carbon 1 of glyceraldehyde phosphate directly, whereas in the modified version carbon 3 of sedoheptulose is derived from carbon 3 of fructose and therefore should have the same labeling at all times as carbon 4 of the sedoheptulose. Since this is not the case, the original version of the rearrangement seems more likely, especially since at very short exposures of the plant to $\mathrm{C}^{14} \mathrm{O}_{2}$, carbon 3 is much more highly labeled than carbon 4 , as would be expected 
if it were derived from carbon 1 of the more recently formed dihydroxyacetone phosphate.

It is to be noted that the difference in labeling between carbons 3 and 4 of fructose (hence 4 and 5 of sedoheptulose) was explained as arising from the formation of dihydroxyacetone phosphate (from which carbon 3 of fructose is derived) subsequent to the formation of glyceraldehyde phosphate (the precursor of carbon 4 of fructose).

Of all the enzymes indicated in Figure 1 only two, the enzyme for the carboxylation of RuDP and the enzyme required for the phosphorylation of ribulose monophosphate, have not been found so far in tissues other than green plants. The preparation of the carboxylation enzyme in a cell-free extract has been reported by Quayle and co-workers (45), and Weissbach et al. have isolated an enzyme preparation which causes the phosphorylation of RuMP to RuDP (63). It appears that the phosphorylation and the carboxylation may be unique steps in the carbon reduction cycle. If these steps are unique to photosynthesis, they may be so only because they either require enzymes not found outside green plants or because the particular concentrations of metabolites within the chloroplast are required if these reactions are to proceed at a finite rate. Some evidence bearing on this point has been obtained in this laboratory. The purple photosynthetic bacterium, Rhodopseudomonas, is not only capable of photoreducing $\mathrm{CO}_{2}$ with $\mathrm{H}_{2}$ but it can also reduce $\mathrm{CO}_{2}$ in the dark with $\mathrm{H}_{2}$ or with organic substrates provided $\mathrm{O}_{2}$ is present $(53,58)$. This is usually spoken of as an energetic coupling of some of the energy released in the reaction

$$
2 \mathrm{H}_{2}+\mathrm{O}_{2} \longrightarrow \mathrm{H}_{2} \mathrm{O}
$$

to help accomplish the reduction reaction

$$
2 \mathrm{H}_{2}+\mathrm{CO}_{2} \longrightarrow\left(\mathrm{CH}_{2} \mathrm{O}\right)_{x}+\mathrm{H}_{2} \mathrm{O}
$$

Since the free energy change for the latter reaction as written is negative, it appears that the presence of a stronger oxidizing agent than $\mathrm{CO}_{2}$ is required to produce some component or 
reagent necessary to permit the reaction to proceed. This reagent may be ATP, and the oxidant required may be either $\mathrm{O}_{2}$ or another oxidant produced photochemically. It is the reaction of this oxidant (or $\mathrm{O}_{2}$ ) with the activated $\left(\mathrm{H}_{2}\right)$ which can produce ATP by the mechanism almost certainly similar to that ordinarily known as "oxidative phosphorylation." Only under special conditions such as these, in which a high level of $\mathrm{H}$ (from $\mathrm{H}_{2}$ ) and ATP are present simultaneously, may we expect the photosynthetic carbon cycle to operate (53).

The precise detailed mechanism of the carboxylation reaction is not yet known with certainty. The first step may be the enolization of the ribulose diphosphate to form a double bond between carbon atoms 2 and 3 . An addition of the carbon dioxide may then take place with the shift of an electron from the hydroxyl hydrogen on carbon 3 to one of the carboxyl oxygens. This hydrogen would come off as hydrogen ion leaving a carbonyl group at carbon 3 . The resulting compound would be a $\beta$-keto acid.
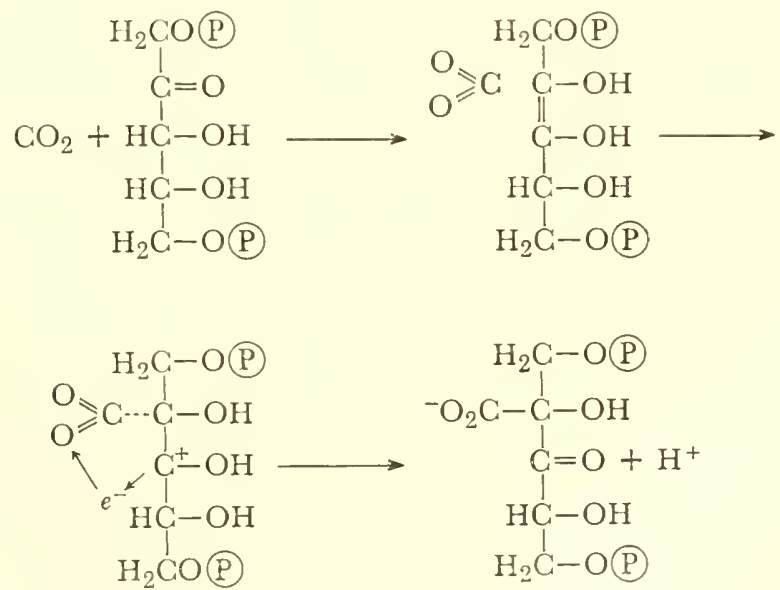

Once formed, the $\beta$-keto acid appears to undergo "acid splitting" into two molecules of PGA. This is analogous to the splitting of acetoacetate to two molecules of acetate. "Ketone 
splitting" would produce $\mathrm{CO}_{2}$ and a 3-keto pentose. Other possibilities are hydrogenation of the carbonyl group followed by a rearrangement which would result in one molecule of PGA and one molecule of phosphoglyceraldehyde; or a direct splitting or "reverse benzoin" type reaction which would result in the formation of one molecule of phosphoglyceraldehyde and one of 3-phosphohydroxypyruvic acid.

Since PGA is known to be one if not the only product of the carboxylation reaction in vivo, it is necessary to consider only the "acid splitting" and reductive splitting. There was considerable evidence for the acid split leading to PGA only, even before the enzyme was studied in vitro. Studies were made of the change in concentrations of RDP and PGA which occur in algae immediately after turning off the light (18 and Bradley, private communication). It was found that the concentration of PGA rose very rapidly under these conditions while that of RuDP dropped very rapidly. This was explained as resulting from a cessation in the reduction of PGA due to the sudden decrease in photochemically formed reducing agent, but at the same time, a continuation of the carboxylation of RuDP leading to the formation of PGA. The latter reaction, therefore, was not apparently affected at once by the lack of illumination. This result indicates that the formation of PGA from $\mathrm{RuDP}$ and $\mathrm{CO}_{2}$ does not necessarily involve a reduction, although it is possible to postulate for this reaction a reducing agent with a longer half life in the dark than the reducing agent required for the reduction of PGA.

Finally, recent work in this laboratory (J. Mayaudon, private communication) with a somewhat more purified enzyme preparation, and with $\mathrm{C}^{14}$-labeled $\mathrm{RuDP}$ and $\mathrm{CO}_{2}$, indicates that the only product of the carboxylation reaction in vitro is $\mathrm{PGA}$.

Despite these arguments, there still remains some possibility that the first product of the carboxylation reaction, the sixcarbon $\beta$-keto acid, might undergo different fates in the light and in the dark. In the dark, splitting to two molecules of PGA would proceed as discussed above; but in the light, with 
reducing agent (TPNH or DPNH) in plentiful supply, the $\beta$-keto acid might be reduced and then split by a rearrangement to one molecule of PGA and one of phosphoglyceraldehyde. This would provide a route for the formation of one molecule of triose phosphate without the requirement of an ATP molecule. This possibility should be kept in mind during the following discussion of the energy requirements of the carbon reduction cycle, which is based on the assumption that each molecule of triose phosphate formed requires the supply of a molecule of ATP.

Estimation of the free energy change for the reaction of RuDP with $\mathrm{CO}_{2}$ and water to produce two molecules of PGA showed a net free-energy change at physiological conditions which was either zero or negative (4), indicating that the reaction would proceed under the influence of a suitable catalyst without energetic coupling with some other reaction. It thus appears that energy is supplied to the cycle in the form of TPNH or ATP. Both are involved in the reduction of PGA to phosphoglyceraldehyde, whereas only ATP is needed for the phosphorylation of RuMP. For each carboxylation, using one molecule of $\mathrm{CO}_{2}$ and producing two molecules of PGA, two TPNH molecules and two ATP molecules would be converted to $\mathrm{TPN}^{+}$and $\mathrm{ADP}+\mathrm{PO}_{4}^{-}$in the subsequent reduction of PGA to phosphoglyceraldehyde. The enzymatic formation of RuDP from ribulose monophosphate has been shown by Weissbach et al. (63) to require a molecule of ATP. Since the enzymatic conversion of triose phosphate to ribulose monophosphate probably does not require the expenditure of any ATP or other substances which supply energy through energetic coupling reactions the net supply of energy to the carbon reduction cycle for each molecule of $\mathrm{CO}_{2}$ reduced is that required for the formation of two molecules of reduced TPN and three molecules of ATP as follows:

$$
\begin{array}{ll}
2\left(\mathrm{TPN}^{+}+\mathrm{H}_{2} \mathrm{O} \longrightarrow \mathrm{TPNH}+\mathrm{H}^{+}+1 / 2 \mathrm{O}_{2}\right) & \Delta F=+103 \mathrm{kcal} . \\
3\left(\mathrm{ADP}+\mathrm{PO}_{4}^{-} \longrightarrow \mathrm{ATP}\right) & \Delta F=+32 \mathrm{kcal} .
\end{array}
$$

which adds up to $135 \mathrm{kcal}$. (If one molecule of triose phosphate 
is obtained without consuming ATP, then the total requirement is 2 TPNH and 2 ATP molecules, or $124 \mathrm{kcal}$.) Since the energy required for the reaction

$$
\mathrm{CO}_{2}+\mathrm{H}_{2} \mathrm{O} \longrightarrow\left(\mathrm{C}_{6} \mathrm{H}_{12} \mathrm{O}_{6}\right)_{1 / 6}+\mathrm{O}_{2}
$$

is about $+116 \mathrm{kcal}$, about $19 \mathrm{kcal}$. apparently have to be expended to make the cycle operate at a high rate. The 135

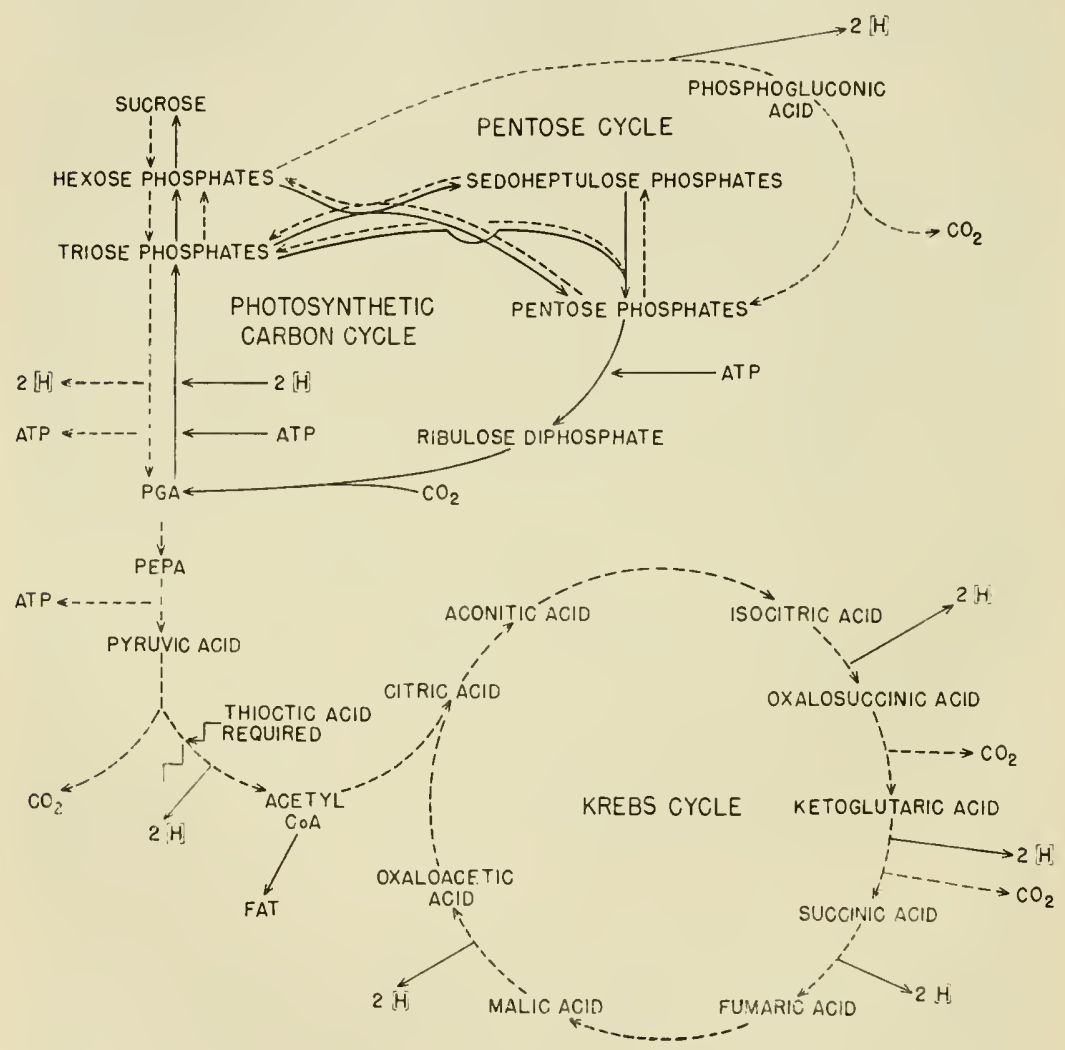

Fig. 2. The relation of carbon paths in photosynthesis and respiration.

kcal. (or $124 \mathrm{kcal}$.) requirement, on the other hand, represents the absolute minimum of energy, which must be obtained from light, exclusive of any losses in the photochemical and other 
energy transfer reactions. We shall return to a discussion of the energy requirements for these other processes later.

The relation between photosynthesis and respiration is shown in Figure 2, where respiration is indicated by dotted lines and photosynthesis by solid lines. The points at which reducing agents and ATP are utilized in photosynthesis or produced in respiration are indicated.

It can be seen that the path of carbon reduction in photosynthesis is far from a simple reversal of its path in respiration. However, the conversion of PGA to hexose in photosynthesis and the reverse in respiration appear to follow similar if not identical paths. Moreover, some of the pentose cycle path seems to have some steps in common with photosynthesis.

\section{THE LIGHT REACTION}

In considering the most characteristic reaction of photosynthesis, the light reaction, it is necessary to kecp in mind the physical arrangement of the chloroplast structure as it is now thought to exist. The fine structure of the chloroplast (or of the grana into which some chloroplasts seem to be divided) is believed to be laminar, with very thin, perhaps monomolecular layers of chlorophyll alternating with thicker layers of proteins and lipoproteins. Although the organization and thickness of these layers seem to vary with the species, particularly in some algae as compared with higher plants, it seems likely that the electrochemical fields that exist at the chlorophyll-protein and lipoprotein interfaces are similar in all cases. Thomas et al. (56) have recently studied the Hill reaction in particles of sublaminar size. Measurcment of oxygen evolution as a function of the particle size showed an ability of particles as small as $10^{6} \mathrm{~A}^{3}$ in volume to carry out the Hill reaction with about $50 \%$ the specific activity of intact grana, but a rapid decrease in such ability with smaller particles, and no activity with particles with volumes of less than $2 \times 10^{5} \mathrm{~A}^{3}$ at a given light intensity. However, higher light intensities resulted in Hill reaction activity with even smaller particles, so it was concluded that if there is a physical 
unit of about $10^{6} \mathrm{~A}^{3}$ volume, or $100 \mathrm{~A}$ diameter, it is capable of producing oxygen in the Hill reaction even if partially fragmented.

Earlier, Milner et al. (42), using the photochemical reduction of 3,6-dichlorobenzene indophenol as a measure of activity, studied the Hill reaction with particles of subgranar size and obtained activity with a mixture of particles, the majority of which were about $20 \mathrm{~A}$ in diameter. The same workers (43), after measuring Hill reaction activity with these particles of about one-fourth that of intact chloroplasts, found that the particles could be aggregated by precipitation with a variety of salts in the presence of $15 \%$ to $20 \%$ methanol in such a way as to produce particles with increased activity. Loss of lipid material resulted in loss of activity.

These experiments indicate that there are physical units, about $100 \mathrm{~A}$ in diameter and containing about 200 chlorophyll molecules, which are capable of carrying out the Hill reaction nearly as efficiently $(\sim 60 \%)$ as intact chloroplasts. If these units are broken down further, Hill reactivity falls off rapidly, but is present to some extent with considerably smaller particles, especially with high light intensities. Moreover, much of the original activity can be restored by reaggregation. The requirements for photodecomposition of water with chlorophyll seem to be some aggregation of chlorophyll, lipids, and protein. Rodrigo (51) has studied associations of a few molecules of chlorophyll, finding no shift of the red absorption peak to 6800 A. However, when chlorophyll was mixed with some ground-up leaves which contained no chlorophyll initially, some shift of the red absorption peak toward $6800 \mathrm{~A}$ and some oxygen evolution in light with quinone were observed. The implication of this result seems to be that a degree of aggregation of the chlorophyll molecules sufficient to shift the red peak as far as $6800 \mathrm{~A}$ is required before the Hill reaction can function. It is interesting to note in this connection that the absorption spectra of chlorophyll in crystals of varying size formed from ethyl chlorophyllide in acetone has been studied by Jacobs and Holt (37) and a shift 
to the longer wavelengths with increasing crystal size observed. A maximum shift of the red peak to about 7450 A was found, beyond which there was no further shift with larger crystals. This shift is ascribed to resonance interaction between identical chromophores and to migration of the resonance energy through the array.

The phenomenon of energy migration through the pigment aggregate brings us to a consideration of the light absorption process. According to present views (24) it appears that most of the energy absorbed by plant pigments for subsequent conversion to chemical energy is either absorbed directly by chlorophyll $a$ or transferred to chlorophyll $a$. The excited state is presumed to differ in energy from the ground state by only about $42 \mathrm{kcal} . /$ mole, corresponding to $6800 \mathrm{~A}$ light, the longest wavelength light that brings about photosynthesis with high efficiency. It is postulated that the extra energy that is absorbed at shorter wavelengths, either by chlorophyll $a$ or other pigments, is converted to vibrational energy and eventually lost as heat. Thus the course of energy transfer from chlorophyll on would be unaffected by the wavelength of the light absorbed. It has long been known, in fact, that the yield of oxygen evolved per quantum of light absorbed is as high for red light as at any other wavelength.

On the other hand, light absorbed at wavelengths around 4800 A produces a relatively lower quantum yield, indicating that pigments which absorb in that range may transfer their energy to chlorophyll inefficiently or may transfer some of their energy to other chemical reactions inefficiently. In the latter case, the course of subsequent steps in photosynthesis should be to some extent affected by the light energy converted to chemical energy without passing through the excited chlorophyll $a$ stage. Such an effect has recently been reported by Voskrenskaya (59), who has studied the products of carbon reduction, using $\mathrm{C}^{14}$, as a function of the wavelength of the incident light. She reports an enhanced ratio of protein to carbohydrate in blue light as compared with red light. This effect seems to be more pro- 
nounced at longer periods of time than those required for the early steps in the reduction of carbon described earlier. It seems likely that such effects are due to changes in the relative rates of transformation for various photosynthetic intermediates into other substances. These changes in rate of specific reactions are probably photocatalytic and the light energy is used only in the activation or deactivation of an enzyme.

Another photoactivation has been reported by Warburg et al. recently (61). In this case it was found that the manometrically measured quatum yield with either red or green light was greatly affected by catalytic amounts of added blue light. Some rather special conditions for the culturing of the algae used in the measurements appear to be necessary for this effect to be seen, since in other experiments reported by Warburg, high quantum efficiencies were obtained with red light only, so that the role of the blue light again appears to be in the activation of an enzyme, but in this case, one which affects the efficiency of the energy conversion path. Another possible interpretation of this effect will be presented in the section on the quantum requirement.

The step in photosynthesis which is perhaps most characteristic is the efficient conversion of energy of an excited state of chlorophyll $a$ to the stored energy of new chemical bonds. The first point to consider is the quantity of energy actually available for transformation. It has been frequently proposed that the primary excited state of chlorophyll $a$ has such a short half life $\left(10^{-13} \mathrm{sec}\right.$.) that direct conversion of the electronic energy of this state to some chemical reaction or the transfer to some other pigment might not take place before the energy was lost by fluorescence. Consequently, it was believed that transition to a metastable triplet state with concurrent loss of some of the electronic energy must first occur, followed by conversion or transfer of the energy of the triplet state. However, according to Scheibe (52), transfer of electronic energy in a condensed pigment system can occur in $10^{-14} \mathrm{sec}$. It seems possible, therefore, that in the aggregated chlorophyll-lipid-protein 
system, the full $42 \mathrm{kcal}$. might be transferred to a suitable proximate acceptor, at least insofar as competition with fluorescence is concerned.

The conversion of electronic energy to chemical energy may involve either the transfer of electronic energy to some acceptor molecule other than chlorophyll followed by energy conversion, or the transfer of an electron from the chlorophyll aggregate (array) to some other molecule and a corresponding recovery of an electron by the positively charged chlorophyll molecule group from some other source. Either of these types of conversion seems possible at the present time, and we will consider both.

In order to accept electronic energy from chlorophyll and convert it to chemical energy, a compound, which could be called the quantum converter, should possess several properties. It would have to be closely associated with, or incorporated in, the chlorophyll aggregate, and should possess some state differing from its ground state by about 30 to $40 \mathrm{kcal}$., in order to accept the energy of a quantum from the chlorophyll. It would convert this energy by some process which occurs in a time that is short compared to the time required for the return of the energy to chlorophyll or its dissipation in a nonuseful way, and in so doing would form new chemical configurations which would store most of the energy received from the chlorophyll. By new chemical configurations, we mean only that some nuclei would have moved sufficiently to prevent the loss of the received energy either by its return to the donor or conversion to heat; that is, the energy would now be trapped in a new configuration which could not return easily to the former one.

When the energy had undergone this conversion, the quantum converter, in its new form, would pass the energy on. Since, in this picture, the most energetically difficult step, the photolysis of water, has yet to occur, we will suppose that the new form of the quantum converter would then react with water to produce a reducing agent and some form of hydroxyl or peroxide compound which can ultimately liberate oxygen. A requirement for this reaction is that the resulting bond energies 
plus about 30 to $40 \mathrm{kcal}$. be about the same as the bond energies of broken bonds, if the reaction is to provide a means of efficient energy conversion. The products of the reaction should not be able directly to recombine easily in such a way as to produce water again (back reaction) but should be able to react separately to produce ultimately oxygen and reducing power. Finally, the quantum converter molecule should be able to return to its orginal state, after having transferred its electrons (reducing power) and oxygen to other molecules.

In addition to the requirements imposed by the mechanism, there is the necessity that the quatum converter be present in sufficiently high concentration in the chloroplast to account for the observed rates of quantum conversion, both in steady-state photosynthesis and in flashing-light experiments. This requirement of concentration will depend, of course, on the time required for the quantum converter to undergo one cycle, from acceptance of the quantum of electronic energy and to return to its original state.

Thioctic acid (lipoic acid) has been proposed as a compound which might satisfy all the above requirements $(3,16)$. It was suggested that following the absorption of a quantum by chlorophyll, this energy is transferred to thioctic acid, causing the $\mathrm{S}-\mathrm{S}$ bond of the latter molecule to break to give a diradical. It was postulated that this diradical then reacts with water, forming a sulfhydryl and a sulfhydroxyl group. Dismutation of this reaction product results in a dithiol molecule and a disulfenic acid. The dithiol would then reduce DPN or TPN and would itself be reoxidized to thioctic acid, while the disulfenic acid would undergo a series of reactions resulting in the reformation of thioctic acid and the liberation of oxygen. All of these reactions from the dismutation onwards would probably involve catalysis by metalloproteins.

The lipophilic properties of thioctic acid and its small molecular size would permit close association with the chlorophyll aggregate and might account for the apparent lipid requirement of the Hill reaction. The formation of the diradical 
by breaking the strained ring has been suggested as the mechanism for storing the accepted electronic energy. Estimates of the bond energy for the $\mathrm{S}-\mathrm{S}$ bond in simple open-chain molecules ranging from 50 to $70 \mathrm{kcal}$. together with estimates for the reduction of the dissociation energy of the $\mathrm{S}-\mathrm{S}$ bond due to ring strain in the 6,8-trimethylene disulfide ring of 10 to $25 \mathrm{kcal}$. indicate the possibility that formation of the free radical could store 30 to 40 kcal. (3). A number of studies with model systems (6,8-trimethylene disulfide in u.v. light) were presented which indicated, among other things, an ability of the trimethylene disulfide to react with $\mathrm{ROH}$ (alcohol) or water under illumination to form a thiol and a sulfenic acid or ester.

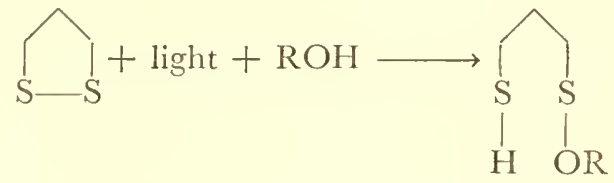

Two pieces of biological evidence were offered to support the suggestion that thioctic acid participates in the quantum conversion. It had already been observed that in photosynthesizing plants the conversion of photosynthesis intermediates to Krebs cycle intermediates is inhibited during illumination, but occurs rapidly in the dark. Since this conversion involves the formation of acetyl CoA from pyruvic acid, a reaction which requires thioctic acid as a cofactor $(14,33,44,48,49)$, it had been suggested (18) that this reaction is blocked in the light because most of the thioctic acid is maintained in the reduced dithiol form within the chloroplast. The reduction of thioctic acid could occur, of course, as a secondary reaction resulting from the formation of some other primary reducing agent in the lightenergy conversion. However, the favorable characteristics for quantum conversion which the thioctic acid possesses together with the indication that it was reduced in the light led to the supposition that it might be in the primary reaction.

When algae are allowed to take up added thioctic acid in the dark for several minutes and then killed with quinone and the 
Hill reaction studied in the light (9) it was found that the initial rate of oxygen evolution is increased by as much as $50 \%$ over the rate observed in the control in which no thioctic acid was added. This result could be interpreted as evidence for the participation of thioctic acid in the primary conversion step or as an acceleration of a dark reaction transfer of reducing power to quinone. Thus the biological evidence for the role of thioctic acid as a quantum converter is suggestive but not unequivocal. However, since the oxygen evolution in the Hill reaction is insensitive to inhibition by parachloromercuribenzoate, a more limited role of thioctic acid in which it acts only as an acceptor of an electron from chlorophyll seems the more likely than the above role, in which it removes this electron from oxygen after receiving energy from excited chlorophyll.

Of the various possible chemical reactions of chlorophyll under the influence of light we shall consider only the transfer of an electron. Since there is evidence that the light absorption process functions with chlorophyll in an aggregated system, it is interesting to consider, instead of the reaction of a single chlorophyll molecule, the possibilities that exist with some sort of orderly array of chlorophyll molecules. This array is probably not actually crystalline chlorophyll but may well be an orderly arrangement of chlorophyll molecules associated with other molecules and protein or lipoprotein. We may think of the electronic system of such an aggregate as a single unit in which the $\pi$ electrons of the chlorophyll molecules interact. The absorption of an electromagnetic quantum will raise one electron of this system from the ground state in which it is confined to a single molecule into a state in which it may migrate throughout the array, i.e., into a conduction level. If there is built into this structure a permanent polar character such as exists at a "p-n" junction, for example, these photoconduction electrons will diffuse toward the positive end of the permanent dipole, leaving a positive hole to diffuse in the opposite direction.

Thus a separation of charge will be induced by the light which may be neutralized by a suitable electron acceptor at one 
end, and a corresponding electron donor at the other end, to drop electrons into the positive holes that have been photocreated. In order to complete the separation of the electron and the positive charge and make use of the electrical energy available, all that is needed is either a semiconductor which will transmit only electrons or positive charges, or else an acceptor molecule which can accept either an electron or a positive charge (i.e., contribute an electron) or both, in such a way as to produce an irreversible change.

The possibility of a semiconductor is an interesting one in that it provides a possible function for the lipid constituents. One might consider most of the lipid material as an insulator with occasional conductor molecules to pass electrons through. Such a function might be served by a conjugated molecule like a carotenoid which could accept an electron at one end and give an electron to a suitable acceptor at the other end. At the same time, the positive charges left behind would be reacting with water, probably through the agency of some metalloprotein, to produce oxygen. The electron acceptor, in the case of the Hill reaction, could be the supplied oxidant such as quinone, while in photosynthesis the acceptor could be the primary carrier of reducing power (thioctic acid).

The conductor function for a carotenoid compound might explain the stimulation of photosynthesis by catalytic amounts of blue light observed by Warburg et al. (61). Warburg has suggested the participation of carotenoid somewhere in the transfer of electrons between the photochemical reaction and the reduction of carbon dioxide as an explanation of the bluelight effect. It should be noted, however, that Stanier (R. Stanier, private communication) has studied mutants of Rhodospirillum which contain no carotenoids but which nevertheless are able to carry out reduction of carbon dioxide during photosynthesis.

The advantage of the semiconductor arrangement would be the physical separation of the points at which reducing agent and oxidizing agents are formed. Also the nonspecificity of 
oxidants required for the Hill reaction could be explained, since they could be reduced directly by electrons supplied by the photoactivation of chlorophyll rather than by some specific primary reducing agent. During actual photosynthesis, a specific reducing agent would undoubtedly be formed and would provide a more efficient transfer of the energy available from the electrons obtained from the photochemical reaction. We would expect that this compound would have special properties which would particularly suit it to the task of accepting electrons and, in its reduced form, transferring energy to the carbon reduction apparatus. It might be expected that this compound would stimulate the Hill reaction owing to its special qualifications for receiving electrons. For example, if thioctic acid were the compound receiving the electrons from the chlorophyll during photosynthesis, it might be expected that thioctic acid would stimulate the Hill reaction under suitable conditions even though not required for the Hill reaction to function.

Such a stimulation has been observed by Bradley and Calvin (9), who studied the rate of oxygen evolution in the Hill reaction as a function of added thioctic acid. When quinone was added as an oxidant at a concentration which produced the highest rate of evolution of oxygen with quinone alone, it was found that the addition of thioctic acid in a molar concentration only 0.1 as great as that of the quinone produced a $35 \%$ stimulation of the initial rate. Moreover, this stimulation was observed with 6,8-dithiooctanoic acid (6-thioctic acid) only, whereas 5-thioctic acid and 4-thioctic acid were not effective. The reduced form (dithiol) of thioctic acid and the more oxidized form (the sulfoxide) were also found to be ineffective. It appears that, barring some enzymatic specificity which seems unlikely in the Hill reaction, the special property of 6-thioctic acid which is responsible for its activity is the ring strain of the five-membered ring which facilitates breaking of the sulfur-sulfur bond.

One advantage of considering the chlorophyll as an aggregated system is that it permits a more reasonable mechanism for the transfer of one electron at a time, with each electron re- 
quiring one quantum of light energy. This corresponds to the well-known four-quantum theory in the primary step, since the transfer of four electrons, requiring four quanta, are required to form one molecule of $\mathrm{O}_{2}$.

Reactions between a single molecule of chlorophyll, water, and hydrogen or electron acceptor are difficult to formulate, since one is faced in that case with the necessity either of absorbing two consecutive quanta in one chlorophyll molecule in order to have enough energy to form oxygen and reducing agents of the strength of TPNH (about $51 \mathrm{kcal}$.) or else of forming oxygen and a reducing agent of insufficient strength which could be used in subsequent reactions involving dismutations of energy to form better reducing agents. Another alternative is that a single molecule of chlorophyll, activated by one quantum, reacts with some activated, hydrated compound in which the $\mathrm{O}-\mathrm{H}$ bond could be more readily broken, and thus has left over enough energy to form a good reducing agent. It may well be that water is in fact incorporated into some compound to weaken the $\mathrm{O}-\mathrm{H}$ bond before the $\mathrm{O}-\mathrm{H}$ bond is broken regardless of the mechanism of electron transfer, but it is doubtful if this activation is sufficient to permit the formation of oxygen and reducing power of the strength of TPNH at the same time by one quantum.

Recent proposals involving reactions in which one molecule of chlorophyll provides both electrons in a given $\mathrm{H}_{2} \mathrm{O}$ photolysis include the one by Levitt (39), who suggests that a chlorophyll molecule, excited by one quatum of light, gives up an electron to thioctic acid forming oxidized chlorophyll, after which the molecule of oxidized chlorophyll absorbs a second quantum and transfers a second electron. The resulting dipositive chlorophyll then reacts with water to produce hydrogen ion and oxygen. In the meantime, the thioctic acid has been reduced by the two electrons to the dithiol.

On the other hand, Wessels (64) has proposed a one quantum per two electrons reaction in which a much weaker reducing agent, reduced vitamin $\mathrm{K}$, is produced. This reducing agent is 
then used in part to produce ATP and in part to produce a reducing agent of the level of TPNH through the expenditure of ATP in a coupled reaction. Since the formation of reduced vitamin $\mathrm{K}$ and oxygen from water and vitamin $\mathrm{K}$ is said to require $39 \mathrm{kcal}$., the energy of one quantum would have to be used with nearly $100 \%$ efficiency. Such an efficient mechanism will be especially attractive if the very low quantum requirements reported by Warburg prove correct. The presence of vitamin $\mathrm{K}$ in chloroplasts and its concurrent formation with chlorophyll (23) are also favorable to this suggestion. Finally, its oxidation-reduction potential is close to that measured with illuminated chloroplast preparations (50). The mechanism proposed by Wessels for the formation of ATP from reduced vitamin $\mathrm{K}$ is reasonable but energetically very inefficient in that only one ATP is formed for each molecule of reducing agent used. No specific mechanism was proposed for the formation of TPNH from $\mathrm{TPN}^{+}$by the oxidation of reduced vitamin $\mathrm{K}$ and the conversion of ATP to ADP and inorganic phosphate, but this reaction would be a bit uphill energetically, since the difference in redox-free energies between TPNH and reduced vitamin $\mathrm{K}$ is apparently about $12.5 \mathrm{kcal}$., slightly more than the 10 or $11 \mathrm{kcal}$. now thought to be available from ATP hydrolysis. Besides this, the proposal of Wessels requires the steps in the liberation of $\mathrm{O}_{2}$ from whatever intermediates may be formed in the reaction of oxidized chlorophyll with water to proceed with virtually no change in free energy. In other words, this proposed mechanism includes one very inefficient step and a number of steps which are nearly $100 \%$ efficient in energy transfer. Although this is entirely possible, we find it slightly more satisfying, from a thermodyamic viewpoint, to suppose that most of the steps involved in the energy transfer from one system to another proceed efficiently but with a small loss of energy in each, thus providing a smooth driving force throughout the entire process which will not require the enzymes at any stage to cope with infinitesimal concentrations of substrates. 


\section{INTERMEDIATE TRANSFER SYSTEMS}

We have already anticipated, in the discussion of both the carbon reduction cycle and the light reaction, some of the reactions involved in the transfer of reducing power and energy from the light reaction to carbon reduction and in the evolution of oxygen from whatever products are formed in the breaking of the $\mathrm{O}-\mathrm{H}$ bond. Let us assume, for the time being, that the theories above, which require that the absorption of a quantum for each electron taken from water (whether by quantum conversion by thioctic acid or by transfer of an electron by the chlorophyll aggregate from water to reducing agent) are correct. Then there is ample energy in four quanta of $6800 \mathrm{~A}$ light (168 $\mathrm{kcal} . /$ mole) to bring about the photolysis of two water molecules and the formation of two molecules of reducing agent of strength equal to TPNH:

$2\left[\mathrm{H}_{2} \mathrm{O}+\mathrm{TPN}^{+}=1 / 2 \mathrm{O}_{2}+\mathrm{TPNH}+\mathrm{H}^{+}\right] \quad \Delta F=+103 \mathrm{kcal}$

The entire excess of energy, some $65 \mathrm{kcal}$. minus whatever was lost in the primary absorption and conversion processes, will be available for the evolution of oxygen from the intermediates formed from the oxidation of water. It is possible that some of this energy might be used in the formation of some ATP from ADP and inorganic phosphate. Whether or not this occurs is very important in the evaluation of possible quantum requirements. We have already arrived at a quantum requirement of two molecules of TPNH and three molecules of ATP for each molecule of carbon dioxide reduced. If one molecule of the primary reducing agent must be oxidized in order to form two or three molecules of the required ATP by some reaction similar to that which couples the energy of the oxidation of DPNH to the formation of ATP (38), then the total requirement for equivalents of reducing agent will be seven or six, requiring seven or six quanta (4). If all the ATP molecules could be supplied from the energy left over from the evolution of oxygen as suggested above, then the quantum requirement will obviously be only four. 
The decomposition of water will require by far the greater portion of the energy available from the primary photochemical reaction. From the half-reaction potential of the primary reducing agent, which in our scheme must be about $0.3 \mathrm{v}$., we can say that the relative energy stored by the transfer of one electron to the reducing agent is about $7 \mathrm{kcal}$./mole (taking the energy required to transfer an electron to $1 \mathrm{NH}^{+}$in contact with $\mathrm{H}_{2}, \mathrm{~g}$, $1 \mathrm{~atm}$., as zero). If there is a loss of about $5 \mathrm{kcal}$. in the transfer of the electron from the chlorophyll aggregate, there is left, from a $42 \mathrm{kcal} . / \mathrm{mole}$ quantum, about $30 \mathrm{kcal} . / \mathrm{mole}$ to be stored in each positive charge ("hole"). This would then be a potential of about $1.3 \mathrm{v}$. The potential required for the half reaction

$$
2 \mathrm{H}_{2} \mathrm{O}=\mathrm{H}_{2} \mathrm{O}_{2}+2 \mathrm{H}^{+}+2 e^{-}
$$

is about $1.2 \mathrm{v}$. at $p \mathrm{H} 7$ and $10^{-6} M \mathrm{H}_{2} \mathrm{O}_{2}$. Thus the reaction will go as written, provided the very high activation energy required for the removal of electrons from water-oxygen atoms, which would result in formation of hydroxyl radicals in solution, can be overcome. We may suppose that the hydration of a suitable surface on the granar fragment, perhaps resulting in actual hydrated compounds, results in an orientation of $-\mathrm{OH}$ groups which permits the formation of $\mathrm{O}-\mathrm{O}$ bonds concurrently with the removal of the electrons from the water.

The formation of the positive and negative potentials discussed above requires some mechanism for obtaining just the right distribution of energy to achieve the necessary oxidation and reduction. This can be accomplished by extending somewhat further the proposal for the separation of charges through the agency of semiconductors. We may think of the subgranar unit as a photoelectric battery. The driving force for this battery is the light energy absorbed by the chlorophyll. The absorption of light produces in the aggregate conduction electrons and their corresponding positive "holes." This part of the structure can be considered a conductor after light absorption. On either side of the chlorophyll aggregate is a layer of semiconducting material. This material may be lipid or lipoprotein. One layer contains a 
permanent structural excess of electrons ( $n$ volume), permitting the conduction of positive charges. The other layer would contain a permanent structural deficiency of electrons ( $p$ volume), permitting the conduction of electrons. With the creation of mobile charges by light absorption there would follow a flow away from the chlorophyll aggregate and across the semiconducting layers. If the potential between these two layers could be measured, it would be found equivalent to $\sim 42 \mathrm{kcal}$./mole minus the 5 $\mathrm{kcal} . / \mathrm{mole}$ loss postulated above, or $1.6 \mathrm{v}$. If the circuit between these two "electrodes" is completed by chemical reactions, as it is in photosynthesis, the potential at each electrode, relative to the ground state of the system, will be simply that required by the oxidants or reductants with which the electrons and positive charges must react. Thus, in terms of our arbitrary "ground," which is the standard hydrogen electrode potential, the $1.6 \mathrm{v}$. potential across the "battery" will be distributed as $1.3 \mathrm{v}$. positive and $0.3 \mathrm{v}$. negative, since these are the potentials, relative to our "ground," with which the electrodes must react. From this point of view it appears that the recently developed solar battery (22) may have been preceded by a similar but much more efficient process in photosynthetic organisms!

The evolution of oxygen from hydrogen peroxide according to the reaction

$$
\mathrm{H}_{2} \mathrm{O}_{2}=1 / 2 \mathrm{O}_{2}+\mathrm{H}_{2} \mathrm{O}
$$

proceeds with a positive potential of $0.37 \mathrm{v}$. for $10^{-6} M$ peroxide, so that another $8.5 \mathrm{kcal}$./mole of excess energy is expended per electron. There is some question as to whether this reaction could be catalyzed by catalase (55), owing to the apparent lack of inhibition of the Hill reaction by cyanide. However, this reaction can be catalyzed by a number of simple inorganic compounds, so perhaps this problem is not too serious. Moreover, inhibition of oxygen evolution in the Hill reaction by orthophenanthroline has been observed (29), so some ironcontaining enzyme may be involved. Finally, the recent work of Chance (21) has demonstrated the formation of catalase- 
$\mathrm{H}_{2} \mathrm{O}_{2}$ complexes under aerobic conditions. It appears that this complex can form with concentrations of $\mathrm{H}_{2} \mathrm{O}_{2}$ as low as $10^{-8} \mathrm{M}$.

Consideration of the above mechanism of oxygen evolution suggests that the place where energy might possibly be available for use in forming ATP, or perhaps additional reducing agent, is in the liberation of oxygen from peroxide. Some $17 \mathrm{kcal}$. are available from each molecule of peroxide. However, it is difficult, with only our present knowledge, to visualize the possible mechanism of this energetic coupling. On the other hand, it is attractive to suppose that the peroxide may be used in part to oxidize some ferrocytochrome (19) in a reaction catalyzed perhaps by peroxidase (19). Thus the requirement for an oxidizing agent to react ultimately with the primary reducing agent could be met without requiring oxygen. This is rather useful because of the evidence that molecular oxygen is not required for photosynthesis. Allen (1) has reduced the concentration of molecular oxygen in contact with photosynthesizing organisms to a value of $0.004 \mathrm{~mm}$. $\mathrm{Hg}$ and finds, at this level, no diminution of the rate of photosynthesis. The absence of a back reaction involving molecular oxygen was also shown by the studies of Brown (10) with the mass spectrometer and isotopically labeled oxygen.

It is interesting to suppose that the ferrocytochrome oxidized by $\mathrm{H}_{2} \mathrm{O}_{2}$ is reduced cytochrome $f$, discovered by Hill and Scarisbrick (35). It was found that this compound was present in considerable amounts in green chloroplast material and that it had an oxidation-reduction potential of $+0.365 \mathrm{v}$., about $0.1 \mathrm{v}$. better as an oxidizing agent than cytochrome $c$. Since the oxidizing potential of $\mathrm{H}_{2} \mathrm{O}_{2}$ for the physiological conditions as given above is $+1.2 \mathrm{v}$., there is a potential difference between these two half reactions of $0.8 \mathrm{v}$., or a negative free energy change of $37 \mathrm{kcal} . /$ mole for the oxidation of two reduced cytochrome $f$ molecules with one $\mathrm{H}_{2} \mathrm{O}_{2}$ molecule.

The oxidized cytochrome could then react with other electron carriers, perhaps other cytochromes, and eventually, with the primary reducing agent, or with TPNH. Since the 
oxidation-reduction potential of the latter is now considered to be $-0.324 \mathrm{v}$. (15), there is a potential difference between cytochrome and $\mathrm{TPN}^{+}$of $0.65 \mathrm{v}$., or a negative free energy of 30.3 kcal. for the oxidation of one mole of TPNH by oxidized cytochrome $f$.

Very little is known about the mechanism of formation of ATP during the oxidation of TPNH. There is enough energy available in each of the steps suggested above to bring about the formation of 2 moles of ATP with ease. We can suppose that the general type of mechanism for the oxidative formation of ATP might involve esterification of a hydroxyl group with inorganic phosphate, dehydrogenation of an adjacent $\mathrm{C}-\mathrm{C}$ or $\mathrm{C}-\mathrm{N}$ bond with a suitable oxidizing agent to form a "highenergy phosphate group," a reaction with ADP to form ATP and an unsaturated alcohol, and finally, reduction of the unsaturated alcohol to the orginal substance.

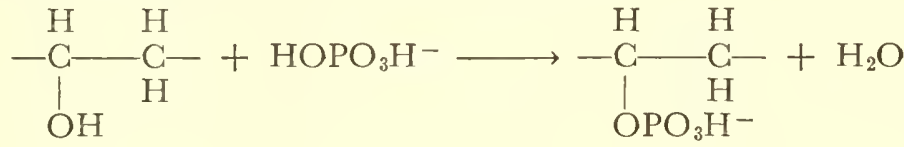

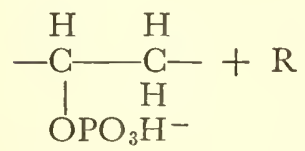

$-\underset{\mathrm{OPO}_{3} \mathrm{H}^{-}}{\mathrm{H}} \mathrm{C}-\mathrm{ADP}$

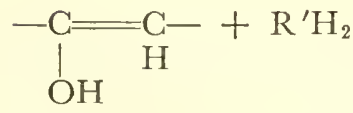

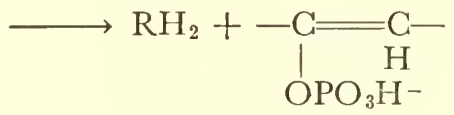
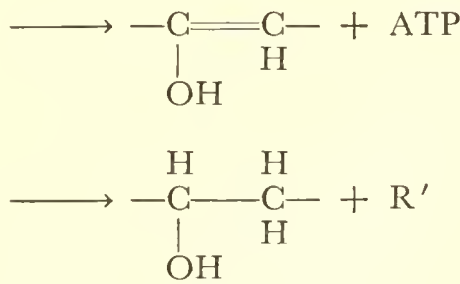

$\mathrm{R}+\mathrm{R}^{\prime} \mathrm{H}_{2}+\mathrm{ADP}+\mathrm{H}_{2} \mathrm{PO}_{4}^{-}$ $\rightarrow \mathrm{RH}_{2}+\mathrm{R}^{\prime}+\mathrm{ATP}$

The $-\mathrm{CH}_{2}$ group of the reacting alcohol can also be - NH- Considering the number of steps involved in the above mechanism, we could expect that at least $5 \mathrm{kcal}$. negative free- 
energy change would be required for the entire process, so that about $15 \mathrm{kcal}$./mole might be required for the formation of ATP by such a mechanism. It is thus possible that anywhere from 1 to 4 molecules of ATP might be formed for each molecule of primary

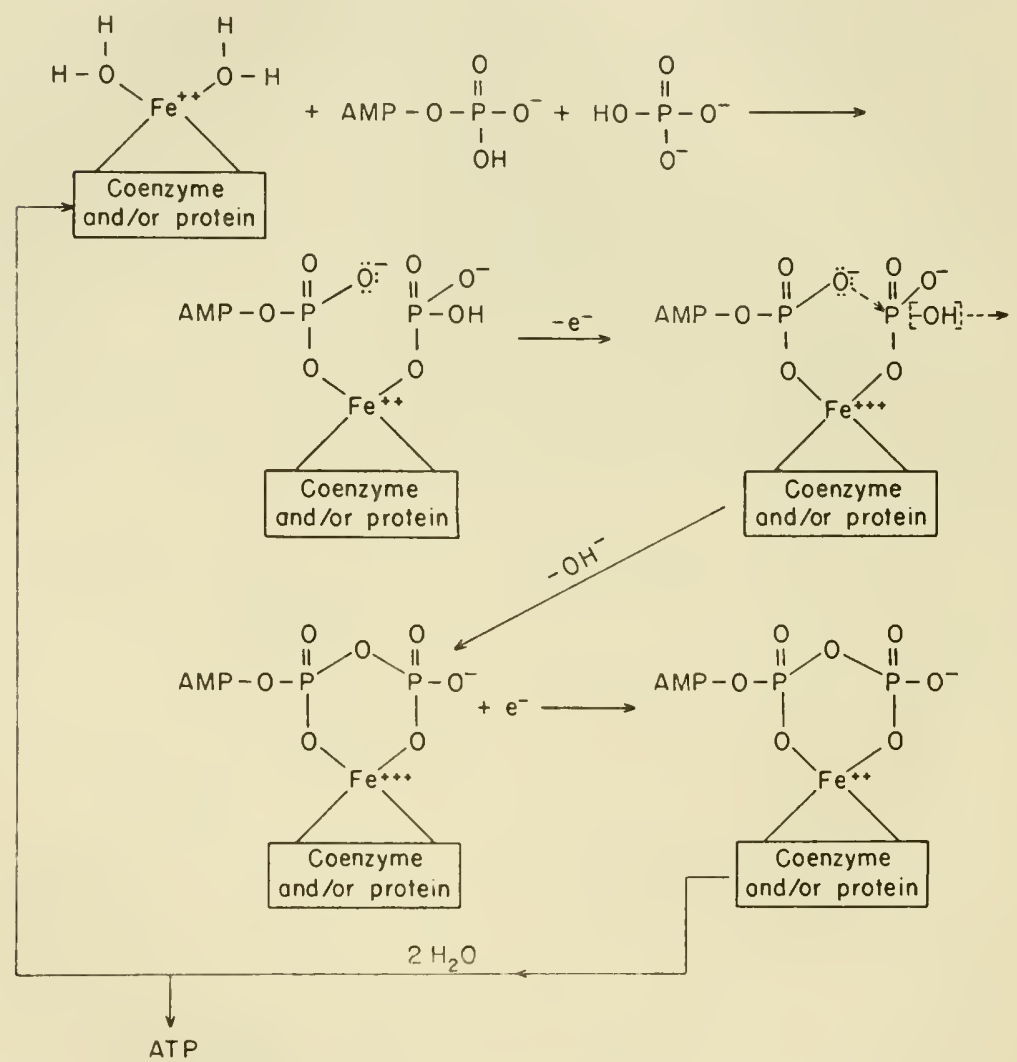

Fig. 3. A suggested mechanism for ATP formation via oxidation and reduction of a metalloenzyme.

reducing agent used, provided electron carriers of suitable intermediate potentials are available. What these might be is difficult to say, but some cytochromes, for example, cytochrome $b$, lie intermediate between cytochrome $f$ and TPNH. It is 
conceivable that there is still another type of process for the generation of ATP which differs fundamentally from the one outlined above, involving the intermediate formation by oxidation of a "high-energy phosphate group" in the form of an enol ester or anhydride. This is suggested by the increasing knowledge of "oxidative phosphorylation" and the participation of metalloproteins in this process, particularly metalloflavins and/or porphyrin, together with the fact that tripositive (higher valence state) ions form stronger and more compact complexes than do dipositive ions (lower valence state). An example of the manner in which such a process might operate is shown in Figure 3. The metal (in this case Fe) in its reduced form $\left(\mathrm{Fe}^{+2}\right)$ could bind into a complex the terminal phosphate of ADP and one orthophosphate ion. This complex, upon oxidation to the higher valence state $\left(\mathrm{Fe}^{+3}\right)$, would contract and induce the displacement of an $\mathrm{OH}^{-}$from orthophosphate by the - $\mathrm{O}^{-}$atom of the terminal phosphate of ADP, thus producing the stable ATP chelate of the $\mathrm{Fe}^{+3}$. In order that the ATP be liberated for other uses, the $\mathrm{Fe}^{+3}$ must be reduced again to $\mathrm{Fe}^{+2}$, for which the chelation constant is much smaller. The cycle is thus complete, the net result being the transfer of an electron from the reducing agent to some oxidizing agent of higher potential via the $\mathrm{Fe}$ atom with the trapping of some (if not all) of the energy of this transfer in the form of ATP. It is interesting to note that Chance (20) has observed an apparent requirement of the reduction step for the liberation of ATP in the course of oxidative phosphorylation.

The process postulated for the formation of primary reductant and $\mathrm{H}_{2} \mathrm{O}_{2}$ has a quantum requirement of 4 for each $\mathrm{H}_{2} \mathrm{O}_{2}$ formed or 2 for each $\mathrm{RH}_{2}$ formed. The over-all quantum requirement will depend on the number of $\mathrm{RH}_{2}$ molecules which must be used to form ATP. If all required ATP can be supplied from respiration reactions outside the chloroplast, as may be the case at very low light intensities, the over-all quantum requirement will be 4 . At high light intensities the over-all quantum requirement will be $10,7,6$, or 5 , depending on 
whether the number of ATP molecules formed per $\mathrm{RH}_{2}$ burned is $1,2,3$, or 4 , respectively.

The controversy regarding the minimal requirement of photosynthesis has not been settled. The recent experiments reported by Warburg et al. (60) are very convincing, since quantum yields of four and even three are reported with high light intensities for long periods of time and without corrections for respiration, thus effectively answering criticisms based on the possibility that the reported quantum requirements are due to contribution of respiratory energy to photosynthesis.

This leaves only criticisms based on the evaluation of the manometric technique. No such evaluation will be attempted here, but it may be worth while to point out one possible difficulty. Calculations of oxygen evolution and carbon dioxide uptake by the two-vessel method depend on the assumption of constant solubilities of these gases. However, the solubility of carbon dioxide may change significantly if the $p \mathrm{H}$ of the medium changes, and this in turn could be influenced by the secretion of acid by the algae. For example, it has been observed in this laboratory that in high light intensities, algae produce glycolic acid. Tolbert (N. E. Tolbert, private communication) has found that glycolic acid formed in strong light by algae is secreted into the medium. One might speculate that perhaps blue light might activate some acid-secreting enzyme system, though there is at present no evidence for this.

In view of this and many other difficulties inherent in the manometric determination of quantum yields, it has seemed desirable to try other methods of measuring oxygen liberation for quantum requirement calculations. One such study is that of Brackett et al. (8), who used a polarographic determination of oxygen and calculated quantum requirement as low as six. A relatively simple and straightforward experiment has now been carried out using an oxygen analyzer employing paramagnetic measurement of oxygen (5).

A suspension of algae was placed in a thin plastic cell of large area. A mixture of $4 \% \mathrm{CO}_{2}$ was passed through this 
suspension, then through an oxygen analyzer and a carbon dioxide analyzer. The circulation of gas through this closed system was accomplished by means of a pump. The indications from the analyzers were continuously recorded on a multipoint recorder. A uniform light field of 6300 A light was obtained from a spiral neon tube with suitable filters and incident and transmitted light intensities measured with a bolometer, which was frequently calibrated against three standard lamps. Small variations in the light field were mapped by a small photoelectric cell and suitable corrections made.

The measurements of photosynthetic rate were dependent only on the measured change in the percentage oxygen in the system and the known volume of the system. Both of these are directly measured quantities which can be, and were, checked frequently with standard gas mixtures. Virtually no variation was found from time to time. The energy measurements were also simple and straightforward, since they involved essentially the measurement of energy absorption in a thin layer of large area.

The algae, Chlorella pyrenoidosa, were grown according to previously described conditions (6) in $4 \% \mathrm{CO}_{2}$. The quantum requirements of these algae were tested after a variety of preconditions. The best condition found was selected and determinations were made as a function of light intensity. The values of the quantum requirement were determined both for the uncorrected rate of photosynthesis and for the rate, which we will call photosynthesis, obtained by subtracting the dark respiration rate from the uncorrected rate.

This correction seems justified in view of Brown's (10) study in which isotopic oxygen was used to demonstrate that no significant change in rate of respiration of Chlorella pyrenoidosa took place during alternate 15 or 20 minutes of light and dark. The same paper, as well as the earlier ones by Emerson and Lewis (25), Weigl et al. (62), and Brackett et al. (8), showed an increase in the dark respiration rate (and the light rate as well in Brown's paper) which is produced by conditioning the plants with photo- 
synthesis as compared with leaving them in the dark for several hours. This indicates a photosynthesis enhancement of the rate

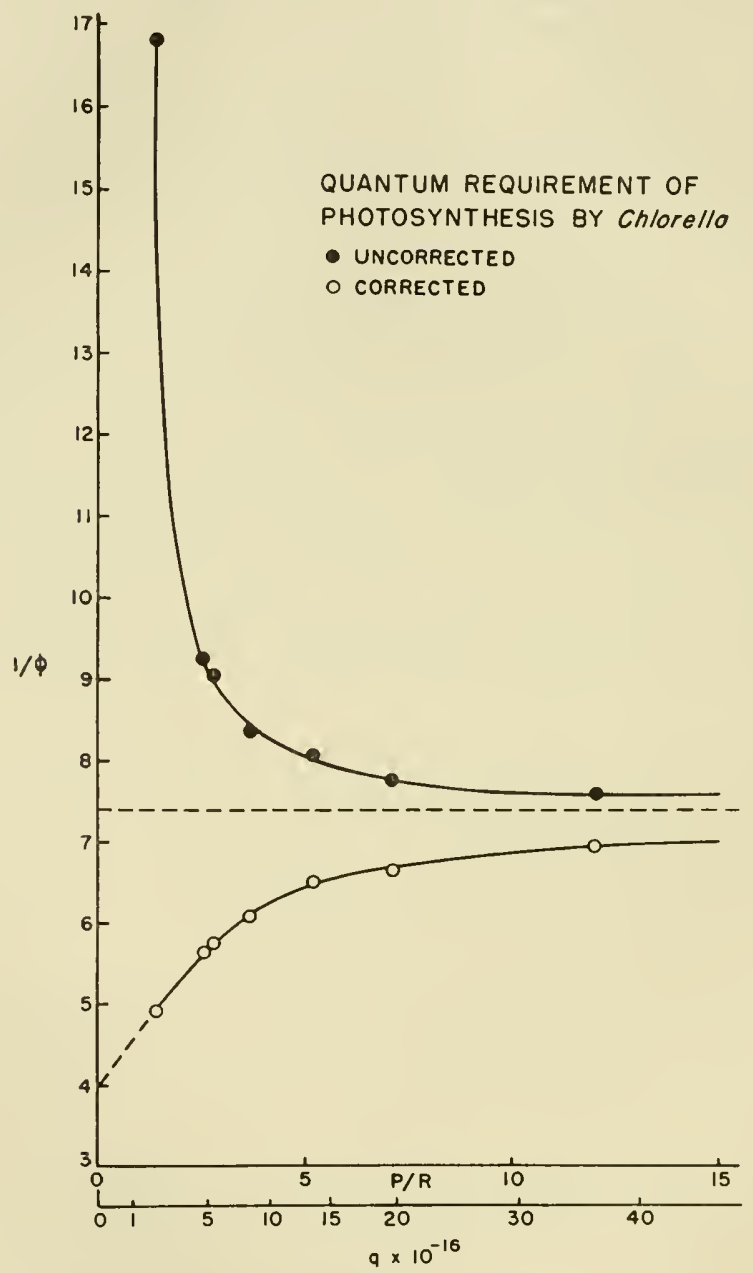

Fig. 4. Relation between quantum requirement in photosynthesis and the ratio of photosynthesis to respiration rate.

of respiration over the rate required by the plants while resting in the dark. 


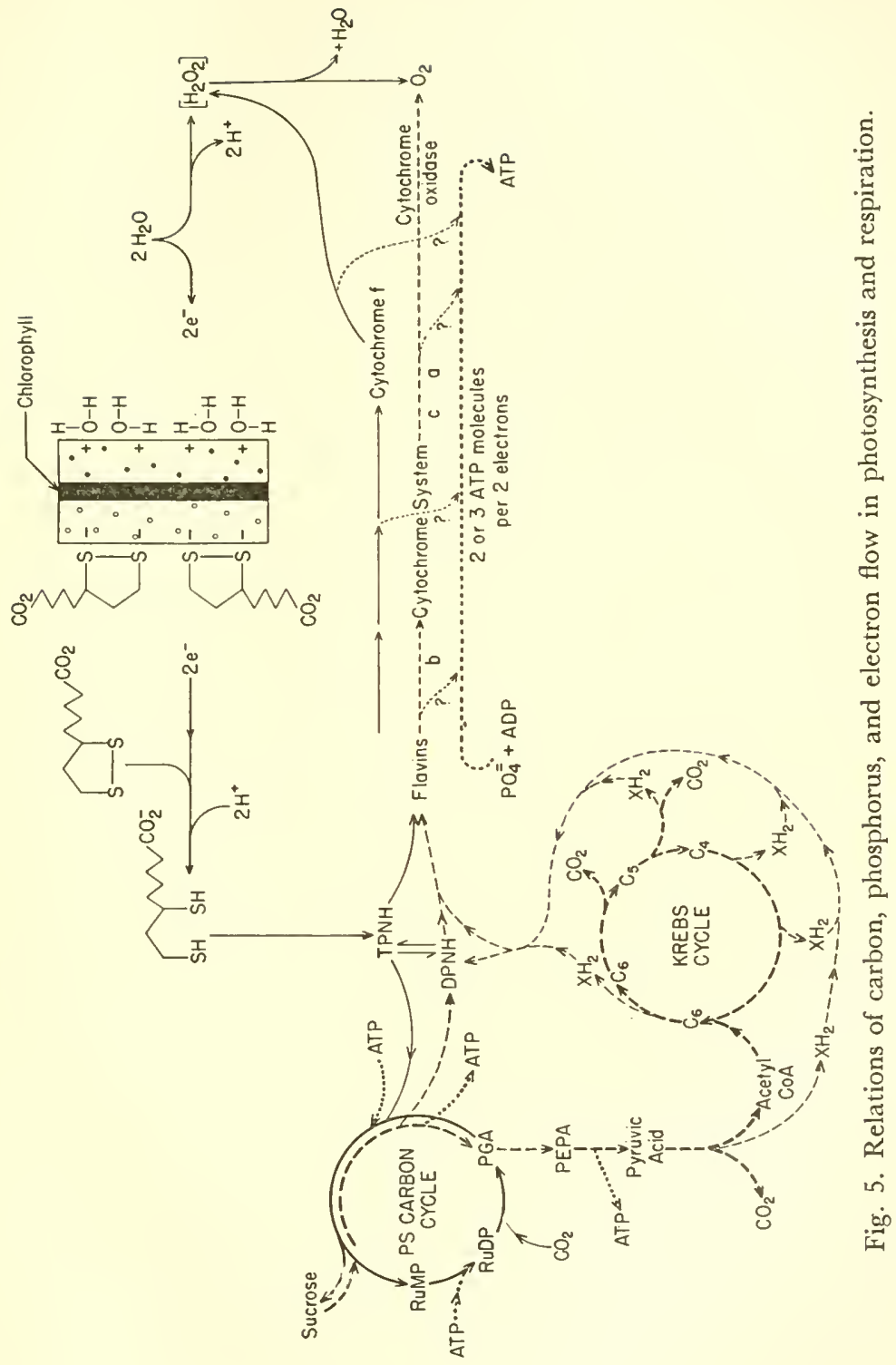


If each of the experimentally determined quantum requirements is plotted against the ratio of photosynthesis to respiration $(p / r)$, an interesting result is obtained. At high ratios of $p / r$, where it would be expected that respiration could contribute relatively little of the ATP required for photosynthesis, both corrected and uncorrected quantum requirements approach the same value, about 7.4. At low values of $p / r$, where respiration could contribute relatively more ATP, the corrected quantum requirement approaches four while the uncorrected quantum requirement becomes very great. This result, shown in Figure 4 , lends credence to the theory that the two molecules of primary reductant required for the reduction of one molecule of $\mathrm{CO}_{2}$ are generated by four quanta but that when the ATP required for the reduction of $\mathrm{CO}_{2}$ must be formed by reactions consuming the reducing agent, there is a net requirement of about six or seven quanta for each $\mathrm{CO}_{2}$ molecule reduced.

When the reduction in quantum requirement at low light intensities is multiplied by the total number of molecules of oxygen evolved and when the product (number of quanta "saved" by respiration) is compared with the enhancement of respiration due to photosynthesis, it is found that about seven quanta are "saved" for each extra molecule of oxygen taken up by photosynthesis enhancement of respiration over the resting dark respiration. Since about seven molecules of ATP are produced by each molecule of oxygen consumed in respiration, this result is consistent with the theory that respiration contributes energy to photosynthesis in the form of the reactivity of ATP and is also consistent with the requirement discussed earlier, of about one quantum for each molecule of ATP formed by burning photochemically produced reducing agent.

The relationships of energy transfer in respiration and in photosynthesis are shown in Figure 5. It will be seen that in this scheme the principal "innovations" required for photosynthesis as compared with respiration are the photochemical "battery" and the use of perhaps two specialized electron carriers, thioctic acid and cytochrome $f$. As more is known about details of the 
energy-transferring processes, we may expect that additional special steps will be found.

\section{References}

1. Allen, F. L., Doctoral Thesis, University of Chicago, Chicago, Illinois, 1953.

2. Arnon, D. I., M. B. Allen, and F. R. Whatley, Nature, 174, 394 (1954).

3. Barltrop, J. A., P. M. Hayes, and M. Calvin, J. Am. Chem. Soc., 76, 4348 (1954).

4. Bassham, J. A., A. A. Benson, L. D. Kay, A. Z. Harris, A. T. Wilson, and M. Calvin, J. Am. Chem. Soc., 76, 1760 (1954).

5. Bassham, J. A., K. Shibata, and M. Calvin, Biochim. et Biophys. Acta, 17, 332 (1955).

6. Benson, A. A., M. Calvin, V. A. Haas, S. Aronoff, A. G. Hall, J. A. Bassham, and J. W. Weigl, in J. Franck and W. E. Loomis, eds., Photosynthesis in Plants, p. 381. Iowa State College Press, Ames, Iowa, 1949.

7. Boychenko, E. A., and V. I. Baranov, Doklady Akad. Nauk. S. S. S. R., 95, 1025 (1954).

8. Brackett, F. S., R. A. Olson, and R. G. Crickard, J. Gen. Physiol., 36, 529 (1953).

9. Bradley, D. F., and M. Calvin, Arch. Biochem. and Biophys., 53, 99 (1954).

10. Brown, A. H., Am. J. Botany, 40, 719 (1953).

11. Brown, A. H., and J. A. Johnston, Plant Physiol., 29, 177 (1954).

12. Brown, A. H., A. O. C. Nier, and R. W. van Norman, Plant Physiol., 27, 320 (1952).

13. Brown, A. H., and G. C. Webster, Am. J. Botany, 40, 753 (1953).

14. Bullock, M. W., J. A. Brockman. Jr., E. L. Patterson, J. V. Pierce, and E. L. R. Stokstad, J. Am. Chem. Soc., 74, 3455 (1952).

15. Burton, K., and T. H. Wilson, Biochem. J. (London), 54, 86 (1953).

16. Calvin, M., and J. A. Barltrop, J. Am. Chem. Soc., 74, 6153 (1952).

17. Galvin, M., J. A. Bassham, A. A. Benson, and P. Massini, Ann. Rev. Phys. Chem., 3, 215 (1952).

18. Calvin, M., and Peter Massini, Experientia. 8, 445 (1952).

19. Chance, B., in J. T. Edsall, ed., Enzymes and Enzyme Systems, p. 93. Harvard University Press, Cambridge, Mass., 1951.

20. Chance, B., Abstracts of the American Chemical Society Meetings, New York, Fall, 1954, p. 1F.

21. Chance. B., in W. D. McElroy and B. Glass, eds., The Mechanism of Enzyme Action, p. 399. Johns Hopkins Press, Baltimore, 1954.

22. Chapin, D. M., C. S. Fuller, and G. L. Pearson, J. Appl. Phys., 25, 676 (1954). 
23. Dam, H., Advances in Enzymol., 2, 285 (1942).

24. Duysens, L. N. M., Nature, 168, 548 (1951).

25. Emerson, R., and C. E. Lewis, Am. J. Botany, 30, 165 (1943).

26. Franck, J., Ann. Rev. Plant Physiol., 2, 53 (1951).

27. French, C. S., and H. W. Milner, Symposia Soc. Exptl. Biol., 5, 232 (1951).

28. Frenkel, A., J. Am. Chem. Soc., 76, 5568 (1954).

29. Gaffron, H., Biochem. Z., 292, 241 (1937).

30. Gaffron, H., Autotrophic Microorganisms, p. 152. Cambridge University Press, Cambridge, England, 1954.

31. Gaffron, H., and E. W. Fager, Ann. Rev. Plant Physiol., 2, 87 (1951).

32. Gerretsen, F. C., Plani and Soil, 3, 1 (1951).

33. Gunsalus, I. C., L. Struglia, and D. I. O'Kane, J. Biol. Chem., 194, 859 (1952).

34. Hill, R., Advances in Enzymol., 12, 1 (1951).

35. Hill, R., and R. Scarisbrick, New Phytologist, 50,98 (1951).

36. Hill, R., and C. P. Whittingham, Photosynthesis. Methuen, London, 1954.

37. Jacobs, E. E., and A. S. Holt, J. Chem. Phys., 20, 1326 (1952).

38. Lehninger, A. L., in W. D. McElroy and B. Glass, eds., Phosphorus Metabolism, Vol. 1, p. 344. Johns Hopkins Press, Baltimore, 1951.

39. Levitt, L. S., Science, 120, 33 (1954).

40. Leyon, H., Exptl. Cell Research, 7, 265 (1954).

41. Lumry, R., J. D. Spikes, and H. Eyring, Ann. Rev. Plant Physiol., 5, 271 (1954).

42. Milner, H. W., C. S. French, M. L. G. Koenig, and N. S. Lawrence, Arch. Biochem., 28, 193 (1950).

43. Milner, H. W., M. L. G. Koenig, and N. S. Lawrence, Arch. Biochem., 28, 185 (1950).

44. Patterson, E. L., J. A. Brockman, Jr., F. P. Day, J. V. Pierce, M. E. Macchi, C. E. Hoffman, C. T. O. Fong, E. L. R. Stokstad, and T. H. Jukes, J. Am. Chem. Soc., 73, 5919 (1951).

45. Quayle, J. R., R. C. Fuller, A. A. Benson, and M. Calvin, J. Am. Chem. Soc., 76, 3610 (1954).

46. Rabinowitch, E. I., Photosynthesis and Related Processes, Vols. 1 and 2 , part 1. Interscience, New York, 1945, 1951.

47. Rabinowitch, E. I., Ann. Rev. Plant Physiol., 3, 299 (1952).

48. Reed, L. J., and B. G. DeBusk, J. Am. Chem. Soc., 74, 3457 (1952).

49. Reed, L. J., B. G. DeBusk, I. C. Gunsalus, and G. H. Schnakenberg, J. Am. Chem. Soc., 73, 5920 (1951).

50. Riegel, B., P. G. Smith, and C. E. Schweitzer, J. Am. Chem. Soc., 62, 992 (1940).

51. Rodrigo, F. A., Biochim. et Biophys. Acta, 70, 342 (1953). 
52. Scheibe, G., Z. Elektrochem., 52, 283 (1948).

53. Stoppani, A. O. M., R. C. Fuller, and M. Calvin, J. Bacteriol., 69, 491 (1955).

54. Strehler, B. L., Arch. Biochem. and Biophys., 43, 67 (1953).

55. Tamiya, H., Studies of Tokugawa Inst. (Tokyo), 6, 1 (1949).

56. Thomas, J. B., O. H. Blaauw, and L. N. M. Duysens, Biochim. et Biophys. Acta, 10, 230 (1953).

57. Thomas, J. B., L. C. Post, and N. Vertrect, Biochim. et Biophys. Acta, 13, 20 (1954).

58. van Niel, C. B., Bacteriol. Revs., 8, 1 (1944).

59. Voskrenskaya, N. P., Duklady Akad. Nauk. S. S. S. R., 93, 911 (1953).

60. Warburg, O., G. Krippahl, W. Buchholz, and W. Schroder, Z. Naturforsch., 8(b), 675 (1953).

61. Warburg, O., G. Krippahl, and W. Schroder, Z. Naturforsch., 9(b), 667 (1954).

62. Weigl, J. W., P. M. Warrington, and M. Calvin, J. Am. Chem. Soc., 73, 5058 (1951).

63. Weissbach, A., P. Z. Smyrniotis, and B. L. Horecker, J. Am. Chem. Soc., 76,5572 (1954).

64. Wessels, J. S. C., Rec. trav. chim., 73, 529 (1954).

65. Whittingham, C. P., Botan. Rev., 18, 245 (1952). 


\section{BACTERIAL FERMENTATIONS}

H. A. BARKER, Department of Plant Biochemistry, University of California, Berkeley. California

Early interest in bacterial fermentations was stimulated mainly by a need to determine the chemical changes occurring in food, soil, and other materials under anaerobic conditions or to discover and produce compounds that might have industrial applications. Abundant information on these aspects of fermentation has accumulated over the years. More recently, the center of interest has shifted to the analysis of the chemical mechanism of fermentations, since anaerobic bacteria frequently provide relatively simple and convenient systems for the study of basic metabolic processes.

The usefulness of bacteria for metabolic studies is dependent in part upon two characteristic properties, the high rate and the extraordinary degree of specialization of their energy-generating mechanisms. These properties are perhaps even more highly developed in anaerobic than in aerobic bacteria. They facilitate the analysis of problems of intermediary metabolism by providing a biological material that is relatively free of complicating side reactions.

Although individual species use highly specialized catabolic reactions, anaerobic bacteria as a group possess the ability to 
attack a great variety of substrates including carbohydrates, polyalcohols, amino acids, purines, pyrimidines, and many organic acids. Consequently they provide a wide range of specialized catabolic processes for study.

\section{Fermentation Patterns}

Not all organic compounds are fermentable. The question arises as to why some compounds are utilized by anaerobic bacteria and others are not. No answer is possible in terms of molecular structure because the fermentability of a compound depends both on its structure and on the enzymatic make-up of the organism to which it is offered. A partial answer can be given only in terms of the nature of the reactions which a compound can undergo in a particular enzymatic system.

An essential but not always sufficient requirement for fermentability is that the compound or products derived from it must be able to serve both as a reductant and as an oxidant. Furthermore, the potential difference between the oxidant and reductant systems must be great enough $(\bar{\Sigma} 0.25 \mathrm{v}$.$) to provide$ the energy necessary to synthesize the various structural and functional components of the cell from simple molecules. Since most biologically important organic compounds are susceptible to enzymatic oxidation, the more unique requirement for fermentability is the formation of a suitable oxidant system.

The oxidizing and reducing systems of bacterial fermentations are provided in several more or less distinct ways. In the simpler fermentations the substrate itself or a phosphorylated derivative is both oxidized and reduced. Examples of this are the dismutation of pyruvate to lactate, acetate, and carbon dioxide by some lactic acid bacteria, and the fermentation of glycerol by Escherichia freundii, in which the oxidation of glycerophosphate to dihydroxyacetonephosphate and other subsequent products is coupled with the reduction of glycerol to trimethylene glycol (22). In a few fermentations the substrate is converted to two different compounds, one of which is oxidized and the 
other reduced. This is true in sugar fermentations by lactic acid bacteria which produce glycerol. The dihydroxyacetonephosphate initially derived from carbons 1 to 3 of glucose presumably is reduced, and the glyceraldehyde initially derived from carbons 4 to 6 is oxidized.

A fermentation pattern more frequently encountered is that in which part or all of the substrate, usually after some preparatory reactions, is oxidized and then is transformed into an hydrogen acceptor. The simple lactic acid fermentation of glucose is of this type; glyceraldehydephosphate is oxidized and the product is converted to pyruvate, which is reduced to lactate. A variation of this pattern is known to occur in two fermentations in which the reduction precedes rather than follows the oxidation in the path of substrate degradation. This situation is encountered in the fermentations of uric acid (30) and of orotic acid (18). In the former, the first step is the reduction of uric acid to xanthine, which is probably coupled with oxidation of glycine and pyruvate derived from xanthine. In the orotic acid fermentation, an initial reduction of orotic acid to dihydroorotic acid is coupled with the oxidation of an as yet undetermined compound.

Besides the true fermentations just described, several other closely related catabolic processes sometimes referred to as "anaerobic oxidations" are utilized by anaerobic bacteria. These processes are similar to the true fermentations in that they provide energy for growth, and they frequently produce acids, gases, and odors characteristic of fermentations. They differ from true fermentations in that two major substrates are required, one to serve as an oxidant, the other as a reductant. The latter is usually an organic compound, although hydrogen gas and carbon monoxide are used as reductants by some species. The oxidant may be either an organic or an inorganic compound according to the nature of the organism. The best known example of the use of organic oxidants is in the so-called Stickland reaction (23) catalyzed by Clostridium sporogenes and several other clostridia. In this reaction an amino acid such as alanine or 
valine is oxidized, and another amino acid such as glycine or proline is reduced.

The main inorganic oxidants used by anaerobic bacteria are nitrate, sulfate, and carbonate. The ability to use one of these compounds as a major oxidant is generally restricted to specialized groups of bacteria. Nitrate is reduced to nitrous oxide and nitrogen by denitrifying bacteria, sulfate is converted to hydrogen sulfide by sulfate-reducing bacteria, and carbon dioxide is reduced either to methane by the strictly anaerobic methane-producing bacteria (32) or to acetic acid by organisms such as C. aceticum (37) and C. thermoaceticum (38). Inorganic oxidants are advantageous in that they make possible the utilization of a wider variety of organic compounds, and the more complete oxidation of individual substrates, than is possible in a true fermentation.

\section{Selection of Organisms}

The selection of an organism capable of attacking a given compound or performing a particular reaction sequence can often be made by consulting a reference book on systematic bacteriology. However, because bacteriologists have generally shown a strong preference for the use of a limited number of substrates, of which the sugars are most prominent, it sometimes happens that no organism is known which possesses a highly developed ability to attack a particular compound in which an investigator is interested. Under these circumstances a suitable organism frequently may still be obtained by use of the enrichment culture method.

The advantages of the enrichment culture method, originally developed by Winogradsky and Beijerinck in the last century, appear not to be widely recognized; certainly, the method has not been applied as extensively as might be desirable. The method depends on the principle that organisms capable of decomposing a given compound can be obtained from a mixed population such as that in soil by using a culture medium in 
which the compound in question constitutes the main energy source. In such a medium only organisms that can multiply at the expense of the added substrate become numerous. Aerobic or anaerobic species can be selected by supplying or removing oxygen, and other conditions such as the nitrogen source, the $p \mathrm{H}$, or the temperature may be varied so as to favor specific groups of bacteria. By making several transfers in the appropriate medium, organisms with the desired substrate specificity and environmental requirements can be "enriched" to the extent that their isolation in pure culture can be achieved by conventional methods.

The application of the enrichment culture method to the study of the fermentation of nitrogenous compounds, for example, has resulted in the isolation of several previously unknown bacteria that are potentially useful for metabolic studies. With uric acid as an enrichment substrate under anaerobic conditions, two closely related purine-fermentating bacteria, Clostridium acidi-urici and C. cylindrosporum, can be isolated from soil (3). These bacteria show a high degree of biochemical specialization, since they decompose uric acid, xanthine, and guanine with great vigor but do not attack a variety of other common substrates (2). Enrichment cultures with orotic acid have yielded an organism, Zymobacterium oroticum, which has been used to study the enzymatic synthesis and degradation of the pyrimidine ring (18,35). Other bacteria fermenting uracil, thymine, or allantoin have been obtained by the same method. A number of amino acid fermenting bacteria have also been isolated from enrichment cultures. Examples of such organisms are Clostridium tetanomorphum, which attacks histidine and glutamate (34), C. propionicum, which ferments alanine, serine, and threonine (8), and Diplococcus glycinophilus, which uses only glycine and certain of its di- and tripeptides (8).

Many similarly specialized bacteria, fermenting other compounds, undoubtedly can be obtained by the enrichment method. It is a useful tool with which to isolate organisms favorable for the study of a variety of biochemical reactions.

The remainder of this article will be devoted to a discussion 
of the way in which our knowledge of specific aspects of some bacterial fermentations has been arrived at.

\section{Initial Stages of Carbohydrate Fermentation}

One of the most significant developments in the field of fermentation biochemistry in the past few years has been the recognition of the existence of several nonglycolytic pathways of carbohydrate breakdown. Some indications of nonglycolytic pathways were obtained long ago by balance experiments, but only since the application of tracer techniques has the evidence for such pathways become conclusive and generally been recognized.

Two fermentative bacteria have been shown to use predominantly nonglycolytic pathways, namely Leuconostoc mesenteroides and Pseudomonas lindneri.

Leuconostoc mesenteroides catalyzes a so-called heterolactic fermentation of glucose, the main products being equimolar amounts of lactic acid, ethanol, and carbon dioxide. The significant feature of this fermentation, from the point of view of its mechanism, is the constancy in the yields of the three products. In other bacterial fermentations giving the same products, the ratio of lactate to ethanol, for example, varies considerably with $p \mathrm{H}$ and other environmental factors. In the Leuconostoc fermentation, on the contrary, half of the glucose is always converted to lactate and half to ethanol and carbon dioxide. This result is inconsistent with the glycolytic mechanism, in which both halves of the glucose molecule are funneled through a common intermediate, pyruvate, which can be converted either to lactate or to ethanol.

More definitive evidence for a nonglycolytic mechanism of glucose fermentation in Leuconostoc was obtained by Gunsalus and Gibbs (14). They found that the fermentation of glucose$1-\mathrm{C}^{14}$ gives labeled carbon dioxide, and glucose-3,4- $\mathrm{C}^{14}$ gives carboxyl-labeled lactate and ethanol-1- $\mathrm{C}^{14}$. Their data indicate that the carbon dioxide is derived from glucose carbon 1, the methyl and carbinol groups of ethanol from glucose carbons 2 
and 3, respectively, and the lactate carbons, starting with the carboxyl group, from carbons 4, 5, and 6 of glucose. The detailed mechanism of glucose fermentation by Leuconostoc has not yet been worked out, but there is considerable evidence that glucose-6-phosphate and 6-phosphogluconate are intermediates.

The alcoholic fermentation of $P$. lindneri differs from that of yeast in respect to the fate of the first three carbons of glucose (13). Whereas in yeast glucose carbons 1 and 2 are converted to ethanol and carbon 3 to carbon dioxide, in the $P$. lindneri fermentation carbon 1 goes to carbon dioxide and carbons 2 and 3 to ethanol. The carbinol carbon of ethanol is derived from carbon 2 of glucose, in contrast to the Leuconostoc fermentation in which the methyl carbon of ethanol comes from the carbon 2 of glucose. The carbon distribution pattern of the $P$. lindneri fermentation is sufficiently similar to that observed in glucose oxidation by Pseudomonas saccharophila to suggest that the underlying mechanisms of the two processes may be the same. Glucose-6-phosphate, 6-phosphogluconate, and 2-keto, 3-deoxy, 6-phosphogluconate have been shown to be intermediates in glucose breakdown by $P$. saccharophila $(11,20)$.

Two other fermentative bacteria that use nonglycolytic mechanisms to some extent are Escherichia coli and Propionibacterium pentosaceum. E. coli possesses enzymes catalyzing both the glycolytic (10) and the nonglycolytic ribulose-phosphate $(29,31)$ mechanisms of glucose breakdown. The available evidence indicates that the glycolytic path is used for anaerobic decomposition of sugar, whereas both paths are used in the oxidation of glucose. The experiments of Cohen (9) with glucose$1-\mathrm{C}^{14}$ indicate that at least 14 to 37 per cent of the glucose decomposed aerobically is metabolized via the pentose pathway. The remainder of the glucose presumably is oxidized by the glycolytic path, although specific and conclusive evidence for this has not been obtained.

Propionic acid bacteria probably use both glycolytic and nonglycolytic pathways of glucose fermentation to a significant extent. Evidence for the glycolytic pathway includes demon- 
strations of the phosphorylation of glucose by ATP (4) and the conversion of glucose or fructose-1,6-diphosphate to phosphoglycerate by dried or toluene-treated cell suspensions (36), and the conversion of phosphoglycerate to the normal fermentation products by proliferating cells. More direct evidence for the distinctive steps of the glycolytic pathway, the formation of fructosediphosphate and its conversion to triose phosphate by the aldolase reaction, is required, particularly in view of the fact that all previous studies with propionic acid bacteria have been done with crude enzyme preparations. Alternate interpretations of the experimental data are therefore possible. For example, the observation that toluene-treated cell suspensions converted fructose-1,6-diphosphate to phosphoglycerate in the presence of fluoride and an oxidant, does not definitely establish the existence of the aldolase reaction. An alternative interpretation is that fructosediphosphate is converted to glucose-6phosphate which is oxidized to phosphoglycerate via phosphogluconate, pentose phosphates, and glyceraldehyde phosphate.

The recent tracer experiments of Leaver and Wood (17) have demonstrated rather conclusively that the glycolytic path is not the only mechanism of glucose fermentation by propionic acid bacteria. The fermentation of glucose-1- $\mathrm{C}^{14}$ gave carbon dioxide with a higher specific activity than that of any carbon of the other products. The quantitative data indicate that at least 20 per cent and possibly considerably more of the glucose is decomposed by oxidation of carbon 1 to carbon dioxide, as would occur in a nonglycolytic pathway. The fermentation of glucose-3,4- $\mathrm{C}^{14}$ resulted in the appearance of $\mathrm{C}^{14}$ in noncarboxyl positions of acetate, propionate, and succinate. This result, when considered in conjunction with other information concerning the propionic acid fermentation, is inconsistent both with a glycolytic pathway and with the Leuconostoc mesenteroides and ribose phosphate pathways of glucose breakdown. The isotope distribution data can be interpreted in terms of glucose oxidation by the 2-keto-3-deoxy-6-phosphogluconate pathway of Pseudomonas saccharophila (20). However, no specific information is 
available concerning the occurrence of this pathway in propionic acid bacteria.

The glycolytic pathway is probably used as a fermentation mechanism by several bacteria, in addition to $E$. coli. For example, several reactions of this pathway have been demonstrated with Streptococcus faecalis (24), particularly the formation and decomposition of fructose diphosphate. Clostridium perfringens has been shown to contain the enzyme aldolase, indicative of a glycolytic mechanism (1). With several other bacteria, such as C. thermoaceticum (39), Lactobacillus casei (12), and Butyribacterium rettgeri (25), the distribution patterns of $\mathrm{C}^{14}$ in products obtained from the fermentation of $\mathrm{C}^{14}$-labeled glucose indicate a predominantly glycolytic pathway, although this has not yet been verified by critical enzymatic experiments.

The examples given above demonstrate that at least three different pathways exist for the anaerobic decomposition of glucose to three-carbon compounds by bacteria. There is no reason to believe that these are the only such pathways. Of the many anaerobic bacteria that are known, only a very few have been studied extensively enough so that any definite conclusion can be reached concerning their mechanisms of glucose decomposition. Also virtually nothing is known about the energyutilizing mechanisms that are associated with nonglycolytic sugar decomposition. Only a good beginning has been made in the study of this fundamental aspect of bacterial fermentations.

The discovery of several alternate pathways of sugar fermentation and other biochemical processes has had a considerable effect on the comparative biochemical point of view. Not long ago, many microbiologists in particular believed that all bacteria are built on essentially the same basic metabolic pattern. The conspicuous differences in fermentation products which were known to exist were thought to be attributable to differences in relative rates of various reactions or to the omission or addition of specific enzymatic steps. The possibility that a given process, such as the conversion of glucose to pyruvate, might occur by more than one mechanism was not seriously 
considered; the existence of two such pathways appeared superfluous and even unreasonable. Therefore the discovery of various alternative pathways for single processes has required a revision of the concept of a basic metabolic pattern in terms of processes rather than in terms of specific chemical mechanisms.

It may also be noted that alternate metabolic pathways provide a useful set of characters for the analysis of phylogenetic relationships among microorganisms. Past attempts to deduce phylogenetic relationships from gross morphology or fermentation product patterns have not been particularly successful, in part because the numbers of characters available were too restricted. This difficulty at least can be overcome by the comparison of complex metabolic pathways which provide many enzymatic steps linked together in process patterns that probably represent the culmination of long sequences of evolutionary development.

\section{Butyric Acid Fermentation}

Clostridium kluyveri is a good example of an anaerobic bacterium that has been useful in the study of a fundamental biochemical process, namely, the synthesis of fatty acids (5). This organism was discovered more or less fortuitously while making enrichment cultures for methane-producing bacteria with ethyl alcohol as the sole organic substrate. Examination of the fermentation products in such cultures revealed that butyric and caproic acids were frequently formed in large yields along with acetic acid, and were always associated with the presence of a clostridium later called $C$. kluyveri. The formation of these acids from ethyl alcohol provided direct confirmation for the old theory that fatty acids containing an even number of carbon atoms are built up from a $\mathrm{C}_{2}$ compound.

Further study of the substrate requirements for butyrate synthesis by pure cultures of $C$. kluyveri demonstrated that both ethanol and acetate are essential for the process, which occurs stoichiometrically according to the equation $\mathrm{CH}_{3} \mathrm{CH}_{2} \mathrm{CH}_{2} \mathrm{COOH}+\mathrm{H}_{2} \mathrm{O}$ 
Tracer experiments indicated that the ethanol is oxidized to the acetate level before being converted to butyrate. Thus acetate or an activated derivative was identified as a major precursor of butyrate. Later, Lynen's acetyl-coenzyme A (19), formed from acetaldehyde and coenzyme A by a DPN-dependent oxidation, was shown to be the activated form of acetate.

Even before the isolation of acetyl-CoA by Lynen, the evidence for the existence of such a compound was greatly strengthened by Stadtman's discovery that the exchange of inorganic phosphate with acetyl phosphate in $C$. kluyveri extracts is catalyzed by an enzyme, phosphotransacetylase, which is coenzyme A dependent (33). This exchange could be most easily interpreted by means of the following reaction

acetyl phosphate $+\mathrm{CoA} \longrightarrow$ acetyl-CoA + phosphate

which was subsequently shown to occur. The phosphotransacetylase reaction has been very useful in enzymatic studies of acetate metabolism, since it provides a convenient method of forming acetyl-CoA.

A central problem in the metabolism of fatty acids was the identity of the intermediates between acetate and butyrate. Early investigations of fatty acid metabolism in animals suggested that acetoacetate and $\beta$-hydroxybutyrate might be involved, but conclusive evidence for or against the participation of these compounds was not forthcoming until the development of cell-free enzyme systems that metabolize fatty acids. With extracts of $C$. kluyveri, acetoacetate, $\beta$-hydroxybutyrate, and other possible $\mathrm{C}_{4}$ compounds in the same oxidation states were shown definitely not to be intermediates in the interconversion of acetate and butyrate (16). This and other evidence led to the development of the idea that the intermediates are not simple $\mathrm{C}_{4}$ molecules but are $\mathrm{C}_{4}$-coenzyme compounds. This hypothesis has been fully confirmed by the discovery of the role of the acetyl, butyryl, crotonyl, $\beta$-hydroxybutyryl, and acetoacetyl derivatives of coenzyme $\mathrm{A}$ in both animal and bacterial systems $(19,21,33)$. 
Although many of the steps in the enzymatic conversion of ethanol and acetate to butyrate and caproate are now known, our understanding of the role of this process in the energy transformation of the bacteria is very inadequate. Presumably the formation of the $\mathrm{C}_{4}$ and $\mathrm{C}_{6}$ fatty acids, which involves a freeenergy change of approximately $12 \mathrm{kcal}$. per mole of ethanol consumed, constitutes the main source of energy for synthetic activities of the organism. This implies that the catabolic reaction should result in a net formation of high-energy phosphate or other suitable energy donor. But as yet there is no evidence that this occurs. The oxidation of acetaldehyde to acetyl-CoA yields a high-energy thioester bond which can be converted to a high-energy phosphate bond by the phosphotransacetylase reaction. However, the thioester bond is not available for this purpose, since it is required for the formation of acetoacetyl-CoA from acetyl CoA.

There appear to be two ways in which useful energy might be provided during the $C$. kluyveri fermentation. One is by an oxidation of acetaldehyde which is not coupled with butyrate synthesis but with the formation of hydrogen gas as illustrated in the following reaction, where $\mathrm{Pi}$ represents phosphate:

$$
\mathrm{CH}_{3} \mathrm{CHO}+\mathrm{Pi}+\mathrm{ADP} \longrightarrow \mathrm{CH}_{3} \mathrm{COOH}+\mathrm{ATP}+\mathrm{H}_{2}
$$

It should be noted that hydrogen is formed in appreciable amounts during the C. kluyveri fermentation. The free-energy change is unfavorable (approximately $4 \mathrm{kcal}$.) for the above reaction to proceed from left to right as written. However, by coupling this reaction with strongly exergonic reactions of ATP, such as the hexokinase reaction, which are involved in the synthesis of cellular constituents, the over-all reaction would be slightly exergonic (approximately $-2 \mathrm{kcal}$.).

A second possible mechanism for net ATP formation in the C. kluyveri fermentation is an oxidative phosphorylation dependent on electron transport from ethanol and acetaldehyde to the unsaturated fatty acid electron acceptors such as crotonylCoA. The potential difference between the ethanol-acetalde- 
hyde and the butyryl-CoA-crotonyl-CoA system (21) appears to be of the order of $0.2 \mathrm{v}$., which is more than enough to permit the formation of one high-energy phosphate bond from orthophosphate. Such a coenzyme-linked phosphorylation could account for the importance of butyrate and caproate synthesis in the metabolism of $C$. kluyveri. However, as yet there is no evidence for such a mechanism. In fact, it has not been possible to observe a reduction of crotonyl compounds by reduced DPN and TPN, which are formed in the oxidation of ethanol and acetaldehyde.

\section{Purine Fermentations}

The fermentation of purines by $C$. acidi-urici and $C$. cylindrosporum presents some interesting and as yet partially unsolved biochemical problems.

The main products of the fermentation of uric acid, xanthine, or guanine are ammonia, carbon dioxide, and acetate, sometimes accompanied by small amounts of formate and glycine $(2,27)$. Early experiments with cell suspensions indicated that the mechanism of the purine fermentation is quite different from that of uric acid oxidation via allantoin in animals and aerobic bacteria. This conclusion was based in part on the observation that neither allantoin nor urea is decomposed under conditions in which uric acid is rapidly fermented.

The first definite indication of the path of uric acid breakdown was the discovery that glycine is formed in appreciable amounts and is also decomposed when uric acid is simultaneously available to the organism (2). The dependence of glycine decomposition on the presence of uric acid suggested some sort of interaction between the two substances. This was further supported by the observation that certain cell suspensions of $C$. acidi-urici exhibit a conspicuous lag in uric acid decomposition, which can be eliminated completely by the addition of glycine. Later work (30) suggests that the oxidation of glycine is coupled with a reduction of uric acid to xanthine. 
The formation of glycine and formate from purines points to a similarity between the mechanisms of the bacterial fermentation and the synthesis of purines in animals. This similarity has been further defined by the use of tracer methods $(15,26)$. Nitrogen atoms in the 1, 3, and 9 positions of the purine have been shown to be converted to ammonia, and the nitrogen in position 7 appears in glycine. Carbon from positions 2 and 6 goes to carbon dioxide, from positions 4 and 5 to the carboxyl and methylene carbons of glycine, and from position 8 to formate. The only conspicuous difference between the fermentation and synthetic processes with respect to the tracer data is in the fate or source of the carbon in position 2. In the uric acid of the pigeon this carbon atom is derived from formate, whereas in the fermentation it is converted to carbon dioxide by a path not involving formate. This suggests that the purine which is actually degraded by the bacterial system, as contrasted with the purine added as a substrate, is more oxidized in the 2 position than is the hypoxanthine ribotide which appears to be the first purine derivative synthesized from nonpurine precursors in animals (7).

Several types of evidence, which will not be given here, indicate that uric acid, guanine, and hypoxanthine are converted to xanthine before the purine ring structure is disrupted. The decomposition of xanthine has been studied with crude cell-free extracts $(6,27,30)$, and has been shown to proceed in accordance with the following equation

1 xanthine $+6 \mathrm{H}_{2} \mathrm{O}$

3 ammonia +2 carbon dioxide +1 formate +1 glycine

which represents a nonoxidative process. Unlike intact cells, the cell-free extracts used in these experiments do not form appreciable amounts of acetate.

A question of special interest in xanthine decomposition is the point of attack on the purine molecule. Previous studies on purine synthesis in birds indicated that the ribotide of 4-amino5-carboxamidoimidazole combines with "formate" to yield a 
hypoxanthine derivative. If a similar but reverse process occurs in the fermentation of xanthine, an initial attack on the bonds between the 1,2 or 2,3 positions in the purine would be required. Actually all available evidence is against an initial splitting of these bonds. For example, 4-amino-5-carboxamidoimidazole is not attacked nor does it accumulate under conditions favorable

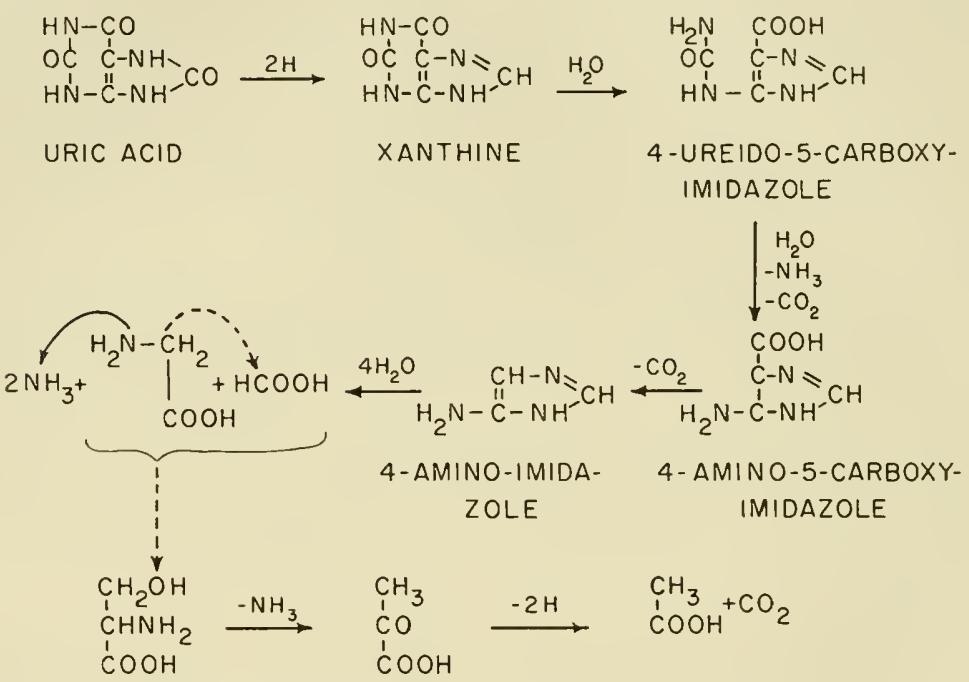

Fig. 1. Fermentation of uric acid. The solid arrows indicate known reactions, the dotted arrows postulated reactions.

for xanthine decomposition. Moreover, in the presence of suitable inhibitors the major product of xanthine decomposition by extracts has been identified as 4-ureido-5-carboxyimidazole (28) which is formed by a rupture of the bond between the 1 and 6 positions. The ureido group of this compound is next attacked enzymatically with the formation of ammonia, carbon dioxide, and 4-amino-5-carboxyimidazole (27). The latter is decarboxylated and then the imidazole ring of the resulting 4-amino derivative is cleaved to give glycine, ammonia, and formate in the cell-free system. This is undoubtedly a multistep process, the details of which have not yet been worked out. 
In the fermentation of purines by living cultures of $C$. acidi-urici, glycine and formate do not accumulate in considerable amounts, whereas acetate is a major product. Tracer experiments have established that both glycine and formate are converted in part to acetate. Formate carbon goes into the methyl group of acetate. The methylene carbon of glycine enters both carbons of acetate, whereas the carboxyl carbon of glycine is converted mainly to carbon dioxide. The mechanism of acetate formation from glycine and formate has not been established definitely, but a pathway involving serine is indicated by indirect evidence (30). Serine is rapidly converted to pyruvate by cell-free extracts and pyruvate is oxidized to acetate and carbon dioxide. Also glycine can be oxidized to acetate.

A schematic representation of uric acid fermentation by $C$. acidi-urici and $C$. cylindrosporum as presently understood is given in Figure 1.

Many aspects of the purine fermentation remain to be elucidated. The steps in the conversion of 4-amino-imidazole to glycine are not known. It is likely that this process involves an "active formate" which facilitates the conversion of glycine or a glycine precursor to serine. Energy useful for synthetic purposes may also be generated during the conversion of xanthine to glycine; otherwise it is difficult to see what purpose this process serves. It is unlikely that this reaction sequence serves only to provide precursors of serine and pyruvate, because these compounds cannot replace purines as fermentation substrates. At present there is no indication of the participation of purine or imidazole nucleotides in the breakdown of purines, but in view of the general similarity between the mechanisms of the fermentation and purine syntheses in animals, a possible role of nucleotides in the purine fermentation should be investigated more closely.

\section{References}

1. Bard, R. C., and I. C. Gunsalus, J. Bacteriol., 59, 387 (1950).

2. Barker, H. A., and J. V. Beck, J. Biol. Chem., 141, 3 (1941). 
3. Barker, H. A., and J. V. Beck, J. Bacteriol., 43, 291 (1942).

4. Barker, H. A., and F. Lipmann, J. Biol. Chem., 779, 247 (1949).

5. Barker, H. A., Harvey Lectures, Ser. 45, 242 (1949-50).

6. Bradshaw, W., and J. V. Beck, Bacteriol. Proc., 86 (1953).

7. Buchanan, J. M., Phosphorus Metabolism, 2, 406 (1952).

8. Cardon, B. P., and H. A. Barker, Arch. Biochem., 12, 165 (1947).

9. Cohen, S. S., Nature, 168, 746 (1951).

10. Elsden, S. R., Enzymes, 2, part 2, 791 (1952).

11. Entner, H., and M. Doudoroff, J. Biol. Chem., 196, 853 (1952).

12. Gibbs, M., R. Dumrose, F. A. Bennett, and M. R. Bubeck, J. Biol. Chem., 184, 545 (1950).

13. Gibbs, M., and R. D. DeMoss, J. Biol. Chem., 207, 689 (1954).

14. Gunsalus, I. C., and M. Gibbs, J. Biol. Chem., 194, 871 (1952).

15. Karlsson, J. L., and H. A. Barker, J. Biol. Chem., 778, 891 (1949).

16. Kennedy, E. P., and H. A. Barker, J. Biol. Chem., 191, 419 (1951).

17. Leaver, F. W., and H. G. Wood, J. Cellular Comp. Physiology, 41, 225 (1952).

18. Lieberman, I., and A. Kornberg, Biuchem. et Biophys. Acta, 12, 223 (1953).

19. Lynen, F., Federation Proc., 12, 683 (1953).

20. MacGee, J., and M. Doudoroff, Bacteriol. Proc., 108 (1954).

21. Mahler, H. R., Federation Proc., 12, 694 (1953).

22. Mickelson, M. N., and C. H. Werkman, J. Bacteriol., 39, 709 (1940).

23. Nisman, B., Bacertiol. Revs., 18, 16 (1954).

24. O'Kane, D. J., and W. W. Umbreit, J. Biol. Chem., 142, 25 (1942).

25. Pine, L., V. Haas, and H. A. Barker, J. Bacteriol., 68, 227 (1954).

26. Rabinowitz, J. R., unpublished data.

27. Rabinowitz, J. R., and H. A. Barker, Federation Proc., 12, 255 (1953).

28. Rabinowitz, J. R., and W. E. Pricer, Jr., Federation Proc., 13, 278 (1954).

29. Racker, E., Federation Proc., 7, 180 (1948).

30. Radin, N. S., and H. A. Barker, Proc. Natl. Acad. Sci. U. S., 39, 1196 (1953).

31. Scott, D. B. M., and S. S. Cohen, Biochem. J. (London), 55, 33 (1953).

32. Stadtman, T. C., and H. A. Barker, J. Bacteriol., 61, 67 (1951).

33. Stadtman, E. R., Record Chem. Progr. (Kresge-Hooker Sci. Lib.), 15, 1 (1954).

34. Wachsman, J. T., and H. A. Barker, J. Bacteriol., 69, 83 (1955).

35. Wachsman, J. T., and H. A. Barker, J. Bacteriol., 68, 400 (1954).

36. Werkman, C. H., R. W. Stone, and H. G. Wood, Enzymologia, 4, 24 (1937).

37. Wieringa, K. T., Leeuwenhoek, J. Microbiol. Serol., 6, 251 (1939-40).

38. Wood, H. G., J. Biol. Chem., 194, 905 (1952).

39. Wood, H. G., J. Biol. Chem., 199, 579 (1952). 


\title{
SOME ASPECTS OF VITAMIN AND GROWTH FACTOR RESEARCH
}

\author{
ESMOND E. SNELL, Biochemical Institute, University of Texas, \\ Austin, Texas
}

The fifteen years since 1940 have been marked by an unprecedented rate of increase in our knowledge of the nature and metabolic role of vitamins and similar growth factors. The perfection of chromatographic methods of purification, together with the partial substitution of rapid microbiological assays for the more tedious bioassay procedures with higher animals, has contributed much to this increase. Detailed accounts of the current status of knowledge of the vitamins have appeared $(54,57,81)$; here no such account will be attempted. Rather shall we be content to emphasize a few aspects of recent nutritional research (and consequently slur others) and their implications for future progress in this field.

\section{Microbiological Techniques in Vitamin Research}

Some fourteen years ago Peterson (43) and Williams (80) emphasized the growing importance of microorganisms as tools in nutritional research by pointing out that work with microorganisms had resulted in discovery of certain of the B-vitamins (pantothenic acid, biotin) and in the first demonstration of the 
nutritional importance of several others ( $p$-aminobenzoic acid, inositol, nicotinic acid). Since 1942, as illustrated in Table I and discussed more fully below, microorganisms (chiefly those of a single group, the lactic acid bacteria) have played a dominant role as test organisms in the discovery and isolation of new vitamins and related factors.

But the discovery, isolation, and chemical characterization of a new vitamin represent only the more glittering facets of a many-sided problem. How is the vitamin distributed in nature? In what forms does it occur? What essential role does it play in metabolism? Investigation of these and related questions also has been immensely furthered by use of microorganisms. Since its introduction by Snell and Strong (71) in 1939 the technique of comparing the growth response of microorganisms to tissue extracts and to pure vitamin as a quantitative method for estimation of vitamins in natural materials (reviews, 2,64,65) has become fully accepted and has increased greatly our knowledge of the distribution of the vitamins in nature. Complications sometimes arise in such assays from the presence of previously unsuspected vitamin derivatives or of metabolically related substances, and the unraveling of such difficulties can provide information relative to the latter two questions posed above.

The study of metabolic roles of the vitamins also has been facilitated greatly through use of artificially induced nutritional mutants of microorganisms (reviews, 13,36) and through detailed study of the action of inhibitors structurally related to vitaminsinhibition analysis (reviews, 58,81). It is the thesis of this essay that bacterial nutrition still has much to contribute to the study of nutrition of higher animals. Some directions in which we may look for such contributions will be pointed out in the sequel.

\section{Vitamins and Growth Factors Identified since 1942}

A brief and incomplete review of progress in recognition of vitamins and growth factors since the similar review by Peterson 
in 1941 (43) will serve to illustrate the outstanding role played by investigations with microorganisms.

A tabulation of vitamins and growth factors discovered, isolated, or synthesized since 1942, together with the test organisms employed during their isolation or characterization, is given in Table I. The difficulties in assigning individual names and dates to the discovery of vitamins is obvious especially where, as with folic acid and vitamin $B_{12}$, approaches from several different directions with different test organisms have been made, and where the specificity of assay procedures has been improved over a period of years by several different investigators. The popular tendency to assign a given scientific advance to a specific individual, although sometimes justified (depending upon the magnitude of the advance), more often represents an injustice to other workers whose important contributions remain thereby unacknowledged. So it is with discovery, isolation, and characterization of the vitamins-never yet a one-man job! The names associated with the assay methods of Table I, therefore, represent not necessarily the original discoverers of the growth factor but rather the devisers of the first reasonably specific assay procedures that permitted real progress toward isolation and characterization of that growth factor.

\section{PYRIDOXAL AND PYRIDOXAMINE}

Discovery of these two forms of vitamin $\mathrm{B}_{6}$ resulted from an attempt to devise a quantitative microbiological method for the determination of pyridoxine, which at that time was considered synonymous with vitamin $\mathrm{B}_{6}$. When lactic acid bacteria were the test organisms and pyridoxine the reference standard, impossibly high values for the "pyridoxine" content of natural materials were obtained (73). Obviously, substances other than pyridoxine were promoting growth in the vitamin $\mathrm{B}_{6}$ deficient medium. The observation (61) that the growthpromoting activity of pyridoxine increased upon heating with the growth medium and that pyridoxine itself was essentially inactive in promoting growth $(61,73)$ suggested that closely related 


\section{TABLE I}

Progress in Recognition of Vitamins and Growth Factors, 1942-1955

I. Substances known to serve as vitamins for both animals and microorganisms.

A. Pyridoxal and pyridoxamine

1. Bioassay organisms used in detection: Streptococcus faecalis (lactis) R: Snell, Guirard, and Williams, 1942 (73)

2. Characterization and synthesis: Snell, 1944 (62); Harris, Heyl, and Folkers, 1944 (20)

B. Folic acid (pteroylglutamic acid) and its conjugates

1. Bioassay organisms used in isolation and characterization:

Lactobacillus casei: Snell and Peterson, 1939, 1940 (69)

Streptococcus faecalis: Mitchell, Snell, and Williams, 1941 (37)

Chicks: Hogan and Parrott, 1939 (23)

2. Isolation

Pfiffner, Binkley, Bloom, Brown, Bird, Emmett, Hogan, and O'Dell, 1943 (45)

Hutchings, Stokstad, Bohonos, and Slobodkin, 1944 (26)

3. Characterization and synthesis

Angier and co-workers, 1946 (1)

C. Folinic acid (leucovorin) and its conjugates.

1. Bioassay organisms used in isolation and characterization:

Leuconostoc citrovorum: Sauberlich and Baumann, 1948 (56)

Streptococcus faecalis and Lactobacillus casei: Bond, Bardos, Sibley, and Shive, 1949 (4)

2. Characterization and synthesis

Pohland, Flynn, Jones, and Shive, 1951 (47)

Cosulich, Roth, Smith, Hultquist, and Parker, 1952 (11)

3. Isolation

Keresztesy and Silverman, 1951 (27)

D. Pantetheine-Pantethine

1. Bioassay organism used in detection:

Lactobacillus bulgaricus: Williams, Hoff-Jørgensen, and Snell, 1949 (82)

2. Purification and characterization

Peters, Brown, Williams, and Snell, 1953 (41)

Brown, Craig, and Snell, 1950, 1953 (8)

3. Synthesis

Snell, Brown, Peters, Craig, Wittle, Moore, McGlohon, and Bird, 1950 (72) 
TABLE I (Continued)

E. Biocytin

1. Bioassay organisms used in detection:

Lactobacillus arabinosus and Lactobacillus casei: Wright and Skeggs, 1944 (85)

2. Isolation

Wright, Cresson, Skeggs, Wood, Peck, Wolf, and Folkers, 1952 (86)

3. Characterization and synthesis

Wolf, Valiant, Peck, and Folkers, 1952 (40)

F. Vitamin $\mathrm{B}_{12}$

1. Bioassay organisms used in detection:

Man: Castle, 1929 (10)

Lactobacillus lactis Dorner: Shorb, 1947 (59)

2. Isolation

Rickes, Brink, Koniusy, Wood, and Folkers, 1948 (53); Smith, 1948 (60)

II. Microbial growth factors of undetermined nutritional significance for animals

A. Lipoic acid (thioctic acid)

1. Bioassay organisms used in detection

Lactobacillus casei: Guirard, Snell, and Williams, 1946 (17)

Streptococcus faecalis (enzymatic): O'Kane and Gunsalus, 1947 (38)

Tetrahymena geleii: Stokstad, Hoffman, Regan, Fordham, and Jukes, 1949 (78)

2. Isolation

Reed, DeBusk, Gunsalus, and Hornberger, 1951 (52)

Patterson, Brockman, Day, Pierce, Macchi, Hoffman, Fong,

Stokstad, and Jukes, 1951 (46)

3. Characterization and synthesis

Hornberger, Heitmiller, Gunsalus, Schnakenberg, and Reed, 1952 (25)

Bullock, Brockman, Patterson, Pierce, and Stokstad, 1952 (9)

III. Bacterial growth factors of dubious significance for animal nutrition
Growth factor
Test organism
Investigators
A. D-Alanine
B. Putrescine

Lactic acid bacteria

Snell, 1945 (63)

Hemophilus parainfluenzae

Herbst and Snell, 1948

(21)

Table continued 
TABLE I (Continued)

\begin{tabular}{|c|c|c|}
\hline $\begin{array}{l}\text { C. } N \text {-Acetylglucosamine } \\
\text { glycosides }\end{array}$ & Lactobacillus bifidus & $\begin{array}{l}\text { György et al., } 1954 \\
\text { (19a) } \\
\text { Kuhn, } 1952 \text { (31) }\end{array}$ \\
\hline $\begin{array}{l}\text { D. D-Lactic acid (or other } \\
\alpha \text {-hydroxy acids) }\end{array}$ & $\begin{array}{l}\text { Lactobacillus casei } \\
\text { (mutant) }\end{array}$ & $\begin{array}{l}\text { Camien and Dunn, } \\
1953 \text { (9a) }\end{array}$ \\
\hline E. $p$-Hydroxybenzoic acid & Escherichia coli (mutant) & Davis, 1950 (12) \\
\hline F. Various peptides & Lactic acid bacteria & $\begin{array}{l}\text { Sprince and Woolley, } \\
1945(77) \\
\text { Snell, } 1945 \text { (63) } \\
\text { Kihara and Snell, 1952, } \\
1955(28)\end{array}$ \\
\hline $\begin{array}{l}\text { G. Thymidine and mis- } \\
\text { cellaneous nucleosides } \\
\text { and nucleotides }\end{array}$ & Lactic acid bacteria & - \\
\hline H. Coprogen & Pilobolus sp. & $\begin{array}{l}\text { Hesseltine, Pidacks, } \\
\text { Whitehill, Bohonos, } \\
\text { Hutchings, and } \\
\text { Williams, } 1952 \text { (21a) }\end{array}$ \\
\hline Ferrichrome & “ & Neilands, 1952 (37a) \\
\hline I. Biopterin & $\begin{array}{l}\text { Crithidia } \\
\text { fasciculata }\end{array}$ & $\begin{array}{l}\text { Patterson, Broquist, } \\
\text { Albrecht, von Saltza, } \\
\text { and Stokstad, } 1955 \\
\text { (38a) }\end{array}$ \\
\hline
\end{tabular}

derivatives were the active substances, and a variety of chemical treatments combined with microbiological testing showed that partial oxidation of pyridoxine to an aldehyde, or its amination to an amine, yielded highly active substances (62). Synthesis (20) of the limited number of possible structures (62) divulged the structures of two active compounds (formulas I and II), which were named pyridoxal and pyridoxamine $(20,62,70)$.<smiles>Cc1ccc(O)cn1</smiles><smiles>CCOC(=O)OCc1cccc(O)c1</smiles>

This was the first instance in which one of the B-vitamins had been shown to exist in more than a single simple (i.e., 
unconjugated) form, and many investigators still have not adjusted themselves to the changed situation but persist in speaking of the "pyridoxine content" of foodstuffs (although in many foodstuffs no detectable pyridoxine is present, all of the vitamin $\mathrm{B}_{6}$ being present as pyridoxal and pyridoxamine (49)) or of "pyridoxine-deficient" rations (yeast, an excellent source of vitamin $B_{6}$, is pyridoxine-deficient-i.e., it contains little or no pyridoxine* (49)). If accuracy in nomenclature is a prerequisite to accuracy in thought, situations such as this, which occur frequently in the vitamin field (cf. vitamin A vs. vitamin A activity, folic acid vs. folic acid activity, etc.), should receive more attention. The matter is not entirely academic, for whereas pyridoxine is a very stable compound in foodstuffs, pyridoxal because of its reactive carbonyl grouping is not, and it is possible that an inaccurate nomenclature has contributed to the delay in recognition of the destruction of vitamin $B_{6}$ that may occur in food processing.

Immediately following synthesis of pyridoxal, one coenzymatic form of vitamin $B_{6}$ was recognized by Gunsalus and coworkers (19) to be pyridoxal phosphate (formula III); subsequently pyridoxamine phosphate (formula IV) also was discovered by Rabinowitz and Snell (48). The latter product was later found to serve as an essential growth factor for certain lactic acid bacteria (e.g., Lactobacillus delbrueckii (34)), which cannot<smiles>Cc1cc(O)c(C=O)cn1</smiles>

(III) Pyridoxal phosphate<smiles>NCc1cccnc1OP(O)O</smiles>

(IV) Pyridoxamine phosphate

* This statement may appear in conflict with the reported isolation (32) of pyridoxine from yeast. This procedure has not been reported in detail; in answer to a query Professor Kuhn revealed that nitric acid was used throughout this procedure, and volunteered the opinion that the pyridoxamine originally present probably was converted (by nitrous acid) to pyridoxine during the course of the isolation. Most other isolations of pyridoxine were from rice-bran extract, in which this form of vitamin $B_{6}$ predominates. 
utilize pyridoxal or pyridoxamine efficiently, and to serve also as a coenzyme in enzymatic transamination (35).

\section{FOLIG AND FOLINIG ACIDS}

The tangled investigational threads that met in the isolation and characterization of folic acid (formula $\mathrm{V}$ ) have been traced

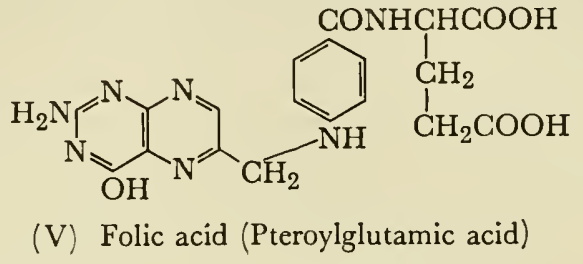

elsewhere $(54,57,44)$. Although the earliest observation of an experimental deficiency of this substance was in monkeys (vitamin M), the discovery failed to provide a practicable assay procedure for its isolation. Such procedures were discovered entirely independently, with chicks (vitamin $\mathrm{B}_{\mathrm{c}}$ ) as the assay organism in one instance, and lactic acid bacteria (eluate factor, folic acid) in the other. The bacterial assays were used at one or another stage in all of the successful isolations.

All the organisms that respond to folic acid respond similarly to folinic acid (formula VI). Thus, the original observations of

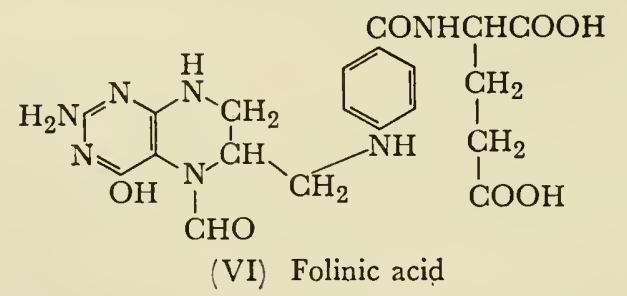

growth responses in various organisms to crude supplements included the response to both substances. Some evidence indicates that the amount of folinic acid in tissues surpasses the amount of folic acid. To what extent the latter is an artifact, formed from the more labile folinic acid (or the even more 


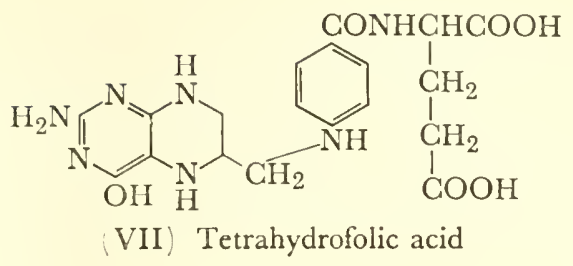

labile di- and tetrahydrofolic acids (formula VII)) during the isolation procedure, remains to be established. Pfiffner and co-workers (45) in their paper describing isolation of folic acid (vitamin $\mathrm{B}_{\mathrm{c}}$ ) demonstrated clearly the existence of a more labile compound which promoted growth of Streptococcus faecalis and which closely resembled in properties those later established for folinic acid. However, the clear-cut differentiation of this latter substance required a specific assay method. This was discovered by Sauberlich and Baumann (56) who found that Leuconostoc citrovorum (more recently identified as Pediococcus cerevisiae) required extremely high levels of folic acid for growth, and then grew only slowly, but grew rapidly in the presence of small amounts of liver extracts. A second assay procedure was independently discovered by Shive and co-workers (4), who found that inhibition of $S$. faecalis by a folic acid analogue was prevented much more effectively by liver extracts than could be accounted for by their folic acid content. Just as pyridoxamine and pyridoxal were earlier produced by empirical procedures from pyridoxine, and their structure clarified without their isolation from natural materials, so it proved possible to form folinic acid from folic acid by empirical procedures, isolate the active reaction product, and establish its structure $(11,47)$. In this instance, however, two difficultly separable diastereoisomeric modifications of the growth factor were produced, and final proof of the identity of the naturally occurring and synthetic growth factor required separation of the diastereoisomers and comparison with the product isolated from natural materials.

Incomplete evidence indicates that in addition to these two substances with folic acid activity, di- and tetrahydrofolic acid 
also may occur naturally. In addition, there appear to occur also conjugates of each of these compounds, that contain up to (and possibly more than) six additional glutamic acid residues and that vary in growth activity depending upon the number of conjugated glutamic acid residues and the test organism. Which if any, of these compounds represents the coenzyme form of the vitamin is not known. The difficulty presented to the analyst by the occurrence of a vitamin in so many forms is great, and the problem of accurate and convenient assay has not been entirely solved.

\section{PANTETHEINE-PANTETHINE}

During an attempt to culture Lactobacillus bulgaricus in a chemically defined medium this and a number of related organisms were found to require an unidentified substance for growth. Highly purified concentrates of the substance were prepared from<smiles>CC(C)(CO)C(C=O)C(=O)NCCC(=O)NCCS</smiles>

(VIII) Pantetheine<smiles>CCCCCCNC(=O)CCNC(=O)OC(C)C(C)(C)CO</smiles>

(IX) Pantethine

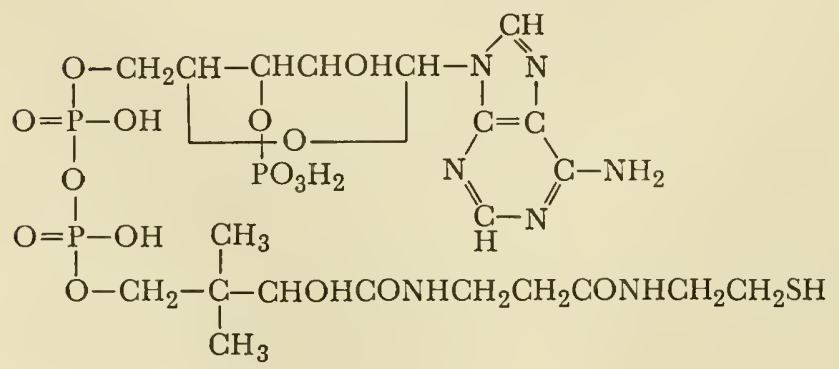

(X) Coenzyme A 
fermentation residues. It was noted that these concentrates replaced pantothenic acid for some organisms (e.g., L. arabinosus) but not for others (e.g., Saccharomyces carlsbergensis), and, conversely, very large amounts of pantothenic acid replaced the unidentified substance as a growth factor for L. bulgaricus. Thus the substance appeared to be a combined form of pantothenic acid, and further work revealed its structure to be that illustrated in formulas VIII and IX. These findings came just as the structure of coenzyme A (formula $\mathrm{X}$ ) was under active investigation, and just as Lynen had shown the acyl-transferring role of coenzyme A to take place via a sulfhydryl grouping. The postulate $(8,72)$ that pantetheine represented the then chemically unidentified portion of coenzyme A was rapidly borne out by the enzymatic resynthesis of coenzyme A from pantetheine and adenosine triphosphate (16). This was the first recognition of the occurrence of the $\beta$-mercaptoethylamine residue in nature. The fact that the growth factor for $L$. bulgaricus occurs naturally in several chromatographically distinct forms was shown to result from the presence of a variety of mixed disulfides formed by oxidative cross-linking between pantetheine and other sulfhydryl compounds (5).

Just as pyridoxal or pyridoxamine and folinic acid are more closely related to the coenzyme form of these vitamins than pyridoxine or folic acid, so then is pantetheine intermediate between coenzyme $\mathrm{A}$ and pantothenic acid. In each case, a specific requirement for these compounds by certain organisms appears to result from inability or a markedly lowered ability to effect conversion $a$ in the sequence:

$\left.\begin{array}{l}\text { pyridoxine } \\ \text { folic acid } \\ \text { pantothenic acid }\end{array}\right\} \stackrel{a}{\longrightarrow}\left\{\begin{array}{l}\text { pyridoxal } \\ \text { folinic acid } \\ \text { pantetheine }\end{array}\right\} \stackrel{b}{\longrightarrow}\left\{\begin{array}{l}\text { pyridoxal phosphate } \\ \text { coenzyme F } \\ \text { coenzyme A }\end{array}\right.$

It sometimes happens that conversion $b$ is also difficult. We have referred to the fact that for some lactic acid bacteria pyridoxamine phosphate is many hundred times more active than pyridoxamine or pyridoxal in promoting growth. Similarly, for a strain of Treponema pallidum neither pantothenic acid 
nor panthetheine serves as a growth factor; panthetheine4 '-phosphate (formula XI, an intermediate between pantetheine and coenzyme A) or coenzyme A itself must be supplied to permit growth (77a).

Do conditions exist in which higher animals show similar, synthetic disabilities, where the coenzymes or their more immediate (and more readily available) precursors would be more effective therapeutic agents than the vitamin forms of commerce? We do not know. In experimentally produced deficiency states (usually produced in young, undiseased animals) no evidence for this exists, but it would be unwise to overlook the possibility while the problems of pathology of all types, of aging, etc., are still with us.

When we say that $L$. bulgaricus has a specific requirement for pantetheine because of an insufficient capacity on the part of the cell to convert pantothenic acid to pantetheine, it is important to understand just what is meant. Experimentally, all that one observes is that to permit growth, pantothenic acid must be supplied in the medium at several hundred times the concentration that suffices when pantetheine is supplied. But with sufficient pantothenic acid, growth does occur, showing that some of it has been converted to coenzyme A. Thus the conversion enzymes do occur, and can function. Is the conversion limited because (1) insufficient of the conversion enzyme(s) is present, or (2) because the enzyme, though present, has a lowered affinity for its substrate, or (3) because activity of the<smiles>CCC(C)(CO[P+](=O)[O-])C(C)OC(=O)NCCC(=O)NCCS</smiles>

(XI) Pantetheine-4'-phosphate

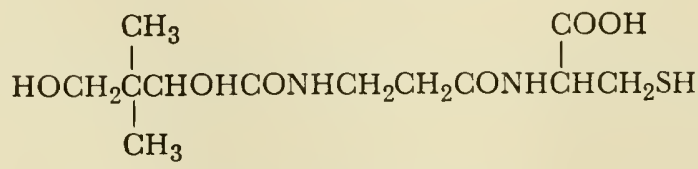

(XII) Pantothenylcysteine 
enzyme is in some way inhibited by presence of other substances, or (4) because pantothenic acid, in contrast to pantetheine, is not readily absorbed by this organism? All of these are possibilities, and any one of them would suffice to permit the observed result. The growth experiment by itself does not favor one explanation over the other.

Without implying preference for any particular explanation in this instance, it may be useful to cite evidence showing that insufficient attention has been paid in the recent past to cell permeability as an explanation for differing activities of closely related growth factors. Current evidence $(c f .6,7)$ indicates that conversion of pantothenic acid (or pantoic acid) to coenzyme A occurs via the following pathway:

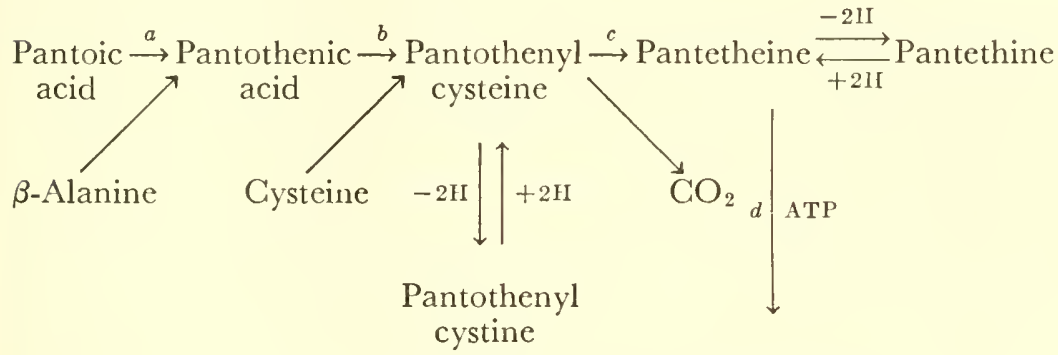

Coenzyme A $\underset{\text { ATP }}{\stackrel{e}{\longleftarrow} \text { P'-phosphate }}$

Acetobacter suboxydans does not grow in the absence of one of the substances shown along the main line of this conversion. Pantoic acid and pantothenic acid have equal activity but are much less active than pantothenylcysteine, pantetheine, 4'phosphopantetheine, or reduced coenzyme A. This could be interpreted as an inability to carry out reaction $b$ readily. However, further investigation showed that the activity of pantothenic acid (and pantoic acid), but not of pantetheine, was greatly increased by lowering the $p \mathrm{H}$ of the medium; this increased activity approximated the increased concentration of undissociated pantothenic acid that resulted from the drop in $p \mathrm{H}$, and the undissociated acids very nearly equalled coenzyme $\mathrm{A}$ in 
growth activity (7). Thus it appears that cells of $A$. suboxydans are relatively impermeable to the anions of pantoic and pantothenic acids, but not to the undissociated acids. Pantothenylcystine and pantethine are without activity, but the reduced compounds, pantothenylcysteine (formula XII) and pantetheine, show full activity. The presence of the - $\mathrm{SH}$ group permits ready absorption despite presence of an ionized carboxyl group, for the activity of pantothenylcysteine is not $p \mathrm{H}$-dependent as is that of pantothenic acid, pantoic acid, or pantothenylglycine (7). This indicates that the - $\mathrm{SH}$ group is concerned in absorption of these compounds through the cell membrane.

Are similar phenomena of importance in animal nutrition? We do not know, but fragmentary evidence suggests that they may be. Thus the conversion of pantothenic acid to coenzyme A appears at present to occur in animals by the same pathway shown above, and cell-free extracts of rat liver convert pantothenylcysteine to pantetheine (22). Yet, whereas pantothenic acid and pantetheine have equal activities in promoting growth of rats, pantothenylcysteine has none (79). The result is difficult to explain except by assuming either that the biosynthetic route is incorrect or that pantothenylcysteine does not get into the cells. Again, the observation that relatively massive doses of vitamin $B_{12}$ given by mouth are required in the absence of intrinsic factor to produce remissions in pernicious anemia, whereas very small amounts are required by injection, may indicate difficulty in absorption of this substance through the intestinal wall. Does the intrinsic factor, by combining with vitamin $\mathrm{B}_{12}$, provide a "handle" by means of which receptors in the intestinal wall can transfer this vitamin to the circulation, much as a free - $\mathrm{SH}$ group in pantothenylcysteine permits efficient absorption of this substance by $A$. suboxydans despite the deleterious effects of an ionized carboxyl group?

BIOGYTIN

When yeast extract is assayed for biotin with Lactobacillus arabinosus, the result is much lower than that obtained with $L$. 
casei; following acid hydrolysis the value given by the former organism rises to the constant value obtained with the latter. This observation of Wright and Skeggs (85) demonstrated the natural occurrence of a biotin conjugate and illustrates again the utility of quantitative comparative bioassays in the detection of naturally occurring vitamin derivatives. Isolation (86) and characterization (40) of the compound, which has the structure illustrated in formula XIII, was followed by means of this same assay procedure.

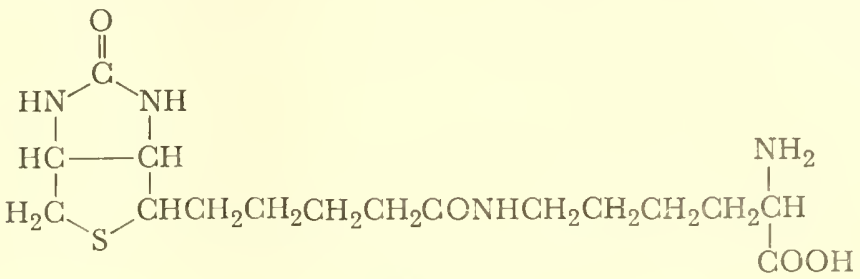

(XIII, Biocytin

This compound replaces biotin as a growth factor for animals, and for some but not all bacteria (84). Its metabolic significance is not yet known, but its unusual structure and the high proportion of the total biotin in some materials comprised by it indicate that it is more than a metabolic oddity.

\section{VITAMIN $B_{12}$}

This spectacular substance is exceptional in all ways: in the complexity of its structure, in the variety of active forms that can be obtained from nature, in its content of a metal ion (cobalt), in its unusally high (even for vitamins!) biological activity, and in the fact that one group of investigators succeeded in isolating it through the exclusive use of man as a test animal (aided greatly in the latter phases, undoubtedly, by the distinctive red color of the vitamin!). Nonetheless, its isolation in this country was greatly aided by discovery that certain lactic acid bacteria required concentrates of it for growth under some conditions-an observation first made by Shorb (59). Because of its activity in controlling pernicious anemia in man, in partially alleviating 
delayed growth in children, in stimulating growth of farm animals fed rations based chiefly or exclusively on plant products, and, from the biochemical side, because of its relationship to nucleic acid synthesis and other biosynthetic reactions, it has stimulated unusual interest, and many review articles dealing with it are available ( $c f .3,15)$.

The structure of vitamin $B_{12}$ is not yet known in detail, but it contains an atypical porphyrin skeleton (4a) the nitrogen atoms of which occupy four of the six coordination positions of cobalt (formula XIV).* The fifth is occupied by a nitrogen of a nucleo-

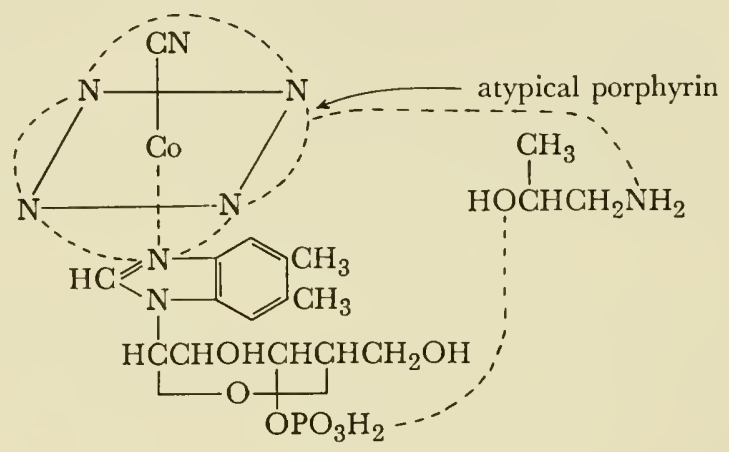

(XIV) Vitamin $B_{12}$ (partial formula)

tide, the nature of which can vary in vitamin $B_{12}$ isolated from different source materials. Coordination position six is occupied typically (but not exclusively) by an anionic grouping-cyanide hydroxide, oxalate, sulfate, nitrite, etc.- so that for any one parent structure containing a single nucleotide, a family of closely related compounds of slightly different properties can be obtained. Recent results (15a) show that by incorporating any of ten or so different nitrogen bases in the medium, Escherichia coli 113-3 will produce vitamin $\mathrm{B}_{12}$-like substances containing the corresponding nucleotides in the molecule-a finding that

* Since this was written, the probable complete structure of vitamin $B_{12}$ has been clarified by a combination of chemical and $x$-ray diffraction methods (D. G. Hodgkin, et al., Nature, 176, 325 (1955) and R. Bonnett, et al., Nature, 176, 328 (1955)). 
multiplies indefinitely the number of possible vitamin-like structures that can be obtained. These vary in their activities for different test microorganisms and for animals. The first compound isolated, first called vitamin $\mathrm{B}_{12}$ and later cyanocobalamin, contained dimethylbenzimidazole as the nucleotide base, and $\mathrm{CN}^{-}$as the anion. It is fully active in animals and all microorganisms so far known, and is the vitamin $B_{12}$ of commerce. Pseudovitamin $\mathrm{B}_{12}$, which is similarly constituted but contains adenine in place of dimethylbenzimidazole, has full activity for some (not all) microorganisms, but little or none for animals. The significance of this variability in structure remains to be clarified.

Similarly, the relationship of the intrinsic factor to vitamin $\mathrm{B}_{12}$ remains to be clarified. This substance appears to be a glycoprotein present in normal individuals but not in those with pernicious anemia, and is necessary for the absorption of the small amounts of vitamin $B_{12}$ ordinarily present in foodstuffs. Whether it functions by combining with the vitamin and preventing its absorption by intestinal bacteria, thus conserving it for the host, or whether it functions otherwise is not known. The possibility that it promotes direct absorption through the intestinal wall has been mentioned earlier (see p. 100).

\section{LIPOIC ACID (THIOCTIC ACID)}

As early as 1937, Snell, Tatum, and Peterson (76) observed the marked stimulating effects of acetate on growth of lactic acid bacteria, and included this substance in the nutrient medium for the purpose of clarifying other nutritional requirements of these bacteria. It was observed (75) that the substance not only served as a buffer but played some more specific role in growth, and that it was not required in media supplied with yeast extract (76). Following elucidation of several other growth requirements of these organisms, Guirard, Snell, and Williams (17) returned in 1941 to an investigation of this substance and demonstrated the existence in yeast and other natural materials of a substance that duplicated the growth-promoting effects of 
acetate for Lactobacillus casei but was far more active on a weight basis. This observation thus provided an assay method of known specificity for the substance. Independently, O'Kane and Gunsalus (38) had observed that cells of Streptococcus faecalis grown in synthetic medium required an unidentified substance in yeast extract to permit oxidation of pyruvate to acetate. Placed in juxtaposition, these findings suggested identity between the two unidentified substances, and following comparative tests of concentrates, Snell and Broquist (68) concluded that the two substances were identical.

In an independent line of investigation, Kidder and Dewey had successfully sorted out the complex nutritional requirements of a protozoan, Tetrahymena geleii, and showed it to require one or more unidentified substances. In 1949, Stokstad and coworkers (78) showed that this unidentified substance contained pyridoxal, copper ions, and an unidentified growth factor that was named protogen, all of which were necessary for growth in the basal medium. Supplementation of this medium with the former two substances thus permitted development of an assay of known specificity for protogen, and the preparation of concentrates. Such concentrates also were highly active in promoting growth of $L$. casei - a fact that led Snell and Broquist (68) to conclude that the acetate-replacing factor for $L$. casei and protogen were identical.

By use of these assay methods, isolation of an active crystalline substance was first achieved by Reed et al. (52), who<smiles>O=C(O)CCCCC(S)CCS</smiles><smiles>O=C(O)CCCCC1CCSS1</smiles>

(b)

(XV) Lipoic acid, $(a)$ reduced and $(b)$ oxidized 
named the crystalline substance lipoic acid, and shortly thereafter by Patterson et al. (46), who later renamed the product thioctic acid. Chemical characterization and synthesis $(9,25)$ showed the substance to be 6,8-dithiooctanoic acid (formula XV); it can exist in both the cyclic structure and as an open-chain dithiol, the interconversion of the two being explicitly involved in its enzymatic role as a cofactor in the oxidative decarboxylation of pyruvate to acetate and other $\alpha$-keto acids to the corresponding carboxylic acids containing one less carbon atom (18,51). Such oxidative decarboxylation also requires thiamin pyrophosphate, and it appears an unsettled point whether the two are combined into a single coenzyme (51).

Most nutritional investigations with higher animals have failed to show a growth response to added lipoic acid. However, DeBusk and Williams (14) have recently observed both increased growth and increased efficiency of food utilization in young rats and chicks fed trace amounts of the substance. The reasons for the differing results are not yet known. If these results are confirmed, this will provide another instance of a vitamin discovered, isolated, and characterized as a microbial growth factor before its role in the nutrition of higher animals was suspected. However, in a sense it is academic whether the substance is or is not a vitamin, i.e., required in the diet of animals. There is no doubt that it is a biocatalyst of great importance in animal as in microbial and plant life, and the genetic accident that determines whether the substance can or cannot be synthesized in animals, though it has important implications for nutrition, hardly changes the intrinsic biochemical importance of the substance.

\section{OTHER MICROBIAL GROWTH FACTORS}

A variety of additional substances found essential for growth of one or another microorganism during the past few years is listed in Table I. Although they are obviously important for the organisms that require them, little is known of their metabolic significance. D-Alanine is required for growth of many lactic acid bacteria, but only in the absence of vitamin $B_{6}$. It is now 
known that this interchangeability results from the fact that vita$\min B_{6}$ is required for synthesis of this amino acid; when the latter is supplied preformed, the requirement for vitamin $B_{6}$ disappears, provided each of the L-amino acids required for growth also is supplied $(24,83)$. This remains the only known instance of a D-amino acid being essential for growth, although these substances, once thought not to occur naturally, have now been frequently encountered in various microbial products, such as antibiotics and capsules. A closer investigation (74) shows the D-alanine occurs chiefly in the cell wall, of which it is apparently an essential component, and in soluble cell extractives. Recent investigation of the composition of bacterial cell walls shows them to be rich in carbohydrate materials, including amino sugars (55). It is possible that the requirement of Lactobacillus bifidus for $N$-acetylglucosamine derivatives (19a,31) and of some lactobacilli, grown with pentoses, for traces of glucose (39) may reflect a requirement for these substances as structural materials for cell wall formation.

$p$-Hydroxybenzoic acid and D-lactic acid (or its homologues) are examples of growth requirements found so far only in artificially induced mutants; this does not lessen their importance but is mentioned to illustrate the utility of mutant organisms in the detection of previously unrecognized essential metabolites. The technique has been especially valuable in permitting detection and isolation of intermediates in biosynthetic reactionscompounds which normally have such a transient existence that their natural occurrence would be difficult to detect in any other way. Space limitations do not permit tabulation and discussion of the many compounds discovered in this fashion-each of which can serve as essential organic nutrients for certain mutant organisms under a restricted set of conditions.

Appropriate peptides stimulate or are even essential for growth of many bacteria, and recent investigations have begun to throw light on the reasons these compounds show activities not shared by their component amino acids. Three model instances have so far been observed: (1) assimilation of one amino 
acid, $a$, may be inhibited by a second amino acid, $b$. Under these circumstances, peptides of $a$, perhaps because of their greater structural dissimilarity to the inhibitor, may greatly surpass the free amino acid in activity. For example, D-alanine inhibits utilization of $\mathbf{L}$-alanine by Lactobacillus casei, but not that of peptides of L-alanine. Such peptides far surpass free L-alanine in growth-promoting activity under these conditions and may become obligatory requirements for growth (28). Many examples of this type of behavior are now known (28). (2) An essential free amino acid may be subject to a degradation reaction that rapidly destroys it before much of it can be used for growth, i.e., protein synthesis. If its peptides are not subject to the degradation reaction, they will greatly surpass the free amino acid in growth-promoting activity. For example, tyrosine peptides are much more active than free tyrosine in promoting growth of $S$. faecalis under conditions such that free tyrosine can be destroyed by tyrosine decarboxylase, but peptides and free amino acid are equally active when conditions are so arranged as to make this enzyme nonfunctional, e.g., by growth in the absence of vitamin $B_{6}$ (29). (3) For reasons still unknown absorption of one free amino acid may take place with difficulty or may require the presence of unusually high concentrations of a second amino acid. Under these conditions, peptides of the amino acid may be much more effective than the free amino acid in promoting growth. For example, Lactobacillus delbrueckii 730 requires relatively tremendous quantities of histidine for growth, but very small amounts of histidine peptides. In this same organism, tyrosine peptides are much more active than free tyrosine in promoting growth when the histidine concentration is low, but equally active when the histidine concentration is raised (42), and these phenomena cannot be accounted for in terms of either mechanism (1) or (2).

None of the instances cited above need be interpreted as indicating direct utilization of the peptides without prior hydrolysis; indeed some evidence suggests that hydrolysis occurs before utilization. In none of these experiments, further, is there 
evidence for a special catalytic role of the stimulatory peptides in metabolism, although the possibility of such a role for other peptides is not, of course, excluded.

\section{Variability in Nutritional Requirements with Conditions}

Cursory reading of the nutritional literature, especially that dealing with animal nutrition, leaves the impression that the nutritional requirements of a given species for vitamins, etc., is a rather constant thing, that these requirements can be determined and expressed in terms of a quantitative figure for the average daily requirement which has validity quite apart from the conditions used in assessing the requirement.

From parts of the foregoing discussion it should be clear that this is far from the case in microbial nutrition. Lactobacillus casei grows well in the absence of both $\mathrm{D}$-alanine and L-alanine peptides if pyridoxal is supplied; in the absence of pyridoxal, both $\mathrm{D}$-alanine and such peptides are required, the former because it cannot be synthesized in the absence of vitamin $B_{6}$, the latter because $\mathrm{D}$-alanine inhibits utilization of free $\mathrm{L}$-alanine (which also cannot be synthesized in the absence of vitamin $\mathrm{B}_{6}$ ) but not that of L-alanine peptides. Again, $L$. casei requires relatively large amounts of biotin if aspartate and unsaturated fatty acids are omitted from the medium, much less if aspartate (but not the fatty acid) is supplied, and none at all if both products are supplied. Such sparing effects of one nutrient on the requirement for another frequently indicate important metabolic roles played by the vitamins. In the instances cited above, a role for vitamin $\mathrm{B}_{6}$ in the synthesis of $\mathrm{D}$ - and $\mathrm{L}$-alanine is indicated, and the necessity for biotin in the synthesis of aspartate and unsaturated fatty acids likewise appears highly probable. Similarly, the necessity for folic acid in the synthesis of the purine and pyrimidine bases and serine early became evident from the fact that in appropriate media these substances greatly reduced or eliminated completely the requirement of $L$. casei and $S$. faecalis for folic acid. 
Examples of this nature could be multiplied indefinitely, and a more complete discussion has been presented elsewhere $(66,67)$. They have their basis in the fact that the vitamins are required only to permit the functioning of enzymes that in turn manufacture products essential for growth. Frequently, if these products are supplied preformed, or the necessity for the enzymic functioning is otherwise bypassed, need for the enzyme (and hence for the vitamin) is reduced, and with some simple organisms can be eliminated completely. One strongly suspects that closer investigation would show a similar variability (though less marked because of the greater organization and range of metabolic reactions present) in higher animals. The well-authenticated variability in the thiamin requirement of animals with the proportion of the caloric intake supplied as fat, and in the vitamin $B_{12}$ requirement with the choline intake, are straws indicating the direction research may take in these matters.

As the number of new nutrients available for study decreases, perhaps animal nutritionists will turn more toward the study of such interrelationships between nutrients and their significance for practical nutrition.

\section{Some Future Areas for Nutrition Research}

The last few years have seen a decrease in the rate of discovery of new vitamins and growth factors. Some experimental animals can now be grown on essentially synthetic diets, and it is becoming more and more difficult to detect entirely new bacterial growth factors. Does this mean we are approaching the time when no more dietary factors remain to be discovered? Although this time must eventually arrive, it appears unlikely that it is yet here. Some of the readily available (though experimentally more difficult) experimental animals have not been grown on defined diets as yet, and there are indications of unidentified dietary requirements for some of these (e.g., monkeys (78a)). Similarly, little is known of the nutritional requirements 
of thousands of microbial species. Only a dent has been made in the problem of the nutrition of protozoa and insects.

The discovery by Fraenkel (15b) of vitamin $\mathrm{B}_{\mathrm{T}}$ as an essential growth factor for the meal worm, Tenebrio molitor, and its identification as carnitine by Carter (9b), a compound that also occurs in higher animals but whose function is unknown, illustrates the fruitfulness of investigations of the nutrition of the currently neglected lower animals. Having discovered the factors required in common by most living species, we may be on the verge of discovering those nutrients characteristically required by a restricted number of species ( $c f$. D-alanine). If such nutrients exist, knowledge of them would be invaluable in permitting design of antimetabolites directed against specific groups of organisms.

In bacteria, appropriate nutrients can sometimes partially overcome the deleterious influence of certain drugs ( $c f$. the effect of glutamine on alcohol tolerance in some bacteria (50)). Similar relationships should hold in animals and provide a large area for investigation. Proper nutrition practice has already reduced the incidence of many of the infirmities of old age. Can further progress along these lines be made; can, indeed, the aging process itself be slowed?

Such questions suggest that, far from being an obsolescent field, the future may hold triumphs for experimental nutrition equal to those of the past.

\section{References}

1. Angier, R. B., J. H. Boothe, B. L. Hutchings, J. H. Mowat, J. Semb, E. L. R. Stokstad, Y. SubbaŔow, C. W. Waller, D. B. Cosulich, M. J. Fahrenbach, M. E. Hultquist, E. Kuh, E. H. Northey, D. R. Seegar, J. P. Sickels, and J. M. Smith, Jr., Science, 103, 667 (1946).

2. Barton-Wright, E. C., The Microbiological Estimation of the Vitamin B Complex and Amino Acids. Pitman, London, 1952.

3. Bessey, O. A., H. J. Lowe, and L. L. Saloman, Ann. Rev. Biochem., 22, 545 (1953).

4. Bond, T. J., T. J. Bardos, M. Sibley, and W. Shive, J. Am. Chem. Soc., 71, 3852 (1949).

4a. Brink, C., D. C. Hodgkin, J. Lindsey, J. Pickworth, J. H. Robertson, and J. G. White, Nature, 174, 1169 (1954). 
5. Brown, G. M., and E. E. Snell, J. Biol. Chem., 198, 375 (1952).

6. Brown, G. M., and E. E. Snell, J. Bacteriol., 67, 465 (1954).

7. Brown, G. M., M. Ikawa, and E. E. Snell, J. Biol. Chem., 213, 855, (1955).

8. Brown, G. M., J. A. Craig, and E. E. Snell, Arch. Biochem., 27, 473 (1950); Brown, G. M., and E. E. Snell, J. Am. Chem. Soc., 75, 1691 (1953).

9. Bullock, M. W., J. A. Brockman, Jr., E. L. Patterson, J. V. Pierce, and E. L. R. Stokstad, J. Am. Chem. Soc., 74, 1868, 3455 (1952).

9a. Camien, M. N., and M. S. Dunn, J. Biol. Chem., 201, 621 (1953); 217, 593 (1954).

9b. Carter, H. E., P. K. Bhattacharyya, K. R. Weidman, and G. Fraenkel, Arch. Biochem. and Biophys., 35, 241 (1952).

10. Castle, W. B., Am. J. Med. Sci., 178, 748 (1929); Castle, W. B., W. C. Townsend, and C. W. Heath, ibid., 180, 305 (1930).

11. Cosulich, D. B., B. Roth, J. M. Smith, Jr., M. E. Hultquist, and R. P. Parker, J. Am. Chem. Soc., 74, 3252 (1952).

12. Davis, B. D., Nature, 166, 120 (1950).

13. Davis, B. D., 2nd Congr. intern. biochim., Chim. biol. V, Symposium metabolisme microbien (Paris), 1952, 32-40.

14. DeBusk, B. G., and R. J. Williams, Arch. Biochem. and Biophys., 55, 587, (1955).

15. Folkers, K., Intern. Z. Vitaminforsch., 23, 429 (1952).

15a. Ford, J. E., E. S. Holdsworth, and S. K. Kon, Biochem. J. (London), 59, 86 (1955).

15b. Fraenkel, G., and M. Blewitt, Biochem. J. (London), 41, 469 (1947); Fraenkel, G., Nature, 161, 981 (1948).

16. Govier, W. M., and Gibbons, A. J., Arch. Biochem. and Biophys., 32, 347 (1951); King, T. E., and F. M. Strong, J. Biol. Chem., 189, 325 (1951).

17. Guirard, B. M., E. E. Snell, and R. J. Williams, Arch. Biochem., 9, 381 (1946).

18. Gunsalus, I. C., J. Cellular Comp. Physiol., 41, Suppl. 1, 11 (1953).

19. Gunsalus, I. C., W. D. Bellamy, and W. W. Umbreit, J. Biol. Chem., 155, 685 (1944).

19a. György, P., R. F. Norris, and C. S. Rose, Arch. Biochem. and Biophvs., 48, 193 (1954); György, P., R. Kuhn, C. S. Rose, and F. Zilliken, ibid., 48, 202 (1954); György, P., J. R. E. Hoover, R. Kuhn, and C. S. Rose, ibid., 48, 209 (1954); Gauhe, A., P. György, J. R. E. Hoover, R. Kuhn, C. S. Rose, H. W. Ruelius, and F. Zilliken, ibid., 48, 214 (1954); Tomarelli, R. M., J. B. Hassinen, E. R. Eckhardt, R. H. Clark, and F. W. Bernhart, ibid., 48, 225 (1954).

20. Harris, S. A., D. Heyl, and K. Folkers, J. Biol. Chem., 154, 315 (1944); J. Am. Chem. Soc., 66, 2088 (1944). 
21. Herbst, E. J., and E. E. Snell, J. Biol. Chem., 176, 989 (1948); ibid., 181, 47 (1949).

21a. Hesseltine, C. W., C. Pidacks, A. R. Whitehill, N. Bohonos, B. L. Hutchings, and J. H. Williams, J. Am. Chem. Soc., 74, 1362 (1952).

22. Hoagland, M. B., and G. D. Novelli, J. Biol. Chem., 207, 767 (1954).

23. Hogan, A. G., and E. M. Parrott, J. Biol. Chem., 128, xIvii (1939).

24. Holden, J. T., R. C. Wildman, and E. E. Snell, J. Biol. Chem., 191, 559 (1951).

25. Hornberger, C. S., R. F. Heitmiller, I. C. Gunsalus, G. H. F. Schnakenberg, and L. J. Reed, J. Am. Chem. Soc., 74, 2382 (1952).

26. Hutchings, B. L., E. L. R. Stokstad, N. Bohonos, and N. H. Slobodkin, Science, 99, 371 (1944).

27. Keresztesy, J. C., and M. Silverman, J. Am. Chem. Soc., 73, 5510 (1951).

28. Kihara, H., and E. E. Snell, J. Biol. Chem., 197, 791 (1952); ibid., 212, 83 (1955).

29. Kihara, H., O. Klatt, and E. E. Snell, J. Biol. Chem., 197, 801 (1952).

30. Knight, B. C. J. G., Bacterial Nutrition. H. M. Stationery Office, London, 1935.

31. Kuhn, R., Angew. Chem., 64, 493 (1952).

32. Kuhn, R., and G. Wendt, Ber., 71, 780, 1118, 1534 (1938).

33. Lwoff, A., L'Evolution physiologique. Etude des pertes de fonction chez les microorganisms. Herman et Cie, Paris, 1943.

34. McNutt, W. S., and E. E. Snell, J. Biol. Chem., 173, 801 (1948); ibid., 182, 557 (1950).

35. Meister, A., H. A. Sober, and E. A. Peterson, J. Am. Chem. Soc., 74, 2385 (1952).

36. Mitchell, H. K., Vitamins and Hormones, 8, 127 (1951).

37. Mitchell, H. K., E. E. Snell, and R. J. Williams, J. Am. Chem. Soc., 63, 2284 (1941); ibid., 66, 267 (1944).

37a. Neilands, J. B., J. Am. Chem. Soc., 74, 4846 (1952).

38. O'Kane, D. J., and I. C. Gunsalus, J. Bacteriol., 54, 20 (1947); 56, 499 (1948).

38a. Patterson, E. L., H. P. Broquist, A. M. Albrecht, M. H. von Saltza, and E. L. R. Stokstad, J. Am. Chem. Soc., 77, 3168 (1955).

39. Patterson, E. L., M. H. von Saltza, H. P. Broquist, and J. V. Pierce, Proc. Soc. Exptl. Biol. Med., 81, 205 (1952).

40. Peck, R. L., D. E. Wolf, and K. Folkers, J. Am. Chem. Soc., 74, 1999 (1952); Wolf, D. E., J. Valiant, R. L. Peck, and K. Folkers, ibid., 74, 2002 (1952).

41. Peters, V. J., G. M. Brown, W. L. Williams, and E. E. Snell, J. Am. Chem. Soc., 75, 1688 (1953).

42. Peters, V. J., J. M. Prescott, and E. E. Snell, J. Biol. Chem., 202, 521 (1953). 
43. Peterson, W. H., Biol. Symposia, 5, 31 (1941).

44. Peterson, W. H., Ann. N. Y. Acad.Sci., 48, 257 (1946).

45. Pfiffner, J. J., S. B. Binkley, E. S. Bloom, R. A. Brown, O. D. Bird, A. D. Emmett, A. G. Hogan, and B. L. O'Dell, Science, 97, 404 (1943).

46. Patterson, E. L., J. A. Brockman, F. P. Day, J. V. Pierce, M. E. Macchi, C. E. Hoffman, C. T. O. Fong, E. L. R. Stokstad, and T. H. Jukes, J. Am. Chem. Soc., 73, 5919 (1951).

47. Pohland, A., E. H. Flynn, R. G. Jones, and W. Shive, J. Am. Chem. Soc., 73, 3247 (1951).

48. Rabinowitz, J. C., and E. E. Snell, J. Biol. Chem., 169, 643 (1947).

49. Rabinowitz, J. C., and E. E. Snell, J. Biol. Chem., 176, 1157 (1948).

50. Ravel, J. M., B. Felsing, E. M. Lansford, R. H. Trubey, and W. Shive, J. Biol. Chem., 274, 497 (1955).

51. Reed, L. J., and B. G. DeBusk, Federation Proc., 13, 723 (1954).

52. Reed, L. J., B. G. DeBusk, I. C. Gunsalus, and C. S. Hornberger, Science, 174, 93 (1951).

53. Rickes, E. L., N. G. Brink, F. R. Koniusy, T. R. Wood, and K. Folkers, Science, 107, 396 (1948).

54. Robinson, F. A., The Vitamin B Complex. Wiley, New York, 1951.

55. Salton, M. R. J., Biochim. et Biophys. Acta, 10, 512 (1953).

56. Sauberlich, H. E., and C. A. Baumann, J. Biol. Chem., 776, 165 (1948).

57. Sebrell, W. H., Jr., and R. S. Harris, The Vitamins: Chemistry, Physiology, Pathology, Vols. 1-3. Academic Press, New York, 1954.

58. Shive, W., Ann. N. Y. Acad. Sci., 52, 1212 (1950).

59. Shorb, M. S., J. Biol. Chem., 169, 455 (1947); Science, 107, 397 (1948).

60. Smith, E. L., Nature, 167, 638 (1948); ibid., 762, 144 (1948).

61. Snell, E. E., Proc. Soc. Exptl. Biol. Med., 51, 356 (1942).

62. Snell, E. E., J. Biol. Chem., 154, 313 (1944); J. Am. Chem. Soc., 66, 2082 (1944).

63. Snell, E. E., J. Biol. Chem., 158, 497 (1945).

64. Snell, E. E., Physiol. Revs., 28, 255 (1948).

65. Snell, E. E., in György, P., ed., Vitamin Methods, Vol. 1, p. 327. Academic Press, New York, 1950.

66. Snell, E. E., in Skoog, F., ed., Plant Growth Substances, p. 431. University of Wisconsin Press, Madison, 1951.

67. Snell, E. E., in Werkman, C. H., and Wilson, P. W., cds., Bacterial Physiology, p. 214. Academic Press, New York, 1951.

68. Snell, E. E., and H. P. Broquist, Arch. Biochem., 23, 326 (1949).

69. Snell, E. E., and W. H. Peterson, J. Biol. Chem., 128, xciv (1939); J. Bacteriol., 39, 273 (1940).

70. Snell, E. E., and A. N. Rannefeld, J. Biol. Chem., 157, 475 (1944).

71. Snell, E. E., and F. M. Strong, Ind. Eng. Chem., Anal. Ed., 11, 346 (1939).

72. Snell, E. E., G. M. Brown, V. J. Peters, J. A. Craig, E. L. Wittle, J. A. 
Moore, V. M. McGlohon, and O. D. Bird, J. Am. Chem. Soc., 72, 5349 (1950); cf. also J. Am. Chem. Soc., 75, 1694 (1953).

73. Snell, E. E., B. M. Guirard, and R. J. Williams, J. Biol. Chem., 143, 519 (1942).

74. Snell, E. E., N. Ikawa, and N. Radin, J. Biol. Chem., 277, 803 (1955).

75. Snell, E. E., F. M. Strong, and W. H. Peterson, Biochem. J. (London), 31, 1789 (1937).

76. Snell, E. E., E. L. Tatum, and W. H. Peterson, J. Bacteriol., 33, 207 (1937).

77. Sprince, H., and D. W. Woolley, J. Am. Chem. Soc., 67, 1734 (1945).

77a. Steinman, H. G., V. I. Oyama, and H. O. Schulze, Federation Proc., 13, 512 (1954).

78. Stokstad, E. L. R., C. E. Hoffman, M. A. Regan, D. Fordham, and T. H. Jukes, Arch. Biochem., 20, 75 (1949).

78a. Tappan, D. V., and C. A. Elvehjem, J. Nutrition, 57, 469 (1953).

79. Thompson, R. Q., and O. D. Bird, Science, 120, 763 (1954).

80. Williams, R. J., Science, 93, 412 (1941).

81. Williams, R. J., R. E. Eakin, E. Beerstecher, Jr., and W. Shive, The Biochemistry of B-Vitamins. Reinhold, New York, 1950.

82. Williams, W. L., E. Hoff-Jørgensen, and E. E. Snell, J. Biol. Chem., 777, 933 (1949).

83. Wood, W. A., and I. C. Gunsalus, J. Biol. Chem., 790, 403 (1951).

84. Wright, L. D., E. L. Cresson, K. V. Liebert, and H. R. Skeggs, J. Am. Chem. Soc., 74, 2004 (1952).

85. Wright, L. D., and H. R. Skeggs, Proc. Soc. Exptl. Biol. Med., 56, 95 (1944).

86. Wright, L. D., E. L. Cresson, H. R. Skeggs, T. R. Wood, R. L. Peck, D. E. Wolf, and K. Folkers, J. Am. Chem. Soc., 72, 1048 (1950); ibid., 74, 1996 (1952). 


\title{
THE SIGNIFICANCE OF INDUCED ENZYME FORMATION*
}

\author{
S. SPIEGELMAN and A. M. CAMPBELL, $\dagger$ Department of \\ Bacteriology, University of Illinois, Urbana, Illinois
}

\section{Introduction}

It is no historical accident that a renewed interest in the phenomenon of "enzymatic adaptation" came hard on the heels of the explosive birth of modern microbial genetics. Two good reasons can be advanced for this virtual concurrence. In the first place, the work with Neurospora, yeast, and the bacteria provided the genetically controlled material necessary for biologically intelligent and interpretable experiments. Secondly, from its very inception, microbial biochemical genetics posed the gene-enzyme problem in terms of a rapidly accumulating multitude of experimental facts which could not be ignored. Their existence demanded an immediate search for tools which would permit the experimental analysis of such statements as "genes control enzymes."

There were two obvious directions which a quest of this nature could, and did, take. One started with the gene and led to

* The original experiments reported here from the authors' laboratory were supported by grants from the National Cancer Institute of the U. S. Public Health Service and the Office of Naval Research.

$\dagger$ Present address: Department of Bacteriology, University of Michigan Ann Arbor, Michigan. 
examination of the enzymatic consequences of individual modifications in the genome with the hope that the interrelations revealed by their careful analysis would ultimately yield a clue to the underlying mechanisms of genetic control. The other, starting from the enzyme, adopted the premise that fruitful speculation on the role of the gene was unlikely in the absence of a relatively detailed understanding of the mechanism of enzyme formation. Those who felt inclined toward the second approach were necessarily forced to look for a system in which the process of enzyme synthesis could be experimentally analyzed. It is not surprising that the attention of these workers was immediately turned to the phenomenon known as "enzymatic adaptation." There had existed in the microbiological literature since 1888 a number of instances subsumed under this designation in which the presence of a particular compound apparently resulted in a well-defined change in the enzymatic activities. However, before these or similar examples could be accepted as systems suitable for the study of enzyme synthesis, the following criteria had to be rigorously satisfied.

1. The changed enzymatic activity observed must not be due to the selection of preexistent mutant types but rather to an induced enzymatic modification against a constant genetic background.

2. The observed change in enzymatic activity must be a reflection of the appearance of an active apoenzyme, rather than due to the accumulation of cofactors or intermediates unique to the metabolism of the inducing substrate.

The use of recently evolved genetic methodology adapted to the analysis of microbial populations, and of modern enzymological procedures, made it possible to demonstrate in certain instances that both criteria could be met. These researches have been thoroughly summarized and discussed in recent reviews $(55,75,90)$ and need not be detailed again here. Certain consequences $(71,72)$ may, however, be noted. Theoretically these findings possess obvious implications for the problem of gene function. They establish that possession by a 
cell of a particular gene in its nucleus does not thereby guarantee that the corresponding enzyme will be found in the cytoplasm in utilizable amounts. Thus, predictive description of phenotype from a knowledge of genotype alone is impossible, even at the basic level of enzymatic constitution. It was necessary therefore to revise such statements as "genes control enzyme synthesis" to read "genes control the potentiality of enzyme synthesis."

Whereas such theoretical implications are of great interest, it was the emergent experimental consequences which proved to be of greater importance. In successfully meeting the two criteria mentioned above, an unequivocal demonstration was provided that the induced synthesis of specific enzymes could be attained under relatively simple and controllable conditions against a constant genetic background.

It was evident that the system had been found and defined which converted into experimental reality the possibility of inquiring into the mechanism of enzyme synthesis. It is the purpose of the present essay to summarize the information which has emerged from the study from such systems. A complete detailed account of the findings of the field will not be found here. On the contrary, the prerogatives of the essayist have been exercised to select and interpret that which is most pertinent according to the writers' own peculiar bias. Those readers who find themselves in agreement with both the selection and the interpretation will label the result an example of good judgment and analytical perception. Others will label it differently.

The very nature of the phenomenon virtually dictates the kinds of questions which are initially asked of it. The presence of certain agents called inducers can, in the presence of a suitable energy supply, stimulate the formation of specific enzymes. The use of inducible systems to elucidate the mechanism of enzyme formation resolves itself quite naturally into attempts to provide adequate answers to the following questions:

a. What is the nature of the precursor material which is transformed into active enzyme molecules? 
$b$. What is the nature of the enzyme-forming mechanism which converts the precursor material into active enzyme?

c. What is the role of the inducer, the presence of which specifically stimulates the appearance of the corresponding enzyme?

We can now turn our attention to an estimation of the extent to which answers can be provided to the questions just proposed.

\section{The Nature of the Precursor}

The problem of the precursor is perhaps most dramatically exhibited by considering inductions carried out under the simplest circumstances. It has been shown by a number of workers with a variety of enzyme systems $(55,75,90)$ that enzyme synthesis can be induced in the absence of a nitrogen source in cells suspended in a buffer solution of the inducer. That the appearance of enzyme activity actually involves the formation of enzyme has been established in these cases by exhibiting the homologous enzyme in extracts prepared from the induced cells. In such inductions, the nitrogen employed by the cell in fabricating the new enzyme molecule must come from some preexisting nitrogenous components, and one is immediately faced with the obvious necessity of identifying the components so employed.

Before considering the most recent experiments which have led to a satisfactory resolution of this problem, it is of interest to note briefly some of the earlier work which, although inconclusive, nevertheless exerted a strong influence on the subsequent development of this aspect of the problem. Monod (53), in his classic investigation into the growth of bacteria, discovered the existence of a severe interaction between enzyme-forming systems, which was expressed by the fact that simultaneous synthesis of two metabolically unrelated enzymes did not occur on exposing cells to a mixture of the two relevant inducers. In general, only one of the enzymes was formed at a time. A similar situation was uncovered in the yeasts by Spiegelman and Dunn (80). 
With yeast the presence of an external nitrogen supply greatly suppressed the severity and the extent of this interaction, and indeed, under certain circumstances simultaneous formation to two otherwise interfering enzymes was made possible.

Although these interactions were discovered relatively early in the renewed investigation of the phenomenon of enzymatic adaptation, their detailed significance remains to be delineated. Nevertheless, at the time, they were interpreted to suggest a competition for some commonly required nitrogenous material and therefore implied the existence of a precursor not as yet specified as to its ultimate enzymatic function. To these findings may be added those which established that the induction of enzymes requires the participation of a functional and utilizable energy-generating mechanism. Agents such as 2,4-dinitrophenol (50), sodium azide (74), and arsenate (93) which prevent the utilization of energy generated by metabolism also inhibit the induction of enzyme activity.

The importance of these early observations derived essentially from the fact that they made unlikely one quite obvious and attractive possibility of explaining the phenomenon of induced enzyme formation. This hypothesis suggests that the mechanism involved is one akin to the activation of trypsinogen to trypsin. Of course, the fact that energy is required for the induction precludes at once any simple application of this concept. In addition, such a model would suppose the preexistence in a cell of inactive forms of enzymes already fully determined as to their specificity and their function. The inhibitory interactions observed would be difficult to explain under these circumstances. Although observations of this nature could not definitely decide the issue, they clearly encouraged the search for a nitrogenous precursor not as yet restricted in its specificity and potentially convertible into more than one kind of enzyme molecule.

In designing experiments which seek to reveal the nature of this precursor, one can be guided by the fact that, in principle, three mechanisms of enzyme synthesis can be written down. They are as follows: 


$$
\text { complex precursor } \longrightarrow \text { active enzyme }
$$

complex precursor + free amino acids $\longrightarrow$ active enzyme

$$
\text { free amino acids } \longrightarrow \text { enzyme }
$$

Reaction (1) assumes the preexistence in the noninduced cell of a complex precursor which can be converted into active enzyme without the involvement of the free amino acids. This property distinguishes it from mechanisms (2) and (3) and permits an experimental decision.

Evidently what we are asking is whether it is possible for the precursor to become active enzyme without the participation of the free amino acid pool. Putting the question this way suggests immediately the necessity for examining the effect on the synthesis of enzyme of any experimental condition which decreases the availability of the free amino acids. Several methods are available and have been employed for achieving a restriction of this nature and they may be listed as follows:

a. The use of amino acid analogues as specific agents to prevent the incorporation of the free amino acids into protein.

$b$. In the case of those cells which possess an internal amino acid pool, to examine the effect of depletion and replenishment of this pool under conditions which would minimally disturb other components of the cell.

c. The use of amino-acid-deficient mutants which would make unavailable specific components.

Experiments along all these lines have been realized with yeast and the bacteria. The following paragraphs summarize briefly the evidence obtained.

THE EFFECT OF AMINO ACID ANALOGUES ON ENZYME SYNTHESIS

Halvorson and Spiegelman (32) carried out a study with a series of more than 40 analogues of amino acids for their effects on induced formation of alpha glucosidase in Saccharomyces cerevisae. A parallelism was established between the capacity of an analogue to inhibit net protein synthesis, as measured by growth, and its ability to suppress enzyme synthesis. In the case 
of the effective analogues, complete and specific reversal of the inhibition was achieved by the addition of the homologous amino acid. The generality of these findings was extended by the independently performed experiments of Lee and Williams (47) who demonstrated that the administration of ethionine to the intact rat prevented the formation of tryptophan peroxidase. In the experiments with the yeast $(32,34,36,83)$, it was possible to demonstrate by direct analysis that the presence of an effective amino acid analogue inhibits incorporation from the free amino acid pool into the protein fraction. One interesting feature which emerged from these experiments is that the presence of any one of the active amino acid analogues prevents the incorporation not only of its homologue but virtually of all the other amino acids as well. Further, no peptide fragments, unique to pools derived from cells incubated with an amino acid analogue, could be found.

In these studies, no evidence for an amino-acid-independent transformation of a complex precursor into active enzyme was obtained. The data rather led to the conclusion that the primary pathway of the induced formation of enzyme in nondividing cells of yeast involves the compulsory utilization of the internal free amino acids. The fact that the utilization of nonhomologous amino acids was blocked concurrently suggested further that the first stable intermediate formed in the synthesis of an enzyme molecule is of such a complexity as to demand the simultaneous availability of a large portion of the component amino acids.

THE EFFECT OF THE AVAILABILITY OF FREE AMINO AGIDS

ON ENZYME SYNTHESIS

If the conclusions derived from the experiments with amino acid analogues are correct, it would be expected that the ability of cells to form enzyme should parallel the availability of free amino acids for protein synthesis.

One striking difference between the enzyme-forming capacity of yeast as distinguished from that of many Gram-negative bacteria receives simple explanation in these terms. Thus, 
yeasts are able to form enzymes when suspended in nitrogen-free solutions of inducer, whereas the Gram-negative bacteria in general require an exogenous supply of nitrogen as a necessary concomitant of enzyme synthesis. The work of Taylor (96) suggests a reasonable explanation for this apparent independence of the yeast enzyme-synthesizing mechanism. This investigator surveyed a variety of yeast and bacteria for the presence of free amino acids in their internal environment. Of the three yeast types examined, all possessed detectable quantitics of the five amino acids looked for. Among the bacteria, the Gram-positives possess primarily glutamic acid and lysine. None of the Gram-negatives included in the survey contained detectable free amino acid by the procedures employed.

The possession of an internal pool was subsequently found to be a universal attribute of a wide variety of yeasts (85). It would appear that the ability of yeast to get along without an external source of nitrogen for enzyme synthesis is due to the fact that they have internal supply. To examine the question then of the effect of free amino acid availability on enzyme formation in the yeast it was necessary to devise and employ procedures capable of modifying these pool levels both quantitatively and qualitatively. Using such procedures, Halvorson and Spiegelman (33) demonstrated a strong correlation between enzymeforming capacity and pool level in both depletion and replenishment cycles. The results obtained in these studies supported the conclusion that free amino acids constitute the quantitatively predominant source of nitrogen in the formation of new enzyme molecules. Again, no evidence was uncovered which suggested the existence of a detectable amino-acid-independent transformation of a preexisting complex prescursor into active enzyme molecule.

ENZYME FORMATION BY AMINO ACID AUXOTROPHIC MUTANTS

The third approach mentioned which could provide relevant information involves the use of the auxotrophic mutants deficient in the ability to synthesize one or another of the amino 
acids. It was obvious that organisms such as yeast which accumulate an internal free pool would be difficult to employ in such studies, and not uncxpected difficulties arose when they were attempted with the yeasts. Fortunately, however, this approach was successfully applied almost simultaneously in two laboratories, and it is interesting to note that in both instances the organisms employed, Escherichia coli and Aerobacter aerogenes, possess a vanishingly small internal supply of free amino acids.

One of these investigations stems from the illuminating studies of Monod and Cohn (55) and their collaborators into the formation of beta-galactosidase by the ML strain of $E$. coli. It is interesting to note that in the course of these studies Cohn and Torriani $(19,20)$ had discovered the existence of an enzymatically inactive protein $(\mathrm{Pz})$ which was serologically related to the beta-galactosidase. In addition to this obvious structural relationship, they established a suggestive correlation between the distribution of the $\mathrm{Pz}$ protein and the capacity of the cells to synthesize the beta-galactosidase. Finally, they also showed that a significant decrease in $\mathrm{Pz}$ occurred in cells during the induced synthesis of beta-galactosidase. Although not the only possible hypothesis entertained by the authors, it is clear that all of these observations would receive ready explanation if $\mathrm{Pz}$ were indeed the precursor of the beta-galactosidase. In any event, taken together, the observations noted offered the most impressive evidence existent in the literature to support the suggestion that a preexistent complex specific precursor is involved in the synthesis of a known enzyme.

There was, however, one fact that militated against the acceptance of this view. Nitrogen-starved cells, though possessing normal amounts of $\mathrm{Pz}$, showed no ability to snythesize the beta-galactosidase unless an external source of nitrogen was provided. It was nevertheless still possible to imagine that the starvation procedure interfered in some way with the metabolic step required for transformation of $\mathrm{Pz}$ into active enzyme.

Monod, Pappenheimer, and Cohen-Bazire (56) undertook to investigate this question further by employing a series of 
mutants each of which was deficient in the ability to synthesize a single amino acid. These mutants were subjected to a "specific starvation" by being grown in a medium in which the required amino acid was present in limiting quantities, whereas all other compounds were in excess. Immediately upon the cessation of growth which attended the exhaustion of the amino acid, an inducer of the beta-galactosidase was introduced. It was found that little or no enzyme was synthesized by cells so treated, despite the fact that they contained normal amounts of $\mathrm{Pz}$. Such cells do, however, form enzyme immediately upon the addition of the amino acid they are unable to synthesize. These results made it necesssary to abandon any interpretation of the relation between $\mathrm{Pz}$ and the beta-galactosidase which involves a direct, amino-acid-independent conversion of $\mathrm{Pz}$ into active enzyme. The large number of amino acid auxotrophs employed in this study would suggest further that if $\mathrm{Pz}$ is indeed the precursor, a considerable number and variety of amino acids must be added to it before it is converted into active enzyme.

Ushiba and Magasanik (98) employed essentially the same approach in their study of the adaptive utilization of myoinositol by mutants of Acetobacter aerogenes. The results obtained led these authors also to the conclusion that the induced formation of the enzymes they were studying involved extensive synthesis from the amino acids. In a subsequent study, Rickenberg et al. (63) reported similar experiments and results with $E$. coli strain K12.

\section{IS THERE A PREEXISTENT COMPLEX PRECURSOR?}

The experiments cited thus far make unlikely any mechanism of synthesis which presumes the conversion of a preexistent precrusor into enzyme by a process which is independent of the free amino acids. The only alternatives left are, either that there is no preexistent complex precursor, or that one does exist but becomes active enzyme only after the incorporation of amino acids. The most obvious experimental approach aimed at a choice between these alternatives would appear to be the use of isotopic 
labels. Thus, the induction of enzyme synthesis in uniformly labeled cells suspended in unlabeled medium should provide the necessary data, providing the enzyme synthesized can be isolated in a pure state and its isotopic content determined. Rotman and Spiegelman (66), and Hogness, Cohn, and Monod (38) independently undertook to provide data relevant to this issue, using the beta-galactosidase system $E$. coli.

Rotman and Spiegelman (66) secured uniformly labeled cells by growth in $\mathrm{C}^{14}$ lactate. Enzyme was induced for short periods while the cells were suspended in nonradioactive medium. The beta-galactosidase synthesized was isolated and purified by means of zone ionophoresis through starch columns. Further purification was achieved with the aid of specific precipitation with purified antiserum. The results obtained revealed that less than 1 per cent carbon of the newly formed enzyme molecules could have been derived from any cellular components existing prior to the moment of the addition of the inducer. In the experiments of Cohn, Hogness, and Monod, $\mathrm{S}^{35}$ was employed as the isotopic label and similar methods were used for the isolation of the enzyme. Identical results and conclusions were obtained.

These findings virtually eliminate any hypothesis which assumes the preexistence of a complex precursor material which is convertible into enzyme. It is evident that a mechanism suggesting the de novo formation of enzyme from amino acids is at present the only one which has received experimental support.

A consequence of considerable importance issuing from this last conclusion is that induced enzyme synthesis is thereby equated to the process of protein synthesis. It follows that data derived from the study of enzyme induction are pertinent and relevant to the more general problem of protein formation. Further, the use of inducible enzymes as model systems of protein formation can, in principle and fact, confer two significant operative advantages. In the first place, one is assured that the synthesis of a protein is being examined-a certainty not available to experiments dependent solely on incorporation studies. 
Secondly, the formation of as little as $0.01 \mu \mathrm{g}$ of new protein can be detected with ease and precision.

\section{Precursor and the Nature of the Enzyme-Forming System}

In the present discussion, the term "enzyme-forming system," hereinafter referred to as EFS, will be used to designate that structure in the cell which is directly and "personally" involved in the process of fabricating the enzyme molecule. This verbal device is employed to isolate the EFS conceptually from all the other cellular components which can and probably do intervene more or less indirectly in the synthetic process. It will, of course, be noted that there is an assumption made here. By so stating the problem we do presume the existence of such a unique structure and, at least implicitly, ignore the possibility that proteins and enzymes are formed by a multitude of cooperating and sequential reactions.

In thinking about the possible nature of the EFS and in designing experiments to clarify the mechanism of its functioning, it is difficult to avoid being influenced by the results of the investigations into the precursor question. In a sense, these findings force an active search for EFS. The data we have reviewed relevant to the precursor problem are satisfyingly clearcut, almost distressingly so. They lead compellingly to the conclusion that in fabricating a new enzyme molecule, the cell prefers to weave it rather than to stamp it into existence. In this process, the simplest components are employed. Further, we find no evidence for any stable intermediates smaller than that requiring the presence and utilization of all the amino acids. A stepwise formation beginning with simple peptides and proceeding through polypeptides of intermediate lengths would appear to be eliminated. From one point of view, this is of course a pessimistic conclusion. It suggests that a successive approximation to an understanding of how proteins are synthesized will not be achieved in terms of a gradually better insight gleaned from the study of intermediate pieces of increasing complexity as they approach the final stage of synthesis. A 
door is thus slammed upon an extremely attractive approach for tackling the question of protein formation. Those who accept these conclusions obviously are faced with the necessity of finding a new approach to the solution of the problem of protein synthesis.

Adopting a mechanism of protein synthesis which involves the simultaneous availability of the constituent amino acid leads one quite naturally to consider a template-type mechanism. It is unnecessary here to undertake an extensive discussion of the recent information $(3,4,12,13,21)$ obtained from radioactive experiments with intact animals and tissues which have attempted to decide between template and stepwise mechanisms. In general, unequal labeling of the protein has been taken as an argument against the template hypothesis.

A serious difficulty is introduced by the realization that the incorporation of label compounds need not provide us with information relevant solely to the question of total protein synthesis. Exchange reactions are necessarily included in the information obtained from such experiments. That a very real difficulty exists is well illustrated by the recent experiments reported by Gale and Folkes $(25,26)$ in which a dissociation of net protein synthesis from incorporation by exchange reactions has been demonstrated. As had been shown with yeast (32), it was found that $p$-chlorophenylalanine can prevent new protein synthesis in Staphylococcus aureus. In addition, Gale and Folkes discovered that although the presence of this analogue effectively prevents the incorporation of phenylalanine into the protein, it has relatively little effect on the exchange incorporation of glutamic acid.

The existence of such phenomena makes it difficult to interpret with certainty the significance and uniqueness of data derived solely from incorporation studies.

\section{On the Role of the Inducer}

One of the most dramatic features of the phenomenon of enzyme induction is the requirement for the presence of the 
inducer and the apparent precision with which it specifically stimulates the formation of a particular enzyme.

Monod and Cohn (55) have recently reviewed the bulk of the published information relevant to inducer function. Much of this knowledge we owe primarily to the efforts of these two men and their collaborators. Certain experiments are particularly pregnant with interesting implications for the nature of EFS and it is these which will occupy our attention.

\section{SPEGIFICITY AND THE RELATION OF AN INDUGER TO THE} HOMOLOGOUS ENZYME

There are two possible mechanisms of inducer action which can be formulated with sufficient exactitude to permit their experimental elimination. These have been labeled by Monod and Cohn (55).

1. The Functional Hypothesis. The synthesis of an enzyme is presumed to be linked to its activity, from which hypothesis one would conclude that effective inducers would necessarily be substrates.

2. The Equilibrium Hypothesis. It is assumed that inducers must form complexes with enzyme synthesized as a sine qua non of enzyme formation.

A number of observations eliminated the first hypothesis. Thus, Spiegelman, Reiner, and Sussman (88) demonstrated that maltose can induce alpha-glucosidase at $p \mathrm{H}$ values that preclude detectable metabolism of the maltose even by fully induced cells. Analogues of natural substrates have been employed for similar purposes. Thus, alpha-methyl-glucoside can be shown $(76,82)$ to be an inducer of maltase under conditions in which utilization cannot be detected. Further, Monod, Cohen-Bazire, and Cohn (54) have demonstrated similarly that thio-methyl-beta-D-galactoside and melibiose, neither of which is detectably metabolized by strain ML of $E$. coli, are nevertheless inducers of beta-galactosidase.

The second hypothesis was proposed in its simplest form first by Yudkin (103) who did so in terms of a mass action hypothesis in which combination between inducer and enzyme is 
assumed to drive the reaction in the direction of precursor conversion into enzyme. This complexing concept leads to several predictions, the experimental violation of any one of which would require either its revision or complete abandonment. We may list some of these as follows:

1. Substances which can complex with enzymes should be effective inducers.

2. Substances shown to be incapable of complex formation with a given enzyme likewise should be unable to induce this enzyme.

3. The dissociation constant of the substance measured in terms of its inductive effect on enzyme synthesis should be comparable in magnitude to the value obtained in experiments in which the constant is derived from complexing properties of the inducer with enzyme.

It should be noted that the term "complex formation" employed here is meant to subsume both the specific type, characterized by combination between an enzyme and its substrate or competitive inhibitor, and the nonspecific type, represented by a complex between an enzyme and a noncompetitive inhibitor. Thus, proof that an inducer does not form a specific complex with enzyme does not eliminate it as an enzyme complexant, since it leaves open the possibility of nonspecific complex formation. There is no a priori reason for believing that nonspecific combinations cannot function in the process of enzyme synthesis.

It is difficult to provide convincing exceptions to the second deduction mentioned, involving as it does only negative properties. However, violations of both (1) and (3) have been found. Thus, Lederberg (45) exhibited a mutant of E. coli which fails to respond to lactose as an inducer of beta-galactosidase, despite the fact that lactose is a substrate and therefore a specific complexant.

Monod, Cohen-Bazire, and Cohn (54) subjected these considerations to the first systematic and thorough analysis. They revealed that thio-phenyl-beta-D-galactoside, although a potent 
competitive inhibitor of the beta-galactosidase, was nevertheless incapable of inducing the formation of this enzyme. Other specific complexants, e.g., ncolactose and phenyl-beta-Dgalactoside, were similarly found devoid of inductive capacity.

An instance of an apparent violation of the third deduction was provided by Spiegelman and Halvorson (82). The dissociation constant of methyl-alpha-D-glucoside, an inducer of alpha-glucosidase synthesis in Saccharomyces cerevisiae, was determined and compared with the constant derived from its complexant properties with enzyme. The two values were found to differ by a factor of about two hundred.

It must be emphasized that the types of hypothesis made unlikely by the experiments cited are highly restricted in nature. All of these experiments had in common an examination of the effect of the inducers as enzyme complexants in terms of measurable effects on enzymatic function. By their very nature they could not preclude the possibility of combination at some site which possessed no consequences in terms of a detectable modification of enzyme activity.

It must further be noted that such experiments do not eliminate the possibility that inducer serves as a coupling agent which leads to the formation of a stable complex among enzyme, inducer, and a third component. If the inducer would combine with enzyme effectively only in the presence of the third component, violation of all three predictions could obtain.

THE QUESTION OF STOICHIOMETRY OF INDUCER ACTION

Until the advent of the resourceful exploitation by Pollock of the penicillinase-forming system of $B$. cereus, the question of stoichiometry was one which could be formulated but hardly resolved. Pollock (59-61) discovered that fleeting exposures of cells to minute concentrations of penicillin at $0^{\circ} \mathrm{C}$. can lead to the specific adsorption of enough inducer molecules to permit penicillinase formation even subsequent to the destruction of all unbound penicillin. With the use of $S^{35}$-labeled penicillin it was found that specific fixation is saturated at about 80 atoms 
per cell. Having purified the penicillinase and determined the minimum turnover number, Pollock and Torriani (cited in 62) were able to demonstrate that each molecule of penicillin thus fixed can cause the formation of at least ten molecules of penicillinase. These observations then establish that an inducer can act catalytically.

The unique feature of virtually irreversible adsorption of inducer has thus far not been exhibited in other enzyme-forming systems, and hence this type of experiment is at present feasible only in the penicillinase-producing system.

\section{The Relationship of Inducer to EFS}

The data reviewed thus far indicate that enzyme molecules are fabricated on a template. Further, inducer molecules appear to be specifically bound to some site concerned with enzyme synthesis. Identification of the inducer-complexing site with the template is the simplest and most economical hypothesis at present worthy of further exploration. The question naturally arises whether combination between template and inducer leads immediately to full function.

The least complicated variety of such an "activator" hypothesis would assume that a full complement of templates preexists and that the addition and adsorption of inducer molecules is all that is required to convert them to complete activity. This relatively simple model possesses several easily testable predictions. It would, for example, suggest that, providing other conditions are not limiting, enzyme synthesis should proceed at its maximal rate immediately subsequent to adsorption of inducer. Further, once inducer molecules are adsorbed, one would not expect on a priori grounds to find the emergence of any strikingly different properties of the EFS as a consequence of the accumulation of enzyme molecules.

It is not too difficult to marshal evidence pointing up the inadequacy of such activator mechanisms as suitable models of induction. We may now consider the nature of this evidence 
with a view to seeing whether it circumscribes the nature of the induction mechanism sufficiently to serve as a useful guide in the construction of a more suitable model.

THE KINETICS OF ENZYME SYNTHESIS

AND ITS INTERPRETATION

One of the most obvious ways of examining the adequacy of mechanisms analogous to the activator hypothesis is in terms of its kinetic consequences, and historically this was the first type of data provided. The kinetics found for the induced synthesis of maltase and galactozymase in yeast were found to be autocatalytic and could be accurately described by the logistic equation $(71,72)$. Subsequently other systems were found to obey the same kinetics (41).

The results obtained suggested that in the early phases of the induction, enzyme-forming capacity increases as new enzyme molecules are produced. The data are clearly inconsistent with a simple activator hypothesis, and on the same ground the Yudkin mass action model (103) for inducer function was rejected (71), since it also predicts maximal rates of enzyme formation from the onset of induction. To explain the autocatalytic kinetics, as well as certain data of a genetic nature to which we will have reference below, the so-called "plasmagene" hypothesis was proposed (72). In this it was assumed that the inducer functions by stabilizing a complex between enzyme and the "plasmagene" and that this complex is capable of replicating itself and producing enzyme. The widespread implications of this hypothesis and the admittedly limited experimental support available at its formulation acted as a stimulatory irritant. Equally plausible alternatives for explaining the logistic kinetics were quickly offered by a number of authors.

Thus, it has been argued (53) that enzyme formation was being followed in these studies employing metabolizable inducers which could serve as an energy source. Under such circumstances, obviously the more enzyme there is present, the greater is the rate of energy generation, and the more rapid therefore 
would be the formation of enzyme. As a consequence, the kinetics of enzyme formation would be autocatalytic independently of the actual kinetic details of the enzyme-forming process. Although eminently plausible, it was pointed out $(76,90)$ that an argument of this nature could not be used to explain away the autocatalytic formation of hydrogenlyase, the functioning of which could hardly yield sufficient energy for protein synthesis. Further, this criticism turned out on subsequent investigation (35) not to be applicable to the system against which it was directed. An examination of the relation between the kinetics of enzyme formation and the rate of energy supply revealed that alpha-glucosidase synthesis is exponential only when the rate at which energy is delivered exceeds a certain critical value. Below this it is linear with time. Thus, if one actually performs the induction in such a manner (e.g., anaerobically, with highly purified maltose) as to insure that the inducer is the sole energy source, linear and not autocatalytic kinetics are obtained for the early phases of the induction. The original experiments were performed aerobically under conditions in which a relatively high endogenous respiration was the principal energy source, the contribution of inducer metabolism being negligible.

Monod and Cohn (55) have quite correctly emphasized that wherever possible, the complications which can attend the utilization of an energy-generating inducer should be avoided by the use of what they call "gratuitous induction." Conditions of gratuity require the use of a nonutilizable inducer and a neutral noninductive energy source. The discovery that alpha-methylglucoside is a nonutilizable inducer of the alpha-glucosidase system made it possible to reexamine the question of the kinetics of alpha-glucosidase synthesis under gratuitous conditions. Again exponential kinetics were obtained (35).

There exists another interesting possibility which, if it obtained, would make interpretation of the kinetics of induction difficult and uncertain. This stems from the fact that in following the appearance of enzyme in a population cell, an over-all average property is measured. Were the process of enzyme 
synthesis in individual cells relatively more rapid than the time of the experiment, and if a lag period in the onset of enzyme formation were normally distributed in the cell population, one would obtain a cumulative curve of enzyme with time which would have the appearance of a logistic. Benzer (8) was able to demonstrate that this was not true under conditions of gratuity by the ingenious use of bacteriophage as a tool. The virus was employed here both to stop enzyme formation and to release the enzyme by lysis of those cells which had accumulated enough enzyme during the period of induction to support virus multiplication. Using this device he demonstrated that enzyme synthesis is uniform in all cells in gratuitous inductions but is heterogeneous if the inducer is the primary or sole energy source.

This question has not been completely resolved in yeast. An interesting attempt to do so was, however, made by Terui and Okada (97), who photomicrographically examined the distribution of the division times in a population of cells in which the energy required for the division was derived from the inducing substrate maltose. They come to the conclusions that the kinetics of the over-all adaptation is explainable on the basis of a normal distribution of lag times in the population. There are certain features of the experiment which make for uncertainty. By its very nature, it is performed under nongratuitous conditions which, according to Benzer's results, lead to inhomogeneities. Further, onset of division may not be an adequate measure of induction level. Finally, as has already been noted, it is relatively easy to change the kinetics of enzyme formation in the very system these authors studied from the logistic to one which is linear with time. Whereas one can explain logistic kinetics on the basis of a normal distribution of lag times, it is virtually impossible to explain linear kinetics on a similar basis, since it would involve assuming a completely unrealistic distribution.

Monod, Pappenheimer, and Cohen-Bazire (56) examined the kinetics of beta-galactosidase formation in $E$. coli by the use 
of an interesting device involving a relative plot in which increment in enzyme is plotted against increment in cell mass. This procedure has many advantages for studying relative synthetic rates, since direct comparisons can be made between slow- and fast-growing cultures. The data provided by certain inducers yielded linear curves when plotted in this manner. They suggest, therefore, that their findings are not consistent with an autocatalytic mechanism. It should be noted, however, that whereas the relative plot is extremely useful for the study of relative rates of synthesis, it is a comparatively insensitive procedure for the kinetic examination of the early phases of the induction. The time interval required for a definably measurable increment in mass could well be long enough to make impossible the examination of the kinetically interesting period of induction. That this is not a remote possibility is easily shown with yeast which form enzyme quite a bit more slowly than bacteria. If the data of yeast induction are plotted relatively, a linear phase is observed. However, the early points show an exponential departure from linearity. Were attention confined solely to the later points, complete agreement with the data of Monod, Pappenheimer, and Cohen-Bazire would have been obtained. Finally, it must be noted that recent information on inducer fixation (15) in the beta-galactosidase system is difficult to reconcile with linear kinetics.

A word may be interposed here about time linear kinetics since a number of authors have taken its existence to nullify the significance and interpretability of the autocatalytic type. It is a fact that one can convert a synthetic reaction which is inherently autocatalytic to one which is linear with time, and the possibility should neither occasion surprise nor engender confusion. Thus, there is little doubt that under the usual conditions the growth of bacterial populations is exponential. Nevertheless, linear growth of a streptomycin-requiring strain was reported by Schaefer (67). In discussing this observation Monod (52) pointed out that a similar type of kinetics should be obtainable artificially by establishing a constant limited supply 
of an essential metabolite. He thus forecast his own discovery (51) of the "bactogen" as well as the independent development by Novick and Szilard (57) of what they call the "chemostat."

It is not surprising that whenever limitations are imposed, whether they be of energy (35), inducer (60), or of a required metabolite, linear rates of enzyme synthesis are observed. One must therefore agree that enzyme synthesis need not be always autocatalytic. However, linear and other (24) deviations from autocatalytic kinetics may signify the presence of restrictions which prevent the exhibition of the exponential kinetics of which the system is capable. In any case, such deviations cannot be accepted as compelling evidence against the existence of exponential rates of enzyme formation.

The presence of a rising rate of enzyme synthesis means that some self-reinforcing activation of the enzyme-forming system is occurring during the induction. A further analysis of the mechanism and the role of the inducer in it requires the application of other analytical devices. One of these approaches is genetic, and we turn now to the data derived from its use.

THE INHERITANCE OF ENZYME-FORMING CAPACITY AND

ITS SIGNIFICANCE FOR THE ROLE OF THE INDUCER

Kinetic arguments, no matter how refined, cannot of themselves be decisive. It was fortunately possible to examine the question of the autocatalytic nature of enzyme formation by completely different means and one which provided a more subtle and surprisingly fruitful method for further probing into the question of inducer function. The potentiality of examining induction by essentially genetic methods was suggested by the discovery of a new and pathological type of induction. Winge and Roberts (102) labeled their new phenotype "long term adapter" because it was much slower in adapting to galactose than the normal type. They further showed that the "slow" character was determined by an allele $\left(g_{s}\right)$, which was recessive to the wild type $(G)$.

Spiegelman, Sussman, and Pinska (89) undertook an an- 
alysis designed to uncover the mechanism underlying the "slowness." They came up with the surprising finding that the vast majority $(99.9 \%)$ of $g_{s}$-cell populations were noninducible. When plated on galactose-EMB test plates they yielded small negative clones. If the one out of a thousand positives were not simply mutants, a situation of signal significance would be available, for it would mean heritably different enzyme-forming capacity against a constant genetic background. This turned out to be the case since fluctuation tests designed to determine the nature of the origin of the positives were inconsistent with a mechanism involving random mutation. The Poisson variance obtained strongly suggested that contact with galactose was necessary and induced in a small proportion of the cells a heritable modification leading to the ability to form the enzyme. Once acquired, enzyme-forming ability was transmitted indefinitely from one cell generation to the next, providing galactose was present. However, growth of the positive cells in the absence of the substrate resulted after six or seven generations in a mass reversion to the original negative phenotype. The mass reversion feature virtually eliminated any mutational interpretation of the phenomenon.

A detailed examination of the kinetics of the reversion was made. The data obtained were most simply explained by assuming that positives contained units necessary for enzyme formation lacking in the negatives. These units or elements could increase only in the presence of the inducer galactose. Growth of positives in the absence of galactose leads therefore to a dilution of the relevant units, and a point is finally reached at which cells are produced possessing either none or an insufficient number of these elements.

The nature of the conclusions and the potential usefulness of the system for the study of the formation of enzyme-synthesizing units warranted an attempt to obtain more critical evidence. A more directly interpretable analysis of the nature of the reversion phenomenon was undertaken (79) by the serial isolation and characterization of single-cell progeny produced by 
positives during division in the absence of substrate. These single-cell analyses confirmed the earlier experiments cited, in demonstrating that abruptly after about five generations cells of the negative phenotype are produced with increasing frequency. The data were uniquely amenable to a probability analysis. It was found that a fully induced positive cell contains in the neighborhood of a hundred particulate units and that the minimal number of particles required for a cell to exhibit the positive phenotype on a test plate is in the neighborhood of one. The probability of a given particle passing into the daughter cell was closely approximated by one half.

It proved further possible to offer evidence for the extrachromosomal nature of the particles in this particular instance by the comparative study of the transmission of the enzyme-forming capacity during reductional segregation (78). The method used was identical to that employed in a previous study involving the inheritance of the ability to ferment melibiose (86), with a similar result. The effect of prior induction of $(G g)$ heterozygotes to galactose on the segregation of the capacity to form the enzyme was studied. Noninduced heterozygotes, with few exceptions, yield a $1: 1$ ratio of positive to negative spores on galactose test plates. However, if the heterozygotes are induced prior to segregation, and the latter occurs in the presence of substrate, all four segregant spores exhibit the positive phenotype in over 90 per cent of the asci dissected. Growth in the absence of substrate for seven to twelve divisions of four such positive spore clones, leads to a reversion of two of them to the negative phenotype, thus restoring the Mendelian ratio of the phenotype. The fact that homozygous recessives carried through the same procedure do not yield positive spores suggests that the presence of both the dominant gene $G$ and the substrate leads to the uniform production of the extrachromosomal elements which are necessary for enzyme formation. When, in the course of the segregation, elements are incorporated into the cytoplasm of spores carrying the $g_{s}$ allele, they are converted to the positive phenotype. The 
abrupt reversion of such positive cultures to the negative phenotype upon growth in the absence of inducer is in agreement with the previous studies noted.

The autocatalytic property of the enzyme-forming system or some necesssary portion of it is here dramatically illustrated in terms of the transmission of enzyme-synthesizing capacity from mother to daughter cell. At least one is needed to make more since, if by chance a cell is produced with none, it and its progeny are negative.

The results obtained on the inheritance of the ability to form galactozymase parallel in many respects the prior investigations of Ephrussi (23) and his co-workers into the "petite" mutant. The data of these workers also suggest the existence of randomly distributed particulate elements in the cytoplasm possessing autocatalytic properties and responsible for the production of cytochrome oxidase. The autocatalytic nature is again exhibited by the spontaneous occurrence of cells lacking the particles which lead to the irreversible loss of the ability to form the corresponding enzymes.

It has been possible to demonstrate (89) that the particulate elements involved in the two instances are distinct entities, since the two enzyme-forming capacities are lost separately. In the "petite" mutant case control of the number of elements of the cell is apparently not achieved by variation of inducer availability. However, cells lacking particles can be produced by treatment with the acridine dye, euflavine. It is of interest to note that Slonimski $(69,70)$ has been able to demonstrate that this compound which is so effective in preventing the transmission of the particles from mother to daughter cells also specifically inhibits their ability to form enzyme.

One can go beyond an independent exhibition of the autocatalytic nature of the enzyme-forming mechanism. The possibility of controlling active particle numbers in the case of the slow adaption to galactose provided an experimental system which permitted a further analysis on the role of inducer and its relation to the active particles and enzyme. 
Let us first consider an hypothesis (55) which would explain the autocatalytic property in terms of enzyme function analogous to that advanced to explain the kinetic data. Under this hypothesis, the possession of a small amount of galactozymase can effect the generation of more galactozymase by virtue of the fact that this enzyme system is involved in the energy-generating mechanism to the cell. The role of the galactozymase in this hypothesis is confined to the supply of energy. The active particles would then be "aggregates" of sufficient amounts of galactozymase to serve as energy generators. Campbell and Spiegelman (11) used a respiratory deficient strain to demonstrate that functional enzyme is lost long before the autocatalyst necessary for further enzyme synthesis, and hence these two cannot be identical.

The quantitative results which were obtained in the study of the reversion from the positive to the negative state led to the formulation of a relatively precise hypothesis concerning the detailed nature of the reversion process. The hypothesis may be summarized in the form of the following series of statements: (1) the distribution of active particles among cells is assumed to be normal, (2) during growth in an inducer-free medium these particles neither decrease nor increase in absolute number and hence the average number per cell decreases exponentially, (3) the particles are distributed at each division randomly with equal probability to the mother cell and to the bud, and (4) the minimal number of active particles that a cell must contain in order to score as a positive is close to one.

These properties were derived solely from a study of the transformation from the positive to the negative state. Analysis of the reverse process quickly led to other features which illuminated further details of the process. Rotman and Spiegelman (65) were able to convert a large percentage of negatives to the positive phenotype by treatment with fractions of yeast extract. It was possible to show that each of the positive cells so obtained acquired only a few active particles as a result of the conversion. On the basis of the properties of the conversion it 
was proposed that the transformation of a negative into a positive cell does not involve the de novo formation of active particles but rather the activation of preexistent inactive units. We thus have two categories of particles, active and inactive. A third type was revealed through an analysis by Campbell and Spiegelman (10) of the growth of active particles. This third kind is intermediate to the active and inactive varieties and is characterized by being easily converted by inducer to an active unitan event which is rare with the inactive particles. We will designate this intermediate type as convertible particles. Their presence is indicated by an abrupt rise in active particle number when reverting positive cells are exposed to inducer. Subsequent increase of active particles obeys an approximately exponential law. The convertible particles occur with increasing frequency as positive cells are allowed to go through dilution growth in the absence of inducer. Further, they appear to suffer decay, since if they are not brought into contact with inducer soon after their appearance, activation by inducer is no longer possible.

Another peculiar feature which emerges from a study of the late portion of the reversion is an anomalous change in stability of active particles. It was shown in one of the earlier studies noted (89) that active particles are perfectly stable in the early reversion divisions. However, it was found (81) that this stability disappeared after about the seventh division in the absence of inducer.

\section{A Model of Enzyme-Forming System Relating Template, Enzyme, and Inducer}

In discussing the information derived from genetic experiments we have deliberately used such neutral words as particles, units, and elements.

We should now like to essay a synthesis of the biochemical, kinetic, and genetic information we have reviewed thus far in terms of the simplest model consistent with the known observations. 
The model to be presented is essentially the one designed by Campbell and Spiegelman (10) in an attempt to explain certain paradoxical aspects of the growth kinetics of active particles in long-term adapting stocks. Its major features are most easily seen and developed in terms of "slow" induction. Once these have been exposed, the scheme will be generalized and applied to normal enzyme synthesis.

The biochemical investigation of the precursor aspect of enzyme synthesis leads to the conclusion that a template is involved. The kinetics of normal induction and the genetic data obtained with the aid of "slow" strains both suggest that induction is characterized in its early stages by an autocatalytic activation. All the data can therefore be described in a unified fashion by the assumption that initially the templates are relatively inactive and are autocatalytically converted to full activity during the course of the induction.

We are thus led to identify the autocatalytic active particle defined by the genetic operations with the autocatalytically activated template demanded by the biochemical studies.

We now inquire where in our model is the inducer likely to fit and how it is to function. Both kinetic and genetic experiments indicate that active templates increase in number during growth in the presence of inducer. Removal of the inducer results in complete cessation of the increase, but the number present at the moment of removal remains constant for many hours. In our opinion the simplest explanation which can be offered to explain this effect is that inducer, or some derivative of it, is irreversibly incorporated into the structure of the active template. If one proceeds along this line of reasoning, one difference between an active template and an inactive one would be that only the former contains galactose. However, if this were the only difference, exposure to galactose should convert negative "slow" cells into positive ones, because in one generation half of the templates would have been formed in the presence of galactose. This suggests then that an active template differs from the inactive form in some property other than 
the possession of the inducer. The possibility is therefore provided for a third template type, neither active nor inactive, possessing the second property but not the galactose. A description is thus provided for the "convertible" template, which becomes stabilized in the active form on the addition of inducer.

We now turn to a consideration of the likely nature of this "second property" possessed in common by active and "convertible" templates, but lacking in the inactive variety. For simplicity and ease of following the argument, we may here summarize the properties which any model constructed on the basis of the above discussion must exhibit.

1. Each cell contains a certain number of templates specific for the synthesis of some enzyme of the galactose system. They may exist in "active," "inactive," or "convertible" forms.

2. A cell containing one or more active or convertible templates will give rise within a few hours of growth in a galactose medium to progeny in which most or all of the templates are active.

3. A cell containing no active or convertible templates may grow for many generations on galactose without any template ever becoming active. If activation does occur it is a rare event. It is heritable on the cellular level as required by property (2).

4. Active templates differ from convertible ones in that the former contain galactose. Convertible templates are readily transformed to active ones by the addition of galactose.

5. Active templates form the enzyme mentioned under (1); inactive ones do not.

From properties (2) and (3) it is clear that the presence of some active templates in the cell greatly accelerate the activation of other templates. Whether this includes other templates already present or only those subsequently formed is not deducible from the data. The question is, however, how this activation might take place.

The two simplest possibilities are $(a)$ the active templates self-duplicate, the new ones being, so to speak, descended from 
the one originally present and $(b)$ active templates produce something which activates inactive templates. Both may operate; however, the functioning of the second mechanism is directly implied by the experiments of Rotman and Spiegelman (65) which show that inactive sites preexist and can be converted to active ones. Under the circumstances, the self-duplicating property can be abandoned as being superfluous for a description of enzyme-forming system as a protein-synthesizing machine. What the active templates might produce which would activate other ones is also not deducible from the data. However, the only thing the template can be presumed to produce heterocatalytically is enzyme. One is then led from this model to conjecture that the enzyme molecule itself is the activator of inactive templates.

The picture of the mechanism emerging is clear, and we may now generalize and formalize it. Let us denote the relevant templates by $\mathrm{T}$, inducer molecules by $\mathrm{I}$, and enzyme by $\mathrm{E}$. We postulate then the following.

1. When cells have been grown for many generations in the absence of inducer, their templates are all, or nearly all, in the simple and inactive form $\mathrm{T}$.

2. The complex T-E is unstable and can occur in either one of two ways. In a noninduced cell each $\mathrm{T}$ has a small probability of fabricating spontancously an enzyme molecule. Secondly an induced cell allowed to grow in the absence of inducer will, when the inducer is sufficiently diluted, produce T-E from decomposition of the inducer-T-E complex, mentioned in the next statement.

3. The reaction

$$
\mathrm{T}-\mathrm{E}+n \mathrm{I} \longrightarrow \mathrm{T}-\mathrm{I}_{n}-\mathrm{E}
$$

is rapid and relatively irreversible. Here, $n$ is the number of inducer molecules bound per T-E complex; $n$ may be unity in some cases and greater in others.

4. A population of cells grown in an inducer-containing medium has most, or all, of its sites in the form of $\mathrm{T}-\mathrm{I}_{n}-\mathrm{E}$. 
5. Of the forms mentioned above, only $\mathrm{T}-\mathrm{I}_{n}-\mathrm{E}$ can effectively and rapidly catalyze enzyme synthesis.

In the nomenclature of Cohn and Monod (18) T would be an apo-organizer, I, a co-organizer; and T-I, an organizer. The complexes $\mathrm{T}-\mathrm{E}$ and $\mathrm{T}-\mathrm{I}_{n}-\mathrm{E}$ represent new entities not embraced or employed by their terminology.

The reversion from positive to negative in "slow adaptors" can be interpreted in terms of the above model in the following manner. As soon as growth in a galactose-free medium occurs, all newly formed templates appear as either T or T-E and consequently no enzyme synthesis takes place at them. The templates which were present initially remain as $\mathrm{T}-\mathrm{I}_{n}-\mathrm{E}$ and continue to synthesize enzyme. They are the autocatalysts or active particles and are diluted out by growth. As the reversion growth proceeds, one gets, as a result of the consequent dilution of inducer, some convertible particles, T-E. The ultimate and irreversible decay of active particles if the reversion proceeds too long receives therefore simple explanation in terms of the instability of T-E. The anomalous change in stability of active particles (mentioned at the end of $I V, B$ ) which occurs late in the reversion is explained simultaneously.

The conversion to the positive state occurs when a cell containing one or more templates of the form $\mathrm{T}-\mathrm{I}_{n}-\mathrm{E}$ or $\mathrm{T}-\mathrm{E}$ is placed in a medium containing inducer. Any T-E complexes are first converted to $\mathrm{T}-\mathrm{I}_{n}-\mathrm{E}$ and then in both cases free enzyme is formed. By virtue of the enzyme produced other templates can be rapidly converted to the stable $\mathrm{T}-\mathrm{I}_{n}$-E state, provided excess inducer molecules are present. The infrequent spontaneous production of positives from negatives in the "slow" strains might be explained on the basis of the rare occurrence of enzyme formation by unoccupied $\mathrm{T}$ or $\mathrm{T}-\mathrm{I}_{n}$.

The novel function attributed to the enzyme and inducer molecules recalls the formally similar hypothesis of the "plasmagene theory." It should be emphasized, however, that the present model exploits a "feed-back" feature which was actually inherent in the plasmagene model but not used. It is the 
recognition that enzyme molecules can serve to activate unoccupied templates that permits an explanation of autocatalysis which does not invoke self-duplication. There are no data existent to our knowledge which demand this latter property as an integral part of the enzyme-forming process. It would, however, seem necessary to retain self-duplication as a method of template maintenance to explain the instances of cytoplasmic transmission noted, particularly in the case of the "petite" mutants.

\section{Stages in the Induced Formation of Enzyme and Their Interpretation}

The particular appeal of the model just proposed lies in the fact that it is in principle applicable to systems other than "slow adaptation" and permits an instructive interpretative comparison among them. A central feature is the assumption that the active enzyme-synthesizing unit is the triple complex between template, enzyme, and the inducer. The difference between the slow adaptors and the normal variety will then be explained in terms of the very much lower probability of an unoccupied template of the slow type producing an enzyme molecule in the absence or the presence of inducer. In normal varieties this event occurs sufficiently frequently in the absence of inducer so that at any given moment it is highly probable that there is at least one T-E complex present per cell. This represents therefore the constitutive mechanism for the synthesis of the low basal enzyme levels frequently observed in inducible strains. In the same vein, one can explain the so-called "constitutive mutants" $(17,46)$ which make enzyme in inducer-free medium even faster than the normals do in the presence of inducer. Thus, one can suppose that "constitutive mutants" possess templates that possess an even higher than normal probability of spontaneously forming enzyme molecules. This has the obvious consequence that constitutive enzyme formation of this variety should more closely approximate noninduced enzyme synthesis. Some constitutive enzyme formation is of course referable to internal inducer production, as is beautifully shown 
by the work of Vogel and Davis (99) with $N$-acetyl-ornithine deacetylase and by Stanier's (91) and Suda's (92) skillful use of sequential induction.

It will be noted that comparatively irreversible combinations of inducer are assumed to occur only when both template and a replica are present. This situation possesses interesting predictive consequences which are sufficiently unique to permit experimental examination. It is evident immediately that the state of a noninduced cell, with the majority of its templates unoccupied by enzyme, should be very different from one which is induced to the condition where a considerable portion of the templates are already in the active state, and where enzyme molecules are freely available for the formation of new active complexes.

In the first place, differences in response to inducer concentration in noninduced as compared with partially induced cells would not be unexpected. Induced cells would contain the $\mathrm{T}$-E complexes permitting the relatively irreversible combination with inducer molecules. This is of course most dramatically exhibited by the long-term adapting instance. Here, no level of inducer concentration is capable of stimulating enzyme formation in the vast majority of cells. However, once this is successful and an induced cell is produced, normal inducer levels are sufficient to maintain enzyme formation indefinitely. An analogous difference can also actually be seen in normal enzyme formation. Thus, with alpha-methyl-glucoside as an inducer it is found that the concentration levels required to initiate induction are considerably higher than those needed to maintain enzyme formation once it is started (77). The same observation has been made independently by Monod (cited in ref. 15) and Spiegelman and Gilmour (81) with respect to beta-galactosidase synthesis in $E$. coli. Here enzyme formation does not commence at thio-methyl-D-galactoside levels of $1 \times 10^{-6} \mathrm{M}$ but will if the concentration is raised to $5 \times 10^{-5} M$. However, once enzyme formation is begun, $1 \times 10^{-6} M$ is perfectly adequate to maintain the induction. 
Pollock (62) has observed interesting differences between induced and noninduced cells in terms of sensitivity to ultraviolet. He found that prior to the onset of penicillinase formation in the presence of inducer, enzyme synthesis is extremely sensitive to inactivation by exposure to UV. Once, however, enzyme formation has commenced, it becomes increasingly more resistant to this type of inhibition. Halvorson and Jackson (31) showed an exactly similar situation in the induced formation of alpha-glucosidase by $S$. cerevisiae.

These are findings which are not unexpected if, as seems reasonable, empty templates are more sensitive to inactivation by UV than those combined with protein in a complex stabilized by inducer molecules.

Finally, another difference between the induced and noninduced state has been observed (84) in terms of response to amino acid analogues. It has already been noted that it is possible to stop enzyme formation by means of amino acid analogues. However, this inhibition is effective only if one adds the amino acid analogue in the lag period of enzyme formation, prior to the appearance of new enzyme molecules. If the induction is allowed to proceed to the point where enzyme is already being formed, suppression of enzyme synthesis with the antagonist becomes increasingly difficult (84). We can explain this apparently puzzling finding quite readily in terms of the model just proposed. In noninduced cells the analogue is competing with its homologue for an unoccupied position on the template. It is not surprising that the competitive situation for the antagonist should be markedly modified when the empty templates are filled with enzyme, for of necessity homologous amino acids now sit bound at, or close to, the sites of competition.

Our discussion of the biochemical, kinetic, and genetic information available on enzyme induction has led us to postulate three components of the enzyme-forming system, inducer, protein, and template. In any given case the first two can be reasonably well defined in terms of known chemical entities. We turn now to the question of the identification of the chemical nature of the third member of the triad, the template. 


\section{The Chemical Nature of the Template}

A template which is to serve as a device for protein synthesis must be at least as complicated and as large as the molecule which it is forming. Other than the protein moleculc itself, there are relatively few candidates one can propose which can satisfy the two criteria of size and informational complexity. With these restrictions in mind, the two known possibilities are deoxyribonucleic acid (DNA) and ribonucleic acid (RNA).

In the following paragraphs we will briefly examine the available evidence for and against the possibility that one or the other of these substances is associated with the enzyme-forming mechanism.

\section{DNA AS A COMPONENT OF EFS}

Evidence from work with the transformation principles $(39,105)$ offers convincing evidence that genetic information can be stored in and transmitted through DNA. The potentiality, therefore, of forming any specific kind of protein molecule must ultimately be referable to the DNA of the cell. The question, however, which we would like to entertain at present, is whether DNA is directly and personally involved in the synthesis of protein or whether it effects its influence via an intermediary. Definitive evidence one way or the other is, at present, not available. Presumably, an unequivocal demonstration will ultimately emerge from experiments analogous to those being performed in the laboratories of Brachet (9) and Mazia (40,49) with enucleated fragments of ameba. At present, the best that can be offered is a series of experiments inquiring whether a correlation can be established between the metabolic activity or state of DNA and the act of protein synthesis.

There exists a variety of experiments in which it is possible to demonstrate a complete dissociation of DNA metabolism from protein synthesis. DNA formation is known (1) to be far more sensitive to inhibition by radiation with $\mathrm{x}$-rays than is protein formation. Baron, Spiegelman, and Quastler (5) have shown 
that $x$-ray dosages far exceeding those expected to stop the formation of DNA completely permit normal enzyme formation in yeast. Similar dissociations have been achieved with other systems and by different means $(30,58)$.

Kelner's (43) studies on photoreactivation of $E$. coli following exposure to ultraviolet have provided an elegant method for a virtually complete separation of RNA and protein formation from net DNA synthesis. Halvorson and Jackson (31) employing yeast have recently repeated and confirmed these results. The results obtained suggest again that protein and RNA continue to be synthesized subsequent to UV doses which completely inhibit the DNA formation.

Cohen and Barner (16) have reported the ability of a thymine-less mutant of $E$. coli which can synthesize xylose isomerase in the absence of an added supply of thymine. This important finding was confirmed in our laboratory (85) using the same mutant and examining beta-galactosidase-forming capacity. It was found that cells of this strain synthesized considerable amounts of enzyme when suspended in a synthetic medium lacking thymine. This behavior is in striking contrast to that observed with mutants possessing other metabolic deficiencies. Thus, in our own experience and in that of others $(56,58,85)$, adenine-less, uracil-less, or amino-acid-deficient mutants form little or no enzyme in the absence of the required metabolite.

An interesting apparent exception to the information cited is that of Allfrey's (2) observation with isolated nuclei. He found that treatment with DNase suppressed the ability of his preparations to incorporate labeled amino acids, whereas RNase had little or no effect. The situation observed here may, however, be a reflection of the low nuclear RNA content. If, as seems likely, RNA is derived from DNA, destruction of the latter would eliminate protein synthesis in such systems.

The data cited above demonstrate that drastic interference with DNA synthesis is often not accompanied by very striking effects on the formation of protein. Whereas such findings cannot eliminate DNA as an active component of the EFS, they 
hardly lend support to the supposition that it is. The credence assignable to such negative conclusions with respect to DNA gains further weight from similar cxperiments examining RNA metabolism which yielded strikingly different results

\section{RNA AS A GOMPONENT OF EFS}

Many have postulated RNA as a key substance in protein synthesis. Chantrenne (14) has succinctly summarized such speculations and the supporting evidence. Here we would like to confine our attention to the information derived from the study of enzyme synthesis. Again, as in the case of DNA, correlative experiments have been performed with intact cells examining the effects on enzyme formation of agents or conditions which influence RNA metabolism.

1. Experiments with Ultraviolet Light. Swenson and Giese $(94,95)$ demonstrated that exposure to ultraviolet dosages far exceeding those required to stop DNA formation, results in the inhibition of induced enzyme synthesis in yeast. Examination of the action spectrum of the inhibition revealed that it coincided with the absorption spectrum of nucleic acid. Halvorson and Jackson (31) extended these interesting observations. They examined the effects of various dosages on the synthesis of alphaglucosidase, the ability to use free amino acid pool components, and the incorporation of $\mathrm{P}^{32}$ in to the nucleotides of RNA. Their results established an excellent parallelism between the loss in capacity to utilize the free amino acids and the ability to synthesize enzyme. It was further found (85) that even slight damage of RNA metabolism, as measured by ability to incorporate $\mathrm{P}^{32}$, had profound effects on enzyme-forming ability. Thus, at a dose which achieved a 22 per cent inhibition of RNA metabolism enzyme formation was suppressed to the extent of 95 per cent.

2. The Effect of a Uridine Analogue on Enzyme Synthesis. One obvious approach which could in principle yield information pertinent to the role of RNA is to examine the effects of various analogues of uracil and its derivatives on enzyme forma- 
tion. Ben-Ishai and Spiegelman (7) undertook such a study. One of the most effective compounds found was 5-OH-uridine, which the experiments of Roberts and Visser (64) suggest is able to prevent the utilization of uracil for the synthesis of RNA. The presence of as little as $5 \mu \mathrm{g} . / \mathrm{ml}$. of this compound results in virtual cessation of beta-galactosidase formation by $E$. coli. Further, this inhibition can be achieved even if the OH-uridine is introduced subsequent to the addition of inducer, at a time when maximal rate of enzyme formation has been attained.

Several illuminating facts emerged from these experiments. One was that the $\mathrm{OH}$-uridine could effect a complete inhibition of beta-galactosidase formation at concentrations which had no effect on over-all protein synthesis. The apparent greater sensitivity of the beta-galactosidase-forming system suggests that it requires a larger effective supply of RNA precursors than other protein-synthesizing systems. A second fact of interest is the ability of the $\mathrm{OH}$-uridine to prevent enzyme formation even after its onset. This would suggest that continued synthesis of RNA is required for the uninterrupted production of enzyme. The same conclusion is derivable from the observation $(56,58)$ that, unlike the previously noted experience with thymine-less mutants, uracil-less mutants cease making enzyme immediately upon the exhaustion of externally supplied uracil.

COMPETITIVE INTERACTIONS AMONG PROTEIN SYNTHESIS SYSTEMS FOR RNA PRECURSORS

The marked response of the beta-galactosidase-forming systems to 5-OH-uridine and its interpretation in terms of an elevated requirement for RNA precursors suggest other types of experiments for exhibiting this kind of interaction. E. coli cells growing logarithmically in a synthetic medium with ammonia as the sole source of nitrogen do not accumulate a detectable internal pool of amino acids. The rate of protein formation is apparently limited by the synthesis of amino acids, since an immediate increase in growth rate follows the addition of an external supply of amino acids. It would be expected that the 
sudden stimulation of protein synthesis caused by the amino acid addition would exert an exhaustive demand on the metabolic devices which supply the derivatives needed for ribonucleic acid synthesis. In view of the suggested sensitivity of the beta-galactosidase-forming system to the supply level of the RNA precursors, the addition of amino acids might be expected to result in an inhibition of beta-galactosidase formation. This prediction is experimentally realized (30). Thus, the presence of inducer fails to stimulate enzyme formation if amino acids are added simultaneously. The suppression is virtually complete for a period of a half hour, following which some recovery of enzyme-forming capacity occurs. That the inhibition is related to RNA precursor supply is supported by the ability of purine and pyrimidine bases to reverse it.

This dependence of enzyme formation on an adequate supply of nucleic acid precursors has also been exhibited (7) in the case of alpha-glucosidase formation in $S$. cerevisiae In additon to their free amino acid pool, yeasts also possess a considerable internal supply of nucleotides and their polyphosphate derivatives (68). It was found possible (7) to specifically deplete the nucleotide pool by incubation in the presence of an external supply of amino acids and energy. This treatment leads to a loss of enzyme-forming capacity while leaving the free amino acid pool intact. If cells are first partially induced and their nucleotide pool then depleted, they fail to form enzyme on being again exposed to inducer. If their nucleotide pool is, however, replenished, enzyme synthesis proceeds normally. These experiments illustrate in a different manner and with another system the apparent requirement that RNA synthesis be possible if enzyme formation is to continue.

\section{EXPERIMENTS WITH SUBCELLULAR FRACTIONS}

The experiments thus far described strongly implicate RNA as the template in the process of enzyme synthesis. They cannot, however, be taken as conclusive. It is painfully obvious that although interesting and perhaps even ingenious experiments can 
be performed with intact cells, the distance between the data and the conclusions derived fr $\mathrm{m}$ them is too great for certainty. Definitive identification of the chemical nature and the mode of action of the template is not likely until the latter has been physically isolated in a functional state. In vitro performance of its function by the isolated enzyme-forming system may be suggesting the impossible, since it demands even more than that which has already been accomplished in the case of transformation in the bacteria. In the latter, genetically competent material has been separated from other cell $\mathrm{c}$ smponents. However, the transforming principles have been asked to function only after reinsertion into an intact living organism.

Nevertheless, that the ideal in vitro situation may be attainable in the not too distant future is prophetically foreshadowed by the striking successes which have recently been recorded with subcellular fractions. Many of these deal primarily with incorporations studies. To this extent it is uncertain that they necessarily represent model systems which will permit the further dissection of the protein-synthesizing mechanism. The data must therefore be interpreted with caution, but their uniqueness and potential value command consideration.

Zamecnik and Keller (104) succeeded in preparing a microsome fraction which actively incorporates amino acids when supplemented with some component of the supernate and an ATP-generating system. Subsequent work on the supernate fraction by Keller and Zamecnik (42) indicated the presence of an enzyme which generated guanosine-triphosphate, a derivative of which functions in the insertion of the amino acids into peptide linkage. The work of Hoagland (37) and DeMoss and Novelli (22) strongly suggests that polyphosphate derivatives of nucleotides activate amino acids prior to their incorporation.

Lester (48) and Beljanski (6) examined the ability of lysozyme-treated preparations of Bacillus megaterium to incorporate amino acids labeled amino acids. Both authors found that treatment with RNase abolished this ability, whereas exposure to DNase was stimulatory. 
The most extensive investigation on the properties of subcellular fractions has come from Gale's $(27,28)$ laboratory. In these studies cells of Staphylococcus aureus are disrupted by sonic disintegration and a fraction obtained by differential centrifugation which is relatively low in viable cells, and therefore presumably in intact cells. Although it is unlikely that this preparation is homogeneous, it nevertheless is of the greatest interest, since it is amenable to enzymatic and extractive resolution. Removal of the nucleic acid from such disrupted cell preparations leads to a marked lowering in their ability to incorporate amino acids. This loss can be restored by the addition of nucleic acids from the same species, DNA being more active than RNA on a dry-weight basis. This latter finding may be merely a consequence of the greater stability of DNA to isolation procedures. The data are consistent with the concept that the RNA made from the DNA supplied is the active agent.

A most interesting recent development has been the discovery by Gale and Folkes (29) that the presence of specific di- and trinucleotides is extremely active in promoting the incorporation of specific amino acids. Thus, for example, dinucleotides containing adenine and cytosine can completely replace the intact RNA in promoting the incorporation of aspartic acid. Indeed, on an equivalent weight basis the dinucleotide is more than a hundred times as active as the intact RNA. Interpretation of these findings is yet uncertain. It may indeed be, as suggested by Gale and Folkes (29), that these small fragments represent that part of the RNA template which is concerned with the insertion of the corresponding amino acid into peptide linkage. An argument which can be raised against this assertion stems precisely from the observed high activity of the dinucleotide. It seems unlikely that nucleotide pairs are sufficient to specify the relevant amino acids, since only 16 possibilities are uniquely determined. At least three bases of the RNA template would have to be involved in the specification of a given amino acid since 20 or more choices have to be made. This rea- 
soning assumes, of course, that the four bases are the only components of the code.

An alternative explanation of these findings can be offered. It may be that the active fragments of Gale-Folkes may, by transfer reactions, generate the nucleotide components functioning in the activating mechanism suggested by the work of Hoagland (37) and DeMoss and Novelli (22). Whatever the interpretation, it nevertheless remains true that these results are pregnant with many possibilities.

As distinguished from incorporation studies, the attainment of protein synthesis has been reported in only two sorts of subcellular fractions. One is the system of Gale and Folkes (28) in which the development of "glucozymase," catalase, and the inductive formation of beta-galactosidase have been demonstrated. When these preparations are sufficiently resolved either by removal of RNA or DNA, it is found that both DNA and RNA stimulate the formation of both catalase and betagalactosidase.

Again, the relative high activity of the DNA may be a consequence of greater stability to extractive procedures. No limits to the potentialities of this system are apparent. It is difficult to believe that its further study can fail to provide definitive answers to the basic problems of template function and specificity.

Another system which gives great promise of future fruitfulness are the so-called "protoplasts" of $B$. megaterium. Weibull (100) showed that these could be prepared by treatment of cells with lysozyme in hypertonic medium. Wiame et al. (101) showed that these preparations were able to synthesize arabinokinase as demonstrated by an increased $Q_{\mathrm{O}_{2}}$ during incubation. Simultaneously Landman and Spiegelman (44) isolated a lactose positive mutant of $B$. megaterium and devised a stabilizing medium for protoplasts which permits synthesis of beta-galactosidase. Virtually all of the enzyme-forming capacity is recovered in the protoplasts. When supplemented with amino acids, hexose-diphosphate, and inducer, they synthesize enzyme 
at rates comparable to those of intact cells. The beta-galactosidase formed has been isolated in soluble form and purified. These preparations are amenable to enzymatic resolution, their enzyme-forming activity being abolished by RNase. This treatment does not destroy them physically but selectively removes 80 to 90 per cent of the RNA.

It is evident that the search for a system which would permit the further experimental probing of protein-synthesizing systems is at present in an exciting but preliminary stage. There seems little doubt, however, that a new era is being opened which will ultimately permit a description in chemically defined terms of the nature of the protein-synthesizing machinery.

\section{Summary and Concluding Remarks}

We have here surveyed the data which have accumulated on the phenomenon of "enzymatic adaptation," with particular emphasis on the efforts of the past decade. In view of the complexity of the problem posed initially, and the difficulties which could easily have hindered understanding or led to irrelevant confusion, the progress which can be recorded is satisfying.

Operationally diverse disciplines have provided the data from which a picture of the enzyme-forming mechanism has evolved. The kinetic, biochemical, and genetic information on induced enzyme production all lead to and can be interpreted in terms of one model. They suggest that the enzyme-forming system is a complex between RNA, inducer, and enzyme.

The problem has been brought to the point where further questions must be posed in terms of the chemical structures and reactive interrelations of the components identified. From the experiments reviewed in the last section it would appear that the systems needed for the experimental resolution of precisely such questions are now well on the way to development.

It seems likely at the present writing that the next decade will provide the necessary answers. Attention can then be profitably turned to the problem which initiated much of the work described, i.e., what is the nature of gene function? 


\section{References}

1. Abrams, R., Arch. Biochem., 30, 90 (1951).

2. Allfrey, V. G., Proc. Natl. Acad. Sci. U. S., 40, 881 (1954).

3. Anfinsen, C. B., and M. Flavin, Federation Proc., 12, 170 (1953).

4. Anfinsen, C. B., and D. Steinberg, J. Biol. Chem., 189, 739 (1951).

5. Baron, L. S., S. Spiegelman, and H. J. Quastler, J. Gen. Physiol., 36, 631 (1953).

6. Beljanski, M., Biockim. et Biophys. Acta, 15, 425 (1954).

7. Ben-Ishai, R., and S. Spiegelman, in manuscript, 1955.

8. Benzer, S., Biochim. et Biophys. Acta, 11, 383 (1953).

9. Brachet, J., and H. Chantrenne, Nature, 168, 950 (1951).

10. Campbell, A. M., and S. Spiegelman, J. Gen. Physiol., in press.

11. Campbell, A. M., and S. Spiegelman, Bacteriol. Proc., p. 91 (1953).

12. Campbell, P. N., and T. S. Work, Biochem. J. (London), 52, 217 (1952).

13. Campbell, P. N., and T. S. Work, Nature, 171, 997 (1953).

14. Chantrenne, H., in Adaptation in Microorganisms, Third Symposium of the Society for General Microbiology. Cambridge University Press, Cambridge. 1953.

15. Cohen, G. N., and H. V. Rickenberg, Compt. rend., 240, 466 (1955).

16. Cohen, S. S., and H. D. Barner, Proc. Natl. Acad. Sci. U. S., 40, 885 (1954).

17. Cohen-Bazire, G., and M. Jolit, Ann. inst. Pasteur, 84, 937 (1953).

18. Cohn, M., and J. Monod, in Adaptation in Microorganisms, Third Symposium of the Society for General Microbiology. Cambridge University Press, Cambridge, 1953.

19. Cohn, M., and A. M. Torriani, Biochim. et Biophys. Acta, 10, 280 (1953).

20. Cohn, M., and A. M. Torriani, J. Immunol., 69, 471 (1952).

21. Dalgliesch, C. E., Nature, 171, 1027 (1953).

22. DeMoss, J. A., and D. Novelli, Bacteriol. Proc., p. 125 (1955).

23. Ephrussi, B., Cold Spring Harbor Symposia Quant. Biol., 16, 75 (1951).

24. Ephrussi, B., and P. Slonimski, Biochim. et Biophys. Acta, 6, 256 (1950).

25. Gale, E. F., and J. P. Folkes, Biochem. J. (London), 53, 483 (1953).

26. Gale, E. F., and J. P. Folkes, Biochem. J. (London), 53, 493 (1953).

27. Gale, E. F., and J. P. Folkes, Biochem. J. (London), 59, 661 (1955).

28. Gale, E. F., and J. P. Folkes, Biochem. J. (London), 59, 675 (1955).

29. Gale, E. F., and J. P. Folkes, Nature, 175, 592 (1955).

30. Gros, F., and S. Spiegelman, unpublished experiments, 1955.

31. Halvorson, H. O., and L. Jackson, Bacteriol. Proc., p. 117 (1954).

32. Halvorson, H. O., and S. Spiegelman, J. Bacteriol., 64, 207 (1952).

33. Halvorson, H. O., and S. Spiegelman, J. Bacteriol., 65, 496 (1953).

34. Halvorson, H. O., and S. Spiegelman, J. Bacteriol., 65, 601 (1953).

35. Halvorson, H. O., and S. Spiegelman, J. Gen. Physiol., in press. 
36. Halvorson, H. O., S. Spiegelman, and R. Hinman, Arch. Biochem. and Biophys., 55, 512 (1955).

37. Hoagland, M. B., Biochim. et Biophys. Acta, 16, 288 (1955).

38. Hogness, D. S., M. Cohn, and J. Monod, Biochim. et Biophys. Acta, 16, 99 (1955).

39. Hotchkiss, R. D., Phosphorus Metabolism, 2, 426 (1952).

40. James, T. W., Biochim. et Biophys. Acta, 15, 367 (1954).

41. Jebb, W. H. H., R. Knox, and A. H. Tomlinson, J. Gen. Microbiol., 4, 376 (1950).

42. Keller, E. B., and P. C. Zamecnik, Federation Proc., 14, 234 (1955).

43. Kelner, A., J. Bacteriol., 65, 252 (1953).

44. Landman, O. E., and S. Spiegelman, Proc. Natl. Acad. Sci. U.S., 41, 698 (1955).

45. Lederberg, J., J. Bacteriol., 60, 381 (1950).

46. Lederberg, J., Genetics in the Twentieth Century, p. 634. Macmillan, New York, 1951.

47. Lee, N. D., and R. H. Williams, Biochim. et Biophys. Acta, 9, 698 (1952).

48. Lester, R. R., J. Am. Chem. Soc., 75, 5448 (1953).

49. Mazia, D., and D. M. Prescott, Biochim. et Biopys. Acta, 17, 23 (1955).

50. Monod, J., Ann. inst. Pasteur, 70, 381 (1944).

51. Monod, J., Ann. inst. Pasteur, 79, 390 (1950).

52. Monod, J., Ann. Rev. Microbiol., 3, 371 (1949).

53. Monod, J., Growth, 11, 223 (1948).

54. Monod, J., G. Cohen-Bazire, and M. Cohn, Biochim. et Biophys. Acta, 9, 648 (1951).

55. Monod, J., and M. Cohn, Advances in Enzymol., 13, 67 (1952).

56. Monod, J., A. M. Pappenheimer, Jr., and G. Cohen-Bazire, Biochsm. et Biophys. Acta, 9, 648 (1952).

57. Novick, A., and L. Szilard, Proc. Natl. Acad. Sci. U. S., 36, 708 (1950).

58. Pardee, A. B., Proc. Natl. Acad. Sci. U. S., 40, 263 (1954).

59. Pollock, M. R., Brit.J. Exptl. Pathol., 31, 739 (1950).

60. Pollock, M. R., Brit.J. Exptl. Pathol., 32, 387 (1951).

61. Pollock, M. R., Brit. J. Exptl. Pathol., 33, 587 (1952).

62. Pollock, M. R., "Stages in Enzyme Adaptation," Adaptation in Microorganisms, Third Symposium of the Society for Microbiology, Cambridge University Press, Cambridge, 1953.

63. Rickenberg, H. V., C. Yanofsky, and D. M. Bonner, J. Bacteriol., 66, 683 (1953).

64. Roberts, M., and D. W. Visser, J. Biol. Chem., 19.4, 695 (1952).

65. Rotman, B., and S. Spiegelman, J. Bacteriol., 66, 492 (1953).

66. Rotman, B., and S. Spiegelman, J. Bacteriol., 68, 419 (1954).

67. Schaefer, W., Ann. inst. Pasteur, 74, 458 (1948).

68. Schmitz, H., Biochem. Z., 325, 555 (1954). 
69. Slonimski, P. P., Recherches sur la Formation des Enzymes Respiratoires chez la Levure, p. 203. Paris 1953.

70. Slonimski, P. P., in Adaptation in Microorganisms, Third Symposium of the Society for General Microbiology. Cambridge University Press, Cambridge, 1953.

71. Spiegelman, S., Ann. Missouri Botan. Garden, 32, 139 (1945).

72. Spiegelman, S., Cold Spring Harbor Symposia Quant. Biol., 11, 266 (1946).

73. Spiegelman, S., Cold Spring Harbor Symposia Quant. Biol., 16, 87 (1951).

74. Spiegelman, S., J. Cellular Comp. Physiol., 30, 315 (1947).

75. Spiegelman, S., in S. Myrbäck, ed., The Enzymes, Vol. 1, Part 1, pp. 267-306. Academic Press, New York, 1950.

76. Spiegelman, S., Symposia Soc. Exptl. Biol., 2, 286 (1948).

77. Spiegelman, S., unpublished observations, 1955.

78. Spiegelman, S., and W. F. DeLorenzo, Proc. Natl. Acad. Sci. U. S., 38, 583 (1952).

79. Spiegelman, S., W. F. DeLorenzo, and A. M. Campbell, Proc. Natl. Acad.Sci. U.S., 37, 513 (1951).

80. Spiegelman, S., and R. Dunn, J. Gen. Physiol., 31, 153 (1947).

81. Spiegelman, S., and M. Gilmour, unpublished observations, 1955.

82. Spiegelman, S., and H. O. Halvorson, J. Bacteriol., 68, 265 (1954).

83. Spiegelman, S., and H. O. Halvorson, Adaptation in Microorganisms, Third Symposium of the Society for General Microbiology. Cambridge University Press, Cambridge, 1953.

84. Spiegelman, S., and H. O. Halvorson, unpublished observations, 1955.

85. Spiegelman, S., H. O. Halvorson, and R. Ben-Ishai, Amino Acid Metabolism, p. 124. Johns Hopkins Press, Baltimore, 1955.

86. Spiegelman, S., C. C. Lindegren, and G. Lindegren, Proc. Natl. Acad. Sci. U.S., 31, 95 (1945).

87. Spiegelman, S., and J. M. Reiner, J. Gen. Physiol., 31, 175 (1947).

88. Spiegelman, S., J. M. Reiner, and M. Sussman, Federation Proc., 6, 209 (1947).

89. Spiegelman, S., R. R. Sussman, and E. Pinska, Proc. Natl. Acad.Sci. U. S., 36, 591 (1950).

90. Stanier, R. Y., Ann. Rev. Microbiol., 5, 35 (1951).

91. Stanier, R. Y., J. Bacteriol., 54, 339 (1947).

92. Suda, M., O. Hayashi, and Y. Oda, Med. J. Osaka Univ., 2, 21 (1949).

93. Sussman, M., and S. Spiegelman, Arch. Biochem., 29, 54 (1950).

94. Swenson, P. A., Proc. Natl. Acad.Sci. U.S., 36, 699 (1950).

95. Swenson, P. A., and A. G. Giese, J. Cellular Comp. Physiol., 36, 369 (1950).

96. Taylor, E. S., J. Gen. Microbiol., 1, 86 (1947).

97. Terui, G., and H. Okada, Tech. Repts. Osada Univ., 4, 425 (1954). 
98. Ushiba, D., and B. Magasanik, Proc. Soc. Exptl. Biol. Med., 80, 626 (1952).

99. Vogel, H. J., and B. D. Davis, Federation Proc., 11, 485 (1952).

100. Weibull, C., J. Bacteriol., 66, 688 (1953).

101. Wiame, J. M., R. Storck, and E. Vanderwinkel, personal communication, 1955.

102. Winge, O., and C. Roberts, Compt. rend. trav. lab. Carlsberg, Ser. physiol., 24, 263 (1948).

103. Yudkin, J., Biol. Revs. Cambridge Phil. Soc., 13, 93 (1938).

104. Zamecnik, P. G., and E. B. Keller, J. Biol. Chem., 209, 337 (1954).

105. Zamenhof, S., H. E. Alexander, and G. Leidy, J. Exptl. Med., 98, 373 (1953). 


\section{CERTAIN PROBLEMS IN THE BIOCHEMICAL STUDY OF DISEASE}

DeWITT STETTEN, JR., National Institute of Arthritis and Metabolic Diseases, National Institutes of Health, Bethesda, Maryland

The study of normal metabolic processes may be pursued with profit at many levels of biological organization, or, more precisely, of biological disorganization. The investigator may elect to employ the minimally disturbed intact animal, the isolated perfused organ or tissue of that animal, the sliced, teased, or minced preparation of such a tissue, the cell-free homogenate, or the solution of enzyme or enzymes. Each level, as here listed, represents a further degree of disorganization of an initially highly integrated system, and the disorganization is in each case imposed by the experimenter. At each successive level of disorganization something is gained in the form of greater simplicity, greater control over variables, greater facility in establishing the necessary components of the system or reaction under scrutiny. However, at each successive level of disorganization something of importance is also lost.

It may be worth while to consider briefly what is lost as one proceeds with this dissection, first surgical and then chemical, of the intact organism. As an example, the functioning of the liver in situ is influenced by the normally continuous supply of substrates, the normally continuous removal of products of its 
activity. These operations depend in turn upon the activities of remotely situated organs and tissues. Remote organs also influence endocrinologically the function of liver in situ. In addition, other influences, nervous, circulatory, and thermal, remote in origin, may affect the functioning of the liver in its normal habitat.

Isolation and perfusion of this liver at once eliminates many of these determinants of liver function. Humoral, including hormonal, regulation is now determined by the experimenter, not by the animal. Nervous regulation is usually lost. If the liver is now sliced and shaken in a bath, further loss of organization occurs. A fraction of the cells is necessarily incised, releasing normally intracellular contents to the extracellular fluid. The remaining cells are nourished in a rather haphazard fashion, splashing about in a vessel instead of being supplied with a steady flow of a regulated nutrient fluid pumped past each cell through a system of venous sinuses. The process under study is confined by the walls of the vessel, and unless special and elaborate conditions are fulfilled, the concentration of substrate continuously declines while that of product continuously rises.

Homogenization of the tissue and disruption of the cellular architecture is still a further insult. The microscopically visible intracellular components, nucleus, mitochondria, nucleolus, and microsomes, all bear, in the intact cell, geometrical relationships to each other which are doubtless of functional significance in the intact cell and which are destroyed in the process of homogenization. It may further be postulated that the submicroscopic particles, the molecules of the soluble enzymes, are likewise in the intact cell not entirely free-swimming, but become so when the cell is disrupted. It is possible that in the geographical arrangement of enzymes within the cell resides the answer to the typically polar or unidirectional character of epithelial cells (14), and this is clearly lost in the process of homogenization. Finally, the separation and purification of individual enzymes from such a cell-free homogenate, though very rewarding, inevitably divorces the enzyme from such inhibitory or excita- 
tory agents as may be closely associated with it in real life. Whereas the determination of the equilibrium constant of an enzyme-catalyzed reaction usually rests upon preliminary purification of the enzyme, it must be recognized that within the cell within the liver within the peritoneal cavity of the intact animal, most reactions of interest in metabolic studies do not proceed to equilibrium. Indeed it is a characteristic of the living organism that in general it operates quite remote from the equilibrium point.

Disease, for purposes of the present argument, may be defined as the manifestation of alterations in metabolic processes. In some cases, such alterations are of a qualitative nature, involving the appearance in the organism of compounds which are apparently totally lacking in the normal animal. The preserice of the abnormal hemoglobin found in sickle-cell anemia (15) or of the Bence-Jones protein of multiple myeloma (23) are examples of such qualitative changes. In more cases, the change appears to be of a quantitative nature, involving alterations in the rates of normally occurring processes. The chemical change observed in such a patient is one of a deviation from normal in the concentration of some blood, urine, or tissue constituent, reflecting a change in the rate of production or of removal of some tissue component. The concentration of glucose or of uric acid in the blood may be elevated, the quantity of depot lipid may be increased, or the concentration of serum albumin may be lowered.

The first question which is presented by such a situation is an analysis of the crude mechanism upon which the change in concentration depends. Most of the tissue constituents of interest in this regard are, in the normal subject, in a dynamic steady state, subject to an elegantly balanced turnover wherein the rate of generation is closely matched to the rate of destruction or elimination. A rise in the quantity of such a constituent may result equally readily from an increase in the synthetic rate or from a decrease in the rate of destruction. Conversely, either an increase in destruction or a decreased synthesis will result in a fall in quantity. It is today possible to design experiments, in 
certain cases, which will differentiate these two types of disturbances and to show, for instance, that diabetic hyperglycemia is a consequence chiefly of impaired glucose utilization, whereas the hyperuricemia in certain types of gout is a consequence of excessive production of uric acid.

Such experiments are most securely conducted on the intact animal, and indeed, in the case of gout, the experimenter is restricted to man, since no analogous disease in experimental animals is available. The reasons for this preference for the intact animal lie in the fact that the disease process is itself a disintegration of some phase of that highly integrated system which is the organism as a whole. Any further disorganization imposed by the experimenter will necessarily confuse the picture and may obscure the primary disturbance which is due to the disease itself. Furthermore, the normal constancy of composition of the blood with respect to glucose, uric acid, albumin, etc., of the subcutaneous tissues with respect to lipid, or of the liver with respect to glycogen, fatty acids, or cholesterol is a resultant of many processes occurring in many tissues. The very separation of the several tissues may result in disappearance of the abnormality of rate the explanation of which is being sought.

The foregoing argument must not be construed to mean that only experimental results derived from the intact animal are of use in the elucidation of disease processes. On the contrary, all levels of biological disorganization have contributed significantly to this body of knowledge. What is intended is merely to point out that of such results as are secured with isolated enzyme preparations or isolated tissues of diseased animals, only those which are compatible with observations on the intact diseased animal are of value in explaining the disease process.

The contributions of the several experimental approaches may be exemplified by an analysis of the various abnormalities which may influence the concentration of glucose in the blood. Glucose enters the portal blood by intestinal absorption and since 
most of the dietary carbohydrate of mammals is polysaccharide, this implies a preliminary digestion. Glucose enters the hepatic vein by the hydrolysis of glucose-6-phosphate and this compound may in turn arise (1) from glucose itself, (2) from glucose1-phosphate derived from glycogen or (3) from hexosediphosphate arising in turn from triose phosphate, etc. These processes undoubtedly occur also in tissues other than the liver, notably the kidney. Glucose may also arise hydrolytically within cells, and as an example of this process may be cited the action of amylo-1,6-glucosidase in muscle. This latter contribution is probably quite small.

Glucose is utilized, so far as is known, by every mammalian cell. For blood glucose to be utilized, it must first cross cell membranes. It is generally held that simultaneously with or immediately succeeding such passage, glucose undergoes hexokinase-catalyzed phosphorylation. From the hexose phosphate thus produced at least four distinct metabolic sequences may arise.

(1) Glucose-6-phosphate $\rightarrow$ glucose-1-phosphate $\rightarrow$ glucosides (e.g., glycogen) or rearrangement products (e.g., galactose1-phosphate).

(2) Glucose-6-phosphate $\rightarrow$ 6-phosphogluconate, initiating the "oxidative pathway."

(3) Glucose-6-phosphate $\rightarrow$ fructose-6-phosphate, initiating the "glycolytic pathway."

(4) Glucose-6-phosphate $\rightarrow$ glucose + inorganic orthophosphate.

Of interest in this regard is the question of the possible utilization of glucose without preliminary phosphorylation. This view rests largely upon the demonstration of a glucose dehydrogenase activity in mammalian liver extracts which forms gluconic acid from glucose in excellent yield (6). Attempts to demonstrate the occurrence of this particular transformation in the intact animal have thus far yielded negative results (20). Provisionally this enzyme activity must be considered inoperative 
in the intact mammal, and its presence must be regarded as an unexplained biochemical curiosity. Whether hepatic glucose dehydrogenase is a vestigial compound or whether it is a truly important enzyme, the proper substrate of which has not been identified, remains to be determined.

In addition to the foregoing fates, glucose may be lost to the mammalian organism in the intestinal and urinary tracts. In the intestinal tract, failure of adequate polysaccharide digestion or excessive bacterial fermentation deprives the mammal of a portion of ingested carbohydrate. Whereas normal sugar losses in the urine are negligible, these become significant if either the normal renal threshold is exceeded by an abnormal hypergiycemia or normal blood is circulating through kidneys with an abnormally low glucose threshold.

In consideration of the numerous sources and fates of blood glucose, it is certainly not surprising that there are many disease states which result in alterations, positive or negative in sign, of glucose concentration in the blood. Still other defects in one or another mechanism might be anticipated to produce alterations in blood glucose concentration but fail to do so as a consequence of homeostatic mechanisms that are invoked. Thus simple failure to ingest carbohydrate or failure to digest polysaccharide, as may occur in pancreatic disease or diarrhea, will not, in general, cause profound hypoglycemia. This is at least in part explained by a compensatory decline in glucose utilization by cells of the liver and is reflected in a diminished consumption of glucose for hepatic lipogenesis in the intact animal (3) as well as in the liver slice (24). Failure of the intestinal mucosa actively to transport glucose from the lumen to the portal blood is seen in sprue, and this situation also rarely leads to hypoglycemia. The site of the defect is readily demonstrable clinically, however, by the finding of a normal intravenous glucose tolerance in the face of a failure of blood glucose to rise after oral administration. It is noteworthy that the defect in sprue can be simulated by the application of phlorhizin to the intestinal mucosa (10).

The liver normally supplements the intestinal tract as a 
source of blood glucose. The immediate hepatic source of glucose is from the hydrolysis of glucose-6-phosphate in the presence of a specific glucose-6-phosphatase. Glucose-6-phosphate arises in liver from various sources, and among these are glycogen, via glucose-1-phosphate, and such noncarbohydrate compounds as are capable of contributing organic fragments to the several intermediates of the glycolytic sequence and the citric acid cycle. These latter contributions derive ultimately from the glycogenic amino acids, glycerol, and other compounds, and the over-all process under consideration is termed glyconeogenesis.

Of the disease processes which influence the generation of glucose in the liver, the best defined is glycogen storage disease (von Gierke). In an elegant analysis of these patients (5), it has been shown that in many instances the defect is attributable simply to a relative or absolute deficiency of glucose-6-phosphatase activity in the liver. Such an abnormality makes the liver, like normal muscle, incapable of contributing significant amounts of glucose to the blood, but in no wise interferes with the steps of glycogenesis. Other patients suffering from glycogen storage disease exhibit normal glucose-6-phosphatase activity but appear to deposit glycogen which deviates markedly from normal liver glycogen in its branching pattern. Such glycogen seems, in some cases, to be less readily degraded by the combined action of phosphorylase and amylo-1,6-glucosidase. A consequence of either type of glycogen storage disease is a failure to elicit the usual hyperglycemia in response to administered epinephrine.

Recent evidence indicates that a mode of action of epinephrine, as well as of glucagon, is to favor the conversion of inactive phosphorylase into active phosphorylase (21). Whereas this finding, is, of itself, not a complete explanation of the mode of action of these agents, it is highly suggestive that these stimulants of glycogenolysis operate at the initial steps of glycogen breakdown. The failure of the blood glucose to rise in response to epinephrine in von Gierke's disease is best explained by the 
hypothesis either that the hexose phosphate which arises is not hydrolyzed or that, owing to its structural abnormality, the glycogen is not sensitive to attack even by active phosphorylase. In this regard it is of interest that patients deficient in pancreatic islet $\alpha$-cells, the apparent source of glucagon, have recently been described (11). Hypoglycemia was noted to occur in these subjects.

Even assuming all the requisite enzyme systems in the liver to be intact, clearly if there is no glycogen in the liver, glycogenolysis must cease to play a role as a source of blood glucose. Virtually total depletion of hepatic glycogen ensues upon relatively brief periods of inanition, and marked decreases in the content of glycogen in the liver have been observed in a variety of conditions, including exposure to low oxygen tension or intoxication with thyroxin or thyroglobulin. It follows that under these circumstances, hepatic glycogenolysis cannot be relied upon as a source of blood sugar.

An apparently distinct mechanism which can be altered in disease but which also affects the rate at which the liver contributes glucose to the blood is gluconeogenesis. Profound changes may be elicited in the experimental animal, and analogous situations may be observed in clinical material wherein variations in adrenocortical activity result in changes in the rate at which amino acids are deaminated and glucose is formed. In general terms, the presence of excessive amounts of the 11oxysteroids of the adrenal cortex results in the generation of large amounts of glucose from noncarbohydrate precursors (22), a rise in the total carbohydrate content of the organism, and incidentally a rise in blood glucose concentration. Conversely, in the adrenalectomized animal or Addisonian patient, with glucogenesis from noncarbohydrate sources impeded, hypoglycemia may be encountered. In contrast to the state of present knowledge of the mode of action of epinephrine or of glucagon, no generally acceptable hypothesis can be offered to account for this action of the corticosteroids.

Defects in the utilization of blood glucose are if anything 
more frequent than defects in its sources and generation. Whereas all tissues in the mammal, so far as is known, are capable of assimilating glucose, attention has been directed largely to the liver and to the skeletal musculature. Although wide fluctuations in glucose utilization may be induced in these tissues by alteration of the hormonal or nutritional state, certain other tissues, e.g., brain, myocardium, fetus, appear to be insulated against drastic changes in rate of glucose consumption. Thus whereas the liver or muscle is sensitive to the concentration of circulating insulin, brain is not, although it obviously cannot tolerate extremes of hypoglycemia.

The rate of assimilation of glucose by muscle is critically dependent upon the activity of the islets of Langerhans, the anterior pituitary gland, and possibly also the adrenal cortex. Space does not permit an analysis at this time of the various conflicting lines of evidence relating to the exact sites of action of the several participating hormones. For purposes of the present discussion it matters little whether the role of insulin is to release muscle hexokinase from a physiological inhibition (16) or to facilitate the entry of unesterified glucose from the extracellular space into the intracellular compartment of muscle (9). It will suffice, for the present argument, to select the area of agreement between the conflicting experiments and define the net result of insulin action as increasing the quantity of intracellular glucose-6-phosphate at the expense of extracellular, ultimately plasma, glucose. Similarly we need not concern ourselves too much, at this time, with the question of whether the anterior pituitary gland secretes an agent that directly inhibits hexokinase (16) or whether it interferes in some fashion with the operation of insulin which is bound to muscle (18). Growth hormone, or some biological product derived therefrom, appears to render muscle insensitive to the action of insulin in the intact animal.

Again in the intact animal the situation is complicated by the fact that, in addition to influencing what is happening in muscle, each endocrine may exert influences upon other endocrine 
glands. An example of this situation is the finding of an increase in gluconeogenesis observed in alloxan-diabetic animals (7). These animals have enlarged adrenal cortices, and it is possible that this effect is not directly attributable to hypoinsulinism but is rather a consequence of a secondary hyperadrenalism.

It is generally agreed today that the major effect upon blood glucose of hypoinsulinism is a decrease in glucose utilization by such tissues as liver and muscle. Conversely the primary effect of hyperinsulinism is an enhancement of glucose utilization in these tissues. Many of the complex consequences of diabetes may be explained upon this basis alone, in that, with the generation of intracellular glucose-6-phosphate impeded, all products derived from glucose-6-phosphate, such as lactate, pyruvate, or $\mathrm{CO}_{2}$, will not be formed from glucose at normal rate. Other reactions, which are coupled to those of glycolysis less directly, such as peptide bond synthesis (8) and fatty acid synthesis $(19,4)$, will also be retarded. There is ample experimental evidence that these effects are observed in the diabetic organism and are reversed by administration of insulin.

Many diabetic patients require dosages of insulin far in excess of what is generally considered the production of the normal pancreas, and in various situations, most strikingly in severe ketosis, the insulin tolerance may increase enormously. Under these circumstances one must suppose that an "antagonist" to insulin is present in undue amount in the blood (12) or tissues. Whether this antagonist is in the nature of a pituitary hormone (2), an antibody (1), or a specific destructive enzyme (insulinase) (13) remains to be determined.

The last fate of glucose to be discussed is urinary loss. The normal operation of renal tubular reabsorption of glucose may be overwhelmed by excessively high concentrations of glucose in the blood. This may occur secondary to a variety of disturbances which interfere with glucose utilization, such as insulin deficit, or which cause excessive glucose production, such as excessive epinephrine or excessive corticosteroid. Further, the mechanism of tubular reabsorption may itself be defective 
and result in renal glycosuria, as is seen in Fanconi's syndrome, or experimentally in phlorhizin poisoning.

The purpose of the above discussion has been to stress that the concentration of glucose or of any other constituent of the

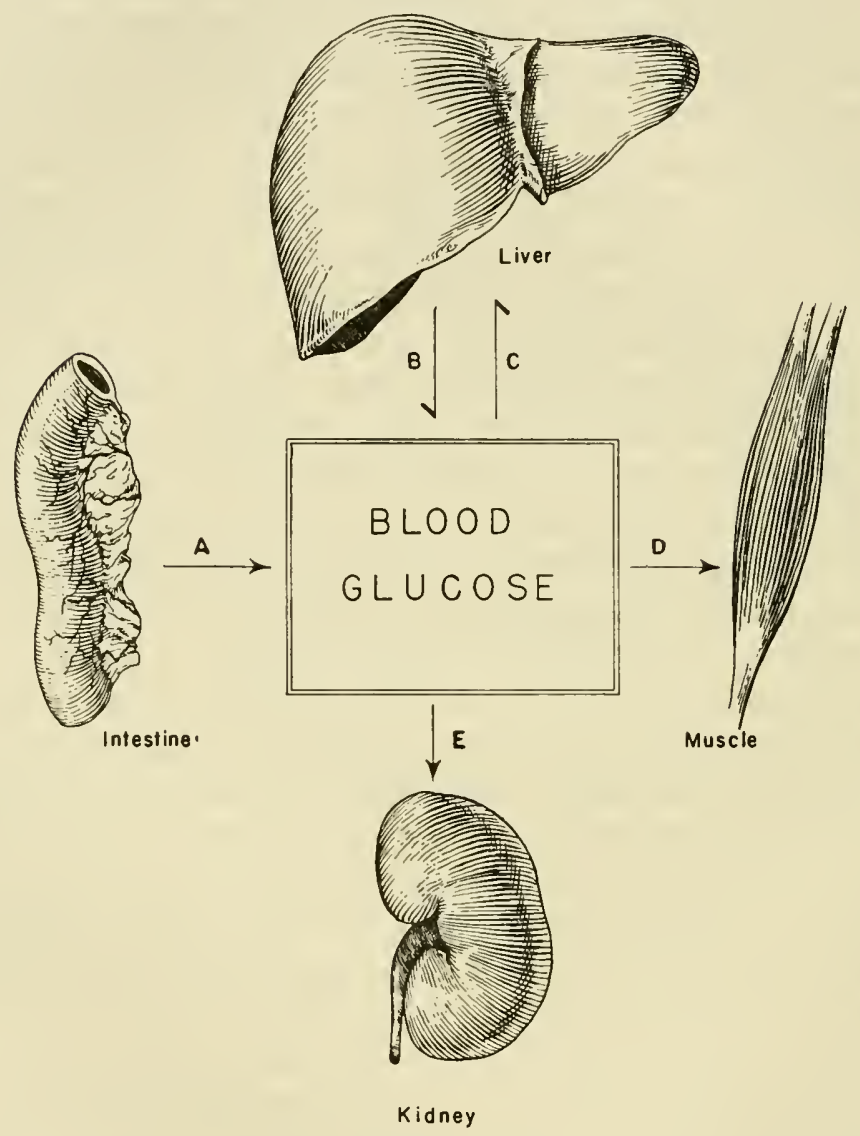

Fig. 1.

blood is the resultant of numerous vectors. Disturbances of any of these vectors may result in abnormalities of concentration but may also cause secondary disturbances in other vectors. If these secondary processes are compensatory in nature, analogous 
to negative feedback, homeostasis results. The secondary disturbances may, however, amplify the defect. As an cxample may be cited the nausea, emesis, and coma of diabetic ketosis, all leading to cessation of food intake and thereby contributing further to the ketosis. Such a situation is analogous to positive feedback, and if uncorrected, may lead to a fatal outcome.

The several vectors that have been discussed in relation to blood glucose are diagrammed in Figure 1. The magnitude of vector $A$ is determined by the abundance of ingested carbohydrate, the duration and effectiveness of intestinal digestion, and the integrity of the intestinal mucosa. It may be markedly diminished in starvation, in disease of pancreatic acinous tissue, or in sprue. Vector $B$ depends upon a supply of hepatic glycogen of normal constitution and an intact enzyme system to convert it to glucose. It is exaggerated by excess epinephrine or glucagon and is diminished in von Gierke's disease. It is likewise influenced by the gluconeogenic rate which is in turn under adrenocortical regulation, is diminished in Addison's disease, and is exalted in hyperadrenalism. Vectors $C$ and $D$ depend critically upon insulin, the anterior pituitary gland, and possibly other anti-insulin agents. Both of these vectors decrease in diabetes and are exaggerated in hyperinsulinism. Vector $E$ depends upon the concentration of glucose in the blood and the integrity of the enzyme architecture of the renal tubule.

This analysis of factors affecting the concentration of blood glucose in health and disease is of course merely exemplary. Given sufficient information, the deviation from normal in the concentration of any tissue constituent might be subjected to a similar analysis. It is the purpose of this discussion to emphasize the complex and highly integrated nature of the normally operating intact organism and to point up the difficulties inherent in the elucidation of the disturbances which are encountered in clinical medicine.

Much has been learned about diabetes from comparative studies of liver slices (4) or enzyme solutions (17) derived from normal and from diabetic animals. The ability of such prepara- 
tions to synthesize fatty acids is far less if they are derived from diabetic rather than normal animals, and these findings are compatible with what is known to happen in the intact animal (19). It is probably incorrect, however, to refer to such preparations as "diabetic liver slices." If by "diabetes" is meant the relative or absolute deficiency of insulin, then all liver slices, regardless of source, are necessarily diabetic. The difference between isolated livers from diabetic and nondiabetic animals is chronological, not diagnostic. Slices prepared from livers of pancreatectomized or alloxanized animals have been deprived of their source of insulin, have been "diabetic," for a somewhat longer period of time than have similar slices prepared from tissues of a normal animal.

\section{References}

1. Bernstein, C., Jr., J. B. Kirsner, and W. J. Turner, J. Lab. Clin. Med., 23, 938 (1938).

2. Bornstein, J., and C. R. Park, J. Biol. Chem., 205, 503 (1953).

3. Boxer, G. E., and D. Stetten, Jr., J. Biol. Chem., 153, 607 (1944).

4. Chernick, S. S., I. L. Chaikoff, E. J. Masoro, and E. Isaeff, J. Biol. Chem.. 186, 527 (1950).

5. Cori, G. T., Harvey Lectures, Ser. 48, 145 (1954).

6. Harrison, D. C., Biochem. J. (London), 26, 1295 (1932).

7. Ingle, D. J., E. H. Morley, and D. Stetten, Jr., Am. J. Physiol., 182, 263 (1955).

8. Krahl, M. E., J. Biol. Chem., 200, 99 (1953).

9. Levine, R., M. S. Goldstein, B. Huddlestun, and S. P. Klein, Am. J. Physiol., 163, 70 (1950).

10. Lundsgaard, E., Biochem. Z., 264, 209 (1933).

11. McQuarrie, I., E. T. Bell, B. Zimmermann, and W. S. Wright, Federation Proc., 9, 337 (1950).

12. Marsh, J. B., and N. Haugaard, J. Clin. Invest., 31, 107 (1952).

13. Mirsky, I. A., R. H. Broh-Kahn, G. Perisutti, and J. Brand, Arch. Biochem., 20, 1 (1949).

14. Patterson, W. B., and D. Stetten, Jr., Science, 109, 256 (1949).

15. Pauling, L., Proc. Am. Phil. Soc., 96, 556 (1952).

16. Price, W. H., C. F. Cori, and S. P. Colowick, J. Biol. Chem., 160, 633 (1945).

17. Shaw, W., and S. Gurin, Arch. Biochem. and Biophys., 47, 220 (1953). 
18. Stadie, W. C., N. Haugaard, and M. Vaughan, J. Biol. Chem., 200, 745 (1953).

19. Stetten, D., Jr., and G. E. Boxer, J. Biol. Chem., 156, 271 (1944).

20. Stetten, M. R., and D. Stetten, Jr., J. Biol. Chem., 187, 241 (1950).

21. Sutherland, E. W., and C. F. Cori, J. Biol. Chem., 188, 531 (1951).

22. Welt, I. D., D. Stetten, Jr., D. J. Ingle, and E. H. Morley, J. Biol. Chem.. 197, 57 (1952).

23. Wilson, D. W., J. Biol. Chem., 56, 203 (1923).

24. Wyshak, G. H., and I. L. Chaikoff, J. Biol. Chem., 200, 851 (1953). 


\title{
THE HORMONES, THEIR PRESENT SIGNIFICANCE, THEIR FUTURE
}

\author{
GREGORY PINCUS, The Worcester Foundation for Experimental \\ Biology, Shrewsbury, Massachusetts
}

\section{Introduction}

With the accelerating increase of our knowledge of the biochemistry of the hormones, any summary assessment of their role as biologically active substances is fated to be overmiserly in description and hazardous in extrapolation. The description is overmiserly not merely because of the necessity for condensation, but also because the acquaintance of any single individual with the diverse and heterogeneous fields of investigation involved is bound to be limited. The extrapolation is hazardous because one is in the position of a mathematician attempting to write the equation of an S-shaped curve which has not yet exhibited its inflection point. To compensate to some extent for inadequate acquaintance with all the data, this writer will confine his observations to animal hormones and chiefly to those which are active in mammals, and as a substitute for precise extrapolation he will offer speculations as well based as possible on established fact.

To indicate the diversities which confront any student of hormonology I have compiled Table I, which lists those substances having fairly established claims to being active internal secretions in animals. A number of additional substances 
HORMONES

TABLE I

A List of Animal Hormones, Their Chemical Nature, and Some of Their Biological Activities

\begin{tabular}{|c|c|c|c|}
\hline Name & $\begin{array}{l}\text { Tissue of } \\
\text { origin }\end{array}$ & $\begin{array}{l}\text { Chemical } \\
\text { nature }\end{array}$ & $\begin{array}{l}\text { Principal } \\
\text { known } \\
\text { biological } \\
\text { activities }\end{array}$ \\
\hline $\begin{array}{l}\text { Growth and de- } \\
\text { velopment (GD) } \\
\text { hormone }\end{array}$ & $\begin{array}{l}\text { Corpus cardia- } \\
\text { cum, ring } \\
\text { gland, } \\
\text { prothoracic } \\
\text { gland }\end{array}$ & Unknown & $\begin{array}{c}\text { Facilitates metamor- } \\
\text { phosis in insects }\end{array}$ \\
\hline Juvenile hormone & $\begin{array}{l}\text { Corpus allatum, } \\
\text { ring gland }\end{array}$ & Unknown & $\begin{array}{l}\text { Inhibits adult differ- } \\
\text { entiation in insects }\end{array}$ \\
\hline $\begin{array}{l}\text { Stimulant of GD } \\
\text { secretion }\end{array}$ & Brain & Unknown & $\begin{array}{l}\text { Induces GD hor- } \\
\text { mone secretion in } \\
\text { insects }\end{array}$ \\
\hline $\begin{array}{l}\text { Insect gonad stim- } \\
\text { ulant }\end{array}$ & Corpus allatum & Unknown & $\begin{array}{l}\text { Stimulates female } \\
\text { gonad activity }\end{array}$ \\
\hline $\begin{array}{l}\text { Voltinism hor- } \\
\text { mone }\end{array}$ & $\begin{array}{l}\text { Subesophageal } \\
\text { ganglion }\end{array}$ & Unknown & $\begin{array}{l}\text { Causes diapausing } \\
\text { eggs }\end{array}$ \\
\hline $\begin{array}{l}\text { Stimulants of } \\
\text { chromatophores }\end{array}$ & $\begin{array}{l}\mathrm{X} \text {-organ, sinus } \\
\text { gland, central } \\
\text { nervous system }\end{array}$ & $\begin{array}{l}\text { Intermedin-like } \\
\text { polypeptides }\end{array}$ & $\begin{array}{l}\text { Induce lightening or } \\
\text { darkening of pig- } \\
\text { mented end or- } \\
\text { gans in crustacea }\end{array}$ \\
\hline $\begin{array}{l}\text { Retinal pigment- } \\
\text { stimulating hor- } \\
\text { mone }\end{array}$ & $\begin{array}{l}\text { Central nervous } \\
\text { system }\end{array}$ & Unknown & $\begin{array}{l}\text { Stimulates contrac- } \\
\text { tion and disper- } \\
\text { sion of retinal pig- } \\
\text { ment granules in } \\
\text { crustacea }\end{array}$ \\
\hline Molting inhibitor & $\begin{array}{l}\mathrm{X} \text {-organ and } \\
\text { sinus gland }\end{array}$ & Unknown & $\begin{array}{l}\text { Prevents molting in } \\
\text { crustaceans }\end{array}$ \\
\hline $\begin{array}{l}\text { Crustacean gonad } \\
\text { stimulant }\end{array}$ & $\begin{array}{l}\mathrm{X} \text {-organ and } \\
\text { sinus gland }\end{array}$ & Unknown & $\begin{array}{l}\text { Stimulates gonad ac- } \\
\text { tivity in crusta- } \\
\text { ceans }\end{array}$ \\
\hline Secretin & $\begin{array}{l}\text { Intestinal mu- } \\
\text { cosa }\end{array}$ & Polypeptide & $\begin{array}{l}\text { Stimulates secretion } \\
\text { of pancreatic juice } \\
\text { and bile }\end{array}$ \\
\hline Pancreozymin & $\begin{array}{l}\text { Intestinal mu- } \\
\text { cosa }\end{array}$ & Unknown & $\begin{array}{l}\text { Enhances secretin ef- } \\
\text { fect }\end{array}$ \\
\hline Cholecystokinin & $\begin{array}{l}\text { Intestinal mu- } \\
\text { cosa }\end{array}$ & Unknown & $\begin{array}{l}\text { Stimulates gall blad- } \\
\text { der contraction }\end{array}$ \\
\hline Enterogastrone & $\begin{array}{l}\text { Intestinal mu- } \\
\text { cosa }\end{array}$ & Unknown & $\begin{array}{l}\text { Inhibits gastric mo- } \\
\text { tility }\end{array}$ \\
\hline
\end{tabular}


TABLE I (Continued)

\begin{tabular}{|c|c|c|c|}
\hline Name & $\begin{array}{l}\text { Tissue of } \\
\text { origin }\end{array}$ & $\begin{array}{l}\text { Chemical } \\
\text { nature }\end{array}$ & $\begin{array}{l}\text { Principal } \\
\text { known } \\
\text { biological } \\
\text { activities }\end{array}$ \\
\hline Vasopressin & $\begin{array}{l}\text { Posterior pitui- } \\
\text { tary }\end{array}$ & Polypeptide & $\begin{array}{l}\text { Antidiuretic, periph- } \\
\text { eral vasocon- } \\
\text { strictor }\end{array}$ \\
\hline Oxytocin & $\begin{array}{l}\text { Posterior pitui- } \\
\text { tary }\end{array}$ & Polypeptide & $\begin{array}{l}\text { Stimulates uterine } \\
\text { muscle contrac- } \\
\text { tion }\end{array}$ \\
\hline Insulin & Pancreas $\beta$-cells & Protein & $\begin{array}{l}\text { Hypoglycemic, gly- } \\
\text { cogenic }\end{array}$ \\
\hline Glucagon & Pancreas $\alpha$-cells & Protein (?) & $\begin{array}{l}\text { Hyperglycemic, gly- } \\
\text { cogenolytic }\end{array}$ \\
\hline Thyroid hormone & Thyroid gland & $\begin{array}{l}\text { Tetraiodothyro- } \\
\text { nine in thyro- } \\
\text { globulin }\end{array}$ & $\begin{array}{l}\text { Increases tissue oxy- } \\
\text { gen consumption }\end{array}$ \\
\hline $\begin{array}{l}\text { Parathyroid hor- } \\
\text { mone }\end{array}$ & $\begin{array}{l}\text { Parathyroid } \\
\text { gland }\end{array}$ & $\begin{array}{l}\text { Unknown (per- } \\
\text { haps one or } \\
\text { more polypep- } \\
\text { tides) }\end{array}$ & $\begin{array}{l}\text { Regulates calcium } \\
\text { and phosphate } \\
\text { metabolism }\end{array}$ \\
\hline Epinephrine & Adrenal medulla & Catechol amine & $\begin{array}{l}\text { Vasoconstrictor, gly- } \\
\text { cogenolytic }\end{array}$ \\
\hline Norepinephrine & Adrenal medulla & Catechol amine & $\begin{array}{l}\text { Vasoconstrictor, } \\
\text { neurohumoral }\end{array}$ \\
\hline Cortisol & Adrenal cortex & $\begin{array}{l}11,17,21-\text { Hydroxy- } \\
\text { lated progester- } \\
\text { one }\end{array}$ & $\begin{array}{l}\text { Glycogenetic, anti- } \\
\text { phlogistic, thymo- } \\
\text { lytic, protein cat- } \\
\text { abolic }\end{array}$ \\
\hline Corticosterone & Adrenal cortex & $\begin{array}{l}\text { 11,21-Hydroxy- } \\
\text { lated progester- } \\
\text { one }\end{array}$ & $\begin{array}{l}\text { Glycogenetic, thy- } \\
\text { molytic, protein } \\
\text { catabolic }\end{array}$ \\
\hline Aldosterone & Adrenal cortex & $\begin{array}{l}\text { 11,21-Hydroxy- } \\
\text { lated, } 18 \text { alde- } \\
\text { hydic progester- } \\
\text { one }\end{array}$ & $\begin{array}{l}\text { Sodium-retaining, } \\
\text { weakly thymolytic }\end{array}$ \\
\hline $\begin{array}{l}\text { Deoxycorticoster- } \\
\text { one }\end{array}$ & Adrenal cortex & $\begin{array}{l}\text { 21-Hydroxylated } \\
\text { progesterone }\end{array}$ & $\begin{array}{l}\text { Sodium-retaining, } \\
\text { potassium-excret- } \\
\text { ing }\end{array}$ \\
\hline $\begin{array}{l}\text { Hydroxyandros- } \\
\text { tenedione }\end{array}$ & Adrenal cortex & $\begin{array}{l}\text { 11-Hydroxylated } \\
\Delta^{4} \text {-androstene- } \\
\text { 3,17-dione }\end{array}$ & $\begin{array}{l}\text { Weakly androgenic } \\
\text { and protein ana- } \\
\text { bolic }\end{array}$ \\
\hline Testosterone & Testis & $\begin{array}{l}\text { 17-hydroxylated- } \\
\Delta^{4} \text {-androstene- } \\
\text { 3-one }\end{array}$ & $\begin{array}{l}\text { Androgenic, protein } \\
\text { anabolic }\end{array}$ \\
\hline
\end{tabular}




\begin{tabular}{|c|c|c|c|}
\hline Name & $\begin{array}{l}\text { Tissue of } \\
\text { origin }\end{array}$ & $\begin{array}{l}\text { Chemical } \\
\text { nature }\end{array}$ & $\begin{array}{l}\text { Principal } \\
\text { known } \\
\text { biological } \\
\text { activities }\end{array}$ \\
\hline Androstenedione & $\begin{array}{l}\text { Testis and } \\
\text { adrenal cortex }\end{array}$ & $\begin{array}{c}\Delta^{4} \text {-Androstene- } \\
\text { 3,17-dione }\end{array}$ & $\begin{array}{l}\text { Weakly androgenic, } \\
\text { protein anabolic }\end{array}$ \\
\hline Estradiol & $\begin{array}{l}\text { Ovary, testis, } \\
\text { adrenal cortex, } \\
\text { placenta (?) }\end{array}$ & $\begin{array}{l}\text { Estratriene-3,17- } \\
\text { diol }\end{array}$ & $\begin{array}{l}\text { Estrogenic, protein } \\
\text { anabolic in fe- } \\
\text { males, thymolytic, } \\
\text { growth-stimulat- } \\
\text { ing }\end{array}$ \\
\hline Estriol & Placenta & $\begin{array}{l}\text { 16-Hydroxy estra- } \\
\text { diol }\end{array}$ & Estrogenic \\
\hline Progesterone & $\begin{array}{l}\text { Ovary, adrenal } \\
\text { cortex, pla- } \\
\text { centa }\end{array}$ & $\begin{array}{l}\Delta^{4} \text {-Pregnene-3,20- } \\
\text { dione }\end{array}$ & $\begin{array}{c}\text { Progestational, ovu- } \\
\text { lation-inhibiting }\end{array}$ \\
\hline Relaxin & Ovary & Unknown & $\begin{array}{l}\text { Relaxes pubic liga- } \\
\text { ments }\end{array}$ \\
\hline $\begin{array}{l}\text { Adrenocorticotro- } \\
\text { phin }\end{array}$ & $\begin{array}{l}\text { Anterior pitui- } \\
\text { tary }\end{array}$ & Polypeptide & $\begin{array}{l}\text { Stimulates adrenal } \\
\text { cortex secretion }\end{array}$ \\
\hline Growth hormone & $\begin{array}{l}\text { Anterior pitui- } \\
\text { tary }\end{array}$ & Protein & $\begin{array}{l}\text { Protein anabolic, } \\
\text { stimulates bone } \\
\text { growth }\end{array}$ \\
\hline $\begin{array}{l}\text { Follicle-stimulat- } \\
\text { ing hormone } \\
\text { (FSH) }\end{array}$ & $\begin{array}{l}\text { Anterior pitui- } \\
\text { tary }\end{array}$ & Glycoprotein & $\begin{array}{l}\text { Stimulates ovarian } \\
\text { follicle }\end{array}$ \\
\hline Intermedin & $\begin{array}{l}\text { Anterior pitui- } \\
\text { tary }\end{array}$ & Polypeptide & $\begin{array}{l}\text { Disperses chromato- } \\
\text { phore granules in } \\
\text { skin }\end{array}$ \\
\hline $\begin{array}{l}\text { Luteinizing hor- } \\
\text { mone (LH) }\end{array}$ & $\begin{array}{l}\text { Anterior pitui- } \\
\text { tary }\end{array}$ & Protein & $\begin{array}{l}\text { Induces ovulation } \\
\text { and gonadal hor- } \\
\text { mone secretion }\end{array}$ \\
\hline Prolactin & $\begin{array}{l}\text { Anterior pitui- } \\
\text { tary }\end{array}$ & Protein & $\begin{array}{l}\text { Stimulates milk pro- } \\
\text { duction and ma- } \\
\text { ternal behavior }\end{array}$ \\
\hline $\begin{array}{l}\text { Thyrotrophin } \\
\text { (TSH) }\end{array}$ & $\begin{array}{l}\text { Anterior pitui- } \\
\text { tary }\end{array}$ & Protein & $\begin{array}{l}\text { Stimulates thyroid } \\
\text { hormone produc- } \\
\text { tion }\end{array}$ \\
\hline
\end{tabular}

might well have been included, but the listing clearly indicates the chemical variety of hormones and their broad range of activities. The latter, however, are inadequately indicated, since only the chief activities are listed. There is, in fact, no 
tissue in the mammalian body which is exempt from some sort of hormonal influence either in the course of its development or in its functional activities. Investigations of hormone physiology have quite properly been centered on the more specific actions of these substances, but it becomes more and more evident that the spheres of action are often extremely broad, and frequently far beyond the narrow limits suggested by the tissue of origin and its known interrelationships with other organs and tissues. Thus the expected action of ovarian estrogen as a promoter of female reproductive tract growth and of estrous behavior is accompanied by multitudinous activities quite outside the reproductive realm; estrogens are also hair and bone growth regulators, thymolytic, euphoria-inducing in the menopause, mitogenetic in the epidermis, enzyme inhibitory in the adrenal cortex, phagocyte-stimulating, alkalosis-inducing, antiatherogenic, tumorigenic, antigoitrogenic, antihyperglycemic, enhancers of cortisone-induced weight loss and glycosuria, hyphothalamic threshold lowering, anemia-inducing in dogs, etc., etc. Similar multiplicities of action may be cited for most hormones. Out of this welter of activities there arises the concept of an array of hormonal substances circulating to the various tissues of the body and interacting in a vast complex of functional processes.

It is not necessary to labor the point that the hormones are chemically a heterogeneous lot and biologically ubiquitously active. The only thing that unites them is a definition of them as internal secretions. How then may one consider them as biochemical entities? I suggest that for any and every single hormone there are five fundamental questions to be answered: (1) What is its chemical structure? (2) How does it originate? (3) What does it do? (4) How does it do it? What is its fate? In the case of no single hormone do we have a complete answer to all these questions. An inspection of Table I indicates that the chemical structures of most hormonal substances are either unknown or only partially defined. If we consider those with known chemical structure we find that even 
in the best instances the answers to questions (2), (3), (4), and (5) are either incomplete or lacking. Let us consider an outstanding example.

\section{Cortisol as a Hormone Protolype}

Cortisol is firmly established as an adrenocortical hormone. Because of its medical utility, cortisol, and, interchangeably, its transformation product cortisone, have been during the past five years objects of extraordinarily intensive investigation. How does it originate? The best information available suggests that its biosynthesis as a specific adrenocortical product is accomplished by at least two major biogenetic pathways $(7,10)$. These are depicted in Figure 1. Adrenocortical enzyme systems responsible for steps $(d),(e),(f)$, and $(g)$ have been described and partially characterized. The transformations involved in $(a)$, the nature of $\mathrm{X}$, and the events occurring in $(b)$ and $(h)$ are unknown. The scission of the side chain of cholesterol to yield $\Delta^{5}$-pregnenolone (reaction $(c)$ ) is known to be expedited by adrenocorticotrophic hormone (ACTH), but $(h)$ is apparently ACTH-independent. It is not certain that these two biosynthetic pathways (one via cholesterol, the other cholesterol-independent) are the only ones occurring in the adrenal cortex.

When we ask ourselves what does cortisol do we are confronted with a plethora of data. As in the case of the estrogens a great diversity of physiological effects are known. Cortisol is not only glycogenetic, antiphlogistic, protein catabolic, it is also eosinopenic, lymphopenic, an inhibitor of estrogen action on the uterus, edematogenic, glycosuric, hypertensive, abortifacient, an adjuvant in fat mobilization, a maintainer of vascular tone and vasomotion in the capillary bed, antianaphylactic, diabetogenic, muscle-work sustaining. It is not possible to ascribe these diverse effects of cortisol to a single basic action, e.g., its neoglycogenetic activity. Nor can we as yet define a circumscribed set of actions as fundamental, although there have 
been attempts to do so (cf. Russell and Wilhelmi (13)). Thus the therapeutic effects of cortisol do not correlate with its metabolic effects; indeed several of the latter have been called

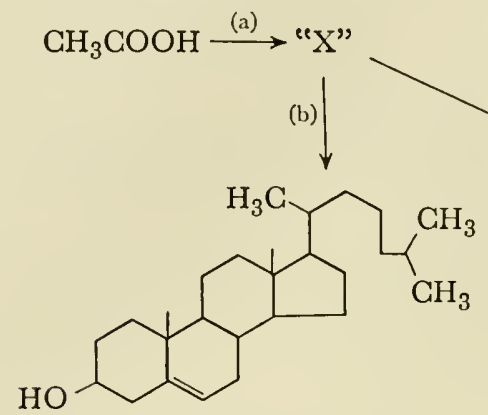

Cholesterol<smiles>CC(=O)C1CCC2C3CC=C4CC(O)CCC4(C)C3CCC12C</smiles>

$\Delta^{5}$-Pregnenolone

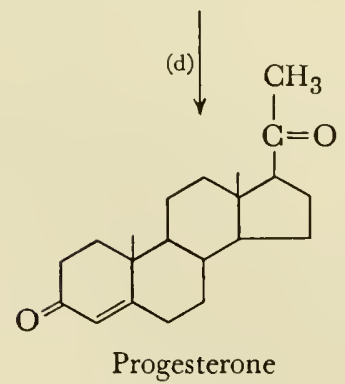

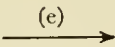

Fig. 1. A representation of the biosynthesis of cortisol.

"side effects." Though the antiphlogistic (or "anti-inflammatory") effects appear to be primary to therapeutic effects, the ameliorations observed in a wide variety of diseases lead one to 
suspect as responsible a combination of actions, some of them tissue-specific, others nonspecific. Even in the fairly simple muscle-work test, the increase of efficiency caused by cortisol is in part due to its making glucose available to the muscle, but there is also a component ascribable to a direct effect of cortisol on the muscle itself.

In considering how cortisol exerts its characteristic effects the problem we face is its biochemical mode of action. Here we are confronted with a rather specific puzzle and a general dilemma. The specific issue rests on the fact that cortisol given orally to men and animals is very nearly as effective as when it is given by the parenteral route. In being absorbed from the gut cortisol passes through the liver, in which it is subjected to extraordinarily rapid chemical transformation (6). Is it possible then that one or more transformation products are the agents responsible for the various effects of cortisol, and that we must search for these if we are to investigate the substance truly active biochemically? May a variety of metabolites in fact be the basis of the diversity of effect? A number of attempts to obtain the characteristic lymphocytopenic effect of cortisol by incubating it with lymphocytes in vitro have met with failure. Is this because a liver-formed metabolite is indeed the effective agent? Even in those instances where direct local action has been demonstrated, e.g., on direct intra-articular or intradermal injection, there is evidence of rapid transformation of cortisol to subsidiary products (12). This possibility of "active" hormone being something else at the site of its action applies to most if not all of the hormones.

The general dilemma is that which involves all who are interested in the mechanism of hormone action. It is first of all the problem of disentangling direct from indirect effects. Thus cortisol is extraordinarily potent in effecting glycogen deposition in the liver of adrenalectomized animals. Yet this effect cannot be obtained by incubating cortisol in vitro with liver tissue. Glycogen deposition represents the end result of a series of events in which cortisol plays a role; the best evidence available sug- 
gests that liver glycogen deposition results from glucose produced from certain tissue protein catabolized under the influence of cortisol (13). An examination of the mechanism of cortisol action must therefore involve not liver glycogenesis but gluconeogenesis from protein. Since the disentanglement of indirect effects has been in itself a task of major proportions, it is perhaps not surprising that studies of the mechanism of cortisol effects have lagged. The fact is that we do not have an explanation of how cortisol exerts its direct specific effects, nor indeed do we have this for any of the hormones.

A number of possible hypotheses have been advanced to explain the mode of cortisol action. It is interesting that these hypotheses might apply to the discussion of any hormone action. They may be summarized as follows: (1) cortisol acts as a cofactor to an enzyme system controlling the rate of a specific process; (2) cortisol acts to affect the permeability of the cell to substances necessary for certain enzymatic processes; (3) cortisol acts upon the processes controlling the movement of electrolytes in such a way that the significant concentrations of these electrolytes, both intra- and extracellular, are the ratecontrolling factors (9). Thus far every attempt to demonstrate that cortisol is a component of an identifiable enzyme system has failed. A meaningful characterization of the cellular permeability alterations that may be ascribed to cortisol has not been made. Although certain aspects of the general metabolism of $\mathrm{K}$ and $\mathrm{Na}$ as influenced by cortisol have been well described, the specific effects of their alterations upon cortisollabile metabolic systems have not been established. If we find ourselves with no explanation of the mechanism of cortisol action, it is not completely because we lack biochemical reactions in which cortisol must play a fairly direct role. Gluconeogenesis from protein, the effect on muscle-work, the interesting cooperative role played by cortisol with nor-adrenalin in the maintenance of vasomotion in the capillary bed, its function as an antihyaluronidase, its actions on connective tissue elements and the release of effector substances in inflammatory 
reactions-all these offer the investigator opportunities for analytic dissection. Sooner or later by the study of these or other reactions a definite role for cortisol will be assigned. That none has been found as yet is perhaps not too surprising. Outstanding metabolic effects of insulin have been known for over thirty years, but the means whereby these effects are exerted are still not definitively established; similarly, no biochemical mechanisms have been elucidated for the long-known actions of epinephrine, thyroxin, estrogens, and androgens.

Our final question concerning cortisol-what is its fate?may be partially answered. Its metabolism has been studied in men and animals, in vivo and in vitro. Certain chemical transformations which it undergoes have been determined, and the enzyme systems responsible for these transformations have been at least partially characterized (4). The chief known pathways of cortisol catabolism are presented in Figure 2. The reactions indicated are based principally upon studies of urinary products obtained after the administration of cortisol, but these data are also in accord with in vitro studies involving cortisol incubation with liver slices or homogenates. Quantitatively major transformations are indicated by heavy arrows, minor ones by the lighter arrows. Compounds II to XIV are products characteristic of cortisol metabolism in human subjects, compounds $\mathrm{XV}$ to XVI appear in guinea pig urine following cortisol administration (1) and have not been seen in the urines of other species, whereas II to XIV, if they exist in guinea pig urine, have not yet been identified therein. Actually in man the 11oxygenated 17-ketosteroids (compounds VI, VIII, XI, and XIV) represent $2 \%$ to $3 \%$ of administered cortisol and compounds $\mathrm{V}$ and VI are the major urinary transformation products, accounting for approximately $30 \%$. In man, therefore, some two-thirds of administered cortisol is unaccounted for in terms of identifiable metabolites. In the guinea pig compounds XV to XVII account for approximately $4 \%$ of administered cortisol, with the probability that a larger percentage may be represented by compounds similar to V to VIII, although it should be 

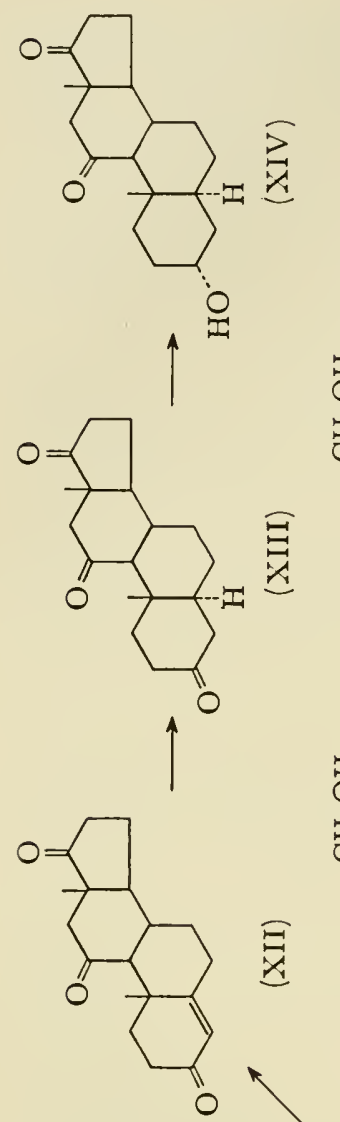

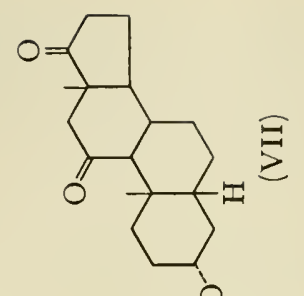

요

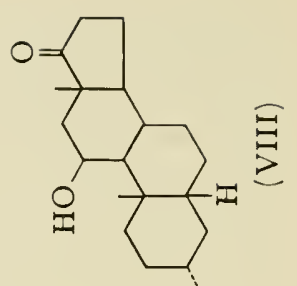

언<smiles>c1ccccc1</smiles>

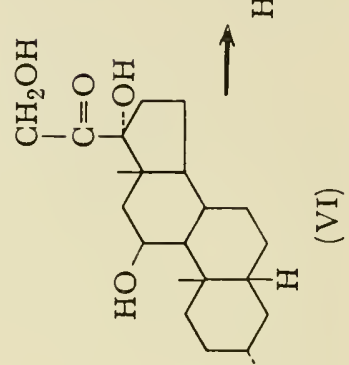<smiles>[123I-]</smiles><smiles>CCCCCCC</smiles>
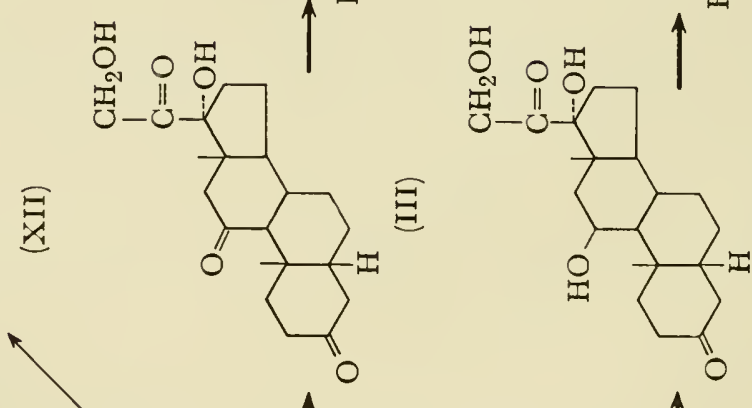

$$
\text { (l) }
$$




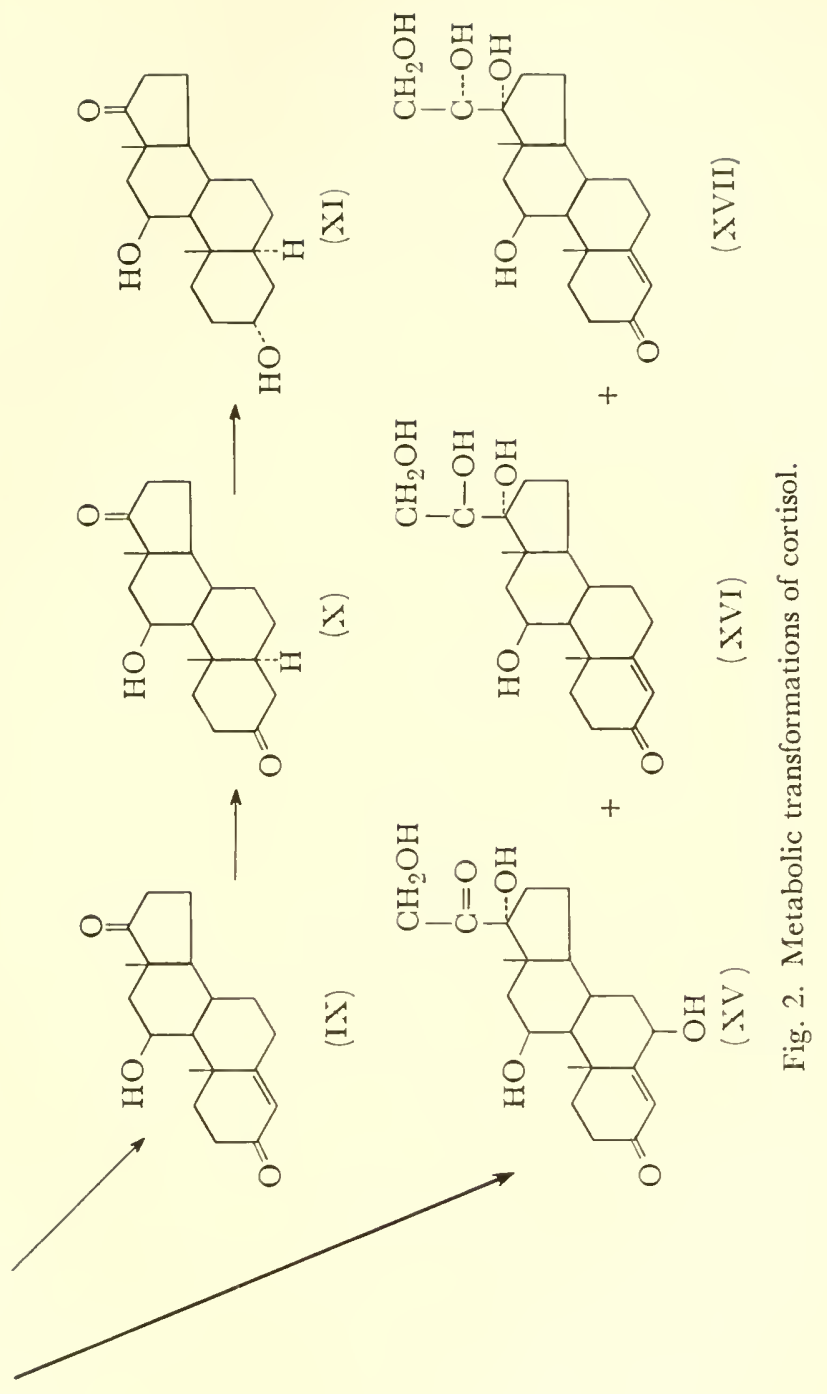


noted that in the guinea pig the reaction $\mathrm{I} \rightarrow \mathrm{II}$ does not appear to occur to an appreciable extent, whereas $\mathrm{II} \rightarrow \mathrm{I}$ occurs readily (2). In all species examined thus far a considerable proportion of the transformation products of cortisol are unknown. In recent studies with $\mathrm{C}^{14}$-labeled cortisol there have been indications that a variety of products may occur, some of which may be nonsteroidal. Furthermore, there are indications of species differences in the mode of metabolism; thus in the rat and mouse there is considerable excretion of fecal metabolites, whereas in man up to $80 \%$ of the $4-\mathrm{C}^{14}$-cortisol radioactivity has been excreted into the urine (5).

In the case of the steroid hormones the search for metabolites generally has paralleled what has been described for cortisol. No complete balance sheet is possible but a variety of transformation products are well established. When we consider the hormones the chemical nature of which is less well established, our knowledge of their fate in the body is meager indeed. Thus it is known that certain anterior pituitary hormones disappear extremely rapidly from the blood stream, but where they go and what their chemical fate is, remains a mystery.

Having discussed in summary fashion the major biochemical problems of a fairly intensively investigated hormone, I think I have made evident some of the hiatuses in our knowledge concerning it. Similar considerations apply to other hormonal substances. What they do is known to a greater or lesser extent, but it may be safely stated that there is no complete knowledge of all the actions of any single hormone. How they do it is for the most part a mystery, at least in the ultimate sense of mechanism of action. And the biochemical fate of most of the hormones is unknown, and for those concerning which we have some specific information a complete balance sheet has not been established.

\section{Synergism and Antagonism}

There are other aspects of hormone biochemistry not encompassed by our five leading questions. For example, sub- 
sidiary perhaps to the problem of mechanism of action are the problems of inhibition and synergism in hormone action. Apparently straightforward examples of hormone antagonism may be cited: the inhibition of androgens by estrogens, the antagonism between pituitary growth hormone and ACTH in a number of their effects, the anti-insulin effect of certain corticosteroids, diuretic and antidiuretic hormones. Synergism is less easy to exemplify-estrogen and progestin seem to synergize in certain effects, not in others; and augmentation of androgen stimulation has been observed with thyroxin administration; a horse pituitary extract in itself inactive appears to sensitize the adrenal to ACTH action. When one examines these types of hormone interaction somewhat more closely, it becomes difficult to be certain that the same process is affected by the interacting hormones. Thus androgen and estrogen both stimulate prostate growth; the former acts upon the secretory tissue, the latter on the utricle; yet estrogen is employed in prostatic disease as an androgen antagonist. Estrogen sensitizes the uterus to progestin action and is thus synergistic, but large estrogen doses may actually inhibit pseudopregnant proliferation due to progesterone. The crux of the matter lies in the identification of the specific chemical processes labile to the various interacting hormones, and this, as we have said before, still eludes us.

\section{Hormone Therapy}

I have scarcely remarked on the therapeutic actions of the hormones, particularly their role in affecting various pathological processes. The earliest uses of hormones in therapy were for replacement in obvious cases of deficit. The wide usage of estrogen in the menopause is considered replacement therapy, as is insulin for the control of diabetes, thyroid in hypothyroidism, and corticosteroid in Addison's disease. With the advent of new and more potent preparations of various types and particularly with the discovery of the antiphlogistic actions of ACTH and corticosteroids, experimental investigations of hormone ef- 
fects in practically every known disease have multiplied. At the moment it is hard to see the forest for the trees, but a concept of "regulatory" therapy appears dimly. The most vocal protagonist of such a concept has been Professor Hans Selye, whose various publications on "diseases of adaptation" suggest a disturbance of the normal pituitary-adrenal relationship as one etiologic factor in a great variety of diseases (14). It is impossible to summarize adequately the voluminous discussion and the shifts and changes which have marked this general theory. The endocrine basis has been a notion of imbalance between mineralocorticoid and glycocorticoid hormones of the adrenal cortex which in turn involves imbalances in mineralocorticotrophic and glycocorticotrophic pituitary hormones. Thus far no mineralocorticotrophin has been established as a distinct entity, although Selye believes that pituitary growth hormone serves that function. ACTH acts as the glycocorticotrophin. The theory demands that continued stress induces an alteration in adrenocortical output of such a nature that mineralocorticoids tend under chronic stress to preponderate among the secretory products. Certain recent findings are worth mentioning. First of all, the discovery of aldosterone as an extremely potent adrenal mineralocorticoid (15) suggests that this substance may be the prophlogistic adrenal hormone. Secondly, the excretion of aldosterone-like sodium-retaining activity in human urine appears to be increased by growth hormone administration and unaffected by ACTH administration (17). Thirdly, chronic ACTH administration, the presumed equivalent of chronic stress, significantly alters the nature of adrenocortical secretion; in the rabbit, primarily a secretor of corticosterone, chronic ACTH treatment tends to increase cortisol and decrease corticosterone secretion (8). Since corticosterone is more of a mineralocorticoid than cortisol (the typical glycocorticoid), $\mathrm{ACTH}$, in the rabbit at least, tends to alter adrenocortical secretion toward prophlogistic hormone output. A detailed demonstration of the nature of aldosterone biogenesis and of the nature of adrenal secretion in chronic stress is clearly needed. 


\section{Hormones and the Future}

In describing certain aspects of the present biochemical status of the hormones I think I have indicated by inference some of their future prospects. Gaps in our knowledge concerning them are most obvious, and these will one day be filled. Instead of examining specific deficiences and what their correction will imply, it may be worth while to take a broad look ahead.

Basic to this broad look is the expectation that one specific set of deficiencies must and will be corrected-our knowledge of the chemical nature of the hormones must be advanced so that either the chemical structure of known hormones will be established, or that, in the case of complex substances, chemically pure preparations will be available. Much of the controversy and confusion in hormone research may be traced to the properties of impure hormone-concentrating extracts. Thus the paradoxical hyperglycemic action of insulin has been established as due to the presence of glucagon in insulin concentrates. Much controversy concerning the salt-retaining activities of adrenal extract appears to be resolved by the isolation and identification of aldosterone. A number of mysteries in thyroid hormone biochemistry have been solved with the establishment of triiodothyronine as an active principle. Many more such instances could be cited. The chemical nature of the hormones listed as unknown in Table I should be known, and among those known many should be better known.

Essential also to a broad look is a realization of the pervasiveness of the hormones in body economy. If we consider the known products of the glands of internal secretion and their known active metabolites, it may be calculated that over 50 biologically active substances circulate continuously in the blood of mammals as hormones. There is no vital process which is exempt from their influence, and yet, with few exceptions, these substances are not essential for life. In the rat, for example, neither thyroidectomy, nor gonadectomy, nor hypophysectomy, 
nor adrenalectomy is fatal. What happens in these events is that the rates of certain processes are reduced to a minimum. When the need arises for a speeding up of these rates it cannot be fulfilled, for the sources of the prime accelerators are gone. The margins of safety afforded by hormone-paced homeostasis are reduced to a minimum. But we are indeed concerned with more than safety alone; the physical and mental dullness of the hypothyroid individual, the asthenia of the Addisonian, represent deficits in an optimal rate of living. One of the extraordinary features of adequate hormone balance is the mental and physical vigor of the organism, its adaptability, its drive.

One of the questions to be asked therefore is whether optimal rates of living are a function of a specific balance of hormonal secretion. Must this large conflux of circulating effector substances contain precise quotas of each component? What is the long-run effect of even minor excesses and deficits? We have developed a tolerable understanding of the daily requirements for a good number of vitamins. Are there similar daily hormone requirements? Are there individual as well as average requirements? One obvious result of our assessment of vitamin requirements has been the administration of adequate amounts to aging individuals. Somewhat less certainly geriatric hormone administration is practised. But even before the obvious deficiencies of age are apparent, may there not be subtler imbalances contributing to inadequacies and inefficiencies of general metabolism, of nervous function? In a recent study of steroid excretion in over 600 men and women of various ages (11), we were struck by the remarkable consistency of individual secretion patterns. Each individual has a characteristic quantitative output of certain steroids and therefore a characteristic ratio of one type to another type. This consistency of personal patterns has been demonstrated in fine chemical detail for the individual metabolites in the urinary ketosteroid array by Dobriner and his colleagues (3). What is its significance? Broadly speaking there is an implication of set secretory activity by steroidogenic organs along with fixed modes of metabolism of 
the hormones by extraglandular tissue. How do these patterns become fixed?

There is at present no valid answer to this question. One reason why an answer evades us is our inability to assess in even approximate quantitative fashion the degree of interdependence of the various hormonogenic systems of the body. From laboriously attained bits and pieces of evidence we have the beginnings of an understanding of such interdependencies. We know, for example, that adequate estrogen action requires adequate thyroid hormone, that the secretion of ACTH may be suppressed by various corticosteroids, that pituitary gonadotrophin release depends in the rat on an estrogen-progestin balance. Is there a key pacemaker to this system of checks and balances? Or are we dealing with a complex servel mechanism? Are there endogenous antihormones which play a role in the complex hormone equilibrium? Certainly in any consideration of the effectiveness of hormone secretion it is important to evaluate those influences which tend to inactivate the hormones. For every known hormone there exists in vivo one or more inactivating mechanisms. These are enzymatic systems which oxidize, reduce, detoxify. What is their quantitative and qualitative contribution to the endogenous hormone pattern? What regulates their rate of functioning? We have recently examined a blood-borne enzyme system that inactivates ACTH. Its concentration in the blood of certain individuals is negligible, whereas in the blood of others it is so high as to effect extremely rapid loss of ACTH activity. What is the biochemical basis of this large individual difference?

We have indicated that both the rate of secretion and the rate of destruction are key processes in the setting of hormone balance. One other functional phenomenon still scarcely explored requires mention-the phenomenon of action of the end organ. One characteristic of the biological response of organisms to hormones is variability. Certain individuals show very low response thresholds, others very high ones, and there is a characteristic distribution in between, exemplified by the 
well-known dosage-response curve. In intact organisms this may be reflective of quantitative variations in hormone-inactivating systems; but one may observe this variability in response in isolated tissues or cells, e.g., in the glycogenolytic action of glucagon or epinephrine on liver cells in vitro, in the effects of insulin on glycogenesis of the isolated diaphragm. What is the basis of this variable effectiveness, which in certain instances may amount to nearly complete ineffectiveness? One may of course postulate hormone-inactivating mechanisms of varying efficiency present in the end organs themselves. Another possibility inheres in the quantitative variations of substrates or cofactors of the hormone-labile systems. A third, and most intriguing, possibility is the existence in the end-organ tissue of native hormone analogues as inhibitors. I may cite as an example the recent demonstration by Velardo and his collaborators (16) of the pacemaking action of estriol. When estradiol, which is a much more potent uterus-stimulating estrogen than estriol, is administered along with estriol, the degree of stimulation over a considerable dosage range is determined by the estriol, not by estradiol. It is as if estriol has an estradioldisplacing action at the effector sites. Is it possible that for many, most, or even all of the hormones endogenous antagonists are produced either as extraglandular metabolites of the active compounds or by the secretory tissues themselves? One of the most interesting phases of the adaptation syndrome is the socalled stage of adaptation. Effects characteristic of the initial stage of alarm cannot be duplicated at this time by identical stresses. May not the stage of adaptation be characterized by a shift to secretion of, for example, corticoid antagonistic substances which saturate the sites of action at the end organs and render the corticoids ineffective.

Thus far in attempting a broad look at the future of the hormones we have apparently been occupied in asking questions. But I feel that these questions are keys to the future. In the case of any individual hormone we must continue to ask our five leading questions. But this is not the limit of our inquiry. We 
still have the larger questions of hormone interaction. There are complex balance sheets to be set up, monograms, and individual accountings. We must account for pathological and personal variations in function and for declines in function attributed to "normal" aging. If this seems a gigantic task, I think its magnitude is exceeded by its fascination.

In our discussion we have considered the hormones as regulators of a great variety of vital processes. I should like to emphasize that these processes may vary from intracellular chemical reactions to complex behavior. Androgens affect protein anabolism, but also the pecking order of cockerels. Cortisone inhibits protein synthesis, induces euphoria, and is, under certain circumstances, psychotogenic. Progesterone prevents water intoxication and induces broodiness in hens. We are concerned not only with intimate biochemical events but also with the organism as a whole. In their practical application, therefore, the hormones must be considered not only as the agents of specific biochemical effects but also as governors of behavior and perhaps even ideation. Hormonal treatment as now practised for premenstrual tension and menopausal vasomotor symptoms is designed not only to enable women to function better but to feel and behave better. Perhaps this is just another way of stating that hormones have considerable effects on the central nervous system.

If, then, we visualize the hormones as continuing to contribute more and more to the metabolic efficiency of the organism in health and disease, to its adaptability to stress, to its resistance to involution, to its emotional well-being, to its better prenatal and postnatal development and growth, have we come to the end of our prophecy? I suspect not. It may not be entirely pertinent to a biochemical essay, but I cannot forbear mentioning certain contributions to the livestock industry. Hormonal caponization has become a standard practise in poultry husbandry. I dare say that fairly simple hormone treatments will shortly be used to improve the quality of beef, pork, mutton. It is possible that control of the sex ratio in 
animals by hormonal means may one day be attained. Certainly hormone therapy in veterinary medicine will be increasingly practised. Then there is anthropology. The measurement of physical and emotional differences between the so-called races of men has disclosed differences that may have rather profound endocrine bases. What about endocrine mechanisms in the fat metabolism of the Eskimo, pituitary and androgen function in the Pigmy, adrenal function in ceremonial rites? Are there endocrine bases to personality and behavioral tribal characteristics?

I have thus far limited this discourse to known animal hormones. I should like to remark finally and briefly on prospects of discovery. Mammalian endocrinology has been the most intensively exploited field, particularly because of medical implications. At this writing the probability of the discovery of many new mammalian hormones appears limited. I am not unmindful of the need for further exploration of pituitary factors (adipokinin may be established as the latest) and of gastrointestinal tract hormones and of the parathyroid mystery and of prospects of new neurohumors. But I do believe that our knowledge of significant hormones in the lower vertebrates and invertebrates is at best elementary. A few faithful and extraodinarily able investigators have discovered fascinating hormonal mechanisms in insects and crustacea, with the result that the problems that they have uncovered can keep many people busy for a long time. Comparative endocrinology as a science is not even an infant, it is a conceptus. If you would like to be the discoverer of a new animal hormone, apply your biochemistry to any one of innumerable species of nonmammalian forms. There lies the untouched treasure.

I hope it is evident that the trajectory of the curve describing hormone research has not yet reached its inflection point. Certainly evident to the scientific pioneer are the prospects of wide-open spaces, of intriguing new phenomena, and of mysterious natural events. There is no stage in individual development from conception to senility which is exempt from 
hormonal influence. There is even dying itself. Moreover, there is a diversity of problems which is unparalleled. In the study of any single hormone the resources of organic chemistry, of cellular and general physiology, of enzyme biochemistry, and even of neuropharmacology and psychology may be called into action. As biologically active substances the hormones have an often baffling ubiquity. For a long time they will continue to be inciters of curiosity and stimulants to investigation. To judge by the scientific rewards which have been reaped, there are greater rewards to come.

\section{References}

1. Burstein, S., and R. I. Dorfman, J. Biol. Chtm., 213, 581 (1955).

2. Burstein, S., and R. I. Dorfman, personal communication.

3. Dobriner, K., J. Clin. Invest., 10, 950 (1953).

4. Dorfman, R. I., and F. Ungar, Metabolism of Steroid Hormones. Burgess, Minneapolis, 1953.

5. Gallagher, T. F., H. L. Bradlow, D. K. Fukushima, C. Beer, T. H. Kritchevsky, M. Stokem, M. L. Eidinoff, L. Hellman, and K. Dobriner, Recent Progr. Hormone Research, 9, 411 (1954).

6. Hechter, O., Ciba Foundation Colloquia on Endocrinol., 7, 272 (1953).

7. Hechter, O., and G. Pincus, Physiol. Revs., 34, 459 (1954).

8. Kass, E. H., O. Hechter, I. A. Macchi, and T. W. Mow, Proc. Soc. Exptl. Biol. Med., 85, 583 (1954).

9. Mechanism of Corticosteroid Action in Disease Processes, Annals $\mathrm{N}$. Y. Acad. Sci., 56, 623 (1954).

10. Pincus, G., in Paul Kallos, ed., Progress in Allergy. Little, Brown, Boston, 1955.

11. Pincus, G., L. P. Romanoff, and J. Carlo, J. Gerontology, 9, 113 (1954).

12. Pincus, G., E. B. Romanoff, and L. P. Romanoff, Ciba Foundation Colloquia on Endocrinol., 7, 240 (1953).

13. Russell, J. A., and A. E. Wilhelmi, in F. D. W. Lukens, ed., Medical Uses of Cortisone, Vol. 1. Blakiston, New York, 1954.

14. Selye, H., The Story of the Adaptation Syndrome. Acta Montreal, 1952.

15. Simpson, S. A., J. E. Tait, A. Wettstein, R. Neher, J. von Euw, O. Schindler, and T. Reichstein, Helv. Chim. Acta, 37, 1163 (1954).

16. Velardo, J. T., F. G. Hisaw, and C. M. Goolsby, Federation Proc., 12, 68 (1953).

17. Venning, E. H., Ciba Foundation Colloquia on Endocrinol., 8, 190 (1955). 


\section{PROBLEMS OF CELLULAR BIOCHEMISTRY}

CARL F. CORI, Department of Biological Chemistry, Washington University School of Medicine, St. Louis, Missouri

If the present era of biochemistry needs a general characterization, it might be said that the central problems are those concerned with enzyme action. The reason for this is based on the recognition, in the last fifty years, that most of the chemical reactions which occur in living organisms are enzyme-catalyzed and that synthesis and degradation of organic molecules generally involve a series of enzymes acting in sequence. From this would follow the present trend of obtaining as much relevant information as possible about each enzymatic reaction a particular organism or tissue is capable of carrying out. When one considers the whole array of living forms and the great diversity of organic molecules which occur in nature, it seems clear that work along these lines will progress actively for a long time to come, subject only to the limitation of available methods.

The level of significance, the depth to which one can penetrate into a problem, is severely limited by methods. It can be clearly seen how recent methodological advances have led to solutions of problems which were previously unattainable. Among others, the use of isotopes, of chromatography, and of precision instruments for physical measurements come to mind. 
The proposition that most biological problems, if one probes deeply enough, can be reduced to enzyme problems, would seem to justify a certain amount of optimism about the possible attainments of the future. Each scientific era sets up its own criteria of what is regarded as significant. We would regard as deeply significant even the partial solution of such problems as that of nucleic acid and protein synthesis, which are in turn connected with the much larger problem of self-duplication of genetic material and its somatic expression through the control of the formation of specific enzymes.

The example just given indicates the degree to which the boundaries between various disciplines, once sharply delineated, have been blurred. The widening territory in which it is possible to operate with biochemical methods enhances the feeling of the unity of science and facilitates cross-fertilization between different disciplines; it also has the effect of counteracting the much decried tendency to overspecialization. Remarkable as the advances are in the elucidation of cellular structure through the use of the electron microscope, biochemists would not have paid much attention if there had not been present an awareness of the importance of structural elements for biochemical events in intact cells. Here one needs refer only to investigations on the role of mitochondria for the energy metabolism of the cell. The study of the biochemical potentialities of these and other cellular particles is important in relation to the activity of the cell as a whole.

\section{Cellular Organization}

The rapidly increasing information about enzymatic reactions is accompanied by attempts to apply this knowledge to the intact cell, the ultimate goal being an understanding of the processes which determine the integration of enzymatic activities at the cellular or even organismic level of organization. Biochemistry is moving but slowly in this direction, because there are many difficulties, but there can be no doubt that such 
understanding will have far-reaching consequences for biology as a whole and that it will be of great benefit in the treatment of disease. In a sense the problem has been worked on extensively in the past on a variety of unicellular organisms. There is also available a large amount of information which is based on work on the intact animal, with a variety of methods, including more recently the use of isotopes. The study of regulatory mechanisms and the endocrine control of metabolism fall into this category.

In this article an attempt will be made to outline some of the problems which arise in this field, and to point out certain areas where additional information is needed. It is not intended to cope with a vast, quite heterogeneous, and widely scattered literature or to treat any given problem exhaustively. The field will be narrowed down further by considering mainly mammalian cells.

\section{Rate of Penetration of Sugars into Cells}

One of the first questions which arises is that of rate of penetration of various substances through the cell membrane. For example, is it the rate of entrance of metabolites, more or less common to all cells, such as sugars, amino acids, fatty acids and their keto derivatives, which determines the rate of metabolism within the cell, or are the rate-limiting steps the enzymatic reactions in which these metabolites are used as substrates? In the first case the cell membrane would have a controlling influence over the rate of metabolism; in the second case the effective enzyme concentration would be decisive. In spite of a very large literature on the subject of permeability, information on this question is very scanty.

Work carried out recently in this laboratory by Drs. Field, Heimberg, and Crane and not as yet reported has given a definite answer to this question for the Ehrlich ascites tumor cells metabolizing various sugars. These washed, single-cell preparations show one of the highest rates of glucose catabolism 
known for mammalian cells (anaerobic lactic acid production equivalent to 25 per cent of dry weight of cells per hour at $37^{\circ}$ C.); their rate of lactic acid production remains linear for many hours under anaerobic conditions, and there is little if any lag period when glucose is introduced at zero time, and initial acid production is measured titrimetrically with a glass electrode assembly. The final distribution of various sugars (some of which were utilizable and some of which were not), based on the aqueous phase of medium and cells, was close to unity, and equilibrium was reached within one minute at $37^{\circ} \mathrm{C}$. over a wide range of sugar concentrations. Only by lowering the temperature to $20^{\circ} \mathrm{C}$. could the actual rate of penetration be measured, which was found to be rapid enough, even at that temperature, to sustain the observed rate of lactic acid formation at $37^{\circ}$ C. Furthermore, rates of lactic acid formation, determined at different concentrations of glucose, gave typical Lineweaver-Burk plots and $K_{m}$ values which were of the same order as $K_{m}$ determined on hexokinase extracted from the cells $\left(K_{m}\right.$ was close to $1 \times 10^{-5} M$ per liter in both cases). These and other observations make it quite certain that the rate of sugar metabolism of these cells is determined by the rate of activity of the intracellular enzymes and not by the rate of penetration of the sugars through the cell membrane.

It is possible to decrease the rate of lactic acid formation in these cells by means of sugars which, while not being phosphorylated, have sufficient affinity for a common transport system to act as competitive inhibitors. Thus galactose and 3methyl glucose inhibit the rate of lactic acid formation from glucose, fructose, and mannose, and the degree of inhibition, at different concentrations, is determined by the relative affinities of these sugars for the transport system.

The question of the rate of penetration of sugars into the tissues of the intact animal is not so easily analyzed. In a study carried out some twenty years ago on rats it was noted that the ratio, fermentable tissue sugar/plasma sugar, was lowest for skeletal muscle, intermediate for diaphragm, and highest for 
heart muscle. Following glucose injection, heart muscle proved to be much more permeable than skcletal muscle. This was attributed to the difference in the number of open capillaries in the active cardiac muscle as compared to resting skeletal muscle. In heart, after glucose injection, the ratios, tissue sugar/plasma sugar, ranged from 0.4 to 0.6 . Had the sugar been present only in the extracellular fluid space, the ratio would have been about 0.2 . Part of the sugar was therefore intracellular, and the increase in the intracellular concentration with rising plasma concentration indicated that the rate of penetration was greater than the rate of utilization. When skeletal muscle was stimulated, thereby imitating heart muscle, an intracellular distribution of sugar could be demonstrated (6).

Following the intravenous injection of a nonutilizable sugar into a nephrectomized animal, to take relatively simple experimental conditions, the following factors come into play in the distribution of the sugar in the body: (1) rate of penetration through capillary wall; (2) rate of distribution in the extracellular fluid space; (3) rate of penetration into different tissue cells; and (4) selectivity of various cell membranes. In general, (1) is a very rapid process, to judge from the initial rate of disappearance of the sugar from the blood. The rate of (2) and hence of (3) will depend on the cross section of open capillaries, on blood pressure, and on rate of blood flow; for this reason different tissues will attain a state of equilibrium at different rates.

In regard to (4), definite information is available with respect to erythrocytes, small intestine, kidney tubules, and peritoneal cavity. The latter shows no selectivity with respect to the rate of absorption of various hexoses and pentoses, whereas intestine and kidney are highly selective. The capillaries of the choroid plexus (representing the so-called blood-brain barrier) are also selective, whereas the glomerular capillaries allow the passage even of substances of relatively large molecular weight, such as inulin.

Whether the mechanism of uptake of sugars by tissue cells 
is similar to that in intestine and kidney is an open question. In intestine the more rapid absorption of galactose and glucose as compared with pentoses is attributed to an "active" process, specifically phosphorylation of the hexoses, whereas the absorption of the pentoses is attributed to a simple process of diffusion. This phosphorylation (and by implication) -dephosphorylation mechanism is also supposed to operate in the proximal convoluted tubules of the kidney. Without attempting to examine the evidence for this hypothesis in detail, certain difficulties may be pointed out at this time. Carefully controlled experiments carried out by Sols (18) with extracts of rat intestinal mucosa have failed to show the presence of enzymes capable of phosphorylation of galactose and 3-methyl glucose (contrary to statements found in the literature). Yet these two sugars are absorbed as rapidly as glucose. A hexokinase capable of phosphorylating glucose, fructose, and mannose and having a specificity similar to that of brain hexokinase was always found in these extracts. On the dephosphorylation side there is the difficulty that glucose-6-phosphatase is missing in the kidney of children with severe glycogen storage disease of liver and kidney, but these children do not have glycosuria (5).

Keston (14) has recently described the occurrence of a mutarotase in kidney, an enzyme which has previously been found in molds and which is specific for certain sugars. On the assumption that a form of low abundance, presumably an openchain form of sugar, penetrates preferentially into cells, a role is ascribed to this enzyme in sugar transport in all tissues. Mutarotase, according to this theory, would control an important rate-limiting step in carbohydrate metabolism, the rate at which sugar enters the cell, and would also be implicated in diabetes and the action of insulin. Levine's (16) theory of insulin action is also based on the assumption that the rate of glucose utilization in tissues is controlled by the rate of sugar transport across the cell membrane. An alternative hypothesis is that the rate of glucose metabolism is controlled by intracellular enzymes. Important as a decision between these two 
alternatives may be, rigorous proof for either concept has not been obtained so far.

\section{Compartments of the Cell}

The control of rates of metabolism by intracellular enzymes may be illustrated in the following examples. When isolated frog muscle is compared at rest and during tetanic contraction, a more than 100-fold difference in the rate of lactic acid formation can be demonstrated. The mechanism underlying this increase in enzymatic rates has not been explained. Recent work (9) indicates that at $0^{\circ} \mathrm{C}$., even if there is no demonstrable splitting of ATP or phosphocreatine during contraction, there occurs nevertheless an increase in the concentration of inorganic phosphate which may arise from an unknown precursor. The concentration of inorganic phosphate in resting muscle is high enough to saturate phosphorylase, the first enzyme in the chain of reactions from glycogen to lactic acid. If the reaction catalyzed by this enzyme were the rate-limiting step in glycolysis in resting muscle, and if it were then accelerated 100 -fold by the formation of inorganic phosphate during contraction, one would have to make the additional assumption that at rest the enzyme is almost completely separated from inorganic phosphate. A determination of intermediates of glycolysis in resting muscle shows that hexosemonophosphate is present in a concentration which would saturate phosphofructokinase. During contraction hexosemonophosphate increases (8), and in order to make phosphofructokinase the rate-limiting step, the same additional assumption would have to be made as in the case of phosphorylase- a compartment of the muscle cell which does not permit contact between the enzyme and its substrate-a barrier which is broken down during contraction and is re-established during rest. Because of the possible existence of such barriers, it may be misleading to draw conclusions from steady-state concentrations of intermediates. For example, exposure of isolated frog muscle to epinephrine causes an approximate doubling of the 
hexosemonophosphate concentration, but this is associated with a minimal increase in the rate of lactic acid formation (11). Instead of envisaging a "barrier," one could also assume that certain muscle enzymes exist as inactive precursors in resting muscle and are changed to active forms during contraction. In fact, epinephrine has such an effect on muscle phosphorylase, which consists in the conversion of an inactive to an active form (see below). For want of a better one, the term "compartment" will be used to describe these deviations from the usual kinetics of enzymes as obtained in cell-free systems, and it will be understood that explanations for this phenomenon are highly speculative.

Compartments can also be invoked to explain glycogen formation in the liver. The equilibria of the enzymes involved are such that if the steady-state concentration of inorganic phosphate in contact with phosphorylase were $1 \mu \mathrm{M}$ per gram liver, the steady-state concentration of glucose-6-phosphate would have to be about $7 \mu \mathrm{M}$ for glycogen synthesis to occur. This is a much higher concentration than is found in the liver. Even if one assumes that inorganic phosphate is not in contact with phosphorylase so that the concentration of glucose-6-phosphate could be lower, one is still faced with the problem of how glucose-6-phosphate formed by hexokinase escapes the action of glucose-6-phosphatase. On the other hand, when glycogen is being broken down, the glucose-6-phosphate formed is accessible to the phosphatase. It is as if glycogen synthesis and degradation were completely separated in the liver cell, in spite of the fact that there is a common intermediate step, catalyzed by phosphoglucomutase. We are at present completely in the dark as to how such cellular operations are accomplished. The examples could be multiplied, since many other intermediates are known which can enter different metabolic pathways.

To judge from work carried out with isolated mitochondria the complexity is very great even on the subcellular level. Factors of permeability are encountered owing to the presence of a membrane and of internal subdivisions as revealed by electron 
microscopy. Several enzymes have been shown to be latent in mitochondria; this implies either that the enzymes were present in an inactive form or, if active, that they were not in contact with their substrates. Furthermore, the metabolic activity of the mitochondria must be integrated with that of the cell as a whole. The relationship is such that metabolites, e.g., pyruvic acid, prepared by other enzyme systems must be supplied to the mitochondria as oxidizable substrates, while the cell must in turn be supplied with ATP which is being regenerated in the mitochondria. The functioning of the cell depends to a large extent on this interrelationship.

The complexity of the system consists not only in the large number of diverse chemical operations which are carried on simultaneously in the same cell but also in the control of the speed of these operations. One might speculate that the necessary catalysts for a particular operation are associated into a unit within the cell. For example, hexokinase, phosphoglucomutase, and phosphorylase in loose combination, perhaps in a separate compartment in the cell, would be a unit for glycogen synthesis, while other molecules of phosphorylase and phosphoglucomutase in combination with glucose-6-phosphatase would form a unit for glycogen degradation in the liver. Glucose-6phosphatase, according to DeDuve et al. (7), is bound to the microsomes. Although phosphorylase and phosphoglucomutase appear to be present in a soluble form in a liver homogenate, this does not exclude loose combinations which would be broken up by present methods used for the disintegration of cells. Crane and Sols (4) have shown that over 90 per cent of the hexokinase in a brain homogenate is bound to particles which sediment at relatively low centrifugal speeds and which resemble mitochondria, whereas homogenates of other tissues contain a considerable part of hexokinase in soluble form. No information is available at the present time as to whether these findings have any physiological meaning. It would seem that new methods will be needed to explore this territory. 


\section{Complexities of Organization}

Even if it is granted that the different chemical operations of the cell are carried out as if they were spatially separated, one has dealt with only a small part of the complexity of the system. The inherent capacity of the cell to duplicate itself and to exert a seemingly purposeful control over its function has made it difficult to assert that the ordinary laws of physics and chemistry apply - a difficulty which the microcosm shares with the macrocosm. One may take as an example the following dilemma. The accretion of material of exactly the same molecular structure prior to division of the chromosomes and the control exerted by this structure on the formation of specific enzymes starts an unending causal chain, where one enzyme is necessary for the formation of another enzyme and so on. In order to break this chain, it is necessary to introduce a nonenzymatic step, either in the process of self-duplication of genetic material or in the formation of the first enzyme of a chain. When faced with such complexities, it is not surprising that some biologists prefer to invoke the operation of special vital forces which they believe are peculiar to living matter. This point of view also finds its expression in statements to the effect that enzymes studied in vitro after extraction from the cells are not the same entities which act in the intact cell. Granted that this may be true in certain cases, one would rather investigate the reason for such deviations than be discouraged about the validity of the methods that enzyme chemists have used.

The higher forms of organization in multicellular organisms are characterized by the increasing complexity of regulatory mechanisms on the evolutionary scale. One finds specialization of chemical operations according to cell types assembled in organs, increasing control through the central nervous system, improved transport of materials to and from the cells through the development of the circulatory system, and finally the elaboration of highly active and specific substances, the hormones, which exert a control over enzymatic processes. A remarkably constant internal environment has been achieved through the 
development of regulatory systems and the organism has gained in performance, but at the same time the different parts of the organism have become highly interdependent.

\section{Hormone Effects}

As a general outline, the following factors are known which enter into a regulatory system such as that of the blood sugar level: (1) specialized tissues which respond to changes in the blood sugar concentrations, e.g., the sympathetic centers in the hypothalamic region and the islet tissue of the pancreas; (2) effectors, which are partly nervous, partly humoral; and (3) targets, which are the enzymatic reactions specifically influenced by the effectors. It is on the last point that a few additional comments may be made. Although phosphorylase has been implicated in the metabolic action of epinephrine, hexokinase in that of insulin, and oxidative phosphorylation in that of thyroxin, none of these systems is as yet sufficiently well understood to make one confident that the results obtained so far explain the actions of these hormones in the intact animal.

\section{Epinephrine and Glucagon}

The characteristic metabolic action of epinephrine in the intact animal consists in a rapid breakdown of glycogen in liver and muscle, even when at the time of injection the reaction is proceeding in the opposite direction. Thus, epinephrine not only causes increased phosphorylase activity but also has an effect on the direction in which phosphorylase activity is proceeding.

Phosphorylase occurs in liver and muscle in an active and inactive form, and there are enzymes present which can convert one form into the other with great rapidity. The active form in muscle, phosphorylase $a$, is a dimer of the inactive form, phosphorylase $b$ (13). Although phosphorylase $b$ can be activated 
by adenylic acid, the concentration needed $\left(1 \times 10^{-3} \mathrm{M}\right)$ is higher than that found in muscle. Epinephrine, when added to liver slices or to diaphragm muscle incubated aerobically in phosphate-Ringer's solution, causes a rapid increase in the concentration of active phosphorylase and at the same time the glucose output of the liver slices and the lactic acid production by the diaphragm are increased (19). No effect of epinephrine has so far been described in a cell-free system. In order to measure the relative amounts of the two forms of phosphorylase in resting muscle, special precautions are necessary. The technique recently used involved freezing the gastrocnemius muscle in situ in amytalized rats. The muscle was rapidly homogenized in the cold in a Waring Blendor in a solution containing 0.001 $M$ versene and $0.02 M$ fluoride, which inhibits the interconversion enzymes but has no effect on phosphorylase activity. Under these conditions rat muscle was found to contain 30 to 50 per cent phosphorylase $a$, the remainder being phosphorylase $b .^{*}$ Following the subcutaneous or intravenous injection of epinephrine the phosphorylase $a$ content of muscle rose to nearly 100 per cent within a few minutes, whereas the total phosphorylase content remained unchanged. In isolated rat diaphragm Sutherland (19) observed an increase in phosphorylase $a$ content within 4 minutes after the addition of epinephrine. Unexplained is the mechanism of the reversal of phosphorylase action. This occurs in an isolated rat diaphragm which is actively synthesizing glycogen from the glucose in the medium. As soon as epinephrine is added, glycogen breakdown exceeds glycogen synthesis so that there is either no gain or an actual loss of glycogen (20). The hexosemonophosphate

* Similar values were obtained when muscle not frozen in situ was homogenized within a few seconds after excision from the animal. When fluoride was omitted from the solution used for homogenization, almost all of the phosphorylase was present in the inactive or $b$ form. An amount of homogenate corresponding to 4 to $5 \mathrm{mg}$. of muscle was used in the phosphorylase tests. The activity in a test without adenylic acid, expressed as per cent of the activity with adenylic acid, corresponds to the per cent of phosphorylase $a$. 
content of muscle is increased by epinephrine, which should favor glycogen synthesis rather than breakdown. No indication has been found for an increase in inorganic phosphate which would favor breakdown. One could refer here again to the compartments of the cell, but this is not a satisfactory explanation since it is not amenable, at the present time, to experimental investigation.

Glucagon, a protein recently isolated from the pancreas in crystalline form, increases the phosphorylase activity of the liver in the same way as epinephrine (19). In contrast to epinephrine, glucagon does not cause an increase in blood lactic acid in the intact animal, and it also has no action on the phosphorylase $a$ content of the isolated diaphragm. It is possible that glucagon cannot penetrate into the muscle cell. What the common denominator, if any, might be between epinephrine and glucagon in their action on the liver is unknown; possibly glucagon causes the liberation of a sympathomimetic amine in the liver. In conclusion it might be pointed out that epinephrine and glucagon probably act on the enzymes which maintain a balance between active and inactive phosphorylase rather than on phosphorylase itself, but this awaits verification in a cell-free enzyme system.

Muscular activity also influences the relative amounts of active and inactive phosphorylase in muscle. Continuously active muscles, such as heart and diaphragm, contain a higher percentage of phosphorylase $a$ than resting skeletal muscle. Stimulation of isolated frog gastrocnemius at a rate of 60 single shocks per minute for 1 to 2 minutes, i.e., conditions which permit work at a steady state without development of fatigue, resulted in a marked increase in the percentage of phosphorylase a. On the other hand, repeated tetanic stimulation during 1 to 2 minutes until the muscle showed fatigue, resulted in an almost complete disappearance of phosphorylase $a$. These experiments, which have not as yet been reported, illustrate again the dynamic balance which exists in muscle between inactive and active phosphorylase. 


\section{Insulin}

Less definite statements can be made about the mechanism of insulin action than about that of epinephrine. There is indirect evidence, obtained by a variety of methods on isolated tissues and on intact animals, that the first step in glucose utilization is accelerated by insulin. The same step presumably is inhibited by a substance of pituitary origin which is present in the serum of diabetic animals, which disappears after hypophysectomy and reappears again when the diabetic hypophysectomized rats are injected with growth hormone plus cortisone (3). The diabetic serum was tested on isolated rat diaphragm and the inhibition of glucose uptake could be counteracted by the addition of insulin. A lipoprotein fraction prepared from diabetic plasma and from anterior pituitary produced a strong inhibition of the hexokinase reaction in a cellfree system, but the reversal of this inhibition by insulin was incomplete and did not have the desired degree of reproducibility (15). In isolated rat diaphragm the inhibition of glucose uptake produced by a lipoprotein fraction from serum of diabetic rats was completely reversed by insulin. It should be pointed out that the effect of injection of these lipoprotein fractions in intact animals has not so far been reported.

The severe disturbance in fat metabolism in the liver of the diabetic animal is now regarded as secondary to the inhibited glucose utilization (10). In the reversible series of reactions represented by acetyl CoA $\rightleftarrows$ fatty acid, there are two reductive steps in the direction to the right, for each $\mathrm{C}_{2}$ fragment which is added in the lengthening of the fatty acid chain (17). These reductive steps require DPNH which can be supplied by glycolysis. Whether the DPNH formed during the operations of the Krebs cycle is available for fatty acid synthesis is uncertain. In the absence of sufficient glucose utilization in a liver depleted of its glycogen reserves, the steady-state concentration of DPNH diminishes, and this shifts the equilibrium to the left, i.e., fatty acids are now broken down and ketosis results (12). It should be mentioned that the liver in ketosis responds very sluggishly 
to insulin, since it takes some time until secondary changes are repaired. That the normal liver responds rapidly to insulin, as do other tissues, follows from observations made on normal and diabetic subjects with the hepatic vein catheterization technique (2). On injection of insulin the hepatic output of glucose diminishes within a short time. Another secondary disturbance is an increased glucose-6-phosphatase activity in the liver, which results in increased dephosphorylations (1). This nullifies to some extent the beneficial effect of fructose in diabetes. This sugar is phosphorylated to fructose-1-phosphate by a separate enzyme and so is able to bypass the metabolic block at the glucose level, but in its metabolic transformations fructose-1-phosphate is in equilibrium with glucose-6-phosphate, and thus is converted to glucose by the increased activity of the phosphatases.

There are some cells which are not influenced by insulin. The ascites tumor cells which were discussed earlier have been tested under a great variety of conditions, but a clear-cut effect of insulin on the rate of sugar uptake could not be shown. The conditions which make a cell respond to insulin are therefore not clearly defined. In general, an enzymatic step, such as that mediated by phosphorylase or hexokinase, which is at the beginning of a series of consecutive reactions, cannot, by being speeded up, increase the rate of the over-all reaction unless it is the rate-limiting step. It has been shown by several methods that phosphorylase is the rate-limiting step for glucose formation in the liver (19). The first step may be rate-limiting under the following conditions which, if changed, will permit an increase in the over-all rate. (1) Part of the enzyme is present in an inactive form. This is the condition under which epinephrine is able to exert its effect, by converting an inactive form of phosphorylase to an active form. (2) The substrate concentration is below that which saturates the enzyme. (3) Product inhibition occurs because a subsequent enzymatic step in a reversible chain of reactions is relatively slow. (4) The enzyme is not fully active because it is combined with an inhibitor. Conditions (2) and (4) have each been suggested as the condition under which 
insulin is able to exert its effect, i.e., by increasing permeability of the cell membrane for glucose or by displacing an inhibitor. Animal hexokinase, in contrast to that of yeast, is noncompetitively inhibited by glucose-6-phosphate, and competitively inhibited (with respect to ATP) by ADP (4). If (3) were the necessary condition, insulin would accelerate an enzymatic step other than the first, or in some other way counteract the product inhibition.

\section{Thyroxin}

The effect of thyroxin and triiodothyronine in uncoupling aerobic phosphorylation in isolated mitochondria is obtained not only with dinitrophenol but also with a number of other unrelated substances, none of which can replace thyroxin in thyroid deficiency. It may be difficult to show that this is the primary effect in the intact animal. The greatly increased oxygen consumption in the hyperthyroid animal may well more than compensate for the decreased efficiency of aerobic phosphorylation and could be the primary effect.

This discussion about the action of epinephrine, insulin, and thyroxin makes it clear how difficult a problem it is to unravel the mechanism of their actions, not to speak of a number of other hormones the study of which has just begun. The task is made somewhat easier if one adopts the point of view that hormones are specific in their action, that they influence only one kind of reaction, and that the apparent multiplicity of their effects is secondary to a primary effect. The metabolic hormones which have been most extensively studied from a biochemical point of view have more or less conformed to this pattern.

\section{References}

1. Ashomore, I., A. B. Hastings, and F. B. Nesbitt, Proc. Natl. Acad. Sci. U. S., 40, 673 (1954).

2. Bearn, A. B., B. H. Billing, and S. Sherlock, Ciba Foundation Colloquia on Endocrinol., II, 250 (1953). 
3. Bornstein, J., and C. R. Park, J. Biol. Chem., 205, 503 (1953); Bornstein, J., J. Biol. Chem., 205, 513 (1953).

4. Crane, R. K., and A. Sols, J. Biol. Chem., 203, 273 (1953); ibid., 206, 925 (1954).

5. Cori, G. T., Harvey Lectures, Ser. 48, 145 (1952-53).

6. Cori, G. T., J. O. Closs, and C. F. Cori, J. Biol. Chem., 103, 13 (1933).

7. DeDuve, C., J. Berthet, H. G. Hers, and L. Dupret, Bull. soc. chim. biol., 37, 1242 (1949).

8. Fischer, R. E., and G. T. Cori, Am. J. Physiol., 172, 5 (1935).

9. Fleckenstein, A., J. Janke, R. E. Davies, and H. A. Krebs, Nature, 774, 1081 (1954).

10. Gurin, S., in V. A. Najjar, ed., Fat Metabolism. Johns Hopkins Press, Baltimore, 1954.

11. Hegnauer, A. H., and G. T. Cori, J. Biol. Chem., 705, 691 (1934).

12. Helmreich, E., H. Holzer, W. Lamprecht, and S. Goldschmidt, $Z$. physiol. Chem., 297, 113 (1954).

13. Keller, P. J., and G. T. Cori, Biochim. et Biophys. Acta, 72, 235 (1953).

14. Keston, A. S., Science, 720,355 (1954).

15. Krahl, M. E., and J. Bornstein, Nature, 173, 949 (1954).

16. Levine, R., and M. S. Goldstein, Brookhaven Symposia in Biology, 5, 73 (1952).

17. Lynen, F., Federation Proc., 12, 683 (1953); Mahler, H. R., Federation Proc., 12, 694 (1953).

18. Sols, A., Third International Congress of Biochemistry, Summaries of Communications, p. 53, Brussels, 1955; Biochim. et Biophys. Acta, 19, 144 (1956).

19. Sutherland, E. W., and C. F. Cori, J. Biol. Chem., 188, 531 (1951); Sutherland, E. W., Phosphorus Metabolism, 7, 53 (1951).

20. Walaas, O., and E. Walaas, J. Biol. Chem., 787, 769 (1950). 


\section{ENZYMES AS REAGENTS}

EFRAIM RACKER, Division of Nutrition and Physiology, The Public Health Research Institute of the City of New York, Inc., New York
$O$ end to which our currents tend Inevitable sea
To which we flow, what do we know What shalt we guess of thee?

Arthur Hugh Clough

During the past ten years our concept of enzymes has undergone a noteworthy change. Enzymes are no longer the mysterious catalysts of a decade ago. They have become the mysterious reactants of today. Fifty years ago, kinetic experiments were performed which suggested the formation of enzymesubstrate intermediates during catalysis, but only in recent years has a direct demonstration of their existence been achieved. Now, an extensive search for these enzyme-substrate compounds is under way in various laboratories. The enzymologist of yesterday who isolated a crystalline enzyme and recorded a few of its properties considered his work a task well done. The enzymologist of today lacks this satisfaction. He realizes that with the isolation of the enzyme his task has just begun, and, depending on his own background and direction of interest, he will approach the purified enzyme as a protein of unknown chemical structure, or as a catalyst with specific kinetic properties, or as a reactant which combines with the substrate. As a protein chemist, he will determine the amino acid composition; he will attempt a study of the amino acid sequence; he will modify the protein and search for a relationship between its 
structure and biological activity. As "kineticist," he will study the process of substrate activation; he will explore the conditions governing the efficiency of catalytic performance; and he will derive equations compatible with his data which might give him clues to the mechanism of enzyme action. As a progressive enzyme "reactionist," he will use the enzyme as a stoichiometric reactant; he will explore the active center of the enzyme and the points of enzyme-substrate interaction.

Some of these investigations require rather large quantities of highly purified enzymes, and it is not surprising that those enzymes which are readily available in large amounts have been studied most extensively. For example, crystalline glyceraldehyde-3-phosphate dehydrogenase (TDH) can be prepared in gram quantities either from rabbit muscle or from yeast in the course of a few days. During the past years it has become the subject of study in numerous laboratories all over the world. Since these studies have been conducted with a relatively pure protein, and because of the multifunctional activities of $\mathrm{TDH}$, this enzyme will be frequently cited as an example in the following pages.

\section{Enzymes as Proteins}

The biosynthesis of proteins, their molecular structure, and the relationship of structure to biological activity are among the unsolved and most challenging problems in biochemistry. Enzymes are particularly suited for such studies because their biological activity can be rapidly and accurately determined by very sensitive methods. The specificity of these methods permits the detection of a particular enzyme in a mass of other proteins. It was possible in this way to detect the formation of new enzyme proteins in cell-free systems, although little increment in total protein nitrogen had occurred (10).

Studies on the composition and sequence of amino acids of various enzymes having the same action but obtained from different sources may represent the beginning of a new kind of comparative biochemistry. A remarkable similarity in amino 
acid composition, molecular weight, DPN binding, turnover number, and other catalytic properties is exhibited by preparations of glyceraldehyde-3-phosphate dehydrogenase from yeast and from rabbit muscle (50). An unidentified crystalline protein from papaya latex was "diagnosed" as lysozyme because of a resemblance in amino acid composition to the egg white enzyme (42). It is difficult to conceive that these similarities are coincidental. Although some differences in the biological and chemical properties of these related proteins exist, these seem minor compared to the great similarities, particularly in view of the fact that even enzyme preparations from a single source reveal microheterogeneity on rigid examination (6). For example, TDH from yeast was fractionated into proteins of different electrophoretic behavior with apparently identical biological activity (22). Such minor variations actually serve to make us more fully aware of the remarkable precision of the cellular machinery responsible for the production of these complex polymers. It seems quite logical therefore to assume that the specific assembly of amino acids takes place with the aid of a structural guide.

There are, on the other hand, enzymes from different sources, for example, alcohol dehydrogenase from yeast and liver, which have vastly different properties although they catalyze the same reaction. It would be interesting to learn whether a discrepancy in catalytic efficiency (alcohol dehydrogenase from yeast is over 50 times as active as the liver enzyme) is mirrored in pronounced differences of amino acid composition or sequence.

The relation between protein structure and biological activity of enzymes has been studied mainly by (1) chemical alterations of the protein; (2) partial proteolytic degradation of enzymes; (3) construction of enzyme models; and (4) investigations of the active centers of the enzymes.

MODIFICATION OF PROTEINS BY CHEMICAL ALTERATIONS

Essentiality or nonessentiality of certain groups for enzymatic activity has been deduced from chemical alterations, such 
as acetylations, oxidations, reductions. The workers in this field have been fully aware of the diffculties of this approach and have emphasized the importance of demonstrating the purity of the new protein derivative and, if possible, the reversibility of the induced modification. Extensive acetylation of pepsin (13) resulted in loss of enzyme activity which was fully restored after removal of the acetyl groups. Among other types of modifications, two of more recent data may be quoted. Diisopropyl fluorophosphate (DFP) inhibits certain esterases as well as proteolytic enzymes with esterase activity, whereas other enzymes appear unaffected ( $c f$. ref. 1b). Cholinesterase and chymotrypsin are completely inactivated after reacting with one mole of inhibitor per mole of enzyme. From the inactive proteins, $O$-phosphoryl serine was isolated after hydrolysis (40). Serine is therefore strongly implicated as part of the active site, provided steric hindrance, which will be discussed below, can be ruled out. Since an acyl shift from nitrogen to the hydroxyl group of serine may have occurred secondarily during the preparative procedure, the hydroxyl group may not be the primary reactor ( $c f$. ref. 6a). It is significant that no phosphothreonine was found in the hydrolyzates. What determines the specificity of the interaction between DFP and enzyme-serine is unknown. Free serine or enzyme-threonine does not react with DFP. Perhaps sequence analysis of peptides containing the radioactive phosphate of $\mathrm{DFP}^{32}$, obtained after partial hydrolysis, may yield information on this point. The biological reactivity of hydroxy amino acids has been recently demonstrated by an entirely different approach. A rapid incorporation of labeled inorganic phosphate $\left(\mathrm{P}^{32}\right)$ was shown to take place into the phosphoprotein fractions from which phosphorylated hydroxy amino acids were isolated $(1,18)$.

The susceptibility of TDH to iodoacetate (IAA) has been known for many years. Since it has been shown (23) that TDH contains firmly bound glutathione (GSH), which can be liberated by proteolytic enzymes, the interaction with IAA was reinvestigated (24). A very rapid reaction was found to take place 
between IAA and enzyme-bound GSH, which was dependent on the presence of DPN. By comparison, the interaction between IAA and free GSH was quite slow. With three equivalents of IAA, enzymatic activity of TDH was completely blocked within a few minutes, and after proteolytic digestion of the modified protein, no unreacted glutathione was liberated. Furthermore, addition of acetyl phosphate to the enzyme led to the formation of acyl enzyme, from which a thiol ester was liberated by proteolytic digestion. Formation of acyl enzyme did not take place after treatment with IAA, but if acetyl phosphate was added prior to the inhibitor, the alkylation of the enzyme by IAA was delayed. These findings with IAA and similar experiments with $N$-ethyl maleimide quite conclusively identified GSH as part of the active center of TDH, and ruled out the possibility that IAA inhibits enzymatic activity by steric hindrance of enzyme-substrate interaction.

In the case of inhibitors of enzymes which act on large molecular substrates, steric hindrance may, however, play an important role. Some of the immunological antienzymes probably act in this manner. The inhibition of trypsin by ovomucoid inhibitor is dependent on groups which are nonessential for proteolysis. This was shown by the fact that acetylated trypsin, which is enzymatically active, is not readily inhibited by ovomucoid inhibitor (9). It seems reasonable to assume that steric hindrance plays a major role in the inhibition of trypsin activity on proteins by ovomucoid, particularly since both inhibitor and substrate are rather large molecular substances $(2 \mathrm{a})$.

It is possible, on theoretical grounds, to differentiate three types of chemical modifications which may result in altered biological activity: (1) interaction with the active center; (2) interaction with sites in the vicinity* of the active center leading to steric hindrance (if the inhibitor is a large molecule, the site

* In a coiled protein structure of the enzyme, a site in the vicinity of the active center may be far removed in terms of the amino acid sequence of the extended protein. 
of interaction may actually be far removed); and (3) partial inhibition due to interaction with sites removed from the active center, "activating groups" in the sense of Langenbeck (27). The fact that only a limited number of specific blocking agents is available which can be used without leading to protein denaturation has seriously hindered progress in this area of investigation.

\section{MODIFICATIONS OF PROTEINS BY PARTIAL DIGESTION}

The effect of partial proteolytic digestion on the activity of enzymes has not been extensively explored. It has been apparent for many years that, in some instances, biological activity of proteins may be retained after fragmentation, as was first demonstrated in the case of proteolytic digestion of diphtheria antitoxin (cf. 35). More recently (17), phosphorylase was cleaved into two inactive fragments and the lost activity was restored by addition of adenosine-5-phosphate. After exposure to proteolytic enzymes, TDH lost its activity, which was restored by adding $\mathrm{SH}$ compounds (23). ATPase activity of myosin was retained after proteolytic digestion (11). Ribonuclease, incubated with carboxypeptidase, contained over $70 \%$ of its original activity, although $20 \%$ of its nitrogen was split off. Further exposure to carboxypeptidase had no effect (29,cf. also $1 \mathrm{a}$ and 15a). The activation of proenzymes such as chymotrypsinogen is another example of proteolytic modification which has yielded information about the active center. The splitting of a single peptide bond between arginine and isoleucine appears to suffice for the activation of chymotrypsinogen (6b). Of special interest is the demonstration of enzymatic activity in dialyzable fragments of autodigested pepsin (34). These small "enzymes" were found to contain only $3 \%$ of the specific proteolytic activity of native pepsin, but to retain $64 \%$ of its catalytic activity toward the synthetic substrate acetyl-L-phenylalanyldiiodo-L-tyrosine. Elucidation of the structure and amino acid sequence of the active fragments may yield significant information about the active 
center as well as about the role of the activating groups in the native protein. This knowledge may also lead to the synthesis of the enzyme models not too far removed from biological reality.

\section{ENZYME MODELS}

From the earliest organic enzyme model recognized by Liebig (30), to the recent models of spccific esterase activity exhibited by SH compounds (33) and the polyfunctional catalysts of mutarotation (47), a very large number of enzyme models have been studied ( $c f .27$ ). Some of them, e.g., the carboxylase models of Langenbeck, show remarkable similarity to enzymes in regard to certain kinetic properties, product inhibition, and inactivation of the catalyst. The polyfunctional catalysts of Swain and Brown exhibit a high substrate specificity and form substrate complexes resembling those formed by enzymes.

Study of model reactions has often clarified our thinking about the active center of enzymes and their mechanism of action, but a word of caution should be added concerning the indiscriminate use of model reactions. For example, the chemical oxidation of aldehydes in the presence of phosphates has been used as a model of enzymatic dehydrogenation of aldehydes (52), in spite of the fact that the course and products of the chemical and enzymatic oxidation are quite different.

\section{Enzymes as Catalysts}

The basic concepts of Michaelis concerning enzyme-substrate interactions have stood the test of time, although the simple original formulation has undergone some essential alterations. Among the more important factors which required consideration were: the presence of more than one active center, multiple points of substrate attachment, the occurrence of more than one enzyme-substrate intermediate, the participation of multiple substrates, including cofactors and water, and the effect of specific buffers and of $p \mathrm{H}$ on the active center as well as on activating groups. 
The kinetic complexities of metabolic processes, such as glycolysis and the citric acid cycle, which are catalyzed by multienzyme systems, have frequently been pointed out. In order to analyze the individual enzymatic steps, it was necessary to separate the catalysts from each other. Now it slowly becomes apparent that the surface of the single enzyme may mirror some of the complexities encountered in multienzyme systems. The fact that a reaction catalyzed by a single enzyme includes the formation of several enzyme-substrate intermediates, required the introduction of the "Michaelis compound" as the ratelimiting step $(4,5)$. It may be confusing at first to learn that pure crystalline glyceraldehyde-3-phosphate dehydrogenase can catalyze five apparently different reactions (cf. 37). One can explain oxidation-reduction, acyl transfer, phosphorolysis, and perhaps even the phosphatase activity exhibited by this enzyme in terms of the over-all process of aldehyde oxidation coupled to phosphorylation. However, there is at present no plausible explanation for the destruction of DPNH in the presence of the enzyme (36). It is very difficult to obtain accurate kinetic data for the individual steps catalyzed by $\mathrm{TDH}$, since some of the "side reactions" interfere with the measurements. Moreover, small modifications in the protein molecule, such as the blocking of the $\mathrm{SH}$ groups, may change the ratios of the different catalytic activities. Since oxidation of $\mathrm{SH}$ groups occurs during the purification of $\mathrm{TDH}$, a microheterogeneity is introduced which is more treacherous for kinetic studies than gross impurities with unrelated proteins. This difficulty cannot be overcome by the addition of cysteine or GSH, which not only reduce the enzyme incompletely but also interact with the aldehyde substrate. However, fully reduced enzyme, suitable for kinetic studies, can be isolated from rabbit muscle with the aid of ethylenediamine tetraacetate (23).

A change in $p \mathrm{H}$ may determine which of a number of possible products is formed by an enzyme. In the case of papain and the cathepsins (15) a decrease in the hydrogen ion concentration alters the enzyme-catalyzed reaction in such a 
manner that the transfer of an acyl group to an acceptor other than water predominates. The kinetics of an enzyme-catalyzed reaction may also be altered by $p \mathrm{H}$ because of a shift to a new rate-limiting Michaelis compound (43).

SUBSTRATE-ENZYME INTERAGTIONS

In the enzyme-catalyzed reaction:

$$
\begin{gathered}
\mathrm{E}+\mathrm{S} \underset{k_{2}}{\stackrel{k_{1}}{\rightleftharpoons}} \mathrm{ES} \stackrel{k_{3}}{\longrightarrow} \mathrm{E}+\text { product } \\
K_{m}=\frac{k_{2}+k_{3}}{k_{1}}
\end{gathered}
$$

the dissociation constant $K_{D}$ for the enzyme-substrate complex (ES) is equal to the Michaelis constant $K_{m}$ only when $k_{3}$ is very small compared to $k_{2}$; then $K_{m}=k_{2} / k_{1}=K_{D}$. Experimental data are accumulating which demonstrate that $K_{D}$ cannot be equated to $K_{M}$. It was shown for some peroxidases $(c f .4,5)$ and for succinic dehydrogenase (41) that $k_{3}$ is considerably larger than $k_{2}$, so that $K_{D}$ may differ from $K_{M}$ by several orders of magnitude. Similar discrepancies have been recorded for enzyme-coenzyme reactions (49). It appears necessary therefore to evaluate the different rate constants independently. Ingenious methods for the direct $(4,5)$ and indirect (41) determination of the velocity constants have been devised.

In a theoretical paper by Foster and Niemann (8), it was pointed out that in multifunctional catalysis, which involves the formation of several reactive intermediates, the experimental values for $k_{3}$ may not represent the velocity constant for the decomposition of the enzyme-substrate complex.

Various experimental approaches have been made to the problem of multiple points of substrate attachment. The use of isotopes in the case of symmetrical substrates, the effect of modified substrates, of inhibitors, and of high substrate concentrations on rate of enzyme activity are among the best known examples. If the enzyme contains two spatially independent points of interaction with the substrate, as shown in Figure 1, 
the presence of substrate excess may lead to the formation of inactive enzyme. A modified Michaelis-Menten equation was derived for this case by Foster, McRae, and Bonner (7):

$$
v=\frac{V_{e x}(\mathrm{~S})}{K_{s}^{\prime}+(\mathrm{S})+(\mathrm{S})^{2} / C}
$$

in which $K_{s}^{\prime}$ and $V_{e x}$ are analogous to $K_{s}$ and $V_{\max }$ of the Michaelis-Menten equation. The term $(S)^{2} / C$ is an index of the probability of two substrate molecules combining with the
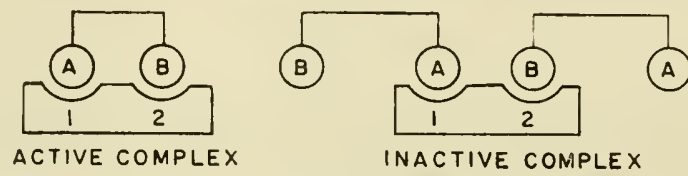

Figure 1.

enzyme, resulting in the formation of inactive enzyme substrate complex. At low concentrations of substrate the term $(\mathrm{S})^{2} / C$ is negligible; with increasing substrate concentrations it becomes appreciable, the velocity $v$ decreases. When the concentration of substrate reaches the value of $C$, the velocity becomes half of maximal; thus one half of the enzyme molecules is in the form of the inactive, oversaturated enzyme complex. Experimental data on the effect of indoleacetic acid concentration on the growth of oat coleoptile sections fit in a remarkable manner the theoretical curve for a two-point attachment. Indeed, studies on the effectiveness of various indoleacetic acid analogues revealed that two specific groupings on the molecule are required for biological activity.

A similar interpretation has been given to the inhibition of acetylcholinesterase at high substrate concentration ( $c f .55)$. An extensive analysis of the mechanism of action of this enzyme has been carried out by Nachmansohn, Wilson, and their collaborators $(31,54)$. They investigated the formation of the enzyme-substrate complex with the aid of ionizable and nonionizable substrates and inhibitors at various $p \mathrm{H}$ values. These kinetic studies revealed some interesting properties of the two sites of the enzyme, which are referred to as the anionic and 
esteratic sites, according to their interactions with the quaternary ammonium and the ester moiety of the substrate. A theory of a two-step mechanism of action was proposed which included the rate-limiting formation of an acyl enzyme as an intermediate. The acyl group can be hydrolyzed or transferred to an acceptor other than water, e.g., hydroxylamine or alcohol. The inactivation of acetylcholinesterase by inhibitory phosphate esters, such as tetra alkyl pyrophosphate, was shown to be an enzymatic process reversible by suitable nucleophilic replacing agents.

\section{SUBSTRATE-SUBSTRATE INTERACTIONS}

Theories of the mode of action of glyceraldehyde-3-phosphate dehydrogenase and glutamic dehydrogenase which assume interaction between substrates prior to enzyme action have been widely accepted, although no experimental evidence for chemical addition products as intermediates is available. In the case of glyceraldehyde-3-phosphate, it was assumed that phosphate adds to the aldehyde prior to its oxidation to an acyl phosphate (cf. 52). A useful kinetic approach to this problem has been made by Bücher and Garbade (3). The experimental data on the effect of phosphate or arsenate on the apparent $K_{m}$ value for the substrate (glyceraldehyde or glyceraldehyde-3-phosphate) seem to rule out the formation of a glyceraldehyde diphosphate as intermediate. Strecker has presented (45) evidence of a similar nature against the chemical formation of the $\alpha$-imino compound as intermediate in the reductive amination of $\alpha$-ketoglutarate to glutamate.

\section{Enzymes as Analytical Tools}

Analysis of biochemical events is dependent on the availability of analytical tools. Isolation procedures and chemical determinations often lack accuracy and specificity. Enzymologists have naturally turned to enzymes as an aid in assays of intermediary metabolites. Kjeldahl, over 70 years ago (19), was probably the first to propose the use of an enzyme as an analytical tool (invertase for sucrose determination). Since then, numerous enzymes have been used for the assay of substrates, coenzymes, and enzymes. The obstacle of an unfavor- 
able equilibrium, which leads to incomplete utilization of the substrate, can be overcome by the removal of products with secondary enzymes. The availability of spectrophotometers has fostered the development of many sensitive and rapid assay methods. If an enzyme-catalyzed reaction results in no changes in light absorption, it is usually possible to link it to a reaction with suitable spectral properties. Occasionally several enzymes have to be added in excess, while the enzyme to be assayed is added in limiting amounts. The number of complications due to side reactions increases, however, with the number of auxiliary enzymes used.

\section{"ENZYMATIC PURITY" OF ENZYMES}

In the course of these studies the enzymologist became aware that a new kind of purity is required for his analytical enzyme tools, since the criteria of chemical purity had little meaning in this case. An enzyme preparation though homogeneous according to the most rigid physicochemical measurements may contain an enzyme impurity which represents only a fraction of $1 \%$ of the total protein. The preparation may be worthless for his analysis if the contaminating protein happens to be (and frequently is) a very active enzyme which catalyzes a side reaction in his assay system. On the other hand, another preparation less pure according to physicochemical determinations may be quite suitable as analytical reagent. Thus, the presence or absence of contaminating enzymes which give rise to side reactions with substrate, product, or coenzyme determines whether or not an enzyme is usable. Therefore, new criteria of "enzymatic purity" must be established for each enzyme reagent.

An example encountered in our laboratory may serve to illustrate the complexities of some of these assays. A preparation of glucose-6-phosphate dehydrogenase from brewers' yeast (20) has been widely used for the quantitative determination of glucose-6-phosphate and of TPN. The enzyme can be used to regenerate TPNH in TPN-linked oxidation-reductions. In combination with hexokinase and glucose it is used for the de- 
termination of ATP. It can be used in an assay for hexokinase or phosphohexose isomerase. In the course of several years during which this enzyme served as a useful reagent in many laboratories, differences in the properties of various preparations probably due to minor variations in the purification procedure were encountered. Thus, some preparations contained traces of a TPNH oxidase or of phosphogluconic dehydrogenase which prevented the achievement of a sharp end point at the time of the complete reaction. Variable amounts of hexokinase, phosphohexose isomerase, adenylate kinase, and glutathione reductase were found in these preparations. Accurate values for ATP could not be obtained in the presence of adenylate kinase, and an analysis of glucose-6-phosphate or ATP in a sample which contained some oxidized glutathione gave erroneous values owing to glutathione reductase. Occasionally it is possible to make a preparation suitable as a reagent by the use of an inhibitor. For example, treatment with $N$-ethyl maleimide has been found to eliminate adenylate kinase activity without inactivating glucose-6-phosphate dehydrogenase (25).

The suitability of an enzyme for quantitative substrate determination is dependent on an appropriate substrate affinity and absence of inhibitory side reactions. Preparations of glucose-6-phosphate dehydrogenase from Torula yeast, although of much higher specific activity than the preparations from brewers' yeast, were found to react much more sluggishly at low glucose-6-phosphate concentrations and were inadequate for assay purposes (44).

\section{ANALYSIS OF MULTIENZYME SYSTEMS}

The determination of an enzyme in crude tissue extracts in which complex chains of metabolically linked reactions take place often poses great difficulties. Measurements of formation of the product or of disappearance of the substrate are complicated by the presence of the other members of the multienzyme systems which act on the same compounds. Although blocking of secondary reactions may be successfully used in some instances, in other instances new complications, e.g., "product inhibition," 
may arise. Perhaps the most reliable of all the methods of enzyme assay in crude systems is one used least frequently, since it requires the availability of auxiliary enzymes of "enzymatic purity." The method consists of adding a large excess of the other enzymes of a multistep assay system to allow a more accurate determination of the rate-limiting enzyme.

Perhaps one should not conclude the discussion of these "practical" aspects of enzymes as reagents without mentioning their usefulness as analytical tools in the determination of chemical structures. This approach, clearly envisaged by Emil Fischer, has served as the key method in the determination of the structure of many coenzymes and is increasingly being used in the elucidation of the structure of proteins and other biological polymers.

\section{Enzymes as Reactants}

In the early years of enzymology, the oxidation of an aldehyde by a DPN-linked dehydrogenase was presented as:

$$
\text { Aldehyde } \underset{\mathrm{DPN}}{\stackrel{\text { Enzyme }}{\longrightarrow}} \text { Acid }
$$

When DPN became available in gram quantities and was widely used as an hydrogen acceptor instead of as a catalyst, the reaction was written:

$$
\text { Aldehyde }+\mathrm{DPN}^{+} \stackrel{\text { Enzyme }}{\longrightarrow} \text { Acid }+\mathrm{DPNH}+\mathrm{H}^{+}
$$

Now that an aldehyde-oxidizing enzyme, glyceraldehyde-3phosphate dehydrogenase, has become available in gram quantities and the participation of its $\mathrm{SH}$ group in the catalysis of aldehyde oxidation has been demonstrated, the first stages of the reaction sequence can be written as:

Aldehyde $+\mathrm{DPN}^{+}+\mathrm{SH}$-enzyme

$$
\text { Acyl-S-enzyme }+\mathrm{DPNH}+\mathrm{H}^{+}
$$

From the physiological point of view, expressions (2) and (3) must be considered as representing Beckman cell artifacts 
far removed from true cellular reality. But the enzymologist has learned a great deal about the mechanism of enzyme action from studies with coenzymes and enzymes as reactants.

\section{METHODS OF ANALYSIS OF ENZYMES AS REACTANTS}

In most instances, enzyme-substrate compounds appear to be unstable. The first direct demonstrations of their existence depended on the use of rapid spectrophotometric methods. The appearance of new absorption bands in the case of peroxidases (4) and the disappearance of the DPN-enzyme absorption band of glyceraldehyde-3-phosphate dehydrogenase (38) on addition of the respective substrates, are examples of such studies. On the other hand, the few enzyme-coenzyme compounds investigated so far appear to be quite stable $(38,49,50)$. Inhibitors such as p-chloromercuribenzoate, iodoacetate, and hydroxylamine have been most useful for the analysis of enzyme-substrate and enzyme-coenzyme interactions $(16,38,49)$. Isotopically labeled substrates have also been valuable tools in these studies. With $\mathrm{C}^{14}$-labeled acetyl phosphate, radioactive acyl glyceraldehyde-3-phosphate dehydrogenase was prepared. Hydrolysis with proteolytic enzymes resulted in the release of a thiol ester with the properties of acetyl glutathione (24). Formation of a phospho-enzyme in the case of phosphoglucomutase was first suggested by studies with $\mathrm{P}^{32}$ (14). The use of isotopically labeled substrates or cofactors in incomplete systems (omitting either a substrate or a cofactor), which frequently results in an enzyme-catalyzed redistribution of the label, helps to elucidate reaction sequences. Emphasis should be placed, however, on the use of pure systems for such studies so that exchange reactions, due to either contaminating coenzymes or contaminating enzymes, can be ruled out. Important conclusions regarding the mode of enzyme action have been deduced from studying the point of cleavage with isotopically labeled substrates ( $c f .21)$.

Finally, valuable information has been obtained from kinetic investigations of the complexes formed between enzymes, coenzymes, and substrates. It has made feasible a bold study of 
these specific interactions in intact cells (5). This investigation represents a new and important approach to the steady-state kinetics of intracellular metabolism.

\section{THE MICROCYCLES OF ENZYME-SUBSTRATE INTERAGTIONS}

Catalysis may be looked upon, in many instances, as a cyclic process in which the catalyst undergoes reversible changes. Evidence for this thesis has been obtained in the case of the few enzymes which have been closely examined from this point of view. The enzymologist, hardened by his experiences with complex metabolic cycles, now begins to turn to the exploration of enzymes as "microcycles."

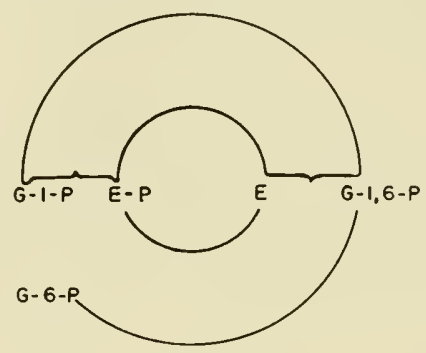

Figure 2.

Phosphoglucomutase catalyzes the reversible transformation of glucose-1-phosphate (G-1-p) to glucose-6-phosphate (G-6-p). The reaction was first written as $\mathrm{G}-1-\mathrm{p} \rightleftarrows \mathrm{G}-6-\mathrm{p}$. When the function of glucose-1,6-diphosphate (G-1,6-p) as a coenzyme was

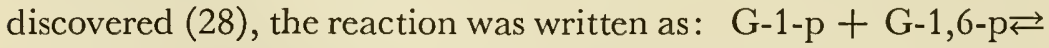
G-1,6-p + G-6-p. With the elucidation of the role of the enzyme protein as phosphate acceptor (32), the reaction sequence can be represented by a cyclic process which includes all reactants, as shown in Figure 2. The enzyme occurs in muscle extracts as phosphoenzyme (E-p). In the presence of the substrate (either G-1-p or G-6-p), the phosphate group is transferred and glucose-1,6-diphosphate is formed. The latter then returns the phosphate to the enzyme and forms the product (G-6-p or G-1-p). 
Another example of a microcyclic sequence of interactions is catalyzed by glyceraldehyde-3-phosphate dehydrogenase and its pyridine nucleotide coenzyme (Figure 3).

DPN combines with the SH-enzyme to form a DPN-enzyme complex (DPN-E) which can be measured by its absorption band at $360 \mathrm{~m} \mu$. The aldehyde interacts with the DPN-enzyme to yield acyl enzyme and reduced DPN. In the presence of phosphate, the acyl enzyme is phosphorolyzed to acyl phosphate and SH-enzyme, which combines again with DPN to regenerate the DPN-enzyme complex (38).

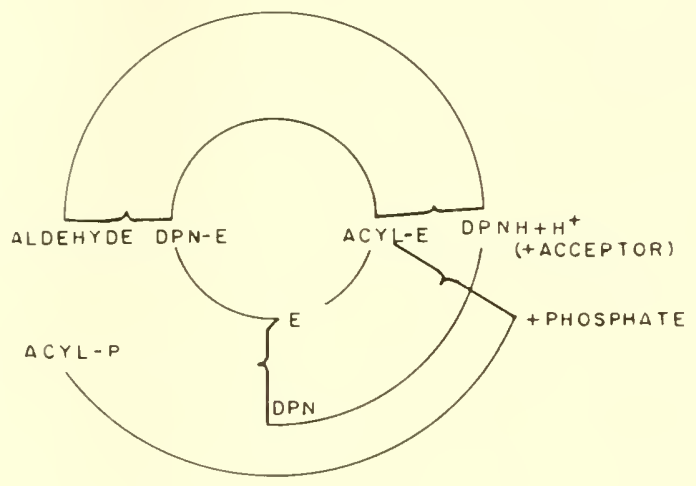

Figure 3.

The enzyme crystallizes from rabbit muscle in the presence of ethylenediamine tetraacetate as the DPN-enzyme complex with 3 moles of DPN per mole. After removal of DPN by treatment with charcoal, the enzyme is somewhat less stable and crystallizes only with difficulty. Exposure of the charcoaltreated enzyme to acetyl phosphate results in the formation of acyl enzyme. Crystallization of the protein from this mixture occurs again quite readily and the crystalline material contains the acetyl enzyme: It gives a positive hydroxylamine test and oxidizes DPNH, thereby forming free acetaldehyde from the acetyl group. With 1,3-diphosphoglycerate, the formation of a phosphoglyceryl enzyme has been demonstrated (26). 
Thus, every one of the intermediates of the microcycles of glyceraldehyde-3-phosphate dehydrogenase and phosphoglucomutase can be isolated. It should be pointed out that in both instances this feat has been made possible by the artificial interruption of the cyclic process of catalysis, either by removal of the coenzymes, as with glyceraldehyde-3-phosphate dehydrogenase, or by omission of a phosphate acceptor, as with phosphoglucomutase. With many enzymes, however, neither of these two approaches is feasible, and, in order to change the course of events, alternative methods, such as the use of inhibitors, must be explored.

\section{INHIBITORS}

An outstanding biochemist once remarked that only uninhibited investigators use inhibitors in complex biological systems. Pitfalls similar to those encountered in inhibitor studies of multienzyme systems also confront the investigator of a microcycle which is catalyzed by a single enzyme. On the other hand, unambiguous studies with inhibitors such as sodium fluoride and iodoacetate have been landmarks in the history of the biochemistry of glycolysis and of muscular contraction. Inhibitors, used cautiously, have also been valuable tools in the study of enzymecoenzyme-substrate interactions.

The protective action of coenzymes or substrates against inhibitors has yielded important information about the mode of their interaction with the enzyme. Early studies of Rapkine on $\mathrm{TDH}$ with several SH inhibitors indicated an interaction between the SH groups of the enzyme and DPN. Alcohol dehydrogenase from yeast can be protected against IAA by DPN or ethyl alcohol ( $c f .37$ ); testosterone dehydrogenase can be protected by DPN against $p$-chloromercuribenzoate (48). It should be pointed out that more complex effects may be encountered. Thus, DPN, which protects TDH against $N$-ethyl maleimide, increases markedly the susceptibility of the enzyme to IAA. Glyceraldehyde-3-phosphate protects TDH against IAA, but so do some other phosphorylated compounds (e.g., 3-phospho- 
glycerate) which are not substrates and do not react directly with SH groups (24).

The inhibitor may block a partial reaction of the cyclic process of enzyme catalysis and pcrmit a functional separation of a multistep system. For example, iodoacetate inhibits the oxidoreduction catalyzed by TDH, but arsenolysis of the acyl enzyme can still take place (38). Potassium cyanide stabilizes acyl enzyme even in the presence of DPN, without interfering with arsenolysis (26). Tetra alkyl pyrophosphate has been shown (54) to inhibit cholinesterase by forming a phospho-enzyme instead of an acyl enzyme. In contrast to the acyl enzyme, the phospho-enzyme is not readily hydrolyzed, and nucleophilic replacement agents must be used to reactivate the active center of the enzyme.

Oxidized (S-S) TDH, which is devoid of dehydrogenase activity, has been shown to hydrolyze acetyl phosphate (12). A stimulation of the phosphatase action of TDH has also been noted after treatment with iodoacetate (26). An inhibitor can, therefore, not only block a cyclic process but channel it into a side reaction. Iodoacetate alters the properties of this catalyst so fundamentally that instead of acting as an acyl transfer agent, it becomes a catalyst of hydrolysis. This exhibition of new catalytic activities in the presence of inhibitors requires most cautious interpretation of observations obtained with enzymes which have been exposed to maltreatment by enzymologists or histochemists. The activation of unspecific phosphatases by iodoacetate or by maleate, reported in the older literature (cf. 53), may represent a similar alteration of functional SH groups resulting in a change in catalytic activity.

\section{EXCHANGE REACTIONS}

The use of isotopically labeled compounds in biochemical research has led to the discovery of a new group of reactions often referred to as exchange reactions. They may be defined as reactions in which an isotopic label is incorporated into a compound, although no synthesis of the latter has occurred. 
There appear to be at least two different kinds of enzyme catalyzed exchange reactions; these will be referred to as (1) transferexchange reactions, and (2) metabolic exchange reactions.

(1) The transfer-exchange reactions represent a special case of a general reaction, usually referred to as a transfer reaction:

$$
\mathrm{AB}+\mathrm{C} \leftrightarrows \mathrm{AC}+\mathrm{B}
$$

These transfer reactions are catalyzed by enzymes which are widely distributed in nature. Transaminases, transglycosidases, transketolase, and transaldolase are a few examples of this type of reaction. These enzymes are assumed to form a complex with the substrate and then catalyze the transfer of a portion of the substrate to another acceptor molecule. For example, the amino group of glutamate is transferred to oxaloacetate by a transaminase. If, in the above equation, free $\mathrm{B}$ can substitute for $\mathrm{C}$ as an acceptor, we are dealing with a transfer-exchange reaction which results in no synthesis but can be detected by incorporation of a labeled compound. The exchange takes place at the same atom of the donor molecule $\mathrm{A}$, in contrast to the metabolic exchange reactions discussed below.

(2) The metabolic exchange reactions occur in the course of a cyclic process and are due to the peculiarity of certain reaction mechanisms that illustrate a special aspect of enzymes as reactants. The cyclic process of enzyme catalysis may proceed in such a manner that an exchange of atoms takes place between the substrate and the catalyst or coenzyme. For example, a phosphate exchange reaction is catalyzed by phosphoglucomutase (see Figure 2). In experiments with $\mathrm{P}^{32}$-labeled glucose-1phosphate, the coenzyme G-1,6-diphosphate becomes labeled (46) because the phosphate is transferred from C-1 of G-1, 6-p to C-6 of the substrate G-1-p, which becomes the coenzyme. An analogous action takes place with oxaloacetate, which acts as a catalyst in oxidation of acetyl CoA in the Krebs cycle. The acetyl group of acetyl CoA condenses with one end of OAA, while the other end is actually oxidized in one turn of the cycle. This is not necessarily an essential feature of the cyclic process but is 
due to the peculiarity of the aconitase enzyme, which dehydrates the oxaloacetate-end of citrate rather than its acetate-end.

\section{PHYSIOLOGICAL SIGNIFICANCE OF EXCHANGE REAGTIONS}

It is apparent from the above that one must differentiate between exchange reactions and incorporation due to the net synthesis of $\mathrm{AB}^{*}$ :

$$
\mathrm{A}+\mathrm{B}^{*} \longrightarrow \mathrm{AB}^{*}
$$

Carbon 14 from carboxyl-labeled acetate will appear in oxaloacetate which on decarboxylation can yield pyruvate or phosphoenol pyruvate. From these three-carbon compounds, glycogen containing the carbons of acetate will be formed. It has been known to physiologists for many years that acetate cannot be used for the biosynthesis of glycogen. We are dealing here with an exchange reaction. It is also apparent that it is impossible to draw conclusions about quantitative aspects of biosynthetic pathways from incorporation studies with isotopes only, unless exchange reactions can be ruled out.

Although no net synthesis of a peptide bond, for example, can be achieved by transfer exchange reactions, these reactions may participate in biosynthetic pathways. It is very probable that transaminases and transpeptidases contribute to the biosynthesis of amino acids and peptides. It is nature's privilege to delegate the specific function of reductive amination to glutamic dehydrogenase. The glutamate formed by this enzyme from ammonia and $\alpha$-ketoglutarzte acts as the key middleman for numerous transfer reactions to other keto acids which lead to new amino acids.

The physiological role of the metabolic exchange reactions is not as apparent. Since in the course of these curious reactions a spatial redistribution of certain groups (e.g., acetyl or phosphate) takes place, it might be conceived that reactions of this type play a role in the transportation of groups and compounds across the cell membrane or from one intracellular structure to another. If work is to be accomplished, the exchange must be catalyzed by two different kinds of reactions as shown in Figure 
4, (a) and (b), e.g., phosphorylation with ATP and dephosphorylation by hydrolysis.

This brings us to the general problem of enzymes participating as reactants in specific physiological functions. Interaction of aldehydes with proteins in the process of vision (51), interaction

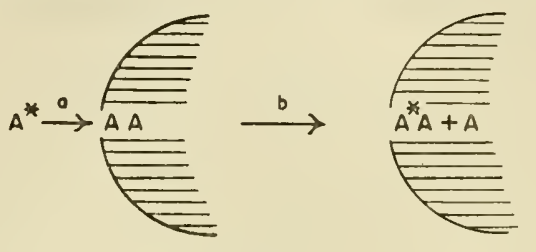

Figure 4.

of nucleotides with the proteins involved in muscular contraction (39), and the role of cholincsterase in the process of transmission of nerve impulses (31) may represent examples of the participation of enzymes as reactants in physiological processes.

\section{Concluding Remarks}

We can look upon enzymes as active centers embedded in a protein matrix. The function of the protein molecule is to provide activating groups which increase the rate of catalysis and permit specificity of interaction between the enzyme and its substrate. Perhaps the most striking illustration for this differentiation between the active center and the activating group of the protein is the example of the "little enzymes" obtained from hydrolyzed pepsin. These dialyzable fragments catalyze efficiently the hydrolysis of a small molecular substrate, but have lost most of their activity with proteins as substrate. Thus, the activating groups of the native enzyme are required for the specific and efficient hydrolysis of the large molecular substrate.

Perhaps another function of the protein is to provide the catalytic center with the anchors and the biochemical stability which is needed for intracellular localization.

Kinetic investigations have received a new impetus during the past ten years. The concept of the polyaffinity of substrates, 
first clearly formulated by Bergmann (2), the kinetic ramifications of the polyaffinity concept of a stepwise interaction between substrate and enzyme, the re-evaluation of the velocity constants, and the existence of complex multiheaded enzymes with multifunctional catalytic activities are among the major developments in this area. They have stimulated new experimental approaches and at the same time contributed to a more timid approach regarding the interpretation of kinetic data. It is self-evident from the above considerations that it is very difficult to interpret unambiguously kinetic data obtained with crude enzyme preparations which may contain inhibitors or interfering enzymes. Moreover, the meaning of painstaking studies, even with highly purified enzymes, may be obscured by the presence of side reactions. For example, several times recrystallized pyruvic kinase contains adenylic kinase, and triose phosphate isomerase may be found in several times recrystallized aldolase. Even pure enzymes with multiple heads, such as glyceraldehyde3-phosphate dehydrogenase, catalyze side reactions which complicate the life of the kineticist.

Enzymes have become accepted as analytical tools. Some enzymatic methods used for determination of metabolic intermediates (e.g., the various hexose phosphates) have a degree of specificity rarely attained by colorimetric and isolation procedures. However, the drawbacks of these enzymatic methods are by no means negligible because of the rigid criteria of purity which have to be applied to the reagents used.

In recent years a few crystalline enzymes have become available in large quantities and have permitted an investigation of their participation as reactants in the reactions they catalyze. It has been possible to show that a cyclic process takes place in which enzyme, coenzyme, and substrate interact to produce a sequence of new intermediates. Glyceraldehyde-3-phosphate dehydrogenase can be isolated as an acyl enzyme and phosphoglucomutase as a phosphorylated enzyme.

These are some of the currents and undercurrents in present enzymology. What are the perspectives? Hundreds of enzymes 
have been described which await further study; many more enzymes will be discovered in the future; nearly endless seems the number of enzymes which might be induced by adaptive processes or might be selected in microorganisms fished from the mud of California. It is clear that the availability of enzymes will not be the limiting factor in the future of enzymology. The purification of enzymes is gradually becoming a routine procedure and biochemists are beginning to attach to it the stigma of a necessary evil. The protein chemists are turning to sequence analysis and to a search of the chemical properties of the active centers and their neighborning groups. The kineticists, after collecting essential data on the interaction between the substrate and various intracellular and surface enzymes, are beginning to return to more complex multienzyme systems and even approach the kinetics of the intact cell. Their studies may bring new clues to the great physiological mystery of the mode of substrate entry into the cell. Enzymes as analytical tools will undoubtedly infiltrate further into the laboratories at the extreme wings of biochemistry: physical chemistry and medical diagnostics. Many more enzymes will be investigated as reactants, and cycle after cycle will appear on the surface of the deep waters of metabolism.

\section{References}

1. Agren, G., C. H. deVerdier, and J. Glomset, Acto Chem. Scand., 8, 1570 (1954).

1a. Anfinsen, C. B., Biochim. et Biophys. Acta, 17, 593 (1955).

1b. Balls, A. K., and E. F. Jansen, Advances in Enzymol., 13, 321 (1952).

2. Bergmann, M., Harvey Lectures, Ser. 37, 37 (1936).

2a. Bier, M., J. Sri Ram, and F. F. Nord, Nature, 176, 789 (1955).

3. Bücher, T., and K. Garbade, Biochim. et Biophys. Acta, 8, 220 (1952).

4. Chance, B., in Modern Trends in Physiol. and Biochem., 1952, 25.

5. Chance, B., in W. D. McEIroy and B. Glass, eds., Mechanism of Enzyme Action, p. 399. Johns Hopkins Press, Baltimore, 1954.

6. Colvin, J. R., D. B. Smith, and W. H. Cook, Chem. Revs., 54, 687 (1954).

6a. Dixon, G. H., S. Go, and H. Neurath, Biochim. et Biophys. Acta, 19, 193 (1956).

6b. Dryer, W. J., and H. Neurath, J. Biol. Chem., 217, 527 (1955). 
7. Foster, R. J., D. H. McRac, and J. Bonner, Proc. Natl. Acad. Sci. U. S., 38, 1014 (1952).

8. Foster, R. J., and C. Niemann, Proc. Natl. Acad. Sci. U. S., 39, 371 (1953).

9. Fraenkel-Conrat, H., R. S. Bean, and H. Lineweaver, J. Biol. Chem., 177, 385 (1949).

10. Gale, E. F., and J. P. Folkes, Nature, 773, 1223 (1954).

11. Gergely, J., Federation Proc., 9, 176 (1950).

12. Harting, J., and S. F. Velick, J. Biol. Chem., 207, 867 (1954).

13. Herriott, R. M., and J. H. Northrop, J. Gen. Physiol., 18, 35 (1934).

14. Jagannathan, V., and J. M. Luck, J. Biol. Chem., 779, 569 (1949).

15. Johnston, R. B., M. J. Mycek, and J. S. Fruton, J. Biol. Chem., 185, 629 (1950).

15a. Kalnitsky, G., and E. E. Anderson, Biochim. et Biophys. Acta, 76, 302 (1955).

16. Kaplan, N. O., and M. M. Ciotti, J. Biol. Chem., 211, 431 (1954).

17. Keller, P. J., and G. T. Cori, Biochim. et Biophys. Acta, 12, 235 (1953).

18. Kennedy, E. P., and S. W. Smith, J. Biol. Chem., 207, 153 (1954).

19. Kjeldahl, J., Compt.rend. trav. lab. Carlsberg, 7, 189 (1881).

20. Kornberg, A., J. Biol. Chem., 782, 805 (1950).

21. Koshland, D. E., in W. D. McElroy and B. Glass, eds., Mechanism of Enzvme Action, p. 608. Johns Hopkins Press, Baltimore, 1954.

22. Krebs, E. G., J. Biol. Chem., 200, 471 (1952).

23. Krimsky, I., and E. Racker, J. Biol. Chem., 198, 721 (1952).

24. Krimsky, I., and E. Racker, Federation Proc., 13, 245 (1954).

25. Krimsky, I., and E. Racker, unpublished experiments.

26. Krimsky, I., and Racker, E., Science, 122, 319 (1955).

27. Langenbeck, W., Ergeb. Enzymforsch., 13, 207 (1954).

28. Leloir, L. F., R. E. Trucco, C. E. Cardini, A. C. Paladini, and R. Caputto, Arch. Biochem., 79, 339 (1948).

29. Levy, M., and O. Blumenfeld, personal communication.

30. Liebig, J. von, Ann., 173, 1 (1860).

31. Nachmansohn, D., and I. B. Wilson, Advances in Enzymol., 12, 259 (1951).

32. Najjar, V. A., and M. E. Pullman, Science, 119, 631 (1954).

33. Perenyi, L., Acta Physiol. et Pharmacol. Neerl., 5, 87, 97, 103 (1954).

34. Perlmann, G. E., Nature, 173, 406 (1954).

35. Petermann, M. L., and A. M. Pappenheimer, Jr., J. Phys. Chem., 45, 1 (1941).

36. Rafter, G. W., S. Chaykin, and E. G. Krebs, J. Biol. Chem., 208, 799 (1954).

37. Racker, E., Physiol. Revs., 35, 11955.

38. Racker, E., and I. Krimsky, J. Biol. Chem., 798, 731 (1952).

39. Szent-Györgyi, A., in Chemistry of Muscular Contraction. Academic Press, New York, 1951. 
40. Schaffer, N. K., S. C. May, Jr., and W. H. Summerson, J. Biol. Chem., 206, 201 (1954).

41. Slater, E. C., and W. D. Bonner, Biochtm. J. (London), 52, 185 (1952).

42. Smith, E. L., J. R. Kimmel, D. M. Brown, and E. O. P. Thompson, $J$ Biol. Chem., 215, 67 (1955); and E. L. Smith, personal communication.

43. Smith, E. L., in W. D. McElroy and B. Glass, eds., Mechanism of Enzyme Action, p. 210. Johns Hopkins Press, Baltimore, 1954.

44. Srere, P. A., unpublished experiments.

45. Strecker, H. J., Arch. Biochem., 46, 128 (1953).

46. Sutherland, E. W., W. Cohn, T. Posternak, and C. F. Cori, J. Biol. Chem., 780, 1285 (1949).

47. Swain, C. G., and J. F. Brown, Jr., J. Am. Chem. Soc., 74, 2538 (1952).

48. Talalay, P., and M. M. Dobson, J. Biol. Chem., 205, 823 (1953).

49. Theorell, H., and B. Chance, Acta Chem. Scand., 5, 1127 (1951).

50. Velick, S. F., in W. D. McElroy and B. Glass, eds., Mechanism of Enzyme Action, p. 491. Johns Hopkins Press, Baltimore, 1954.

51. Wald, G. W., Ann. Rev. Biochem., 22, 497 (1953).

52. Warburg, O., H. Klotsch, and K. Gawehn, Z. Naturforsch., 9b, 391 (1954).

53. Williams, H. L., and E. M. Watson, J. Biol. Chem., 135, 337 (1940).

54. Wilson, I. B., in W. D. McElroy and B. Glass, eds., Mechanism of Enzyme Action, p. 642. Johns Hopkins Press, Baltimore, 1954.

55. Zeller, E. A., Advances in Enzymol., 8, 459 (1948). 


\section{ATTEMPTS AT THE FORMULATION OF SOME BASIC BIOCHEMICAL QUESTIONS}

FRITZ LIPMANN, Biochemical Research Laboratory, Massachusetts General Hospital and the Department of Biological Chemistry, Harvard Medical School, Boston, Massachusetts

The fogginess in which the physical-chemical aspects of the inner working of the living organisms had in the past been shrouded, inevitably invited mystification of one kind or another. In counterreaction, the past generation of biochemists was almost religiously concerned with the task of demystifying life. Through their pioneering, the fog lifted during the twenties and thirties. But now we witness the development of a new phase, in which the hesitant, skeptical, and largely analytical approach is being discarded. The depth to which the understanding of organismic methodology has reached demands reorientation. At this stage it seems most important to ask the right questions. I will not attempt much more than to try to raise a few such relatively naive questions and even these in not too explicit a manner.

The approach toward clarification appears to be most hopeful by way of defining those distinctive attributes of the living state which have been thought to indicate a novel and specifically "living" phenomenon. We will first consider briefly the energy problem, which is relatively the farthest advanced, and then try problems specifically related to duplication. 


\section{The Energy Problem}

The recognition of the relative simplicity of the energy supply system of the living organism has made it possible to understand a large number of biosynthetic mechanisms (15). We now feel justified to state with confidence that the energy problem can be related to a boundary condition of the living system as a whole and does not represent an intrinsic characteristic of the life process except that it is a necessary premise for its existence. The living organism is to be defined as a system with a boundary condition of a constant influx of energy from the outside. Such a system, however, in no way transcends the laws of thermodynamics. In the past, a suspicion often crept in that need might arise for a modification of the second law. Recently a tendency developed to build into a definition of life the concept of a negative entropy (20) which, however, is not a characteristic of life but rather of the environmental conditions which permitted life to develop. The earth is indeed, if isolated and considered as a quasi-autonomous system, appropriately represented by including the incidental radiation energy as a boundary condition, abstracting it, as we mostly tend to do anyway, from the solar system and the universe. Although, in its elaboration of metabolic work, the living organism acts in the capacity of a refinery, the essential boundary condition almost always refers back to the influx of energy from the outside into the earth sphere.

\section{Patternization}

Much emphasis is generally placed on our recently acquired capacity to understand the biochemical mechanisms for building up structures such as steroids, carotenoids, and similar complex molecules, which had previously been elucidated through organic chemical analysis. Although these complex molecules have their important significance for organismic life, the most important problem is the problem of the building up of specific and unique structures by fusing a number of otherwise 
unlike units through a uniform linking mechanism. This creates unique and specific surface and structure patterns which we call proteins, nucleic acids, and mucopolysaccharides. The primary determinants are a fixed sequential arrangement.

So far this type of problem has not occurred in man-made chemistry and it is rather puzzling. Energy is needed first for the joining of the common links in the backbone. Secondly, the lining up of a defined sequential arrangement, the patternization, represents a structural energy equivalent or rather, an increase in entropy (Maxwell's 'demon"), which is not quite easily defined with our present notions. Patternization, I feel, is dominant in duplication, replication, and mutation. It is possibly important to reiterate that the energy requirement for patternization may be divided into (1) a well-defined caloric equivalent for the joining of the links in the respective backbone structures and (2) an "energy of position," the directing into a specific order. The latter is biologically the most important. We meet here a novel situation, the biochemical definition of which has no well-understood examples to fall back upon. It has become the meeting ground between biochemistry and genetics. To define the problem in all its facets, a fusion between biochemical and genetic principles will be needed.

It is most likely not mere coincidence that metabolic chemistry has come to a point where the problem of energy distribution apparently is entering a new stage. The great successes of the past twenty years were obtained by a methodology which aimed at extracting enzymes, coenzymes, and such from the cell. In this manner, rather complex systems could be separated and reunited successfully as, for example, the glycolytic system. The limitation, however, of this approach becomes apparent now. The old methodology of cleaving and separating seems to fall short because the complex and more intrinsic functions of the cell appear to depend on macromolecular arrangements of greater specificity. Therefore, from the analytical point of view, the intrinsic involvement of structure in certain metabolic systems requires new approaches which are slowly developing. It is in 
part the previous success of the methodology of cleavage into the smallest possible units which now acts almost like a mental block.

The common denominator in many of our difficulties seems our inability to understand structure in biochemical terms. This is particularly evident in the approach to protein synthesis. With the analysis of protein synthesis, the energy problem now tangibly merges with the propagation problem. This is obvious if one realizes that many forms of specificity are an expression of a particular sequential arrangement of the amino acids, a sequential arrangement into chains of various shapes, together with an interlocking through interchain links such as, e.g., disulfide links.

A lively discussion of the manner in which these sequences are built up has recently sprung up, motivated in particular through ingenious models for deoxynucleic acids (23). These nucleic acids appear to carry a directing force, often referred to as the code of replication. Metabolically speaking, there is still a link missing from predetermined nucleic acid chain sequences leading to determination of amino acid chain sequences, although dependence of protein synthesis on nucleic acid structures appears to be quite real. In spite of still meager facts, there is a need to start discussion in this biochemical vacuum.

Before embarking on particulars, it seems important to realize that the synthesis of a fixed sequence can be effected methodologically either by a predominant space pattern, a template, or by a timing device analogous to the assembly line. In reality, it most likely will be effected by a mixture of both. It is my impression that presently template processes have been in the foreground of discussions, including my own (16), because of the greater ease in devising template models. The assembly line procedure, however, is really met with mostly in biochemical systems, including a few cases with sequential arrangement on a relatively small scale $(10,21)$.

These problems are now in the foreground because it is here that genetics and biochemistry meet, namely, where the trans- 
mission of the code laid down in the genetic material is translated into chemical mechanisms. A chemical methodology of code transmission, it appears, consists of arranging specific sequences through standard links in the line-up. Possibly a master methodology may exist for a specific sequential arrangement of unlike units through a common linkage system which forms the backbone structure of the resulting compound. However that may be, patternization or sequential arrangement includes, but largely transcends, what we have been mostly concerned with so far, namely, mere mechanisms of linking.

\section{Synthesis of Peptidic Links}

It has at times appeared that the main problem in understanding protein synthesis is to tackle the mechanism of peptide bond formation. However, to understand protein synthesis truly, the understanding of peptide bond formation is a premise rather than an end in itself. It will be wise, nevertheless, first to obtain a background by reviewing our present understanding of the mechanism of peptide bond formation. It seems that uniformly, peptide linking starts with the activation of the carboxyl group rather than of the amino group. The primary source of energy appears to be the energy-rich phosphate bonds of ATP, the energy currency. Unfortunately, the finer mechanism of the conversion of phosphate into active acyl grouping is still only imperfectly understood. There are different subtypes which are of considerable interest. The conversion of phosphoryl to active acyl may occur (7) by a pyrophosphate elimination from ATP, directly or possibly through other nucleoside triphosphates (NTP) or (2) by phosphorolysis of the terminal phosphate of ATP or other NTP's. These two mechanisms at first looked like interchangeable types of procedure. On close inspection, however, they might represent two distinct and rather different mechanisms. The pyrophosphate elimination, as it seems to develop more and more clearly, leads to a linking between the acyl grouping and the nucleotide part of the NTP (2) 
with the elimination of a molecule of pyrophosphate $(1,3,12,18)$, while phosphorolysis apparently leads in some manner to the formation of acyl phosphate with elimination of nucleoside diphosphate (NDP) $(5,7,13,21)$. In other words, the pyrophosphate elimination represents an activation through linking the carboxyl to the nucleoside by way of a monophosphate bridge, forming an NMP acyl. On the other hand, phosphorolysis seems to represent a discarding of the nucleotidic part of the energy carrier and a more or less direct linking of the carboxyl to the phosphate $(5,13)$. Such a pyrophosphate elimination scheme was first observed in a CoA-linked acyl activation. A similar mechanism appears to apply to the synthesis of the peptidic bond in pantothenic acid (18). But more important, an analogous mechanism has now been found to operate in the activation of normal amino acids (11). In both cases, the acyl phosphonucleotide seems not to be freely diffusible but rather tightly linked to the activating protein. This interpretation is mainly based on isotope exchange reactions and should rather be considered as somewhat preliminary. Nevertheless, it may serve as background for a discussion of the mechanism of peptide bond formation in protein synthesis.

\section{Protein Synthesis}

With this still sketchy information on mechanisms for peptide bond synthesis, we now proceed much less securely to possible mechanisms of lining up the different amino acids into a specific protein pattern. Sequential arrangement of the amino acids obviously is the dominant problem before us. If the apparatus is available to line up a predetermined sequence of amino acids most of which will be quite long, twenty, forty, or more links, the final shaping of the molecule might be expected to be wholly or partly a spontaneous one. In any case, this phase of final shaping will be neglected at the present, and our focus will be exclusively on sequential arrangement as a background for specificity, even though this almost certainly is a somewhat precarious simplification. 
There are good indications for the assumption that the carboxy-activated amino acids enter directly into the mechanism of sequential arrangement $(8,22,24)$. Furthermore, the amino acids may enter as an amino acid $\sim \mathrm{P}$-nucleoside. The activated amino acid seems, however, to approach the assembly line not isolated but still fixed to the amino acid specific enzyme protein (11). As a first approximation to a mechanism, we then consider a specific enzyme for each amino acid which as a warhead carries the amino acid linked to the phosphoryl of a mononucleotide, eventually to effect selective linking into chains.

There is, on the other hand, a certain likelihood that the amino acid chain is deposited and grows along on ribonucleic acid. The incorporation of radioactive amino acids takes place in special microsomes rich in nucleic acid $(4,24)$. Recent observations (14) have indicated that the microsome carrying out amino acid synthesis contains approximately equal amounts of nucleic acid and protein. This observation seems to counterindicate a nucleoprotein as responsible for the direction of the amino acids into sequences. The amount of protein would be too small to permit such an interpretation. But the particular activated amino acid approaches the assembly line in association with its specific enzyme. An enzyme-mononucleotide amino acid might react with a specific nucleic acid which by its own sequential arrangement of nucleotides is supposed to direct the sequential patternization of a protein.

One process of progressive chain elongation has been successfully analyzed, namely, fatty acid synthesis (17). It appeared in that case that chain elongation always occurred between two activated molecules. But the energy of only one energy-rich link is utilized, namely, the thioester bond to the carboxyl which enters the $\cdot \mathrm{COCH}_{2} \cdot$ link. To compare this methyl-carboxyl linking to the amino-carboxyl linking in a peptide is not too far fetched, since it has been found (6) that the methylene-carbonyl bond is hydrolyzed by chymotrypsin, which makes the biochemical comparison between $\cdot \mathrm{CO}-\mathrm{CH}_{2} \cdot$ and - $\mathrm{CO}-\mathrm{NH} \cdot$ linking rather plausible. The importance of this 
consideration lies in the fact that here we have a case where chain growth is terminal and is maintained in such a manner that each addition prepares for the following by permitting reaction only between two activated carboxyl derivatives, the energy of only one of which is used in the process. The terminal activated carboxyl remains intact for the next step of chain elongation. Furthermore, although fatty acid synthesis impresses as a homogeneous process where long, straight, uniform chains are synthesized from indentical two-carbon fragments, obviously during the chain elongation the growing chain is changing constantly. At least three different enzymes participate with different specificity for activation of short, intermediate, and long fatty acids (19). The process, therefore, is not a homogeneous process, but a handling of a changing molecule which changes through the process of elongation itself. Although a longchain fatty acid is homogeneous as compared to a polypeptide chain, the two processes of chain elongation may have common features. It is possible, though no indication has appeared so far, that chain elongation of the polypeptide chain proceeds similarly through linking of an activated carboxyl waiting at the polypeptide terminal for a newly arriving, likewise activated amino acid.

However that may be, such considerations do not help much in solving the cardinal question of how activated amino acids are lined up in the specific sequence particular for the special protein or enzyme. In all present considerations the mechanism of the linking process still presses into the foreground because there is some information present. The mechanism of directing into proper sequence must be determined through some kind of coding mechanism which seems to involve a translation, in Gamow's terminology (9), of specific nucleotide sequential into amino acid sequences.

\section{Conclusion}

The understanding of the basic mechanisms of joining molecules together has opened the possibility of approaching the 
problems of duplication, reproduction, and individualization which I like to bring together under the term patternization. This means that essentially they all may be reduced to the problem of joining building stones into a specific reproducible sequence or pattern. With the energy problem more or less out of the way, it becomes more apparent that patternization is distinct from, although superimposed upon, the process of linking.

It is astonishing to realize that the more one proceeds with the understanding of the workings of the organism, the more one becomes concerned with methodological problems. Strangely, the prying into the mystery of life reduces more and more to an unraveling of a sometimes rather unusual and unexpected methodology of the cell. On the other hand, it is significant that the complexity and methodology of man-made instruments seem to converge increasingly towards the complexity of organismic methodology and instrumentation.

\section{References}

1. Beinert, H., D. E. Green, P. Hele, H. Hift, R. IV. Van Korff, and C. V. Ramakrishnan, J. Biol. Chem., 203, 35 (1953).

2. Berg, P., J. Am. Chem. Soc., 77, 3163 (1955).

3. Boyer, P. D., O. J. Koeppe, W. W. Luchsinger, and A. B. Falcone, Federation Proc., 14, 185 (1955).

4. Borsook, H., C. L. Deasy, A. J. Haagen-Smit, G. Keighley, and P. H. Lowy, J. Biol. Chem., 787, 839 (1950).

5. Cohn, M., Phosphorus Metabolism, 7, 374-376 (1951).

6. Doherty, D. G., and L. Thomas, Federation Proc., 13, 200 (1954).

7. Elliott, W. H., J. Biol. Chem., 201, 661 (1953).

8. Gale, E. F., in W. D. McElroy and B. Glass, Amino Acid Metabolism, pp. 171-192. Johns Hopkins Press, Baltimore, 1955.

9. Gamow, G., Sci. American, 193, 70 (1955).

10. Green, D. E., Biol. Revs. Cambridge Phil. Soc., 29, 330 (1954).

11. Hoagland, M. B., Biochim. et Biophys. Acta, 16, 288 (1955).

12. Jones, M. E., S. Black, R. M. Flynn, and F. Lipmann, Biochim. et Biophys. Acta, 12, 141 (1953).

13. Koshland, D. E., Federation Proc., 14, 238 (1955).

14. Littlefield, J., E. B. Keller, J. Gross, and P. C. Zamecnik, J. Biol. Chem., 217,111 (1955). 
15. Lipmann, F., Am. Scientist, 43, 37 (1955).

16. Lipmann, F., in W. D. McElroy and B. Glass, eds., Mechanism of Enzyme Action, pp. 599-604. Johns Hopkins Press, Baltimore, 1954.

17. Lynen, F., Harvey Lectures, Ser. 48, 212 (1954).

18. Maas, W. K., 3rd Intern. Congr. Biochem. Abstr. of Commun. Brussels, in press, 1955.

19. Mahler, H. R., Federation Proc., 12,694 (1953).

20. Schroedinger, E., What is Life? Macmillan, New York, 1945.

21. Snoke, J. E., S. Yanari, and K. Bloch, J. Biol. Chem., 201, 573 (1953).

22. Spiegelman, S., H. O. Halvorson, and R. Ben-Ishai, in W. D. McElroy and B. Glass, eds., Amino Acid Metabolism, pp. 124-170. Johns Hopkins Press, Baltimore, 1955.

23. Watson, J. D., and F. H. C. Crick, Nature, 171, 964 (1953).

24. Zamecnik, P. C., and E. B. Keller, J. Biol. Chem., 209, 337 (1954). 


\section{ENZYME COMPLEXES AND COMPLEX ENZYMES}

HENRY R. MAHLER, ${ }^{*}$ Institute for Enzyme Research, University of Wisconsin, Madison, Wisconsin

"During the past ten years a good deal of successful effort has been expended on the elucidation of the properties, the structure, and the biochemical function of isolated, carefully fractionated subcellular particulates." This was to be the opening sentence, the springboard of my presentation whence I was to launch myself directly into the topics suggested and summarized by my title. But when I paused after putting this first sentence on paper I realized that it in itself could serve as the summary of one of the main currents in our contemporary biochemical thinking and as such deserved at least a passing comment.

The existence of practical, rapid, and reproducible methods for the separation of the various formed elements of the cell, relatively free from each other and of the soluble cytoplasmic constituents, is basic to many of our experiments and to much of our hypothesizing. Yet these methods barely existed ten years ago. The idea that a large number of enzymes complete with all their coenzymes and cofactors, acting jointly and con-

* Present address: Department of Chemistry, Indiana University, Bloomington. 
secutively to bring about the complete multistep conversion of a substrate, are linked together to form part of the mitochondrion seems commonplace to us today. Yet neither the thought nor its experimental verification had been attempted ten years ago. I do not wish to belabor the point-reviews on the topic of the mitochondrial enzymes are plentiful $(14,22,37)$. I simply wish to stress the thought that there is the germ of novelty inherent already in the term "mitochondrial enzyme." The subsequent discussion is fully intended to be provocative rather than enumerative. ${ }^{*}$ The interpretations of the experimental work presented lay no claim either to uniqueness or correctness. They are merely intended to point at possibilities, rather than probabilities; possibilities which may at least have the virtue of suggesting fruitful approaches for further experimentation.

Let us assume then that enzyme complexes exist within the mitochondrion, and that their primary purpose is to effect efficient multistep transformations subject to the homeostatic and control mechanisms of the cell. What are the methods available to us to study the nature of these transformations; how are we to approach the individual enzymatic process; and how are we to arrive at an understanding of the interaction of the several individual processes making up the whole?

The first method, an essentially dynamic one, proposes to refine our observational tools to such an extent that we may observe individual or sequential reactions with the mitochondrion in situ, without disturbing the array at all. These tools, honed to fine perfection in the hands of Chance (7) then permit us to study the kinetics not only of over-all reactions but also of the individual component reactions. As with all studies of mechanisms based on kinetics a study of this sort limits the number of mechanisms to those which are consistent with the kinetic data but does not allow us to make a choice between the various permissible mechanisms. Individual reactions can

* No review of the literature will be attempted. The only references actually cited are to reviews or to those papers which present a particular point of view cogent to the argument at hand. 
be studied only within the context of the over-all process. If we wish to isolate any single reaction and study it separately this is possible only if we have available ( $/$ ) a mechanism for the generation of the substrate(s), (2) a method for introducing the substrate(s) into the over-all pathway, and (3) a means of blocking off subsequent changes by the use of inhibitors. Analysis of individual enzymes within the array with respect to their characteristic chemical composition or to their prosthetic groups or cofactors is possible only if such an assignment can be made in an unambiguous manner on the basis of supplementary evidence. If we know for instance that (7) all flavoproteins absorb at $450 \mathrm{~m} \mu$, (2) the value of the molecular extinction coefficient for flavoproteins in general at this wavelength, and (3) that under certain conditions a certain definite measurable change at $450 \mathrm{~m} \mu$ can be due only to the reduction of a particular flavoprotein, then we cannot only assay for this flavoprotein within the cell but also follow the kinetics of its reduction and reoxidation.

If I am to be allowed a horribly anthropomorphic analogy, we may look at our problem as similar to one which asks us to account for the location and movements of a large number of individuals within a building. Evidently from the very outset these positions and movements will be restricted by the nature of the building and by its internal architecture, the size and location of the rooms, the position of stairways, elevator shafts, etc. By placing a large number of telescopes and mirrors external to the building, we may hope to keep all the inhibitants under constant observation and plot their movements as lines on a plan of the building. Nevertheless there will always be spots within the structure which are hidden from our view, notably in doorways, stairs, and elevators, especially when relatively large numbers of the inhibitants are in motion simultaneously, even if this motion be perfectly orderly and according to some prearranged plan. But even more fundamentally our problem cannot possibly become completely determinate without an appreciation of the various individuals involved and a careful analysis of their individual properties. 
This is, of course, the approach used in the second of our methods: the isolation, purification, and exhaustive study of the individual enzymes involved in a mitochondrial reaction sequence. In general the next step is an attempt at reconstructing the sequence by the use of a combination of the individual enzymes, their coenzymes, cofactors, etc. These steps were used with spectacular success in the elaboration and reconstruction of the three great metabolic cycles: the tricarboxylic acid cycle (32), the urea cycle (35), and the fatty acid cycle $(15,23)$, as well as such other complex processes as purine synthesis. This is the method par excellence for arriving at a complete appreciation of each individual enzymatic activity and of its potentialities. Note first that I say enzymatic activity and not enzyme. The two are not necessarily synonymous. An enzymatic activity is determined by the assay system employed in its measurement. It is of course associated with a definite protein component-but whether this component as such had an equally definite existence prior to its isolation, or whether it originally formed part of a different or of a larger molecular or catalytic unit may not always be readily discernible or even capable of determination. This point of view will become less obscure as the discussion proceeds, but it must be apparent that the use of artificial electron acceptors in oxidative reactions (such as dyes and quinones), a usual, nay a necessary, experimental device, does not lead to the isolation of "natural" enzymes or at least does not need to. For the introduction of an "artificial" acceptor imposes an element of artificiality into the system, and as far as the study of an oxidative enzyme is concerned, an artificial acceptor is no whit different from the use of an artificial substrate or an artificial coenzyme. Yet frequently we do not even know the nature of the "natural" acceptor, and thus we find ourselves impaled upon the horns of a dilemma from the very outset.

The second point to be raised here concerns the use of the word potentialities. The study of the mechanism and the fine structure of individual enzymatic activities is limited only by our 
conceptual and experimental inadequacies. How these potentialities are translated into actualities, however, can be determined only in the presence of the other components of the system. It is trite to state here and it has been argued more convincingly by others that different observational principles are operative, and different experimental approaches are fruitful, at different levels of organization. I should like to go one step further. I believe it to be inherently impossible (or at least to be accompanied by such formidable experimental and arithmetical hurdles as to be essentially and pragmatically fruitless) to arrive at a completely determined description of the interaction of individual units at one observational or abstractional level using only the observations and abstractions of that level. Thus I doubt whether it is possible to arrive at a knowledge of molecular structure and inter- and intramolecular interactions on the basis of a knowledge, no matter how thorough, of the properties and structures of the individual atoms making up the molecules, in the complete absence of any observations on molecules. Similarly an all-embracing study of individual enzymatic reactions can tell us very little about their interactions and about the more complex array of enzymes from which they must have been derived. Investigations solely of this kind do not permit us to form a clear picture of the catalytic efficiency of this array, the nature of the forces holding together the array, and the control mechanisms operative within the array. Yet it is precisely these concepts of efficiency and control that are probably the most distinguishing features in any comparison of a group of enzymes acting jointly within an array as compared to a mixture of these same enzymes in solution. Efficiency means increased turnover at a given substrate concentration: the array permits the transfer of key coenzymes (such as CoA) and of intermediates (the products of any preceding enzymic step which become the substrates of the succeeding one, as in the reaction catalyzed by pyruvic dehydrogenase, vide infra) in an essentially intramolecular manner, from one site to the next. Free coenzyme or intermediate in solution never comes into 
play at all; thus their effective concentration is equal to their total concentration. With the same enzymes placed together in true solution all the enzymic sites must be occupied for maximal rates; thus the effective concentration of coenzyme or intermediate will be only a fraction of the total that has to be added. Control by the action of some metabolic regulator similarly can be effected in a more direct and efficient manner intramolecularly: the selection of one of two or several possible pathways, say of electron transport, is conditioned by placing a block at, or by the addition of an alternate carrier to, a critical junction point in the array, e.g., a metal atom. We might postulate a scheme such as that shown in Figure 1. In this

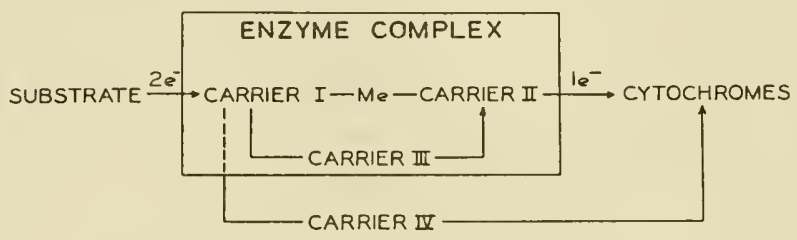

Figure 1.

scheme carrier I might be one flavin prosthetic group linked to one protein, say a dehydrogenase, carrier II a cytochrome component linked directly to this metalloflavoprotein, and carrier III an electron transport enzyme also contained within the same complex, capable of linking the flavin to the cytochrome either by a different metal or by another unspecified means. Blocking at the metal site between I and II will divert electrons through carrier III. If the internal cytochrome component were to be blocked, electron transport to the cytochrome system as a whole or to some other electron acceptor(s) can be made possible by the addition of a carrier added externally (IV), say a quinone of appropriate potential. Schemes very similar to that postulated here actually do seem to play a role in electron transport as shown in subsequent sections.

Thus it becomes evident that there are inherent weaknesses in both approaches: a study of unmodified enzyme complexes 
can give us information about relationships and over-all metabolic sequences. It is not designed to increase our knowledge about the fine structure of individual enzyme-catalyzed reactions too far. Little can be learned about the fundamental chemical changes within the enzyme-substrate complex which must be the prime cause of this catalysis. For studies of this sort we must rely on observations on highly purified individual enzymes. But we must realize that by so doing we may lose sight of the role of this one specified catalysis in relation to other catalytic events, and that in the process of isolation we may have changed the very nature of the catalysis as it originally existed. Thus the proper experimental and conceptual approach demands the use of successive approximations derived in an alternating and synergistic manner both from studies on integrated systemswith their inherent inadequacies-and on highly purified, isolated enzymes-with their attendant dirawbacks.

Can we find footholds in this continuous process of oscillation between the grand but blurred vista of the whole and the sharply defined but myopic study of the individual? In enzyme chemistry, more precisely in the study of oxidative, mitochondrial enzymes there fortunately are such halfway points. There too it has, of course, frequently been possible to isolate highly purified enzyme preparations carrying out just one reaction of a sequence in a completely determined manner, in line with the tenets of classical enzyme chemistry. The remarkable fact is that it has also been possible, in certain instances at least, to isolate just as well-defined and characterized complex enzymes capable of catalyzing complex, sequential reactions.

Such enzymes frequently contain more than one prosthetic group or coenzyme and in all cases almost by definition, must contain more than one site involved in enzymatic activity. This fact, together with the observation that if the enzyme is obtained from a different source - frequently bacterial in origin-or if a different isolation procedure is followed, it is sometimes possible to resolve the preparation into more than one protein component, has been used to argue against a definite unitarian 
designation of these enzymes. This brings us into the realms of nomenclature, semantics, and philosophy and once again raises the spectre of having to determine how and in what form enzymes preexisted in the cell. For practical purposes the aim should be to isolate these activities in a reproducible manner with constant composition with respect to the characteristic chemical components and tending toward homogeneity by physical, chemical, and biological criteria.

\section{Pyruvic and $\alpha$-Ketoglutaric Dehydrogenases}

These were the first complex oxidative enzymes to be isolated from animal tissues in a homogeneous form. The reaction catalyzed by them, the nature of the prosthetic groups and coenzymes involved, and other aspects of the catalytic mechanism have been studied intensively $(14,32)$ and will not be covered here in extenso. The highly purified enzyme carries out at least the following activities: (1) that of a carboxylase: decarboxylation of the $\alpha$-keto acid to a diphosphothiamin (or diphosphothiamin-lipoic)-aldehyde complex, (2) oxidation to an acetyl-lipoic complex (with the lipoic moiety reduced from the disulfide to the dithiol), (3) and (4) the reoxidation of reduced lipoic acid by DPN, in conjunction with the transfer of an acyl moiety from lipoic acid to CoA. Electron transport from substrate to DPN is thus mediated by lipoic acid, and it is at that level that alternate and bypath mechanisms might come into play (e.g., the oxidation of pyruvate or $\alpha$-ketoglutarate by ferricyanide, dyes, or oxygen in the presence of the dehydrogenase, but in the absence of either CoA or DPN).

\section{Metalloflavoproteins}

A large group of flavoproteins has recently been shown to contain metals in close association with their flavin prosthetic groups and as an integral part of the electron transport mechanism of these enzymes (29). Among the enzymes so classified 
are butyryl CoA dehydrogenase (Cu), DPNH-cytochrome reductase $(\mathrm{Fc})$, nitrate reductase $(\mathrm{Mo})$, xanthine oxidase $(\mathrm{Mo}, \mathrm{Fe})$, aldchyde oxidase (Mo), hydrogenase (Mo). The metal appears to be involved in linking not only flavin to protein but also the acceptor to the other two components during the lifetime of the enzyme acceptor complex (27). During this period there is evidence that all four components become part of the same resonating system with a free flow of $\pi$-electrons from reduced enzyme (or substrate) to acceptor. Among the acceptors in this class are nitrate and cytochrome $c$. Thus, for instance for the oxidation of a reduced normal flavoprotein there is observed an optimal reactivity with only one member of a series of closely related electron acceptors (e.g., substituted 1,4-naphthoquinones) (28). This acceptor has a definite redox potential (called the optimum interaction potential $\epsilon$ ). If the logarithm of the reaction rate for reduction of quinone is plotted against potential of quinone, the rate of interaction falls off sharply and linearly on either side of $\epsilon$. If instead the enzyme is in the metalloflavoprotein form, then not only is $\epsilon$ shifted to a more positive or more negative value, but also the drop-off of rates at either side of $\epsilon$ is much less rapid. Let us briefly develop one of a number of possible interpretations which may be consistent with these observations. In accordance with current views it is assumed that (1) reduced flavoprotein and acceptor must interact within a complex and (2) that $\pi$-bonding or the interaction between two such mobile electronic systems can take place only if both partners possess certain closely matched energy levels $(2,10,21)$. For the interaction of a series of quinones with one given flavoprotein, matching might well be optimal for only one member of the series and less efficient in a constant and predictable manner if the inherent electronic structure of the quinone is modified by a variation of substituents. This inherent difference in energy levels will manifest itself then (1) in a variation of the $E_{0}{ }^{\prime}$ of the various members of the series and (2) in a variation of the ability to match energy levels with a constant interacting system (the flavoprotein), as observed. 
The metalloflavoprotein model may constitute the simplest example of a system capable of linking the areas of $\mathrm{H}$-transport enzymes (pyridinoproteins and flavoproteins) and true electron transport enzymes (the cytochrome system). Where true hydrogen transport (or a $2 \mathrm{e}^{-}$transfer) is possible from flavin to oxidizing agent, i.e., in the interaction of flavoproteins with dyes, quinones, or directly with oxygen, the presence of the metal is not necessary and its absence does not affect the mechanism of the catalysis observed. Metalloflavoproteins are of interest not only because of their inherent importance as electron transport enzymes but also because of the fact that certain extensions of principles first observed and easily tested with this class of enzymes may be possible to more complex enzymatic arrays as well.

\section{Hemo-Flavoproteins}

In the case of the metalloflavoprotein enzymes just described the interaction between reduced flavin, and the preceding $\mathrm{H}$-transport system, with the cytochrome system is mediated by a metal, and this linkage between the flavoprotein and the isolated cytochrome (cytochrome $c$ ) is produced in a transient manner only during the existence of the enzyme-acceptor complex. As an extension of this principle, possibly depicting a status more closely akin to that to be found in the mitochondrion, it has been possible in at least three instances to isolate enzymes containing flavin and an iron-porphyrin linked in a more permanent manner. One such example is the crystalline lactic dehydrogenase of yeast, containing FMN and cytochrome $b_{2}$, isolated by Appleby and Morton (1).

Another example is the aldehyde oxidase of mammalian liver (30), which has the following four prosthetic groups: thiol groups, FAD, molybdenum and iron-protoporphyrin (with a spectrum very similar to that of cytochrome $b$ ). Finally solubilized enzymes have been obtained from DPNH oxidase, which will be described below (25), and from complex succinic dehydro- 
genase preparations which contain flavin, iron, and cytochrome $b$. In the light of these observations it would be of great interest to determine whether the yeast enzyme contains a metal instrumental in linking flavin to cytochrome $b_{2}$ * The potentialities for alternate schemes of electron transport and for their control by external factors with an enzyme such as aldehyde oxidase are manifold. Electrons may funnel into the cytochrome chain "directly" through the "built-in" cytochrome. They may be diverted to cytochrome $c$ and into alternate channels in the cytochrome system through interaction with external cytochrome $c$, or they may be removed by oxygen or by an autooxidizable quinone, in both cases bypassing the metal and the cytochrome system entirely. Finally, the quinone may act as carrier between the enzyme and some cytochrome. The analogy to the hypothetical case exemplified by Figure 1 is thus almost perfect. As far as selective inhibitors are concerned, the - $\mathrm{SH}$ groups of the enzyme may be blocked by the usual inhibitors and the enzyme protected from their action by substrate; the flavin may be blocked by atabrine, this inhibition being reversed by FAD; the molybdenum may be blocked by chelating agents, this inhibition being reversed by excess Mo; whereas the iron-porphyrin is irreversibly blocked by cyanide, azide, etc.

Finally, soluble succinic dehydrogenase preparations, capable of interacting with one-electron acceptors (phenazine, ferricyanide) and having the properties of ferroflavoproteins, have been isolated (38).

\section{The Electron-Transferring Flavoprotein (ETF)}

During their studies of the three enzymes concerned with the oxidation of saturated fatty acyl CoA derivatives to the corresponding $\alpha$ - $\beta$ unsaturated compounds, Crane and Beinert (9) discovered a new flavoprotein concerned in the electron

* This has been verified experimentally. Yeast lactic dehydrogenase contains 8 atoms Fe per mole of flavin or cytochrome $b_{2}$. (E. Boeri, private communication.) 
transport from these dehydrogenases to the cytochrome system. This enzyme utilizes enzyme-bound reduced FAD (of the dehydrogenase) as substrate and a cytochrome (cytochrome $c$ is the one actually employed) as electron acceptor. Although it had been shown previously that an enzyme-bound electron transport coenzyme (i.e., the DPNH of D-glyceraldehyde phosphate dehydrogenase) could become the substrate or coenzyme for a second enzyme $(8,26)$, this novel reaction marks the first such in the flavin field, is the first obligatory case, and provides us with yet another possible pathway between the flavoprotein and cytochrome systems. There is considerable evidence that the three acyl CoA dehydrogenases (one specific for the lower fatty acids - the green copper-containing butyryl CoA dehydrogenase-and two yellow, iron-containing, more general acyl CoA dehydrogenases) and ETF originally formed part of a more elaborate enzyme complex (3). Within this complex the copper associated with the isolated butyryl CoA dehydrogenase may have been used to bind the four components by complex formation and to provide the link between this complex flavoprotein and the cytochrome system.

\section{DPNH Oxidase}

The most complicated, well-defined enzyme complex which has been subjected to investigation is the DPNH oxidase complex isolated by Green et al. (17) and exhaustively studied by Mackler $(24,25)$. This particulate enzyme can be obtained in a highly purified, highly active form and then carries out only the antimycin-sensitive reaction $\mathrm{DPNH}+\mathrm{H}++1 /{ }_{2} \mathrm{O}_{2} \rightarrow \mathrm{DPN}^{+}+$ $\mathrm{H}_{2} \mathrm{O}$. In this state the enzyme apparently contains but little cytochrome $c$, and does not catalyze either the reduction of ferricytochrome $c$ by DPNH or the oxidation of ferrocytochrome $c$ by oxygen. On prolonged standing or on treatment of this "closed" enzyme by deoxycholate or other surface-active agents, the ability to catalyze the aerobic oxidation of DPNH by $\mathrm{O}_{2}$ disappears. In its stead, this "open" enzyme, indistinguishable 
from the "closed" enzyme in appearance and composition, is now capable of catalyzing the two reactions involving cytochrome c just described. The "open" or "closed" enzyme of molecular weight of approximately 2,000,000 (lipid free) contains flavin, non-heme iron, copper, a hematin component spectroscopically indistinguishable from cytochrome $b$, and one indistinguishable from the heme component of cytochrome oxidase. On treatment of this preparation with trypsin two fractions may be obtained. One (A Fraction) contains flavin, the bulk of the iron, and the cytochrome $b$, and catalyzes the antimycinsensitive reduction of cytochrome $c$. The other (B Fraction)

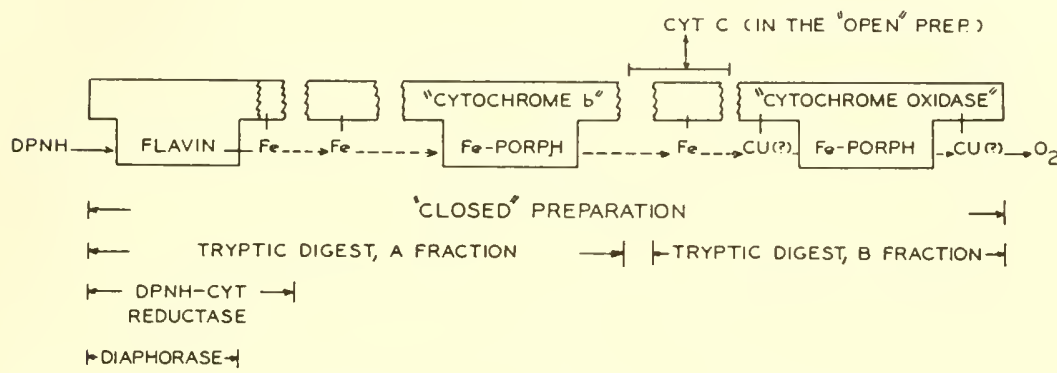

Figure 2.

contains the remaining iron, the enzyme-bound copper, and the heme component of cytochrome oxidase. It functions only as a cytochrome $c$ oxidase. Further modification of the antimycinsensitive, hematin-containing cytochrome reductase leads to the isolated ferroflavoprotein DPNH-cytochrome reductase, whose relationship to diaphorase had already been established (27). Thus we can achieve the complete and continuous transformation of a mitochondrial electron transport enzyme into isolated, soluble electron transport enzymes of any desired degree of complexity. This transformation is then "closed" $\rightarrow$ "open" $\rightarrow$ hemometalloflavoprotein $\rightarrow$ metalloflavoprotein $\rightarrow$ flavoprotein. The different preparations and their properties may be pictured as in Figure 2. 


\section{Interaction between Flavoprotein and Cytochromes}

We might then summarize and extend the possible means of interaction between enzymes at the flavoprotein and those at the cytochrome level in the following manner (see Figure 3). Probably the most significant feature of this scheme is its inherent complexity. Instead of one straight-line path from substrate to oxygen we find the electrons traveling several paths in parallel.

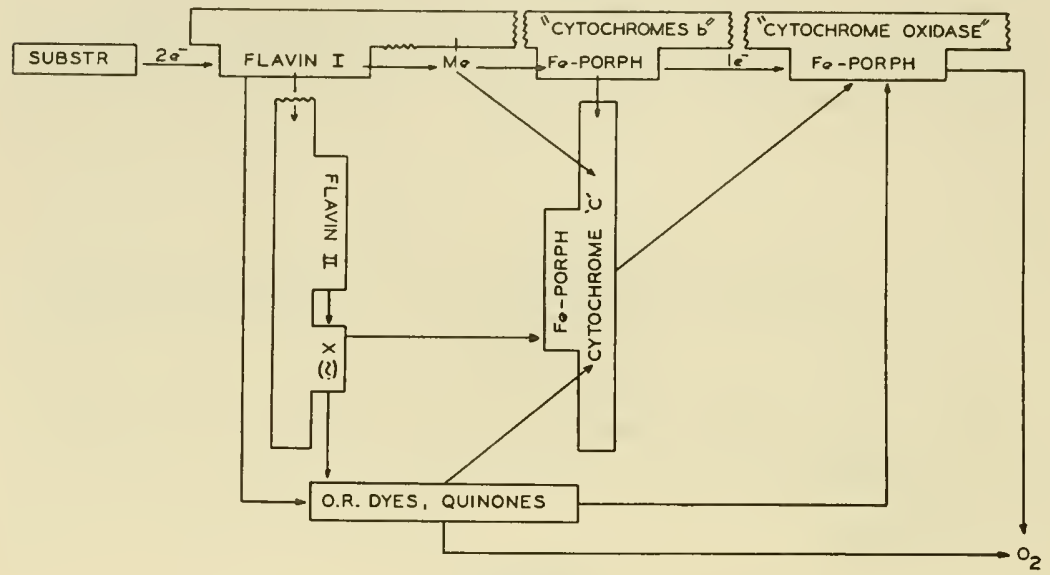

Figure 3.

This is not to suggest that all paths will be operative at any one time. During any one set of physiological parameters probably only one of the several alternatives will actually be in use. The switches are set for this particular alternative. But the other possibilities are always present. Under the influence of metabolic controls at one or several junction points an alternate path may become obligatory. Thus the electrons may be diverted from say a relatively slow but energetically efficient to a more rapid but inefficient route, for example, during uncoupling of oxidative phosphorylation (20). Another feature worth noting may be that quinones (e.g., vitamin K) and cytochrome $c$ lie on parallel paths. The scheme also illustrates a second point: electron transfers from the flavin stage onward, at least in the native 
enzyme complex, are largely intramolecular in nature. We are therefore dealing with electronic shifts from one part of one molecule to another part rather than with electronic transfers between two molecules - a vastly more rapid and efficient process. Thus this representation may lead us toward an explanation of those intramitochondrial events which we had previously characterized by the twin attributes of efficiency and control.

\section{Biochemical Functions of Metal Ions in Electron Transport and in the Formation of Enzyme Complexes}

\section{FORMATION OF BONDS BY COMPLEX FORMATION}

Intuitively the most obvious role of metal ions, and one susceptible to experimental verification in many instances, is that of linking different parts of one enzyme, or of an enzymesubstrate complex (39), or of several functionally related proteins. In this category we have already discussed the probable function of metals in metalloflavo-proteins as sites of attachment of the flavin nucleotide to the protein. This type of complexing is similar to that observed by Klotz (19) in his experiments on the linking of neutral dyes to protein by means of metal ions. The metal has been shown to be involved in forming flavoprotein-cytochrome $c$ complexes in the case of DPNH cytochrome reductase (27) and is probably so involved in the reaction of all the other metalloflavoproteins with cytochrome $c$ as well. As a further extension of this concept it is postulated that the metal is also involved in the bonding of the flavoprotein and the hemoprotein moieties in the isolated instances of aldehyde oxidase, yeast lactic dehydrogenase, and the transformed DPNH oxidase. The implication of these findings is then that in the mitochondrion we have similar flavin-metalhemin complexes held together at least in part by virtue of chelate bonds between the various components and the metal. Various cytochrome components in turn might be complexed 
together again by means of nonporphyrin metal chelates to form even larger units such as the "native" DPNH oxidase.

\section{IMPLICATION OF METALS IN OXIDATIVE PHOSPHORYLATION}

The finding that metals are involved in electron transport has given rise to different speculations as to how these cationic constituents might be the locus of primary phosphate bond formation during electron transport. A possible mechanism might envisage the uptake of phosphate during the reduction of the metal in the course of the catalytic cycle (28). This reduced phospho-metal-enzyme complex might be more stable thermodynamically with respect to hydrolysis than the corresponding oxidized complex. If, (1) the difference in the free energy of hydrolysis of the two complexes were of the order of $-10,000$ cal.* and (2) some mechanism for the transfer of this metal bound phosphate to yield eventually a nucleotide pyrophosphate were available, then this process would constitute oxidative phosphorylation. The cycle reduction $\rightarrow$ phosphate uptake $\rightarrow$ oxidation $\rightarrow$ phosphate release is believed to be obligatory in the case of the molybdoflavoproteins, but there phosphate release by hydrolysis is so rapid that a search for phosphate transfer is probably doomed to failure. The isolated DPNH-cytochrome reductase, on the other hand, does appear capable of phosphate uptake during catalysis, without rapid hydrolysis of the phosphate, but no mechanism for phosphate transfer has so far been discovered and thus this phosphate uptake does not lead to oxidative phosphorylation but only to phosphate inhibition of the enzyme. It is, however, conceivable that a search for a system capable both of an uptake of phosphate to form a metal phosphate during catalysis and of phosphate

* This assumption at least in the case of iron would not appear to be unreasonable by analogy with the known properties of the corresponding inorganic iron compounds (4). Thus the formation constant of the ferric hydroxy complex is considerably higher than that of the ferrous complex, whereas the solubility of ferric phosphate is higher than that of ferrous phosphate. 
release to a nucleotide acceptor may lead to the discovery of an enzyme system capable of carrying out oxidative phosphorylation.* Another attractive mechanism for oxidative phosphorylation during metalloflavoprotein oxidation by cytochromes, involving a phosphate bridge between the two components, has been postulated by Boyer (5).

\section{A POSSIBLE ROLE OF METAL IONS IN ELECTRON TRANSPORT}

Studies with metalloflavoproteins have demonstrated that the metals involved in this type of enzyme are capable of undergoing reversible valence changes, of contributing to the light absorption of the molecule, and of influencing not only the rates and the type of catalytic action but also the energy content of the molecule. These different lines of evidence taken in conjunction have been interpreted to mean that the metal orbitals are in direct electronic interaction with the other parts of the catalytically active molecular structure and that they form part of a resonating, mobile $\pi$-electron system consisting of flavin, acceptor, and those parts of the protein adjacent to and interacting with the catalytically active site $(21,28)$. Oxidation of reduced flavin by cytochrome $c$ therefore entails simply the intra-molecular movement within the enzyme-acceptor complex of one of the $\pi$-electrons from the flavin system to the acceptor system, with the metal as an essential feature of the circuit. The metal functions as the site of attachment for the acceptor and provides a linking place or nexus for the two $\pi$-electron systemsthat of the flavoprotein and that of the acceptor. This concept is capable of extension first of all to the hemoflavoproteins, the group of flavoproteins containing a "built-in" cytochrome

* A possible variant of this scheme (suggested by Dr. B. M. Strehler) may involve uptake of a nucleoside pyrophosphate instead of phosphate by the metal enzyme. After reoxidation the original aquoenzyme would then be reformed by phosphorolysis leading to the simultaneous production of a nucleoside triphosphate. The corresponding cycle would then be: reduction $\longrightarrow$ nucleoside pyrophosphate uptake $\longrightarrow$ oxidation $\stackrel{\mathrm{HPO}^{-}}{\longrightarrow}$ nucleoside triphosphate release. 
component, and then to such complex and complete electron transport enzymes as the DPNH oxidase. Here the cytochrome component is presumably linked to the conductive system of the protein moiety by means of the histidine residues bonded to the iron-porphyrin (40).

Let us reflect for a moment and consider whether such a radical and highly speculative step as the suggestion that electron transport between individual cytochromes may be metalmediated is at all necessary or whether more classical explanations may not be equally satisfactory. Now in the picture presented here, i.e., the sequential arrangement of individual electron transfer enzymes as part of a larger self-contained supramolecular unit, some method of connecting the individual parts both structurally and electronically must obviously be made. But this picture is certainly not the only possible one. Chance (7), for instance, has suggested that simple collisions of individual cytochromes, each anchored to a matrix but able to interact freely with its neighbor, can account for the kinetic data obtained by him. Although simple and kinetically unobjectionable, this picture may also require a possible role for metal ions. For if we are to take cytochrome $c$ as our model it is apparent that all the coordination valencies of iron are permanently occupied ( 4 by the porphyrin ring and 2 by histidine residues of the protein). Thus the primary interaction of the cytochrome with electron donor or acceptor must of necessity take place at a locus removed in space from the iron atom. Therefore we must here too look for a possible mechanism for the transmission of electrons from one part of the protein to another, i.e., from that spot where collision with donor has taken place to the iron porphyrin and thence to the spot where collision with acceptor is to take place. This problem appears to be only quantitatively and not qualitatively different from that to be considered in our model. In all cases it reduces to the question of possible transmission of electronic interactions through proteins. This is a problem which arises in connection with a wide variety of observations in biochemistry. Various theories, most of which 
regard the protein as a semiconductor or as a macro resonating system involving the hydrogen bonds of the polypeptide chain, have been proposed by others and have been critically reviewed recently $(6,21)$.

Semiquantitative calculations have actually been carried out by Evans and Gergely (12) and have led to the assignment of at least partially metallic character to some of the valence electrons associated with the polypeptide structure. The present suggestion would certainly imply further increase in the conductive nature of the protein or provide a possible alternate explanation of such conductive phenomena. Once again as in the metalloflavoprotein case the introduction of a metal atom, or as here of a large number of metal atoms, should lead to a much larger variety and a smearing-out of possible energy states in the complex molecule and to an enhancement of the possibilities and rates of interaction with external donor and acceptor systems (see also ref. 21, pages 32-40).

The speculative picture which I should like to advance, in order to focus attention on a possible new parameter to this problem, is based on some observations on the composition of DPNH oxidase $(24,25)$ and on some aspects of the present-day thinking concerning metallic conduction (18). The enzyme contains a large number of non-heme iron and copper ions (15 of the former per flavin and approximately 4 per cytochrome $b$ hemin; 2 to 3 of the latter per cytochrome oxidase hemin) tightly bound to the protein. It is possible that electron transport from flavin to cytochrome $c$ or from one cytochrome to the next is mediated at least in part by a conducting core of metal ions linked to and surrounded by the polypeptide helix (34). The latter would then function in some measure like an insulating sheath. The metal ions may form a complex with one or more of the nitrogen and one or more of the oxygen atoms of the helix by actual bond formation but some of the orbitals of the metal will remain unfilled and free to interact with similar unfilled orbitals of neighboring metal atoms. This property then might impart a metal-like character to the chain of metal 
ions imbedded in the polypeptide chain. Some or all of the metal ions may not even be linked to the helix by classical bonds; instead they may be simply immersed inside the helix and held there trapped by the geometry of the situation.

Let us now consider whether the quantitative information at our disposal is capable of demonstrating the consistency or inconsistency of this model. This information is of two kinds: first we must see whether the atoms of iron are of the proper size to fit into the Pauling-Corey helix (34) as the most likely protein model. Obviously if the radius of iron (which will be of partially covalent and of partially metallic character) is too large to be fitted into the dimensions of the helix, we would have to look at the model with deep misgivings. Similarly if the radius of the metal ion were too small, then tight bonding of the metal could not take place and once again the model would be open to question a priori. Fortunately the covalent diameters of iron and copper are 2.46 and $2.7 \mathrm{~A}$, respectively, while their metallic diameters are about $2.5 \mathrm{~A}$ each (33), of just the right dimensions to fit snugly into the circular cross-sectional area produced by one turn of the helix (the distance between nitrogen and oxygen being between 2.5 and $2.6 \mathrm{~A}$ ), providing bonding to the metal occurs.

Next we must see whether the number of metal atoms associated with one enzyme molecule is sufficient to account for transmission along a significant proportion of the chain, assuming one metal atom per turn of the helix. Here we must make certain assumptions. The first concerns the molecular weight of a "typical" cytochrome molecule. Clearly not all portions of the enzyme molecule will be concerned with its primary purpose, the transport of electrons, nor will the distance between the two prosthetic groups to be linked be necessarily identical with the over-all length of the molecule. Let us boldly assume that the "effective minimum molecular weight" of a hemoprotein, i.e., that which is directly related to its function, is that of cytochrome $c$ or of myoglobin, i.e., about 15,000. This then, assuming but one polypeptide chain, leads to about 130 amino 
acid residues or 35 turns of the $\alpha$-helix. Assuming one metal atom for each turn we would therefore require 35 atoms to account for complete and sole electronic transmission by our model. The actual number of iron atoms per flavin in the isolated antimycin-sensitive, DPNH oxidase equals 15; thus a considerable portion of the over-all molecule could be involved in the electron transfer of the pcstulated type. This model is based on a catalytic unit consisting of one flavin and four hemin prosthetic groups (similar to the conditions in hemoglobin or catalase). Another estimate can be arrived at from the beautiful work of Perutz and his collaborators on the structure of the hemoglobin molecule. This molecule, of molecular weight 68.000 , is believed to be $65 \mathrm{~A}$ long with the other two dimensions being 55 and $55 \mathrm{~A}$, respectively (13.18). On the basis of x-ray diffraction evidence, the presence of rods, each having the structure of $\alpha$-keratin, i.e., the $\alpha$-helix of Pauling and Corcy, extending in the $65 \mathrm{~A}$ direction is indicated. These rods are packed in a pseudohexagonal array with the centers $10.5 \mathrm{~A}$ apart. Now let us consider the make-up of one individual rod: the $\alpha$-helix has 48 amino acid residues per 13 turns of the helix, corresponding to a distance of about $72 \mathrm{~A}$. Thus a rod $65 \mathrm{~A}$ long would consist of about 12 turns. Assuming only one of the rods to be actually concerned with electron transport, "semimetallic" transmission of electrons could therefore extend over most of the distance occupied by a hemoprotein molecule constructed like hemoglobin. Two further observations may have some bearing on the proposed distribution of metal atoms throughout the protein chain of an enzyme. (1) The molybdo-flavoprotein xanthine oxidase contains more than one flavin nucleotide prosthetic group per mole of enzyme. Thus the possibility of interaction of these various prosthetic groups in an interdependent fashion must receive some consideration. This enzyme has been shown by Richert and Westerfeld (36) to contain several iron atoms per mole.

(2) The succinic dehydrogenase complex can be degraded in a serial fashion by tryptic digestion and other means in a 
manner entirely analogous to that described for DPNH oxidase (16). These fragments we might call A, AB, ABC to describe their increasing order of complexity and multiplicity of prosthetic groups. They show different specificity toward electron acceptors: A interacts well only with phenazine, $A B$ with phenazine and ferricyanide, ABC with phenazine, ferricyanide, and methylene blue, etc. It can be shown that iron atoms are associated with each of the fragments described, in such a manner that $\mathrm{AB}$ contains a higher number than $\mathrm{A}, \mathrm{ABC}$ a higher number than $\mathrm{AB}$, and so forth. Thus in both the cases mentioned protein-bound iron may be conceived as linking together several prosthetic groups of an enzyme molecule.

It is obvious that this is a crude picture, a possible lead toward a first approximation at best. There is no reason to assume that this is the most probable means of electron transport along a polypeptide chain. It is merely a possible one. Such a partially metallic model of electron transport would lead to transfers with rate constants of the order of $10^{11} \mathrm{M}^{-1} \mathrm{sec}^{-1}$. These values are several orders of magnitude more rapid than Chance's observed constants of $10^{6}$ to $10^{7} \mathrm{M}^{-1} \mathrm{sec} .^{-1}$. This alone does not constitute a valid objection to the theory, however. The constants were calculated assuming a truly metallic conductor. It is obvious that there will be differences between a series of metal ions inside a protein helix and a true metal. These differences will lead to a slowing down of the speed of transmission. Nor is there any reason to assume that coupling between the electronic system inherent in a prosthetic group, say an iron porphyrin, and the protein-bound chain of metals will be perfect, nor even coupling from iron atom to iron atom, nor will the distances between individual metal atoms be invariant. There may be areas within the helix without metal atoms. All these effects and many more due to the imperfection of both the conductor and the insulator, i.e., the protein, will lead to retardation. Part of the circuit may consist of yet another type, neither resonating electrons in a prosthetic group $\pi$-system, nor metal ions but instead it may be part of a thin conducting 
sequence of aromatic amino acids, etc. The encouraging aspect of the problem is, on the contrary, that even with all these tendencies toward slower rates, the rate of interaction between cytochromes, using the metallic model, may still be fast enough to be consistent with the observed rates. Only relatively short portions of the over-all system may be actually metal-mediated. These conducting sequences may be inserted only in the vicinity of or between iron-porphyrins and other prosthetic groups. Their main purpose may well be to provide junction points and plugs along the main line sequence for the multitude of parallel and accessory paths.

\section{References}

1. Appleby, C. A., and R. K. Morton, Nature, 773, 749 (1954).

2. Bayliss, N. S., Quart. Rers. (London), 6, 319 (1952).

3. Beinert, H., and F. Crane, in W. D. McElroy and B. Glass, eds., Inorganic Nitrogen Metobolism: Role of Flavoproteins. Johns Hopkins Press, Baltimore, 1956.

4. Bjerrum, J., Chem. Rev's., 16, 381 (1950).

5. Boyer, P. D., A. B. Falcone, and W. H. Harrison, Nature, 17t, 401 (1954).

6. Bucher, Th., Advances in Enzymol., 14. 21 (1953).

7. Chance, B., Nature, 169, 215 (1952); Science, 120, 767 (1954); also in W. D. McElroy and B. Glass, eds., The Mechanism of Enzyme Action. Johns Hopkins Press, Baltimore, 1954.

8. Cori, C. F., S. F. Telick, and G. T. Cori, Biochim. et Biophys. Acta, 4, 160 (1950).

9. Crane, F. L., and H. Beinert, J. Am. Chem. Soc., 76, 4491 (1954).

10. Dewar, M. J. S., Electronic Theory of Organic Chemistry. Oxford University Press, London, 1949.

11. Eley, D. D., G. D. Parfitt, M. J. Perry, and D. H. Taysum, Trans. Faraday Soc., 49, 79 (1953).

12. Evans, M. G., and J. Gergely, Biochim. et Biophys. Acta, 3, 188 (1949).

13. Granick, S., in W. H. Cole, ed., Some Conjugated Proteins. Rutgers University Press, New Brunswick, New Jersey, 1953.

14. Green, D. E., in D. M. Greenberg, ed., Chemical Patherays of Metabolism, Vol. 1, pp. 27-65. Academic Press, New York, 1953; Biol. Revs. Cambridge Phil. Soc., 26, 15 (1951).

15. Green, D. E., Biol. Revs. Cambridge Phil. Soc., 29. 330 (1954).

16. Green, D. E., R. E. Basford, and S. Mii, in W. D. McElroy and B. Glass, eds., Inorganic Nitrogen Metabolism: Role of Flavoproteins. Johns Hopkins Press, Baltimore, 1956.

17. Green, D. E., B. Mackler, R. Repaske, and H. R. Mahler, Biochim. et Biophys. Acta, 15, 435 (1954). 
18. Haurowitz, F., and R. L. Hardin, in H. Neurath and K. Bailey, eds., The Proteins, Vol. 2, Part A. Academic Press, New York, 1954; see also B. Low in the same series, Vol. 1 (Part A).

19. Klotz, I. M., in W. D. McElroy and B. Glass, eds., The Mechanism of Enzyme Action. Johns Hopkins Press, Baltimore, 1954.

20. Lardy, H. A., in L. F. Wolterink, ed., The Biology of Phosphorus, pp. 143144. Michigan State College Press, Lansing, Michigan, 1952.

21. Leach, S. J., Advances in Enzymol., 15, 32 (1954).

22. Lindberg, O., and L. Ernster, in L. V. Heilbrunn and F. Weber, eds., Protoplasmatologia, Vol. 3A. Springer, Vienna, 1954.

23. Lynen, F., and S. Ochoa, Biochim. et Biophys. Acta, 12, 299 (1954).

24. Mackler, B., R. Repaske, P. Kohout, and D. E. Green, Biochim. et. Biophys. Acta, 15, 437 (1954).

25. Mackler, B., Federation Proc., 14, 248 (1955).

26. Mahler, H. R., Biochim. et Biophys. Acta, 14, 100 (1954).

27. Mahler, H. R., and D. G. Elowe, J. Biol. Chem., 210, 165 (1954).

28. Mahler, H. R., A. S. Fairhurst, and B. Mackler, J. Am. Chem. Soc., 77, 1514 (1955).

29. Mahler, H. R., and D. E. Green, Science, 120,7 (1954).

30. Mahler, H. R., B. Mackler, and D. E. Green, J. Biol. Chem., 270, 465 (1954).

31. Mrozowski, S., Phys. Rev., 85, 609 (1952).

32. Ochoa, S., Advances in Enzymol., 15, 183 (1954).

33. Pauling, L., The Nature of the Chemical Bond. Cornell University Press, Ithaca, New York, 1950.

34. Pauling, L., and R. B. Corey, Proc. Natl. Acad. Sci. U. S., 37, 205-211, 235 ff. (1951).

35. Ratner, S., Advances in Enzymol., 15, 319 (1954).

36. Richert, D. A., and W. W. Westerfeld, J. Biol. Chem., 209, 179 (1954).

37. Schneider, W. C., and G. H. Hogeboom, Cancer Research, 11, 1 (1954).

38. Singer, T. P., and E. Kearney, Federation Proc., 14, 282 (1955).

39. Smith, E. L., in W. D. McElroy and B. Glass, eds., The Mechanism of Enzyme Action. Johns Hopkins Press, Baltimore, 1954; also in F. R. N. Gurd, Enzymes and Enzyme Systems. Harvard University Press, Cambridge, Massachusetts, 1951.

40. Theorell, H., Ergeb. Enzymforsch., 9, 231 (1943). 


\title{
RELATIONS BETWEEN PROSTHETIC GROUPS, COENZYMES AND ENZYMES
}

\author{
HUGO THEORELL, Department of Biochemistry, Medical Nobel \\ Institute, Stockholm
}

The reactions of proteins-most of which seem to be enzymes-are fundamental for biological life, since they play a primary role in both of its essential features: reproduction and metabolism. From a chemical point of view cell division is a complicated and as yet largely unknown chain of reactions between proteins and nucleic acids which occur in cell structures. Metabolism is promoted and governed by a great number of interactions between proteins and their cofactors (for example nucleotides or hematins), and various substrates.

Whereas the chemical structure of the known prosthetic groups and coenzymes is relatively well established, the structure of proteins is in many respects unknown. Only recently has sufficient progress in protein chemistry been made to permit the hope of being able to find the "active" groups in the proteins, and of formulating in chemical terms what happens when proteins react with their cofactors and substrates. We shall discuss here some of the recent results obtained in this field, and essentially confine ourselves to three oxidation enzymes of different type: cytochrome $c$, the "old yellow enzyme," and liver alcohol dehydrogenase. 
It is still common practice to distinguish between "prosthetic groups" and "coenzymes." As recently discussed by the author (21), there are certain historical reasons for maintaining this terminology which are based upon some simple preparative differences. Whereas prosthetic groups are so tightly bound to the protein that they remain attached to the protein even if this is denatured, for example, by boiling the coenzymes, before anything was known about their chemical nature, were defined as heat-stable, dialyzable activators remaining in solution after the heat denaturation of the proteins.

At the present time there is an obvious tendency to another distinction from a chemical point of view, since the hematin group of the hemoproteins is practically always called "prosthetic group"--but never "coenzyme"-whereas the group of substances always called coenzymes are the well-known phosphorylated compounds DPN, FMN, FAD, ${ }^{*}$ and so on.

It would perhaps not be practical or even feasible to give up the name "prosthetic groups" in favor of "coenzymes" for various reasons, the main one being that some hemo-proteins, like hemoglobin or myoglobin, are not usually regarded as enzymes. But from a general point of view there is no need to keep up a distinction that is based mercly upon how strong the linkages are between the parts of a conjugated protein, since very great variations in this respect occur within both groups.

The prosthetic groups, as far as we yet know, always remain attached to their proteins during their functional cycles; the coenzymes may either remain "immobile" during their function, as is the case with FMN in the old yellow ferment, or pass through different parts of the functional cycle in combination with different proteins, as is very probably the case with DPN in the liver alcohol dehydrogenase system ("mobile" coenzyme). The proteins, as well as coenzymes and prosthetic groups, are inactive when separated. This is just the reason why the coupling reactions between the proteins and their cofactors are

$*$ DPN $=$ diphosphopyridinenucleotide $; \mathrm{FMN}=$ flavinmononucleotide; FAD = flavindinucleotide. 
so important. It is especially important to learn about the groups functioning as binding sites for cofactors and substrates, and the groups that are close enough to influence the reactions. The more remote parts of the protein molecule are probably less important. For instance, the mode of action of hemoglobin has at least partly been explained on the basis of the chemical nature of its "heme-linked" groups.

\section{Cytochrome c, a Protein with Immobile Prosthetic Group}

Cytochrome $c$ is from some points of view a suitable material for investigating the chemistry of the coupling between hematin and protein. It is comparatively readily available and is stable over a very wide range of $p \mathrm{H}$. As in all hemo-proteins the porphyrin moiety puts light absorption bands at our disposal for spectrophotometry. In addition, the iron atom under different conditions has $0,1,3$, or 5 unpaired electrons; therefore magnetometric determinations are useful for structure studies.

There have been some advances in our knowledge of the structure of the cytochrome $c$ molecule. 'The $\alpha$-carbon atoms of the side chains in the 2- and 4-positions of the porphyrin are linked by thioether bonds to sulfur atoms in two cysteine residues of the protein. This was demonstrated by Theorell in 1938-39 (19). Paul (13), in this institute, further corroborated the $\alpha$ position of the thioether bonds to the porphyrin side chains by isolating an optically active hematoporphyrin after splitting the thioether bonds with silver acetate. In 1941 (22) the theory was advanced that the groups forming a hemochromogen with the iron atom were both imidazoles from histidine residues; this was based upon titrimetric, spectrophotometric, and magnetic data. Support to this theory was given by Paul's titration experiments on the cytochrome apoprotein, liberated by the silver-splitting method

Reduced cytochrome $c$ is not autoxidizable. This fact, 
exceptional for a hemochromogen, was tentatively explained by the assumption that the hematin is built into a crevice of the protein molecule in such a way as to prevent oxygen from reacting with the iron. It was further pointed out that if imidazoles were attached to the iron, electrons from suitable substrates could be led to or from the iron by radical reactions in the imidazole rings (20). However, obviously much more experimental evidence was needed before it could be regarded as conclusively proved that imidazole groups are attached to the iron. Some recent experiments have shed some more light upon this question.

The thioether bonds are strong enough to keep the cysteine residues attached to the hematin even during acid or enzymatic degradation of the protein. Under such circumstances the hematin part may be regarded as a colored label on the hemelinked peptide fragment produced by a partial degradation. Furthermore, since the molecule contains none but the two heme-linked cysteine residues, these serve as indicators to tell where the hematin is attached to the peptide chain. Tsou (29) obtained a brown-red split-product of cytochrome $c$ by peptic digestion. This "hemopeptide" contained the hematin part of cytochrome $c$ linked to a peptide fragment of the native molecule. The iron content $(2.21 \%)$ indicated a molecular weight of 2500 . The peptide was insoluble in weakly acidic solution, and the enzymatic properties of cytochrome $c$ had been profoundly altered by the digestion procedure. This was also the case with the hemopeptide obtained by digestion with papain, trypsin, and chymotrypsin.

Tuppy and Bodo (30) very recently published some interesting papers on the amino acid sequence in hemopeptides produced by partial hydrolysis of cytochrome $c$ with mineral acids, or by digestion with trypsin or subtilisin. The Sanger DNP technique was used after removing the hematin part by Paul's silver-splitting procedure. The sequence of ten constituent amino acids was established as a result of this work (Nos. 312 , see below): 
$\begin{array}{lllllllllll}2 & 3 & 4 & 5 & 6 & 7 & 8 & 9 & 10 & 11 & 12\end{array}$

Val-Glu $\left(\mathrm{NH}_{2}\right)$-Lys-Cy-Ala-Glu $\left(\mathrm{NH}_{2}\right)$-Cy-His-Thr-Val-Glu-Lys

$$
\mathrm{S} \text { hematin } \mathrm{S}
$$

Interestingly enough the bemopeptides from cytochromes $c$ of horse, beef, and pig had the same structure. In this part of the molecule near the prosthetic group, therefore, the cytochromes $c$ from these different sources are identical, though species differences may of course occur in other parts of the molecule.

Since Paléus, working in this institute, had produced pure peptic degradation products of the cytochromes $c$ from beef. chicken, and salmon. Tuppy came to Stockholm for some time to continue his research with Paléus (31).

These peptic hemopeptides were considerably purer than Tsou's. The iron content was $2.76 \%$, and there were 20 nitrogen atoms per one $\mathrm{Fe}$. The position of two additional amino acids in the molecule was established, and the sequence of amino acids in the immediate vicinity of the prosthetic group in the case of beef and salmon cytochromes $c$ can now be confidently asserted to be:

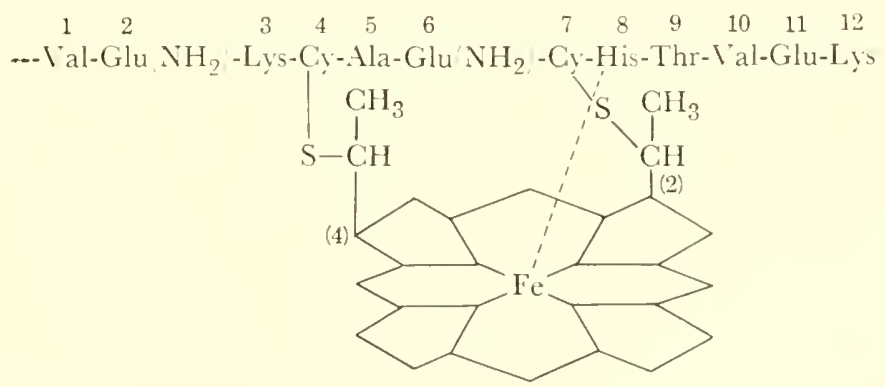

The chicken cytochrome $c$ showed a remarkable difference from the other ones: it contained serine in the place of alanine at position 5. The presence of a polar hydroxymethyl group instead of the nonpolar methyl group of alanine so close to the prosthetic group might be of functional importance, apart from the interesting example that it provides of a species difference.

Since two amino acids are interposed between the two heme- 
linked cysteine residues, it appeared to us that if the peptide chain has the shape of an $\alpha$-helix with 3.7 amino acid residues per turn, the cysteine residues would occur at the same side of the helix, perhaps in a favorable position for the thioether bonds.

When Professor Pauling visited Stockholm to receive his

(a)

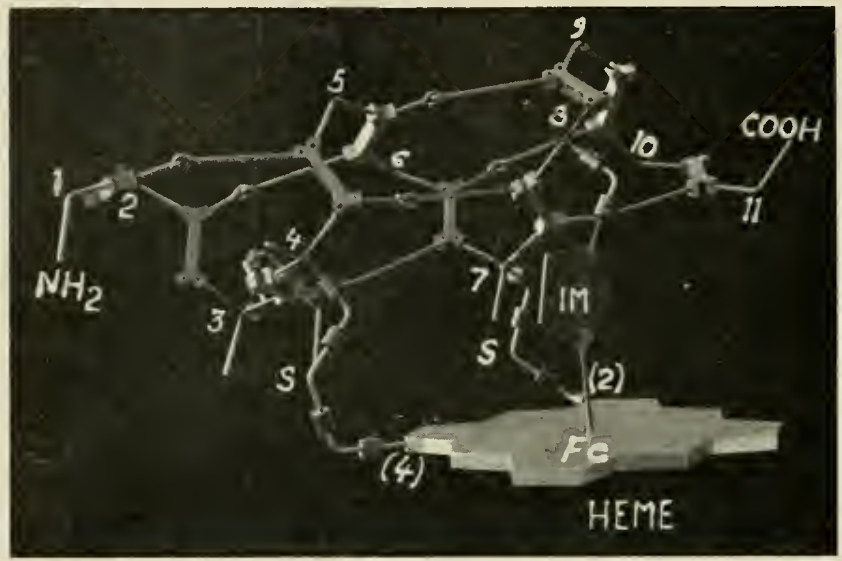

(b)

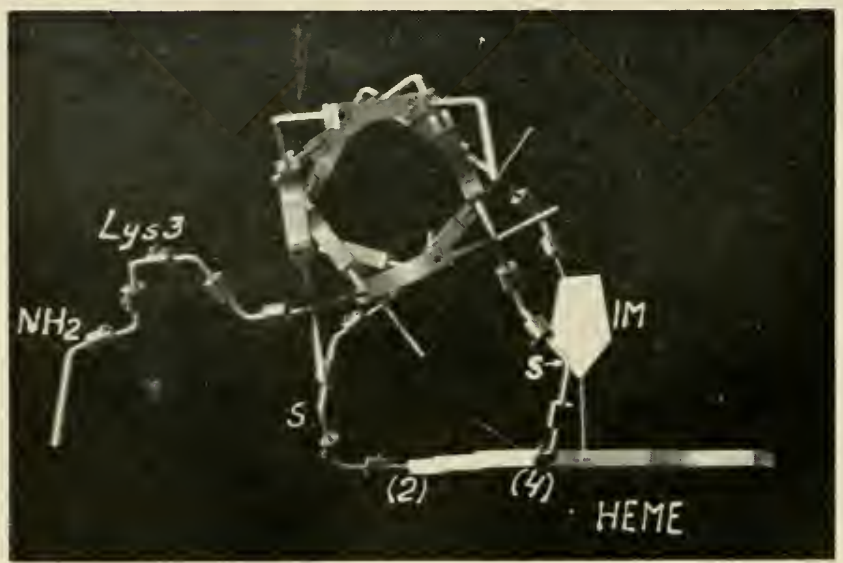

Fig. 1. Hemopeptide of cytochrome $c$ arranged as left-hand $\alpha$-helix. When the imidazole is bound to the Fe-atom, the heme is nearly parallel with the axis of the $\alpha$-helix. (a) Seen perpendicularly to helix axis. (b) Seen parallel with helix axis. $\alpha$-Carbons of the amino acids numbered as in formula. 
Nobel Prize, he kindly presented us his new model of an $\alpha$-helix. We then attached a model of a heme-molecule to the $\alpha$-helix, using the side chains of the cysteine residues of the hemepeptide (25). The heme was found to coincide easily with the cysteine residues to form the thioether bonds. In both left- and righthand $\alpha$-helices histidine imidazole in position 8 could be arranged sterically so that it could form a covalent bond with iron perpendicular to the plane of the heme. There are two theoretical ways in which the heme could be attached: either the heme side chain in position 2 to either cysteine residue number 4 or 7 . With a right-hand screw both structures seemed to fit equally well, but this kind of screw has so far not been found to exist in Nature. The left-hand screw, which probably is present, for example, in keratin, fits well only when the cysteine residue number 7 is attached to the heme side chain in the 2position, as indicated in the formula. In this case the axis of the peptide helix was practically parallel with the plane of the heme. See Figure 1.

Helices with 4.4 (" $\pi$ ") and 5.1 (" $\gamma$ ") amino acids per turn were also constructed, and it was found that in the $\pi$-helix the imidazole could with some strain form a covalent bond with the iron atom. In the $\gamma$-helix the imidazole was not able to form a bond with the iron atom. In none of the helices could any of the other side chains form a bond with the iron opposite to the bond with imidazole. On the other hand, if the Fe-imidazole bond was broken, the free amino group of the lysine residue in position 3 could form a covalent bond with the iron.

Even if the hydrogen bonds in the $\alpha$-helix were broken, it was hardly possible for any two of the side chains to form covalent bonds at both sides of the heme-disc. The question then arises how the hemopeptide can give typical hemochromogen absorption bands, indicating covalent bonds to the iron on both sides of the heme. When the hemopeptide is titrated with acid the two hemochromogen-forming groups are split off in two steps with $p \mathrm{~K}$ 's 3.5 and 5.8 (12). This is of course to be expected since in this case the iron-linked groups must be chem- 
ically different. In cytochrome $c$, both the iron-linked groups are liberated simultaneously, with $p \mathrm{~K}^{\prime} 2.12$ and $n=2$ (1). This means that the liberation of one of the groups, by the addition of a proton to the nitrogen atom, favors the disruption of the other bond, too, perhaps by changing the shape of the molecule. Chloride ions in acid solution give an interesting

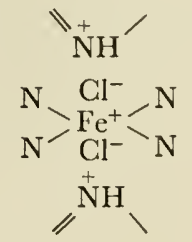

compound, "Cyt-2 $\mathrm{H}+2 \mathrm{Cl}-$," presumably by entering between the positively charged iron and nitrogen atoms. The magnetic measurements revealed the iron to have 3 unpaired electrons, and thus to be held by square $\mathrm{dsp}^{2}$ bonds, essentially covalent in the plane of the heme disc, but more ionic in the perpendicular direction.

The hemopeptide was studied in the Spinco ultracentrifuge

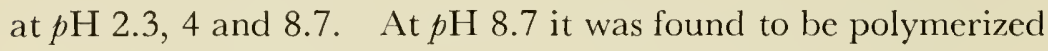
to a penta- or hexamer, whereas it was a monomer at $p H 2.3$. This finding explains the occurrence of hemochromogen spectral bands in alkaline solution; the iron may be coupled on one side to the imidazole of its own peptide, on the other side to the terminal valine- $\mathrm{NH}_{2}$, or the $\epsilon$-amino group of lysine, of another peptide, so that a five- or six-membered ring of hemopeptides is formed. The spectral bands of the reduced hemopeptide polymer were a little different from those of ferrocytochrome; however, when free histidine was added, the absorption bands became spectrophotometrically indistinguishable from those of ferrocytochrome $c$, and furthermore, depolymerization to monomers was observed in the ultracentrifuge. These results scem to speak strongly in favor of our earlier assumption that imidazole is attached to the iron atom on both sides in the native cytochrome $c$. 
Cytochrome $c$ has a remarkably low partial specific volume, $V=0.702-0.707$. After quantitative analysis of all the amino acids we could calculate the $V$ of the protein component; the value found was 0.737 . If now the weight of the heme moiety was added to the protein part under the assumption that it was built into the protein in a way not to increase its volume, $V$ would be equal to 0.700 . In fact this seems to be very nearly the case. As seen from Figure 1b, the peptide chain left after pepsin digestion occupies one quadrant of the space around the heme disc. If the other quadrants are filled with $\alpha$-helices in square packing around the cytochrome $c$ heme, this arrangement would explain as well the experimental value for the partial specific volume as the fact that the iron atom is inaccessible to oxygen. Electrons could be led to and from the iron via the free nitrogen atom of the imidazole ring, because this atom, according to our model, would be situated nearer to the surface of the molecule and probably could react with suitable oxidizing or reducing agents, perhaps by chelate formation with heavy metal atoms of other enzyme systems.

The recent advances in our knowledge of the cytochrome $c$ structure encourage us to believe that the whole problem can be solved in the not too distant future. A few years ago such a hope would have appeared overly optimistic.

\section{The Old Yellow Enzyme, a Conjugated Protein with}

\section{Dissociable but Functionally Immobile Coenzyme}

This enzyme is interesting from a historical point of view, since it was the first oxidation enzyme that was crystallized; the first that was reversibly split into protein and coenzyme: and the first from which a pure coenzyme was prepared; and still its physiological function is entirely unknown.

Warburg and Christian (35), the discoverers of the "O.Y.E.," observed that a yellow dye, which was liberated from a high molecular moiety by treatment with methanol, showed a greenish fluorescence. Kuhn and Moruzzi (7) observed that 
the fluorescence of riboflavin disappeared in alkaline solution, and that the disappearance followed a dissociation curve with $p \mathrm{~K}^{\prime}=10.2$ and $n=1$. The corresponding acid group was considered to be the imino group NH in the 3-position, and in analogy with the quenching of the fluorescence by the dissociation of a proton from this group, Kuhn and Boulanger (6) explained the absence of fluorescence in the O.Y.E. by a coupling of this imino group to an unknown group in the protein.

In 1937 (18), Theorell used the disappearance of the fluorescence of FMN upon recombination with the apoprotein as the basis for "titrating" this protein with solutions of FMN.

Since nowadays very sensitive photomultipliers and rapid recorders are available, we recently decided to study the kinetics of the dissociation and reassociation of the O.Y.E. by the aid of fluorescence measurements (26). No doubt it would have been technically possible to carry out such studies spectrophotometrically with the aid of the rapid-flow technique, since the absorption band at $445 \mathrm{~m} \mu$ of free FMN shifts to $465 \mathrm{~m} \mu$ in the O.Y.E. Such combination experiments had been carried out some years ago by Theorell and Chance (24) in the case of DPNH + alcohol dehydrogenase (ADH), where the absorption band of DPNH at $340 \mathrm{~m} \mu$ shifts to $325 \mathrm{~m} \mu$ when DPNH is combined with $\mathrm{ADH}$.

In the case of the O.Y.E. we actually found the same $k_{1}$ by the aid of a recording spectrophotometer, at $495 \mathrm{~m} \mu$, and by fluorescence measurements on the same solutions. The experiments had to be carried out in concentrations high enough to give enough light absorption, and with high concentrations of phosphate to reduce the rate of the association reaction to measurable dimensions.

It was thus found that the fluorescence method has some distinct advantages over spectrophotometrical methods. The reason is that spectrophotometry is practically possible only down to concentrations around $10^{-6} \mathrm{M}$, whereas by the aid of 
fluorescence measurements it is possib)le to work in much more dilute solutions, in the case of FMN down to $10^{-9} \mathrm{M}$. As a matter of fact the region $10^{-6}$ to $10^{-9} M$ is even optimal for quantitative fluorescence work, since at concentrations higher than $10^{-6} M$ certain deviations caused by light absorption in the cuvette become appreciable.

The possibility of working in very dilute solutions and varying the concentrations by a factor of $10^{3}$ simply by changing the sensitivity of the amplifying system was found to be surprisingly valuable. The combination reaction ("on reaction") between FMN and apoprotein is very rapid, under certain conditions of the same order of magnitude as for DPNH $+\mathrm{ADH}$. But at the high dilutions that we can now apply, the reaction velocity could be accurately studied without the aid of "rapidflow" apparatus and oscillograph.

From the simple relationship

$$
1 \text { FMN + } 1 \text { apoprotein } \underset{k_{2}}{\stackrel{k_{1}}{\rightleftharpoons}} 1 \text { O.Y.E. }
$$

one might expect $k_{1}$ to be of second order, $k_{2}$ of first order. However, this could not be taken for granted since more than one group in FMN combines with the protein. Thus it was conceivable that half-coupled intermediates could be formed and disturb the simple kinetic relations. However, this was found not to be the case. It was concluded that if half-coupled intermediates existed, they reacted far too rapidly to interfere with the kinetics of the over-all process.

\section{THE DISSOCIATION VELOCITY (27)}

First of all, to our surprise we found that the dissociation velocity and the degree of dissociation of O.Y.E. in pure water or in solutions with anions which form weak acids was immeasurably low. Increasing the acidity down to $p \mathrm{H} 4$ in acetate (at still lower $p \mathrm{H}$ the O.Y.E. begins to get destroyed), or increasing the $p \mathrm{H}$ to 11 in glycine, still caused no dissociation of O.Y.E. However, when salts of strong acids were added, the enzyme was found to dissociate readily. 
Dissociation of O.Y.E. By Different Anions $(0.1 M)$ at $p$ H $4.40,23.5^{\circ} \mathrm{C}$.

\begin{tabular}{lc}
\multicolumn{1}{c}{ Salt } & $\begin{array}{c}k_{2} \times 10^{4} \\
\text { sec. }\end{array}$ \\
\hline Na formate & 0 \\
$\mathrm{Na}$ acetate & 0 \\
$\mathrm{Na} \mathrm{F}^{\mathrm{NaH}} \mathrm{PO}_{4}$ & 0 \\
$\mathrm{Na}_{2} \mathrm{SO}_{4}$ & 1 \\
$\mathrm{NaCl}$ & 9 \\
$\mathrm{NaNO}$ & 33 \\
$\mathrm{NaBr}$ & 63 \\
& 99 \\
\hline
\end{tabular}

When anions of strong acids are present, the value of $k_{2}$ is greatly influenced by the hydrogen ion concentration (see Figure 2). In the acid range $k_{2}$ was found to be proportional both to $\left[\mathrm{H}^{+}\right]$and $\left(\mathrm{Cl}^{-}\right)$.

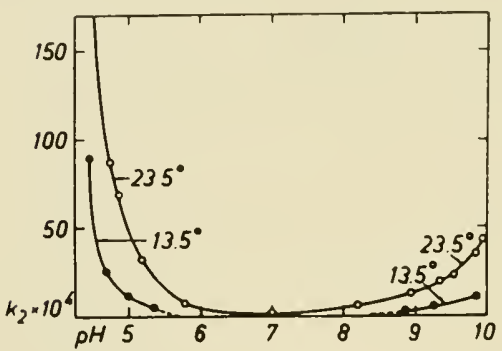

Fig. 2. The value of $k_{2}$ for O.Y.E. as a function of $p \mathrm{H}$ in $0.4 \mathrm{M}$ $\mathrm{NaCl}$ (from ref. 24).

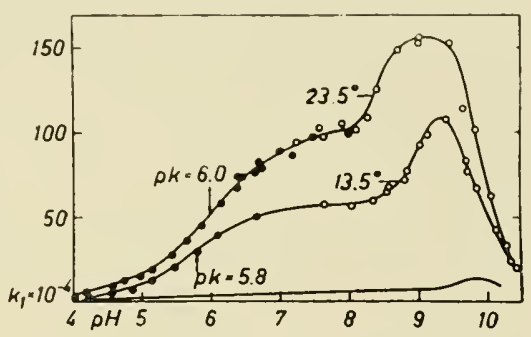

Fig. 3. The value of $k_{1}$ for FMN + apoprotein as a function of $p \mathrm{H} . \bullet \bullet-$ in $0.1 M$ acetate buffer. ○०० in $0.1 M$ glycine buffer. The lower curve (experimental points omitted) is $k_{1}$ for riboflavin + apoprotein at $13.5^{\circ} \mathrm{C}$. (from ref. 24 ).

The first splitting of the O.Y.E., in 1934 (17), was accomplished by dialysis against weak hydrochloric acid. As we can see now, this was a fortunate choice, since the velocity of splitting is proportional to the square of the concentration of 
hydrochloric acid. The value of $k_{2}$ increases fourfold from 13.5 to $23.5^{\circ} \mathrm{C}$.

\section{THE ASSOCIATION VELOCITY (27)}

In order to study the association velocity constant, $k_{1}$, as a function of $p \mathrm{H}$ we used acetate from $p \mathrm{H} 4$ to 7, and glycine from $p \mathrm{H} 7$ to 10.5 , since neither of these buffer substances was found to interfere with the "on" velocity. The value of $k_{1}$ was found to vary with $p \mathrm{H}$ in a most interesting way (see Figure 3 ). From $p \mathrm{H} 4$ to $8 k_{1}$ at $23.5^{\circ} \mathrm{C}$. followed a monovalent dissociation curve with $p \mathrm{~K}^{\prime}=6$. This coincides with the second dissociation constant of the phosphoric acid residue of FMN. From $p \mathrm{H} 8$ to 9 there is a new increase in $k_{1}$ up to a maximum around pH 9 at $23.5^{\circ} \mathrm{C}$. or 9.3 at $13.5^{\circ} \mathrm{C}$. With increasing alkalinity

\section{TABLE II}

The Inhibition of $k_{1}$ By Polyvalent Anions and Its Counteraction by MonoVALENT ANions $\left(p \mathrm{H}=7.0,23.5^{\circ} \mathrm{C}\right.$.)

\begin{tabular}{lcc}
\hline & Salts & $\begin{array}{c}k_{1} \times 10^{-4} \\
M^{-1} \mathrm{sec}^{-1}\end{array}$ \\
\hline None & & 90 \\
Na-phosphate, $\Gamma / 2=0.1$ & & 14 \\
" & & 64 \\
$0.033 M \mathrm{Na}_{2} \mathrm{SO}_{4}$ & & 50 \\
$0.05 \mathrm{M} \mathrm{Na}$ citrate & & 10 \\
" & $+0.4 M \mathrm{NaCl}$ & 40 \\
$0.01 M \mathrm{Na}$ versene & $+0.4 M \mathrm{Na}$ acetate & 25 \\
" & $+0.4 M \mathrm{NaCl}$ & 19 \\
\hline
\end{tabular}

$k_{1}$ falls off very rapidly; $k_{1}$ is $=1.5 \times 10^{6} M^{-1} \mathrm{sec}^{-1}$ at $p \mathrm{H} 9$, thus roughly half as great as $k_{1}$ for $\mathrm{DPNH}+\mathrm{ADH}$, and five times greater than the "on" velocity for DPN + ADH (cf. below). Anions decrease $k_{1}$; the higher the charge, the stronger the effect. Secondary phosphate, for example, inhibited $k_{1}$ very considerably, whereas primary phosphate did not. The inhibitory effect of polyvalent anions was to a great extent counteracted by monovalent anions. 
These peculiar effects are likely to be explained by the fact that polyvalent anions are more strongly bound to many proteins than are monovalent anions, but they can be displaced by higher concentrations of monovalent anions. In the same manner, FMN itself is displaced from the protein by chloride ions (increased dissociation velocity).

The influence of the chloride ion in the absence of polyvalent anions on the association velocity was studied at varied $p \mathrm{H}$ and temperature (see Figure 4).

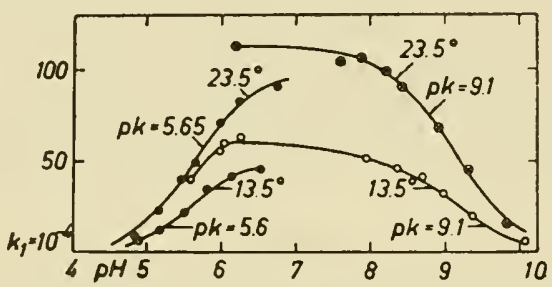

Fig. 4. The value of $k_{1}$ as a function of $p \mathrm{H}$ in $0.4 \mathrm{M} \mathrm{NaCl}$.

$M$ acetate buffer. $\otimes \otimes \otimes$ and $000,0.1 \mathrm{M}$ glycine buffer (from ref. 24).

At alkaline reaction, $0.4 M$ chloride moves the part of the $k_{1}$ curve descending above $p \mathrm{H} 9$ toward lower $p \mathrm{H}$ values and abolishes the $k_{1}$ maximum at $p \mathrm{H} 9$, and gives a $k_{1}$ following a monovalent dissociation curve with $p \mathrm{~K}=9.1$. We think this is a salt effect on the $p \mathrm{~K}$ of primary amino groups attached to the phosphoric acid residue of FMN.

Complete reversibility of the reaction

$$
\text { FMN + apoprotein } \underset{k_{2}}{\stackrel{k_{1}}{\rightleftharpoons}} \text { O.Y.E. }
$$

requires that

$$
\frac{k_{2}}{k_{1}}=K
$$

where $K$ is the equilibrium constant. We were able to determine all three of the constants separately. The values of $k_{2}$ and 
$k_{1}$ were determined both from the initial velocities at $t=0$ and from different points on the curves before equilibrium was reached; $K$ was, of course, determined from the amount of free FMN at equilibrium starting from different concentrations of

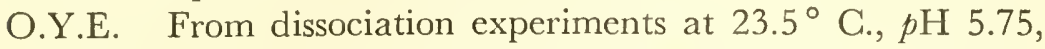
$0.4 \mathrm{M} \mathrm{NaCl}$ we found $k_{2}=13.5 \times 10^{-4} \mathrm{sec} .^{-1}$, and $K=0.71 \times$ $10^{-8}$. From these values

$$
k_{1}=\frac{13.5 \times 10^{-4}}{0.71 \times 10^{-8}}=19 \times 10^{4} M^{-1} \text { sec. }^{-1}
$$

The direct determination of $k_{1}$ from the corresponding association experiment was

$$
k_{1}=17 \times 10^{4} \mathrm{M}^{-1} \text { sec. }^{-1}
$$

These experiments thus gave confirmation by kinetic methods of the perfect reversibility of the reaction.

\section{INTERACTION OF RIBOFLAVIN WITH THE APOPROTEIN}

In 1936, Kuhn and Rudy (8) found that apoprotein and an excess of riboflavin could substitute for O.Y.E. in the enzymatic activity test. This observation made it likely that riboflavin could form an active compound with the apoprotein, but experimental proof has been lacking. It was therefore of interest to investigate whether the apoprotein could extinguish the fluorescence of free riboflavin. This was found to be the case. As seen from the lowest curve in Figure 3, $k_{1}$ is approximately 10 times lower than for FMN, and there is nothing which simulates a dissociation curve in the $p \mathrm{H}$ region 4 to 8 , just as would have been expected. A maximum appears at $p \mathrm{H}$ 9.8, and $k_{1}$ decreases at higher $p \mathrm{H}$ values. The riboflavin-apoprotein compound is much more dissociated than O.Y.E., mainly depending on the fact that $k_{2}$ is comparatively high, $1000 \times 10^{-4} \mathrm{sec} .^{-1}$ in acetate $p \mathrm{H} \mathrm{7,} \mathrm{where} \mathrm{the} k_{2}$ for O.Y.E. is certainly lower than 0.01 $\times 10^{-4} \mathrm{sec}^{-1}$. This illustrates the importance of the phosphoric acid group for establishing functioning holoenzymes at reasonable levels of coenzymes. 
THE CHEMICAL NATURE OF THE FMN-BINDING GROUPS IN THE PROTEIN OF O.Y.E.

The strong influence of anions on the stability of the FMNprotein bonds directly suggests that the bonds between the phosphoric acid residue of FMN and the protein are saltlike themselves. The effect of $p \mathrm{H}$ and anions on the FMN-apoprotein system indicates that FMN is linked to the protein in very much the same manner as an anion to a weakly basic ion exchanger.

There are several reasons to believe from the kinetic experiments that primary amino groups in the protein serve as binding sites for the phosphoric acid residue.

1. Above $p \mathrm{H} 9$ a sharp drop of $k_{1}$ occurs, corresponding to the expected discharge of $-\mathrm{NH}_{3}^{+}$groups in this region.

2. Lowering the temperature by $10^{\circ}$ shifted the $k_{1}$ curve upwards on the $p \mathrm{H}$ scale by approximately the amount expected for amino groups $(0.3 p \mathrm{H}$ unit).

3. $0.4 M \mathrm{NaCl}$ shifted this part of the $k_{1}$ curve in the opposite direction, as expected from the known influence of the ionic strength on the $p \mathrm{~K}$ of primary amino groups.

When we move from neutrality toward acid reaction the positive charge of the amino groups keeps constant, but the second negative charge of

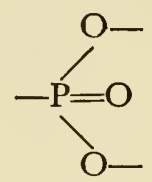

begins to disappear by the addition of a proton $(p \mathrm{~K} \sim 6)$. The observed effects of temperature change and ionic strength were the same on the $k_{1}$ curve and on the titration curve of FMN. The decrease in $k_{1}$ toward the acid side thus is attributed to the change of FMN from the divalent to the monovalent state.

It is uncertain how the peculiar change in $k_{1}$ between $p \mathrm{H} 8$ and 9 should be interpreted; the protein could change shape, 
increasing the effectiveness of the collisions with FMN molecules, but other explanations are also possible.

Some recent experiments confirmed the role of primary amino groups (11) in a convincing way.

Acetylation of a small percentage of the primary amino groups of the apoprotein with acetic anhydride in acetate solutions resulted in a strong reduction of the combination velocity. If O.Y.E. is acetylated in the same way, the enzyme dissociates. Neither of these effects is due to acetylation of thiol or tyrosine$\mathrm{OH}$ groups, and must therefore depend upon acetylation of amino groups.

Formaldehyde in water did not cause any rapid appearance of free FMN from O.Y.E. This was expected, since formaldehyde reacts only with uncharged $-\mathrm{NH}_{2}$ groups, and in the O.Y.E. the

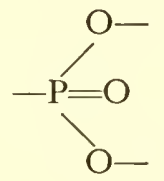

stabilizes the positive charge of the bound $-\mathrm{NH}_{3}^{+}$groups. In the presence of sodium chloride, however, formaldehyde increased the dissociation velocity. Association experiments gave very clear results: a decrease in $k_{1}$ was observed immediately with formaldehyde concentrations as low as $0.02 M$. Just as might be expected, the inhibition increased with $p \mathrm{H}$.

The strong and immediate effect of low concentrations of formaldehyde suggests that the FMN-binding amino groups are in an exposed position on the surface of the protein molecule. This is not surprising, since it may be a prerequisite for their ability to combine with FMN.

The question arose which protein group is attached to the imino group in the flavine ring system, thus quenching the fluorescence. Weber (36) suggested it might be a tyrosine hydroxyl group, without giving any experimental evidence in this direction. We have experimental results which show that 
tyrosine may be important in the quenching of FMN. O.Y.E. does not contain any reactive sulfhydryl groups. Therefore, if iodine were added to the apoprotein it could be expected to enter mainly into the 3- and 5-positions in the benzene ring of tyrosine, thereby greatly increasing the acidity of the tyrosine hydroxyl group. In fact it was found that very small quantities of iodine were sufficient to decrease the coupling velocity of FMN to apoprotein very substantially. Ninety per cent of the disappeared amount of iodine was recovered in diiodotyrosine. The results suggest that in the O.Y.E. tyrosine-OH may be attached to the imino group (13) - or its enol form-presumably by a hydrogen bond. This does not exclude the possibility that other groups as well are important in this binding.

There is at present no method available for studying the kinetics of the reaction between leuco-FMN $\left(\mathrm{FMN}-\mathrm{H}_{2}\right)$ and the apoprotein. However, the remarkably high normal potential of the O.Y.E., as determined by Kuhn and Boulanger (6), $-0.06 \mathrm{v}$., as compared with that of free riboflavin, $-0.185 \mathrm{v}$., indicated that the leuco-O.Y.E. should be around 10,000 times less dissociated than the O.Y.E. In the presence of suitable anions, however, measurable values of the dissociation constant could be expected, and some information on the influence of salts on $k_{2} / k_{1}$ for leuco-O.Y.E. could be hoped for. C. S. Vestling, while working in this laboratory in 1954, therefore undertook a reinvestigation of the normal potential of O.Y.E. Vestling (33) found $E_{0}^{\prime}$ at $p \mathrm{H} 7$ and $30^{\circ} \mathrm{C}$. of $-0.123 \pm 0.006 \mathrm{v}$., thus $63 \mathrm{mv}$. lower than Kuhn's and Boulanger's value. The slope, $E_{0}^{\prime} / \Delta p \mathrm{H}$ was found $=-0.06 \mathrm{v}$. from $p \mathrm{H} 4.8$ to 8.9

The new value sets the ratio of the dissociation constants of O.Y.E. and leuco-O.Y.E. a little higher than 100.

As could be expected, the anion effects were soon found to be of more general occurrence. Walaas and Walaas (34), in this institute, found strong inhibitory effects of anions on the activity of $d$-amino acid oxidase in the following order:

$$
\mathrm{I}^{-}>\mathrm{Br}^{-}>\mathrm{NO}_{3}^{-}>\mathrm{Cl}^{-}>\mathrm{PO}_{4}=>\mathrm{SO}_{4}=>\mathrm{CH}_{3} \mathrm{COO}^{-} \gg \mathrm{F}^{-}
$$


The inhibitions were remarkably strong. For instance, the chloride ion in a concentration of $0.085 \mathrm{M}$ gave $50 \%$ inhibition. Increased dissociation of the FAD-protein compound must be the reason for the inhibition, since it could be reversed by raising the concentrations of FAD.

The experiments described here seem to indicate that kinetic measurements of the velocities with which coenzymes and proteins associate or dissociate are going to be a most valuable instrument for revealing the chemical nature of the binding groups. We can expect to find a great variation in binding types in coenzyme-enzyme and enzyme-substrate systems; and this is in fact what we call enzyme specificity.

\section{The Liver Alcohol Dehydrogenase, an Enzyme with Dissociable and Mobile Coenzyme}

In the last few years a great deal of interesting work has been carried out in order to elucidate the reaction mechanism of pyridine enzymes; see, for example, reference (10). It would lead us too far to review this whole field in this paper. Instead we shall select for consideration just one DPN-enzyme, the horse liver alcohol dehydrogenase "ADH," because it has some especially interesting properties and has turned out to be a very suitable object for studies with different methods.

Bonnichsen and Wassén (2) crystallized the enzyme in 1948 in a very satisfactory yield, around $1 \mathrm{~g}$. from one horse liver. The pure enzyme is thus easily available.

It occurred to us (23) that a shift of the position of the 340-m $\mu$ absorption band of DPNH could possibly result from the coupling with $\mathrm{ADH}$, in analogy with the band shift from $445 \mathrm{~m} \mu$ in free FMN to $465 \mathrm{~m} \mu$ in the O.Y.E. This was found to be the case, though the $340-\mathrm{m} \mu$ band of DPNH moved in the opposite direction to $325 \mathrm{~m} \mu$, when $\mathrm{ADH}$ was added (see Figure 5).

Chance and Neilands (4) later observed a similar band shift in DPNH + lactic dehydrogenase. However, it is not a 
general phenomenon, since it does not occur in yeast $\mathrm{ADH}$, triosephosphate, or glutamic dehydrogenases.

The displacement of the band enabled us to establish spectrophotometrically that $1 \mathrm{~mol}$ ADH binds 2 mols of DPNH. For the sake of convenience we shall denote $1 / 2 \mathrm{ADH}$ as $\mathrm{ADH}^{\prime}$.

Furthermore, by using rapid spectrophotometry Theorell and Chance (24) could determine the velocity of the band shift,

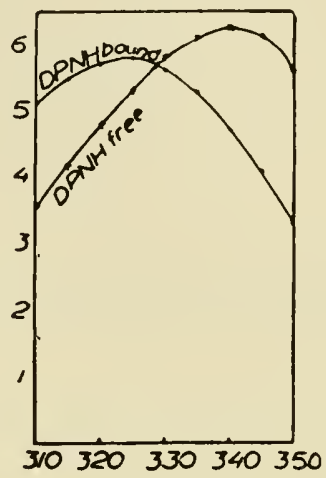

Fig. 5. Millimolar extinction of free and ADH-bound DPNH. Ordinate: $\epsilon \mathrm{cm}^{-1} \times \mathrm{mM}^{-1}$. (From ref. 23.)

and thus obtained the value $4 \times 10^{6} \mathrm{M}^{-1}$ sec. $^{-1}$ for the velocity constant $\left(k_{1}\right)$ of the combination between DPNH and ADH.

In the equilibrium

$$
\frac{[\mathrm{DPNH}]\left[\mathrm{CH}_{3} \mathrm{CHO}\right]\left[\mathrm{H}^{+}\right]}{[\mathrm{DPN}]\left[\mathrm{C}_{2} \mathrm{H}_{5} \mathrm{OH}\right]}=K
$$

Racker (15), using small amounts of yeast ADH, had found $K$ to be constant and independent of $p \mathrm{H}$, as required by the formula. The value for $K$ was close to $10^{-11}$.

Since $K$ is a function of purely thermodynamic parameters, it was no surprise that we found the same value with low concentrations of liver ADH. But in the liver the concentration of the enzyme ( $\sim 1 \mathrm{~g}$. per kilogram wet weight) is not at all low enough to be negligible. 
Determinations of $K$ with varied amounts of $\mathrm{ADH}$ and at $p \mathrm{H} 6.4$ to 10 revealed the fact that $K$ was increased by a factor of about 200 , in the presence of a slight excess of $\mathrm{ADH}$, in the $p \mathrm{H}$ region 6.4 to 7.8 . From $p \mathrm{H} 7.8$ to 10 this factor decreased gradually from 200 to 3 .

On the basis of the theories of Clark and his associates for the redox potential of hemochromogens our findings could be readily explained. The factor, by which the equilibrium constant $K$ is multiplied, is equal to the ratio between the dissociation constants $\left(D_{\text {ox }}\right.$ and $D_{\text {red }}$ ) of the oxidized and the reduced enzyme-coenzyme compounds. And the redox potential of the free coenzyme is changed by the coupling to enzyme protein according to a simple formula

$$
E_{0}^{\prime}(\text { holoenzyme })=E_{0}^{\prime}(\text { free coenzyme })+\frac{R T}{n F} \ln \frac{D_{\text {ox }}}{D_{\mathrm{red}}}
$$

In the case of $\mathrm{ADH}$ this means that $E_{0}^{\prime}$ for the holoenzyme is 70 mv. higher than $E_{0}^{\prime}$ for the free DPN-DPNH system in the physiological $p \mathrm{H}$ range.

The calculated $E_{0}^{\prime}$ values for the holoenzyme at varied $p \mathrm{H}$ indicated the slope $d E_{0}^{\prime} / d p \mathrm{H}$ to be $-0.030 \mathrm{v}$. from $p \mathrm{H} 6.4$ to 7.8 , and $-0.060 \mathrm{v}$. above $p \mathrm{H} 7.8$. This indicated the existence of an acid group with $p \mathrm{~K}^{\prime}=7.8$ in the DPN-ADH. There might be a corresponding group in DPNH·ADH with a $p \mathrm{~K}$ around 10, but convincing experimental data are difficult to obtain at this high alkalinity. The chemical nature of the group with $p \mathrm{~K}^{\prime}=7.8$ has not yet been established, but as we shall discuss below, it is certainly of decisive importance for the kinetics of the whole enzyme system.

From a thermodynamic point of view, DPN would appear very unsuitable for oxidizing ethanol. As easily seen from the value of $K, 10^{-11}$, the reaction between DPN and ethanol plus catalytic amounts of $\mathrm{ADH}$ at $p \mathrm{H}=7$ would come to equilibrium when, for example, the ratio DPN:DPNH is $=1$, and the ratio ethanol:aldehyde is $=10,000$. After the coupling to the protein this last ratio is much more favorable for ethanol oxi- 
dation around 50 at $p \mathrm{H} \mathrm{7,5}$ at $p \mathrm{H} 8$. Since in the liver the aldehyde is rapidly removed by an aldehyde oxidase, alcohol oxidation can proceed smoothly.

In the case of yeast $A D H$ the $D_{\text {ox }}$ and $D_{\text {red }}$ are not very different, and accordingly there is practically no "protein concentration effect" on the equilibrium or the redox potential.

This is an illustration of how enzymes adapt coenzymes for quite different functions. Yeast produces alcohol from acetaldehyde, whereas liver ADH catalyzes the opposite reaction. We might say that the same alcohol that is produced by aldehyde oxidizing DPNH in the yeast is oxidized by DPN back to aldehyde in the liver. For these opposite purposes Nature has been able to use the same DPN-DPNH system, by producing proteins with different properties.

Theorell and Chance derived some useful formulas for the system

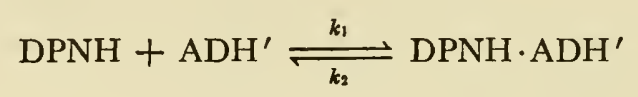

$\mathrm{DPNH} \cdot \mathrm{ADH}^{\prime}+\mathrm{CH}_{3} \cdot \mathrm{CHO}+\mathrm{H}^{+} \stackrel{k_{6}}{\rightleftharpoons} \mathrm{DPN} \cdot \mathrm{ADH}^{\prime}+\mathrm{C}_{2} \mathrm{H}_{6} \mathrm{OH}$

$$
\mathrm{DPN} \cdot \mathrm{ADH}^{\prime} \underset{k_{s}}{\stackrel{k_{t}}{\rightleftharpoons}} \mathrm{DPN}+\mathrm{ADH}^{\prime}
$$

The initial reaction velocity (at $t \sim 0$ ), in terms of reacted substrate and coenzyme molecules per $1 \mathrm{ADH}^{\prime}$-molecule per time unit ("turnover number"), was under these assumptions calculated to be

$$
\frac{1}{e} \times \frac{d a}{d t}=\frac{1}{\frac{1}{k_{1} x}+\frac{1}{\left[\mathrm{H}^{+}\right] k_{4} a}\left(1+\frac{k_{2}}{k_{1} x}\right)+\frac{1}{k_{3}}}
$$

An analogous formula can be derived for the reverse reaction $\left(k_{5}\right.$ instead of $k_{1} ; k_{6}$ instead of $\left[\mathrm{H}^{+}\right] k_{4} ; k_{2}$ instead of $k_{3} ;$ and $a$ then $=$

$$
\left.\left[\mathrm{C}_{2} \mathrm{H}_{5} \mathrm{OH}\right], x=[\mathrm{DPN}]\right)
$$


The formulas (4) and (4a) lead to some new considerations on the nature of the Michaelis constant, $K_{m}$, which had formerly been very often interpreted as representing dissociation constants of enzyme-substrate compounds. This is not at all the case, if formulas (1) to (3) and therefore also (4) and (4a) are valid. It was found, for example, that $K_{m}$ for DPNH is = $k_{3} / k_{1}$; thus the ratio between the dissociation velocity constant for $\mathrm{DPN} \cdot \mathrm{ADH}^{\prime}$ and the association velocity constant of DPNH $+\mathrm{ADH} .^{\prime}$

The $K_{m}$ for acetaldehyde in this system does not represent a dissociation constant of an aldehyde-enzyme complex, but should be $=k_{3} / k_{4}\left[\mathrm{H}^{+}\right]$. $^{*}$

Some other interesting formulas follow directly from equations (1) to (3), when the whole system is considered to be in the equilibrium state:

$$
\frac{k_{1} k_{4} k_{3}}{k_{2} k_{6} k_{5}}=\frac{1}{K}
$$

and

$$
\frac{D_{\text {ox }}}{D_{\text {red }}}=\frac{k_{1} k_{3}}{k_{2} k_{5}}=\frac{k_{6}}{k_{4} K}
$$

The rather uncertain values of $k_{1}$ to $k_{6}$, obtained in 1951, when inserted in (5) gave a value of $K$ that was of the right order of magnitude $\left(0.2 \times 10^{-11}\right)$. Since this was the first time that direct kinetic and equilibrium data had been correlated for an enzyme system, it was of considerable interest to collect more experimental data. It should be emphasized that formulas (5) and (6) give excellent possibilities to check the validity of such data, because $K$ and $D_{\text {ox }} / D_{\text {red }}$ were determined independently from equilibrium data. New determinations of $k_{1}$ to $k_{6}$ have

* It should be noticed that in previous publications the values given for $k_{4}$ include $\left[\mathrm{H}^{+}\right]$as a factor. We shall here use $k_{4}$ for the true reaction velocity constant; thus

$$
\frac{d a}{d t}=k_{4}\left[\mathrm{DPNH} \cdot \mathrm{ADH}^{\prime}\right]\left[\mathrm{CH}_{3} \mathrm{CHO}\right]\left[\mathrm{H}^{+}\right]
$$




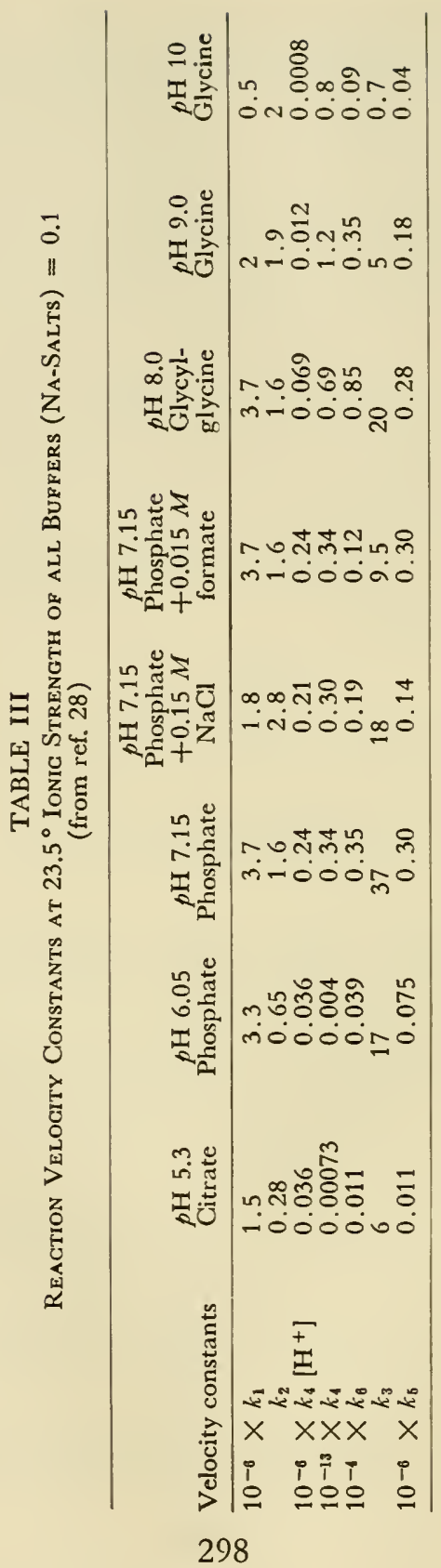


recently been made over the $p \mathrm{H}$ range 5.3 to 10 , and in addition the influence of some electrolytes was studied (28). Again fluorescence measurements were found to be superior to spectrophotometry. DPNH fluoresces but DPN does not. The sensitivity of the method is so great that a reaction cycle involving a concentration change of DPNH less than $1 \mu$ molar can easily be recorded.

It was found possible to calculate all six of the constants from equations (4) and (4a) from initial reaction velocities in a test system with systematic variations in the concentrations of DPN, DPNH, alcohol, and aldehyde. Some data are summarized in Table III.

Let us first consider the influence of varied $p \mathrm{H}$. The "on" velocities for DPNH $\left(k_{1}\right)$ and DPN $\left(k_{5}\right)$ both show a maximum at $p \mathrm{H} 7$ to 8 , and decrease toward alkaline and acidic reaction. The decrease on the alkaline side follows monovalent dissociation curves with $p \mathrm{~K}^{\prime}$ around 9 for both $k_{1}$ and $k_{5}$. This drop is reasonably explained, in analogy with O.Y.E., by assuming that the pyrophosphate group of $\mathrm{DPN}(\mathrm{H})$ is linked to positively charged groups, perhaps again $-\mathrm{NH}_{3}^{+}$, in the protein. Kaplan and Ciotti (5) have recently shown that splitting the pyrophosphate group by snake venom pyrophosphatase releases reduced nicotinamide mononucleotide from DPNH.ADH, and they concluded that the pyrophosphate grouping is essential for the attachment of DPNH to the liver enzyme.

Toward the acidic side $k_{5}$ drops sooner $\left(p \mathrm{~K}^{\prime}=6.5\right)$ than $k_{1}\left(p \mathrm{~K}^{\prime}=5.5\right)$. The reason for this we do not know, nor do we understand entirely why $k_{1}$ is $10-100$ times higher than $k_{5}$. We think both facts are connected with the extra plus charge in the pyridinium ring of $\mathrm{DPN}^{+}$and with the presence of two more or less positively charged atoms of zinc in the ADH molecule.

The "off" velocity of DPNH $\left(k_{2}\right)$ is nearly independent of $p \mathrm{H}$ from 7 to 10 , but this is by no means true for the DPN.ADH' $\left(k_{3}\right)$. The $k_{3}$ values lie very close to a monovalent dissociation curve with $p \mathrm{~K}^{\prime}=7.8$. Both $k_{2}$ and $k_{3}$ decrease below $p \mathrm{H} 7$ 
along monovalent dissociation curves of $p \mathrm{~K}$ 6.3-6.4. The "off" velocities for these coenzyme-enzyme complexes are thus in contradistinction to O.Y.E. maximal around neutrality. This arrangement is obviously favorable for an enzyme system with a mobile coenzyme.

As already mentioned above the independent equilibrium data for $D_{\text {ox }} / D_{\text {red }}$ gave a calculated curve for the redox potential of the holoenzyme with an increase in $d E_{0}^{\prime} / d p \mathrm{H}$ at $p \mathrm{H} 7.8$ from -0.030 to $-0.060 \mathrm{v}$., indicating the presence of an acid group in DPN.ADH that was absent, or at least had a much higher $p \mathrm{~K}^{\prime}$, in DPNH.ADH.

Why is $k_{3}$ so nicely $p \mathrm{H}$-dependent in the alkaline range, whereas $k_{2}$ is not? This is very probably connected with the fact that the pyridine ring in DPN has a positive charge in contradistinction to DPNH. If now a group in the protein, $\mathrm{H}^{+} \mathrm{X}^{-}-\mathrm{R}$, is close to the pyridine and in this position has a $p \mathrm{~K}$ of 7.8 , this would result in an extra stabilization (low $k_{3}$ ) of the DPN-ADH linkage in alkaline solution by the ion attraction in

$$
\mathrm{Py}+\mathrm{X}-\mathrm{R}
$$

When $\mathrm{X}-\mathrm{R}$ adds a proton this stabilization would disappear along a dissociation curve, as is indeed observed.

The proximity of $\mathrm{Py}^{+}$to $\mathrm{X}-\mathrm{R}$ would result in a lowering of the $p \mathrm{~K}^{\prime}$-value for $\mathrm{X}-\mathrm{R}$. In free $\mathrm{ADH}$ this group may very well have a $p \mathrm{~K}^{\prime}$ value of 9 or 10 , and could thus be a-SH or tyrosyl-OH group. The low value for $k_{2}$ indicates that the DPNH-ADH linkage is stabilized by some extra bond in addition to those which must be fairly equal for both the oxidized and the reduced compounds (e.g., pyrophosphate to $-\mathrm{NH}_{3}^{+}$). This is made certain by the fact that the $340 \mathrm{~m} \mu$ band of DPNH moves to $325 \mathrm{~m} \mu$ on coupling to ADH. And this band shift is reversed by low concentrations of $p$-chloromercuribenzoate, which completely inhibits the activity of ADH. There is thus good reason to believe that a sulfhydryl group in the protein interacts with the pyridine ring. How this occurs in the case of 
DPNH we do not know, whether by a hydrogen bond - $\mathrm{SH}$ - - $\mathrm{N}^{+}$, as suggested by Kaplan and Ciotti, or by a covalent linkage, or otherwise.

The whole question of the $340 \mathrm{~m} \mu$ band shifts are probably of very great importance for elucidating the working mechanism of DPN and TPN-enzymes.

Meyerhof, Ohlmeyer, and Möhle (9) already in 1938 observed that DPN forms compounds with cyanide and bisulfite, at that time presumed to be<smiles></smiles>

and these compounds showed a band shift from 340 to 320 or $325 \mathrm{~m} \mu$.

Pullman et al. (14) and Rafter and Colowick (16) have presented very good evidence suggesting that of four possible resonating structures in the pyridine ring of DPN the one with a positive charge on $\mathrm{C}_{4}$ is preferred<smiles>CN1C=C[C+]=CC1</smiles>

This means that the chemical addition should occur primarily at $\mathrm{C}_{4}$ instead of at $\mathrm{C}_{6}$. Kaplan and Ciotti observed that the 325-m $\mu$ band of DPN.CN was shifted further to $310 \mathrm{~m} \mu$ when it was coupled with $\mathrm{ADH}$; and the $315-\mathrm{m} \mu$ band of the DPNhydroxylamine complex, studied by Kaplan and his co-workers, moved further to $300 \mathrm{~m} \mu$ when $\mathrm{ADH}$ was added. This seems to us to indicate that, for example, cyanide and $\mathrm{ADH}$ are bound to different sites at the pyridine ring. Perhaps addition reactions may occur at more than one of the double bonds of the pyridine ring. The developments in this special field are awaited with much interest.

Now, let us turn back to the question of the validity of our 
formulas (1), (2), (3), and (4). When the values for $k_{1} \ldots k_{6}$, given in Table III, were inserted in (5) and (6) the agreement was found to be very satisfactory, the deviations never exceeding the possible experimental errors. The agreement of the kinetic values, measured by initial velocities in $k_{1} k_{3} / k_{2} k_{5}$, with the older values for $D_{\text {ox }} / D_{\text {red }}$ obtained from equilibrium measurements, was practically perfect. This proves that the two DPNH or DPN molecules attached to the same molecule of $\mathrm{ADH}$ act similarly and independently of one another. There is convincing reason to believe that the presence or absence of substrate does not interfere with the reactions (1) and (3), because the value of $k_{1}$, measured spectrophotometrically in 1951 by the aid of the velocity of the band shift from 340 to $325 \mathrm{~m} \mu$, in the absence of substrate, agrees with the new value obtained in the presence of substrate (Table III), $k_{1}=4 \times 10^{6}$, and $3.7 \times 10^{6}$, respectively.

It was clear in 1951 that formula (2) might be an oversimplification, since it does not take into account the possibility that the formation and breakdown of enzyme-substrate compounds may under some conditions be rate-limiting steps. That such compounds must exist became evident in 1953 from the beautiful work of Vennesland, Westheimer, and their associates (32), demonstrating a direct and stereospecific exchange of deuterium between alcohol and DPN in the presence of ADH. According to Burton and Kaplan (3), it seems plausible that (2) should involve the following partial reactions:
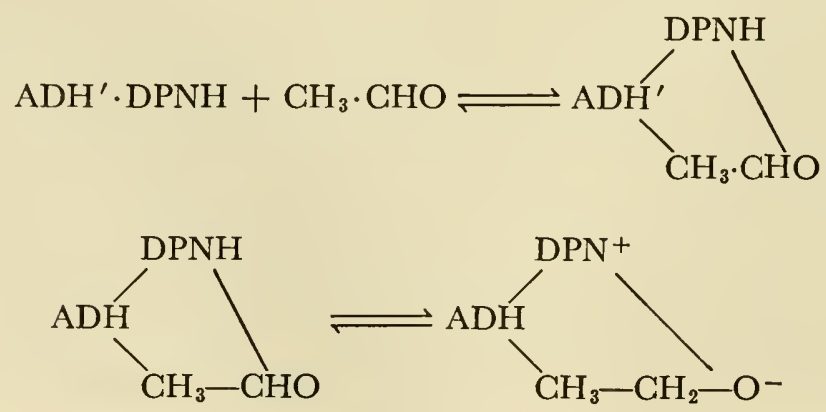

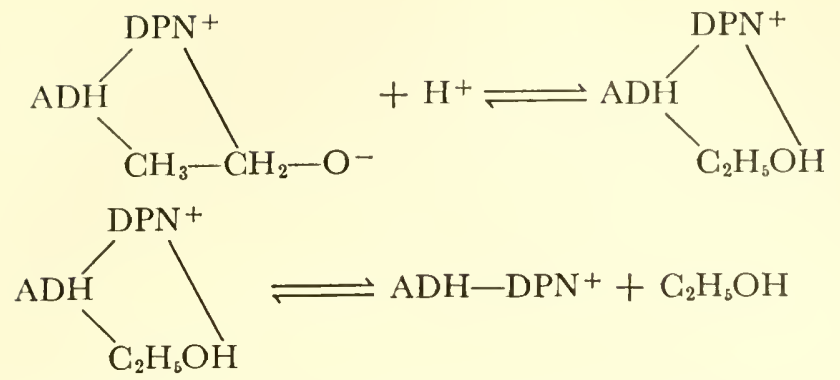

The influence of some sodium salts of different anions on the reaction velocity in the ADH-system was studied and found to be unexpectedly large and interesting. Some values are summarized in Table IV.

\section{TABLE IV}

Influence of Some Sodium Salts, $0.15 M$, on the Reaction Velocity, V/e sec. ${ }^{-1}$, in the ADH-DPN-DPNH System ( $p \mathrm{H}=7.1,23.5^{\circ}$ C.) (ref. 28)

\begin{tabular}{|c|c|c|c|}
\hline \multicolumn{2}{|c|}{$\begin{array}{l}{\left[\mathrm{ADH}^{1}\right]=0.13 \mu \mathrm{M}} \\
{\left[\mathrm{CH}_{3} \mathrm{CHO}\right]=2100 \mu \mathrm{M}} \\
{[\mathrm{DPNH}]=9.6 \mu \mathrm{M}}\end{array}$} & \multicolumn{2}{|c|}{$\begin{array}{l}{\left[\mathrm{ADH}^{1}\right]=0.13 \mu \mathrm{M}} \\
{\left[\mathrm{C}_{2} \mathrm{H}_{\mathrm{B}} \mathrm{OH}\right]=2300 \mu \mathrm{M}} \\
{[\mathrm{DPN}]=8 \mu \mathrm{M}}\end{array}$} \\
\hline Salt & $\mathrm{V} / \mathrm{e} \mathrm{sec}^{-1}$ & Salt & $\mathrm{V} / \mathrm{e} \mathrm{sec}^{-1}$ \\
\hline Glycine & 15 & Glycine & 0.32 \\
\hline Phosphate & 8.7 & Phosphate & 0.26 \\
\hline Phosphate + Versene & 8.9 & & \\
\hline Chloride & 3.5 & Chloride & 0.097 \\
\hline Sulfate & 2.3 & Sulfate & 0.059 \\
\hline Nitrate & 1.6 & Nitrate & 0.013 \\
\hline Bromide & 0.13 & Bromide & 0.055 \\
\hline \multirow[t]{2}{*}{ Formate } & 0.17 & Formate & 0 \\
\hline & & Acetate & 0.059 \\
\hline
\end{tabular}

We think very few people would have considered it likely to predict in advance that, for example, $0.15 M \mathrm{NaBr}$ would inhibit the aldehyde-DPNH reaction by $99 \%$. In general, the table shows how careful we have to be in controlling the ionic milieu when dealing with coenzyme-enzyme reactions. $0.15 M$ chloride and $0.015 \mathrm{M}$ formate were subjected to the more complete set of analyses that allowed calculations of all the six velocity constants (see Table III). $0.15 M$ chloride decreases 
both the "on" velocities, $k_{1}$ and $k_{5}$, to about half. This was not unexpected, since the same kind of effect occurs with FMN + apoprotein in the alkaline $p \mathrm{H}$ range; nor was the increase in $k_{2}$ unexpected for the same reason. But the decrease in $k_{3}$ by $50 \%$ was indeed unexpected. The mechanism leading to this result is hard to understand, but its consequences are worth considering.

First of all, whether chloride will increase or decrease the reaction velocity is found to depend on the concentration of the reaction participants. In a system where DPN and alcohol are both in excess, chloride stimulates the reaction, because $k_{2}$ is then rate-limiting, and $k_{2}$ is increased by chloride. If now the concentration of DPN is decreased, so that $k_{5}$ begins to be ratelimiting, chloride inhibits because of its decreasing effect on $k_{5}$. Thus the Lineweaver-Burke plots for varied DPN cross one another at a point, where chloride apparently has no effect at all.

Suppose we would determine the dissociation constant $\left(D_{\text {ox }}\right)$ of DPN.ADH by equilibrium measurements with and without chloride. Practically the same value would come out in both cases, because $k_{3}$ and $k_{5}$ are both reduced to half. There is, however, a kinetic difference: in chloride it takes a longer time for DPN and ADH to reach equilibrium.

The opposite effect of chloride on $k_{2}$ and $k_{3}$ leads to a change in $D_{\text {ox }} / D_{\text {red }}$. This means that the oxidation-reduction potential of the enzyme complex will be lowered from -0.204 to -0.219 $\mathrm{v}$. if $0.15 M$ chloride is added to a solution of DPN.ADH + DPNH·ADH.

Formate, $0.015 \mathrm{M}$, is found to affect only two of the six constants, $k_{3}$ and $k_{6}$. This means that formate reacts only with $\mathrm{DPN} \cdot \mathrm{ADH}$, but not with $\mathrm{DPN}$ or $\mathrm{ADH}$ before the complex is formed, since $k_{5}$ is not changed. Formate may enter into the binding site for ethanol in DPN.ADH, since it decreased $k_{6}$ competitively. The formate then stabilizes the linkage of DPN to $\mathrm{ADH}$ (decreased $k_{3}$ ).

There thus seems to be a strict analogy between the effect of formate and the effect of hydroxylamine, as studied by Kaplan 
et al., since both substances are competitive inhibitors of the alcohol oxidation, and both increase the stability of the DPNADH linkage. This may seem surprising at first sight. However, Burton and Kaplan emphasize the similarity in molecular shape of ethanol and hydroxylamine, and assume that both have to dissociate off a proton in order to form anions, which in their turn should form addition compounds with the $\stackrel{+}{\mathrm{C}} \mathrm{H}_{\backslash}$ of the

pyridine ring. Now, both ethanol and hydroxylamine have a very weak tendency to dissociate off a proton, whereas formic acid certainly dissociates very readily. Considering the resemblance of the formic acid and the hydroxylamine molecules
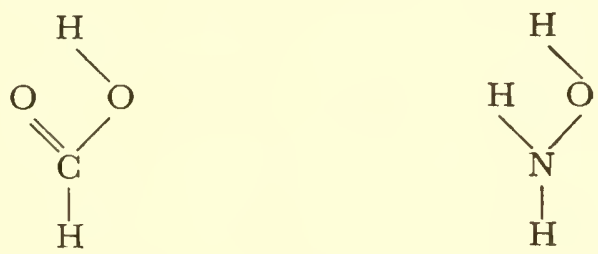

we may say that it is not at all surprising that the formate anion can react with the positively charged pyridine. It is much harder to understand how the ADH can cause ethanol and hydroxylamine to form anions so readily as required by Burton's and Kaplan's theory.

From what has been said above it may be predicted that the activity of coenzyme-enzyme systems is influenced by anions to a greater degree than we would have heretofore believed. Our attention has been far too exclusively focused on the hydrogen ion. A systematic investigation of the salt effects on enzymatic activity will undoubtedly reveal many new and interesting facts.

The effect of chloride, and other anions, on the oxidationreduction potential of the $\mathrm{ADH}$-system led us to the idea that a salt-sensitive system with an oxido-reducible cofactor reversibly attached to a high molecular carrier might be present in 
certain nerve receptors, transforming a change in ion concentration to a potential change and an electrical discharge.

Some recent work by Zotterman et al. (37) on the response in taste fibers from the cat tongue to stimulus by various salt solutions applied on the tongue give some support to this theory. They have found particular "water" fibers that respond to pure water, and to solutions of acetate and formate, when they are rinsed over the tongue. Salts of strong acids ( $\mathrm{NaBr}, \mathrm{NaI}$, $\mathrm{NaNO}_{3}, \mathrm{NaCl}, \mathrm{KCl}$, and $\mathrm{CaCl}_{2}$ ) all abolished the response. It should be recalled that acetate and formate have no effect on the dissociation of O.Y.E., whereas the anions of strong acids all dissociate it.

Finally, the strong effect of substances like cyanide, hydroxylamine, pyruvoxim, and formate on the reaction velocity in the ADH-system is perhaps of considerable interest from a wider point of view. There is no reason to believe that any of these substances, with the possible exception of formate, are present in detectable concentrations in the living cells. But since these chemically very different substances can all react with the pyridine ring we shall have to look for such effects with substances known to occur physiologically in the cells. This may lead to the discovery of mechanisms for the coordination and regulation of the different enzymatic activites in the cells.

\section{References}

1. Boeri, E., A. Ehrenberg, K. G. Paul, and H. Theorell, Biochim. et Biophys. Acta, 12, 273 (1953.

2. Bonnichsen, R., and A. Wassén, Arch. Biochem., 18, 361 (1948).

3. Burton, R. M., and N. O. Kaplan, J. Biol. Chem., 211, 447 (1954).

4. Chance, B., and J. B. Neilands, J. Biol. Chem., 199, 383 (1952).

5. Kaplan, N. O., and M. M. Ciotti, J. Biol. Chem., 211, 431 (1954); N. O. Kaplan, M. M. Ciotti, and F. E. Stolzenbach, ibid., 217, 419 (1954).

6. Kuhn, R., and P. Boulanger, Ber., 69, 1557 (1935).

7. Kuhn, R., and G. Moruzzi, Ber., 67, 888 (1934).

8. Kuhn, R., and H. Rudy, Ber., 69, 2557 (1936).

9. Meyerhof, O., P. Ohlmeyer, and W. Möhle, Biochem. Z., 297, 90 (1938). 
10. McElroy, W. D., and B. Glass, eds., The Mechanism of Enzyme Action. Johns Hopkins Press, Baltimore, 1954.

11. Nygaard, A. P., and H. Theorell, Acta Chem. Scand., 8, 1489 (1954).

12. Paléus, S., A. Ehrenberg, and H. Tuppy, Acta Chem. Scand., 9, 365 (1955).

13. Paul, K. G., Acta Chem. Scand., 5, 389 (1951).

14. Pullman, M. E., A. San Pietro, and S. P. Colowick, J. Biol. Chem., 206, 129 (1954).

15. Racker, E.. J. Biol. Chem., 184, 313 (1950).

16. Rafter, G. W., and S. P. Colowick, J. Biol. Chem., 209, 773 (1954).

17. Theorell, H., Biochem. Z., 272, 155 (1934).

18. Theorell, H., Biochem. Z., 290, 293 (1937).

19. Theorell, H., Enzymologia, 6, 88 (1939).

20. Theorell, H., Arkiv Kem. Mineral. Geol., 16A, no. 14 (1942).

21. Theorell, H., IX Conseil de Chimie, Inst. Int. de Solvay, Bruxelles, 1953, p. 301.

22. Theorell, H., and A. Ákeson, J. Am. Chem. Soc., 63, 1804 (1941).

23. Theorell, H., and R. Bonnichsen, Acta Chem. Scand., 5, 1105 (1951).

24. Theorell, H., and B. Chance, Acta Chem. Scand., 5, 1127 (1951).

25. Theorell, H., and A. Ehrenberg, Acta Chem. Scond., 91193 (1955).

26. Theorell, H., and A. P. Nygaard, Acta Chem. Scand., 8, 877 (1954).

27. Theorell, H., and A. P. Nygaard, Acta Chem. Scand., 8, 1649 (1954).

28. Theorell, H., A. P.Nygaard, and R. Bonnichsen, Acta Chem. Scand., 9, 1148 (1955).

29. Tsou, C. L., Biochem. J. (London), 49, 362, 367 (1951).

30. Tuppy, H., and G. Bodo, Monatsh. Chem., 85, 807, 1024, 1182 (1954).

31. Tuppy, H., and S. Paléus, Acta Chem. Scand., in press.

32. Vennesland, B., and F. H. Westheimer, in W. D. McElroy and B. Glass, eds., The Mechanism of Enzyme Action. Johns Hopkins Press, Baltimore, 1954, p. 357.

33. Vestling, C. S., Acta Chem. Scand., 9, 1600 (1955).

34. Walaas, O., and E. Walaas, Acta Chem. Scand., 10 (1956), in press.

35. Warburg, O., and W. Christian, Biochem. Z., 266, 377 (1933).

36. Weber, G., Biochem. J. (London), 47, 114 (1950).

37. Zotterman, Y., Acta Physiol. Scand., 18, 181 (1949); Andersson, B., and Y. Zotterman, ibid., 20, 95 (1950); Liljestrand, G., and Y. Zotterman, ibid., 32, 291 (1954); Cohen, M. J., S. Hagiwara, and Y. Zotterman, ibid., in press. 


\section{ENZYME-SUBSTRATE COMPOUNDS AND ELECTRON TRANSFER*}

BRITTON CHANCE, Johnson Research Foundation, University of Pennsylvania, Philadelphia, Pennsylvania

The search for intermediates in enzyme reactions began with the hyperbolic relationship between the over-all reaction rate and the substrate concentration: Henri (39), Brown (2), and Michaelis and Menten (52) formulated a mechanism for enzyme action involving a single intermediate compound. Direct experimental studies have identified such intermediate compounds in iron porphyrin and pyridine nucleotide enzyme systems $(4,18,43,60,66-68)$ and experiments on their reaction kinetics have shown them to participate directly in the enzymatic sequence. But other compounds formed in the enzymesubstrate reaction have been found to be definitely inhibitory $(5,6)$ or to have no function $(6,32,42,43)$. Analogous complexities arise in sequences of enzyme reactions, for example, cytochrome $b$ has been identified as a nonfunctional component of the nonphosphorylating DPNH (63) and succinate (12) oxidase system of heart muscle particles, whereas it participates actively in the phosphorylating system. From such a confusing array of reactive and unreactive intermediates we must identify

* Much of the work of this laboratory referred to in this contribution was supported in part by the United States Public Health Service, the National Science Foundation, and the Office of Naval Research. 
the actual participants in enzymatic reaction sequences with critical and incisive methods. Direct study of the reaction kinetics of intermediates has been useful for some relatively simple systems (13), and its current application to further complexities of single- and multi-enzyme reaction sequences is presented here.

This paper also illustrates the multiplicity of physical and chemical methods that may be used in the study of complex enzymatic sequences and the use of the analogue computer in order to verify reaction mechanisms that are proposed to represent the enzyme systems.

\section{One-Enzyme Reaction Sequences}

In the case of catalase and peroxidase the existence of intermediates was suggested by over-all studies and was followed by chemical and kinetic studies of the labile intermediate compounds in the reaction sequence. Such studies have given us a definite framework into which the reaction mechanism must be fitted $(14,23,36)$. It is our purpose to examine the theoretical and experimental data on these enzyme reaction mechanisms in order to determine whether undetected intermediates precede those already studied.

\section{CATALASE AND HYDROGEN PEROXIDE}

In the case of catalase, complex I appears to be the primary product of the reaction of catalase and hydrogen peroxide, and is the "Michaelis" enzyme-substrate compound (3). This conclusion is based largely upon the fact that no intermediate compound is observed to precede complex I, even when the reaction is measured in the flow apparatus at very short times ( 2 millisec.). Studies of the over-all reaction of catalase described below support the idea of direct combination of enzyme and substrate to form complex $I$ in reaction times as short as $2 \times 10^{-8} \mathrm{sec}$. and a similar conclusion is reached on the basis of less extensive data in the case of peroxidase complex I. On 
the other hand, when an oxidizing agent other than peroxide is used to form complex I, kinetic studies show definitely that such agents do not react directly to form complex I. Nevertheless, the possibility of a two-step reaction of catalase or peroxidase involving an intermediate preceding complex I needs to be considered.

\section{THEORETICAL BACKGROUND}

By means of the accelerated-flow method we have observed that complex I of catalase and hydrogen peroxide is the first spectroscopically detectable intermediate to appear upon addition of peroxide to catalase. And this is supported by the fact that the pseudo first-order velocity constant for the formation of the complex has been found to increase linearly with hydrogen peroxide concentration up to times as short as 2 millisec. (Ref. 9, Figure 11). The second-order velocity constant for the combination of catalase and hydrogen peroxide $(0.4 \times$ $10^{7} M^{-1}$ sec. $^{-1}$ at $25^{\circ}$ C.) agrees fairly well with the value $\left(0.6 \times 10^{7} \mathrm{M}^{-1} \mathrm{sec}^{-1}\right)$ independently computed from the overall reaction (23).

The velocity constant for the over-all reaction $\left(k_{1}^{\prime}\right)$ does not depend solely upon the velocity constant for the formation of complex I $\left(k_{1}\right)$, but it depends also upon the velocity constant $\left(k_{4}^{\prime}\right)$ for the reaction of complex I with the second peroxide molecule, which serves as a hydrogen donor (23):

$$
k_{1}^{\prime}=\frac{8}{\frac{1}{k_{4}^{\prime}}+\frac{1}{k_{1}}}
$$

Nevertheless, the constancy of $k_{1}^{\prime}$ with varying peroxide concentrations implies constancy of $k_{1}$ over this range. (Note that if $k_{1}$ decreased while $k_{1}^{\prime}$ remained constant, $k_{4}^{\prime}$ would have to increase. It is unlikely that the second-order velocity constant would increase at high peroxide concentrations.) On this basis experiments proving the constancy of $k_{1}^{\prime}$ from the region of low peroxide concentrations where the kinetics of 
complex I are observed spectrophotometrically up to very high peroxide concentrations would provide evidence that the formation of complex $\mathrm{I}$ is so rapid that no other intermediate than complex I would have time to be formed in the reaction.

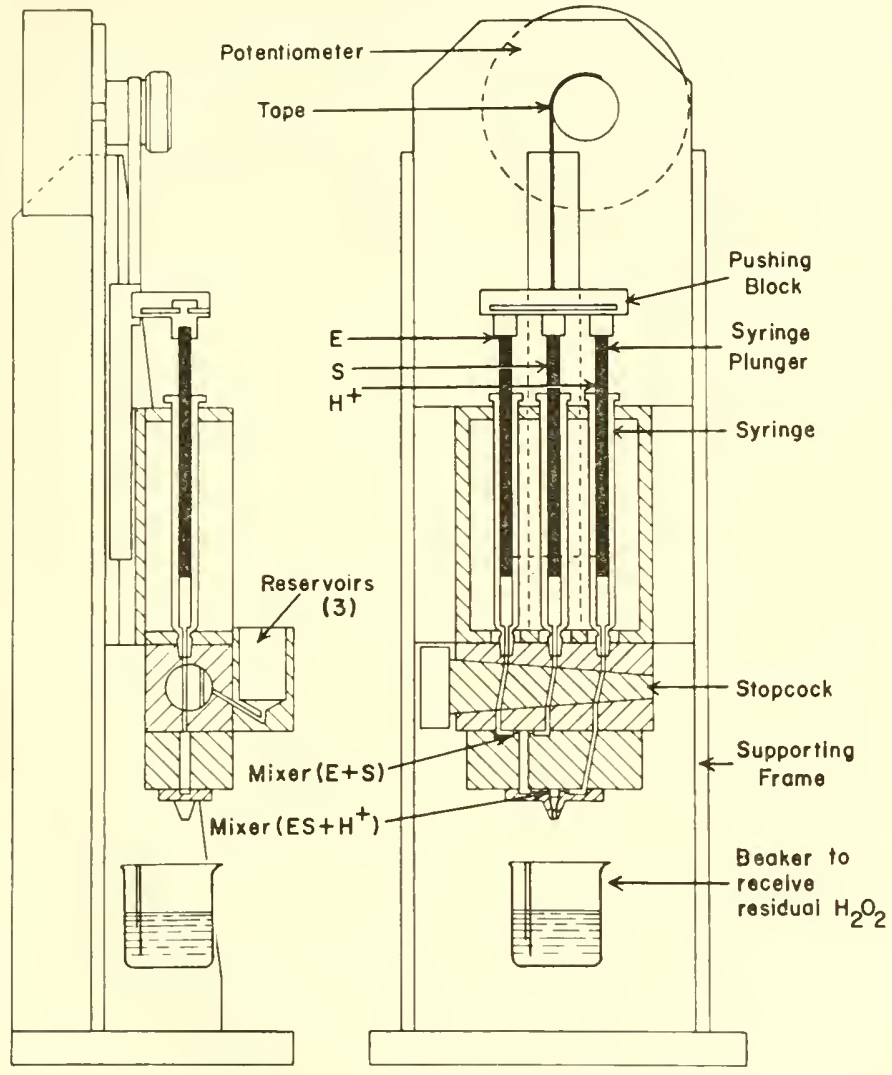

Fig. 1. Quenching type of Hartridge-Roughton flow apparatus used for the investigation of catalase activity at high hydrogen peroxide concentrations (17).

A pair of 2-ml. tuberculin-type syringes contain, respectively, 1-5 $\mathrm{M} \mathrm{H}_{2} \mathrm{O}_{2}$ and about $10^{7} \mathrm{M}$ enzyme. Enzyme and substrate are forced into the mixing chamber by the pushing block and flow down the reaction tube a distance corresponding to 0.1 to $0.4 \mathrm{sec}$. Sulfuric acid is then rapidly mixed to stop the reaction. The remaining peroxide is back titrated with permanganate (FA-20). 


\section{PHYSICAL METHOD}

Under classical conditions for catalase assay (33) the value of $k_{1}^{\prime}$ declines rapidly above $0.02 M$ peroxide concentrations, but activity measured in a minute or less gives constant values of $k_{1}^{\prime}$ up to $0.05 M$ peroxide (1). In studies expressly for the purpose of investigation of catalase activity at high peroxide concentrations, George has measured the over-all reaction with rapid manometric methods (35), but an examination of George's data indicates that the values of $k_{1}^{\prime}$ under these conditions were about one-fifth of the correct value (48). Thus the manometric data are not suitable for evaluating the constancy of $k_{1}^{\prime}$ and are, in fact, of questionable value in relation to the reactions of intact catalase.

We have therefore devised a "quenching" type of rapidflow apparatus especially designed for studies of catalase at high hydrogen peroxide concentrations (19). With this apparatus catalase is rapidly mixed with 1-5 $M$ peroxide, and the reaction is stopped by a second rapid mixing with sulfuric acid a very short time later in order that inactivation of the enzyme be minimized. Figure 1 shows the important features of this apparatus. The plungers are manually driven at a constant rate, and the time required to discharge a given volume is measured with a stop watch. The buffered catalase solution $\left(\sim 10^{-7} M\right)$ is rapidly mixed with the strong peroxide solution, the reaction mixture flows down the observation tube a distance of $2 \mathrm{~cm}$. in a time of 0.1 to $0.4 \mathrm{sec}$, and then it is mixed with about $3 N$ sulfuric acid to stop the reaction. The remaining peroxide is titrated with permanganate.

\section{THE LIFETIME OF INTERMEDIATES}

IN THE CATALASE REACTION

Dr. Y. Ogura of this laboratory has completed a series of catalase studies with this apparatus (55). As Figure 2 shows, the rate of the decomposition of peroxide by catalase reaches a plateau of $4.6 \mathrm{M} / \mathrm{sec}$. above $5 \mathrm{M}$ peroxide. This corresponds to 
a turnover number of $4 \times 10^{7} \mathrm{sec}^{-1}$ at $25^{\circ} \mathrm{C}$., the fastest enzymatic process yet measured. The turnover number is calculated from the quotient of the initial rate of peroxide decomposition and the enzyme concentration $(4.6 \mathrm{M} / \mathrm{sec}$./ $\left.1.2 \times 10^{-7} M=4 \times 10^{7} \mathrm{sec}^{-1}\right)$. This result differs dramatically from that of George, who used the manometric method (35).

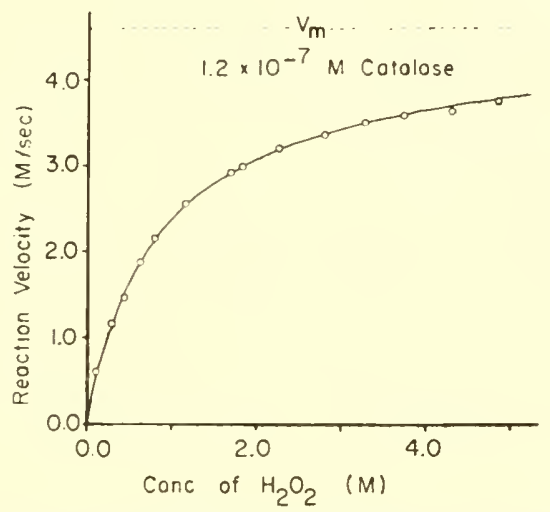

Fig. 2. Experimental study of catalase activity at high $\mathrm{H}_{2} \mathrm{O}_{2}$ concentrations by means of the quenching-flow apparatus (Ogura, unpublished data and ref. 55).

The ordinates represent the initial rate of $\mathrm{H}_{2} \mathrm{O}_{2}$, decomposition in moles per liter per second in the presence of $1.2 \times 10^{-7} M$ liver catalase. The extrapolated maximal rate is $4.6 \mathrm{M} / \mathrm{sec}$. at $30^{\circ} \mathrm{C} ., p \mathrm{H} 7.0$.

At very high peroxide concentrations the theoretical analysis above is inadequate to describe the experimental result and it appears that an unidentified intermediate in catalase action becomes rate-limiting in $5 M$ peroxide. However, the lifetime of such an intermediate is very short; at the plateau of Figure 2 the turnover time is only $0.693 / 4 \times 10^{7}=2 \times 10^{-8}$ sec. at $25^{\circ} \mathrm{C}$. $*$

* In our previous discussion of the existence of ternary complexes in catalase action we set the lifetime at a value less than $3 \times 10^{-8} \mathrm{sec}$. (11). These data indicate that the lifetime may be $2 \times 10^{-8} \mathrm{sec}$, somewhat shorter than our crude estimate. 


\section{POSSIBLE RATE-LIMITING STEPS IN CATALASE ACTION}

Since we are not at present able to make spectroscopic studies of catalase in $5 \mathrm{M}$ peroxide, one must consider hypothetical explanations for the hyperbolic relationship of Figure 2.

1. That the activity is inhibited by the formation of an inhibitory compound not in the reaction sequence (complex II) (10).

2. That the activity is limited by the formation of a ternary complex consisting of complex I and a second peroxide molecule $(11,17,56)$.

3. That a short-lived compound of catalase and peroxide precedes complex I.

Each of these three possibilities leads to hyperbolic relationships between reaction velocity and hydrogen peroxide concentration and cannot be distinguished on this basis. Ogura has, however, shown that inhibition of catalase by cyanide, fluoride, or formate becomes a competitive effect at very high peroxide concentrations. This observation rules out mechanism (3), which should not show a competitive effect of the kind observed. Thus these studies indicate that no intermediate of a life-time longer than $2 \times 10^{-8} \mathrm{sec}$. precedes complex I.

\section{RATE CONSTANTS FOR A REACTION SEQUENCE \\ INVOLVING A TERNARY COMPLEX}

If we accept mechanism (2) above as more likely than mechanism (1), and there is some evidence in favor of this at the present time (55), the molecular statistics of catalase reactions would be as follows:

$$
\begin{aligned}
& \text { catalase }+\mathrm{H}_{2} \mathrm{O}_{2} \stackrel{k_{2}=0.5 \times 10^{7} M^{-1} \text { sec. }^{-1}}{\longrightarrow} \text { complex I } \\
& \text { complex I }+\mathrm{H}_{2} \mathrm{O}_{2} \stackrel{k^{\prime}=1.5 \times 10^{7} M^{-1} \text { sec. }^{-1}}{\longrightarrow} \text { ternary complex } \\
& \text { ternary complex } \stackrel{k_{3}=3 \times 10^{7} \text { sec. }^{-1}}{\longrightarrow} \text { catalase }+\mathrm{O}_{2}+2 \mathrm{H}_{2} \mathrm{O}
\end{aligned}
$$

That the ternary complex is catalase complex III (42) is unlikely because catalase complex III requires complex II for 
its formation and the latter does not participate in the decomposition of hydrogen peroxide (10). The lifetime of such a ternary complex would be so short $\left(2 \times 10^{-8}\right.$ sec. $)$ that considerations of whether or not the two oxidizing and reducing equivalents of the two peroxide molecules were transferred stepwise or simultaneously begin to lose their importance. But direct spectroscopic studies of strong catalase in several molar peroxide are now needed to determine whether the ternary complex actually exists or whether the reaction is inhibited by the formation of inactive complex II, as has been observed spectroscopically in more dilute peroxide (10).

\section{PEROXIDASE AND HYDROGEN PEROXIDE}

Complexes I of catalase and peroxidase appear to be physically and chemically identical, but their protein parts give rise to different mechanisms and specificities in their reactions with hydrogen donors. It is probable that our data on the nature of the combination of catalase and hydrogen peroxide to form complex I apply equally well to peroxidase and direct experiments over a more limited range support this idea.

Direct spectroscopic measurement of the combination of peroxidase and hydrogen peroxide carried out in the rapid-flow apparatus has shown that a second-order reaction occurs $\left(k_{1}=0.9 \times 10^{7} \mathrm{M}^{-1} \mathrm{sec}^{-1}\right.$ at $25^{\circ} \mathrm{C}$. $)$, but the results have been limited to times longer than 2 millisec. (7). There are in addition a number of assay methods for the over-all activity of peroxidase from which the value of $k_{1}$ could be computed. The guaiacol assay method for peroxidase fulfills the conditions for determining $k_{1}$ from the rate of the over-all reaction:

$$
d x / d t=\frac{1}{\frac{1}{k_{1} x}+\frac{1}{k_{4} a}} ;
$$

if $k_{4} a \gg k_{1} x$

$$
d x / d t=k_{1} x e .
$$


When the pseudo first-order velocity constant for the donor reaction $\left(k_{4} a\right)$ is large compared to the corresponding value for the combination of enzyme and substrate $\left(k_{1} x\right)$, then $k_{1}$ can be computed from the rate of the over-all reaction $(d x / d t)$ and the enzyme concentration $(e)$ :

$$
k_{1}=\frac{1}{x e} \frac{d x}{d t} .
$$

With the guaiacol test under these conditions, values of $k_{1}$ of $0.89 \times 10^{7} \mathrm{M}^{-1} \mathrm{sec}^{-1}$ at $20^{\circ} \mathrm{C}$. and $1.03 \times 10^{7} \mathrm{M}^{-1} \mathrm{sec}^{-1}$ at $30^{\circ} \mathrm{C}$. were obtained with pure horse-radish peroxidase (25). These values agree very well with the value obtained in the flow apparatus at $25^{\circ} \mathrm{C}$. $\left(0.9 \times 10^{7} \mathrm{M}^{-1} \mathrm{sec}^{-1}(7)\right)$.

Other studies on the over-all reaction of peroxidase do not permit us to assume as small a lifetime for any unknown intermediates in the peroxidase reaction as in the case of catalase presented above. In the guaiacol assay just mentioned a lifetime of 2 millisec. suffices, while the work of Mann (51) at higher peroxide and guaiacol concentrations indicates that $6 \times 10^{-5} \mathrm{sec}$. suffices. The classical purpurogallin assay method allows $10^{-4} \mathrm{sec}$. for the breakdown of any unknown intermediate (8).

Thus the reaction of enzyme and substrate to form complex I is second order at reaction times of about $10^{-4} \mathrm{sec} .^{*}$ and, in view of the similarity of the reactions of catalase and peroxidase with peroxide, it is unlikely that an unknown intermediate precedes complex I in either case.

\section{REACTION KINETICS WITH OXIDIZING AGENTS OTHER THAN PEROXIDE}

George has found that compounds spectroscopically similar

* A considerable error may be introduced into the determination of $k_{1}$ from the over-all reaction kinetics if there is any possibility that a direct reaction of the oxidizing agent and the hydrogen donor takes place by any pathway in addition to that identified with $k_{1}$. An example of such a possibility is the attempt to determine $k_{1}$ for the reaction of peroxidase and hypochlorous acid by using the guaiacol test (37). 
to complexes I or II can be formed in the reaction of peroxidase and various strong oxidizing agents other than hydrogen or substituted hydrogen peroxides (37). Although considerable destruction of peroxidase occurs with many agents, and although few of these give appreciable enzymatic acitvity, George's results raise interesting questions with regard to the nature and definition of enzyme specificity. If such agents all react with peroxidase by the same mechanism as peroxide, then peroxidase has no absolute specificity for its substrate, any oxidizing agent that can react with the iron-porphyrin structure would seem to be suitable, and the role of the protein in directing the enzymesubstrate combination would seem to be negligible. On the other hand, there is no evidence that the reaction mechanism for these reagents is the same as with peroxide; it is possible that they react with a rather nonspecific end group of the protein and form an intermediate (possibly peroxide) at that site and that this intermediate subsequently interacts with the iron porphyrin group. The kinetic methods described above would seem to be suitable for testing whether or not such an intermediate step exists in the reaction of peroxidase with agents other than peroxide. Mr. R. R. Fergusson of this laboratory (34) has selected for this test a one-electron oxidant of known and relatively stable properties, potassium bromiridate, $\mathrm{K}_{2} \mathrm{IrBr}_{6}$. This reagent is preferable to others in which peroxide formation would a priori be suspected. The one-electron oxidation should form complex I by a reversal (equation (8)) of the one-electron steps in which complex $\mathrm{I}$ is known to react with donor molecules.

$$
\text { HRP } \stackrel{-e}{\longrightarrow} \text { complex II } \stackrel{-e}{\longrightarrow} \text { complex I }
$$

In confirmation of George's preliminary results, we find no trace of complex II to be formed prior to complex I in the reaction with bromiridate, and this result supports the view that a two-electron oxidant must be formed from the one-electron oxidizing agent before formation of complex I can occur.

The kinetics of the formation of complex I upon addition of bromiridate have been recorded spectrophotometrically, and 
the rate of complex I appearance is very little affected by the bromiridate concentration. Apparently an intermediate compound of a half life of about $10 \mathrm{sec}$. at $25^{\circ} \mathrm{C}$. is formed. Furthermore, this intermediate does not transfer oxidizing equivalents solely to the peroxidase heme to form complex I; over twice the theoretical amount of bromiridate is required to form one mole of complex I $\left(>4 \mathrm{~K}_{2} \mathrm{IrBr}_{6} \approx\right.$ complex I) even under optimal reaction conditions.

A possible explanation of this peculiar reaction is afforded by the observation that these strong oxidizing agents react with various amino acids; Fergusson has found in particular that bromiridate reacts with tyrosine alone as rapidly as it reacts with peroxidase which contains tyrosine (49).

It is illuminating to compare the action of bromiridate on the peroxidase system to a coupled oxidation reaction in which peroxide is produced from oxygen. Coupled oxidation reactions can occur by the addition of a separate enzyme system (glucose plus notatin) or by peroxidase itself reacting with dihydroxymaleic acid. In the latter case peroxidase serves as catalyst for both reactions (16). It is not at all unreasonable that a related effect occurs with bromiridate.

Such a reaction mechanism appears to differ considerably from that obtained with hydrogen peroxide in which the kinetic and titration data indicate that peroxide combines with the iron porphyrin group in a 1:1 stoichiometric ratio and in a secondorder reaction over a wide range of times. It is clear that the term "substrate" is suitable for peroxide, while bromiridate is surely not a substrate for the direct formation of complex I. On the other hand, bromiridate could be considered to be a substrate for a reaction with peroxidase protein.

\section{Multi-Enzvme Reaction Sequences}

The classical biochemical approach to the problem of establishing sequences of enzyme reactions has often been the isolation and purification of the enzyme and a reconstruction of the entire sequence in vitro. An excellent example is the recon- 
struction of the glycolytic system with purified enzymes. But such a reconstruction may be faulty with respect to the relative amounts of enzymes and coenzymes that are added, the diffusibility of the coenzymes, and, in addition, the steady-state concentrations of the intermediates in the system, for the intracellular system shows complex reaction patterns (20).

Reconstruction of the respiratory chain appears to be a much more formidable problem. Cytochrome $c$, the only member of the chain that has been purified, has an activity in the reconstructed Keilin and Hartree system of less than $1 \%$ that of the intact system (61), although in recent experiments with the succinic oxidase liver system this value has been raised towards $50 \%$ (R. Estabrook, unpublished data). And such other anomalies beset the reconstruction as the fact that isolated cytochrome $c$ will readily bind cyanide, whereas cytochrome $c$ in the intact respiratory chain does not (69).

Separation of some parts of the oxidase system has not yet been accomplished; although the cytochromes $a_{3}$ and $a$ have been purified in the presence of detergents, no chemical separation of the components has been obtained (64). The degree of purification of this portion of the respiratory chain obtainable when phosphorylative activity is retained, appears to be even less, although a smaller phosphorylating particle has been obtained by digitonin treatment (46a).

In order to evaluate any of these reconstructions it is essential to compare them directly with quantitative measurements of the intact enzyme systems, especially in the case of the respiratory chain involved in oxidative phosphorylation. Spectroscopic methods can be used to detect six intermediates in this respiratory chain, cytochromes $a_{3}, a, c$, and $b$, flavoprotein, and pyridine nucleotide $(26,28)$. Spectroscopic identification plus kinetic and inhibitor studies can be used to: (1) establish the sequence of action of the components of the chain; (2) identify components not detectable spectroscopically if they (a) react specifically with a substrate or inhibitor or $(b)$ can be made to be rate-limiting. 
The application of these methods to the study of the respiratory chains of phosphorylative* and nonphosphorylative DPNH oxidase systems of mitochondria and of mitochondrial fragments is outlined here.

\section{THE SUBSTRATE-INHIBITOR METHOD}

The examination of a respiratory chain by means of specific substrates or inhibitors rests largely upon the generality: components of the respiratory chain above the point of action of the added reagent become more reduced in the case of a substrate, $\uparrow$ and more oxidized in the case of an inhibitor. If the inhibitor is added to a system in which the substrate and oxygen are already present, the components above the site of action of the inhibitor will become more oxidized and those below will become more reduced (see, for example, Figure 4 of Ref. 12). The point in the respiratory chain at which the effect of an inhibitor upon the steady state shifts from an oxidation to a reduction, or vice versa, is called the crossover point. This crossover point locates the site of action of the inhibitor.

\section{CYTOCHROME $b$ AND SLATER'S FACTOR}

A clear example of the substrate-inhibitor technique is afforded by the effect of antimycin-A upon the DPNH oxidase system of phosphorylating liver mitochondria, as illustrated by Figure 3. Since phosphate acceptor is lacking electron transfer is in progress at a slow rate before the addition of the inhibitor. Upon addition of the inhibitor, the spectroscopic record shows an oxidation of reduced cytochrome $c$ (the $550 \mathrm{~m} \mu$ absorption peak of ferrocytochrome $c$ disappears and leaves a trough at $550 \mathrm{~m} \mu$ ) and cytochrome $b$ becomes reduced (the peak of reduced cytochrome $b$ appears at $563 \mathrm{~m} \mu$ ). Thus the crossover point is between cytochromes $b$ and $c$, and the theorem above

* In collaboration with Dr. G. R. Williams.

$f$ "Above" refers to the oxygen side, "below" to the substrate side of the point of action. 
indicates that cytochrome $c$ is above $b$ in the respiratory chain involved in oxidative phosphorylation.

Slater's factor $(41,63)$ is adequately described in nonphosphorylating preparations as the component linking flavoprotein to cytochrome $c$ that is sensitive to BAL. But in phosphorylating preparations it has recently been found (1) that cytochrome $b$ links flavoprotein to cytochrome $c$ (or $c_{1}$ ) in both the DPNH and succinate-linked systems, and (2) that the

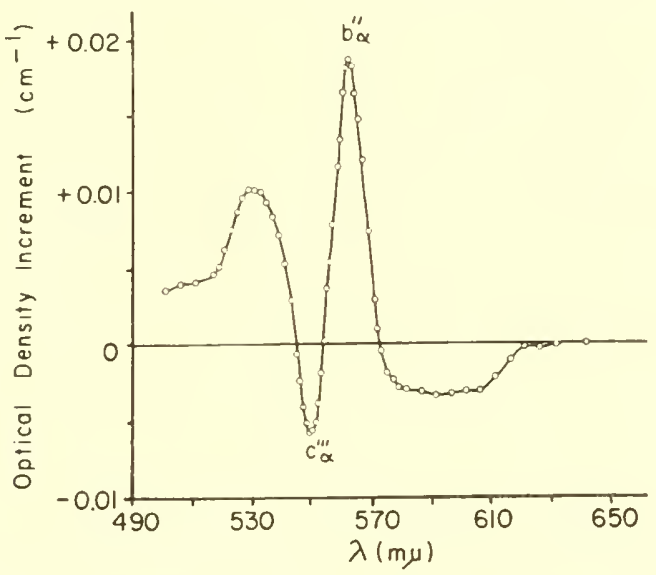

Fig. 3. The spectroscopic changes in an aerobic mitochondrial suspension caused by addition of excess antimycin-A.

Mitochondria were suspended in a reaction medium that contained all substances necessary for oxidative phosphorylation except phosphate acceptor. The base line of the figure corresponds to the material before antimycin-A treatment, the curve, just following the treatment. The peak at $563 \mathrm{~m} \mu$ is caused by the reduction of cytochrome $b$, the trough at $550 \mathrm{~m} \mu$, by the oxidation of reduced cytochrome $c$ (460-1-2).

sensitive point lies between cytochrome $b$ and the component above it in the respiration chain (probably cytochrome $c_{1}$ ). Thus, in the phosphorylating system, cytochrome $b$ fulfills the requirements for Slater's factor. The minimum hypothesis for an explanation of the effect of these inhibitors in the intact system is that they affect the reaction

$$
b^{\prime \prime}+c^{\prime \prime \prime} \longrightarrow b^{\prime \prime \prime}+c^{\prime \prime}
$$


by combining with $b^{\prime \prime}$, since $c^{\prime \prime \prime}$ can participate in oxidative phosphorylation in the presence of antimycin-A (54).

\section{REVERSAL OF INHIBITION :}

\section{LOCALIZATION OF SITES OF OXIDATIVE PHOSPHORYLATION}

The theorem above applies conversely to the reversal of inhibition, and this concept is particularly useful in analyzing

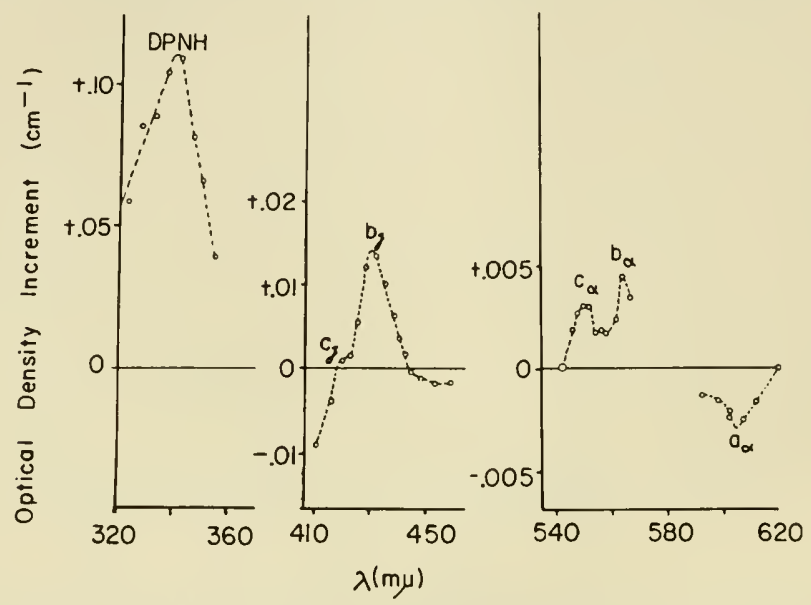

Fig. 4. The spectroscopic changes that occur in an aerobic mitochondrial suspension when added adenosinediphosphate is used up and the respiration slackens owing to lack of phosphate acceptor (29).

The mitochondria were initially carrying out oxidative phosphorylation (spectrum plotted as a horizontal base line), and as the added ADP is exhausted the various absorption bands appear due to reduced cytochromes $b(564 \mathrm{~m} \mu$ and $431 \mathrm{~m} \mu), c(550 \mathrm{~m} \mu$ and $420 \mathrm{~m} \mu)$, and DPNH at $340 \mathrm{~m} \mu$. The absorption band of the oxidized form of flavoprotein which has an absorption band at $465 \mathrm{~m} \mu$ disappears and leaves a trough at $465 \mathrm{~m} \mu$. The absorption band of reduced cytochrome $a$ disappears and leaves a trough at $605 \mathrm{~m} \mu$. The changes at $445 \mathrm{~m} \mu$ due to cytochrome $a_{3}$ are too small to measure under these conditions, but are readily recorded if a small azide concentration $(\sim 100 \mu \mathrm{M})$ is added (Expt. 359).

the effect of ADP addition upon respiration and phosphorylation in a "tightly coupled" mitochondrial preparation. In such a case, electron transport through the system may be increased 
over tenfold by the reversal of some inhibitory process in the respiratory chain (45), and the localization of the sites of action of the inhibitor should be demonstrable in terms of the crossover point for spectroscopic changes in the respiratory pigments caused by ADP addition.

In rat liver preparations, it is found that the addition of ADP causes an oxidation of the steady state of cytochromes $c$ and $b$, flavoprotein, and DPNH and a reduction of cytochromes $a$ and $a_{3}$, the crossover point being between cytochromes $c$ and $a$ (28). The action spectrum for ADP is illustrated by Figure 4. With other substrates, mitochondria from other sources, the crossover point can be moved along the respiratory chain so that it lies between cytochromes $c$ and $b$. In the presence of appropriate concentrations of inhibitors of cytochrome $a_{3}$ such as azide, only DPNH is oxidized when ADP is added.

These crossover points identify pairs of components that can be involved in oxidative phosphorylation. For example, in the oxidation of cytochrome $b^{\prime \prime}$ by $c^{\prime \prime \prime}$

$$
b^{\prime \prime}+c^{\prime \prime \prime} \longrightarrow b^{\prime \prime \prime}+c^{\prime \prime}
$$

a portion of the free-energy change can be conserved and later utilized to add high-energy phosphate to ADP. The crossover theorem does not identify which one of the reaction products acquires this portion of the free-energy change. Current theories of oxidative phosphorylation (63a) suggest, by analogy with glyceraldehyde-3 phosphate dehydrogenase, that the oxidation product is involved (for a recent summary, see Lehninger (46)), but thermodynamics would make no distinction between the two pathways for energy transfer. Our experimental data suggest that the reduced form is involved. We find that DPNH exists in an inhibited form in mitochondria lacking ADP and therefore propose that it is the reduced form of the reaction products that are involved in the free-energy conservation. In order to translate this view into chemical terminology, it is assumed that an unknown ligand (I) of the 
enzyme becomes a high-energy compound ( $\sim$ I) during the oxidative-reduction reaction

$$
b^{\prime \prime}+c^{\prime \prime \prime} \cdot \mathrm{I} \longrightarrow b^{\prime \prime \prime}+c^{\prime \prime} \sim \mathrm{I}
$$

and that further reaction of $c^{\prime \prime} \sim \mathrm{I}$ with the respiratory chain is slow unless transfer of $\sim$ I to another intermediate occurs, whereupon $c^{\prime \prime}$, the rapidly reacting form, is released.

$$
c^{\prime \prime} \sim \mathrm{I}+\mathrm{X} \longrightarrow c^{\prime \prime}+\mathrm{X} \sim \mathrm{I}
$$

$\mathrm{X} \sim \mathrm{I}$, of course, can interact with phosphate and ADP to form ATP.

According to equation (12), the crossover point identifies the component on the oxidation side of the three crossovers mentioned above as sites of oxidative phosphorylation in the intact mitochondrial respiratory chains. The sites are pyridine nucleotide, cytochrome $b$, and cytochrome $c$. If the oxidized form of the respiratory enzymes if found to conserve the increment of free energy, the sites would be flavoprotein, cytochrome $c$, and cytochrome $a$.

\section{RAPID REACTION METHODS}

As in the case of the soluble hemoproteins, the rapid kinetic methods have proved to be of considerable value in identifying the sequence of action of the various components of the intact mitochondrial respiratory chain. For a study of this complex system it has been necessary to refine our methods of mathematical analysis by the use of an electronic analogue computer and to improve the optical and hydraulic aspects of the rapidflow method so that satisfactory results could be obtained with intact mitochondria or whole cells.

\section{THEORETIGAL ASPEGTS}

On suddenly initiating a change in the steady-state level of one member of the respiratory chain, the time sequence of the response of the other members of the chain should indicate the sequence of chemical reactions. For the sequence of reactions 
that occurs when oxygen is suddenly added to the reduced components of the respiratory chain, it is easy to see by inspection that the time sequence of the oxidation reactions will be the chemical sequence for the abbreviated system:

$$
\begin{aligned}
& \mathrm{O}_{2}+a_{3}^{\prime \prime} \stackrel{k_{1}}{\longrightarrow} a_{3}^{\prime \prime \prime}+\mathrm{O}_{2}- \\
& a_{8}^{\prime \prime \prime}+a^{\prime \prime} \stackrel{k_{3}}{\longrightarrow} a_{3}^{\prime \prime}+a^{\prime \prime \prime} \\
& a^{\prime \prime \prime}+c^{\prime \prime} \stackrel{k_{6}}{\longrightarrow} a^{\prime \prime}+c^{\prime \prime \prime}
\end{aligned}
$$

The differential equations are

$$
\begin{gathered}
\frac{d a_{3}{ }^{\prime \prime \prime}}{d t}=k_{1}\left[\mathrm{O}_{2}\right] a_{3}{ }^{\prime \prime}-k_{3} a_{8}{ }^{\prime \prime \prime} a^{\prime \prime} \\
\frac{d a^{\prime \prime \prime}}{d t}=k_{3} a_{3}{ }^{\prime \prime \prime} a^{\prime \prime}-k_{5} a^{\prime \prime \prime} c^{\prime \prime}
\end{gathered}
$$

Near $t=0, a_{3}{ }^{\prime \prime \prime}$ and $a^{\prime \prime \prime}$ are negligible and

$$
\frac{d a_{3}^{\prime \prime \prime}}{d t} \gg \frac{d a^{\prime \prime \prime}}{d t}
$$

In fact, the ratio of these initial reaction rates can approach a value dependent on the ratio $\left[\mathrm{O}_{2}\right] / a^{\prime \prime \prime}$.

For this simple example the terminal component of the respiratory chain is identified, and, by following the time sequence, the chemical sequence of the other components can also be determined. By adding an electron donor to the oxidized system the reaction sequence in the opposite direction can also be determined. A third alternative is to add suddenly a terminal inhibitor such as azide or cyanide and observe the sequence of reduction of the components.

\section{ANALOGUE COMPUTER}

The evaluation of the time separation that may be expected from a sequence containing components of different concentrations and reaction velocity constants can best be determined by means of an electronic analogue computer. In this case it is unnecessary to neglect the concentrations ${a_{3}}^{\prime \prime \prime}$ and $a^{\prime \prime \prime}$ as above. 
In the computer that we have been using the concentrations are represented by voltages, and mathematical operations upon
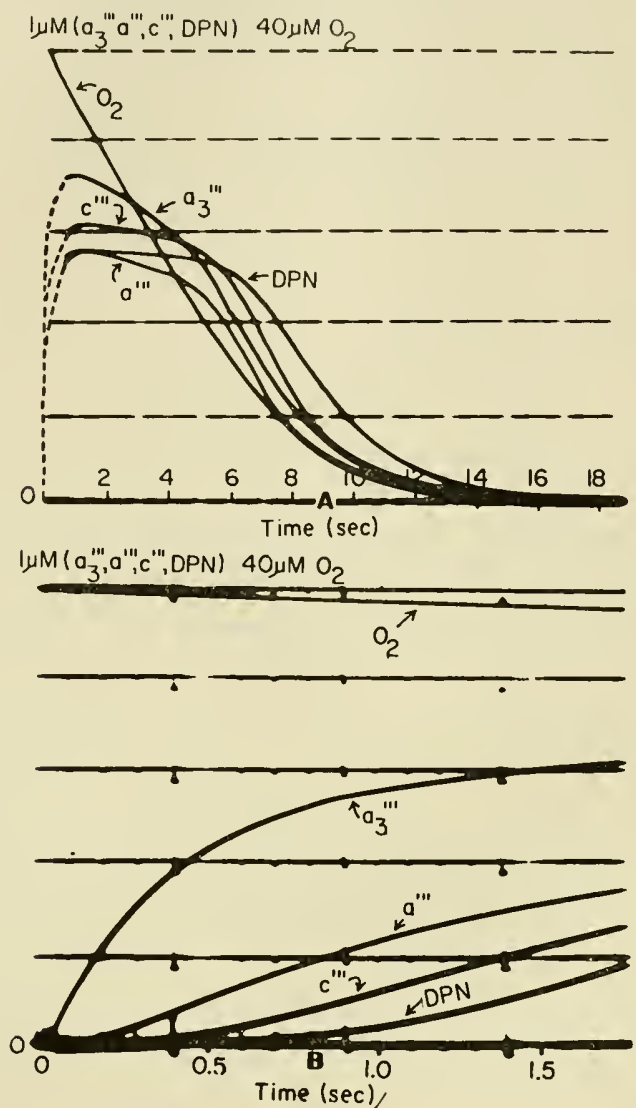

Fig. 5. An analogue computer representation of the kinetics of oxidation and reduction of members of the abbreviated respiratory sequences:

$\mathrm{O}_{2} \longrightarrow a_{3} \longrightarrow \mathrm{c} \longrightarrow \mathrm{DPN} \longrightarrow$ substrate caused by adding oxygen to the anaerobic components.

The top trace (A) is on a slow time scale and shows the complete "cycles" of the four components and the oxygen kinetics as well. The lower trace (B) is on an expanded scale and shows clearly that the time sequence of oxidation of the components is the sequence in which they react in the respiratory chain (AC-64). 
these voltages are carried out in accordance with equations similar to (16) and (17) above. The concentrations of the reactants are multiplied together in an electronic multiplier, the product is multiplied by the fixed value of the reaction velocity constant, the terms corresponding to a particular reaction rate are summed with an "adder," and the sum is integrated with respect to time in an electronic integrator. The concentration of one of the reactants determined in this way is automatically substituted into the appropriate place in the differential equations. A similar process gives the values of concentration of all the other reactants which are substituted into the appropriate places in the equations. The solution then continues, just as does the chemical reaction, until one of the reactants is used up. In equations (13), (14), and (15) we would choose oxygen to be limiting in concentration so that the reaction would halt with the components in the reduced form. The reaction is then readily restarted by adding a voltage proportional to the desired initial oxygen concentration. For convenience the computer solution is restarted every 0.1 sec. so that a continuous pattern of solutions is displayed on the screen of a cathode-ray tube. A typical series of traces for a sequence of four catalytic reactions is illustrated by Fig. $5 \mathrm{~A}$.

The solution of these equations for short times is shown by Figure 5B. In this case it is easy to see that the time sequence is the same as the chemical sequence. It should also be noted that there is a perceptible delay in the oxidation of the components farther from oxygen; one component must achieve a considerable oxidation before the next one in the sequence is oxidized perceptibly.*

\section{REGENERATIVE-FLOW METHOD}

In the study of pure soluble enzymes the chief obstacle has been the supply of material, and for this reason the stoppedflow method has been evolved, which requires only $10^{-10}$ mole of reactants for very rapid reactions. The turbidity of whole

* The studies were largely carried out by Mr. J. Higgins and Mr. W. Holmes. 
cell or mitochondrial suspensions is so great that this high performance is adversely affected and as much as $10^{-8}$ mole of reactants must be expended to measure rapid reactions. At the same time we have discovered a method for increasing the fluid economy by 100 -fold without sacrificing the performance, and thus it is now possible to study the rapid reactions of cytochromes, flavoproteins, and pyridine nucleotides in relatively small quantities of mitochondria or intact cells.

The new method is called the regenerative-flow method $(21,24)$. It depends upon the fact that the reduced state of a respiratory system is regenerated shortly after a low concentration of oxygen has been added, and the process may be repeated in a completely reversible fashion. Thus an enzyme solution that has once been reacted with oxygen can soon be used again for a second reaction with oxygen. A given volume of mitochondrial suspension will suffice for a large number of kinetic experiments if (1) the apparatus is designed for re-use of the regenerated material and (2) the material is diluted only slightly by the addition of oxygen solution.

A practical design of an apparatus for this purpose is illustrated in Figure 6. An 80-ml. syringe containing the anaerobic reduced mitochondrial suspension is discharged at the same time as a $1-\mathrm{ml}$. syringe containing the solution of oxygen. These reactants enter a mixing chamber ( 6 jets) and thence into a 1 $\times 1 \times 1 \mathrm{~cm}$. observation tube by means of a pushing block. The effluent is received in an $80-\mathrm{ml}$. syringe in which the oxidized components are allowed to become reduced again by their own respiration. The solution in the bottom syringe is then discharged into the upper one, the small syringe being refilled with oxygen solution. The measurement of reaction kinetics can be repeated at wavelengths appropriate to another component of the reaction sequence. This process can be repeated until the dilution of the solution becomes objectionableusually 40 experiments are possible with one filling of this apparatus. 


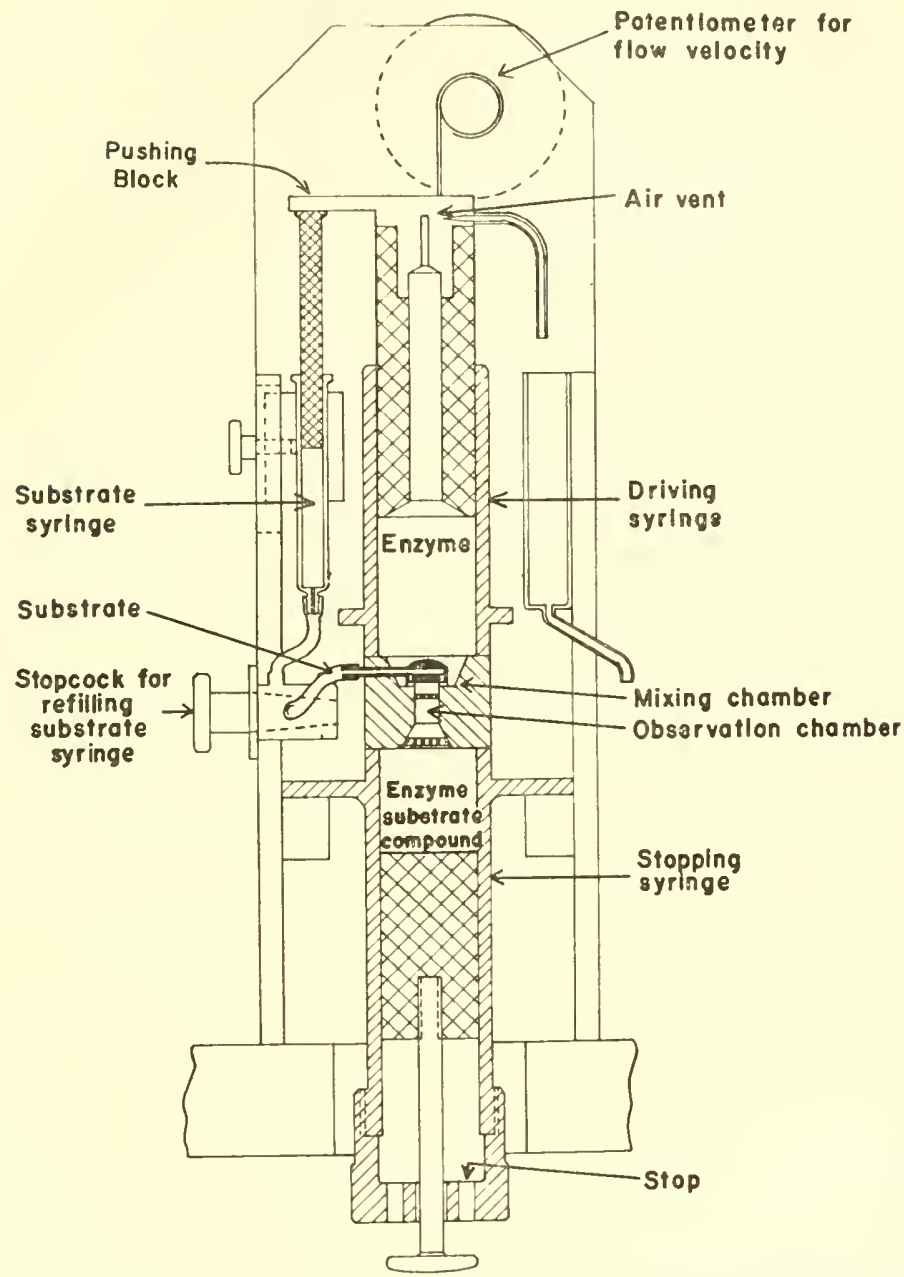

Fig. 6. An apparatus for measuring the time sequence of oxidation of the components of the mitochondrial respiratory chain (24).

Since a series of oxidations and reductions of the mitochondria can be carried out repetitively in this apparatus because exhaustion of the added oxygen occurs automatically with a respiratory system, the mitochondria can be used for a series of oxidations and reductions, and the apparatus is so arranged to take advantage of this. Volume of the "enzyme" syringe is 80 $\mathrm{ml}$., $1 \mathrm{ml}$. for the "substrate" syringe. Mixing time is less than 5 millisec. Satisfactory records can be obtained with cytochrome concentrations of less than $1 \mu \mathrm{M}$ (FA 21). 
THE SEQUENCE OF RESPIRATORY ENZYMES

IN THE RESPIRATORY GHAIN INVOLVED

IN OXIDATIVE PHOSPHORYLATION

We have recently studied the sequence of oxidation reactions that immediately follow the rapid mixing of oxygen and anaerobic mitochondria prepared from liver, and typical results are illustrated by Figure 7 . The top trace gives the complete record from the oxidation of reduced cytochrome $c$ (upward deflection of the trace) to its reduction (downard deflection of the trace) caused by the respiration of the mitochondria. The speed of oxidation is measured from the magnitude of the oxidation of cytochrome $c$ and the time after mixing, as measured from the flow velocity trace. For the particular record shown, cytochrome $c$ is $46 \%$ oxidized at 11 millisec. after mixing $15 \mu \mathrm{M}$ oxygen with anaerobic mitochondria. This corresponds to a pseudo first-order velocity constant of $53 \mathrm{sec}^{-1}$. At pairs of wavelengths appropriate to the difference spectrum of other components, reaction times are measured, and velocity constants are computed (see Table I). It is clear that the components listed in this table are oxidized at speeds consistent with

TABLE I

Sequence of Oxidation of Reduced Enzymes in Phosphorylating and

Nonphosphorylating Preparations. Regenerative Flow Apparatus

\begin{tabular}{llc}
\hline Material & Mitochondria & Small particles \\
Source & Rat liver & Pig heart \\
Substrate & BOHB & Succinate \\
Acceptor & ADP & None \\
[Oxygen], $\mu \mathrm{M}$ & 3 & 3 \\
Temperature, ${ }^{\circ} \mathrm{C}$. & 25 & 25 \\
Experiment & 368 & $255 \mathrm{~b}$ \\
Component & First-order velocity constants, sec. ${ }^{-1}$ \\
$a_{8}$ & 158 & - \\
$a$ & 120 & 180 \\
$c$ & 39 & 87 \\
$b$ & 20 & 11 \\
fp & 13 & - \\
\hline
\end{tabular}


their positions in the respiratory chain; for example, cytochrome $c$ is oxidized much more rapidly than flavoprotein. The

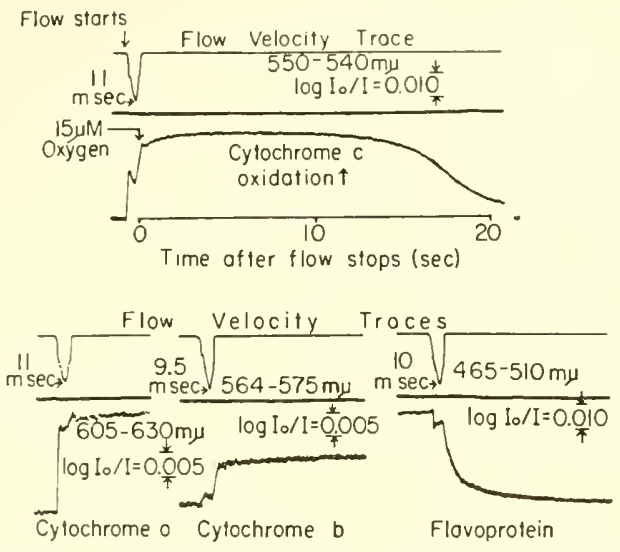

Fig. 7. An experimental study of the time sequence of oxidation of four members of the mitochondrial respiratory sequence.

The top trace of each of the four records represents flow velocity, and a downward deflection from the base line represents an increase of velocity. Since the method involves both accelerated-and stopped-flow principles, the interval of flow is only about $1 / 2 \mathrm{sec}$. The time after mixing corresponding to the shortest time achieved in a particular record is marked on the figure.

The spectrophotometric data are recorded by two monochromator apparatus in which the optical density changes are recorded with respect to a nearby neutral "reference" wavelength. The optical density changes at the "active" wavelengths are registered so that a decrease of optical density gives an upward deflection in all cases (the sense of deflections being the same as in Fig. 5). Thus an oxidation gives an upward deflection in all cases except flavoprotein. The complete cycle of oxidation and reduction is shown for cytochrome $c$; only the initial phases of the reaction are shown for the other three components.

The speed of reaction is computed from the optical density change attained at the highest value of flow velocity recorded by the flow velocity trace. This value is converted into per cent completion of the oxidation reaction by means of the maximum optical density change attained after the flow stops. A summary of data is given in Table I (Expt. 367c).

speeds of oxidation are more than adequate to account for the observed respiration of these preparations. Any other re- 
spiratory chain that is proposed for cellular respiration should be examined critically in view of these data.

It is interesting to note that the oxidation of cytochrome $c$ relative to cytochrome $a$ is faster in the nonphosphorylating preparation than in the phosphorylating one. And the reverse is true of cytochrome $b$. It is very likely that not only can the reactivity of components in the respiratory chain be decreased upon the loss of oxidative phosphorylation (e.g., cytochrome b), but some may also increase.

\section{RESPIRATORY CHAIN}

On the basis of substrate-inhibitor techniques and rapid reaction methods, the respiratory chain of intact mitochondria, whole ascites tumor cells, and baker's yeast are observed to contain the sequence of six spectroscopically identifiable components : cytochromes $a_{3}, a, c$, and $b$, flavoprotein, and DPNH. Other components which cannot be adequately identified and characterized are omitted from the present discussion (Slater's factor, cytochrome $c_{1}$, peroxidase complex II, etc.). If one includes the data obtained on the basis of the ADP action spectrum described above, at least three sites of oxidative phosphorylation should be included in the chain, at cytochromes $c, b$, and DPNH. Other data indicate phosphorylation between cytochrome $c$ and oxygen as well $(50,54)$. According to the mechanism proposed, these phosphorylation reactions affect the intact respiratory chain as follows :*

$$
\begin{aligned}
& \mathrm{O}_{2} \stackrel{k_{1}}{\rightarrow} a_{3}^{\prime \prime \prime} \stackrel{k_{3}}{\rightarrow} a^{\prime \prime \prime} \stackrel{k_{5}}{\rightarrow} c^{\prime \prime \prime} \stackrel{k_{7}}{\rightarrow} b^{\prime \prime \prime} \stackrel{k_{9}}{\rightarrow} \text { ff } \quad \stackrel{k_{11}}{\rightarrow} \text { DPN } \stackrel{k_{13}}{\rightarrow} \text { acetoacetate (19) } \\
& \mathrm{O}_{2}-\stackrel{k_{1}}{\leftarrow} a_{3}^{\prime \prime} \stackrel{k_{8}}{\leftarrow} a^{\prime \prime} \stackrel{k_{5}}{\leftarrow} c^{\prime \prime} \stackrel{k_{7}}{\leftarrow} b^{\prime \prime} \stackrel{k_{0}}{\leftarrow} \operatorname{rfp} \stackrel{k_{11}}{\leftarrow} \text { DPNH } \stackrel{k_{13}}{\leftarrow} \beta \text { hydroxybutyrate }
\end{aligned}
$$

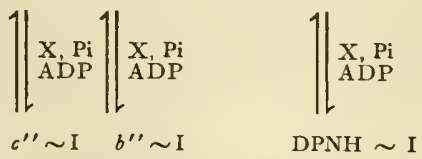

${ }^{*} \mathrm{BOHB}=\beta$ hydroxybutyrate, $\mathrm{fp}=$ flavoprotein, $\mathrm{rfp}=$ reduced flavoproteins. Cytochromes are designated by letter only. 
The members of the reaction chains written one over the other are those that react to give the products following the arrows. A discussion of this chain appears elsewhere $(29,30)$.

\section{OTHER RESPIRATORY CHAINS}

The detailed experiments of Keilin and Warburg afford strong evidence that the CO-sensitive cytochrome $a_{3}$ is a terminal oxidase of cellular respiration. And the evidence for cytochrome $c$ participation in respiration of intact mitochnndria is adequately supported by the cessation of respiration and the oxidation of cytochromes $c, a$, and $a_{3}$ upon addition of antimycin-A (29,31). Nevertheless, there are three variations of the respiratory chain that require consideration.

The first variation is the antimycin-A-insensitive cytochrome $b_{5}$ pathway for DPNH oxidation by oxygen $(27,46,57$, 65). This chain is inoperative in intact mitochondria because the intramitochondrial DPNH is not accessible to the microsomes which contain cytochrome $b_{5}$ (31). But upon hypotonic treatment of the mitochondria and addition of cytochrome $c$, considerable antimycin-A-insensitive respiration is obtained via this pathway $(46,59)$ :

$\mathrm{O}_{2} \longleftarrow$ oxidase $\longleftarrow c \longleftarrow$ microsomes $\longleftarrow$ DPNH $\longleftarrow$ substrate $(b s)$

With intact mitochondria, DPNH must also be added to activate the external pathway.

The possiblity of this pathway illuminates an open question with regard to the physiological function of mitochondria: how do they oxidize cytoplasmic DPNH to which the isolated mitochondria are impermeable? Whether or not the cytochrome $b_{5}$ pathway solves this problem is equally dubious, because the $b_{5}$ pathway involves the oxidation of cytoplasmic cytochrome $c$, which may not exist and to which the mitochondria are relatively inactive. Perhaps the only solution to such a dilemma is that the mitochondria as prepared have a diminished permeability compared to that of their state in nature. The only direct evidence bearing on this point is that when DPN is reduced in 
yeast cells by the action of the cytoplasmic glyceraldehyde- 3 phosphate dehydrogenase, cytochrome $b$ shows a large and rapid reduction (cytochrome $c$ also shows a small reduction) (22 and unpublished data). If the yeast particulates can be assumed to be similar to those of liver, this result indicates that cytoplasmic DPNH is immediately available to the intramitochondrial cytochrome $b$. However, direct experiments on intact liver cells are desirable to prove this point.

A second variation is one described by Martius (53) to explain the possibility that thyroxin could uncouple only one site of phosphorylation.

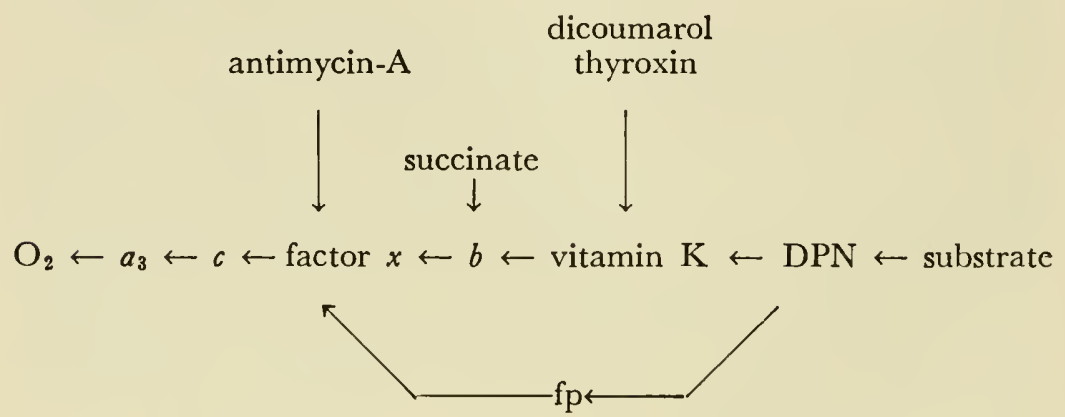

In terms of the spectroscopic studies represented here, Martius's hypothesis proposes that a cytochrome-c reductaseflavoprotein-provides a nonphosphorylating shunt from DPNH to cytochrome $c$, the shunt being sensitive to antimycin-A. Our studies show to the contrary that flavoprotein is involved in the pathway leading to cytochrome $b$. In addition, detailed studies of the effect of triiodothyronine on intact mitochondria failed to show a localization of the effect upon cytochrome $b$ (unpublished data in collaboration with Dr. H. A. Lardy). On a kinetic basis, such a mechanism is unsatisfactory because it requires a decrease of respiration when the $\mathrm{P}: \mathrm{O}$ value falls according to the following reasoning: The system implies that two electron donor systems compete for the factor, one phosphorylating and one nonphosphorylating. In order to give a higher $\mathrm{P}: \mathrm{O}$ value when the vitamin $\mathrm{K}$ pathway is operative, 
it must have a higher flux than the flavoprotein pathway. Thus blockage of the vitamin $\mathrm{K}$ pathway would cause respiration to fall by about $1 / 3$. But uncoupling effects generally cause increases of respiration. Thus the shunt mechanism is at present inadequate.

Another question is, of course, whether the $\mathrm{P}: \mathrm{O}$ value falls by one or whether thyroxin uncoupling gives a continuous decrease of phosphorylative activity to zero $(40,44)$. In the latter case the split respiratory chain is of no great aid in explaining the uncoupling effect.

The third alternative respiratory chain is proposed by Green et al. (38), on the basis of purified nonphosphorylating DPNH oxidase particles. Their contribution is of unusual interest because it states that Keilin's reduced cytochrome $c$ oxidase "must be considered. . .to be artifactual activities. . . ." and that cytochrome $c$ is "not a part of this electron transfer sequence." These statements must now be regarded as incorrect in view of recent work in this laboratory by Estabrook and Mackler (339). The closed DPNH oxidase preparation has cytochrome content, steady-state oxidation reduction levels, and turnover numbers of the components that are so similar to those previously reported for a Keilin and Hartree preparation (12) that the mechanism of electron transport from cytochrome $c$ to oxygen in the two preparations can be considered to be identical and in accord with equations (19) and (20). On the other hand, this material is less turbid than Keilin and Hartree's preparations, thus it has been possible to use faster flow apparatuses than that of Figure 6 in the study of the time sequence of oxidation and reduction of the cytochrome components as well as in the reaction of ferrocytochrome $a_{3}$ with oxygen. These results support the chemical representations of the electron transfer processes discussed here and obviate the necessity of formulating alternative respiratory chains or concepts of electron transport. 


\section{References}

1. Bonnichsen, R. K., B. Chance, and H. Theorell, Acta Chem. Scand., 1 685 (1947).

2. Brown, A. J., Trans. Chem. Soc., 81, 373 (1902).

3. Chance, B., Acta Chem. Scand., 7, 236 (1947).

4. Chance, B., Nature, 167, 914 (1948).

5. Chance, B., J. Biol. Chem., 180, 865 (1949).

6. Chance, B., Arch. Biochem. Biophys., 21, 416 (1949).

7. Chance, B., Arch. Biochem. Biophys., 22, 224 (1949).

8. Chance, B., Arch. Biochem. Biophys., 24, 410 (1949).

9. Chance, B., and D. Herbert, Biochem. J. (London), 46, 402 (1950).

10. Chance, B., Biochem. J. (London), 46, 387 (1950).

11. Chance, B., Advances in Enzymol., 12, 170 (1951).

12. Chance, B., Nature, 169, 215 (1952).

13. Chance, B., Modern Trends in Physiol. and Biochem., 1952, 25.

14. Chance, B., Arch. Biochem. Biophys., 41, 416 (1952).

15. Chance, B., Arch. Biochem. Biophys., 41, 404 (1952).

16. Chance, B., J. Biol. Chem., 197, 577 (1952).

17. Chance, B., in A. Weissberger, ed., Techniques of Organıc Chemistry, Vol. 8, p. 655. Interscience, New York-London, 1953.

18. Chance, B., in W. D. McElroy and B. Glass, eds., The Mechanism of Enzyme Action, p. 438. Johns Hopkins Press, Baltimore, 1954.

19. Chance B., in D. Glick, ed., Methods of Biochemical Analysis, Vol. I, p. 420. Interscience, New York-London, 1954.

20. Chance, B., Harvey Lectures, Ser. 49, 145 (1954).

21. Chance, B., Discussions Faraday Soc., No. 17, 120 (1954).

22. Chance, B., in W. D. McElroy and B. Glass, eds., The Mechanism of Enzyme Action, p. 430. Johns Hopkins Press, Baltimore, 1954.

23. Chance, B., D. S. Greenstein, and F. J. W. Roughton, Arch. Biochem. Biophys., 37, 301 (1952).

24. Chance, B., and V. Legallais, Discussions Faraday Soc., No. 17, 123 (1954).

25. Chance, B., and A. C. Maehly, in S. P. Colomick and N. O. Kaplan, eds., Methods in Enzymology, Vol. 2, p. 764. Academic Press, New York, 1955.

25a. Chance, B., and B. Mackler, Am. Chem. Soc. Spring Meeting, 1956, Dallas, in press.

26. Chance, B., and G. R. Williams, Federation Proc., 13, 633 (1954).

27. Chance, B., and G. R. Williams, J. Biol. Chem., 209, 945 (1954).

28. Chance, B., and G. R. Williams, Federation Proc., 14, 190 (1955).

29. Chance, B., and G. R. Williams, Nature, 176, 250 (1955).

30. Chance, B., and G. R. Williams, J. Biol. Chem., 217, 439 (1955).

31. Chance, B., and G. R. Williams, J. Biol. Chem. 277, 395 (1955).

32. Elliot, K. A. C., in Handbuch den Katalyse, Vol. 3, p. 387. Springer, Berlin, 1941.

32a. Estabrook, R., and B. Mackler, Federation Proc., in press.

33. Euler, H. von, and K. Josephson, Ann., 452, 158 (1927).

34. Fergusson, R. R., and Chance, B., Science, 122, 466 (1955). 
35. George, P., Biochem. J. (London), 44, 197 (1949).

36. George ${ }_{\varsigma}$ P., Biochem. J. (London), 54, 267 (1953).

37. George, P., J. Biol. Chem., 201, 413 (1953).

38. Green, D. E., B. Mackler, R. Repaske, and H. R. Mahler, Biochin. et Biophys. Acta, 15, 435 (1954).

39. Henri, V., Lois generales ou l'action des diastases, 1903.

40. Hoch, F. L., and F. Lipmann, Proc. Natl. Acad. Sci. U. S., 40, 909 (1954).

41. Keilin, D., and E. F. Hartree, Proc. Roy. Soc. (London), B127, 167 (1939).

42. Keilin, D., and E. F. Hartree, Biochem. J. (London), 49, 88 (1951).

43. Keilin, D., and T. Mann, Proc. Roy. Soc. (London), B122, 119 (1937).

44. Lardy, H., and G. F. Maley, Recent Progr. Hormone Research, 10, 129 (1954).

45. Lardy, H., and H. Wellman. J. Biol. Chem., 195, 215 (1952).

46. Lehninger, A. L., Harvey Lectures, Ser. 49, 176 (1954).

46a. Lehninger, A. L., Enzyme Symposium, Detroit, 1956. Academic Press, New York, in press.

47. Mackler, B., R. Repaske, P. M. Kohout, and D. E. Green, Biochim. et Biophys. Acta, 15, 437 (1954).

48. Maehly, A. C., in D. Glick, ed., Methods of Biochemical Analysis, Vol. I, p. 372. Interscience, New York, 1954.

49. Maehly, A. C., and S. Paleus, Acta Chem. Scand., 4, 508 (1950).

50. Maley, G. F., and H. A. Lardy, J. Biol. Chem., 210, 903 (1954).

51. Mann, P. J. G., Biochem. J. (London), 24, 918 (1931).

52. Michaelis, L., and M. L. Menten, Biochem. Z., 49, 433 (1913).

53. Martius, C., Biochem. Z., 306, 26 (1954).

54. Nielson, S. O., and A. L. Lehninger, J. Am. Chem. Soc., 76, 3860 (1954).

55. Ogura, Y., to be submitted to Arch. Biochem. Biophys.

56. Ogura, Y., Y. Tonomura, and S. Hino, J. Biochem. (Japan), 37, 249 (1950).

57. Pappenheimer, A. M., Jr., and C. M. Williams, J. Biol. Chem., 209, 915 (1954).

58. Polis, D. B., and H. W. Shmukler, Abstracts, Am. Chem. Soc., 126th Meeting, New York, 1954.

59. Pressman, B. C., and C. DeDuve, Arch. intern. physiol., 62, 306 (1954).

60. Racker, E. F., and I. Krimsky, J. Biol. Chem., 198, 731 (1952).

61. Slater, E. C., Biochem. J. (London), 44, 305 (1949).

62. Slater, E. C., Biochem. J. (London), 45, 14 (1949).

63. Slater, E. C., Biochem. J. (London), 46, 484 (1950).

63a. Slater, E. C., Nature, 172, 975 (1953).

64. Smith, L., and E. Stotz, J. Biol. Chem., 209, 819 (1954).

65. Strittmatter, C. F., and E. G. Ball, Proc. Natl. Acad. Sci. U. S., 38, 55 (1952).

66. Theorell, H., Enzymologia, 10, 250 (1941).

67. Theorell, H., and R. K. Bonnichsen, Acta Chem. Scand., 5, 1105 (1951).

68. Theorell, H., and B. Chance, Acta Chem. Scand., 5, 1127 (1951).

69. Tsou, C. L., Biochem. J. (London), 50, 493 (1952). 


\section{ON THE NATURE OF HEMOPROTEIN REACTIONS}

PHILIP GEORGE, Department of Colloid Science, Cambridge University*

\section{A Survey}

In biochemistry and physiology, hemoproteins are important because of the roles they play in biological oxidation, on the one hand, transporting and storing oxygen, and on the other, acting as catalysts in certain of the oxidation processes. In physicochemical studies, which have as their aim an understanding of the mechanism by which chemical reactions take place, hemoprotein reactions are important too, because they show a specificity which is more highly evolved than that of any other class of enzyme. Furthermore they exemplify particularly well two of the ways in which a protein can influence the reaction of a prosthetic group-an effect which is to be distinguished from the determination by the protein of the specific reaction undergone by the prosthetic group. Interaction can occur between the prosthetic group and an acidic group on the protein, which on dissociation alters the affinity of the enzyme for its substrate. Interaction can occur between prosthetic groups themselves, if there are more than one on each molecule, the protein in some way providing a "pathway" by which the groups influence each other.

* Present address: Department of Chemistry, University of Pennsylvania, Philadelphia, Pa. 
The greater specificity of the hemoproteins lies in the fact that with the same prosthetic group, iron protoporphyrin, one of four different reactions is especially favored depending on the particular protein to which the prosthetic group is joined (32). The cytochromes play a catalytic role in the main metabolic process by undergoing rapid oxidation and reduction between the ferrous and ferric states. The hemoglobins, of blood, and of muscle tissue, combine reversibly with oxygen in the ferrous state without undergoing rapid oxidation to the ferric state. The peroxidases and catalases are very efficient catalysts for the oxidation of certain reducing agents by peroxides and some other strong oxidizing agents. Peroxidase action proceeds by single equivalent oxidation steps, catalase action by what appears to be a simultaneous two-equivalent oxidation step, each of the enzymes showing a marked preference for one class of reducing agent $(10,11)$. The intermediate compounds formed in both reactions were at one time regarded as "enzyme-substrate complexes," according to the Michaelis-Menten theory of enzyme action (5), but it seems very likely now that they are higher oxidation states of the hemoproteins, in which a more complicated oxidation process than electron transfer has occurred (21).

The interaction effects have been more fully investigated with the hemoglobins. Interaction between prosthetic groups themselves is, in fact, only well established in the case of hemoglobin, which has four heme groups per molecule. The affinity of one particular heme for, say, oxygen depends upon the extent to which the other three are already combined $(32,38,47)$. Interaction between the prosthetic group and an ionizing group on the protein was also first observed in the hemoglobin-oxygen reaction, although this kind of interaction certainly occurs in myoglobin reactions, and very probably in those of catalase and peroxidase too. When hemoglobin combines with oxygen, the acid strength of an ionizing group in the neighborhood of the heme, believed to be on the amino acid residue to which the heme is attached, increases from a $p \mathrm{~K}$ value of about 7.9 in 
hemoglobin to about 6.7 in oxyhemoglobin (47). The liberation of the proton, which accompanies oxygenation in the $p \mathrm{H}$ range between these $p \mathrm{~K}$ values, accounts for the important physiological effect (the Bohr effect) whereby the production of $\mathrm{CO}_{2}$ from $\mathrm{HCO}^{-}$and $\mathrm{CO}_{3}{ }_{3}$ in the blood is enhanced when hemoglobin becomes oxygenated (32). Just as the degree of ionization of this acidic group depends upon the extent of oxygenation, so the affinity of the ferrous iron atom for oxygen depends upon the $p \mathrm{H}$ of the solution. Groups whose reactions are interdependent in this way have been designated "linked groups," and the ionization, a "linked ionization" (47). Evidence has recently been presented showing that the ionization of other groups on the protein as a whole can also influence the reactivity of the heme iron atom, but this effect is perceptible only in solutions of low ionic strength (17).

Three of the specific hemoprotein reactions are obviously oxidation-reduction processes, and similar considerations are entailed in understanding the specific character of the oxygenation reaction, as will be shown later. Yet these specific reactions seem to involve only the iron atom of the heme or hematin, in a valency change, or in replacement reactions at the sixth position in the coordination shell, just like the whole series of nonspecific reactions which hemoproteins undergo. In the ferrous state, for example, combination with carbon monoxide and nitric oxide occurs, and in the ferric state, combination with cyanide, fluoride, azide, thiocyanate, etc. (32).

A detailed knowledge of the mechanism of these nonspecific reactions can therefore help to show how the specificity is achieved in the other reactions, because a comparison of data should enable this specificity to be ascribed to certain thermodynamic and kinetic factors. A comparison of this kind would furthermore provide a basis for deciding to what extent hemoprotein reactions conform with those of simpler coordination compounds and aquated ions, and to what extent the especially complicated hemoprotein structure does, in fact, endow the molecule with new properties. 
The data required are very simple, but scrupulous attention must be paid to the control of temperature, $p \mathrm{H}$, and ionic strength. The possibility of specific salt effects complicating the reactions should be investigated and buffer solutions chosen to minimize them, although, fortunately, the hemoprotein reactions dealt with below appear to be free from such complications (15). The reaction mechanism can be decided from the $p \mathrm{H}$ variation of equilibrium constants $\left(K_{\text {obs. }}\right)$ and forward and back velocity constants $\left(k_{f}, k_{d}\right)$; and the solution of various algebraic equations gives equilibrium constants $(K)$ and velocity constants $(k)$ for the individual reaction steps. A typical example, the ferrimyoglobin-cyanide reaction, will be discussed later. Having obtained $K$ at a series of ionic strengths, extrapolation to zero ionic strength gives $K_{0}$, from which the standard free-energy change for the particular step, $\Delta F^{0}$, can be calculated using the van't Hoff isotherm,

$$
\Delta F^{0}=-R T \ln K_{0}
$$

From values of $K$ at several temperatures, $\Delta H_{0}$, the difference in heat content between products and reactants follows from the van't Hoff isochore,

$$
d / d t \ln K=\Delta H^{0} / R T^{2},
$$

and $\Delta S^{0}$, the entropy change accompanying the reaction, can be obtained from the thermodynamic expression

$$
\Delta F^{0}=\Delta H^{0}-T \Delta S^{0} .
$$

In addition, heats and entropies of activation may be calculated from the variation of velocity constants with temperature, using the equation

$$
k=6.2 \times 10^{12} e^{\Delta S^{*} / R} e^{-\Delta H^{*} / R T}
$$

where $\Delta H^{*}=E-R T, E$ being the Arrhenius activation energy. However there are fewer data of this kind available, so the present discussion will be restricted to equilibrium and velocity constants, and the values of $\Delta F^{0}, \Delta H^{0}$, and $\Delta S^{0}$.

It will appear that the $\Delta H^{0}$ and $\Delta S^{0}$ values are the most 
useful criteria in comparing and contrasting the various reactions, as has already been found in the case of simpler inorganic and organic systems. The $\Delta H^{0}$ values give the balance between the energies of the old bonds broken and the new bonds formed. The $\Delta S^{0}$ values reflect the accompanying changes in configuration and solvation. The structure of the products may entail a looser, or a more closely knit, configuration, and the entropy increases or decreases accordingly. If ionic charges are created or destroyed in the reaction, the solvation or desolvation of water molecules about these charges will result in a substantial decrease or increase in entropy.

\section{Ferrous and Ferric Hemoprotein Complexes}

It is self-evident that the correct elucidation of the mechanism by which these complexes are formed depends on knowing their structure; for this purpose the nature of the amino acid residue in the protein to which the iron protoporphyrin is attached is immaterial, but it is essential to know precisely the nature of the ligand joined to the iron in its sixth coordination position. This is not always obvious. Many years elapsed before oxyhemoglobin was recognized as a complex of ferrohemoglobin in which oxygen was the ligand, partly because the distinction between reversible oxygenation and irreversible oxidation yielding ferrihemoglobin was not readily appreciated. The simplest approach is unfortunately inapplicable. The large size of hemoproteins, whose molecular weights range from 16,200 for myoglobin through 41,000 and 68,000 for peroxidase and hemoglobin to 224,000 for catalase, makes it impossible to determine the composition of the complexes by the analytical methods in use for simpler coordination compounds. Hence there can be no direct evidence for the replacement of a water molecule coordinated to the iron by oxygen when hemoglobin forms oxyhemoglobin, and no direct evidence that $\mathrm{CN}^{-}$and not HCN is bonded when ferrihemoglobin forms its cyanide complex. The hypothesis has long been accepted, however, that in the 
ferrous state complexes are formed preferentially with neutral molecules, e.g., $\mathrm{O}_{2}$ and $\mathrm{CO}$, and in the ferric state with singly charged anionic ligands, e.g., $\mathrm{CN}^{-}, \mathrm{F}^{-}$, and $\mathrm{N}_{3}^{-}(30,32)$. There has been scarcely any need to question its general validity, although there are exceptions, for example, nitric oxide and cyanide combine with both ferri- and ferro-hemoglobin.

The justification for such a hypothesis has received little attention. Two of the four pyrollic nitrogen atoms of the porphyrin ring are negatively charged, and so, provided that the atom in the amino acid residue to which the iron is attached does not itself carry a charge, the iron atom in the ferrous state is uncharged, and in the ferric state carries a charge of +1 . Thus if complex formation was in accord with the hypothesis, both ferrous and ferric complexes would be neutral, and this could be regarded as the required condition for the most stable complex. Furthermore, if the bonding was covalent through $d^{2} s p^{3}$ hybridization, as it is in many of the complexes (28), and there were in addition double bonds utilizing otherwise unshared $d$ electrons of the iron atom, then the iron atom itself could become neutral. Recent considerations of the stability of aquated ions, ammonia complexes, cyanide complexes, and oxy-acids of the transition elements, suggest that this particular charge distribution is the most favorable (35).

There are good thermochemical reasons why this should be so. A large amount of energy, given by the ionization potential, is required to produce an ion by the expulsion of an electron. In forming coordinate links of the covalent type an ion regains electrons, and, depending upon the extent to which this happens, the resultant charge may become zero or even negative. The energy release accompanying the decrease in charge is taken up in endothermic steps necessary for bond formation, the balance appearing as the heat of reaction. The nearer the charge to zero the larger the energy release, which, other factors being equal, would favor an exothermic heat of reaction, and thus a favorable free-energy change.

Considerations of the entropy changes associated with 
complex formation also show that the formation of a neutral complex can be highly favored. In reactions accompanied by a decrease in charge, as there would be if the ferric hemoprotein having a net charge of +1 combined with a singly charged anionic ligand, there is an increase in entropy because the water molecules are less tightly held in the solvation shells of the products than in those of the reactants. The formation of ionpair complexes by inorganic cations, for example, $\mathrm{Fe}^{3+}, \mathrm{Cu}^{2+}$, $\mathrm{Cd}^{2+}, \mathrm{Pb}^{2+}$, provides a very good example (46). $\Delta S^{0}$ values for five such complexes of the ferric ion are given in Table I, where the majority of the values are seen to be large and positive. If one of the reactants, however, is effectively neutral as in the case of a ferrous hemoprotein, then the entropy change does not favor unequivocally any one type of reaction.

TABLE I

Entropy Data for "Ion-Pair" Complexes of the Ferric Ion (46)

\begin{tabular}{llllll}
\hline \multicolumn{1}{c}{ Ligand } & $\mathrm{OH}^{-}$ & $\mathrm{F}^{-}$ & $\mathrm{Cl}{ }^{-}$ & $\mathrm{Br}^{-}$ & $\mathrm{CNS}^{-}$ \\
\hline$\Delta S^{0}($ e.u. $)$ & +50 & +49 & +35 & +23 & +8.0 \\
$\left(\bar{S}_{\mathrm{ML}}^{0}-\bar{S}_{\mathrm{M}}^{0}\right)($ e.u. $)$ & +47.5 & +46.7 & +48.2 & +42.3 & +44 \\
\hline
\end{tabular}

With the ferric form of hemoproteins all three considerations favor combination with a singly charged anionic ligand in preference to a neutral molecule. It is therefore surprising that the $p \mathrm{H}$ variations of velocity constants and equilibrium constants, which provide the best indirect evidence for the structure of the complexes, can be taken to suggest that the HCN molecule is bonded to the iron in the cyanide complexes of both ferrihemoglobin and ferrimyoglobin (2). On the basis of similar evidence it would appear that ferricatalase forms complexes by combining with $\mathrm{HF}, \mathrm{HN}_{3}, \mathrm{HCO}_{2} \mathrm{H}$, and $\mathrm{HCN}$ (2); and the scanty equilibrium data available for the fluoride and cyanide complexes of ferriperoxidase likewise indicate bonding of HF and HCN (17). However, in the analysis of these results, concurrent reaction paths, one via the undissociated acid and one via the anion, were not considered, nor was the ionization of heme-linked groups. 
The $p \mathrm{H}$ dependence of velocity constants and equilibrium constants arising from their ionization is superimposed on the $p \mathrm{H}$ dependence characteristic of the bonding of the ligand. The interpretation of results can therefore be very complicated, and it may be very difficult to decide from such results alone whether the undissociated acid or the anion is bonded to the iron.

This difficulty can be resolved if the acid strengths of the linked ionizing groups are first derived from data on reactions where the effect of $p \mathrm{H}$ comes entirely from the ionization of these groups, for instance, in the oxygenation reaction-

$$
\mathrm{Fe}\left(\mathrm{H}_{2} \mathrm{O}\right)+\mathrm{O}_{2} \rightleftharpoons \mathrm{Fe}\left(\mathrm{O}_{2}\right)+\mathrm{H}_{2} \mathrm{O}
$$

and in the cell reaction involving the standard hydrogen electrode,

$$
\mathrm{Fe}^{+}\left(\mathrm{H}_{2} \mathrm{O}\right)+{ }_{1} /{ }_{2} \mathrm{H}_{2} \rightleftharpoons \mathrm{Fe}\left(\mathrm{H}_{2} \mathrm{O}\right)+\mathrm{H}^{+}
$$

In the case of hemoglobin these two reactions are affected quite markedly by changes in $p \mathrm{H}$ and, within experimental error, to

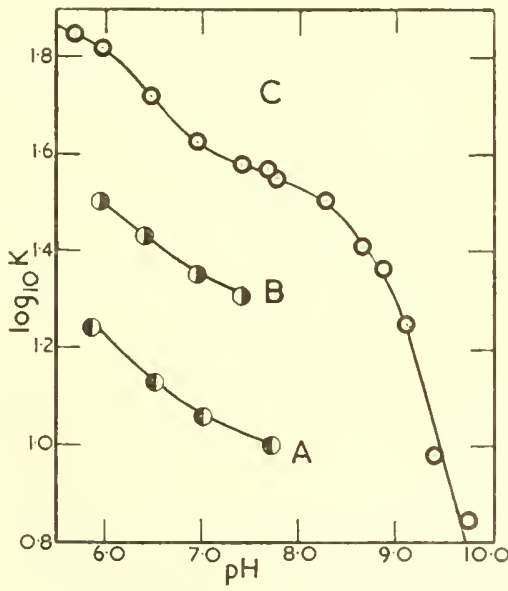

Fig. 1. The logarithm of the equilibrium constant plotted against $p \mathrm{H}$ for $\mathrm{A}$, the myoglobin-oxygen reaction (43), for $\mathrm{B}$, the cell reaction involving the standard hydrogen electrode (42), and for C, the formation of ferrimyoglobin fluoride (18). In $\mathrm{A}$ and $\mathrm{B}, 1.00$ and 0.60 , respectively, have been added to the actual values of $\log _{10} K$ to bring them closer to curve $\mathrm{C}$. 
the same extent $(6,47)$. Analysis of the equilibrium constant data has shown that the effect can be attributed to two hemelinked groups with $p \mathrm{~K}$ values, denoted by $p \mathrm{~K}_{1}$ and $p \mathrm{~K}_{2}$, which are about 5.3 and 7.9 in ferrohemoglobin, changing identically in oxyhemoglobin and ferrihemoglobin to 5.8 and 6.7, respectively. In the case of myoglobin, although it has often been stated that the same two reactions are little affected by $p \mathrm{H}$, a similar analysis has been made only recently (16). The data required were taken from the papers of Taylor and Morgan (42) and of Theorell (43) and are plotted in Figure 1, curves $A$ and $B$, along with some equilibrium data for the fluoride reaction of ferrimyoglobin, curve $C$ (18). The similar trend of all three curves suggests that, despite differences in temperature and ionic strength, the $p \mathrm{~K}$ values for the heme-linked ionizations are quite similar in oxymyoglobin and ferrimyoglobin, and in ferromyoglobin and ferrimyoglobin fluoride. Mathematical analysis of curves $A$ and $B$ shows that one linked group, with $p \mathrm{~K}$ values of about 6.0 for the first two compounds and about 6.4 for the second two compounds, is sufficient to account for the observed variation of $\log K$ with $p \mathrm{H}$ (16).

Having obtained an approximate $p \mathrm{~K}$ value for this single heme-linked ionization in ferrimyoglobin, it was found possible to make a consistent analysis of equilibrium constant data for both the fluoride and cyanide reactions on the assumption that the anion is bonded to the iron in both complexes. The data cover a $p \mathrm{H}$ range which is considerably greater than that for the oxygenation and cell reactions ( $c f$. Fig. 1), and lead to the following provisional $p \mathrm{~K}$ values for the heme-linked group at $20^{\circ} \mathrm{C}$. and $I=0.10: 6.0$ in ferrimyoglobin and 6.4 and 7.0 in its fluoride and cyanide complexes, respectively (16). These results were confirmed by an independent and direct measurement of the $p \mathrm{~K}$ value in the fluoride complex, where the intensities of the absorption bands increase slightly owing to the ionization of the heme-linked group as the $p \mathrm{H}$ rises from 5.5 to $7.0(17,45)$ - a similar change to that found with ferrihemoglobinfluoride in the $p \mathrm{H}$ range 5.4 to 6.2 (17). Additional confirma- 
tion of anion bonding comes from studies of the variation of the equilibrium constant for the fluoride reaction with ionic strength, $I$ (17). If $\log K$ is plotted against $\sqrt{I}$ the slope of the line is -1.2 at low values of $I$, compared to the theoretical slope of -1.0 expected for a reaction of the type

$$
\mathrm{Fe}^{+}\left(\mathrm{H}_{2} \mathrm{O}\right)+\mathrm{F}^{-} \rightleftharpoons \mathrm{FeF}+\mathrm{H}_{2} \mathrm{O}
$$

where the change in charge on the iron is +1 to zero. This result also substantiates the structure assumed for the hemoprotein with the iron joined to a neutral atom in an amino acid residue, for if this atom were negatively charged the net charge on the iron in the ferric state would be zero, and the charge change in the above reaction would be from zero to -1 .

The $p \mathrm{H}$ changes which accompany the formation of ferrimyoglobin fluoride and cyanide in unbuffered solutions, at initial $p \mathrm{H}$ values of about 8 and about 10, were also what would be expected if the anions were bound (16).

Parallel studies of the $p \mathrm{H}$ variation of the velocity constants for the formation and dissociation of the ferrimyoglobin cyanide and fluoride complexes show that both complexes are formed

\section{TABLE II}

Reaction Mechanism and Provisional Kinetic Data for the FerrimyoglobinCyanide Reaction at $25^{\circ} \mathrm{C}$. And $\mathrm{I}=0.10(16,18)$

Symbols: $\quad \mathrm{H}^{+} \mathrm{Y} \mathrm{Fe}^{+}\left(\mathrm{H}_{2} \mathrm{O}\right)$ and $\mathrm{Y} \mathrm{Fe}^{+}\left(\mathrm{H}_{2} \mathrm{O}\right)$ represent ferrimyoglobin with its heme-linked group in the conjugate acid and base forms, respectively, the charge being arbitrarily assigned to the former.

Units:

$$
\begin{aligned}
& k_{1}, k_{1}^{\prime}, k_{2}, k_{2}{ }^{\prime}, k_{-1}{ }^{\prime} \text { and } k_{-2}{ }^{\prime}, 1 . \text { mole }^{-1} \mathrm{sec}^{-1}: k_{-1} \text { and } k_{-2}, \text { sec. }{ }^{-1} \\
& \mathrm{Y} \cdot \mathrm{Fe}^{+}\left(\mathrm{H}_{2} \mathrm{O}\right)+\mathrm{CN}^{-}-\underset{k_{-1}=1.9 \times 10^{-4}}{\stackrel{k_{1}=3.7 \times 10^{3}}{\rightleftharpoons}} \mathrm{Y} \cdot \mathrm{FeCN}+\mathrm{H}_{2} \mathrm{O} \\
& \mathrm{Y} \cdot \mathrm{Fe}^{+}\left(\mathrm{H}_{2} \mathrm{O}\right)+\mathrm{HCN} \underset{k_{-1^{\prime}}=1.0 \times 10^{4}}{\stackrel{k_{1^{\prime}}=1.1 \times 10^{2}}{\rightleftharpoons}} \mathrm{Y} \cdot \mathrm{FeCN}+\mathrm{H}_{3} \mathrm{O}^{+} \\
& \mathrm{H}^{+} \mathrm{Y} \cdot \mathrm{Fe}^{+}\left(\mathrm{H}_{2} \mathrm{O}\right)+\mathrm{CN}^{-} \frac{k_{2}=1.6 \times 10^{5}}{\stackrel{k_{-2}=7 \times 10^{-4}}{\rightleftharpoons}} \mathrm{H}^{+\mathrm{Y}} \cdot \mathrm{FeCN}+\mathrm{H}_{2} \mathrm{O} \\
& \mathrm{H}^{+} \mathrm{Y} \cdot \mathrm{Fe}^{+}\left(\mathrm{H}_{2} \mathrm{O}\right)+\mathrm{HCN} \underset{k_{-2^{\prime}}=4.5 \times 10^{2}}{\stackrel{k_{2}^{\prime}=41}{\rightleftharpoons}} \mathrm{H}^{+} \mathrm{Y} \mathrm{FeCN}+\mathrm{H}_{3} \mathrm{O}^{+}
\end{aligned}
$$


by two distinct reaction paths, one with the undissociated acid, and the other with the anion $(17,18)$. The reaction mechanism is set out for the case of the cyanide complex in Table II. A consistent analysis of results is possible on the basis that the reactions of alkaline ferrimyoglobin, $\mathrm{YFeOH}$, in which a proton has ionized from the water molecule coordinated to the iron, are negligible in the comparison with the other four reactions, $1,1^{\prime}$, 2 , and $2^{\prime}$. The various forward and back velocity constants, $k_{1}$ and $k_{-1}$, etc., have been calculated from the experimentally determined velocity constants for the formation and dissociation of the complex, $k_{f}$ and $k_{d}$, and the over-all equilibrium constant, $K_{\text {obs }}$ using the equations:

$$
\begin{gathered}
k_{f}=\frac{\mathrm{H}^{+}}{\left(K_{r}+\mathrm{H}^{+}\right)\left(K_{\mathrm{HCN}}+\mathrm{H}^{+}\right)\left(K_{\mathrm{Fe}_{\theta}}+\mathrm{H}^{+}\right)} \\
\left\{K_{r}\left(k_{1} K_{\mathrm{HCN}}+k_{1}^{\prime} \mathrm{H}^{+}\right)+\mathrm{H}^{+}\left(k_{2} K_{\mathrm{HCN}}+k_{2}^{\prime} \mathrm{H}^{+}\right)\right\} \\
k_{d}=\frac{1}{\left(K_{p}+\mathrm{H}^{+}\right)}\left\{K_{p}\left(k_{-1}+k^{\prime}{ }_{-1} \mathrm{H}^{+}\right)+\mathrm{H}^{+}\left(k_{-2}+k^{\prime}{ }_{-2} \mathrm{H}^{+}\right)\right\}
\end{gathered}
$$

and

$$
\begin{aligned}
K_{\text {obs. }} & =\frac{k_{f}}{k_{d}} \\
& =\frac{K_{r} \times\left(K_{p}+\mathrm{H}^{+}\right) \times \mathrm{H}^{+} \times K_{\mathrm{HCN}}}{\left(K_{\tau}+\mathrm{H}^{+}\right) \times K_{p} \times\left(K_{F \bullet}+\mathrm{H}^{+}\right) \times\left(K_{\mathrm{HCN}}+\mathrm{H}^{+}\right)} \times\left(\frac{k_{1}}{k_{-1}}\right)
\end{aligned}
$$

$K_{r}$ and $K_{p}$ are, respectively, the ionization constants for the heme-linked group in ferrimyoglobin and the complex, $K_{\mathrm{HCN}}$ and $K_{\mathrm{Fe}}$ are the ionization constants of $\mathrm{HCN}$ and of the coordinated water molecule in acidic ferrimyoglobin. In the analysis it is not possible to distinguish between reactions $1^{\prime}$ and 2, i.e., $\mathrm{YFe}^{+}\left(\mathrm{H}_{2} \mathrm{O}\right)+\mathrm{HCN}$ and $\mathrm{H}^{+} \mathrm{YFe}^{+}\left(\mathrm{H}_{2} \mathrm{O}\right)+\mathrm{CN}^{-}$, because both reactions show an identical $p \mathrm{H}$ variation in the $p \mathrm{H}$ range of the experiments. It has been assumed that each path contributes equally in order to calculate the velocity constants $k_{1}^{\prime}, k_{-1}^{\prime}, k_{2}$, and $k_{-2}$, which are listed in Table II along with those for reactions 1 and $2^{\prime}$. These values are provisional because a more detailed investigation over the entire $p \mathrm{H}$ range 
(5-12) is in progress. Their relative magnitudes are, however, unlikely to change significantly. The effect of the ionization of the heme-linked group on the velocity constants and equilibrium constants for the corresponding reactions can thus be examined, bearing in mind that either $k_{1}^{\prime}$ and $k_{-1}^{\prime}$, or $k_{2}$ and $k_{-2}$, but not both, may have much smaller values and so invalidate one of the comparisons.

When the attacking species is an ion it can be seen that the velocity constant is considerably greater for the path most favored by coulombic attraction (or cancellation of charge), e.g., with $\mathrm{CN}^{-}$as reactant, $k_{2} \approx 44 k_{1}$, and with $\mathrm{H}_{3} \mathrm{O}^{+}$as reactant, $k_{-1}^{\prime} \approx 21 k_{-2}^{\prime}$. But when the attacking ligand is a neutral molecule, the difference between velocity constants is very much smaller, as may be seen by comparing $k_{1}^{\prime}$ and $k_{2}^{\prime}$, $k_{-1}$ and $k_{-2}$. Since the velocity constants change in this way when the group ionizes, ferrimyoglobin has the greater affinity for cyanide when the linked group is in its conjugate acid form, i.e., $K_{2}$ is about 10 times greater than $K_{1}$, and $K_{2}^{\prime}$ about 10 times greater than $K_{1}^{\prime}$. Now the influence of charge on the magnitude of the velocity constants is important in the formation reaction for the path involving $\mathrm{CN}^{-}$, and in the dissociation reaction for the path involving $\mathrm{HCN}$. As a consequence the greater affinity for cyanide shown by the conjugate acid form of ferrimyoglobin $\left(\mathrm{H}^{+} \mathrm{Y}\right)$ arises in a different way for each path. The reaction of the conjugate acid with $\mathrm{HCN}$ is favored with respect to its conjugate base, because the dissociation velocity constant increases more than the formation velocity constant increases upon ionization $\left(k_{-1}^{\prime} \gg k_{-2}^{\prime}, k_{1}^{\prime}>k_{2}^{\prime}\right)$. The reaction with $\mathrm{CN}^{-}$is favored because the formation velocity constant decreases more than the dissociation velocity constant decreases upon ionization $\left(k_{1} \ll k_{2}, k_{-1}<k_{-2}\right)$.

Corresponding kinetic data, summarized in Table III, are available at present only for the fluoride reaction of ferrimyoglobin with the heme-linked group in the conjugate base form $(16,18)$. The most striking difference between this reaction and the cyanide reaction is seen in a comparison of the relative 
magnitudes of $k_{1}$ and $k_{1}^{\prime}$. The cyanide complex is formed by reaction of $\mathrm{CN}^{-}$just a little more readily than by $\mathrm{HCN}$, whereas

TABLE III

Kinetic Data for the Ferrimyoglobin-Fluoride Reaction at $20^{\circ} \mathrm{C}$. and $I=0.40(16,18)^{a}$

$$
\begin{aligned}
& \mathrm{Y} \cdot \mathrm{Fe}^{+}\left(\mathrm{H}_{2} \mathrm{O}\right)+\mathrm{F}^{-} \frac{k_{1}=1.7 \times 10^{-2}}{\stackrel{k_{-1}=4.6 \times 10^{-4}}{\leftrightarrows}} \mathrm{Y} \mathrm{FeF}+\mathrm{H}_{2} \mathrm{O} \\
& \mathrm{Y} \cdot \mathrm{Fe}^{+}\left(\mathrm{H}_{2} \mathrm{O}\right)+\mathrm{HF} \underset{k_{-1^{\prime}}=5.7 \times 10^{5}}{\frac{k_{1^{\prime}}=1.4 \times 10^{4}}{\rightleftharpoons}} \mathrm{YeF}+\mathrm{H}_{3} \mathrm{O}^{+}
\end{aligned}
$$

${ }^{a}$ For units see Table II.

the formation of the fluoride complex by reaction with $\mathrm{HF}$ is about a million times faster than the reaction with $\mathrm{F}^{-}$. A possible explanation lies in the relative magnitudes of the solvation energies of the ions. The fluoride ion is more highly solvated than the cyanide ion, and in forming a complex, one, at least, of the water molecules must be removed from the solvation shell. The energy required to do this, reflected in a high activation energy for the $\mathrm{F}^{-}$reaction, could thus account for the result.

These data provide a quantitative background against which the effect of $p \mathrm{H}$ on the biologically important hemoprotein reactions can be judged. For example, in the oxygenation of hemoglobin (the Bohr effect) it is small in magnitude as with the ferrimyoglobin reactions that involve neutral molecules, viz., $\mathrm{HCN}$ and $\mathrm{H}_{2} \mathrm{O}$. The equilibrium constant for combination with oxygen is about 8 times greater in alkaline solution as a consequence of an 8-fold decrease in the dissociation velocity constant, the formation velocity constant being substantially the same $(8,29)$. The marked contrast between these reactions and those involving ionic species, e.g., $\mathrm{CN}^{-}$and $\mathrm{H}_{3} \mathrm{O}^{+}$, where the ionization of the heme-linked groups affects the velocity constants far more, suggests that the same kind of theoretical treatment (27) which has proved so successful in 
accounting for the kinetic characteristics of inorganic and organic reactions between ions and between an ion and a neutral molecule, may also provide the explanation for the operation of heme-linked groups.

A similar detailed study has not yet been carried out on the ferrihemoglobin-fluoride and -cyanide reactions, but it seems probable that the data referred to earlier for the cyanide reaction (2), which was regarded as evidence for the bonding of HCN in the complex, could be accounted for by the same, more complicated, mechanism developed for the ferrimyoglobin reaction. More recently the $p \mathrm{H}$ variation of the equilibrium constants for the formation of ferrihemoglobin fluoride, azide, cyanate, and thiocyanate has been investigated (41). In interpreting the results it was assumed that the anions are bonded, and no analysis was made taking into account the ionization of heme-linked groups. However the $p \mathrm{~K}$ values for their ionization in ferrihemoglobin are known from data on the cell reaction, and, since the $p \mathrm{H}$ variations are similar to that for ferrimyoglobin fluoride given in Figure 1, it seems very likely that the same analysis used in the case of myoglobin would confirm anion bonding.

The structure of the peroxidase and catalase complexes now presents a very interesting problem, for, as mentioned above, the simplest interpretation of kinetic and equilibrium data again appears to suggest bonding of the undissociated acid $(2,17)$. Yet the physical properties of their fluoride, cyanide, and other complexes, particularly absorption spectra and magnetic susceptibilities, resemble so closely those of the ferrihemoglobin and ferrimyoglobin complexes, that on these considerations alone it has long been accepted that combination occurs with the anion $(30,32)$. One explanation of this apparent contradiction could be that anions are bonded in all the complexes, but that in the case of catalase and peroxiclase, one or more linked groups ionize in such a way as to mask completely the $p \mathrm{H}$ dependence of equilibrium and velocity constants, which would otherwise be characteristic of anion bonding. 


\section{$\Delta F^{0}, \Delta H^{0}$, and $\Delta S^{0}$ for the Formation of Ionic Iron, Myoglobin, and Hemoglobin Complexes}

In the formation of some of their complexes myoglobin and hemoglobin show a greater affinity for the ligand than the ferrous or ferric ion. It is of interest to see how this arises in terms of the relative magnitudes of $\Delta H^{0}$ and $\Delta S^{0}$, for since $\Delta F^{0}=$ $\Delta H^{0}-T \Delta S^{0}$, the more negative the value of $\Delta H^{0}$, and the more positive the value of $\Delta S^{0}$, the greater is the decrease in free energy, $\Delta F^{0}$, which provides the quantitative measure of the affinity between the metal and the ligand.

The data at present available have been assembled in Tables IV and V. For the ferrous complexes no direct comparison is possible yet, but, since NO combines with both ferrous ion and the ferrohemoproteins, data for $\mathrm{FeNO}^{2+}$ may be compared with data for the ferromyoglobin complexes with $\mathrm{O}_{2}$ and $\mathrm{CO}$. In the case of these complexes and the $\mathrm{F}^{-}, \mathrm{CNS}^{-}$, and $\mathrm{N}_{3}^{-}$complexes of ferrihemoglobin, no allowance was made in the analysis for the participation of heme-linked ionizations $(41,43)$, but any discrepancy is unlikely to exceed $2 \mathrm{kcal} . / \mathrm{mole}$ in $\Delta F^{0}$ or $\Delta H^{0}$, or 5 c.u. in $\Delta S^{0}$. Only when the ligand is covalently bonded, vis., $\mathrm{O}_{2}, \mathrm{CO}, \mathrm{CN}^{-}$, and $\mathrm{N}_{3}^{-}$(28), does the hemoprotein have the greater affinity: with the ligands $\mathrm{OH}^{-}, \mathrm{F}^{-}$, and $\mathrm{CNS}^{-}$ferric ion has the greater affinity, decreasing in this order.

TABLE IV

Thermodynamic Data for Ferrous Ion and Ferromyoglobin Complex FormaTION $(33,43)$

\begin{tabular}{lccc}
\hline \multicolumn{1}{c}{ Reaction } & $\begin{array}{c}\Delta F^{0} \\
\text { (kcal./mole) }\end{array}$ & $\begin{array}{c}\Delta H^{0} \\
(\mathrm{kcal} . / \mathrm{mole})\end{array}$ & $\begin{array}{c}\Delta S^{0} \\
(\mathrm{e} . \mathrm{u} .)\end{array}$ \\
\hline $\mathrm{Fe}^{2+}+\mathrm{NO}$ & -3.5 & -9.2 & -19.1 \\
Ferromyoglobin $+\mathrm{O}_{2}$ & -7.5 & -16.4 & -30.0 \\
Ferromyoglobin $+\mathrm{CO}$ & -9.2 & -20.0 & -36.2 \\
\hline
\end{tabular}

An examination of the $\Delta H^{0}$ and $\Delta S^{0}$ values reveals that the differences in affinity arise through opposing effects. The 
TABLE V

Thermodynamic Data for Ferric Ion, Ferrimyoglobin, and Ferriliemoglobin Complex Formation $(15,18,40,43,46)$

\begin{tabular}{l|lllllc}
\hline \multicolumn{2}{c}{ Ligand } & $\mathrm{OH}^{-}$ & $\mathrm{F}^{-}$ & $\mathrm{CN}^{-}$ & $\mathrm{N}_{3}^{-}$ & $\mathrm{CNS}^{-}$ \\
\hline$\Delta F^{0}$ & Ferric ion & -16.1 & -6.9 & $-9.6^{a}$ & -5.7 & -4.0 \\
(kcal./mole) & Ferrimyoglobin & -6.85 & -2.0 & -11.5 & - & - \\
& Ferrihemoglobin & -7.1 & -2.4 & - & -7.5 & -3.0 \\
$\Delta H^{0}$ & Ferric ion & -1.2 & +7.5 & $-4.8^{a}$ & -4.3 & -1.6 \\
(kcal./mole) $)$ & Ferrimyoglobin & -7.65 & -1.5 & -18.6 & - & - \\
& Ferrihemoglobin & -9.5 & -2.5 & - & -12.9 & -8.8 \\
$\Delta S^{0}$ & Ferric ion & +50 & +49 & $+16.1^{a}$ & +5 & +8.1 \\
(e.u.) & Ferrimyoglobin & -2.6 & +1.8 & -24 & - & - \\
& Ferrihemoglobin & -7.9 & -0.6 & - & -18.4 & -19.7 \\
\hline
\end{tabular}

a Calculated from an estimated $K=10^{7}$, a little greater than one-sixth of $K$ for the formation of $\mathrm{Fe}(\mathrm{CN})_{6}^{3-}$, and a value for $\Delta S^{0}$ of 16.1 e.u., based on the assumption that $\bar{S}^{0}$ for $\mathrm{FeCN}^{2}$ is 44.3 e.u., equal to the mean of the ${\overline{S^{0}}}^{0}$ values for the other ferric ion complexes listed in Table I.

values of $\Delta H^{0}$ in all cases favor the hemoprotein reactions, to the extent of between 7 and $14 \mathrm{kcal} . / \mathrm{mole}$. The bonding is stronger in the hemoprotein complexes. If the entropy changes were the same for both ionic iron and hemoprotein complexes, the equilibrium constants for the latter would be between $10^{5}$ and $10^{10}$ times greater. However, this is not so; the entropy changes greatly favor the formation of the ionic iron complexes, to the extent of 11 to $17 \mathrm{e} . \mathrm{u}$. for the ferrous derivatives and 23 to 57 e.u. for the ferric derivatives. On this account alone the equilibrium constants for the ionic iron complexes would be greater by between $10^{2.5}$ and $10^{12.5}$. The actual differences in affinity depend upon whether differences in $\Delta H^{0}$ or $\Delta S^{0}$ predominate, and there is no simple correlation with ionic or covalent bonding within the complex.

This behavior is what would be expected on qualitative considerations, for stronger bonding might be anticipated with the iron in a coordination complex rather than a solvation shell of water molecules, and, correspondingly less desolvation and less change in the tightness of the structure might be anticipated when the iron is already held in the rigid planar ring system of the protoporphyrin. It is of interest, however, to examine the 
entropy data a little more closely because configurational changes within the protein fabric might accompany complex formation and contribute substantially to the observed entropy change.

This effect can best be looked for by comparing the differences between the entropy of the parent compound and that of its complex. Standard entropies of substances in solution are denoted by $\bar{S}^{0}$, referred to as a partial molal entropy, and values are available for most inorganic ions and simple neutral molecules (31). Hence, since entropy is an additive property, in the formation of the complex ML from the parent compound $\mathrm{M}$ and the ligand $\mathrm{L}$,

$$
\mathrm{M}+\mathrm{L} \rightleftharpoons \mathrm{ML}
$$

the entropy change $\Delta S^{0}$ is given by

$$
\Delta S^{0}=\bar{S}_{\mathrm{ML}}^{0}-\bar{S}_{\mathrm{M}}^{0}-\bar{S}_{\mathrm{L}}^{0}
$$

The difference between the partial molal entropies of the complex ML and the parent compound $\mathrm{M}$ is thus:

$$
\left(\bar{S}_{\mathrm{ML}}^{0}-\bar{S}_{\mathrm{M}}^{0}\right)=\Delta S^{0}+\bar{S}_{\mathrm{L}}^{0}
$$

Since the charge does not change in the formation of complexes with neutral ligands, $\left(\tilde{S}_{\mathrm{ML}}^{0}-\bar{S}_{\mathrm{M}}^{0}\right)$ may be compared directly with $\bar{S}_{\mathrm{L}}^{0}$, for it is a measure of the gain or loss in entropy when the ligand is bound to the metal and the water molecule liberated. For example, when the inorganic cations, $\mathrm{Cu}^{2+}, \mathrm{Zn}^{2+}, \mathrm{Co}^{2+}$, $\mathrm{Ni}^{2+}$, etc., combine with ammonia for which $\bar{S}^{0}=26.3$ e.u., $\left(\bar{S}_{\mathrm{ML}}^{0}-\bar{S}_{\mathrm{M}}^{0}\right)$ is 23 e.u. (19). In the formation of $\mathrm{FeNO}^{2+}$, $\left(\bar{S}_{\mathrm{ML}}^{0}-\bar{S}_{\mathrm{M}}^{0}\right)$ is 9.8 e.u., $\bar{S}^{0}$ for NO being 28.9 e.u. (31). Now the partial molal entropies of $\mathrm{O}_{2}$ and $\mathrm{CO}$ are 25.7 and 25.5 e.u., respectively, very similar to the values for $\mathrm{NH}_{3}$ and $\mathrm{NO}$; hence for the ferromyoglobin complexes values of $\left(\bar{S}_{\mathrm{ML}}^{0}-\bar{S}_{\mathrm{M}}^{0}\right)$ ranging between +10 and +25 e.u. might have been expected. The actual values are -4.3 and -10.7 e.u., shown diagrammatically with those for the inorganic complexes in Figure 2. This is evidence that an additional change in configuration or solvation does occur in the formation of the ferrohemoprotein 
complexes resulting in a considerable entropy decrease of about 14 to 36 e.u.

The same effect is apparent with the ferrihemoprotein complexes, although, since the charge changes in the formation of complexes with anionic ligands, the basis of comparison is a little more complicated. For the ion-pair complexes of monatomic cations a simple rule holds. $\left(\bar{S}_{\mathrm{ML}}^{0}-\bar{S}_{\mathrm{M}}^{0}\right)$ is constant for the same metal ion and a series of anions, as shown in Table I for the ferric ion. It is as if the dominant feature is the diminution in charge accompanying complex formation, and the

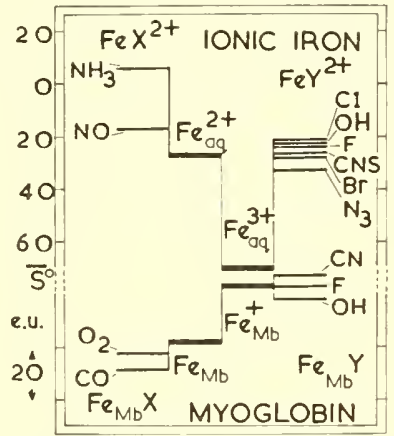

Fig. 2. Values of $\overline{\mathrm{S}}^{0}$ in aqueous solution for ferrous and ferric ions and the relative values for myoglobin in the ferrous and ferric oxidation states, together with the values for some of their complexes. $\mathrm{Fe}_{\mathrm{aq}}^{2+}$ and $\mathrm{Fe}_{\mathrm{aq}}^{3+}$, ferrous and ferric ions; $\mathrm{FeX}^{2+}$, ferrous ion complexes with neutral ligands $\mathrm{X} ; \mathrm{FeY}^{2+}$, ferric ion complexes with anionic ligands $\mathrm{Y}^{-} ; \mathrm{Fe}_{\mathrm{Mb}}, \mathrm{Fe}_{\mathrm{Mb}}^{+}, \mathrm{Fe}_{\mathrm{Mb}} \mathrm{X}$, and $\mathrm{Fe}_{\mathrm{Mb}} \mathrm{Y}$, the corresponding myoglobin derivatives.

composition of the ion-pair complex is of little consequence. The rule also holds reasonably well for ion-pair complexes formed by complex cations (7). $\left(\bar{S}_{\mathrm{ML}}^{0}-\bar{S}_{\mathrm{MI}}^{0}\right)$ values for the complexes of $\mathrm{Co}\left(\mathrm{NH}_{3}\right)_{6}^{3+}$ with $\mathrm{Cl}^{-}, \mathrm{Br}^{-}$, and $\mathrm{I}^{-}$are 34.2, 34.3, and 37.1 e.u., respectively, and for the complexes of $\mathrm{Co}(\mathrm{en})_{3}^{3+}$ with $\mathrm{Br}^{-}$and $\mathrm{I}^{-}, 32.3$ and 34.1 e.u., where en stands for ethylenediamine. There are just enough data now to infer that the same rule also holds approximately for ferrihemoprotein complexes. $\left(\bar{S}_{\mathrm{ML}}^{0}-\bar{S}_{\mathrm{M}}^{0}\right)$ has the values $-5.1,-0.5$, and +4.2 e.u. for the $\mathrm{OH}^{-}, \mathrm{F}^{-}$, and $\mathrm{CN}^{-}$complexes of ferrimyoglobin, and -10.4 , 
$-2.9,+13.6$, and +16.3 for the $\mathrm{OH}^{-}, \mathrm{F}^{-}, \mathrm{N}_{3}^{-}$, and $\mathrm{CNS}^{-}$ complexes of ferrihemoglobin. These values, with those for ferric iron complexes, are shown diagrammatically in Figures 2 and 3. Whereas the values for the triatomic ligands $\mathrm{N}_{3}^{-}$and $\mathrm{CNS}^{-}$are appreciably higher than those for the monatomic $\mathrm{F}^{-}$ and diatomic $\mathrm{OH}^{-}$, which could arise in part through the additional vibration, the values as a whole are extremely negative. Values not very different from those for the cobaltic complex ions might have been anticipated, say between +20 and +30 e.u., since, although the ligand is directly attached to the metal in the ferrihemoprotein complexes, in both instances the metal is already bound within a definite coordination structure in the parent compounds. The very low values are

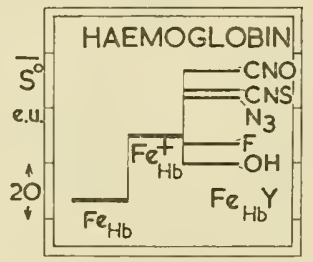

Fig. 3. Relative values of $\overrightarrow{\mathrm{S}}^{0}$ for hemoglobin in the ferrous and ferric oxidation states, together with values for some ferrihemoglobin complexes.

again indicative of a loss in entropy, arising through some change in solvation, or tightening of the prosthetic group and possibly the protein fabric in its immediate vicinity, which is absent in the other cases. A configurational change of this kind, lowering $\Delta S^{0}$ for both ferro- and ferri-myoglobin reactions, would, however, entail a favorable increment in $\Delta H^{0}$, heat being liberated because of the stronger bonds formed as the structure tightens, and so the more positive heats of formation (more negative $\Delta H^{0}$ values) for both sets of hemoprotein reactions are consistent with these entropy effects.

Finally a useful comparison can be made between ferrimyoglobin and ferrihemoglobin. It has been suggested that the equilibrium constants for complex formation are about 10 times greater for the latter (45). This may be true, however, 
only at certain finite ionic strengths; at zero ionic strength the factor is as low as 2 for the $\mathrm{OH}^{-}$complexes (15), corresponding to a difference in $\Delta F^{0}$ of $-0.25 \mathrm{kcal}$. $/$ mole (sec Table $\mathrm{V}$ ). But although the two hemoproteins have such a similar affinity for $\mathrm{OH}^{-}$there are rather greater differences in the $\Delta H^{0}$ and $\Delta S^{0}$ values. As in the case of the previous comparison with ferric ion complexes these values again reflect opposing effects. For the ferrihemoglobin reaction $\Delta H^{0}$ is more favorable by 1.85 $\mathrm{kcal}$./mole, whereas $\Delta S^{0}$ is unfavorable to the extent of -5.3 e.u. The data for the $\mathrm{F}^{-}$complexes show just the same trends, although in the case of ferrihemoglobin small corrections may be necessary to allow for the participation of linked ionizations. The differences in $\Delta F^{0}, \Delta H^{0}$, and $\Delta S^{0}$, are, respectively, -0.4 and $-1.0 \mathrm{kcal} . / \mathrm{mole}$, and -2.4 e.u.

\section{The Reactivity of Cytochrome $\mathrm{c}$ in Complex Formation}

In cytochrome $c$ the heme is bound very differently from that in hemoglobin and myoglobin. A comparison of the data for complex formation should therefore show the extent to which a difference in structure can affect the magnitudes of $k, K$, $\Delta F^{0}, \Delta H^{0}$, and $\Delta S^{0}$, and so give a clue to the kind of correlation to be expected between structure and reactivity in hemoprotein systems.

In the $p \mathrm{H}$ range 4 to 11 ferrocytochrome $c$ does not combine readily with carbon monoxide, nor does ferricytochrome $c$ readily form fluoride and cyanide complexes like the ferric forms of other hemoproteins (44). The absorption spectrum of ferrocytochrome $c$ closely resembles that of a hemochromogen, the coordination complex formed by heme with two molecules of nitrogenous base, such as pyridine or piperidine, one on each side of the porphyrin ring (32). It is therefore thought that in cytochrome $c$ there are two links to amino acid residues binding the heme in a crevice in the protein, and that before complex formation with simple ligands can occur, the weaker link has to be broken. Actually ferricytochrome $c$ does react very slowly 
to form its cyanide complex throughout the entire $p \mathrm{H}$ range, so the phenomenon is one of a difference in degree rather than kind. Kinetic and equilibrium studies (26) suggest that both HCN and $\mathrm{CN}^{-}$react to form the complex as in the case of ferrimyoglobin, but there is no evidence for the presence of a similar linked ionizing group between $p \mathrm{H} 6$ and 8 . The data, listed in Table VI, show that there is relatively little difference between the affinity of ferrimyoglobin and ferricytochrome $c$ for cyanide, the equilibrium constants being, respectively, $2.3 \times 10^{8}$ and $1.2 \times 10^{6} \mathrm{M}^{-1}$, corresponding to a free-energy difference of only $3.1 \mathrm{kcal} . / \mathrm{mole}$. The difference in reactivity

TABLE VI

Kinetic and Equilibrium Data for Cyanide Complex Formation $(16,18,26)$

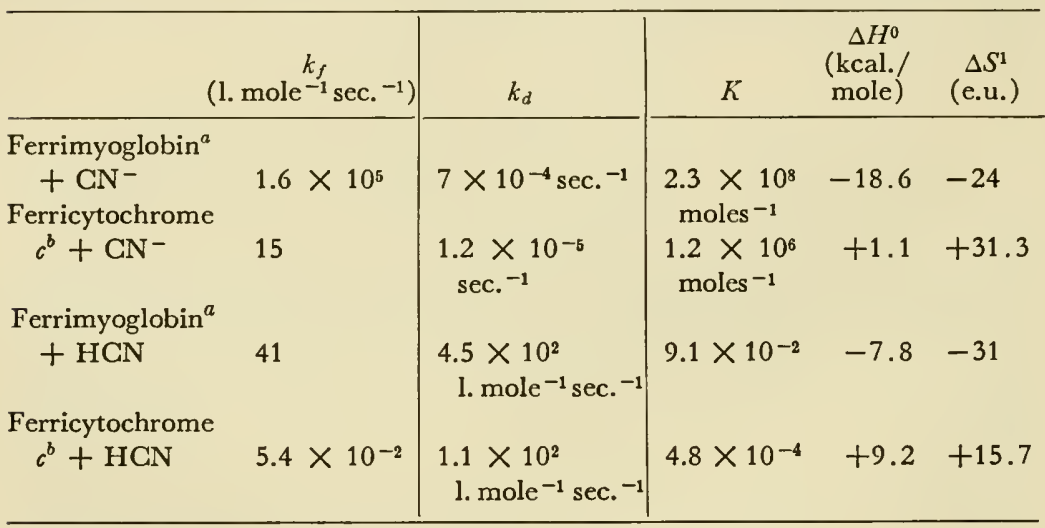

a $25^{\circ} \mathrm{C}$., $\mathrm{I}=0.10$, heme-linked group in conjugate acid form.

b $24.6^{\circ} \mathrm{C}$., $\mathrm{I} \approx 0.15$.

is a kinetic feature. Ferrimyoglobin cyanide is formed by the $\mathrm{CN}^{-}$path about $10^{4}$ times more rapidly, and by the HCN path about $10^{3}$ times more rapidly: dissociation occurs about 10 times and about 4 times more rapidly, respectively (the comparison involving the $\mathrm{CN}^{-}$path for ferrimyoglobin being subject to the proviso mentioned above). In contrast to other hemoprotein complexes of similar affinity, e.g., $\mathrm{O}_{2}$ and $\mathrm{CO}$ complexes, ferricytochrome $c$ cyanide owes its existence to extremely slow dissociation rather than rapid formation. 
Although the affinities of ferrimyoglobin and ferricytochrome $c$ for cyanide are so similar, the heat and entropy data given in Table VI reveal very profound underlying differences. The ferrimyoglobin reaction with $\mathrm{CN}^{-}$is favored by a very large exothermicity of $18.6 \mathrm{kcal} . /$ mole, whereas $\Delta S^{0}$ is very unfavorable, $-24 \mathrm{e.u}$. The ferricytochrome $c$ reaction, in great contrast, is a little endothermic, and the complex forms entirely by virtue of a very favorable entropy change, +31.3 e.u. Such differences between $\Delta H^{0}$ and $\Delta S^{0}$ values are just what would be expected if the heme in cytochrome $c$ was bound in a crevice in the protein. Energy would be required to break the bond to the more weakly held amino acid residue, so diminishing the net exothermicity of complex formation, but the entropy change would be favorable because part of the protein would gain more freedom of movement. A comparison of $\left(\bar{S}_{\mathrm{ML}}^{0}-\bar{S}_{\mathrm{MI}}^{0}\right)$ values, +4.2 e.u. for ferrimyoglobin and +59.5 e.u. for ferricytochrome $c$, shows that this structural difference between the two hemoproteins favors cyanide complex formation by ferricytochrome $c$ relative to ferrimyoglobin by about 55 e.u.; and in the contrary sense this is the entropy loss suffered by cytochrome $c$ itself because of its peculiar structure.

\section{Electron Transfer in Myoglobin and Hemoglobin Systems}

The reversible oxidation and reduction between the ferrous and ferric states is one of the least specific of all hemoprotein reactions. Catalase alone is the exception; it can only be reduced directly in solutions made up from the freeze-dried enzyme. Provisional values (18) have been obtained for the heat and entropy changes associated with the cell reaction

$$
\mathrm{Fe}^{+}\left(\mathrm{H}_{2} \mathrm{O}\right)+{ }^{1} /{ }_{2} \mathrm{H}_{2} \rightleftharpoons \mathrm{Fe}\left(\mathrm{H}_{2} \mathrm{O}\right)+\mathrm{H}^{+}
$$

of both myoglobin and hemoglobin from measurements of the oxidation-reduction potentials at $p \mathrm{H} 6.0$ and ionic strength 0.22 over a range of temperatures. Since $\Delta H^{0}$ for the reaction $\mathrm{H}^{+}+\mathrm{e}^{-} \rightleftharpoons 1 / 2 \mathrm{H}_{2}$ is $82.5 \mathrm{kcal} . /$ mole, the ionization potentials 
of ferromyoglobin and ferrohemoglobin in aqueous solution can also be calculated. This is the energy required to effect electron removal,

$$
\mathrm{Fe}\left(\mathrm{H}_{2} \mathrm{O}\right) \longrightarrow \mathrm{Fe}^{+}\left(\mathrm{H}_{2} \mathrm{O}\right)+e^{-}
$$

and the values are 96 and $97 \mathrm{kcal} . /$ mole, respectively, compared with $95 \mathrm{kcal} . / \mathrm{mole}$ for the ferrous ion itself.

From the entropy change in the cell reaction, the sum $\Delta S^{0}+1 / 2 S_{\mathrm{H}_{2}}^{0}$ gives $\left(\bar{S}_{\mathrm{Fe}\left(\mathrm{H}_{2} \mathrm{O}\right)}^{0}-\bar{S}_{\mathrm{Fe}+\left(\mathrm{H}_{2} \mathrm{O}\right)}^{0}\right)$ because $\bar{S}_{\mathrm{H}^{+}}^{0}$ is, by convention, taken as zero. For myoglobin and hemoglobin the provisional values are -21.4 and -23.4 e.u. These differences in partial molal entropy, which are large and negative, are quite remarkable because the corresponding quantity for ionic iron is $-27.1-(-70.1)=+43$ e.u. (31). Figures 2 and 3 show this contrast very clearly.

The large and positive difference in the case of ionic iron is attributable to two factors. There is a favorable change in the Born charging entropy which is purely electrostatic in origin, arising in the transfer of a charged particle from vacuo to the solvent, and also a favorable change in the entropy of solvation, because around the ion of smaller charge and larger size the water molecules are less tightly bound. Even for complex cations where the ligands are bulky organic molecules, e.g., the tris-dipyridyl complexes of $\mathrm{Fe}^{2+}, \mathrm{Ru}^{2+}$, and $\mathrm{Os}^{2+}$, the corresponding $\bar{S}^{0}$ difference falls only to $+3 \pm 4$ e.u., which suggests that in these coordination complexes, the metal ion, held already in a rigid chelate structure, is shielded to such an extent that solvent interactions are very small indeed (19).

The large negative values of $\left(\bar{S}_{\mathrm{Fe}\left(\mathrm{H}_{2} \mathrm{O}\right)}^{0}-\bar{S}_{\mathrm{Fe}^{+}\left(\mathrm{H}_{\varepsilon} \mathrm{O}\right)}\right)$ for myoglobin and hemoglobin point to a common configurational difference between the ferrous and ferric states, the ferric state having the more random configuration. If the heme were held in a crevice in the protein in the ferrous state, but not in the ferric state, this could account for the large negative values. But this explanation receives no support at all from the $\left(\bar{S}_{\mathrm{ML}}^{0}-\right.$ $\bar{S}_{\mathrm{M}}^{0}$ ) values for the ferrous complexes, which should, on this 
basis, be even more positive than those for ferrous ion complexes, and not some 30 e.u. more negative, as is actually found. The comparison between $\left(\bar{S}_{\mathrm{ML}}^{0}-\bar{S}_{\mathrm{MI}}^{0}\right)$ values for ferrimyoglobin and cytochrome $c$ and their cyanide complexes has shown how the binding on heme in a crevice affects these values. A more likely explanation in the case of hemoglobin and myoglobin is the following. If the ferrous state, although neutral as regards the net charge in the immediate vicinity of the iron atom, were actually negatively charged over that part of the structure which in its entirety determines the entropy change, then a negative value for $\left(\bar{S}_{\mathrm{Fe}\left(\mathrm{H}_{2} \mathrm{O}\right)}^{0}-\bar{S}_{\mathrm{Fe}^{+}\left(\mathrm{H}_{2} \mathrm{O}\right)}\right)$ would follow because the effective charge would increase and not decrease from +1 to zero, as the ferric state is reduced to the ferrous state. This criterion is satisfied if, for instance, the prosthetic group as a whole with its negatively charged propionate side chains is involved in determining the entropy changes, since the charge change on reduction would then be -1 to -2 . This interpretation does not necessarily conflict with the conclusion drawn from studies on the variation of equilibrium constants with ionic strength, which showed that the iron in ferrimyoglobin reacts between $p \mathrm{H} 6.6$ and 7.5 as if the charge were +1 , provided that this particular behavior is determined only by the resultant charge on the iron atom.

\section{Aspects of the Bohr Effect and Heme-Heme Interaction}

These important physiological features of hemoglobin reactions can now be examined in greater detail. The data for the reactions of myoglobin, hemoglobin, and cytochrome $c$ discussed above show how the reactivity of these hemoproteins and their affinity for ligands fit into a certain pattern. In some respects it should therefore be possible now to distinguish a "normal" reaction from one showing "abnormal" characteristics.

With hemoglobin the magnitude of both of the interaction effects depends, first, upon the source of the hemoglobin, secondly, upon the treatment it has received, and thirdly, upon 
the particular reaction studied $(32,47)$. There is no similar evidence for a Bohr effect of different magnitude with myoglobin from different species, nor has the method of extraction or degree of purity been shown to affect it, although a more systematic examination might well reveal these features. Myoglobin, possessing only one heme per molecule, cannot, of course, exhibit the other effect, heme-heme interaction.

It is understandable that the magnitude of the Bohr effect should depend on the particular reaction studied, for it is governed by the difference between the acid strength of the heme-linked ionizing group in reactant and product; this quantity naturally takes on a different value, depending upon the pair of hemoprotein derivatives involved, as a direct consequence of structural differences. Earlier hemoglobin studies suggested that the chief heme-linked group (with $p \mathrm{~K}_{2}$ ), responsible for the marked oxygenation Bohr effect, had an identical value in oxy- and carbonmonoxy-hemoglobin and in ferrihemoglobin of 6.68, compared to the value of 7.9 in ferrohemoglobin (47). A structural interpretation of this was given in terms of a model in which the heme was joined to the tertiary $\mathrm{N}$-atom of a histidine residue (6), and it was to be expected that other covalently bonded complexes would have a $p \mathrm{~K}$ value similar to that of the covalently bonded oxygen and carbon monoxide complexes. Sufficient data are not yet available to judge whether this is true for both ferri- and ferro-hemoglobin derivatives, but in the case of myoglobin, ferrimyoglobin cyanide, with covalent bonding, has a $p \mathrm{~K}$ value of 7.0 compared with 6.0 in oxy- and carbonmonoxy-myoglobin and ferrimyoglobin. It would thus appear that the acid strength is determined by more factors than those taken account of in this generalization based on bond type.

The prospect of identifying a heme-linked ionizing group by its thermodynamic properties is very attractive. The magnitude of the $p \mathrm{~K}$ value and the heat and entropy of ionization can be obtained from the variation of physical properties with $p \mathrm{H}$, from kinetic or equilibrium studies, or from differential 
titration experiments (47). For hemoglobin this last method gives $\Delta H^{0}=6.5 \mathrm{kcal} . /$ mole, supporting the hypothesis that the group with $p \mathrm{~K}_{2}$ is the glyoxalinium $\mathrm{NH}^{+}$of a histidine residue to which the heme is joined. Studies of the slight variation in absorption spectrum of ferrimyoglobin fluoride with $p \mathrm{H}$ and temperature give $\Delta H^{0}=2 \pm 2 \mathrm{kcal} . /$ mole and $\Delta S^{0}=-22 \mathrm{e.u}$. for its linked group (17). These values more nearly resemble those for the ionization of a carboxylic acid than for the $\mathrm{NH}$ of histidine. However, before concluding from these differences that the heme is joined to a different amino acid residue in hemoglobin and myoglobin more confirmatory data for other complexes would be desirable, but in addition there are good reasons for doubting whether such a direct conclusion is valid. Firstly, hemoglobin from different species shows Bohr effects of different magnitudes, and sea lamprey hemoglobin (with apparently only one heme per molecule) has an even larger Bohr effect than most mammalian hemoglobins (36). Secondly, if hemoglobin is reconstituted from heme and native globin the Bohr effect is much smaller (47). These facts mean either that the heme is joined to different amino acid residues in the various hemoglobins, and to a different residue in reconstituted hemoglobin compared with the original, or else that slight differences in structure or configuration about an identical residue to which the heme is joined in all these molecules are responsible for the variable behavior. The former explanation carries with it the assumption that reversible combination with oxygen is a property shared by several amino acid residues, the latter, that it is a very special property. For this reason the latter explanation is to be preferred, and so if the acid strength of an ionizable group, in an identical amino acid residue to which the heme is attached in all hemoglobins, is dependent upon the structure or configuration of adjacent parts of the protein fabric, then the thermodyamic data for its ionization are clearly composite, and cannot be used to identify the group by straightforward comparison with corresponding data for its ionization in the isolated amino acid or simpler peptides. The contrast between the data 
for cyanide complex formation by ferrimyoglobin and ferricytochrome $c$ illustrate, in what is probably an extreme case, how configurational changes can modify the thermodynamic data for a simple reaction.

There can be no question that some kind of configurational change is involved in heme-heme interaction, for otherwise there would be no reason for the free-energy change for the successive addition of oxygen to the four hemes to be different. The interaction is greatest with lysed cell preparations, becomes much less with recrystallized samples (32), and is completely absent in reconstituted hemoglobin (39). But no evidence for interaction has been found in recent potentiometric titrations of recrystallized ferrihemoglobin, or in studies of the ionization of the iron-bound water molecule and complex formation with various ligands $(15,18,41)$. The fugitive nature of the effect can be regarded as experimental evidence for its origin in configurational changes within the molecule; and one of the problems now is to specify precisely what this change is.

The thermodynamic data for the cell reaction, the ionization of the iron-bound water molecule, and fluoride complex formation are almost identical for recrystallized samples of ferrihemoglobin and ferrimyoglobin $(15,18,40)$. This suggests very strongly that the heme is similarly situated in both hemoproteins in these particular preparations. Data given in Table VII for the oxygenation of a recrystallized myoglobin preparation (43) and extremely fresh hemoglobin solutions, in which interaction was very marked (38), show the reaction of the first heme in hemoglobin to resemble most the oxygenation of myoglobin. The data for the oxygenation of the fourth heme are very different. The first oxygen is $7.0 \mathrm{kcal}$./mole (i.e., $12.1-$ 5.1) more strongly bound than the last, whereas the entropy change is unfavorable for the entry of the first ( -22.2 e.u.), but favorable to about 13.0 e.u. for the last. These data do not support the hypothesis that the interaction has its origin in steric effects, whereby, the hemes being "buried" within the protein, the entry of the first oxygen loosens the structure, 
TABLE VII

Thermodynamic Data for Myoglobin and Hemoglobin Reactions at $25^{\circ} \mathrm{C}$.

\begin{tabular}{c|c|c|c|c}
\hline & $K$ (moles $\left.^{-1}\right)$ & $\begin{array}{c}\Delta H^{0} \\
(\mathrm{kcal} . / \mathrm{mole})\end{array}$ & $\Delta S^{0}(\mathrm{e.u.})$ & $\begin{array}{c}\bar{S}_{\mathrm{ML}}^{0}- \\
\bar{S}_{M}^{0}\end{array}$ \\
\cline { 1 - 3 } $\begin{array}{c}\text { Myoglobin } \\
\left(\mathrm{O}_{2}\right)^{a}\end{array}$ & $5 \times 10^{5}$ & -16.4 & -30.0 & -4.3 \\
$\begin{array}{c}\text { Myoglobin } \\
(\mathrm{CO})^{a}\end{array}$ & $8.7 \times 10^{6}$ & -20 & -36.2 & -10.7 \\
$\begin{array}{c}\text { Hemoglobin } \\
(\text { first }\end{array}$ & $1.1 \times 10^{4}$ & -12.1 & -22.2 & +3.5 \\
$\begin{array}{c}\text { oxygen })^{b} \\
\text { Hemoglobin } \\
(\text { last } \\
\text { oxygen })^{b}\end{array}$ & $3.9 \times 10^{6}$ & -5.1 & +13.0 & +38.7 \\
\hline
\end{tabular}

a Calculated from data given in references 34 and 43.

b Calculated from data in Table 6 , reference 38 , corrected by the appropriate statistical factors.

making the entry of successive oxygens easier (39). On this basis, $\Delta S^{0}$ for the first oxygenation should be more positive than $\Delta S^{0}$ for the last, and $\Delta H^{0}$ should be more favorable for the last than the first. Neither do the data support another hypothesisthat the interaction is between unoxygenated hemes, becoming progressively less with increasing oxygenation (48), for on this basis there should be no interaction affecting the entry of the last oxygen, which should therefore resemble most the myoglobin reaction.

These new data for the hemoglobin reaction taken in conjunction with those for myoglobin complexes, and particularly the values of $\left(\bar{S}_{\mathrm{ML}}^{0}-\bar{S}_{\mathrm{M}}^{0}\right)$ suggest that an explanation for the interaction can be sought in the following way. The value of $\left(\bar{S}_{\mathrm{ML}}^{0}-\bar{S}_{\mathrm{M}}^{0}\right)$ is "normal" for the entry of the first oxygen, i.e., $\approx+4$ e.u., but is "abnormally" large and positive for the entry of the last, i.e., $\approx+39$ e.u. Interaction therefore sets in after the first heme has reacted; it increases with increasing oxygenation, in contrast with the predictions of the other hypothesis just referred to. But the "normal" values of $\left(\bar{S}_{\mathrm{ML}}^{0}-\right.$ $\bar{S}_{\mathrm{MI}}^{0}$ ) for myoglobin complexes, which range from -0.5 to 
-10.7 e.u., are themselves low when compared to the values for ionic iron complexes; this can be attributed to a tightening of the prosthetic group and possibly the nearby protein fabric accompanying complex formation. Hence if the structure tightens upon the entry of the first oxygen, and the configuration of the hemoglobin molecule in fresh preparations is such that this tightening influences the structure about the hemes which have not yet reacted, then the same degree of tightening may not be possible in the addition of the second, third, and fourth oxygen molecules. $\Delta S^{0}$ would thus be more positive for the last oxygenation, and $\Delta H^{0}$ more negative for the first because of the stronger bonding-as is found experimentally.

Some aspects of the kinetic data for these oxygenation reactions, however, suggest that the oxygenation of the fourth heme parallels the myoglobin reaction (37), so additional data on other hemoglobin reactions are very desirable for a more thorough appraisal of the problem. In a very recent survey (13a), which includes data for the hemoglobin-CO reaction, it appears that even though there are great similarities between the partial molal entropies of $\mathrm{O}_{2}$ and $\mathrm{CO}$, and great similarities between the physical properties of oxyhemoglobin and carbonmonoxyhemoglobin, the trends in $\Delta H^{0}$ and $\Delta S^{0}$ given in Table VII may be a unique feature of the oxygenation reaction.

\section{The Stability of Oxyhemoglobin and Oxymyoglobin*}

The oxygenation of myoglobin and hemoglobin has been discussed so far only in relation to the formation of other hemoprotein complexes, containing $\mathrm{CO}, \mathrm{F}^{-}, \mathrm{CN}^{-}, \mathrm{OH}^{-}$, etc. But the existence of these particular complexes as stable entities

* In this section and the next, formulas are written with the charge on the iron atom corresponding to ferrous and ferric ions, not according to the net charge as before, so as to reveal more clearly the valency changes and changes in oxidation state. The subscript $p$ is used, denoting porphyrin, for reactions common to several hemoproteins; subscripts $\mathrm{Mb}$, Per, etc., are used for particular myoglobin or peroxidase reactions, etc. 
depends not only on the free-energy change for the binding of oxygen being favorable, but also upon the oxidation to ferrihemoglobin and ferrimyoglobin being a slow reaction in contrast to the rapid oxidation of other coordination complexes of ferrous iron that possess comparable structures and similar oxidation-reduction potentials. For instance, heme itself and the hemochromogens are very readily oxidized by molecular oxygen.

Kinetic studies of the oxidation of hemoglobin and myoglobin by oxygen have shown it to be an extremely complicated process $(1,25)$ with other groups in the molecule becoming oxidized as well as the heme iron. Nevertheless, two factors can be distinguished which contribute greatly to the resistance towards oxidation. Whatever the detailed mechanism of the oxidation process, the fundamental electron transfer reaction

$$
\mathrm{Fe}_{p}^{2+}+\mathrm{O}_{2} \longrightarrow \mathrm{Fe}_{p}^{3+}+\mathrm{O}_{2}^{-}
$$

will be among the most important governing its speed. If this reaction is fast the net oxidation process will also be fast unless there are significant back reactions regenerating the ferrous state. Using the provisional values of 96 and $97 \mathrm{kcal} . / \mathrm{mole}$ for the ionization potentials of ferromyoglobin and ferrohemoglobin obtained from the oxidation-reduction potential study and other thermochemical data, reaction (5) can be shown to be endothermic with $\Delta H^{0}$ values of 20 and $21 \mathrm{kcal} . /$ mole, respectively. Reactions which are endothermic to this extent are usually very slow. In fact the ionization potential of the simple aquated ferrous ion is a little less, $95 \mathrm{kcal}$./mole, which means that on this criterion these ferro-hemoproteins are a little less susceptible to oxidation than ferrous salts. These remarks refer to the bimolecular electron transfer between the ferrous state and oxygen. For the fission of the oxygen complex itself with electron transfer, reaction (6), an additional factor must be considered, namely, its heat of formation.

$$
\mathrm{Fe}_{p}^{2+} \mathrm{O}_{2} \longrightarrow \mathrm{Fe}_{p}^{3+}+\mathrm{O}_{2}^{-}
$$


Since the formation of both oxymyoglobin and oxyhemoglobin are highly exothermic, about 16 and $14 \mathrm{kcal}$./mole, respectively, this has the effect of enhancing the resistance toward oxidation through an increase in the endothermicity of the over-all electron transfer by these large amounts.

The coexistence of the two reaction paths - combination to form the stable oxygen complex and electron transfer giving irreversible oxidation - is an uncommon feature in chemical

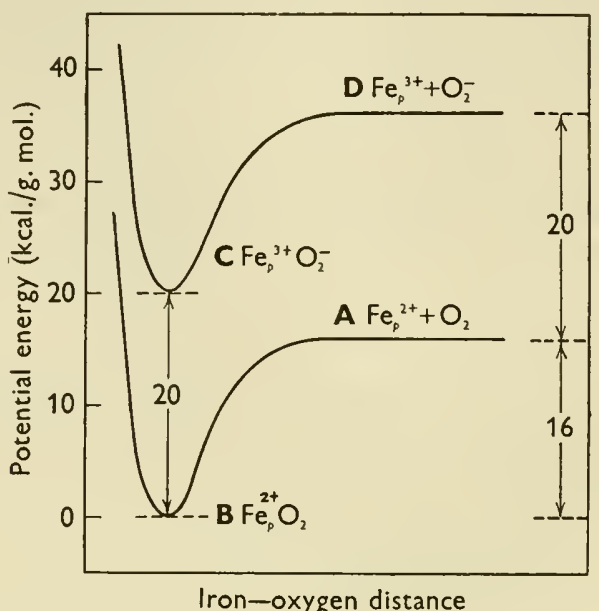

Fig. 4. Schematic potential energy diagram for the reaction of oxygen with ferromyoglobin, giving $\mathrm{O}_{2}^{-}$and ferrimyoglobin (25).

kinetics, and the detailed mechanism can be envisaged with the help of the schematic potential energy diagram given in Figure 4. The positions of state $\mathbf{A}\left(\mathrm{Fe}_{p}^{2+}+\mathrm{O}_{2}\right.$ infinitely separated) and state $\mathbf{B}\left(\mathrm{Fe}_{p}^{2+} \mathrm{O}_{2}\right.$, the oxygen complex) are defined by the heat of formation of the oxygen complex, and that of state $\mathbf{D}$ $\left(\mathrm{Fe}_{p}^{3+}+\mathrm{O}_{2}^{-}\right.$, infinitely separated) by the calculation employing the ionization potential and other thermochemical data referred to above. State $\mathbf{C}$, in which an electron has been transferred within the complex, $\mathrm{Fe}_{p}^{3+} \mathrm{O}_{2}^{-}$, but separation of the products has not yet occurred, has been placed $20 \mathrm{kcal}$./mole above state 
$\mathbf{B}$ on the basis of certain kinetic results (25). Reaction (5) thus involves passing from state $\mathbf{A}$ to $\mathbf{B}$, from $\mathbf{B}$ to $\mathbf{C}$, and finally from $\mathbf{C}$ to $\mathbf{D}$, whereas reaction (6) requires only the change from $\mathbf{B}$ to $\mathbf{C}$ and $\mathbf{C}$ to $\mathbf{D}$. It is clear from the diagram that because of the energy difference between the two initial states, reaction (5) is less unfavorable than reaction (6), because its endothermicity is less by about $16 \mathrm{kcal} . /$ mole, provided of course that the exothermic heat of formation of $\mathrm{Fe}_{p}^{2+} \mathrm{O}_{2}$ (the $16 \mathrm{kcal} . / \mathrm{mole}$ ) is not dissipated before electron transfer to state $\mathbf{C}$ has occurred. If it is dissipated, then no distinction is possible, and only reaction(6) remains.

It thus appears that in contrast to other classes of hemoproteins and heme derivatives, the exothermic formation of an oxygen complex by myoglobin and hemoglobin is in itself a very significant factor in "protecting" the heme from oxidation.

\section{Higher Oxidation States of Ferrimyoglobin, Ferriperoxidase, and Ferricatalase*}

The idea that catalysts form intermediate compounds with one or other reactant antedates the study of enzyme action by many years. In the oxidation of various reducing agents by hydrogen peroxide or alkyl hydroperoxides catalyzed by these hemoproteins, intermediate compounds of this kind are formed, and their nature will now be discussed.

They have often been regarded as "enzyme-substrate complexes," as postulated in the Michaelis-Menten theory of enzyme action, and their structures accordingly have been formulated with, for example, $\mathrm{H}_{2} \mathrm{O}_{2}$ or its anion $\mathrm{O}_{2} \mathrm{H}^{-}$coordinated to the iron just as $\mathrm{O}_{2}, \mathrm{CO}, \mathrm{CN}^{-}, \mathrm{F}^{-}$, etc., are coordinated in the other hemoprotein complexes. With concentration ratios of about unity, ferrimyoglobin gives only one intermediate compound-reddish-violet in color-whereas peroxidase and catalase give two, compound I, which is green and which changes spontaneously into compound II, which is also

* See footnote regarding formulas on page 366. 
red in color but with different absorption bands from the ferrimyoglobin compound $(5,30)$.

More detailed studies have shown that the red intermediate compounds of ferriperoxidase and ferrimyoglobin are not in equilibrium with their parent substrate, peroxide, as a true "enzyme-substrate complex" would be, i.e., $\mathrm{E}+\mathrm{S} \rightleftharpoons \mathrm{ES}$; both are formed in irreversible reactions $(11,20)$. Furthermore spectrophotometric titration with reducing agents showed both compounds to be single equivalent oxidation products of the ferric state of the hemoproteins. Hence the peroxide molecule or its anion, which possess two oxidizing equivalents, cannot be a constituent of these compounds. They do not correspond to "enzyme-substrate complexes" nor hemoprotein complexes in the usual sense, but they react as quadrivalent iron compounds would. The titration results do not, however, distinguish whether the site of the oxidation is at the iron atom or some other group in the molecule-a question which is taken up again shortly. In accord with their nature as higher oxidation states, other strong oxidizing agents were found to form them besides peroxides, for example, $\mathrm{HOCl}, \mathrm{HOBr}, \mathrm{HClO}_{2}, \mathrm{ClO}_{2}$, and $\mathrm{O}_{3}$, etc., with ferriperoxidase, $\mathrm{ClO}_{2}$ and $\mathrm{HClO}_{2}$ with ferrimyoglobin and most effective of all with both hemoproteins, the simple electron-accepting oxidizing agents, potassium chloriridate and molybdicyanide $(12,13,22)$.

The change from the green intermediate, compound I, to the red intermediate, compound II, was recognized to be a single equivalent reduction step, and a new mechanism for peroxidase action was put forward on this basis (9); later work has confirmed this (3). Compound I is thus two equivalents above the ferric state, and therefore, since peroxides possess two oxidizing equivalents, on this evidence alone it could have the true peroxide complex structure. But there is good spectroscopic evidence that the majority of oxidizing agents which give the red compound II of peroxidase also form an intermediate, similar to, or identical with, the green compound I formed by peroxides (12). A particularly stable green intermediate is produced with potassium chloriridate, which for this reason 
was thought originally to differ in some way from compound I in the peroxide systems, possibly by stabilization through complex formation with the chloriridite ion (13). However, a simpler kinetic explanation for the greater stability is to be preferred, for peroxides unlike chloriridate cannot readily oxidize compound II back to compound I, and hence, with chloriridate, compound I will persist all the time excess chloriridate is present. The simplest interpretation of these observations is that compound $\mathrm{I}$ is also a higher oxidation state, which reacts as a quinquevalent iron compound would. In view of spectroscopic and other similarities, the same oxidation-reduction relationship is probably true for compounds I and II of catalase, which has recently been found to react with hypochlorous acid to give intermediate compounds similar to, or identical with, compounds I and II formed by peroxides (14). The specificity shown by peroxidase and catalase evidently follows from minor differences in the reactivity of the two higher oxidation states, and a mechanistic interpretation in terms of a "reaction cage" has been put forward recently (10).

These higher oxidation states, which can be represented in chemical equations by the symbols $\mathrm{Fe}_{\mathrm{Per}}^{\mathrm{V}}, \mathrm{Fe}_{\mathrm{Per}}^{\mathrm{IV}}$ and $\mathrm{Fe}_{\mathrm{Mb}}^{\mathrm{IV}}$, etc., could result either from electron or hydrogen atom abstraction from the porphyrin ring system, or from oxidation at the iron atom itself. The latter is more likely for the following reasons. Experiments in which cyanide or fluoride together with peroxide are added to ferrimyoglobin (24), or ferricatalase, indicate competition between the reagents for the iron atom. The absence of any higher oxidation states of ferricytochrome $c$ is additional evidence that an exposed iron atom is required, and furthermore that the oxidation process is more complicated than electron transfer. This is also substantiated by the observation that none of the intermediate compounds forms a series of derivatives with various ligands, as do the ferric and ferrous oxidation states. This last observation also suggests that both the primary (electrovalent) and the coordination valencies of the iron atom are fully utilized in the higher oxidation states. 
There is evidence for hydrogen atom transfer (or $\mathrm{H}^{+}+$ $\left.e^{-}\right)$in the reactions of both $\mathrm{Fe}_{\mathrm{Mb}}^{\mathrm{IV}}$ and $\mathrm{Fe}_{\mathrm{Per}}^{\mathrm{IV}}(11,23)$. Measurements of the $p \mathrm{H}$ changes that occur in unbuffered solutions show that for each equivalent of acidic ferrimyoglobin $\mathrm{Fe}_{\mathrm{Mb}}^{3+}\left(\mathrm{H}_{2} \mathrm{O}\right)$ oxidized to $\mathrm{Fe}_{\mathrm{Mb}}^{\mathrm{IV}}$ by $\mathrm{IrCl}_{6}^{2-}$, two equivalents of $\mathrm{H}^{+}$are formed; and correspondingly on reduction by $\mathrm{Fe}(\mathrm{CN})_{6}^{4-}$, two equivalents of $\mathrm{H}^{+}$are removed from solution; i.e.,

$$
\mathrm{Fe}_{\mathrm{Mb}}^{3+}\left(\mathrm{H}_{2} \mathrm{O}\right) \rightleftharpoons \mathrm{Fe}_{\mathrm{Mb}}^{\mathrm{IV}}+2 \mathrm{H}^{+}+e^{-}
$$

From a study of the $p \mathrm{H}$ variation of the equilibrium constant for the reaction of ferrimyoglobin with chloriridate, it has been found that these $2 \mathrm{H}^{+}$arise in the oxidation process itself, and not

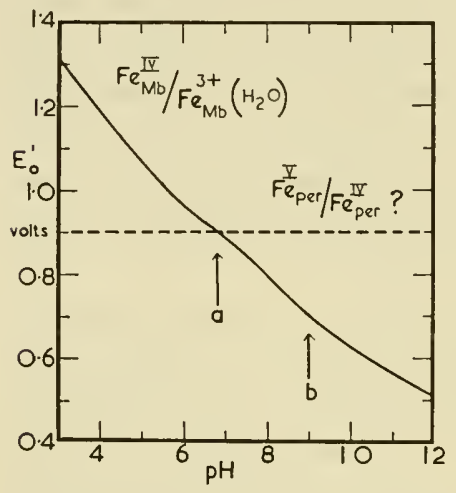

Fig. 5. Full curve: Variation of $E_{0}^{\prime}$ with $p \mathrm{H}$, at $20.4^{\circ} \mathrm{C}$. and ionic strength 0.04 for the couple involving the higher oxidation state of ferrimyoglobin (24), $\mathrm{Fe}_{\mathrm{Mb}}^{\mathrm{IV}}+2 \mathrm{H}^{+}+e^{-} \rightarrow$ $\mathrm{Fe}_{\mathrm{Mb}}^{3}\left(\mathrm{H}_{2} \mathrm{O}\right)$. a, point of inflection due to heme-linked ionizations (compare curve C, Fig. 2); b, line changes slope due to the ionization of the iron-bound water molecule in ferrimyoglobin, $\mathrm{Fe}_{\mathrm{Mb}}^{3+}\left(\mathrm{H}_{2} \mathrm{O}\right) \rightarrow \mathrm{Fe}_{\mathrm{Mb}} \mathrm{OH}^{2+}+\mathrm{H}^{+}, \mathrm{pK}$ $=9.00$ (15). Dotted curve: Hypothetical variation for the Compound I/Compound II couple of peroxidase, which, if the variation for the Compound II/Peroxidase couple is similar to that for myoglobin, would account for the stability relationships in the peroxide system.

through the operation of the heme-linked ionization, the effect of which can readily be recognized by analyzing the data using the equation given in Section II (24). The $p \mathrm{~K}$ of the group in $\mathrm{Fe}_{\mathrm{Mb}}^{\mathrm{IV}}$ is estimated as about 7.5 , compared to 6.1 in ferrimyoglobin, at $20.4^{\circ} \mathrm{C}$. and an ionic strength of 0.04 . Using these figures the $p \mathrm{H}$ variation of $E_{0}^{\prime}$ for the $\mathrm{Fe}_{\mathrm{Mb}}^{\mathrm{IV}} / \mathrm{Fe}_{\mathrm{Mb}}^{3+}\left(\mathrm{H}_{2} \mathrm{O}\right)$ couple can be calculated; the results are plotted in Figure 5 and show that in acid solution $\mathrm{Fe}_{\mathrm{Mb}}^{\mathrm{IV}}$ is quite a strong oxidizing agent with $E_{0}{ }^{\prime}>1.0 \mathrm{v}$. 
The "ferryl ion" structure is the simplest that fits all the evidence,

$$
\mathrm{Fe}_{\mathrm{Mb}}^{3+}\left(\mathrm{H}_{2} \mathrm{O}\right) \rightleftharpoons \mathrm{Fe}_{\mathrm{Mb}} \mathrm{O}^{2+}+2 \mathrm{H}^{+}-e^{-}
$$

and provisionally the higher oxidation state of ferrimyoglobin can be named "ferrylmyoglobin" (23). Bonded oxygen atom complexes are already well known in certain higher oxidation states of simple transition element ions, e.g., manganates, permanganates, vanadyl, and uranyl ions, etc., and also in certain complex ions, e.g., Os $\left(\mathrm{NH}_{3}\right)_{4} \mathrm{O}_{2}^{2+}, \mathrm{U}(\mathrm{N} \text {-base })_{2} \mathrm{O}_{2}^{2+}$, the vanadyl porphyrins and phthalocyanine.

If compound II of peroxidase has this structure too, in spite of its absorption bands being different, which may be a reflection of the heme being joined to a different amino acid residue, then the formation of compound I from compound II can be envisaged as simple electron removal, and the catalytic cycle in peroxidase action represented as follows

$$
\begin{gathered}
\mathrm{Fe}_{\mathrm{Per}}^{3+}\left(\mathrm{H}_{2} \mathrm{O}\right)+\mathrm{H}_{2} \mathrm{O}_{2} \underset{\text { two equiv. }}{\stackrel{\text { oxidation }}{\longrightarrow}} \mathrm{Fe}_{\mathrm{Per}} \mathrm{O}^{3+}+2 \mathrm{H}_{2} \mathrm{O} \\
\mathrm{Fe}_{\mathrm{Per} \mathrm{O}^{3+}+\mathrm{AH}_{2}}^{\stackrel{\text { single equiv. reduction }}{\text { (electron transfer only) }}} \mathrm{Fe}_{\mathrm{Per}} \mathrm{O}^{2+}+\mathrm{AH}^{\bullet}+\mathrm{H}^{+} \\
\mathrm{Fe}_{\mathrm{Per}} \mathrm{O}^{2+}+\mathrm{AH}_{2}+\mathrm{H}^{+} \underset{\text { single equiv. red. }}{\text { (electron } \left.+2 \mathrm{H}^{+}\right)} \mathrm{Fe}_{\mathrm{Per}}^{3+}\left(\mathrm{H}_{2} \mathrm{O}\right)+\mathrm{AH}^{\bullet}
\end{gathered}
$$

The different character of the two single equivalent reduction steps is to be noted, and although these structures are purely speculative in the case of peroxidase, they have the merit of explaining why in the peroxide system compound I is apparently more stable with respect to compound II formation in acid solution (3), whereas the reverse holds in more alkaline solutions. The oxidation-reduction potential of $\mathrm{Fe}_{\mathrm{Per}}^{\mathrm{IV}} / \mathrm{Fe}_{\mathrm{Per}}^{3+}\left(\mathrm{H}_{2} \mathrm{O}\right)$ would vary with $p \mathrm{H}$ in a very similar manner to $\mathrm{Fe}_{\mathrm{Mb}}^{\mathrm{IV}} / \mathrm{Fe}_{\mathrm{Mb}}^{3+}\left(\mathrm{H}_{2} \mathrm{O}\right)$, heme-linked ionizations and the bound water molecule ionization only modifying the slope in different $p \mathrm{H}$ ranges. The potential of the $\mathrm{Fe}_{\mathrm{Per}}^{\mathrm{V}} / \mathrm{Fe}_{\mathrm{Per}}^{\mathrm{IV}}$ couple, apart from the effect of linked ionizations, would be independent of $p \mathrm{H}$. As a consequence, the plots of $E_{0}^{\prime}$ against $p \mathrm{H}$ could cross, as shown in Figure 4, and the free- 
energy change would then favor the following reaction between the two couples

$$
\mathrm{Fe}_{\mathrm{Per}}^{\mathrm{V}}+\mathrm{Fe}_{\mathrm{Per}}^{3+}\left(\mathrm{H}_{2} \mathrm{O}\right) \rightleftharpoons 2 \mathrm{Fe}_{\mathrm{Per}}^{\mathrm{IV}}
$$

in the forward direction at high $p \mathrm{H}$ values and in the reverse direction at low $p \mathrm{H}$ values. On the other hand, if the structures of compounds I and II are such that the two single equivalent reduction steps are the same, i.e., H-atom transfer or its equivalent (electron transfer and $\mathrm{H}^{+}$association), the respective oxidation-reduction potentials would show the same $p \mathrm{H}$ variation. These stability relationships would then have to be explained either through the operation of heme-linked ionizations alone, or by postulating that the speed of reduction of compound I to compound II is less in more acid solution, which seems very unlikely.

Finally, the observation that other strong oxidizing agents as well as peroxides form the higher oxidation states can be seen to have a very important bearing upon biological oxidation. The appearance of these compounds in intact enzyme systems in vivo can no longer be taken as proof that hydrogen peroxide is formed as such during the reduction of oxygen to water, which provides the driving force for the oxidation process (4). It just remains a very likely possibility.

\section{Conclusions}

As more and more data have accumulated, it appears that hemoprotein structures and reactions can be interpreted in terms of the present system of chemical and physical theory. There is no compelling evidence, from the in vitro reactions which have been thoroughly investigated to suggest that fundamentally new properties emerge at this level of molecular complexity, or that fundamentally new principles need be invoked to explain the reactions. Whilst it is true that the especially complicated hemoprotein structure endows individual hemoproteins with a reactivity that is shared by others to a far 
lesser degree, or that appears to be a unique feature, kinetic and equilibrium studies have revealed that the underlying thermodynamic and kinetic quantities, $\Delta I I^{0}, \Delta S^{0}$, etc., have magnitudes which justify treating these reactions on the same basis as those of simpler coordination compounds and aquated ions. A comparison of data has led to certain tentative conclusions about the way the porphyrin prosthetic group and the protein contribute to the reactivity.

In the ligand replacement reactions of myoglobin, the magnitudes of the entropy changes suggest that the protein participates through a tightening of its structure as the water molecule coordinated to the iron is replaced by $\mathrm{O}_{2}, \mathrm{CO}, \mathrm{CN}^{-}$, $\mathrm{F}^{-}$, and $\mathrm{OH}^{-}$. A similar effect in hemoglobin could be responsible for heme-heme interaction. In the reduction of ferrimyoglobin to ferromyoglobin the sign and magnitude of the entropy change are characteristic of a reaction in which the over-all charge on the complex increases. One interpretation is that the prosthetic group as a whole, with its two negatively charged propionate side chains, cooperates in determining the entropy change.

There is strong evidence at present that the higher oxidation state of ferrimyoglobin is a true quadrivalent iron compound possessing the "ferryl ion" structure, $\mathrm{Fe}_{\mathrm{Mb}} \mathrm{O}^{2+}$, and thermodynamic data for its reactions are now very desirable so that a comparison can be made with those for well-established bondedoxygen structures.

The acquisition of similar data for peroxidase, catalase, and other hemoproteins, in particular the elucidation of the part played by heme-linked ionizations, would now contribute greatly to a better understanding of the origin and nature of their specific reactions. 


\section{References}

Review articles and papers containing other essential references to primary sources are denoted by an asterisk.

1. Brooks, J., Proc. Roy. Soc. (London), B109, 35 (1931); ibid., B118, 560 (1935).

2. Chance, B., J. Biol. Chem., 194, 483 (1952).

3. Chance, B., Arch. Biochem. and Biophys., 37, 235 (1952).

4. Chance, B., Science, 116, 202 (1952).

5. Chance, B., Modern Trends in Physiol. and Biochem., 1952, 25.

6. Coryell, C. D., and L. Pauling, J. Biol. Chem., 153, 91 (1940).

7. Evans, M. G., and G. H. Nancollas, Trans. Faraday Soc., 49, 363 (1953).

8. Ferry, R. M., and A. A. Green, J. Biol. Chem., 81, 175 (1929).

9.* George, P., Advances in Catalysis, 4, 367 (1952).

10. George, P., Biochem. J. (London), 52, XIX (1952).

11.* George, P., Biochem. J. (London), 54, 267 (1953); 55, 220 (1953).

12. George, P., J. Biol. Chem., 201, 413 (1953).

13. George, P., Science, 117, 220 (1953).

13a. George, P., and F. J. W. Roughton, "The Physical Chemistry of Enzymes," Discussions Faraday Soc., in press.

14. George, P., and G. H. F. Fraser, unpublished work.

15. * George, P., and G. I. H. Hanania, Biochem. J. (London), 52, 517 (1952); ibid., 55, 236 (1953).

16. George, P., and G. I. H. Hanania, Biochem. J. (London), 56, XXXVII, XXXVIII (1954).

17.* George, P., and G. I. H. Hanania, Nature, 174, 33 (1954).

18. George, P., and G. I. H. Hanania, unpublished work.

19. George, P., G. I. H. Hanania, and D. H. Irvine, J. Chem. Phys., 22, 1616 (1954).

20.* George, P., and D. H. Irvine, Biochem. J. (London), 52, 511 (1952); ibid., 55, 230 (1953).

21.* George, P., and D. H. Irvine, Brit. J. Radiol., 27, 131 (1954).

22. George, P., and D. H. Irvine, Biochem. J. (London), 58, 188 (1954).

23. George, P., and D. H. Irvine, Symposium on Co-ordination Chemistry, Danish Chem. Soc., Copenhagen, 1954, p. 135; Biochem. J. (London), 60, 596 (1955).

24. George, P., and D. H. Irvine, unpublished work.

25. George, P., and G. J. Stratmann, Biochem. J. (London), 51, 103, 418 (1952); ibid., 57, 568 (1954).

26. George, P., and C. L. Tsou, Biochem. J. (London), 50, 440 (1952).

27. Glasstone, S., K. J. Laidler, and H. Eyring, The Theory of Rate Processes, especially Ch. 8. McGraw-Hill, New York, and London, 1941. 
28.* Hartree, E. F., Ann. Repts. on Progress Chem. (Chem. Soc., London), 43, 287 (1946).

29. Hartridge, H., and F. J. W. Roughton, Proc. Roy. Soc. (London), A104, 395 (1923).

30. Keilin, D., and E. F. Hartree, Bi,chem. J. (London), 49, 88 (1951).

31. Latimer, W. M., Oxidation Potentials. Prentice-Hall, New York, 1952; see also Cobble, J. W., J. Chem. Phys., 27, 1451 (1953).

32.* Lemberg, R., and J. W. Legge, "Haematin Compounds and Bile Pigments." Interscience, New York-London, 1949.

33. Manchot, W., and H. Haunschild, Z. anorg. allgem. Chem., 140, 22 (1924).

34. Millikan, G. A., Proc. Roy. Soc. (London), B120, 366 (1936).

35. Pauling, L., Contrib. étude structure moléculoire, Vol. commén. Victor Henri, Liège, 1947-48, 1-14.

36. Riggs, A., and G. Wald, J. Gen. Physiol., 35, 45 (1951-2).

37. Roughton, F. J. W., and Q. Gibson, private communication.

38. Roughton, F. J. W., A. B. Otis, and R. Lyster, Proc. Roy Soc. (London), B744, 29 (1955).

39. St. George, R. C. C., and L. Pauling, Science, 174, 629 (1951).

40. Scheler, W., and F. Jung, Biochem. Z., 325, 515 (1954).

41. Scheler, W., F. Jung, and K. H. Hoffmann, Biochem. Z., 325, 401 (1954).

42. Taylor, J. F., and V. E. Morgan, J. Biol. Chem., 144, 15 (1942).

43. Theorell, H., Biochem. Z., 268, 64, 73 (1934).

44. Theorell, H., J. Am. Chem. Soc., 63, 1820 (1941).

45.* Theorell, H., and A. Ehrenberg, Acta Chem. Scand., 5, 283 (1951).

46.* Uri, N., Chem. Revs., 50,375 (1952), especially Table 10.

47.* Wyman, J., Advances in Protein Chem., 4, 410 (1948).

48. Wyman, J., and D. W. Allen, J. Polymer Sci., 7, 499 (1951). 


\title{
ASPECTS OF PROTEIN STRUCTURE
}

\author{
BARBARA W. LOW, Department of Biological Chemistry, Harvard \\ Medical School, Boston, Massachusetts \\ JOHN T. EDSALL, Biological Laboratories, Harvard University, \\ Cambridge, Massachusetts
}

We propose here to inquire into the configuration of a few important protein molecules. The inquiry is still preliminary and tentative, but it can proceed at a level which would have been unattainable only a few years ago. The last ten years, and more especially the last five, have seen the most remarkable advances ever recorded in our knowledge of protein structure. These advances have illuminated the difficult problems of the configuration of polypeptide chains in space, and of the sequences of amino acid residues in the peptide chains found in natural proteins.

The complete structural formulas of the insulins of beef, sheep, and pig have been deduced by Sanger and his associates $(21,92-96)$ by a powerful chain of detailed circumstantial evidence. The structure of each of the many peptides derived by partial breakdown from either the $A$ or the $B$ chain of the insulin molecule is compatible with one, and only one, sequence in the amino acid residues in each of these chains. It is true that the original insulin used as starting material was not quantitatively accounted for in the split products; conceivably some material in the latter which escaped detection might have represented peptides arising from other sequences than those 
deduced by Sanger to be present in the original peptide chains of insulin. However, the complete and carcful amino acid analyses by Harfenist (46) on these insulins are in full accord with the values to be expected from the structural formulas of Sanger and his associates. The fitting together of a great mass of circumstantial evidence leaves little doubt of the general correctness of the inferred sequences. The great difficulties that attended the determination of the positions of the disulfide linkages, due to the ease of disulfide interchange reactions, appear

(A)

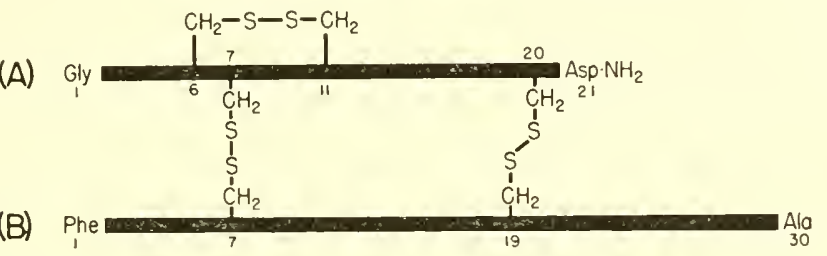

Fig. 1. Outline of the structure of beef, hog, and sheep insulins.

The glycyl (A) chain, with 21 residues, and the phenylalanyl (B) chain, with 30 residues, both have the same orientation, with the free terminal $\alpha$-amino groups at the left-hand side of the diagram. The sequence of residues 3 to 14 inclusive of the $\mathrm{A}$ chain is shown in more detail in Figure 2, and the complete sequences in both chains are given in the text. Possible configurations of the insulin molecule in space are discussed later.

to have been overcome, with decisive evidence in favor of the cross linkage of the $\mathrm{A}$ and $\mathrm{B}$ chains at two points, the third disulfide linkage serving to bridge residues 6 and 11 of the A chain $(21,91 \mathrm{a}, 91 \mathrm{~b}, 93)$. The final proof of structure by synthesis in the laboratory may still be a long way off, but the greatness of the achievement is not thereby diminished.

The outline of the inferred structure of the insulins is given in Figure 1, and the sequences in the $\mathrm{A}$ and $\mathrm{B}$ chains are given in detail below. It is remarkable that the complete sequence of amino acid residues in the $B$ chain is identical for all three species of insulin; the same is true in the A chain except for residues 8,9 , and 10 , which lie within the intrachain disulfide 
loop. This loop, which involves a 20 -atom ring, closed by the disulfide bond, has also been found by du Vigneaud and his associates $(106,107)$ in the very different structures of the smaller polypeptide hormones, oxytocin and vasopressin, with different sets of amino acid residues in the loop. Here, in the case of oxytocin at least, the structure has been completely proved by synthesis. The presence of this unusual ring (Figure 2)

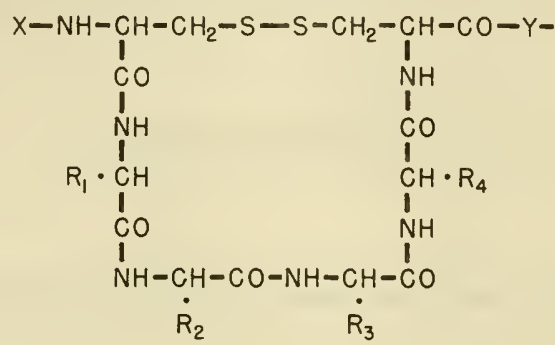

Fig. 2. The peptide-disulfide ring structure of oxytocin, vasopressin, and insulin. The attached groups at positions $\mathrm{X}, \mathrm{Y}, \mathrm{R}_{1}, \mathrm{R}_{2}, \mathrm{R}_{3}$, and $\mathrm{R}_{4}$ are indicated below.

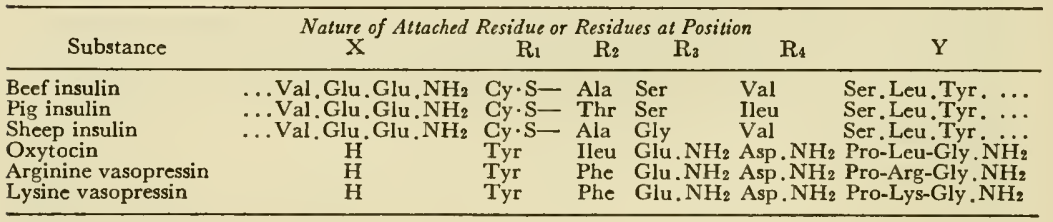

in several different protein or peptide hormones is a striking phenomenon; one cannot help suspecting that it bears a special relation to the physiological activities of these compounds. Yet it is remarkable that the only differences between the three insulins are found among the amino acid residues in this ring; a single unique set of side chains here is clearly not required for the physiological activity of insulin. Moreover there is no reason to suppose that the spatial configuration of this 20 -atom ring is at all the same, in insulin, oxytocin, and vasopressin; indeed the presence of the adjoining disulfide link to the $\mathrm{B}$ chain at 
position 7 in insulin imposes constraints on the possible configurations which are examined further below, and which do not exist in the case of the smaller peptide hormones.

This work on insulin indicates that it is possible to obtain protein preparations which are genuinely homogeneous, all molecules in the preparation being identical in the sense in which we may say that the molecules in pure acetone or glycine are identical. There is no doubt that such purity has seldom been achieved in protein preparations, and increasingly delicate analytical techniques - especially those employed by the physical chemists-have revealed heterogeneity in preparations earlier thought to be pure. On the other hand, the increasing refinement of the techniques of fractionation has also permitted the resolution of mixtures into pure components to an extent unattainable earlier. Thus, two kinds of beef insulin, differing only by the conversion of one carboxyl to an amide group in a molecule with 51 amino acid residues, have been separated by countercurrent fractionation $(46,47)$. If there is no difference of net charge-for instance, if we consider two molecules which differ only by the conversion of one valyl to isoleucyl, and one alanyl to threonyl, as with beef and pig insulins (Figure 2)separation by present techniques would be much more difficult. Even such separations, however, now appear practicable. It remains to be seen whether a given animal makes all its insulin molecules just alike, or whether it can produce several similar but not identical molecules, all more or less equivalent in physiological function. Even if the latter alternative corresponds to the actual situation, the possibility of fractionating the mixture into pure components still remains. Moreover the heterogeneity found in a protein preparation may be due not to natural differences between the molecules but to damage done by the chemist in the process of extracting the material from its natural state-damage which can be avoided by gentler techniques. We express confidence here-confidence based perhaps more on faith than on reason, but with a faith fortified by the results already achieved with insulin-that genuinely pure protein 
preparations will be obtained in future, and their structures determined, in steadily increasing numbers.

We now return to the more general theme of our inquiry into protein structure. The structure of a native protein may be considered at three different but somewhat overlapping levels. The primary level is that of structure in the sense generally employed by the organic chemist; it involves knowledge of the number of polypeptide chains in the protein molecule, the sequence of the amino acid residues in each chain, and the nature and position of the cross links which bridge different chains or form loops involving two different portions of the same chain. Such a structure, even if completely established, may correspond to a vast number of possible spatial configurations all of which are compatible with the structural formula. At the second level of organization we must consider the spatial configuration of individual peptide chains, or segments of chains, the bond distances and bond angles, and the degree of resistance to rotation around various valence bonds. The possible structures include the now familiar $\alpha$-helix, as well as a number of other helical configurations which are theoretically possible. They also include the extended " $\beta$ " configurations, of which the most probable are the "pleated sheets" of Pauling and Corey. Other, more irregular, configurations certainly occur also. At the third level, which is closely related to the second, we consider the folds and turns which may, and indeed must, occur in these already coiled peptide chains in order to fit the entire molecule into a region of the appropriate size and shape. We may briefly consider all of these problems in turn.

\section{Sequences and Cross Linking in Peptide Chains}

The first level of knowledge indicated above is the problem which has apparently been completely solved by Sanger and his collaborators for the three insulins. More recently a virtually complete sequence determination has been achieved for $\beta$ corticotropin of the pig (12) with a single chain of 39 residueslonger than either of the chains of insulin, but lacking in sulf- 
hydryl or disulfide groups. Limited knowledge of some sequences in the peptide chains in other proteins has been achieved. The difficulties of sequence determination are formidable, and become increasingly so as the length of the chain increases. Even for molccules as relatively simple as lysozyme or ribonuclease, the chain length is of the order of 130 residues, and the problem is far more complex than in the case of insulin. However, the pace of progress is so rapid that a complete structure determination, for these relatively small proteins, may be a matter of only two or three years.

We tabulate some of the most important sequences already determined, and invite the reader to contemplate them, to see whether he can make some attempt to read the riddles with which they confront him.

Beef Insulin, A Chain

Beef Insulin, B Chain

\section{Pig Insulin}

Sheep Insulin

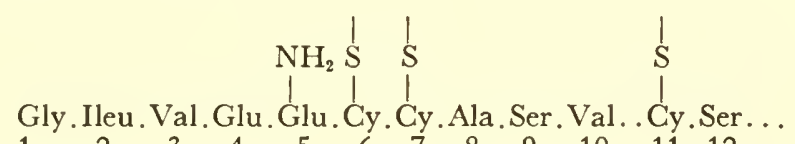
$\begin{array}{llllllllllll}1 & 2 & 3 & 4 & 5 & 6 & 7 & 8 & 9 & 10 & 11 & 12\end{array}$

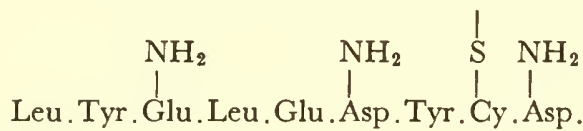
$\begin{array}{lllllllll}13 & 14 & 15 & 16 & 17 & 18 & 19 & 20 & 21\end{array}$

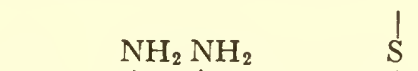
Phe.Val.Asp. Glu. His.Leu. Cy. Gly.Ser.His.Leu. Val... $\begin{array}{llllllllllll}1 & 2 & 3 & 4 & 5 & 6 & 7 & 8 & 9 & 10 & 11 & 12\end{array}$

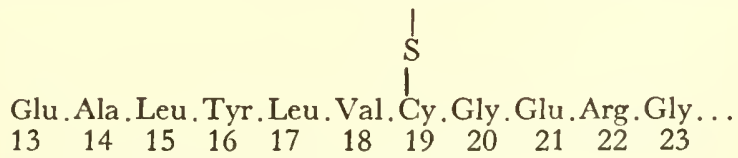

Phe.Phe. Tyr. Thr. Pro. Lys. Ala $\begin{array}{lllllll}24 & 25 & 26 & 27 & 28 & 29 & 30\end{array}$

Sequence same as in beef insulin except for residues 8,9 , and 10 in the $\mathrm{A}$ chain, for which the sequence is Thr.Ser. Ileu.

Sequence same as in beef insulin except for residues 8,9 , and 10 in the A chain, for which the sequence is Ala.Gly. Val. 
The peptide derived from conversion of ovalbumin into plakalbumin (76) Ala.Gly.Val.Asp.Ala.Ala

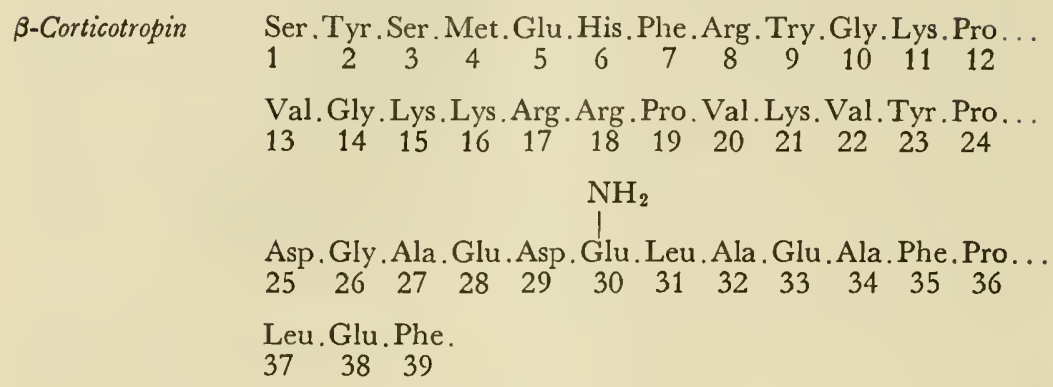

\section{Comparison of Corticotropins}

In addition to pig $\beta$-corticotropin $(12,48 a)$ as shown above, sequences for sheep $\alpha$-corticotropin (60a) and pig corticotropin A (108a) have also been reported. All are made up of a single chain sequence of 39 amino acid residues, and the sequence appears to be identical for all three except within the segment of residues 25-33 inclusive. The comparison of the residue sequence for these residues in the three corticotropins is shown below. Note that the composition of the sequence $25-28$ is the same in all cases; only the order differs. There appears still to be a little uncertainty about the order, especially in the case of corticotropin A.

Residue no.

Sheep $\alpha$-corticotropin*

Pig corticotropin A

Pig $\beta$-corticotropin

Ala.Gly.Glu.Asp.Asp.Glu. Gly.Ala.Glu.Asp.Asp.Glu.

Ala.Ser.Glu Asp.Gly.Ala.Glu.Asp.Glu. $\mathrm{NH}_{2}$.Leu.Ala.Glu

* There are two amide groups in $\alpha$-corticotropin, but the particular Asp. or Glu. residues with which they are associated are still undetermined.

We employ for the amino acid residues the now indispensable abbreviated notation which was originated by the late Dr. Erwin Brand and set forth in a review by Brand and Edsall (20) and later by Sanger (92). Each amino acid residue is denoted by the first three letters of its name, except for isoleucine (Ileu), cysteine (CySH), and a half cystine residue (CyS-). Asparagine and glutamine residues are denoted by $\mathrm{Asp} . \mathrm{NH}_{2}$ and $\mathrm{Glu} . \mathrm{NH}_{2}$, respectively. An $N$-terminal residue, with a free $\alpha$-amino group, is by convention written at the left-hand end of the formula. All optically active amino acid residues are to be taken as having the $\mathrm{L}$ configuration in these formulas. 
To interpret such patterns will be a formidable task in which as yet almost no progress has been made. The observed sequences appear to be compatible with the view that any one of the amino acid residues may be placed next to any other, in substances which nature is capable of producing. However, some of the observed sequences suggest that similar or identical side-chain residues appear to be bunched together more frequently than would be expected on a random basis. Perhaps significant, for instance, is the aromatic side-chain sequence Phe.Phe.Tyr. in residues $24-26$ of the $B$ chain of insulin. The presence of three alanine residues and one valine in the hexapeptide derived from ovalbumin represents a striking predominance of nonpolar side chains in this short series. Three hydroxyamino acid residues - two aliphatic and one aromatic-appear at the $N$-terminal end of the chain of $\beta$-corticotropin. The sequence Lys.Lys.Arg.Arg, containing four successive positively charged side chains, occurs in residues 15-18 of this substance. Indeed all the nine positively charged groups in $\beta$-corticotropin, including the $N$-terminal ammonium group of serine, are located between positions 1 and 21; all but one of the seven negatively charged groups, including the $C$-terminal phenylalanine, lie between positions 25 and 39. The molecule may thus be considered as consisting of a positively charged segment in one half, and a negatively charged segment in the other. Biological studies show that the former-including also the bydroxyl side chains on residues 1,2 , and 3 -is essential to hormone activity; the latter, at least the last 15 residues, is nonessential.

Finally we may mention evidence on a rather different level-the distribution of sulfhydryl groups in horse hemoglobin. The importance of these groups for the oxygen affinity of hemoglobin was first shown by the work of Riggs (89). Binding of the sulfhydryls by a mercurial modifies both the oxygen affinity and the heme-heme interactions, and the effects are reversible on removing the mercurial. Ingram (51) has recently shown in a careful study that four sulfhydryl groups are readily titrated with silver ions in the native hemoglobin; however, these four 
combine quite firmly with two, and only two, mercury atoms. Evidently each mercury forms a bond between a pair of sulfhydryl groups, by a reaction similar to that which occurs in serum mercaptalbumin when two mercaptalbumin molecules are linked through their sulfhydryl groups by a mercury atom $(36,39,50)$. Hence these sulfhydryl groups of hemoglobin must exist in pairs, the members of each pair being very close together. The x-ray work of Bragg and Perutz (18) on these mercurial derivatives, which give crystals isomorphous with those of the original hemoglobin, indicates that the two pairs of sulfhydryl groups are arranged symmetrically around the twofold axis of the hemoglobin molecule. In addition to these four sulfhydryl groups, which are titratable with silver ions in the native hemoglobin, two more become titratable when the hemoglobin is denatured; Ingram has produced evidence that these two additional groups are geometrically closely associated with the other pairs, so that there are in effect two clusters of sulfhydryls in the hemoglobin molecule, each consisting of three - SH groups. These groups, therefore, are not distributed at random in the molecule but are closely concentrated within two very narrow regions. There is of course as yet no evidence that these sets of associated groups are members of the same peptide chain; they may be in different chains, but packed close together for geometrical reasons. Nevertheless this is a striking example of the clustering together of like groups within certain very limited portions of a large and complex molecule.

It is tempting to say that the presence of adjoining sets of similar residues in the sequences of peptide chains was to be expected-that protein molecules are built to do a job, and that the doing of that job requires the concentration of certain sets of side-chain groups-charged groups, or hydroxyl groups, or perhaps nonpolar groups-within a relatively limited region. Probably there is more than a germ of truth in this conception, but the idea as naively stated here is certainly too simple. The order of residues in the sequence of a peptide chain does not in itself specify their spatial relations. If, for example, the chain is 
coiled in the form of an $\alpha$-helix, the $\beta$-carbon atoms of successive residues emerge from the core of the helix at intervals of approximately $1.5 \mathrm{~A}$, along the helical axis, and they are also rotated with respect to each other through an angle of approximately $100^{\circ}$ about this axis. In general the longer side chains have appreciable freedom of rotation around the $\beta$-carbon atom, so that the exact position of the terminal groups of a side chain is adjustable within certain limits, even if the helical core is rigid. A given side chain may, and probably will, approach as closely to another side chain three or four residues away from it in the main peptide chain, as to its nearest neighbor in the sequence. The end of the side chain may also, of course, approach most closely to the end of another side chain arising from a peptide chain which is geometrically adjacent, although perhaps far away in the sequence, or belonging to a different subunit of the molecule. If the chain structure is not that of an $\alpha$-helix, but something different, then another quite different set of possibilities arises. Thus any speculations based on observed sequences of amino acid residues in a single chain are necessarily tentative; the riddle cannot be read without a knowledge of the stereochemistry involved, but hints may be obtained from the sequences nevertheless.

\section{COVALENT CROSS LINKAGES: DISULFIDE AND PHOSPHATE}

The commonest and most important covalent cross links are, of course, the disulfide bonds of cystine residues. They may serve to link two different chains together, or they may link two half cystine residues within the same chain. Both types of linkage are exemplified in the insulins, and the intrachain links by such proteins as serum albumin or ribonuclease, which are discussed later.

Cross linkages through phosphate groups have been revealed as significant in several proteins, by the recent work of Perlmann (85). Phosphate groups may be attached in monoester linkage to one of the hydroxyamino acid residues in a peptide chain: 


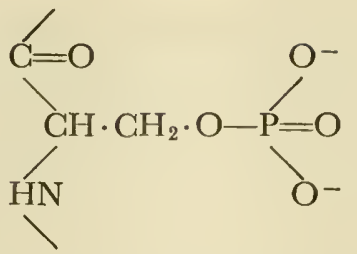

Of course no cross linkage is involved here. They may, however, be involved in a diester linkage between two such chains, or link different parts of the same chain together into a loop. The cross linkage of two seryl residues is illustrated as an example:

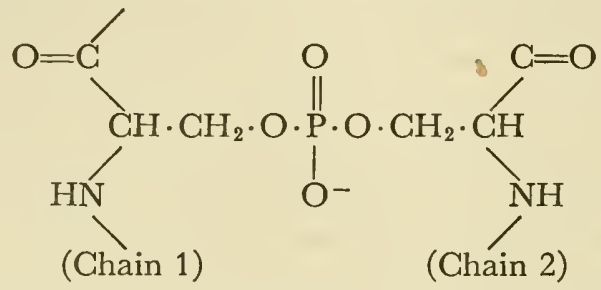

The linkage may also involve an amino or other nitrogenous group on an amino acid residue of one of the two chains, and a hydroxyl group of the other. Such a linkage may be called an $-\mathrm{N}-\mathrm{P}-\mathrm{O}-$ bond, in distinction from the $-\mathrm{O}-\mathrm{P}-\mathrm{O}-$ bond illustrated above. Finally the cross linkage may involve a pyrophosphate, instead of an orthophosphate, bridge between two chains or chain segments; such a bridge may be designated as an $-\mathrm{O}-\mathrm{P}-\mathrm{O}-\mathrm{P}-\mathrm{O}-$ cross link. All of these linkages have been found by Perlmann in proteins, by employing different phosphatases specific for each type of linkage in smaller molecules. Thus in $\alpha$-casein (84) if the molecular weight be taken as 30,000 , there are in all ten atoms of phosphorus per molecule. Four of these are found to be present in the form of simple primary phosphate esters, linked presumably to hydroxyamino acids. Four other phosphorus atoms are held in diester linkages; the specificity of the enzyme used indicates that these linkages are of the $-\mathrm{N}-\mathrm{P}-\mathrm{O}-$ type discussed above. Finally two of the ten phosphorus atoms are involved in a pyrophosphate linkage of the type $-\mathrm{O}-\mathrm{P}-\mathrm{O}-\mathrm{P}-\mathrm{O}-$. The splitting of 
this linkage by pyrophosphatase involves no release of inorganic phosphate into the solution, but such release can be produced by subsequent addition of the prostate phosphatase which splits primary phosphate esters. Thus it appears that this pyrophosphate linkage actually serves as a cross link between peptide chains. Breakage of these cross links results in a separation of the protein molecule into smaller units, indicating that these phosphorus linkages actually hold different peptide chains together. In $\beta$-casein (83) likewise, diester cross linkages are found, but here the specificity of the enzyme involved indicates that the linkages are of the type - $-\mathrm{O}-\mathrm{P}-\mathrm{O}-$, illustrated above. Here again it is found that after removal of the phosphorus, material soluble in trichloroacetic acid is present in the solution, so that a splitting into smaller molecules has occurred, as a result of the breakage of the $-\mathrm{O}-\mathrm{P}-\mathrm{O}$ linkage. Finally Perlmann has pointed out that the single atom of phosphorus present in the pepsin molecule is probably also present in an ester linkage of the $\mathrm{O}-\mathrm{P}-\mathrm{O}$ type (83). Pepsin appears to be a protein consisting of only one peptide chain; in this case, therefore, the phosphorus must cross link a portion of the chain into a cyclic loop. It is still uncertain how general the significance of these phosphate cross linkages in proteins may prove to be. They are certainly much less common than the disulfide cross linkages, but their discovery is an important event in enlarging our general picture of the possibilities of protein structure.

\section{OTHER GROSS LINKAGES}

As yet we have spoken only of cross linkages involving covalent bonds. Cross links involving hydrogen bonding between amino acid side chains, or perhaps between groups in the side chains and $\mathrm{CO}$ or NH groups of the peptide linkages, may also be of great importance in fixing the protein molecule in its native state. A protein molecule may hold together in solution as a functional unit with a high degree of stability, even when it consists of several different peptide chains with no 
covalent cross links between them. This, for instance, appears to be the case with horse hemoglobin, for which Porter and Sanger (87) have shown the presence of $6 \mathrm{~N}$-terminal amino groups per molecule, and therefore, by inference, of 6 different peptide chains. The work of Ingram (51), already mentioned, on the sulfhydryl groups of horse hemoglobin has shown that 6 sulfhydryl groups are present, but no disulfide cross links. Therefore, since there is also no evidence whatever of phosphate cross linkages in hemoglobin, other types of bonds must be invoked to explain the stability of the molecule. Some of these may be the relatively weak linkages which Steinhardt and Zaiser (102) have shown to be broken on acid denaturation with the release of 36 acid and basic groups which are nontitratable in the native molecule. This, however, cannot be the complete explanation, since the acid denaturation is not accompanied by a splitting of the hemoglobin into subunits. Although concentrated urea causes a splitting of hemoglobin into two subunits, each of half the original molecular weight, these half units remain as stable entities even in concentrated urea solution (109). There is no indication of the further breakdown that would occur if the molecule were split into 6 constituent peptide chains. In any case, the presence of large numbers of unreactive acid and basic groups in native hemoglobin, which become available on denaturation as shown by Steinhardt and Zaiser, points to a very special set of stabilizing factors in the native hemoglobin structure. The further characterization of this structure, and its correlation with the rest of our knowledge of hemoglobin, should lead to most important advances. Hemoglobin is probably the most thoroughly investigated of all the proteins, yet it still presents most baffling problems; a synthesis of all our knowledge on the subject is badly needed but has not yet emerged.

In sorne proteins the phenolic hydroxyl groups of tyrosine residues appear to be cross linked with other groups, perhaps by hydrogen bonding with ionized carboxyl groups of aspartic or glutamic acid residues. The simplest analogue for such hydrogen 
bonding is the salicylate ion, for which the $p \mathrm{~K}$ value of the hydroxyl group is displaced from its usual value of approximately 10 to 13.4 at $18^{\circ} \mathrm{C}$., obviously because of the strong hydrogen bonding to the $-\mathrm{COO}^{-}$group in the ortho position $(1,59)$. The $m$ - and $p$-hydroxybenzoic acids show no such effect (Table I). The behavior of ovalbumin, as described in the classical

TABLE I

$p \mathrm{~K}$ Values for Hydroxybenzold Acids and Their Ethyl Esters ${ }^{a}$

\begin{tabular}{lccc}
\hline & $p \mathrm{~K}_{1}^{\prime}\left(18^{\circ}\right)$ & $p \mathrm{~K}_{2}^{\prime}\left(18^{\circ}\right)$ & $p \mathrm{~K}^{\prime}$ ester $\left(25^{\circ}\right)$ \\
\hline Salicylic acid & 2.99 & 13.4 & 10.5 \\
$m$-Hydroxybenzoic acid & 4.06 & 9.99 & 9.1 \\
$p$-Hydroxybenzoic acid & 4.54 & 9.4 & 8.3 \\
\hline
\end{tabular}

a From Larsson, E., Z. anorg. Allgem. Chem., 183, 30 (1929).

Abichandani, C. T., and Jatkar, S. K. K., J. Indian Inst. Sci. A21, 417 (1938) report $p \mathrm{~K}_{2}^{\prime}$ for salicylic acid at $30^{\circ}$ as 12.4 ; the decrease in $p \mathrm{~K}_{2}^{\prime}$ with increase of temperature is in the expected direction, but the uncertainties in the data for such alkaline solutions are too great to warrant calculating a heat of ionization from these data.

study of Crammer and Neuberger (28), is in some respects closely akin to that of the salicylate ion. The ultraviolet absorption spectrum of ovalbumin shows that the phenolic hydroxyls remain unionized up to $p \mathrm{H}$ values of 12 or above, until a critical $p \mathrm{H}$ is reached at which ionization of all the groups occurs more or less simultaneously. The effect is irreversible; the groups remain ionized on returning to $p \mathrm{H}$ values between 11 and 12 , and the protein is found to be denatured. Obviously a system of internal hydrogen bonds has been ruptured. Although the other groups linked to the tyrosine hydroxyls have not been identified, it is reasonable to suspect that carboxyl groups from neighboring dicarboxylic amino acid side chains are involved:

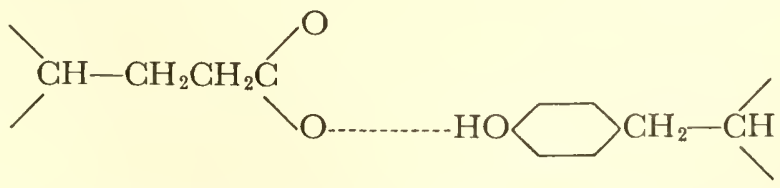

How much strength these bonds actually contribute to the native structure of the protein is still uncertain. It is quite 
possible that other bonds, as yet unidentified, are more important in maintaining the molecule in the native configuration, and that the breakage of the links involving the tyrosine hydroxyls may be a secondary result of the breakage of these other linkages. However, it seems likely that the bonds involving the tyrosine hydroxyls are important in maintaining the native structure. In any case we know that there are other proteins in which this is not true; Crammer and Neuberger found the tyrosine groups in insulin to react as if they were part of a free tyrosine peptide. Tanford and Roberts (103) have found an intermediate state of affairs in serum albumin; the tyrosine hydroxyls appear to be involved in some sort of hydrogen bonding, as judged from their free energies and entropies of ionization, but the breakage of the bonds is readily reversible on lowering the $p \mathrm{H}$, and involves no such drastic alteration of the native structure of the protein as in ovalbumin.

Laskowski and Scheraga (60) have set forth a detailed analysis of the formation and breakage of various types of hydrogen-bonded structures which may occur in proteins, and have pointed out that some of the observed peculiarities of protein titration curves might be readily explained in terms of such bonding. It is difficult as yet to obtain proof or disproof of the validity of their explanation, for any actual protein, but they have pointed out important new possibilities, which deserve careful examination. In any case, it appears that many side-chain ionic and polar groups in many proteins-perhaps most such groups in most proteins-are not tied down but are reactive like similar groups in smaller molecules (52).

The cohesion between nonpolar side chains is probably often important as a stabilizing factor. As Kauzmann (53) has pointed out, these side chains tend to hold together with considerable force. The energy required to separate them is decidedly greater than would be calculated from van der Waals attraction alone, for the separation of two nonpolar side chains involves bringing each of them in contact with additional water molecules, and therefore requires the breaking of some hydrogen 
bonds between water molecules. The stabilization of serum albumin against denaturation by heat (15), or by urea and guanidine hydrochloride (14), by the addition of fatty acid anions with long nonpolar side chains is probably associated with a stabilizing interaction of this sort-the fatty acid anions certainly bind to the albumin, and they probably tie down some potentially loose and unstable points in the albumin structure.

Further reflections on these general problems are contained in the thoughtful essay of Waugh (108). We now turn to a detailed consideration of spatial configurations.

\section{Spatial Configuration of Peptide Chains}

Along a peptide chain $\mathrm{NH}_{2} \mathrm{CH}\left(\mathrm{R}_{\mathrm{I}}\right) \mathrm{CO}$. $\mathrm{NH} \mathrm{CH}\left(\mathrm{R}_{2}\right) \mathrm{CO} . \mathrm{NH}$ $\mathrm{CH}(\mathrm{R}) \mathrm{CO}-.--\mathrm{NH} \mathrm{CH}\left(\mathrm{R}_{n}\right) \mathrm{COOH}$ there is a simple unit repeat, the peptide chain residue (NH.CH.CO.). We know that the ordered regions in fibrous proteins which give $\mathrm{x}$-ray diffraction patterns are regions of skeletal backbone chain order. They arise from regular intra- or inter-chain packing of identical neighboring chain residue units. Short-range or long-range order in the side-chain sequence $\mathrm{R}_{1} \mathrm{R}_{2}$---- $\mathrm{R}_{n}$ may sometimes occur also. In silk fibroin, for example, an ordered sequence appears very probable.

The Astbury classification of fibrous proteins into three groups is based on the three different types of wide-angle $\mathrm{x}$-ray diffraction patterns observed. These correspond to three distinct types of molecular peptide chain configurations and interchain packing. The outstanding features of the $\alpha$-keratin pattern given by proteins of the keratin-myosin-epiderminfibrinogen $(k-m-e-f)$ group are a meridional reflection of $5.1 \mathrm{~A}$ and an equatorial reflection at 9.8 A. X-ray diffraction patterns of fibrous proteins are commonly taken with the x-ray beam normal to the fiber axis. The meridional reflections correspond therefore to spacings along the fiber axis, and the equatorial spacings relate to spacings of planes parallel to the fiber axis. $\alpha$-Keratin is extensible along the fiber axis direction. In the 
stretched-out state keratin gives a $\beta$ pattern with a strong meridional reflection at $3.33 \mathrm{~A}$ and equatorial reflections at 9.7 and $4.65 \mathrm{~A}$. Collagen gives the third distinct pattern with a strong meridional reflection at $2.8 \mathrm{~A}$ and equatorial reflection at 11.6 and $4.6 \mathrm{~A}$. Collagen fibers show only limited shortrange $(\sim 15 \%)$ extensibility. No new diffraction pattern is obtained from the stretched fiber; the spacings of the principal reflections simply show a proportional increase (27). The axial spacing of $\beta$-keratin and of silk fibroin are similar. In silk fibroin Meyer and Mark (75) related the x-ray diffraction diagram to stretched-out polypeptide chains lying parallel to the fiber axis. Although their detailed model has been superseded, this general description characterizes the $\beta$-configuration. The $\alpha-\beta$ transformation thus corresponds (8) approximately to the twofold extension of chains folded or coiled along the fiber axis direction.

The early model structures for both the $\alpha$ and $\beta$ configurations were designed to satisfy the geometry of the x-ray diffraction patterns, the mechanism of $\alpha-\beta$ transformation, and the physical requirements of density and side-chain packing. Detailed and precise dimensions for the bond lengths and bond angles of the intrachain residue were not then available, and adequate chemical stabilization of the configuration was not considered in detail. During the years succeeding Astbury's classification, many investigators put forward new models for the $\alpha$ structure limited by additional chemical requirements, including the importance of hydrogen bonding between $\mathrm{CO}$ and $\mathrm{NH}$ of neighboring residues. These studies have been discussed in some detail by Low (66) and by Kendrew (55) in their recent accounts of work on protein structure and polypeptide chain configuration. It is stimulating to read some of the original papers by Astbury and his co-workers $(7,8)$. The prime physical requirements established by these investigators for the $\alpha$ and $\beta$ chain configurations are still valid.

In attempting to describe an appropriate structure for collagen Astbury and his co-workers suggested that the high 
proline and hydroxyproline content of this protein was responsible for its unique configuration. Proline residues cannot be written NH.CH(R)CO, since instead of a hydrogen atom there is a $=\mathrm{CH}_{2}$ group attached to the amino nitrogen, and a $\mathrm{NH}----\mathrm{O}$ hydrogen bond cannot therefore be formed. The proline (hydroxyproline) ring is only slightly puckered $(34,73)$, and this imposes stereochemical restrictions on the orientation of adjacent peptide chain residues. Models for the collagen structure are therefore dependent upon an assumed residue sequence; they are of the greatest interest at the present time, but we shall not attempt to discuss them further here.

Globular proteins appear most closely related in structure to the $\alpha$-type fibrous proteins. Fibers of denatured globular proteins may be oriented in the stretched-out $\beta$ configuration. In the globular proteins studied the three-dimensional structure is complex and the identification of the dominant peptide chain configuration is only a part, though an important one, of the whole problem. We shall discuss the recent advances in the study of peptide chain configurations and their significance in the $\alpha$ and $\beta$ proteins. This will serve as an introduction to the globular protein problem.

In 1951 Pauling, Corey, and Branson specified the most rigid and coherent set of chemical limitations on plausible polypeptide chain configurations (82). First, on the basis of the results from detailed crystal structure studies of amides, amino acids, and some simple peptides, they defined precise dimensions for the peptide chain residue. These are shown in Figure 3. The amide group must be planar; there is nearly $50 \%$ double bond character in the $\mathrm{C}^{\prime}-\mathrm{N}$ bond, which is consequently shorter $(1.32 \mathrm{~A})$ than the normal single bond length of $1.47 \mathrm{~A}$. The planar requirement gives two possible configurations, either trans as in Figure 3 or cis, as shown here.

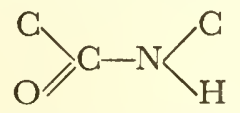

In the polypeptide chain configurations discussed below, which 
appear to satisfy the $\alpha$ and $\beta$ diffraction patterns, trans amide groups are used exclusively. The second Pauling and Corey requirement was that the identical chain residues should all be

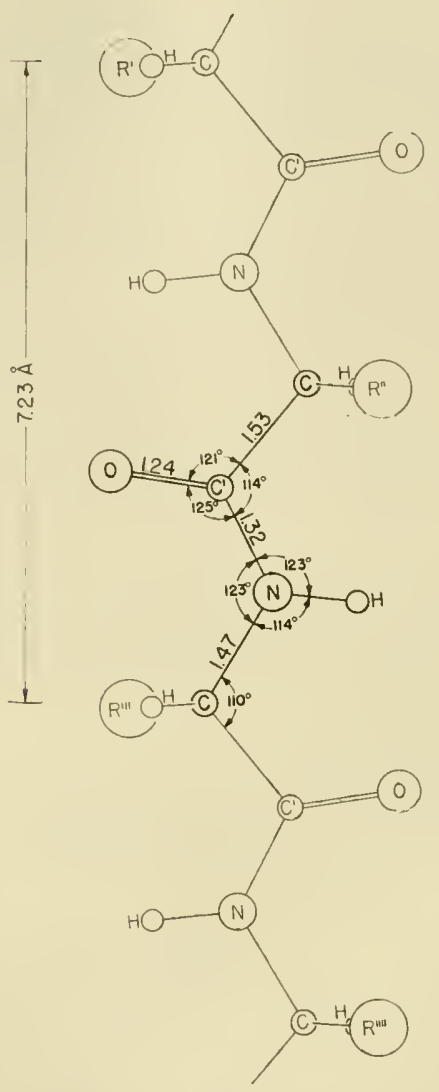

Fig. 3. Fully extended trans polypeptide chain configuration with chain residue bond lengths and bond angles. R. B. Corey and L. Pauling, Proc. Roy. Soc. (London), B141, 10 (1953).

structurally equivalent and should make the maximum number of strong intra- or inter-chain hydrogen bonds. When identical chain residues are arranged so that one common operation serves to translate the first residue position into the second, and 
the $n$th into the $(n+1)$ th, the resulting structure is a helix. The residues in a helical structure all have identical intra chain environments. Once the limiting conditions for strong hydrogen bonding are described for one residue they are defined for all. In the $\beta$ structure, where inter-chain hydrogen bonds are used to provide configurational stability, simple symmetry operations may relate one residue to the next succeeding one along the same chain, or they may relate one residue to another in a neighboring chain. Corey and Pauling (26) have defined a strong hydrogen bond as one in which the O...H-N distance is approximately $2.79 \pm 0.12 \mathrm{~A}$ and where the vector from the nitrogen atom to the hydrogenbonded oxygen atom lies not more than $30^{\circ}$ from the $\mathrm{NH}$ direction.

They also considered the favorable orientation of groups about the HN-CHR and HRC-CO bonds which should lead to maximum bond stability (78). Van der Waals forces between nonbonded atoms also affect the over-all stability of a configuration. The importance of van der Waals forces and the semiquantitative estimates of the instability resulting from deviations from the initial criteria set up by Pauling and Corey will be discussed later.

One helical structure-the $\alpha$-helix-completely satisfies all the chemical criteria for configurational stability (79). It also fits the physical requirements for an appropriate configuration. The structure is shown in Figure 4. Each succeeding residue $\mathrm{C}_{1}(\mathrm{CO} . \mathrm{NH}) \mathrm{C}_{2}, \mathrm{C}_{2}(\mathrm{CO} . \mathrm{NH}) \mathrm{C}_{3}$, etc., lies in one of a zone of vertical planes parallel to the helical axis. Figure 5, which shows a similar helix structure, perhaps demonstrates this relationship more clearly. The plane which contains the residue $\mathrm{C}_{1}(\mathrm{CO} . \mathrm{NH}) \mathrm{C}_{2}$ is parallel to the helical axis (vertical). It projects as a line on the plane normal to the helical axis. The planes containing the $\mathrm{C}_{2}-\mathrm{C}_{3}, \mathrm{C}_{3}-\mathrm{C}_{4}, \mathrm{C}_{4}-\mathrm{C}_{5}$, etc., residues, are all parallel to the helical axis. All intersect the plane normal to the helical axis as shown in Figure 5. These are some of the planes in this zone. When the helix repeats, the plane of the 
residue $\mathrm{C}_{n}-\mathrm{C}_{n+1}$ will coincide with the plane of the residue 1-2. The angle between two successive planes is the helical angle $360 / n=\psi$, where $n$ is the number of residues per complete turn.
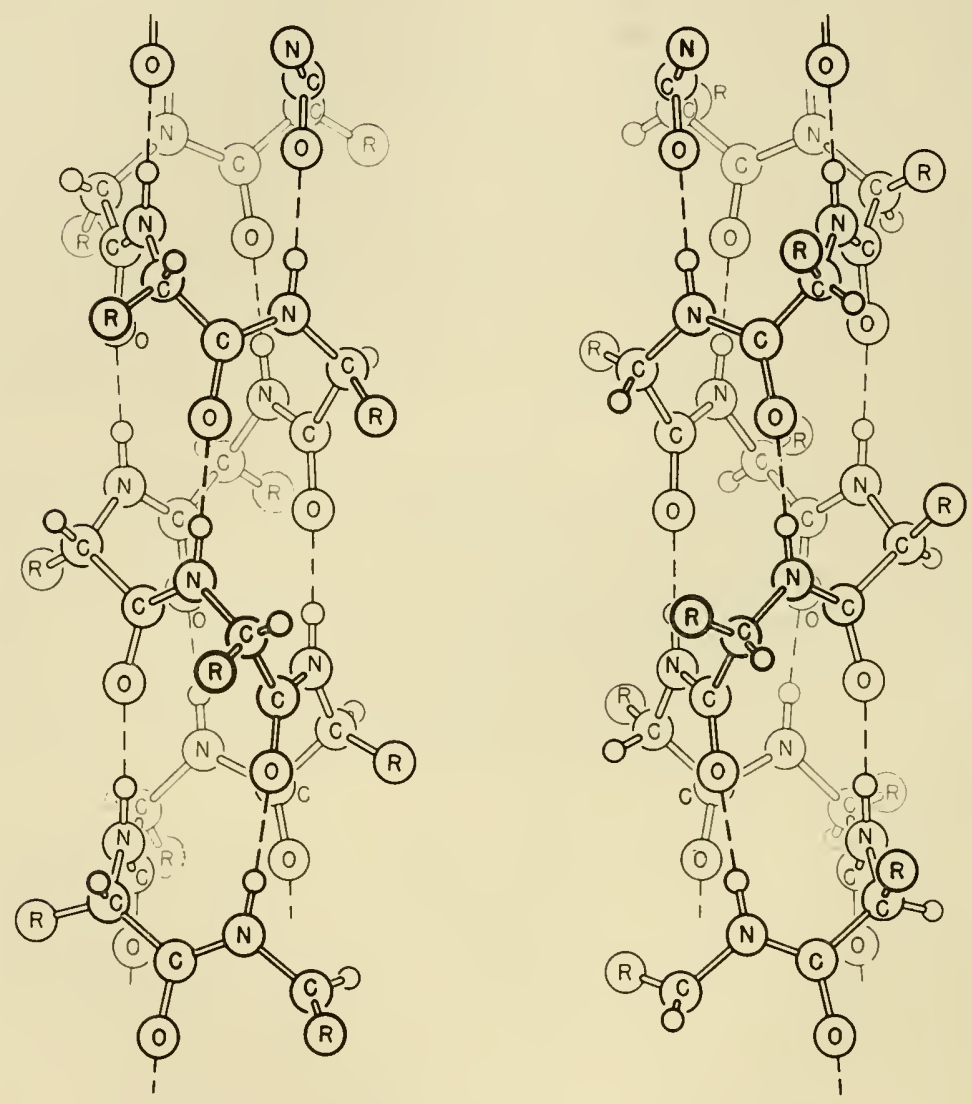

Fig. 4. Drawings of the left-handed and right-handed $\alpha$-helices. The $\mathrm{R}$ and $\mathrm{H}$ groups on the $\alpha$-carbon atom are in the correct position corresponding to the known configuration of the L-amino acids in proteins. L. Pauling and R. B. Corey, unpublished drawings.

Each residue is tipped down (in the plane) from the horizontal position for $\mathrm{C}_{1}-\mathrm{C}_{2}$ through a vertical distance $P / n$, where $P$ is the pitch of the final coil. The resultant of the horizontal (180 - 
$\psi$ ) and vertical components of the angle $\mathrm{N}_{1} \mathrm{C}_{2} \mathrm{C}_{12}^{1}$ is equal to $110^{\circ}$, the tetrahedral carbon angle.

Tilting the residues in this way gives $\mathrm{CO}$ and $\mathrm{NH}$ bond directions closely parallel to the helical axis. Upon detailed examination it is seen that the $\alpha$-helix corresponds to the chemical sequence

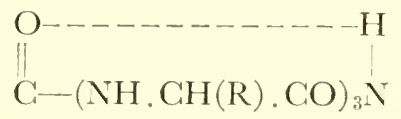

read in either direction, with 13 atoms in the hydrogen-bonded loop so formed and with 3.6 residues per unit turn. It may thus be designated 3.6 13 . Minor changes in the wrap-up angle and pitch will change the number of resiclues in the helix from 3.6 to 3.7 without affecting its over-all agrecment with the detailed chemical criteria set out above. Thus if $n=3.7, P=5.4 \mathrm{~A}, P / n$ $=1.47 \mathrm{~A}$. In the $\alpha$-helix with 3.60 residues per turn and a vertical increment per unit residue $P^{\prime} n=1.47, P=5.3$. The tetrahedral $\alpha$-carbon bond angle is $108.0^{\circ}$. This helix has 18 resiclues in 5 turns. Hence the plane of the $\mathrm{C}_{1}-\mathrm{C}_{22}$ resiclue is also the plane of the $\mathrm{C}_{18}-\mathrm{C}_{19}$.

A helix structure described by Low and Baybutt $(69,70)$ is shown in Figure 5. This corresponds to the sequence

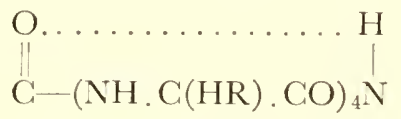

with 16 atoms in the hydrogen-bonded loop so formed. It thus belongs to the " $\alpha$ " series if this be characterized as a sequence

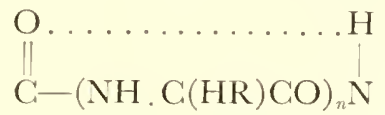

with $(3 n+4)$ atoms in the hydrogen-bonded loop.

Comparison of Figure 4 and Figure 5 shows that the two helices are closely related as adjacent members of the " $\alpha$ " series. In the $\alpha$-helix each amide group forms hydrogen bonds with the third amide group beyond it in either direction along the chain. 
Nitrogen

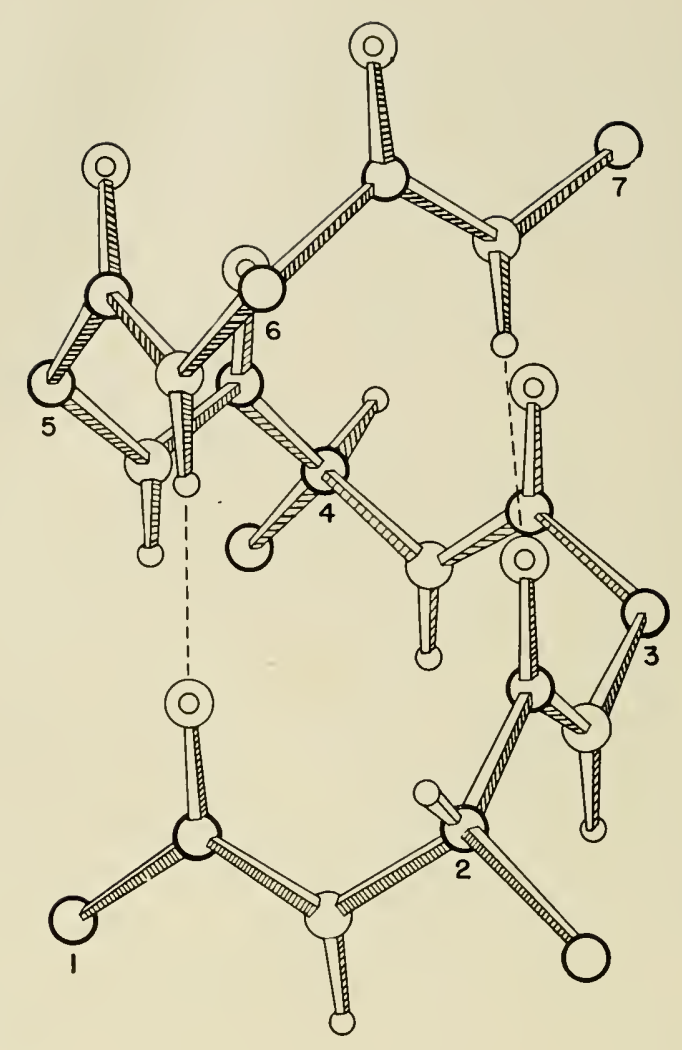

CARBON

(O) OXYGEN

O HYDROGEN

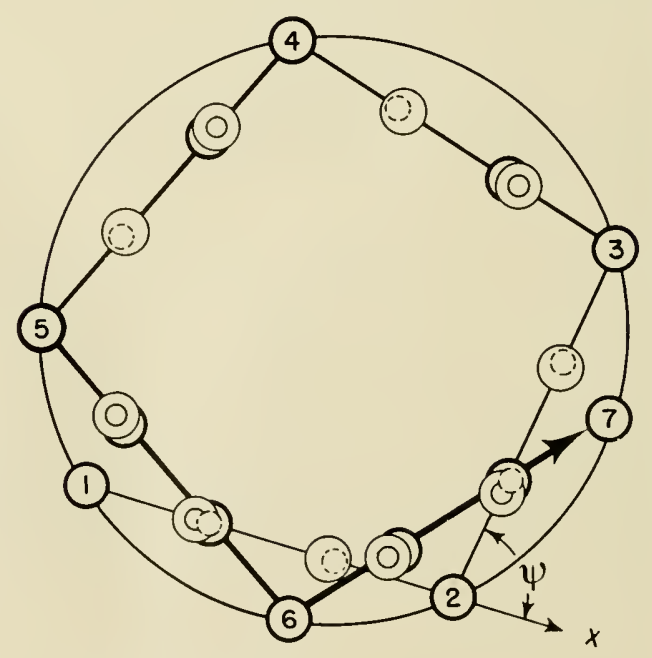

(See opposite page for caption) 
In the $\pi$-helix (Figure 5) the hydrogen bonds are between one amide group and the fourth amide group beyond. The initial vertical tilt is less in the $\pi$-helix, $P / n=1.14 \mathrm{~A}$, and the horizontal component of the $\mathrm{CC}_{2} \mathrm{~N}$ angle is consequently larger than in the $\alpha$-helix.

The $\pi$-helix satisfies the Pauling-Corey criteria if these are modified to permit a $4^{\circ}$ angular distortion in the tetrahedral $\mathrm{CC}_{2.2} \mathrm{~N}$ ( $\alpha$-carbon) angle. Deviations of this magnitude have been observed elsewhere for instance in threonine (100) - and lead to only a slight recluction in relative stability per mole per residuc.

The $\alpha$-helix is wrapped up more tightly than the $\pi$-helix. It is possible to go from one configuration to the other with only limited loss of local hydrogen bond stabilization. The $\alpha$-helix has greater over-all configurational stability than the $\pi$-helix and than all other members of the " $\alpha$ " series. It is the most stable coiled peptide chain structure known.

There is another family of helical configurations to which the Pauling-Corey $\gamma$-helix belongs. This " $\gamma$ " series may be represented by the formula

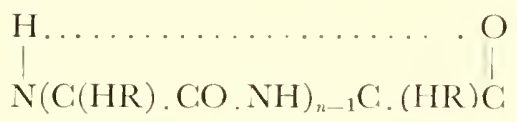

with $3(n-1)+5$ atoms in the hydrogen-bonded loop. For the $\gamma$-helix itself $n=5$. The two series of helices are quite distinct. In the " $\alpha$ " series, as Figure 5 shows, the $\mathrm{C}_{1}-\mathrm{C}_{2}$ line is inclined upwards in such a way that the $\mathrm{CO}$ and $\mathrm{NH}$ bond directions are nearly vertical and parallel to the helical axis. If the $\mathrm{C}_{1}-\mathrm{C}_{2}$ direction is horizontal, the $\mathrm{CO}$ and $\mathrm{NH}$ bonds are

Fig. 5. (a) Perspective drawing of the right-handed $\pi_{R}$-helix. The link-end carbon atoms are numbered consecutively. The $\beta$-carbon and hydrogen atom positions of L-amino acid residues are shown attached to $\mathrm{C}_{2}(\alpha)$ and $\mathrm{C}_{4}(\alpha)$. (b) Plan view of $\pi_{R}$-helix. The hydrogen positions are shown with broken lines. Nitrogen positions are omitted at the two crossover points. B. W. Low and H. J. Grenville-Wells, Proc. Natl. Acad. Sci. U. S., 39, 785 (1953). 
inclined to the vertical and when the resiclue is tilted in the opposite direction (downwards), this angle is increased. Helical chains of the " $\alpha$ " and the " $\gamma$ " series are not interconvertible without unwrapping, nor can a region of one succeed a region of the other along the same axis. Although the $\gamma$-helix satisfies the initial Pauling-Corey criteria, excluding the $\alpha$-carbon single bond orientation effects, it is less stable than the $\alpha$, and some other " $\alpha$ " series, configurations. It appears unlikely that the $\gamma$ structure or any of the other, even less stable, members of this general series are present in protein structure.

The helices (Figures 4 and 5) are shown with $\beta$-carbon atoms in the correct configuration for L-amino acid residues. Bijvoet, Peerdeman, and van Bommel (13) have established the absolute configuration of $\mathrm{D}(+)$-tartaric acid and demonstrated the correctness of the Fischer convention. The $\pi$-helix shown has a right-handed screw $\pi_{R}$. The mirror image of a righthanded screw is a left-handed screw; the mirror image of an $\mathrm{L}$-amino acid residue is a $\mathrm{D}$-amino acid residue. The mirror image of a left-handed helix of L-amino acid residues, is thus a right-handed helix of $\mathrm{D}$-amino acid residues. These are true enantiomorphs. A left-handed helix of L-amino acid residues is different from a right-handed helix of $\mathrm{L}$-amino acid residues. In the latter (Figures 4 and 5) the $\alpha \mathrm{C}-\beta \mathrm{C}$ bond has a vertical component in the same direction as the vertical component of the $\mathrm{NH}$ bond, whereas in the former the two are opposed. There are thus two alternative helical chain configurations possible with L-residues. These two helices are sometimes described in terms of the two possible $\beta$-carbon atom positions. This terminology depends on the enantiomorphous relationship discussed above. X-ray diffraction effects cannot normally distinguish between mirror image structures. A left-handed helix of D-amino acid residues is thus the equivalent of a righthanded helix of L-amino acid residues.

There are three extended $(\beta)$ polypeptide chain structures which satisfy the initial Pauling-Corey chemical criteria and which give the favored stable orientation about the single bonds 
to the $\alpha$-carbon atom (78). In the fully extended planar polypeptide chain configuration (Figure 3) the predicted repeat distance is $7.23 \mathrm{~A}$. When two such chains are packed side by side to give interchain hydrogen bonds there is steric hindrance between opposing side chains unless these are (1) both glycyl residues $(-\mathrm{H})$ or $(2)$ one glycyl and the other alanyl $\left(-\mathrm{CH}_{3}\right)$ or seryl $\left(-\mathrm{CH}_{2} \mathrm{OH}\right)$. Both the other two structures form lateral interchain hydrogen bonds, even if the side chains are bulky. In one arrangement, the parallel pleated sheet structure, all the chains have the same direction. In the antiparallel pleated sheet structure, alternate chains are opposed in direction. The predicted repeat distances along the chain direction in both structures are somewhat shorter than the $7.23 \mathrm{~A}$ of the fully extended structure. The general pattern of the pleated sheet structure is susceptible of some modifications in details of arrangement. In their earlier calculations (78) Pauling and Corey assumed that certain preferred orientations around single bonds in the chains would give the most stable configurations. Later (80) they concluded that this factor was of secondary importance and that configurations would be favored which gave approximately linear hydrogen bonds-that is, bonds in which the $\mathrm{N}-\mathrm{H} \ldots \mathrm{O}$ angle is $180^{\circ}$ or nearly so. The models constructed on this assumption gave a fiber axis period of 7.00 A for the antiparallel, and $6.50 \mathrm{~A}$ for the parallel, chain pleated sheet. The structure of silk fibroin has been studied in great detail by Marsh, Corey, and Pauling (72); the x-ray patterns obtained were in good agreement with expectation for an antiparallel chain pleated sheet structure, in which the predominant chain sequence is Gly.X.Gly.X.Gly, where $\mathrm{X}$ is alanyl or seryl. The structure may be modificd so as to include the larger side chains, such as tyrosyl, in the crystalline component of structure, instead of placing them in amorphous regions as did most earlier investigators who considered this problem.

In the $\beta$-keratin structure the parallel pleated sheet arrangement appears the more reasonable model. The hydrogenbonded sheets in both silk fibroin and $\beta$-keratin must be held 
together by interactions between side-chain groups, which may be polar or nonpolar. The $\beta$ structure is essentially made up of extended peptide chains in parallel array. The two pleated sheet structures provide nicely for the stereochemical requirements of this mode of packing, and one or other fits the observed $\mathrm{x}$-ray diffraction patterns of most of the $\beta$ proteins.

In the $\alpha$-helix structure the side chains bristle out from the chain core. The structure may be thought of as a cylinder of circular cross section. When a group of these structures are arranged in parallel close-packed array an interhelical distance of $\mathrm{X}$ angstroms would give rise to an interplanar spacing of $\mathrm{X} \sqrt{3 / 2 \mathrm{~A}}$. The pitch of the $\alpha$-helix is $5.4 \mathrm{~A}$, and the structure should give rise to a nonmeridional reflection of this spacing. Further such parallel arrays of $\alpha$-helices should give a strong meridional x-ray reflection corresponding to diffraction from planes normal to the helical axis and spaced a unit residue increment apart: $5.4 / 3.6=1.5 \mathrm{~A}$.

A reflection of $1.5 \mathrm{~A}$ was noted in an $\alpha$-keratin pattern from porcupine quill by MacArthur (71) in 1943, but no especial significance was then attached to it. When the $\alpha$-helix structure was first proposed, Perutz (86) recognized that the reflection should show up prominently in $\alpha$-helix structures if the fiber were tilted at the appropriate Bragg angle to the incident $x$-ray beam. He observed it in synthetic polypeptides, in $\alpha$-keratin, and in wet crystals of the globular protein hemoglobin.

The synthetic polypeptides do show the expected nonmeridional $5.4 \mathrm{~A}$ reflection. In fact the synthetic polypeptide poly- $\boldsymbol{\gamma}$-methyl-L-glutamate gives an x-ray diffraction pattern which fits excellently the calculated pattern for an $\alpha$-helix $(9,24,110)$.

In the fibrous protein $\alpha$ pattern there are meridional reflections at $5.1 \mathrm{~A}$ and $1.5 \mathrm{~A}$. The discrepancy between a predicted 5.4 A nonmeridional spacing and the 5.1 A meridional spacing observed cannot be resolved simply by supposing that the $\alpha$-helices are inclined to the fiber axis, for this would put the 1.5 A spacing off the meridian. Crick (29) has shown that if 
the $\alpha$-helix is distorted into a coiled coil with a pitch angle of about $20^{\circ}$, then theoretically such a coiled coil would give diffuse meridional 5.1 and $1.5 \mathrm{~A} x$-ray reflections. Pauling and Corey have suggested a similar scheme shown in Figure 6 (81). The packing together of such coiled coils would give ropelike structures with each $\alpha$-helix a single strand in the final cable. Pauling

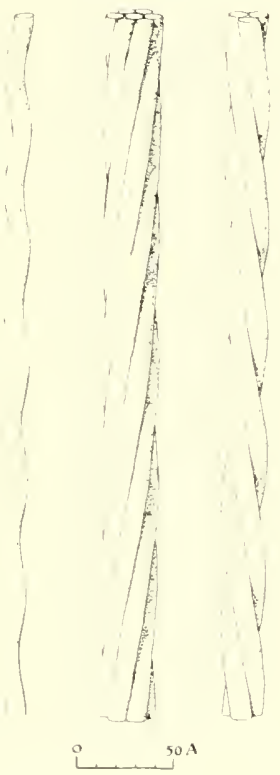

Fig. 6. (a) A representation of a compound helical configuration of polypeptide chains, with the axis of the $\alpha$-helix describing a helical course. (b) A compound $\alpha$-helix (at the left), a 7 -strand $\alpha$ cable (in the center), and a 3 -strand $\alpha$ rope (at the right). L. Pauling and R. B. Corey, Nature, 171, 59 (1953).

(77) considers that local variations in hydrogen bond length along an $\alpha$-helix may be caused by side-chain interactions or interactions between side chains and the carboxyl and amino groups of the chain amide residue. Such minor discontinuities would cause the helical axis to curve. Crick (31) on the basis of model studies has shown that regular systematic "coiled-coil" distortions of the helices would permit the side chain of one helix 
to dovetail into the space between neighboring residues on another helix. Whether the formation of a coiled coil is attributed to such systematic steric effects or to more general polar and nonpolar side chain residue interactions, the coiled coil proposed has the advantage of explaining the observed diffraction effects.

The density of an $\alpha$-helix array is, of course, clependent upon the interaxial distance chosen. An interaxial distance of approximately $10.6 \mathrm{~A}$ would give a reasonable density of about 1.30. Such packing would give rise to a 9.2 A equatorial spacing. Although $9.8 \mathrm{~A}$ is usually quoted as the $\alpha$-pattern spacing, it has been shown in well-resolved photographs that this broad maximum is split into two reflections - with one at $9.2 \mathrm{~A}$ as required.

In $\alpha$-keratin the chains are held together by cystine disulfide linkages. The $\alpha-\beta$ transformation in the $k-m-\ell-f$ group of fibrous proteins is usually considered to take place without $-\mathrm{S}-\mathrm{S}-$ link breakdown. If the interchain cystine linkages are disposed at random the $\alpha$ - $\beta$ transformation could not take place without breaking the disulfide linkages (6). Indeed simple steric hindrance effects of neighboring side chains would appear to make the transformation extremely difficult, even if the disulfide linkages are located in positions favorable to forming the stretched-out $(\beta)$ configuration. This difficulty can be avoided by consigning the $-\mathrm{S}-\mathrm{S}$ linkages to the nonoriented regions of the peptide chain. Polarized infrared radiation studies (2) of keratin show rather low dichroism. They suggest that keratin must contain considerable regions of unknown structure as well as regions where the $\alpha$-hclix structure predominates.

The evidence in support of the $\alpha$-helix and $\beta$ pleated sheet structures as dominant features of the $\alpha$ and $\beta$ fibrous protein structure is in general diverse and formidable. Existence of the $\alpha$-helix structure has been clearly demonstrated in some synthetic polypeptides.

We may summarize the conclusions in rather different terms. The synthetic polypeptides examined so far have been 
monofunctional chains with identical nonpolar $\mathrm{R}_{1}, \mathrm{R}_{2}, \ldots \mathrm{R}_{n}$ residues. In a chain of this kind where side-chain interactions are unlikely to be very strong, we might expect the chain to adopt the most stable intrachain configurations. Interactions between identical nonpolar side chains are not likely to be a major factor in providing configurational stability. In the $\alpha$-keratin structure the situation is more complex. Because of the different side-chain groups, the chain residues can no longer be strictly equivalent. It has been necessary to invoke side-chain interactions to account for distortion of the helix and the formation of coiled coils. Some investigators have also considered it necessary to consign the disulfide linkages to amorphous (noncrystalline) regions along the fiber. Regions of ordered "crystalline" intra- and inter-chain packing appear to be separated by regions in which a regular chain configuration is absent.

When globular proteins are denatured by reagents and under conditions which should not lead to the breaking of covalent intrachain bonds they can be drawn out into fibers with the $\beta$ configuration (66). This observation provides evidence for latent polypepticle chains within the native molecule. It also relates the native state of the globular molecule to a specific molecular configuration the loss of which is associated with loss of biological specificity. A specific configuration is not necessarily one which contains multiple repeats of some small unit of packing. Any three-dimensional array which is uniquely defined is specific. In globular proteins there is evidence that the molecular configuration is an ordered or quasi-ordered array of unit pattern repeats. This evidence comes from two different sources. First there are fibrous proteins $(\alpha)$ which can exist in the globular form. We may perhaps infer that there is a single common mode of protein packing which consists of parallel or quasi-parallel regions of peptide chain in an ordered chain configuration. Studies of the rector structures (Patterson diagrams) of some proteins substantiate this inference. A grossly oversimplified picture of a globular protein would 
represent it as a close-packed array of cylindrical rodlike regions of high electron density, in each of which a length of peptide chain is coiled or folded into a regular chain configuration.

References to individual experimental studies would not in most cases provide the logical basis for the general conclusions outlined here. The reader is referred to the critical accounts of Low (66) and Kendrew (55) mentioned earlier and to other general reviews by Kendrew (56) and Kendrew and Perutz (57). There he will find both extensive and documented exposition of these and other conclusions which have been treated rather sketchily here.

A most striking piece of evidence for the existence of regions of regular chain configurations in hemoglobin is, of course, the 1.5 A spacing observed by Perutz. Polarized infrared radiation studies of some crystalline proteins $(40,43)$ confirm the hypothesis of oriented regions of ordered chain configuration.

The x-ray scattering distribution from a large number of globular proteins, studied as amorphous powders, can be compared with the interference intensity function calculated from the parameters of any well-defined chain configuration. Arndt and Riley $(5,90,91)$ have recently developed this method extensively: they have included $\beta$-carbon atom positions in the calculated functions. Their studies show that the left-handed $\left(\alpha_{1}\right)$ helix is the most plausible predominant chain configuration in globular proteins, and that the helical regions are associated together in some sort of relatively compact array. Both these conclusions are qualitative. To quote their conclusions, "It is, of course, possible that alternative configurations exist over limited segments of the chain or as separate chains in small amounts... The closely related $\alpha_{2}$-helix configuration is a special case; this could occur as an important proportion of the whole molecule, although it is unlikely to be the major constituent" (5, p.437) and elsewhere they comment that "on the basis of the protein data alone, it is impossible to assert that the $\pi_{1}, \pi_{2}$, and $4_{13}\left(\mathrm{C}_{\beta 2}\right)$ helices are markedly inconsistent with observation." (The $4_{13}$ helix (17) is for other reasons highly improbable.) "Equally, the admixture of a certain proportion of $\alpha_{2}$ would not be an impossibility, although a $100 \% \alpha_{2}$ structure 
would seem to be highly improbable" (5, p.430). The identification of an interhelical packing peak does not define rigorous interhelical parallelism. The experimental scattering curves simply indicate that there are certainly considerable regions of interchain order in the molecule.

X-ray crystal structure studies of hemoglobin have led to this same general conclusion. The vector structure for hemoglobin suggests a molecular structure which may be represented

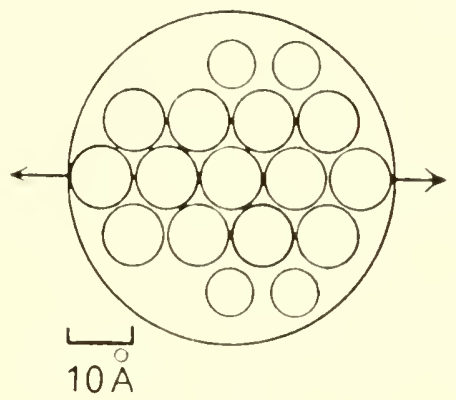

(a)

Fig. 7. Horse methemoglobin. Idealized representation of molecule in a plane projection. The circles correspond to the end-on view of rodlike regions of high electron density in elose-packed array. W. L. Bragg, E. R. Howells, and M. F. Perutz, Acta Cryst., 5, 136 (1952).

in cross section as shown in Figure 7. In this idealized model the circles represent the end-on projection of peptide chains stacked in close-packed array with each chain coiled in some regular configuration. The molecular length parallel to the $a$ axis and normal to this section is approximately $62 \mathrm{~A}$. The molecule is bisccted by a twofold symmetry axis normal to a. An individual single coiled chain could not therefore be longer than $a / 2$, that is $31 \mathrm{~A}$. In considering agreement between this model and the experimental data both Bragg, Howells, and Perutz (16) and Crick (30) have shown that the model is oversimplified. 
To fit the experimental data some deviations are necessary from this simple structurc - that is, chains 30 to $25 \mathrm{~A}$ in length with an $\alpha$-helix configuration cannot all be stacked parallel; irregularities must occur. Several different kinds of irregularities can be postulated: (1) short lengths of chain in an $\alpha$-helix configuration $(\sim 15 \mathrm{~A})$ may be imperfectly aligned so that as a group the coiled chains zigzag about the over-all axial direction; (2) short lengths of $\alpha$-helix may be followed by regions in which the specific local molecular configuration is not associated with any regular peptide chain configuration.

These conclusions underline a further difference between the $\alpha$ fibrous protein model and the globular protein model. In globular proteins the stable discrete unit is a single molecule; here, the third level of molecular organization described earlier is intramolecular. Ordered and disordered regions of chain configurations are confined within and establish the unique three-dimensional molecular structure. Globular proteins are almost always shorter than the model structure which would be derived if peptide chains of average length (calculated from end group determinations) were each coiled into a single cylindrical $\alpha$-helix structure and then stacked together in closepacked array. The peptide chains must therefore turn corners and reverse direction. In horse hemoglobin there are only 6 $N$-terminal end groups per molecule, that is, three per (globin) half-molecule. Even in the simple idealized structure (Figure 7) there are 17 "crystallographic" ends, that is, seventeen closepacked cylindrical regions of coiled configuration. The absolute intensity studies demand even more numerous discontinuities. We referred earlier to the stereochemical discontinuity which a proline residue introduces into regular helical chain configurations which have the maximum number of strong and equivalent hydrogen bonds. In the $\alpha$-helix proline residues cause different problems in left- and right-handed structures. This difference may be understood by considering the closely similar $\pi$-helix. In the drawing (Figure 5), of a right-handed helix a reasonably planar proline ring can be constructed by joining the nitrogen 
atom of the $\mathrm{C}_{1}-\mathrm{C}_{2}$ residue to the $\alpha \mathrm{C}_{2}$, and closing the ring by forming $\alpha \mathrm{C}_{2}-\beta \mathrm{C}_{12} \quad \mathrm{C}_{2} \mathrm{H}_{2}-\mathrm{CH}_{2}-\mathrm{N}$ bonds.

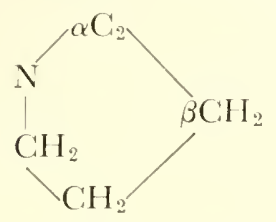

This would eliminate one hydrogen bond and lengthen anotherit would not, however, completely disrupt the helical configuration.

On the other hand, if the $\beta$-carbon were in the alternative position in Figure 5, equivalent to a left-handed helix, the proline ring could not be closed. The presence of a proline residue would completely disrupt the helical sequence in this latter configuration. Proline residues thus provide a possible stereochemical reason for discontinuity in left-handed helix structures. The photographs of scale models (Figure 8) illustrate the two different stereochemical effects of an $\mathrm{L}$-proline residue in left-handed and right-handed $\alpha$-helix structures. In hemoglobin it is suggestive that the number of proline residues per globin (half-molecule) is eleven. Proline residues and the three end groups are together almost adequate in number to account for the number (17) of crystallographic chains pictured in the model if the proline residues do occupy the required positions. Proline residues in left-handed helix structures would certainly lead to a region of irregular chain configurations and could initiate corner turning. However, the partial sequences which have been established for ribonuclease (discussed later) place two of the four proline residues in a short terminal sequence of 15 residues. There are 124 residues in the chain (48) and the turns must occur at fairly regular intervals along the chain to give a relatively compact molecular shape, such as is required by the physical properties of the molccule. This suggests that residues other than proline must sometimes be responsible for initiating a reversal of chain direction. An economical and 
direct stereochemical mechanism for turning the helical axis through $180^{\circ}$ in a left-handed helix has been suggested by

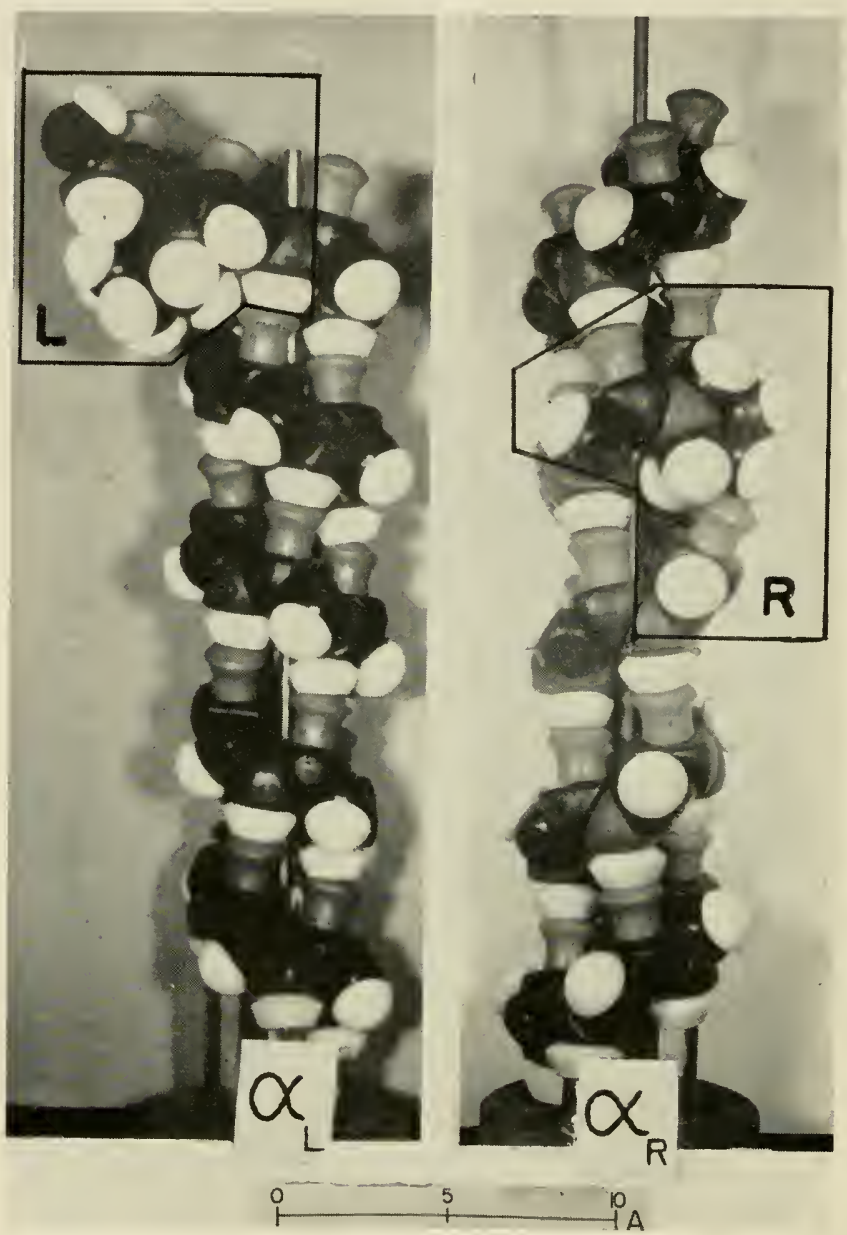

Fig. 8a. Photograph of scale models of left-handed and righthanded $\alpha$-helices. One proline residue has been introduced into each chain configuration. In the left-handed helix the regular chain configuration is completely disrupted, as shown. In the right-handed helix some minor discontinuities are introduced with loss of hydrogen bonding. (See opposite page for exploded drawings of the regions L and R.) 
Lindley (61). This does not involve proline residues. It is dependent upon certain specific residuc sequences and the formation of main-chain-side-chain hydrogen bonds.
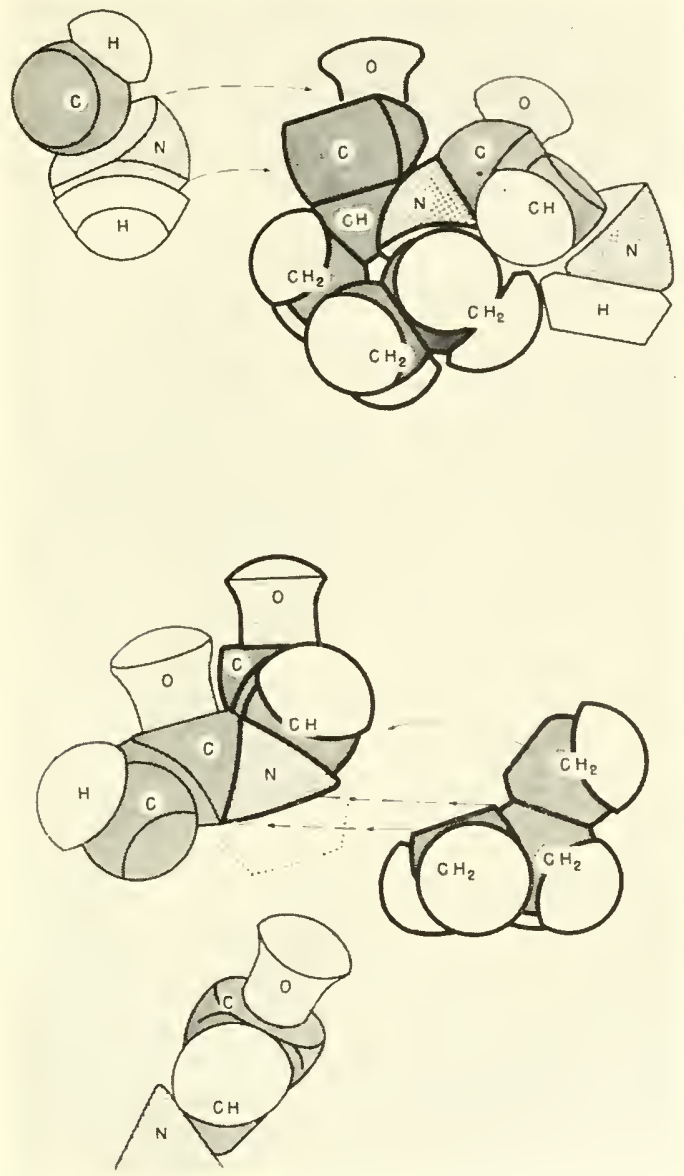

Fig. 8b. Exploded drawings of the regions $L$ and $R$ opposite. In the drawings atoms of the proline residues are in heavy outline. (1) Above: region L. (2) Below: region R. The dotted outline in this drawing represents the hydrogen atom which would be present-and hydrogen-bonded to the oxygen beneath-if the residue were not proline. 
The $\alpha$-helix has been shown to be the dominant feature of the configuration of long chain synthetic polypeptides with identical nonpolar side chains. Similarly, once the coiled coil modification is introduced, the $\alpha$-helix structure satisfactorily describes the major features of the ordered regions in the $\alpha$ fibrous proteins. In the globular proteins, however, the $\alpha$-helix model needs more extensive revision and modification. In this group of proteins a folded or coiled peptide chain is further folded back on itself making many complete $\left(180^{\circ}\right)$ turns. A single molecule appears either to have considerable regions of disordered chain or to be made up of rather short and poorly aligned lengths of coiled chain.

Whatever the mechanism which controls protein synthesis, the stability of a single molecule must depend upon the overall intramolecular three-dimensional packing. Apart from the intrachain amide group interactions (hydrogen bonds) of a coiled chain, only three other types of intramolecular interactions are possible: (1) Interchain amide hydrogen bonds. Ordered regions with regular interchain hydrogen bonds are regions of $\beta$ configuration. There is no evidence for this type of bonding in globular proteins. (2) Side-chain-side-chain interactions. Both inter- and intrachain covalent (e.g., cystine $-\mathrm{S}-\mathrm{S}-$ ) linkages are known to exist. Electrostatic interaction will depend upon the distribution of polar groups. Interaction between adjacent nonpolar side chains may also be expected. These interactions must be responsible for the associations between peptide chains which hold the molecule in its unique compact configuration. (3) Side-chain-main-chain interactions are theoretically possible; for example hydrogen bonds may be formed between polar and hydroxyl-containing side-chain and peptide-chain amide $\mathrm{CO}$. NH groups.

In brief, the kinds of intramolecular bonds possible will be dependent on the precise sequences of amino acid residues along the chains and the details of the interchain arrangement. This puts the cart before the horse; rather we should say that the specific molecular configuration will be determined by the amino 
acid sequences and consequent interchain packing. Since major chain discontinuities do certainly exist in globular proteins, we must conclude that some amino acid sequences inhibit the formation of regular backbone chain configurations.

Protein molecules have specific functions and present-day thinking demands that structure and function be related in a highly specific manner. In fibrous proteins, for example, hair, horn, and muscle, the main function appears to be architectural support or some specialized mechanical flexibility. The jobs which globular proteins are built to do are not associated with "mechanical" function of this kind. Reactive sites or groups on a globular protein molecule are reactants in certain specific chemical reactions: usually when the protein is in aqueous solution. These sites must be accessible; they probably have a complex, highly specific three-dimensional structure. Sidechain group interactions may have a marked effect on the stability of helical configurations as compared with a more randomly coiled configuration. We might expect that in some regions of strong side-chain interactions the $\alpha$-helix would be unstable. The presence of both helical and disordered chain configurations in a globular protein molecule may be an important structural feature. It may provide both structural flexibility and specific interatomic arrangements which cannot be achieved with a continuous regular chain configuration.

Helical configurations of peptide chains may be stable or unstable, as compared with the more randomly coiled configurations which are possible when the $-\mathrm{C}=\mathrm{O} \cdots \mathrm{H}-\mathrm{N}-$ hydrogen bonds are broken and the regular helix structure is unfolded. The free energy of unfolding is of course the determining factor; if this is negative the helix, if it is formed at all, will be unstable. A very important critical examination of the whole problem has recently been given by Schellman (98) in terms of the $\alpha$-helix configuration. Heat is absorbed when a $-\mathrm{CO} \cdots \mathrm{NH}$ - bond is broken. In a medium of low dielectric constant, incapable of hydrogen bond formation with the amide 
$\mathrm{CO} \cdot \mathrm{NH}$ groups in the peptide chain, the reaction involved may be represented as

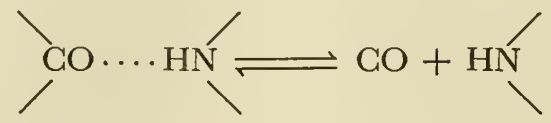

and $\Delta H$, the heat absorbed, is of the order of 7 or $8 \mathrm{kcal}$. per mole of such bonds broken (82). In water, on the other hand, the reaction may be written, following Kauzmann (53)

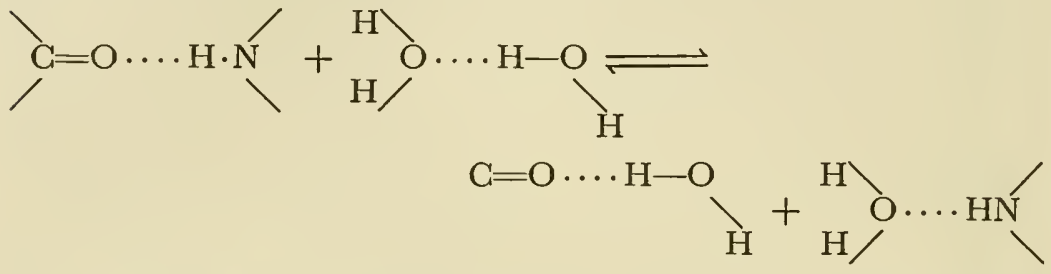

The net value of $\Delta H$ for this reaction, which may be considered as hydrogen bond interchange rather than hydrogen bond breakage, is naturally much less than for the other. Schellman estimates a value of $1.5 \mathrm{kcal}$. per mole per bond, from data on the association of urea molecules in aqueous solution and the temperature coefficient of the association constant.

A regular hydrogen-bonded chain configuration has low configuration entropy. The gain in entropy on unfolding will contribute a negative term to the free energy of unfolding. Schellman has calculated that in general the free energy of unfolding per residue $\Delta F_{\text {res }}$ of a hydrogen-bonded peptide structure at room temperature, neglecting side-chain interactions, is of the order of $R T$ or less, that is about $500 \pm 100 \mathrm{cal}$.

There are $\mathrm{C}=\mathrm{O}$ groups at one end of an $\alpha$-helix and $\mathrm{NH}$ groups at the other which project outwards and cannot form hydrogen bonds. Terminal $(\alpha)-\mathrm{NH}_{2}$ and $(\alpha)-\mathrm{COOH}$ bonds are not held in fixed positions. A helix may be either righthanded or left-handed. These three factors must be allowed for in calculating the over-all free energy of unfolding $\left(\Delta F_{\text {unf }}\right)$ for a chain with $n$ residues. In equation (1)

$$
\Delta F_{\text {unf }}=n \Delta F_{\text {res }}+C
$$


$C$ includes terms which allow for these three effects; $C$ is negative for hydrogen-bonded configurations in both aqueous and nonaqueous solvents at room temperature. There will therefore be a critical chain length below which the helical configuration is unstable. This critical size has been estimated as probably between 8 and 15 peptide residues for a polypeptide chain in aqueous solution, but this calculation neglects side-chain interactions. The critical size is reduced by interchain covalent bonding; thus cystine linkages between coiled chains can be shown to contribute very markedly to the stability of any array of intrinsically unstable coiled chain units (see the discussion on insulin later).

The entropy changes which occur when water molecules are bound to the $-\mathrm{C}=\mathrm{O}$ and $-\mathrm{N}-\mathrm{H}$ groups, as the helix is unfolded, have not been treated by Schellman. Allowance for these effects would probably somewhat decrease the calculated critical size for helix formation, but would not alter Schellman's principal conclusions.

Schellman has also considered the effects of electrostatic and other interactions between adjacent residues. A series of adjacent side chains with charges of the same sign gives rise to repulsive interactions, which favor extended configurations; if the adjacent charges are of opposite sign, if hydrogen bond formation occurs between adjacent side chains, or if a series of adjacent nonpolar side chains is present compact configurations such as the $\alpha$-helix are favored.

When an $\alpha$-helix structure is broken by making an abrupt alteration in the helical axis direction, stabilizing hydrogen bonds are broken but little configurational entropy is gained. "Broken" helices of this kind are therefore unlikely to occur. This would suggest that one of the two alternatives proposed on page 410 for hemoglobin-the zigzag arrangement of consecutive regions of $\alpha$-helix about some common axis-is the less likely solution of the observed experimental discrepancies. A further criticism of the zigzag arrangement is the difficulty, except where the angle between the two segments is large, in forming reasonably strong hydrogen bonds between free $\mathrm{C}=\mathrm{O}$ or $\mathrm{NH}$ groups and water molecules. 
These arguments may obviously be applied to other helical peptide chain structures. In so far as these are initially less stable than the $\alpha$-helix the values for the critical length will be different from the $\alpha$-helix value. Donohue (33) has studied the relative stabilities of a group of several helices which includes the first four members of the $\alpha$ series

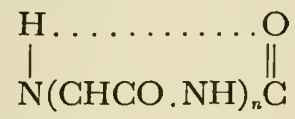

where $n=1,2,3$, and 4 .

The $\alpha$-helix structure shows excellent van der Waals packing across the interior of the coil. None of the other three helices packs so well. Donohue has not included the contributions of van der Waals forces in his estimates of the order of relative stability within the group. He finds that the two most reasonable alternative structures, the $\pi$-helix $(n=4)$ and a ribbon structure (49) with $n=1$ are less stable than the $\alpha$-helix by an amount which is approximately equal to $500 \mathrm{cal}$. per residue per mole. This finding may be of significance in considering the absence of experimental evidence for any helical configuration except the $\alpha$-helix. On the other hand, it should be remembered that most globular proteins are labile and denature easily. The most stable molecular configuration may not be that of the native protein molecule.

The experimental evidence on the configuration of longchain polypeptides in water solution is still meager. Bamford, Elliott, Trotter, and their associates-see for instance $(10,11,41)$ - have made extensive studies of synthetic polypeptide films or fibers cast from various solvents. In general it is true that the long-chain peptides are more likely to be found in the compact $\alpha$ configuration, as judged by x-ray and infrared studies; unfolded chains commonly aggregate to form fibers aligned in the $\beta$ configuration. The tendency to assume the $\alpha$ or $\beta$ formor sometimes a mixture of the two-is naturally much influenced, not only by the chain length, but also by the solvent from which the fibers are cast. Recently Doty, Holtzer, Bradbury, and Blout 
(35) have prepared poly- $\boldsymbol{\gamma}$-benzyl-L-glutamate of widely varying degrees of polymerization, each preparation being of highly uniform chain length. In weakly interacting solvents such as chloroform, these exist in the $\alpha$ form if the molecular weight is high; strongly hydrogen-bonding solvents, such as dichloroacetic acid, lead to unfolding of the polymer into a randomly coiled configuration, regardless of molecular weight. Very low molecular weight polymers, in weakly interacting solvents, exist partly in the $\beta$ form, which dissociates upon dilution to give a solvated form. All this is qualitatively in accord with the theoretical discussion outlined above, but the most interesting field of study-that of the configuration of large water-soluble polypeptides in aqueous solution-is only just beginning.

What is the relation of the considerations we have just discussed to the total structure of a globular protein? Obviously no simple answer can suffice. A complex of polypeptide chain and side-chain interactions will determine the three-dimensional structure of each single molecule. A single molecule is itself in this respect comparable to a crystal structure. Once the crystal structure of a compound has been established and the atomic parameters determined the analysis carries its own persuasive conviction. The whole range of pertinent intraand inter-molecular packing principles is seen to be nicely and effectively balanced. We may expect a similar satisfaction when the three-dimensional structure of a globular protein molecule is determined. The detailed crystal structures, even of relatively simple organic compounds, however, have never yet been determined simply by correct guesswork, even when the probable packing principles were recognized. Many crystal structures, such as those of penicillin (32) and threonine (100), took two years to solve although full use was made of all the evidence available, both chemical information and diffraction data.

The brilliant work of Pauling and Corey has established one of the outstanding packing principles in protein stereochemistry. We know that there are regions of $\alpha$-helix configuration in many proteins. It would, however, be unrealistic to conclude that 
the problem of protein structure is not still largely an enigma. Detailed x-ray diffraction studies of protein crystals may one day succeed in the determination of a detailed molecular structure for some protein. The probable absence of regions of ordered side-chain array-ordered, that is, in the crystalline sense-makes it difficult to attack the remaining problems of three-dimensional structure systematically. Scale models may be helpful even though at this stage they are unfortunately not able to indicate more than the stereochemical plausibility of a trial structure. Electrostatic forces, van der Waals interactions, and lyophobic effects, etc., cannot be or have not yet been built into scale models. The models will not automatically fall into place and throw the structure into its proper form. The kind of information that such investigations do provide is illustrated by the model studies of insulin based on the Sanger sequences discussed below.

\section{Insulin}

The insulin molecule contains one A and one B chain (as described on page 379), the molecular weight being 5734 for beef insulin. The molecules associate in aqueous solution. At $p \mathrm{H}$ values of 2 to 3 the predominating species is a dimer of two molecules. This 12,000 molecular weight unit is usually referred to as the monomer solution unit since it has not been found possible to dissociate insulin further in aqueous solution except under special and unusual conditions (58). At higher $p \mathrm{H}$ values further association occurs and around $p \mathrm{H} 6$ to 7 a trimer unit of molecular weight 36,000 predominates.

At $p \mathrm{H} 6$ to 7 the insulin trimer unit of molecular weight 36,000 crystallizes from zinc-containing solutions (99). The amount of zinc incorporated into the crystal may vary over a wide range (25) and may be considerably less than 3 zinc atoms per 36,000 unit. In zinc-free solutions at $p \mathrm{H} 2.5$ to 3.5 insulin may be crystallized as the sulfate (63), selenate, citrate, or formate. The unit of packing in the orthorhombic crystals of acid insulin sulfate is the solution monomer containing 2 (A and 
B) molecules (65). The peak distribution in the vector structure (Patterson series) suggests that each 12,000 unit is made up of four cylindrical rods of high electron density stacked in closepacked array parallel to the $a$ axis. The intermolecular packing between these 12,000 molecular weight units maintains the parallel close-packed arrangement throughout the structure. The $a$ axis in the air-dried crystals is $44 \mathrm{~A}$ long and thus the maximum possible length of each rod is $44 \mathrm{~A}$. It appeared reasonable therefore to identify each cylindrical region with a length of coiled or folded polypeptide chain. From calculations based on the length and cross-packing area of each rod, the density, and the number of residues in the $\mathrm{B}$ chain, it appears that an $\alpha$-helix might be the most plausible model for the chain configuration $(66,67)$.

In suggesting this relationship Low observed that the length of the $\mathrm{B}$ chain with 30 residues would, when coiled into an $\alpha$-helix configuration, be $45 \mathrm{~A}(30 \times 1.5)$. In this crystal structure model each crystallographic length of chain would correspond to a complete $\mathrm{A}$ or $\mathrm{B}$ chain with parallel interchain packing both within and between molecules.

The $\alpha$-helix structure satisfied the simple geometrical packing requirements of the unit cell. It appeared useful therefore to undertake a scale model study based on the Sanger amino acid sequences. An early model study established that in the two-chain $(\mathrm{A}+\mathrm{B})$ unit a simple model of parallel $\alpha$-helices for both $A$ and $B$ chain required modification. The $-\mathrm{C}_{(\beta)}-\mathrm{S}-$ (half cystine) residues on the $\mathrm{A}_{6}$ and $\mathrm{A}_{11} \alpha$-carbon positions are too far removed in space on a simple $\alpha$-helix structure to permit the formation of an $\mathrm{A}_{6}^{11}$ ] intrachain disulfide bond.

A simple continuous $\alpha$ helix structure is thus ruled out for the A chain of insulin. This limitation does not impose intrinsic restrictions on the configuration of the terminal segments of the A chain. Both these two parts of the A chain and the whole of the $\mathrm{B}$ chain may be in the $\alpha$-helix configuration without affecting the interchain cystine linkages. If the specific configuration of the pentapeptide loop is irregular, then intrachain 
hydrogen bonds between peptide $\mathrm{CO} \cdot \mathrm{NH}$ residues cannot be formed. If we ask whether there is any configuration for this region of the chain which would permit some intrachain hydrogen bonding, or whether it is possible to maintain the $\alpha$-helix configuration in at least some part of the $\left.\mathrm{A}_{6}^{11}\right]$ loop, then these problems can be studied using scale atomic models.

Lindley and Rollett (62) have shown that this region of the chain may be twisted into a more regular configuration if it joins two segments of a chain which have different screw directions. If the $A_{1-9}$ part of the chain is a left-handed coil and the $A_{9-21}$ part is a right-handed coil, then the $A_{6}^{11}$ ] region forms the figure of eight required by this change of hand in the coil. In the detailed model, which they have developed, only two of the main chain NH.... OC hydrogen bonds are broken. The $\mathrm{CO}$ group of residue 8 could, in beef insulin, form an $\mathrm{OH}$... bond with the hydroxyl group of the seryl residue 9 (see Figures 1 and 2). In this model the helical axis of the right-handed $A_{9-21}$ sequence is parallel to a right-handed $B$ chain $\alpha$-helix. The helical axis of the $A_{1-7}$ segment is in the same plane as the $\mathrm{B}$ and $\mathrm{A}_{9-21}$ axes; it is tilted away from $\mathrm{B}$ at an angle of $30^{\circ}$.

Low (68) has constructed a model using a similar change of hand from a left-handed $\mathrm{A}_{1-8}$ segment to a right-handed $\mathrm{A}_{11-21}$ segment. The $\mathrm{B}$ chain in her model is a left-handed $\alpha$-helix. In this structure the two parts of the A chain do not lie in the same plane. There is a lateral shift between them so that the model appears in "end-on" projection as an area of three close-packed helices. Three intrachain hydrogen bonds are broken in this model. Both structures are plausible. The Lindley and Rollett model is the more satisfying solution of the simple stereochemical problem: to find a model structure for the insulin molecule in which all of the B chain and as much as possible of the A chain are in the $\alpha$-helix configuration. The helix structure in this model is predominantly right-handed, whereas the Low model is predominantly left-handed.

Insulin was included in Arndt and Riley's (5) detailed 
study of the globular proteins, in which they concluded that the $\alpha_{1}$ left-handed helix is the most plausible predominant chain configuration. They write, "Our earlier suggestion (4) that the insulin molecule is made up of equal numbers of $\alpha_{1}$ and $\alpha_{2}$ helices remains hypothetical and has not been capable either of substantiation or disproof in the present work."

Calvin (21a) has discussed the stereochemistry of the $-\mathrm{C}_{1}-\mathrm{S}_{1}-\mathrm{S}_{2}-\mathrm{C}_{2}-$ linkage. The position of minimum potential energy corresponds to a configuration in which the dihedral angle between the two $-\mathrm{S}-\mathrm{C}$ bonds is nearly $90^{\circ}$. In other words, the plane defined by the $\mathrm{C}_{1}, \mathrm{~S}_{1}$, and $\mathrm{S}_{2}$ atoms is nearly perpendicular to the plane defined by $S_{1}, S_{2}$, and $\mathrm{C}_{2}$. The resistance to free rotation around the $\mathrm{S}-\mathrm{S}$ bond is very great; surprisingly great for what is ordinarily considered a single bond. This high potential energy barrier must be of great importance in determining the configuration of the insulin molecule in the regions of inter and intra chain disulfide bridges. When the cystine linkage is in the favored configuration it will, by Calvin's estimate, be more stable, by 10 to $20 \mathrm{kcal}$. per mole, than the cis or trans configuration in which all four atoms lie in the same plane. In the Lindley and Rollett model all three cystine $\mathrm{C}_{1}-\mathrm{S}_{1}-\mathrm{S}_{2}-\mathrm{C}_{2}$ linkages have the favored dihedral angle between the $\mathrm{S}-\mathrm{C}$ bonds. However, deviations from the favored configuration do occur. In the crystal structure of $N, N^{\prime}$-diglycyl-L-cystine (110a) a dihedral angle of $101^{\circ} \pm 2^{\circ}$ has been reported.

Both the Low and the Lindley and Rollett models can be stacked in close-packed array. Lindley and Rollett have given a detailed discussion of the possible modes of intermolecular packing in the rhombohedral crystals of zinc insulin. They suggest that the 12,000 unit, which is the stable monomer in solution, may be made up of $2(\mathrm{~A}+\mathrm{B})$ units packed in rectangular array and held together by specific interaction between zinc ions and histidine and glutamic acid residues. This particular packing model unit appears rather unlikely as zinc is not necessary to hold the 12,000 unit intact. Other packing models of $2(\mathrm{~A}+\mathrm{B})$ units can be devised. 
The important question, however, is the relevance of these stereochemical models to the actual insulin structure. The $\mathrm{x}$-ray study of acid insulin sulfate crystals does offer rather persuasive geometrical evidence that a good part at least of the insulin molecule is in a regular configuration. Arndt and Riley's study gives even further evidence of this. Unfortunately, the 1.5 A spacing characteristic of the $\alpha$-helix structure has not been found, despite intensive search in x-ray photographs of wet crystals of acid insulin sulfate. However, the nature of the interchain packing between $\alpha$-helices could reduce the intensity of this reflection very markedly, so that negative observations of this kind carry little weight. It is a little difficult, however, to dismiss so easily the study of Elliott (42), using polarized infrared radiation, on air dried crystals of acid insulin sulfate. For the absorption bands corresponding to the $\mathrm{C}=\mathrm{O}$ and $\mathrm{N}-\mathrm{H}$ vibrations marked dichroic effects would be expected from an arrangement of $\alpha$-helices stacked parallel to the $a$ axis. The dichroism should be reduced somewhat if a part of one helix were tilted at an angle. But the complete absence of obvious dichroism in these crystals is difficult to explain. At this stage in such studies of protein structures, however, these observations should probably be considered merely as anomalies, requiring further investigation. It is interesting to note that when the cystine linkages in insulin are oxidized the A and B chains both appear to be in the $\beta$ configuration (5). Schellman (98) regards this as strong evidence for the favorable effect of interchain covalent bonds in holding a molecule in the coiled $\alpha$ configuration. Linderstrøm-Lang and his co-workers have studied the kinetics of the deuterium exchange of insulin with $\mathrm{D}_{2} \mathrm{O}$. Linderstr $\not \mathrm{m}$-Lang (60b) reports that a certain number of the hydrogen atoms exchange very slowly. The actual number of slowly exchanging hydrogen atoms would suggest that only those regions of the $\mathrm{A}$ and $\mathrm{B}$ chains between the two interchain cystine linkages are in a regular coiled configuration with strong intrachain hydrogen bonds, for dissolved insulin. The imide hydrogens in the intrachain disulfide loop of the A chain are probably free to exchange readily with deuterium. 
On balance, it is obvious that more direct experimental evidence is needed before the dominant features of the insulin structure are completely established, although it seems reasonable to suppose that regions of $\alpha$-helix configuration are probably a feature of the molecular stereochemistry. Extensive $\mathrm{x}$-ray crystal structure studies of insulin are being carried out by Low and her associates here at Harvard. Even for insulin, therefore, in which the chain sequences and cross links are completely known, the exact spatial configuration of the molecule still remains in doubt. For all other proteins the uncertainties are far greater, but there are some for which great progress has been made, and we turn finally to a consideration of two of these-ribonuclease and serum albumin.

\section{Ribonuclease}

This relatively simple protein from pancreas has been intensively studied both by chemists $(3,48)$ and by x-ray crystallographers $(22,23)$. In addition to the $x$-ray work already published the extensive and important work of David Harker and his associates at the Protein Structure Project holds promise of greatly increasing insight into the structure of this molecule. However, it would be premature at the moment to draw extensive conclusions from the $\mathrm{x}$-ray work, although far-reaching developments may emerge shortly. Studies of sedimentation, diffusion, and viscosity, in addition to the x-ray studies, all indicate that the molecule is a highly compact structure. The chemical studies have produced a preliminary but illuminating picture. The evidence appears decisive (3) that the molecule consists of a single polypeptide chain, probably 124 residues long (48) with an $N$-terminal lysyl group and a $C$-terminal valine. No sulfhydryl groups are found, but there are four disulfide bridges, which must therefore cross-link different portions of the chain into loops. It was earlier suggested that the four proline residues present could provide loci for the folding of the peptide chains; a model based on this assumption was published by Anfinsen et al. (3). However, this model has now been aban- 
doned by its proponents, because of the finding that two out of the four proline residues are in a single segment, 15 residues long, at the $C$-terminal end of the chain (C. H. W. Hirs, W. H. Stein and S. Moore, personal communication). To maintain the structure of a highly compact molecule, such as ribonuclease is known to be, there must be loops spaced at more or less regular intervals along the entire chain. Evidence from ultraviolet absorption near $290 \mathrm{~m} \mu$ indicates that three of the six tyrosine hydroxyl groups in ribonuclease are involved in strong hydrogen bonds $(100 \mathrm{a}, 102 \mathrm{a})$ similar to those observed in ovalbumin (28), and these may play a part in maintaining the native molecular configuration. However, no model yet proposed has succeeded in presenting a unified picture of all the very extensive knowledge now available concerning the structure of ribonuclease. Further research is rapidly extending this knowledge, and there is reasonable hope that within a relatively short time an accurate and fairly detailed picture of the structure will emerge.

\section{Serum Albumins and Ovalbumin}

Human and bovine serum albumin are very similar in molecular size and physical properties generally $(37,38)$; the general pattern of amino acid composition for the two is highly similar, although they differ quite definitely with respect to certain amino acids (19). The major portion of both albumin preparations consists of mercaptalbumin (50) (written as ASH for brevity) with a single free sulfhydryl group; mercaptalbumin is readily separated from the rest of the albumin fraction by crystallization as the mercury derivative, AS- $-\mathrm{Hg}-\mathrm{SA}$. The molecular weight has been variously estimated, but certainly lies within a rather narrow range, 65,500 to $69,000(37,38,64,97)$. On the basis of the lower figure there are approximately 550 amino acid residues in either the human or bovine molecule. The most careful determinations of $N$-terminal groups have revealed only one per molecule. This is aspartyl in both proteins $(104,105)$, although the next adjoining residue is alanyl in human, threonyl in bovine serum albumin. There are 17 
disulfide linkages per molecule in both species (19) for 65,000 molecular weight, or 18 if the molecular weight is taken as 69,000 -and the evidence thus suggests that all of these serve to cross-link different segments within a single peptide chain, which must therefore be folded back and forth in many loops. The number of proline residues-29 for human, 32 for bovine albumin, taking the molecular weight as 65,000 -is ample to provide the necessary turning points for reversal of chain direction in the loops; although we must emphasize again that proline is not necessarily involved. This picture has received further support from the work of McDuffie and Hunter (74), who have reduced all the disulfide groups of human albumin to sulfhydryls with thioglycolate, in the presence of a detergent or of concentrated urea solution, and then blocked the sulfhydryls with iodoacetamide to prevent reoxidation:

$$
\mathrm{RSH}+\mathrm{CH}_{2} \mathrm{I} \cdot \mathrm{CONH}_{2} \longrightarrow \text { R.S. } \mathrm{CH}_{2} \cdot \mathrm{CONH}_{2}
$$

The resulting molecule is insoluble in water but forms a soluble complex with detergents such as dodecyl sulfate; the composition and molecular weight of the complex indicated that no breakdown of the albumin molecule into smaller units had occurred, so that a single peptide chain of about 550 units was apparently present. Thus the general pattern of the albumin molecule appears to be not unlike that suggested above for ribonuclease but on a much larger scale, with a peptide chain more than four times as long as that of ribonuclease, and four times as many disulfide cross links.

Reichmann and Colvin (88) have oxidized the disulfide sulfur atoms of bovine albumin to sulfonic acid groups with performic acid, and find a decrease of molecular weight, by light scattering and osmotic pressure, to less than half the original value. No increase in free end groups was observed, however. If only disulfide bonds are broken by performic acid oxidation, these results imply that bovine albumin consists of three or four peptide chains, bound together by disulfide bonds. It is possible, however, that other reactions, involving the breakage of peptide chains, may be produced by this reagent. Final judgment as to whether serum albumin consists of one or of several peptide chains must therefore be postponed. 
Although the portions of the serum albumin molecule are tied together by the 17 disulfide bridges, the structure must possess considerable flexibility (97), as shown by the virtually instantaneous increase in optical rotation and in viscosity which occurs when the molecule is exposed to high concentrations of urea $(45,54)$ or to acid $p \mathrm{H}$ values in the range 1.5 to 3.5 (111). The changes are not only instantaneous but also largely reversible. In contrast, ovalbumin, which has only one or two disulfide linkages per molecule, shows slow changes in optical rotation (101) and in viscosity (44) in urea solutions, which continue over many hours. The native structure of ovalbumin appears to be more rigid; it does not yield easily to stress, but once opened up it is disrupted for good. Serum albumin, on the other hand, yields readily to forces which tend to cause it to unfold-either to the repulsive electrostatic forces produced when the molecule acquires a large net charge or to the penetrating urea molecules which disrupt hydrogen bonds-but returns to something closely approaching its original state when the disturbing force is removed. We have already called attention to a similar difference between the two molecules with respect to the ionization of their tyrosine hydroxyl groups. In ovalbumin (28) these groups are firmly held by hydrogen bonds and do not ionize until a $p \mathrm{H}$ greater than 12 is reached, but the breaking of the bonds, once achieved, is irreversible. In serum albumin (103) some hydrogen bonds involving tyrosine appear to exist, but they are readily broken in alkaline solutions and the process is readily reversed on acidification.

These differences, and many others which could be recorded, indicate a profound difference in structural pattern between these two familiar proteins. We have tried to suggest some of the common recurring features, which appear to be of nearly universal significance in protein architecture, and others which may be related to the individuality - we may almost say the caprice-displayed in the pattern of this or that particular protein. Our vision of these molecular patterns is still, for the most part, uncertain and dim. However, our understanding of 
protein structure today, imperfect as it is, gives increased hope of more definite solutions to the great problems that concern the biologist - the biogenesis of proteins and the mechanisms of their biological functions. We may reasonably believe that the work of the next ten years will go far to deepen our understanding of these fundamental problems.

We are indebted to Mr. J. Ralph Einstein for his detailed and careful work in the drawing of Figure 8, and to Professors Linus Pauling and R. B. Corey for providing us with an unpublished drawing (Fig. 4).

\section{References}

1. Abichandani, C. T., and S. K. K. Jatkar, J. Indian Inst. Sci., 21A, 417 (1938).

2. Ambrose, E. J., and A. Elliott, Proc. Roy. Soc. (London), A206, 206 (1951).

3. Anfinsen, C. B., R. R. Redfield, W. L. Choate, J. Page, and W. R. Carroll, J. Biol. Chem., 207, 201 (1954).

4. Arndt, U. W., and D. P. Riley, Nature, 772, 245 (1953).

5. Arndt, U. W., and D. P. Riley, Trans. Roy. Soc. (London), A249, 409 (1955).

6. Astbury, W. T., Proc. Roy. Soc. (London), B141, 1 (1953).

7. Astbury, W. T., and A. Street, Trans. Roy. Soc. (London), A230, 75 (1931).

8. Astbury, W. T., and H. J. Woods, Trans. Roy. Soc. (London), A232, 333 (1933).

9. Bamford, C. H., L. Brown, A. Elliott, W. E. Hanby, and I. F. Trotter, Nature, 169, 357 (1952).

10. Bamford, G. H., L. Brown, A. Elliott, W. E. Hanby, and I. F. Trotter, Nature, 171, 1149 (1953); ibid., 173, 27 (1954).

11. Bamford, C. H., W. E. Hanby, and F. Happey, Proc. Roy. Soc. (London), A205, 30 (1950); ibid., 206, 407 (1951).

12. Bell, P. H., J. Am. Chem. Soc., 76, 5566 (1954); and personal communication.

13. Bijvoet, J. M., A. F. Peerdeman, and A. J. van Bommel, Nature, 168, 271 (1950).

14. Boyer, P. D., G. A. Ballou, and J. M. Luck, J. Biol. Chem., 162, 199 (1946).

15. Boyer, P. D., F. G. Lum, G. A. Ballou, J. M. Luck, and R. G. Rice, J. Biol. Chem., 162, 181 (1946).

16. Bragg, W. L., E. R. Howells, and M. F. Perutz, Acta Cryst., 5, 136 (1952).

17. Bragg, W. L., J. C. Kendrew, and M. F. Perutz, Proc. Roy. Soc. (London), A203, 321 (1950). 
18. Bragg, W. L., and M. F. Perutz, Proc. Roy. Soc. (London), A225, 315 (1954).

19. Brand, E., Ann. N. Y. Acad.Sci., 47, 187 (1946).

20. Brand, E., and J. T. Edsall, Ann. Rev. Biochem., 16, 223 (1947).

21. Brown, H., F. Sanger, and R. Kitai, Biochem. J. (London), 60, 556 (1955).

21a. Calvin, M., in S. Colowick and others, eds., Glutathione: Proceedings of a Symposium Held at Ridgefield, Connecticut, November, 1953, p. 3. Academic Press, New York, 1954.

22. Carlisle, C. H., and H. Scouloudi, Proc. Roy. Soc. (London), A207, 496 (1951).

23. Carlisle, C. H., H. Scouloudi, and M. Spier, Proc. Roy. Soc. (London), B141, 1 (1953).

24. Cochran, W., and F. H. C. Crick, Nature, 167, 2053 (1951).

25. Cohn, E. J., J. D. Ferry, J. J. Livingood, and M. H. Blanchard, J. Am. Chem. Soc., 63, 17 (1941).

26. Corey, R. B., and L. Pauling, Proc. Roy. Soc. (London), B141, 10 (1953).

27. Cowan, P. M., A. C. T. North, and J. T. Randall, in J. T. Randall, ed., Nature and Structure of Collagen, p. 241. Academic Press, New York, 1953.

28. Crammer, J. L., and A. Neuberger, Biochem. J. (London), 37, 302 (1943).

29. Crick, F. H. C., Nature, 170, 882 (1952).

30. Crick, F. H. C., Acta Cryst., 5, 381 (1952).

31. Crick, F. H. C., Acta Cryst., 6, 689 (1953).

32. Crowfoot, D., C. W. Bunn, B. W. Rogers-Low, and A. Turner-Jones, in H. T. Clarke, J. R. Johnson, and Sir Robert Robinson, eds., The Chemistry of Penicillin, Ch. 11, p. 310. Princeton University Press, Princeton, 1949.

33. Donohue, J., Proc. Natl. Acad. Sci. U. S., 39, 470 (1953).

34. Donohue, J., and K. N. Trueblood, Acta Cryst., 5, 414, 419 (1952).

35. Doty, P. M., A. M. Holtzer, J. H. Bradbury, and E. R. Blout, J. Am. Chem. Soc., 76, 4493 (1954).

36. Edelhoch, H., E. Katchalski, R. H. Maybury, W. L. Hughes, Jr., and J. T. Edsall, J. Am. Chem. Soc., 75, 5058 (1953).

37. Edsall, J. T., in H. Neurath and K. Bailey, eds., The Proteins, Vol. 1, Part B, Ch. 7. Academic Press, New York, 1953.

38. Edsall, J. T., J. Polymer Sci., 12, 253 (1954).

39. Edsall, J. T., R. H. Maybury, R. B. Simpson, and R. Straessle, J. Am. Chem. Soc., 76, 3131 (1954).

40. Elliott, A., Proc. Roy. Soc. (London), A211, 490 (1952).

41. Elliott, A., Proc. Roy. Soc. (London), A226, 408 (1954).

42. Elliott, A., personal communication.

43. Elliott, A., and E. J. Ambrose, Discussions Faraday Soc., No. 9, 246 (1950).

44. Frensdorff, H. K., M. T. Watson, and W. Kauzmann, J. Am. Chem. Soc., 75,5157 (1953). 
45. Frensdorff, H. K., M. T. Watson, and W. Kauzmann, J. Am. Chem. Soc., 75,5167 (1953).

46. Harfenist, E. J., J. Am. Chem. Soc., 75, 5528 (1953).

47. Harfenist, E. J., and L. C. Craig, J. Am. Chem. Soc., 74, 3083, 3087 (1952).

48. Hirs, C. H. W., W. H. Stein, and S. Moore, J. Biol. Chem., 217, 941 (1954); ibid. in press.

48a. Howard, K. S., R. G. Shepherd, E. A. Eigner, D. S. Davis, and P. H. Bell, J. Am. Chem. Soc., 77, 3419 (1955).

49. Huggins, M. L., Chem. Revs., 32, 195 (1943).

50. Hughes, W. L., Jr., Cold Spring Harbor Symposia Quant. Biol., 14, 79 (1950).

51. Ingram, V. M., Biochem. J. (London), 59, 653 (1955).

52. Jacobsen, C. F., and K. Linderstrom-Lang, Nature, 164, 411 (1949).

53. Kauzmann, W., in W. D. McElroy and B. Glass, eds., The Mechanism of Enzyme Action, p. 70. Johns Hopkins Press, Baltimore, 1954.

54. Kauzmann, W., and R. B. Simpson, J. Am. Chem. Soc., 75, 5154 (1953).

55. Kendrew, J. C., in H. Neurath and K. Bailey, eds., The Proteins, Vol. 2, Part B, Ch. 23. Academic Press, New York, 1953.

56. Kendrew, J. C., Progr. Biophys. and Biophys. Chem., 4, 244 (1954).

57. Kendrew, J. C., and M. F. Perutz, in F. J. W. Roughton and J. C. Kendrew, eds., Haemoglobin, p. 161. Butterworth, London, and Interscience, New York, 1949.

58. Kupke, D. W., and K. Linderstrom-Lang, Biochim. et Biophys. Acto, 13, 153 (1954).

59. Larsson, E., Z. anorg. u. allgem. Chem., 183, 30 (1929).

60. Laskowski, M., Jr., and H. A. Scheraga, J. Am. Chem. Soc., 76, 6305 (1954).

60a. Li, C. H., I. I. Geschwind, R. D. Cole, I. D. Raacke, J. I. Harris, and J. S. Dixon, Nature, 176, 687 (1955).

60b. Linderstrom-Lang, K., in Peptide Chemistry: Report of a Symposium held by the Chemical Society March 30, 1955, The Chemical Society, London, 1955. A. Hvidt and K. Linderstróm-Lang, Compt. rend. trav. lab. Carlsberg, Ser. chim. 29, 385 (1955).

61. Lindley, H., Biochim. et Biophys. Acta, 18, 194 (1955).

62. Lindley, H., and J. S. Rollett, Biochim. et Biophys. Acta, 18, 183 (1955).

63. Low, B. W., Cold Spring Harbor Symposia Quant. Biol., 14, 77 (1950).

64. Low, B. W., J. Am. Chem. Soc., 74, 4830 (1952).

65. Low, B. W., Nature, 169, 955 (1952).

66. Low, B. W., in H. Neurath and K. Bailey, eds., The Proteins, Vol. 1, Part A, Ch. 4. Academic Press, New York, 1953.

67. Low, B. W., Nature, 772, 1146 (1953).

68. Low, B. W., Unpublished observations, 1955.

69. Low, B. W., and R. B. Baybutt, J. Am. Chem. Soc., 74, 5806 (1952). 
70. Low, B. W., and H. J. Grenville-Wells, Proc. Natl. Acad. Sci. U. S., 39, 785 (1953).

71. MacArthur, I., Nature, 152, 38 (1943).

72. Marsh, R. E., R. B. Corey, and L. Pauling, Biochim. et Biophys. Acta, 16, 1 (1955).

73. Mathieson, A. McL., and H. K. Welsh, Acta Crvst., 5, 599 (1952).

74. McDuffie, F. C., and M. J. Hunter, Abstracts 126th Meeting, Am. Chem. Soc., New York, September 1954, p. 79C.

75. Meyer, K. H., and H. Mark, Ber., 61B, 1932 (1928).

76. Ottesen, M., and G. Villee, Compt. rend. trav. lab. Carlsberg, Ser. Chim., 27, 421 (1951).

77. Pauling, L., in R. Stoops, ed., Les Proteines: Rabports et Discussions, p. 63. Institut Intern. de Chimie Solvay, Brussels, 1953.

78. Pauling, L., and R. B. Corey, Proc. Natl. Acad. Sci. U.S., 37, 729 (1951).

79. Pauling, L., and R. B. Corey, Proc. Natl. Acad. Sci. U. S., 37, 235-285 (1951).

80. Pauling, L., and R. B. Corey, Proc. Natl. Acad. Sci. U. S., 39, 253 (1953).

81. Pauling, L., and R. B. Corey, Nature, 171, 59 (1953).

82. Pauling, L., R. B. Corey, and H. R. Branson, Proc. Natl. Acad. Sci. U. S., 37, 205 (1951).

83. Perlmann, G. E., Biochim. et Biophys. Acta, 73, 452 (1954).

84. Perlmann, G. E., Nature, 174, 273 (1954).

85. Perlmann, G. E., Advances in Protein Chem., 70, 1, 1955.

86. Perutz, M. F., Nature, 167, 1053 (1951).

87. Porter, R. R., and F. Sanger, Biochem. J. (London), 42, 287 (1948).

88. Reichmann, M. E., and J. R. Colvin, Can. J. Chem., 33, 163 (1955); ibid. in press.

89. Riggs, A., J. Gen. Physiol., 36, 1 (1952-53). A. Riggs and R. A. Wolbach, J. Gen. Physiol., 39, 585 (1956).

90. Riley, D. P., and U. W. Arndt, Nature, 169, 138 (1952). A. Riggs, and R. A. Wolback, J. Gen. Physiol., 39, 585 1956).

91. Riley, D. P., and U. W. Arndt, Proc. Roy. Soc. (London), B141, 93 (1952).

91a. Ryle, A. P., and F. Sanger, Biochem. J., 60, 535 (1955).

91b. Ryle, A. P., F. Sanger, L. F. Smith, and R. Kitai, Biochem. J., 60, 541 (1955).

92. Sanger, F., Advances in Protein Chem., 7, 1 (1952).

93. Sanger, F., L. F. Smith, and R. Kitai, Biochem. J. (London), 58, vi (1954).

94. Sanger, F., and E. O. P. Thompson, Biochem. J. (London), 53, 353, 366 (1953).

95. Sanger, F., E. O. P. Thompson, and R. Kitai, Biochem. J. (London), 59, 509 (1954).

96. Sanger, F., and H. Tuppy, Biochem. J. (London), 49, 463, 481 (1951).

97. Scatchard, G., Am. Scientist, 40, 61 (1952). 
98. Schellman, J. A., Compt. rend. trav. lab. Carlsberg, Ser. chim., 29, 223, 230 (1955).

99. Scott, D. A., Biochem. J. (London), 28, 1592 (1934).

100. Shoemaker, D. P., J. Donohue, V. Schomaker, and R. B. Corey, J. Am. Chem. Soc., 72, 2328 (1950).

100a. Shugar, D., Biochem. J., 52, 142 (1952).

101. Simpson, R. B., and W. Kauzmann, J. Am. Chem. Soc., 75, 5139 (1953).

102. Steinhardt, J., and E. M. Zaiser, Advances in Protein Chem., 10, 151, 1955.

102a. Tanford, C., J. D. Hauenstein, and D. G. Rands, J. Am. Chem. Soc., 77, 6409 (1955).

103. Tanford, G., and G. L. Roberts, Jr., J. Am. Chem. Soc., 74, 2509 (1952). C. Tanford, S. A. Swanson, and W. S. Shore, J. Am. Chem. Soc., 77, 6414 (1955).

104. Thompson, E. O. P., J. Biol. Chem., 208, 565 (1954).

105. Van Vunakis, H., and E. Brand, Abstracts, 119th Meeting, Am. Chem. Soc., Boston (April, 1951), p. 28C.

106. du Vigneaud, V., H. C. Lawler, and E. A. Popenoe, J. Am. Chem. Soc., 75, 4880 (1953).

107. du Vigneaud, V., C. Ressler, J. M. Swan, C. W. Roberts, and P. G. Katsoyannis, J. Am. Chem. Soc., 76, 3115 (1954).

108. Waugh, D. F., Advances in Protein Chem., 9, 325 (1954).

108a. White, W. F., and W. A. Landmann, J. Am. Chem. Soc., 77, 1711 (1955).

109. Wyman, J., Advances in Protein Chem., 4, 407 (1948).

110. Yakel, J. L., Jr., Acta Cryst., 6, 724 (1953).

110a. Yakel, H. L., Jr., and E. W. Hughes, Acto Cryst., 7, 291 (1954).

111. Yang, J. T., and J. F. Foster, J. Am. Chem. Soc., 76, 1588 (1954). 


\section{THE STRUCTURE OF INSULIN}

F. SANGER, Department of Biochemistry, University of Cambridge, Combridge, England

In recent years protein chemistry has been so extensively reviewed from many different points of view that it would seem purposeless to prepare another general article on the subject; rather it is proposed to give an account of our own work of the last decade which has led to the elucidation of the structure of the protein insulin. It is hoped in this way to illustrate the newer methods which are available for the study of proteins and to indicate some of the present trends in protein chemistry.

The foundations of the present study were laid by much painstaking work carried out at a time when insulin was available only in very small quantities and was of doubtful purity. To these early investigations we owe the initial hypotheses of this work, which are that insulin as purchased is virtually a pure substance and that it is a protein containing 16 different amino acids (for references, see ref. 17). We may further assume, following the pioneers of protein chemistry, that the amino acids are joined together through peptide bonds.

\section{Molecular Weight}

When studied under normal conditons in aqueous solution 
by the osmotic pressure or ultracentrifugal methods insulin exhibits a molecular weight of about 48,000 , but the volume of the unit cell, determined by $\mathrm{x}$-ray measurement, suggested a lower value of 36,000 . This discrepancy was resolved by Gutfreund (7), who showed that under certain special conditions insulin dissociated to smaller particles; he suggested that the actual molecular weight was 12,000 , and that the higher values were due to aggregation in solution. Other workers have confirmed this value and agree that it is the minimum molecular weight to which insulin dissociates in aqueous solution.

Recently, however, Harfenist and Craig (8) have applied an entirely new chemical method to this problem. Insulin was treated with fluorodinitrobenzene under conditions which allowed partial but not complete reaction with the amino groups. The partially substituted product was then subjected to countercurrent fractionation. Besides unchanged insulin a number of colored compounds were obtained corresponding to the various dinitrophenyl (DNP) derivatives of insulin. By analyses of each fraction it was shown that the one which fractionated nearest to the unchanged insulin and must therefore be the mono DNP derivative contained one DNP group per equivalent weight of 6000 , the second band two DNP groups per 6000, and so on. There was no evidence of a band containing one DNP group per 12,000 equivalent weight. From this it may be concluded that the minimum particle weight of insulin, representing the smallest unit which is held together by covalent bonds, is 6000 . Recent physical studies $(4,11)$ have confirmed this value using certain special conditions for dissociation, but it does not seem to be universally accepted by physical chemists and it would appear that in aqueous solution the 12,000 unit is held very firmly together, presumably by secondary valency forces, since it is very unlikely that the conditions used by Harfenist and Craig would break any of the covalent bonds found in insulin. This value of 6000 will be assumed for the present discussion, though most of the structural work was in fact carried out when it was considered to be 12,000 . A more accurate value of 5733 may be calculated from the known structure. 


\section{Composition}

Many analyses of insulin have been carried out with the use of various methods, the most recent being that of Harfenist (9), who used the chromatographic method of Moore and Stein. These values are given in Table I. They agree well with the values calculated from the structure (Figure 4), which was determined largely by qualitative methods but which also made use of the analytical values obtained by Chibnall and his coworkers (see Tristram, ref. 24). These are also given in Table I and differ only slightly from the more recent values.

TABLE I

Amino Acid Composition of Insulin

\begin{tabular}{|c|c|c|}
\hline \multirow[b]{2}{*}{ Amino acid } & \multicolumn{2}{|c|}{ No. of residues/mole of insulin (6000) } \\
\hline & $\begin{array}{l}\text { Harfenist } \\
\text { (9) }\end{array}$ & $\begin{array}{c}\text { Tristram } \\
\text { (24) }\end{array}$ \\
\hline Glycine & 4 & 35 \\
\hline Alanine & 3 & 3 \\
\hline Valine & 5 & 4 \\
\hline Leucine & 6 & 6 \\
\hline Isoleucine & 1 & 1.5 \\
\hline Proline & 1 & 1.5 \\
\hline Phenylalanine & 3 & 3 \\
\hline Half-cystine & 6 & 6 \\
\hline Arginine & 1 & 1 \\
\hline Histidine & 2 & 2 \\
\hline Lysine & 1 & 1 \\
\hline Aspartic acid & 3 & 3 \\
\hline Glutamic acid & 7 & 7.5 \\
\hline Serine & 3 & 3 \\
\hline Threonine & 1 & 1 \\
\hline Tyrosine & 4 & 4.5 \\
\hline Amide $\mathrm{NH}_{3}$ & 6 & 6 \\
\hline
\end{tabular}

Besides the 51 amino acid residues, crystalline insulin contains a small amount of zinc (about one atom per 12,000 equivalent weight), which is necessary for crystallization and appears to be bound rather firmly, probably through the imidazole side chains of the histidine residues (22). Whether or not it should 
be regarded as part of the functional hormone is open to question.

\section{$N$-Terminal Residues}

After the amino acid composition of a protein has been ascertained, the next problem is to determine their sequence in the polypeptide chains. One amino acid to which a definite position in the molecule may be assigned is the residue that is situated at the end of the chain, and bears a free amino group. This is known as the $N$-terminal residue and may be distinguished from other residues by suitable chemical reactions involving its free amino group. Thus an initial attack on the insulin molecule was made by developing a general method for the identification and estimation of the $N$-terminal residues of proteins and peptides (14). The reagent used was 1,2,4fluorodinitrobenzene, which reacts with amino groups to form yellow DNP (dinitrophenyl) compounds. Hydrolysis of a DNP-protein yields the DNP derivatives of those amino acids which were present as $N$-terminal residues. Thus in a hydrolyzate of DNP-insulin it was possible to detect DNP-glycine and DNP-phenylalanine, and allowing for hydrolytic loss there was one equivalent of each present. This indicated that there were two polypeptide chains present, a glycyl chain and a phenylalanyl chain.

\section{Oxidation}

Insulin contains a high proportion of cystine, and it seemed probable that the two different chains were joined together through the disulfide bridges of these residues, especially since no other type of covalent cross-linkage is known to occur in proteins. Thus in order to separate the two chains, insulin was oxidized with performic acid (15), splitting the cystine residues and converting them to cysteic acid. From the oxidation mixture two fractions were isolated, which appeared to represent the whole of the insulin. Fraction A was acidic and had only 
glycine as the $N$-terminal residue; it contained no basic amino acids and no threonine, proline, or phenylalanine. Fraction B contained a phenylalanine $N$-terminal residue and all amino acid found in insulin except for isoleucine. All the basic amino acids were present in this fraction. From a quantitative determination of the $N$-terminal residues it was calculated that the molecular weight of fraction A was 2900 and that of fraction B 3800. Sulfur estimation indicated that fraction A contained 4 cysteic acid residues, which had originated from the cystine residues of insulin, whereas fraction $B$, the longer chain, contained only two.

Although not fully realized at the time, this oxidation procedure had specifically split the insulin molecule of 50 residues into two chains containing 20 and 30 residues, respectively, and had thus considerably simplified the problem of structure determination.

From the fact that insulin contained two $N$-terminal residues it was suggested that only two polypeptide chains were present. This, however, was not entirely certain, since there might also have been cyclic chains, which would contain no $N$-terminal residue. These, however, would be expected to appear after oxidation as a fraction containing no end group. The fact that there was no evidence of any such fraction confirmed that the glycyl and the phenylalanyl chains were the only ones present in insulin.

\section{$N$-Terminal Sequences}

Hitherto the position of only one amino acid in each chain had been determined. These were the $N$-terminal residues, which were located by the DNP technique. The method could, however, be extended to the identification of other residues near to this $N$-terminal position (16). Thus, whereas complete hydrolysis of the DNP derivative of fraction B gave DNPphenylalanine, partial hydrolysis gave a mixture of DNPphenylalanyl peptides, which were fractionated by partition 
chromatography, hydrolyzed, and the amino acids identified. The results are shown in Table II. From the composition of these peptides and the fact that peptide B4 on further partial hydrolysis gives rise to the other smaller peptides (B2, B3) it is clear that the $N$-terminal sequence is Phe-Val-Asp.Glu.

TABLE II

DNP-Phenylalanyl Peptides from Insulin

\begin{tabular}{|c|c|c|c|c|}
\hline Peptide & $\begin{array}{l}\text { Hydrolysis } \\
\text { products }\end{array}$ & Structure & $\begin{array}{c}\text { Yield } \\
\text { from } \\
\text { DNP } \\
\text { insulin, } \\
\text { equivalents } \\
\%\end{array}$ & $\begin{array}{c}\text { Yield } \\
\text { from } \\
\text { B4, } \\
\text { equivalents } \\
\% \\
\end{array}$ \\
\hline \multirow{2}{*}{$\begin{array}{l}\mathrm{B} 1 \\
\mathrm{~B} 2\end{array}$} & DNP-Phe & DNP-Phe & 13 & 13 \\
\hline & $\left.\begin{array}{l}\text { DNP-Phe } \\
\text { Val }\end{array}\right\}$ & DNP-Phe $\cdot$ Val & 16 & 14 \\
\hline B3 & $\left.\begin{array}{l}\text { DNP-Phe } \\
\text { Val } \\
\text { Asp }\end{array}\right\}$ & DNP-Phe $\cdot \mathrm{Val} \cdot$ Asp & 13 & 12 \\
\hline B4 & $\left.\begin{array}{l}\text { DNP-Phe } \\
\text { Val } \\
\text { Asp } \\
\text { Glu }\end{array}\right\}$ & DNP-Phe $\cdot$ Val $\cdot$ Asp $\cdot$ Glu & 30 & 55 \\
\hline \multicolumn{2}{|c|}{$\begin{array}{l}\text { Other bands giving B4 on } \\
\text { hydrolysis }\end{array}$} & - & $\begin{array}{r}20 \\
6\end{array}$ & \\
\hline \multicolumn{2}{|c|}{ Total } & & $\overline{98}$ & $\overline{94}$ \\
\hline
\end{tabular}

When this work was carried out it was believed that the molecular weight of insulin was 12,000 and that there were two glycyl and two phenylalanyl chains. It was thus important to know if the two phenylalanyl chains, for instance, were identical. Evidence that this was so was obtained by a quantitative study of the DNP peptides produced by partial hydrolysis of insulin; these yields are given in Table II, expressed as equivalents per cent of the total terminal phenylalanine residues of insulin. The three main peptides (B2, B3, B4) account for 59\% and a further $20 \%$ is present in bands that give B4 on hydrolysis and must therefore contain the same terminal sequence. Thus $92 \%$ of 
the terminal phenylalanine is accounted for in terms of peptides that fit the sequence DNP-Phe.Val-Asp.Glu, and from this it was concluded that there was only one type of phenylalanyl chain. Further support for this was obtained by studying the hydrolysis of peptide B4 under the same conditions that were used for hydrolysis of DNP insulin. If all the terminal phenylalanine in insulin is present as DNP-Phe.Val-Asp.Glu, then the yields of B1, B2, and B3 should be the same from DNP-insulin as from B4. Table II shows that this is in fact the case.

Besides reacting with the $\alpha$-amino group of phenylalanine in fraction $B$, fluorodinitrobenzene also reacts with the $\epsilon$-amino group of the lysine residue, which gives rise on complete hydrolysis to $\epsilon$-DNP-lysine. On partial hydrolysis yellow peptides containing $\epsilon$-DNP-lysine were obtained, and from their structure and yield it could be shown that all the lysine of insulin was present in the sequence Thr·Pro'Lys.Ala.

A similar study on fraction A showed that the only $N$ terminal glycyl sequence was Gly·Ileu·Val-Glu·Glu.

\section{C-Terminal Residues}

The identification of $C$-terminal residues is a somewhat more difficult problem than the determination of $N$-terminal residues, and no generally applicable method has yet been worked out.

When insulin was treated with carboxypeptidase, the first amino acid to be liberated in the free form was alanine (12). From the known specificity of this enzyme it was concluded that a free carboxyl group was located on an alanine residue, and the subsequent determination of the sequence of fraction $B$ indicated that it was the $C$-terminal residue of the phenylalanyl chain. That asparagine was the other $C$-terminal was shown independently from the complete sequence of the fraction $A$ (19) and from the fact that a small amount of asparagine is also liberated by the action of carboxypeptidase (10). Recently a more general method has been developed by Chibnall and Rees 
(2), in which the free carboxyl groups are reduced to hydroxyl groups and the resulting amino alcohols identified. When applied to insulin the presence of alanine and asparagine $C$ terminal residues was confirmed.

\section{Amino Acid Sequence in the Phenylalanyl Chain}

The results with the $N$-terminal sequences showed for the first time that the fractions $\mathrm{A}$ and $\mathrm{B}$ of the oxidized insulin were essentially homogeneous preparations of single polypeptide chains containing 20 and 30 residues, respectively. It thus seemed that it would be worth while to investigate the peptides produced on partial hydrolysis in order to determine amino acid sequences within the chains (20). The method was essentially that of Consden, Gordon, Martin, and Synge (3), which made use of paper chromatography to separate the very complex peptide mixture produced.

Fraction B was subjected to partial hydrolysis with concentrated acid, and preliminary fractionation of the mixture into less complex groups of peptides was carried out by ionophoresis, ion-exchange chromatography, and adsorption on charcoal. The fractions thus produced contained up to about 20 different peptides, and could be further fractionated by twodimensional paper chromatography. The various peptide spots were then cut out, eluted with water, and taken to dryness. The residue was then hydrolyzed and the amino acids present identified. To determine the order of amino acids in the peptides it was necessary to identify the $N$-terminal residues. This was in general carried out by the DNP technique which could be adapted to work on a small scale. In this way the complete structure of dipeptides could be determined, and a considerable amount of information about longer peptides obtained. In certain cases the structure of higher peptides was determined by degrading them further by partial hydrolysis to dipeptides. With such methods 22 dipeptides, 13 tripeptides, and 8 higher peptides were identified in the partial acid hydrol- 
TABLE III

Sequences in Fraction B

1. $\mathrm{Phe} \cdot \mathrm{Val} \cdot \mathrm{Asp} \cdot \mathrm{Glu} \cdot \mathrm{His} \cdot \mathrm{Leu} \cdot \mathrm{CySO}_{3} \mathrm{H} \cdot \mathrm{Gly}$

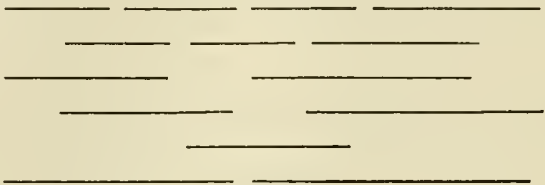

2. Gly $\cdot$ Glu $\cdot$ Arg $\cdot$ Gly

3. Thr $\cdot$ Pro $\cdot$ Lys $\cdot$ Ala

4. $\mathrm{Tyr} \cdot \mathrm{Leu} \cdot \mathrm{Val} \cdot \mathrm{CySO}_{3} \mathrm{H} \cdot \mathrm{Gly}$

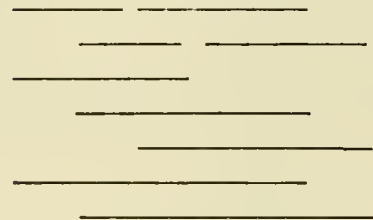

5. $\mathrm{Ser} \cdot \mathrm{His} \cdot \mathrm{Leu} \cdot \mathrm{Val} \cdot \mathrm{Glu} \cdot \mathrm{Ala}$

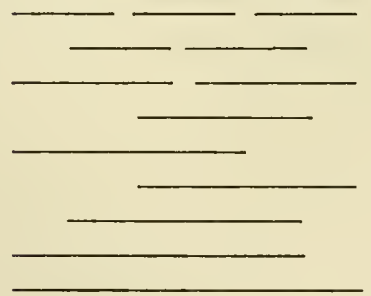

yzate. Hydrolyzates prepared by the action of alkali and of dilute acid were also investigated, but only a few different peptides were obtained. From the combined results it was possible to determine the five sequences listed in Table III as being present in fraction B. The structure of the peptides from which the sequence was deduced is indicated by underlining. 
Thus for instance an unbroken line beneath Phe.Val indicates that the peptide Phe-Val had been detected. These sequences account for all the residucs of fraction $B$ except for one leucine and tyrosine and two phenylalanine residues.

The chief obstacle to working out the complete amino acid sequence from these small peptides was the fact that in fraction $\mathrm{B}$ the less polar residues (phenylalanine, leucine, and tyrosine) are grouped closely together and give rise on hydrolysis to a large number of similar nonpolar peptides which are particularly difficult to fractionate one from another. A further obstacle with both fractions A and B was the great lability of the bonds involving the amino groups of serine and threonine residues, since these bonds were never found intact in any peptide from a partial acid or alkaline hydrolyzate.

In order to determine the complete sequence it was necessary to study hydrolyzates obtained by the action of proteolytic enzymes (21), which show an entirely different and much more exacting specificity than does acid and give rise to a relatively simple mixture of longer peptides containing up to about 15 residues. Such peptides could be separated by paper chromatography although less satisfactorily than the smaller peptides, and were studied by similar methods. Recently paper ionophoresis (13) has proved most useful for the fractionation of larger peptides. In Figure 1 is shown a tracing of an ionophoretic fractionation of a peptic hydrolyzate of fraction $\mathrm{B}$, where the different peptides show a good separation and form well-defined bands; this is frequently not the case with paper chromatography.

As an example we can consider the peptide Bp3 and see what further information may be deduced from its composition concerning the sequence of fraction B. It contained a phenylalanine $N$-terminal residue and the amino acids $\left[\mathrm{CySO}_{3} \mathrm{H}\right.$, Asp, Glu, Ser, Gly, Val, Leu, His], of which the most significant are aspartic acid and serine. Fraction B contains only one aspartic acid residue which is present in the $N$-terminal sequence (sequence 1, Table III). This sequence must be intact in 
peptide $\mathrm{Bp} 3$, since all the requisite amino acids are present. Serine, which is found only in sequence 5 , is also present in $\mathrm{Bp} 3$; whereas amino acids characteristic of the other three sequences

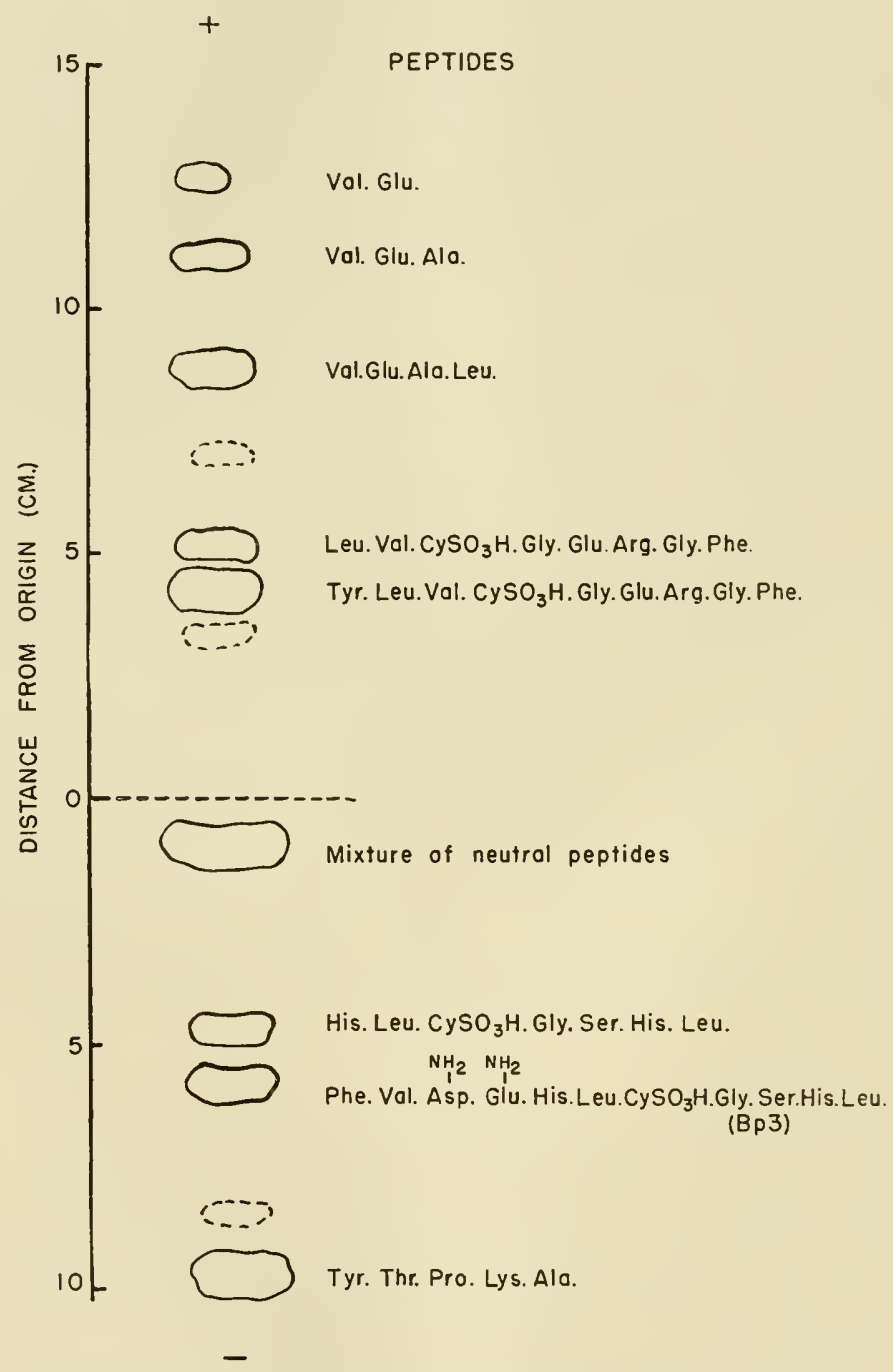

Fig. 1. Ionophoresis of peptic hydrolyzate of fraction $\mathrm{B}$ at pH 6.4 and 1000 v. (3 hours). 
(Arg (2); Thr, Pro, Lys (3); Tyr (4)) are absent. It was thus established that sequence 5 was combined to the $N$-terminal sequence through a Gly.Ser linkage. That no other residue could come between the glycine and serine residues was also indirectly demonstrated. From the results with peptic and chymotryptic hydrolyzates it was possible to deduce the three sequences shown in Table IV.

TABLE IV

Sequences in Fraction B

1. $\mathrm{Phe} \cdot \mathrm{Val} \cdot \mathrm{Asp} \cdot \mathrm{Glu} \cdot \mathrm{His} \cdot \mathrm{Leu} \cdot \mathrm{CySO}_{3} \mathrm{H} \cdot \mathrm{Gly} \cdot \mathrm{Ser} \cdot \mathrm{His} \cdot \mathrm{Leu} \cdot \mathrm{Val} \cdot \mathrm{Glu} \cdot \mathrm{Ala} \cdot \mathrm{Leu} \cdot-$ Tyr

2. $\mathrm{Tyr} \cdot \mathrm{Leu} \cdot \mathrm{Val} \cdot \mathrm{CySO}_{3} \mathrm{H} \cdot \mathrm{Gly} \cdot \mathrm{Glu} \cdot \mathrm{Arg} \cdot \mathrm{Gly} \cdot \mathrm{Phe} \cdot \mathrm{Phe}$

3. Tyr $\cdot$ Thr $\cdot$ Pro $\cdot$ Lys $\cdot$ Ala

When fraction B was treated with trypsin, a peptide (Bt2) was obtained with glycyl $N$-terminal residue and the amino acids [Tyr, Phe, Thr, Pro, Lys]. The last three are found only in sequence 3 (Table IV). The phenylalanine present cannot be the one that is in the $N$-terminal position of fraction $\mathrm{B}$, since it is not $N$-terminal in peptide $\mathrm{Bt} 2$. It must therefore represent the two phenylalanine residues from the $C$-terminal group of sequence 2 (Table IV), thus establishing that sequence 2 is joined to sequence 3 and that the structure of fraction $B$ is as shown in Figure 2.

Besides establishing the structure, the study of enzymic hydrolyzates provided considerable information about the specificity of the proteolytic enzymes used. The main sites of action of these enzymes are shown in Figure 2. Trypsin and chymotrypsin were found to be highly specific and to split only those types of bonds which earlier work with synthetic substrates had indicated would be susceptible. Pepsin, on the other hand, was found to have a much wider specificity which it was difficult to define in terms of the amino acids involved in the susceptible bonds. It seems possible that the specificity of pepsin may not in fact be determined in this way, but rather that the 
F. SANGER

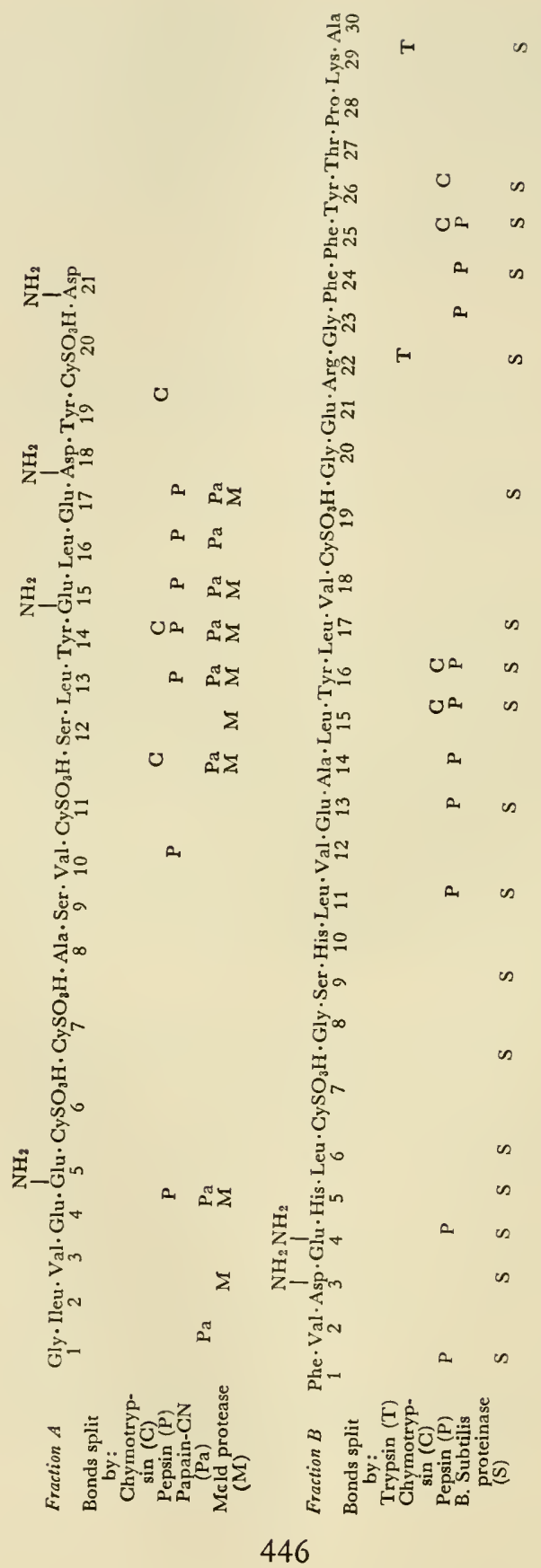

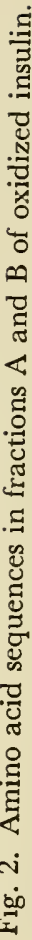


enzyme may become attached to the less polar parts of the substrate and catalyze a more general hydrolysis in that area.

The action on fraction B of the protease obtained from Bacillus subtilis, which catalyzes the specific conversion of ovalbumin to plakalbumin, has been studied by Tuppy (25). These results are also shown in Figure 2. Clearly in its action on fraction $B$ it appears to be very nonspecific in contrast to its action on ovalbumin.

\section{Amino Acid Sequence in the Glycyl Chain}

Although it contains fewer residues, the elucidation of the structure of fraction A proved more difficult than that of fraction B. In fraction B there are several residues such as aspartic acid, serine, and arginine that occur only once in the chain and assist greatly in the interpretation of results. Fraction $A$, on the other hand, contains very few such residues and has relatively large amounts of cysteic acid, glutamic acid, and leucine; this made it necessary to obtain a higher proportion of larger peptides in order to work out the complete sequence.

Particular difficulty was encountered with the large number of cysteic acid peptides, especially those containing two such residues, which are very water-soluble, and could not be well separated by paper chromatography. They could, however, be fractionated by paper ionophoresis in acetic acid $(p \mathrm{H} 2.75)$, for under these conditions the $-\mathrm{SO}_{3} \mathrm{H}$ but not the $-\mathrm{COOH}$ groups are charged. Thus peptides free of cysteic acid residues are basic owing to the positively charged amino group, whereas those containing one cysteic acid residue are neutral and may be separated as a group free from all other peptides; those with two are acidic and may be fractionated according to their rate of migration towards the anode. More recently, the separation has been carried out in a pyridine acetate buffer at $p \mathrm{H} 3.3$, where some fractionation of the individual cysteic acid peptides is obtained. Figure 3 is a diagram of a fractionation of a partial hydrolyzate: of fraction A obtained under these conditions. 
From the study of partial hydrolyzates of fraction A obtained by the action of acid, pepsin, and chymotrypsin the sequence indicated in Figure 2 was deduced $(18,19)$. Hydrolyzates obtained by the action of papain and a mold protease were also studied, largely in connection with the location of amide resi-

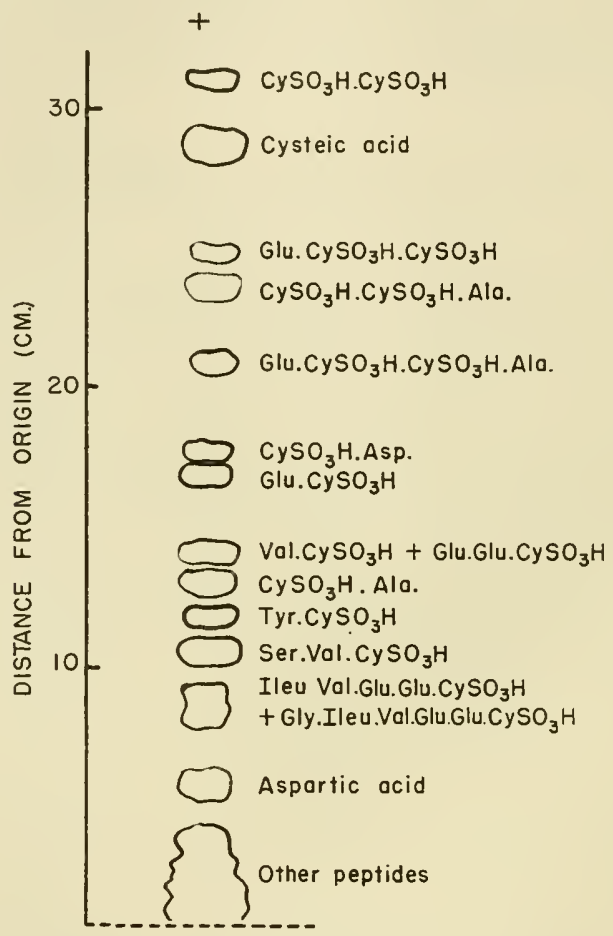

Fig. 3. Ionophoresis of acid hydrolyzate of fraction $\mathrm{A}$ at $p \mathrm{H} 3.3$ and 1500 v. (2 hours).

dues and also with the purpose of confirming the structure. From Figure 2 it can be seen that these two latter enzymes exhibit a rather wide specificity, resembling in some ways the action of pepsin, though definitely different in detail. An unexpected finding was that the bond $\mathrm{CySO}_{3} \mathrm{H} \cdot \operatorname{Ser}(\mathrm{A}, 11-12)$ was split by chymotrypsin. The only other susceptible bonds are 
those involving the carboxyl group of aromatic acid residues, as would be expected from studies with synthetic substrates. It may be that this is due to the presence of another enzyme, though such a structure does not occur in natural proteins. Unoxidized insulin, where residue 11 was present as CyS, was not attacked in this way.

\section{Amide Groups}

When insulin is hydrolyzed with acid there are liberated besides the amino acids 6 moles of ammonia, which are present as amide groups on the $\omega$-carboxyl groups of some of the glutamic and aspartic acid residues. The method of reduction of Chibnall and Rees (2) could be used to determine the distribution of the amide groups between these two types of residues, and in this way it was shown that the three aspartic acid residues were all present as amides, whereas three glutamic acids were amidized and the other four have free $\gamma$-carboxyl groups.

In order to locate these amide groups on the individual dicarboxylic acid residues, two methods were used which depend on the fact that when hydrolysis is carried out with enzymes the amide groups remain intact in the peptides. From the ionophoretic behavior of such peptides it was in general possible to determine whether the carboxyl groups were free to ionize or bound as amides. For instance the peptide containing cysteic and aspartic acids which was obtained from a chymotryptic hydrolyzate of fraction A migrated towards the anode considerably more slowly than the $\mathrm{CySO}_{3} \mathrm{H} \cdot$ Asp obtained from an acid hydrolyzate. It must then have been $\mathrm{CySO}{ }_{3} \mathrm{H} \cdot \mathrm{Asp}\left(\mathrm{NH}_{2}\right)$, with the $C$-terminal aspartic acid residue amidized. Similarly the Gly·Ileu.Val.Glu obtained by the action of papain migrated as an acid, thus establishing that the glutamic acid residue in position A,4 has a free carboxyl group. Positions of the various amide groups located in this way are indicated in Figure 2.

A second method was to determine the amide content of 
the peptides from enzymic digests, by estimating the ammonia liberated from them by acid hydrolysis. Certain difficulties were encountered owing to the small amounts of peptides available, but in general results by the two methods were concurrent.

\section{The Disulfide Bridges}

The oxidized insulin fractions contained cysteic acid residues in place of the three cystine residues of the original insulin. In order to find the distribution of the disulfide bridges of these cystine residues, intact insulin was submitted to partial hydrolysis. The resulting cystine-containing peptides were fractionated from one another and oxidized, and the cysteic acid peptides so obtained were identified. Since the latter peptides could be completely separated from other peptides by ionophoresis at $p \mathrm{H} 3.3$ (Figure 3), it was unnecessary to obtain the cystine peptides free of non-cystine-containing peptides, provided the former could be separated one from another.

In the initial experiments concentrated $\mathrm{HCl}$ was used for hydrolysis, and a large number of cystine peptides were identified in this way. However, the results could not be interpreted in terms of a unique structure for insulin, and it appeared that the disulfide bonds had become mixed up during the procedure. This was found to be due to an interchange reaction of one disulfide with another:

$$
\mathrm{R}_{1} \mathrm{SSR}_{2}+\mathrm{R}_{3} \mathrm{SSR}_{4} \leftrightharpoons \mathrm{R}_{1} \mathrm{SSR}_{3}+\mathrm{R}_{2} \mathrm{SSR}_{4} \text {, etc. }
$$

This reaction was studied using as model system a mixture of cystine and bis-DNP-cystine. On interchange they gave rise to mono-DNP-cystine, which was water-soluble and could thus be estimated independently of the ether-soluble bis-DNP-cystine.

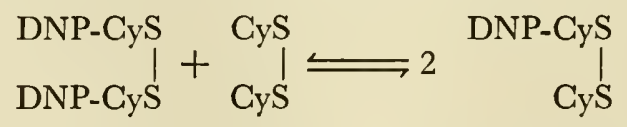

A similar interchange took place in neutral solution. This 
was catalyzed by - $\mathrm{SH}$ compounds and probably is due to initial hydrolysis of the disulfide and a subsequent chain reaction catalyzed by the - $\mathrm{SH}$ compound so produced:

$$
\begin{gathered}
\mathrm{R}_{1} \mathrm{SSR}_{2} \stackrel{\mathrm{OH}^{-}}{\longrightarrow} \mathrm{R}_{1} \mathrm{~S}^{-}+\mathrm{R}_{2} \mathrm{SOH} \\
\mathrm{R}_{1} \mathrm{~S}^{-}+\mathrm{R}_{3} \mathrm{SSR}_{4} \longrightarrow \mathrm{R}_{1} \mathrm{SSR}_{3}+\mathrm{R}_{4} \mathrm{~S}^{-} \text {etc. }
\end{gathered}
$$

At $p \mathrm{H} 7$ to 8 the reaction is not very rapid, so it was possible to hydrolyze insulin with enzymes without any extensive mixing up of the disulfide bonds. In this way the position of one of the bridges could be found. Thus for instance the soluble material from a chymotryptic hydrolyzate was subjected to ionophoresis. The cystine peptides were identified by treating a strip with cyanide-nitroprusside reagent (23), and the bands were oxidized and again fractionated by ionophoresis. From one such cystine peptide two cysteic acid peptides were produced with the following amino acid composition:

$$
\begin{gathered}
{\left[\mathrm{CySO}_{3} \mathrm{H}, \mathrm{Asp}\right]} \\
{\left[\mathrm{CySO}_{3} \mathrm{H}, \mathrm{Glu}, \mathrm{Gly}, \text { Val, Leu, Phe, Arg }\right]}
\end{gathered}
$$

These peptides had been found before in the chymotryptic hydrolyzates of oxidized fractions and were $\mathrm{CySO}{ }_{3} \mathrm{H} \cdot \mathrm{Asp}\left(\mathrm{NH}_{2}\right)$ from the A chain, Leu.Val. $\mathrm{CySO}_{3} \mathrm{H} \cdot \mathrm{Gly} \cdot \mathrm{Glu} \cdot \mathrm{Arg} \cdot \mathrm{Gly} \cdot \mathrm{Phe} \cdot \mathrm{Phe}$ from the $\mathrm{B}$ chain. The structure of the cystine peptide was thus:

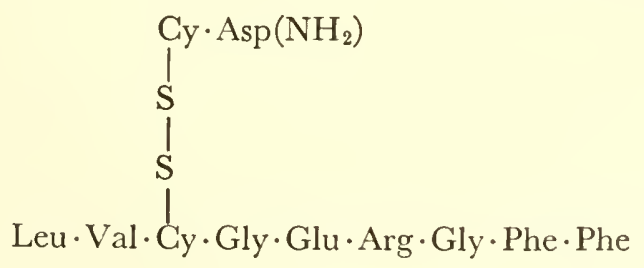

establishing the presence of a disulfide bridge between positions $\mathrm{A} 20$ and $\mathrm{B} 19$ in insulin. The location of the same $-\mathrm{S}-\mathrm{S}-$ bond was confirmed by the structure of a number of other peptides from chymotryptic and peptic hydrolyzates. The remaining two cystine residues were present in the insoluble material (core) from the chymotryptic hydrolyzate and no enzyme could 
be found that would split between these residues. It was thus necessary to use acid hydrolysis to identify these two disulfide bridges.

In contrast to that found in neutral solution, the interchange reaction in acid was found to be markedly inhibited by $\mathrm{SH}$ compounds. The mechanisms of the two reactions thus appear to be different. Moreover, it was possible to make use of this inhibition to find conditions in which insulin could be hydrolyzed to fairly small peptides in acid without any appreciable interchange occurring. To simplify the position somewhat the chymotryptic "core" which contained only two of the three cystine residues was studied. In Table $\mathrm{V}$ are listed the various

\section{TABLE V}

Cysteic Acid Peptides Found in the Oxidized Partial Hydrolyzate of the CHYMOTRYPTIC "CORE" OF INSULIN

$\mathrm{CySO}_{3} \mathrm{H}$
$\mathrm{CySO}_{3} \mathrm{H} \cdot \mathrm{Gly}$
$\mathrm{Leu} \cdot \mathrm{CySO}_{3} \mathrm{H}$
$\mathrm{Leu} \cdot \mathrm{CySO}_{3} \mathrm{H} \cdot \mathrm{Gly}$
$\mathrm{His} \cdot \mathrm{Leu} \cdot \mathrm{CySO}_{3} \mathrm{H}$
$\mathrm{His} \cdot \mathrm{Leu} \cdot \mathrm{CySO}_{3} \mathrm{H} \cdot \mathrm{Gly}$
$\mathrm{Glu} \cdot \mathrm{CySO} \mathrm{H}_{3}$
$\mathrm{Glu} \cdot \mathrm{Glu} \cdot \mathrm{CySO}_{3} \mathrm{H}$

$\mathrm{CySO}_{3} \mathrm{H}$

$\mathrm{CySO}_{3} \mathrm{H} \cdot \mathrm{Gly}$

$\mathrm{Leu} \cdot \mathrm{CySO}_{3} \mathrm{H}$

$\mathrm{Leu} \cdot \mathrm{CySO}_{3} \mathrm{H} \cdot \mathrm{Gly}$

$\mathrm{His} \cdot \mathrm{Leu} \cdot \mathrm{CySO}_{3} \mathrm{H}$

$\mathrm{Glu} \cdot \mathrm{CySO}_{3} \mathrm{H}$

$\mathrm{Glu} \cdot \mathrm{Glu} \cdot \mathrm{CySO}_{3} \mathrm{H}$

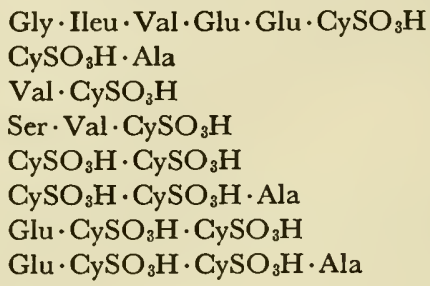

cysteic acid peptides which were present in the hydrolyzate after oxidation. It can be calculated that 95 different cystine peptides would be present in the unoxidized hydrolyzate to give rise to the 16 cysteic acid peptides. Of these 95,12 contain a half cystine residue (CyS-) and thus provide no information about the distribution of disulfide bonds, 72 contain the group CyS.CyS and are also of no use, whereas 11 contain a peptide on either side of the disulfide bond and provide the information required. To obtain as little of the peptides with CyS.CyS as possible, a fairly long period of hydrolysis was necessary, as this group was particularly stable. The conditions eventually chosen were to boil the insulin 45 minutes with $10 \mathrm{~N} \mathrm{H}_{2} \mathrm{SO}_{4}$ in $30 \%$ acetic acid containing $0.005 M$ thioglycolic acid. 
Various methods were studied for fractionating the cystine peptides produced. Conditions were required that would not lead to redistribution of the disulfide bonds and that would provide sufficient material for identification of the oxidation products. Paper chromatography seemed unsatisfactory from both these points of view, and the best results were obtained using high-voltage paper ionophoresis (13) in pyridine-acetate buffers at $p \mathrm{H} 3.3$ and 6.4. At the latter $p \mathrm{H}$ a good separation of the neutral peptides may be obtained owing to the unusually low $p \mathrm{~K}$ of the amino groups of cystine in peptides (6). Thus whereas most peptides of mono-amino acids are neutral at $p \mathrm{H}$ 6.4 , those which have a free amino group on a cystine residue are slightly acidic and those (such as cystinyl diglycine) with two such groups are even more acidic. In general ionophoresis at two different $p \mathrm{H}$ values yielded peptides of reasonable purity, and their structure was determined by oxidation.

In Table VI are listed the significant peptides detected in this work. Although the distribution of the disulfide bridges in insulin could be deduced from any one of these peptides, it was

TABLE VI

Cystine Peptides Identified in Partial Agid Hydrolyzate of Insulin

\begin{tabular}{|c|c|c|}
\hline Cy $\cdot$ Gly & Leu $\cdot \mathrm{Cy}$ & Leu $\cdot$ Cy $\cdot$ Gly \\
\hline $\mathrm{S}$ & $\mathrm{S}$ & $\mathrm{S}$ \\
\hline S & S & S \\
\hline $\mathrm{Cy} \cdot \mathrm{Ala}$ & $\mathrm{Cy} \cdot \mathrm{Ala}$ & Cy $\cdot$ Ala \\
\hline His - Leu - Cy · Gly & Val. Cy & $\mathrm{Ser} \cdot \mathrm{Val} \cdot \mathrm{Cy}$ \\
\hline $\mathrm{S}$ & S & S \\
\hline $\mathrm{S}$ & $\mathrm{S}$ & S \\
\hline $\mathrm{Cy} \cdot \mathrm{Ala}$ & Glu $\cdot$ Cy & $\mathrm{Glu} \cdot \mathrm{Cy}$ \\
\hline
\end{tabular}

necessary to investigate all the cystine peptides present in significant amount to be sure that no rearrangement of bonds had occurred. The peptides all agree and establish the structure of insulin given in Figure 4.

This structure is based on a value of 6000 for the molecular weight but does not prove this value, since the structure shown 
F. SANGER

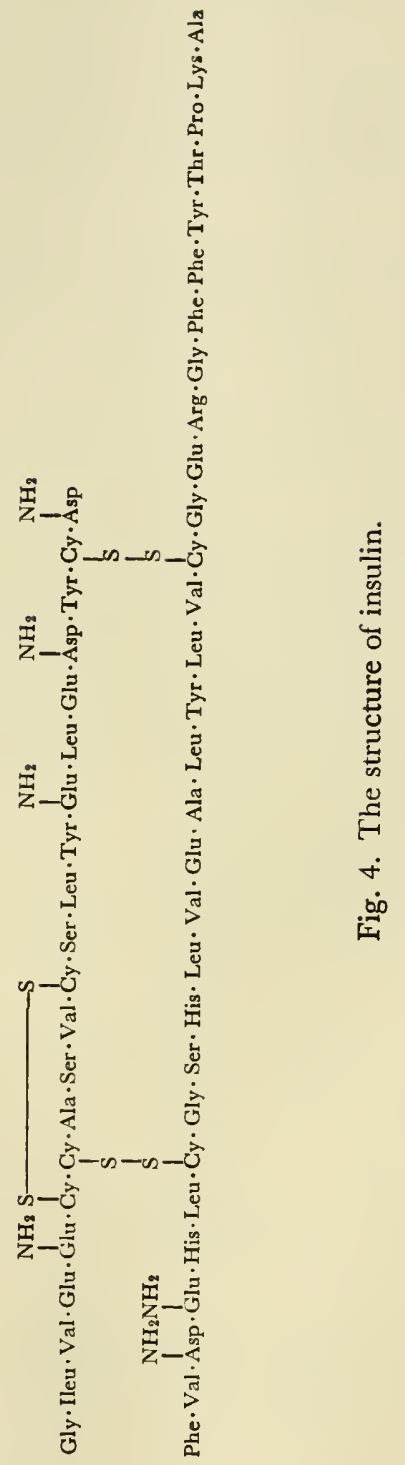


in Figure 5, which has a molecular weight of 12,000, would also fit our experimental results. However, it is most improbable that such a molecule with two disulfide bonds joining the two identical halves would dissociate under the conditions used by Harfenist and Craig to demonstrate a molecular weight of 6000 .

The structure given embodies a disulfide ring joining the two CyS residues in positions $\mathrm{A} 6$ and $\mathrm{A} 11$. This ring is the same size as the disulfide rings found in oxytocin $(26,27)$ and vasopressin $(1,28)$. The three hormones have very different biological functions, but the presence of this common structure

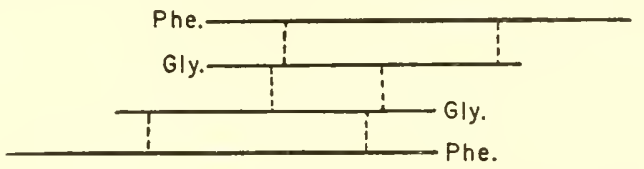

Figure 5.

may have some physiological significance and may be reflected in a mechanism that is common to these polypeptide hormones. The ring disulfide does not show any particular chemical reactivity in vitro and appears to be more stable to reduction and in the disulfide interchange reaction than are cystine or oxidized glutathione. Nothing, however, is known about its reactivity in vivo.

To form the disulfide ring the polypeptide chain must be oriented in such a way that the two CyS residues are close together. This imposes definite limitations on the various configurations that can be considered for this part of the chain. The popular $\alpha$-helix with 3.7 residues per turn can clearly not be present here, and it will be interesting to see what other structures are possible. The stability of the ring and the fact that reduced oxytocin can be readily converted to the disulfide ring form by oxidation suggest that no great distortion of the stable configuration of the polypeptide chain is brought about by formation of the disulfide bond. 
Both oxytocin and partial hydrolyzates of insulin exhibit strong activity as strepogenin, a growth factor for Lactobacillus casei (29). Oxytocin and insulin have no amino acid sequences in common, the only common feature being the disulfide ring, which may thus again assume a biological role.

The structure given in Figure 4 is deduced as the only one that is consistent with all the experimental findings, assuming certain general principles of protein chemistry such as the peptide hypothesis. Although our results do not prove these principles, they do lend considerable support to them. If indeed insulin were not composed solely of $\alpha$-amino acids bound together by peptide linkages and disulfide bonds, one would have expected to find some inconsistency in the results, and it would probably not have been possible to deduce a unique structure.

Various workers have suggested from time to time that other types of structures and bonds such as $\boldsymbol{\gamma}$-glutamyl bonds and diketopiperazine structures may be present in small amounts in proteins. Such structures do exist in some of the bacterial polypeptides, but the evidence for their occurrence in proteins is based largely on the results of rather violent degradative procedures where it is impossible to predict all the ways in which the various amino acid side chains would react. Indeed it would seem unlikely that a mechanism of protein synthesis which specifically joins together amino acids by the peptide bond would produce other types of bonds in occasional positions in protein chains. Thus, until more definite evidence to the contrary is produced, we are probably justified in concluding from the results with insulin that proteins in general will be found to obey the simple laws of protein chemistry and that they are composed of polypeptide chains having a unique arrangement of amino acids. This arrangement differs from protein to protein but does not differ from one molecule to another molecule of the same protein. Each position in the insulin chains is occupied by one and by only one residue, at least to within the limits of the methods used. This implies that all proteins are synthesized by a process which joins the amino acids together by some common 
mechanism, but is unique for each protein in determining the amino acid sequence and possesses in this respect a very high degree of specificity. Such specificity is somewhat difficult to conceive. The mechanism must be able to distinguish not only between a valine and an isoleucine residue, but also uniquely for all the other amino acids. Few enzymes show such a high specificity, and unless the factors involved are very different from those involved in enzyme action a one-stage mechanism for protein synthesis would hardly be expected to give rise to a protein of unique structure. A greater specificity may, however, be expected from a multistage process where differences in reaction rates become magnified through successive stages (see ref. 5). Clearly the present results can provide little information about the mechanisms of protein syntheses, but they do provide certain limitations on those who are speculating on the subject.

The determination of the structure of insulin raises the possibility of similar studies on other proteins. From a consideration of the end groups of a large number of proteins it appears that the chains of insulin with 21 and 30 residues, repectively, are uniquely short. Other simple proteins such as ribonuclease, lysozyme, or hemoglobin contain chains of about 100 residues, whereas many have much longer chains. So the question arises as to whether the methods used for insulin will be sufficient to elucidate the complete structure of these more complex proteins, or whether entirely new methods will have to be developed. In the chains of insulin there are several amino acids that occur only once in the molecule and these greatly help in deducing the structure. In a chain of 100 residues there will be very few, if any, such residues, whereas many will occur 5 to 10 times. This will make it extremely difficult to interpret the results obtained with nonspecific types of degradation, such as acid or peptic hydrolysis, and it will be necessary to obtain a greater number of longer peptides to provide a unique structure. Not only do longer peptides exist in much more complex mixtures than smaller ones but also they are more difficult to 
fractionate. It would seem that the most hopeful method of attacking these larger proteins would be by an initial specific degradation to polypeptide chains of up to 30 residues long, which could then be studied by the methods used for insulin. It should not, however, be assumed that these methods will automatically yield the structure of any polypeptide of 20 to 30 residues long. Special problems are likely to be encountered, as they were in the insulin chains, and will have to be solved by special methods. It does, however, appear that the main lines of progress in this field will be dependent on finding specific methods for degrading proteins and techniques for fractionating the large polypeptides so produced. The enzymes trypsin and chymotrypsin are at present the most specific hydrolyzing agents and will undoubtedly be of great use in this respect, though it is doubtful if they alone will be sufficient for the more complex proteins. Work is at present actively in progress in this field, and it is evident that in the near future we will learn much more about the detailed structure of proteins and of the many biological processes which depend upon their structures.

\section{References}

1. Acher, R., and J. Chauvet, Biochim. et Biophys. Acta, 12, 487 (1953).

2. Chibnall, A. C., and M. W. Rees, Biochem. J. (London), 52, iii (1952).

3. Consden, R., A. H. Gordon, A. J. P. Martin, and R. L. M. Synge, Biochem. J. (London), 41, 596 (1947).

4. Fredericq, E., Nature, 171, 570 (1953).

5. Fruton, J. S., II Congrès International de Biochimie, Symposium sur la Biogénèse des Protéines, p. 5 (1952).

6. Greenstein, J. P., F. W. Klemperer, and J. Wyman, J. Biol. Chem., 129, 681 (1939).

7. Gutfreund, H., Biochem. J. (London), 42, 544 (1948).

8. Harfenist, E. J., and L. C. Craig, J. Am. Chem. Soc., 74, 3087 (1952).

9. Harfenist, E. J., J. Am. Chem. Soc., 75, 5528 (1953).

10. Harris, J. I., J. Am. Chem. Soc., 74, 2944 (1952).

11. Kupke, D. W., and K. Linderstrøm-Lang, Biochim. et Biophys. Acta, 13, 153 (1954).

12. Lens, J., Biochim. et Biophys. Acta, 3, 367 (1949).

13. Michl, H., Monatsh. Chem., 82, 489 (1951). 
14. Sanger, F., Biochem. J. (London), 39, 507 (1945).

15. Sanger, F., Biochem. J. (London), 44, 126 (1949).

16. Sanger, F., Biochem. J. (London), 45, 563 (1949).

17. Sanger, F., Ann. Repts. Progr. Chem. (Chem. Soc. London), 45, 283 (1949).

18. Sanger, F., and E. O. P. Thompson, Biochem. J. (London), 53, 353 (1953).

19. Sanger, F., and E. O. P. Thompson, Biochem. J. (London), 53, 366 (1953).

20. Sanger, F., and H. Tuppy, Biochem. J. (London), 49, 463 (1951).

21. Sanger, F., and H. Tuppy, Biochem. J. (London), 49, 481 (1951).

22. Tanford, C., and J. Epstein, J. Am. Chem. Soc., 76, 2163 (1954).

23. Toennies, G., and J. J. Kolb, Anal. Chem., 23, 823 (1951).

24. Tristram, G. R., Advances in Protein Chem., 5, 84 (1949).

25. Tuppy, H., Monatsh. Chem., 84, 996 (1953).

26. Tuppy, H., and H. Michl, Monatsh. Chem., 84, 1011 (1953).

27. du Vigneaud, V., C. Ressler, and S. Trippett, J. Biol. Chem., 205, 949 (1953).

28. du Vigneaud, V., H. C. Lawler, and E. A. Popenoe, J. Am. Chem. Soc., 75, 4880 (1953).

29. Woolley, D. E., and R. B. Merrifield, J. Am. Chem. Soc., 76, 316 (1954). 


\section{A NEW CONCEPT OF RIBONUCLEIC ACIDS*}

WALDO E. COHN, Biology Division, Oak Ridge National Laboratory, Oak Ridge, Tennessee

When progress in a particular fragment of science is viewed in retrospect, there seem to be periods when advances are so few and widely separated as to give an impression of finality, and other periods when, again in retrospect, new discoveries and interpretations come at a rate which seems to imperil accuracy of experimentation and of interpretation alike. It is tempting to draw an analogy between this semidiscontinuous process and the biological one of gestation and birth. However, this implies a degree of smoothness of progress which, on close inspection, is not warranted. Perhaps a better analogy is that of geyser eruption, in which a quiet period is followed by an apparently uncoordinated discharge of material.

In specific reference to the changes in our concept of the structure of ribonucleic acid (RNA) since 1949, this analogy might be pushed even further to include the mild play of the water between eruptions and the autocatalytic nature of the eruption itself. Certainly there were indications, prior to 1949, that the structure proposed in 1935 (45) (see Figure 1a), linking the ribose moieties with diesterified phosphate from the second

* Manuscript prepared under Contract No. W-7405-eng-26 for the Atomic Energy Commission. 
carbon of one to the third carbon of the next $\left(2^{\prime}, 3^{\prime}\right.$ phosphodiester link), could not accommodate the facts which were accumulating. Chief among these were the hydrolysis of RNA to nucleosides and inorganic phosphate by snake venoms (25-27) containing a phosphomonoesterase specific for $5^{\prime}$ nucleotides, and the reduction of periodate by the mixture of

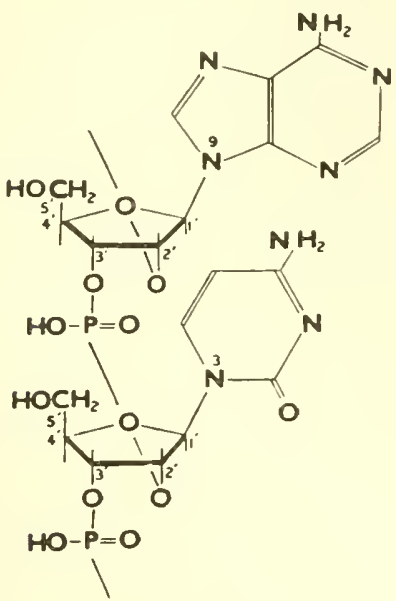

(a)

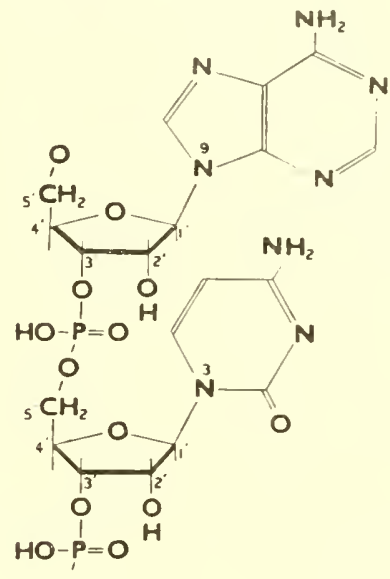

(b)

Fig. 1. Polynucleotide linkages: (a) section of $2^{\prime}, 3^{\prime}$ polynucleotide; (b) section of $3^{\prime}, 5^{\prime}$ polynucleotide.

ribonuclease-produced polynucleotides after removal of end phosphate groups by a phosphomonoesterase (54-56). The venom experiments indicated that $5^{\prime}$ nucleotides are present in the course of the degradation by the mixture of enzymes present in whole venom, and present to an extent approaching the total nucleotide in the digest. Barring transphosphorylation, this was evidence for a $2^{\prime}$ (or $3^{\prime}$ ) to $5^{\prime}$ phosphate linkage. A similar conclusion was warranted by the second observation that ribonuclease produces polynucleotides, each one possessing a monoesterified phosphate end group, which are susceptible to periodate oxidation after removal of these end groups by a monoesterase, and to a degree equivalent to the phosphate removed. Since each such dephosphorylated polynucleotide must retain one phosphate link to the adjacent nucleotide 
residue, this remaining link can only be through the $5^{\prime}$ hydroxyl group of the terminal ribose. Again, barring transphosphorylation, this was evidence for the existence of linkages other than $2^{\prime}$ or $3^{\prime}$ in at least $40 \%$ of the pyrimidine nucleotides.

There were other, less direct warnings that all was not well with the $2^{\prime}, 3^{\prime}$ hypothesis. To explain the enormous difference between RNA and DNA (deoxyribonucleic acid), the latter of $3^{\prime}, 5^{\prime}$ structure (Figure $1 b$ ), in susceptibility to alkaline hydrolysis, it was necessary to assume a special alkali sensitivity on the part of nucleoside 2 -phosphates. However, the presumed synthetic 2 ' nucleotides showed no special lability to alkali. (These substances are now known to have been $5^{\prime}$ nucleotides.) Then, too, the first $\mathrm{x}$-ray diffraction studies (2), coupled with consideration of bond angles and distances, gave support to a $3^{\prime}, 5^{\prime}$ (or $2^{\prime}, 5^{\prime}$ ) linkage and not to the $2^{\prime}, 3^{\prime}$.

The strength of the $2^{\prime}, 3^{\prime}$ hypothesis, which successfully withstood the challenge of the foregoing contradictory evidence, derived largely from the following arguments: (1) the (seeming) recovery of only one nucleotide of each base from alkaline hydrolyzates; (2) the (apparent) identification of the sugar phosphate residue as ribose 3-phosphate; (3) the absence of the acid-stable ribose 5-phosphate (or its pyrimidine derivatives) in acid hydrolyzates; (4) the alkali stability of the necessarily $3^{\prime}, 5^{\prime}$ linked DNA. Yet each of these bulwarks was faulty. It is now known that two isomers of each nucleotide (the $2^{\prime}$ and the $3^{\prime}$ ) are produced by alkaline hydrolysis $(16,17)$ and that the earlier identification of ribose 3-phosphate as the sole sugar phosphate was faulty $(39,40)$. Ribose 5-phosphate nucleotides can be produced from RNA by enzymic hydrolysis $(19,20)$ and the alkali lability of RNA, in contrast to DNA, stems from the presence of an unesterified $2^{\prime} \mathrm{OH}$ group, not from a different internucleotide linkage $(8,10,29,63,64)$. These findings, which were made in rapid succession in 1949-1952, fulfilled the neglected prophecies and warnings of earlier date that all was not well with the $2^{\prime}, 3^{\prime}$ hypothesis of RNA structure $(25,55)$.

The application of a new analytical technique, ion-exchange chromatography, to the analysis of the conventional alkali digest 
of RNA inaugurated this succession of events $(16,17)$. Yet the first obscrvation thus made, that each nucleotide existed in two isomeric forms and that one of these was the established (presumably $3^{\prime}$ ) nucleotide, seemingly strengthened the $2^{\prime}, 3^{\prime}$ hypothesis by uncovering what was thought to be the longmissing $2^{\prime}$ isomer. The finding that acid would catalyze the migration of phosphate from the $2^{\prime}$ hydroxyl to the $3^{\prime}$, and vice versa, whereas no such migration took place in alkali, was not at first appreciated as devaluating the older proof of structure of ribose 3-phosphate, which had been produced by acid hydrolysis of purine nucleotides $(43,44)$. This realization came later, after it had been shown that the same RNA samples that yielded, with alkali, 40:60 mixtures of the $2^{\prime}$ and $3^{\prime}$ isomers could be degraded by enzymes to the $5^{\prime}$ isomers $(10,19,20)$.

The finding that alkali produced $2^{\prime}$ and $3^{\prime}$ isomers whereas intestinal phosphatase yielded $5^{\prime}$ isomers seemed at first inconsistent with the phosphodiester concept of RNA structure, unless the coexistence of two different kinds of RNA were assumed, e.g., $2^{\prime}, 3^{\prime}$ and $3^{\prime}, 5^{\prime}$ (or $2^{\prime}, 5^{\prime}$ ). A repetition of the older degradations with snake venom, which had led to the initial postulate of a $3^{\prime}, 5^{\prime}$ structure, indicated that the $5^{\prime}$ linkage was present in large, if not exclusive, amounts. Thus it became necessary to formulate one diester structure which could give rise, under various conditions of hydrolysis, to the three isomeric types found.

This paradox was promptly resolved in favor of a $3^{\prime}, 5^{\prime}$ (or $2^{\prime}, 5^{\prime}$ ) structure from a consideration of the ability of phosphates esterified to one of a pair of cis hydroxyl groups to cyclize to the other or, if in diester linkage, to substitute such a cyclic position for the bridge position (10). Such shifts of phosphate (by acid in the first case, by alkali or acid in the second) had already been demonstrated in the glycerophosphates $(4,5)$ (see Figure 2), which could be considered the simplest analogues of ribose phosphates. It had also been shown, with radiophosphorus (15), that glycerophosphate isomerization was intramolecular, proceeding through a cyclic phosphate intermediate. With respect to the glycerophosphate esters involving, as in 
RNA, doubly esterified phosphate, the evidence indicated that these, upon alkaline hydrolysis, gave rise to this same cyclic phosphate intermediate which subsequently was hydrolyzed to yield both glycerophosphate isomers $(4,5)$.

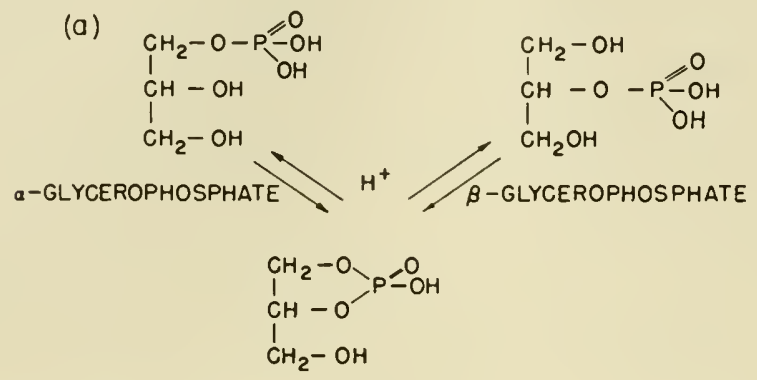

(b)
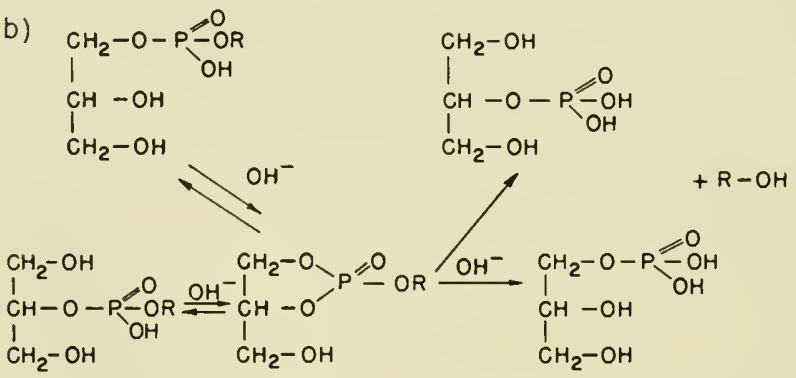

Fig. 2. Phosphomigration in the glycerophosphates, showing intermediate cyclization $(4,5)$ : (a) acid catalyzed phosphomigration in glycerophosphoric acids; intermediate demonstrated (15); (b) acid- or alkalicatalyzed hydrolysis of esterified glycerophosphates with production of both glycerophosphoric acids; intermediate postulated.

Here, then, was a complete parallel to the situation newly uncovered with respect to RNA, regarded as a series of phosphodiesters containing, as in glycerophosphate esters, a free alcoholic hydroxyl cis to the phosphorylated hydroxyl. It was proposed, then (10), that RNA of a $3^{\prime}, 5^{\prime}$ (or $2^{\prime}, 5^{\prime}$ ) linkage could hydrolyze by intermediate cyclization of the phosphate to the $2^{\prime}$ (or $3^{\prime}$ ) 
position, with rupture of the $5^{\prime}$ linkage and thus of the chain. The cyclic esters would subsequently be hydrolyzed randomly to the mixture of the $2^{\prime}$ and 3 ' nucleotides already found.

Although this mechanism was inherently logical, it rested on certain assumptions which it became necessary to verify (as the assumptions of the older hypothesis had not been). The most important of these was that a cyclic $2^{\prime}: 3^{\prime}$ intermediate existed. However, this postulate was promptly verified by synthesis of these compounds (9), by isolation of them from partial hydrolyzates $(46,47)$, and by the testing of both synthetic and isolated phosphodiesters. All this work appeared within the span of one year (1951-1952) and, together with the following correlations, completely established the $3^{\prime}, 5^{\prime}$ (or $2^{\prime}, 5^{\prime}$ ) hypothesis.

The newer structure, to begin with, needs no assumptions of preferential alkali lability of one phosphate bond over the other. Preferential splitting of the $5^{\prime}$ linkage rests on the demonstration of the cyclic $2^{\prime}: 3^{\prime}$ intermediate. The possibility of forming such intermediates simultaneously and randomly throughout the polynucleotide chain gives a logical basis to the observations that RNA is almost completely reduced to nucleotides by mild alkali treatment, whereas DNA, with the same $3^{\prime}, 5^{\prime}$ backbone but no $2^{\prime}$ hydroxyl, is completely resistant to mild alkali. Preferential splitting of the $3^{\prime}$ ( or $2^{\prime}$ ) link by enzymes to yield $5^{\prime}$ nucleotides is demonstrably caused by the specificity of the diesterase enzymes used which also produce $5^{\prime}$ deoxynucleotides from DNA $(14,34)$. The presumed specificity of action has been further verified by the use of synthetic and isolated smaller substrate molecules $(22,49)$. The older observation that RNA polynucleotides reduce periodate only after dephosphorylation could now easily be explained as brought about by the removal of end $3^{\prime}$ phosphates leaving terminal nucleosides esterified only in the $5^{\prime}$ position. Furthermore, the production of nucleosides and $3^{\prime}, 5^{\prime}$ (or $2^{\prime}, 5^{\prime}$ ) diphosphonucleosides in equal amounts in the diesterase degradation of polynucleotide material $(19,20)$ could be accounted for only by a $3^{\prime}, 5^{\prime}$ (or $2^{\prime}, 5^{\prime}$ ) structure. 
The application of paper and ion-exchange chromatographic techniques to the ribonuclease digests of RNA $(49,62)$ permitted the isolation of many of the fragments whose properties had hitherto been known only en bloc. It was soon shown that (1) the pyrimidine mononucleotides are all of the $3^{\prime}$ variety, (2) the polynucleotides end in $3^{\prime}$ pyrimidine nucleotides, and (3) the intervening linkages are of the $3^{\prime}, 5^{\prime}$ (or $2^{\prime}, 5^{\prime}$ ) variety. An extension of great significance was that ribonuclease degradation proceeds through or concomitantly with intermediate $2^{\prime}: 3^{\prime}$ cyclization of pyrimidine nucleoside phosphates which are subsequently degraded by the enzyme to the end products, 3' nucleotides (48). Synthetic substrates for ribonuclease showed that the enzyme hydrolyzes only the esters of $3^{\prime}$ pyrimidine nucleotides through the intermediate $2^{\prime}: 3^{\prime}$ cyclic phosphate, which indicates that the $3^{\prime}$ form probably exists in at least the pyrimidine nucleotides of the original chain (7).

The question as to whether the internucleotide linkage is $3^{\prime}, 5^{\prime}$ or ${ }^{\prime} 2^{\prime}, 5^{\prime}$ or a mixture of both was finally settled by the use of an enzyme from spleen which hydrolyzed RNA to $3^{\prime}$ nucleotides (of both purines and pyrimidines) but without the formation of cyclic nucleotides $(29,30,63)$. The latter observation eliminated consideration of intermediate cyclization, and thus the appearance of $3^{\prime}$ nucleotides (of purines and pyrimidines alike) could be accepted as evidence of the $3^{\prime}, 5^{\prime}$ linkage to the exclusion of $2^{\prime}, 5^{\prime}$.

At the time of the initial postulate of a $3^{\prime}, 5^{\prime}$ (or $2^{\prime}, 5^{\prime}$ ) linkage, necessitated primarily by the degradation of RNA to $5^{\prime}$ nucleotides by enzymes, it was pointed out that the possibility of branching, by phosphorylation of the $2^{\prime}$ (or $3^{\prime}$ ) hydroxyl giving rise to triply esterified ribose, must be considered (10). Attempts to demonstrate, by methylation experiments, the existence in RNA of ribose without free hydroxyls failed to be convincing because of the difficulty of proving that the unmethylated ribose found was not due to failure to methylate a free position (1). Another approach lay in considering the products released from RNA by the action of the diesterase of 
snake venom. These products are largely 5' nucleotides, but equimolar amounts of purine nucleosides and pyrimidine nucleoside $3^{\prime}, 5^{\prime}$-and $-2^{\prime}, 5^{\prime}$-diphosphates (18-20) were also isolated. Although equal amounts of such substances would be expected from the two ends of a simple $3^{\prime}, 5^{\prime}$ (or $2^{\prime}, 5^{\prime}$ ) RNA chain, the large quantities of these would require chain lengths of only seven to ten nucleotides. However, any branches originating in triply esterified ribose and proceeding via the $3^{\prime}, 5^{\prime}$ (or $2^{\prime}, 5^{\prime}$ ) linkage could yield these same products. The difficulty in excluding the possibility of nuclease contamination in the diesterase preparations, which are essentially whole venom minus the $5^{\prime}$ monoesterase activity, has undermined the validity of this argument, as any nuclease present could reduce a long, unbranched chain to short chains which could in turn give rise to the products observed. Plausibility is attached to this latter speculation because the cytidine diphosphate as isolated was shown to be a mixture of $2^{\prime}, 5^{\prime}$ and $3^{\prime}, 5^{\prime}$ isomers, which may have been formed from cytidine $2^{\prime}: 3^{\prime}, 5^{\prime}$-diphosphate, the latter substance arising in turn from nuclease action on a longer chain. Until this source of the diphosphates (and purine nucleosides) can be eliminated from consideration, the diesterase evidence for branching through ribose must be considered equivocal.

Branching originating from triply esterified phosphate has been considered ever since titration experiments became sufficiently accurate to indicate an excess of secondary phosphate groups (that is, singly esterified phosphates having a second ionizable hydrogen with a $p \mathrm{~K}$ value of about 6) over end groups. With increasing attention being paid to the prevention of hydrolytic degradation during the isolation of RNA samples, the amounts of secondary phosphate observed have fallen to less than $10 \%$ of the total phosphate present, but are seemingly still present. The additional observation has been made that the secondary phosphate ionizations are found at the expense of primary, the phosphorus content of the sample being used as the measure of primary ionizations to be expected (57). Since each triply esterified phosphate represents a loss of one primary 
ionization and a gain of one secondary in its place, this has been a favored explanation of the results.

Additional evidence for this hypothesis was at first indicated by the release of small amounts (less than 10\%) of inorganic phosphate from RNA samples by bone phosphatase $(19,20)$. Here again, as in the case of ribose branching just discussed, the ever-present possibility of a nuclease contaminant in the bone phosphatase leads to some reservations about accepting this evidence. It has also been observed that alkaline hydrolysis of RNA previously treated with bone phosphatase leads to the formation of pyrimidine nucleosides in amounts equivalent to the inorganic phosphate released. As in the case of ribose branching discussed, this is consistent with the mode of action of ribonuclease, which causes fission of the phosphodiesters of pyrimidine nucleosides, thus leading to their appearance as unique products upon subsequent hydrolytic cleavage. Therefore, the exclusion of nuclease action is necessary before the enzymic evidence for either type of branching can be accepted. Until then, the most that can be said is that if branching of either type does occur, pyrimidine nucleotides are involved, not purine nucleotides (18).

In arriving at these conclusions, it should be understood that none of the experiments which underlie them are of such quantitative nature as to exclude small (i.e., up to 5\%) amounts of other types of material. The presumption of complete alkali lability, the classic distinguishing characteristic of RNA, has not been quantitatively verified and the persistence of some polynucleotide material beyond a reasonable time limit may indicate a small amount of an alkali-resistant linkage (60). Difficulties in isolating, or even in testing for, all the sugar components of a given preparation, and the necessity of referring all analytical data to phosphorus rather than mass of material, makes difficult the demonstration of a strict $1: 1: 1$ ratio of bases to ribose to phosphate or of the exclusion of all other materials or of other sugars. However, important as these discrepancies and unresolved questions may be, there can be little doubt at 
this time that most of the linkages in RNA are of the $3^{\prime}, 5^{\prime}$ (or $\left.2^{\prime}, 5^{\prime}\right)$ variety, and definitely $3^{\prime}, 5^{\prime}$ in all specimens so far tested by the spleen enzyme referred to earlier $(8,29,30,63)$.

The implications of this structure are many. The similarity in backbone revives speculation as to whether or not there is an interconversion of RNA and DNA; only a reduction at the $\mathrm{C}_{2}$ carbon of ribose (and the methylation of uracil) is necessary to convert one into the other. The spatial considerations which fortify the consideration of DNA as a template for protein (35) now seem applicable to RNA; in the absence of definite knowledge as to which precedes, RNA, DNA, and protein may all be considered as templates of one another. Then there is the possible relation of the $5^{\prime}$ mononucleotides which exist in cytoplasm to consider.

When the $5^{\prime}$ linkage was first demonstrated in RNA, only two varieties of $5^{\prime}$ nucleotides had been identified in tissues: (1) adenosine 5'-phosphate, which occurs alone and in pyrophosphate combination in adenosine triphosphate, di- and tri-phosphopyridine nucleotide, flavinadenine dinucleotide, and coenzyme A; (2) uridine 5'-phosphate, identified in several pyrophosphate combinations in penicillin-treated bacteria (51), and as part of a coenzyme (12). Within the past three years, and at least partially because of the extension of the same ionexchange chromatographic technique to the analysis of the acid-soluble phosphates of tissues, it has become apparent that the 5'-phosphates, -diphosphates, and -triphosphates (all in pyro linkage) of all four ribonucleosides exist generally in tissues $(33,58,59)$. Whereas some of the diphosphates are linked through the pyrophosphate group to another phosphate, as in adenosine triphosphate, some are combined with nonnucleotide groups; examples of this type are now known for cytidine $(3,38)$, uridine $(12,13,21,33,58-61)$, and guanosine $(11,50)$, some having been discovered by ion-exchange chromatography $(3,11,33,59)$. Enzyme-catalyzed equilibria among the triphosphates as well as coenzyme functions of some of these are now known to exist $(53,65)$. The recent demonstra- 
tions of the group transfer activities of guanosine diphosphomannose (50) and of cytidine diphosphocholine (37) extend the list of functional examples of this type of compound from uridine diphosphate derivatives $(12,13,21,36,42,61)$ and the well-known adenosine coenzymes to include at least one representative of each of the four RNA bases. The coenzyme and carrier functions of some of these substances were revealed because modern chromatography made available more highly purified adenosine phosphates, thus exposing the role previously played by an impurity (examples of this type of discovery are: (1) guanosine triphosphate in the succinyl-coenzyme A reaction (53), and in amino acid incorporation in vitro (65); (2) cytidine diphosphocholine in lecithin synthesis (38)). Others have been shown to be substrates for enzymic oxidations or other conversions $(12,21,36,61)$. The possibility that compounds of this type are precursors of RNA, or are in equilibrium with such precursors, is indicated by studies of the rate of incorporation of labeled precursors (32) and by the recent observation of the enzymecatalyzed polymerization of nucleoside diphosphates into polynucleotide structures and the depolymerization (phosphorolysis) of RNA into the diphosphates (24). The latter is a method of synthesis of polynucleotide material considerably different from that involving the reverse of ribonuclease degradation of RNA involving cyclic pyrimidine nucleotides (31), although both involve diesterified phosphate groups. Nor should other $5^{\prime}$ nucleotide precursors, ribose 1-pyrophosphate 5-phosphate $(41,52)$ and the two glycineamideribose $5^{\prime}$-phosphates $(23,28)$, also isolated by ion-exchange chromatography, be overlooked in this listing.

From all of this there arises the concept of coenzymes and transfer agents involving all of the RNA nucleoside 5 '-phosphates, these being in metabolic equilibrium with RNA and also possible precursors, with respect to the phosphodiester linkage, of RNA. It thus becomes possible to consider RNA as a source of coenzymes, or carriers, a possible new function to go with the new structure. Whether this relationship of RNA to intermediary metabolism 
is at variance with or consistent with the template and proteinsynthesizing hypotheses $(6,37,66)$ must await further clarification of the biological role of undegraded RNA (6).

Thus the casual observation of isomerism in the adenylic acids derived from RNA, a consequence of the introduction of ion-exchange chromatography to the problems of nucleic acid chemistry, has had far-reaching repercussions with respect to nucleic acid structure, nucleic acid synthesis, nucleotide coenzymes, and the concept of a dyamic metabolic relationship among these classes of compounds.

\section{References}

1. Anderson, A. S., G. R. Barker, J. M. Gulland, and M. V. Lock, J. Chem. Soc., 1952, 369.

2. Astbury, W. T., Nucleic Acid Symposium, Symposia Soc. Exptl. Biol., 1, 66 (1947).

3. Baddiley, J., and A. P. Mathias, J. Chem. Soc., 1954, 2723.

4. Baer, E., and M. Kates, J. Biol. Chem., 175, 79 (1948).

5. Baer, E., and M. Kates, J. Biol. Chem., 185, 615 (1950).

6. Brachet, J., in E. Chargaff and J. N. Davidson, eds., The Nucleic Acids, Vol. 2. Academic Press, New York, 1955.

7. Brown, D. M., C. A. Dekker, and A. R. Todd, J. Chem. Soc., 1952, 2715.

8. Brown, D. M., L. A. Heppel, and R. J. Hilmoe, J. Chem. Soc., 1954, 40.

9. Brown, D. M., D. I. Magrath, and A. R. Todd, J. Chem. Soc., 1952, 2708.

10. Brown, D. M., and A. R. Todd, J. Chem. Soc., 1952, 52.

11. Cabib, E., and L. F. Leloir, J. Biol. Chem., 206, 779 (1954).

12. Caputto, R., L. F. Leloir, C. E. Cardini, and A. C. Paladini, J. Biol. Chem., 184, 333 (1950).

13. Gardini, C. E., L. F. Leloir, and J. Chiriboga, J. Biol. Chem., 214, 149 (1955).

14. Carter, C. E., J. Am. Chem. Soc., 73, 1537 (1951).

15. Chargaff, E., J. Biol. Chem., 144, 455 (1942).

16. Cohn, W. E., J. Am. Chem. Soc., 72, 1471, 2811 (1950).

17. Cohn, W. E., J. Cellular Comp. Physiol., 38, Suppl. 1, 21 (1951).

18. Cohn, W. E., D. G. Doherty, and E. Volkin, Phosphorus Metabolism, 2, 339-354 (1952).

19. Cohn, W. E., and E. Volkin, Nature, 167, 483 (1951).

20. Cohn, W. E., and E. Volkin, J. Biol. Chem., 203, 319 (1953).

21. Dutton, G. J., and I. D. E. Storey, Biochem. J. (London), 53, xxxvii (1953). 
22. Elmore, D. T., and A. R. Todd, J. Chem. Soc., 1952, 3681.

23. Goldthwait, D. A., R. A. Peabody, and G. R. Greenberg, J. Am. Chem. Soc., 76, 5258 (1954).

24. Grunberg-Manago, M., P. J. Ortiz, and S. Ochoa, Science, 122, 907(1955).

25. Gulland, J. M., J. Chem. Soc., 1938, 1722.

26. Gulland, J. M., and E. M. Jackson, Biochem. J. (London), 32, 590 (1938).

27. Gulland, J. M., and E. M. Jackson, J. Chem. Soc., 1938, 1492.

28. Hartman, S. C., B. Levenberg, and J. M. Buchanan, J. Am. Chem. Soc., 77, 501 (1955).

29. Heppel, L. A., R. Markham, and R. J. Hilmoe, Nature, 171, 1152 (1953).

30. Heppel, L. A., and P. R. Whitfeld, Biochem. J. (London), 60, 1 (1955).

31. Heppel, L. A., P. R. Whitfeld, and R. Markham, Biochem. J. (London), 60,8 (1955).

32. Hurlbert, R. B., and V. R. Potter, J. Biol. Chem., 209, 1 (1954).

33. Hurlbert, R. B., H. Schmitz, A. F. Brumm, and V. R. Potter, J. Biol. Chem., 209, 23 (1954).

34. Hurst, R. O., A. M. Marko, and G. C. Butler, J. Biol. Chem., 204, 847 (1953).

35. Jordan, D. O., in E. Chargaff and J. N. Davidson, eds., The Nucleic Acids, Vol. 1, pp. 461 et seq. Academic Press, New York, 1955.

36. Kalckar, H. M., Science, 119, 479 (1954).

37. Keller, E. B., P. C. Zamecnik, and R. B. Loftfield, J. Histochem. Cytochem., 2, 378 (1954).

38. Kennedy, E. P., and S. B. Weiss, J. Am. Chem. Soc., 77, 250 (1955).

39. Khym, J. X., D. G. Doherty, and W. E. Cohn, J. Am. Chem. Soc., 76, 5523 (1954).

40. Khym, J. X., and W. E. Cohn, J. Am. Chem. Soc., 76, 1818 (1954).

41. Kornberg, A., I. Lieberman, and E. S. Simms, J. Am. Chem. Soc., 76, 2027 (1954).

42. Leloir, L. F., and C. E. Cardini, J. Biol. Chem., 214, 157 (1955).

43. Levene, P. A., and S. A. Harris, J. Biol. Chem., 95, 755 (1932).

44. Levene, P. A., and S. A. Harris, J. Biol. Chem., 98, 9 (1932).

45. Levene, P. A., and R. S. Tipson, J. Biol. Chem., 109, 623 (1935).

46. Markham, R., and J. D. Smith, Nature, 168, 406 (1951).

47. Markham, R., and J. D. Smith, Biochem. J. (London), 52, 552 (1952).

48. Markham, R., and J. D. Smith, Biochem. J. (London), 52, 558 (1952).

49. Markham, R., and J. D. Smith, Biochem. J. (London), 52, 565 (1952).

50. Munch-Petersen, A., Arch. Biochem. and Biophys., 55, 592 (1955).

51. Park, J. T., J. Biol. Chem., 194, 877, 885, 897 (1952), et ante.

52. Saffran, M., and E. Scarano, Nature, 172, 949 (1953).

53. Sanadi, D. R., D. M. Gibson, and P. Ayengar, Biochim. et Biophys. Acta, 14, 434 (1954). 
54. Schmidt, G., R. Cubiles, B. H. Swartz, and S. J. Thannhauser, J. Biol. Chem., 170, 759 (1947).

55. Schmidt, G., R. Cubiles, and S. J. Thannhauser, J. Cellular Comp. Physiol., 38, Suppl. 1, 61 (1951).

56. Schmidt, G., R. Cubiles, N. Zollner, L. Hecht, N. Strickler, K. Seraidarian, M. Seraidarian, and S. J. Thannhauser, J. Biol. Chem., 192, 715 (1951).

57. Schmidt, G., M. Seraidarian, K. Seraidarian, and S. J. Thannhauser, Federation Proc., 17, 283 (1952) (as modified by personal communication from G. Schmidt).

58. Schmitz, H., W. Hart, and H. Ried, Z. Krebsforsch., 60, 301 (1955).

59. Schmitz, H., R. B. Hurlbert, and V. R. Potter, J. Biol. Chem., 209, 41 (1954).

60. Smith, K. C., and F. W. Allen, J. Am. Chem. Soc., 75, 2131 (1953).

61. Strominger, J. L., H. M. Kalckar, J. Axelrod, and E. S. Maxwell, J. Am. Chem. Soc., 76, 6411 (1954).

62. Volkin, E., and W. E. Cohn, J. Biol. Chem., 205, 767 (1953).

63. Whitfeld, P. R., L. A. Heppel, and R. Markham, Biochem. J. (London), 60,15 (1955).

64. Whitfeld, P. R., and R. Markham, Nature, 171, 1151 (1953).

65. Zamecnik, P. C., personal communication.

66. Zamecnik, P. C., and E. B. Keller, J. Biol. Chem., 209, 337 (1954). 


\section{CHEMICAL STRUCTURE AS A GUIDE TO THE STUDY OF BIOCHEMICAL SYNTHESES*}

KONRAD BLOCH, Department of Chemistry, Harvard University, Cambridge, Massachusetts

In the study of biochemical syntheses it would appear reasonable to proceed on the premise that the mechanisms of formation of tissue constituents are implicit in their chemical constitution. Indeed, this line of thinking is responsible for the many attempts to deduce biochemical pathways from typical features of chemical structure and from the in vitro behavior of organic molecules. It is a matter of fact, however, that past efforts to decode the information which is provided by chemical constitution have had only limited success. For example, the discoveries of the role of acetate in steroid biogenesis, of succinic acid and glycine in porphyrin formation, or of formate and glycine as purine precursors were not at all what would have been expected from the background of chemical or biochemical knowledge. They have since been rationalized, but were hardly predictable from any particular structural features of the end products. Though the use of structural arguments to predict biochemical pathways has been perhaps somewhat discredited by its past record, the contribution which chemical theory can

* The author is indebted to Dr. R. B. Clayton for valuable discussion of the contents of this paper. 
make to the understanding of biological phenomena is now becoming increasingly apparent. Because of the widening scope of biochemical experimentation, the theorist can no longer afford to speculate in an experimental vacuum and hence must proceed with greater restraint. On the other hand, the biochemist, though traditionally an empiricist, has learned to accept the view that the reactions he is dealing with are those of organic chemistry, if of a special kind. He is therefore becoming more receptive to the guidance which chemical theory can provide.

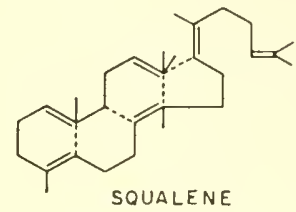

ROBINSON 1934

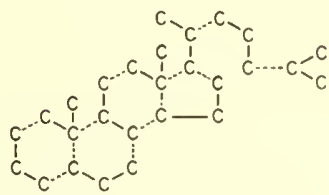

9 MOLS OF TRIOSE REICHSTEIN 1938
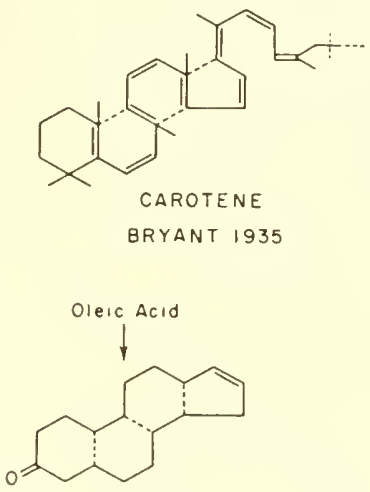

CIVETONE

WINDAUS 1933

Fig. 1. Early hypotheses on cholesterol biogenesis.

Many examples can be cited to illustrate the increasing degree of coordination between structural work in organic chemistry and experimentation in biochemistry, but few are as instructive as the example of steroid biogenesis. The constitution of the cyclopentanoperhydrophenanthrene derivatives has stimulated thoughts as to their biological origin for nearly thirty years, and it is of more than academic interest to recall some of the proposals which have been made in the past. Because of the scarcity of the biochemical information then available, it is not surprising that the early schemes on the origin of cholesterol were purely formalistic in character and that the ideas which 
they expressed were so diverse. These hypotheses, some of which are shown in Figure 1, not only differ from each other in detail but have invoked as precursors a great variety of carbon sources: carotene, squalene, oleic acid, acetone, formate, or triose. The biochemist has been inclined to view these speculative efforts with considerable indifference, because they often tended to disregard the biochemical facts. For example, carotene could be ruled out as a cholesterol precursor because steroids are synthesized in the animal body while carotenes are not. Similarly, the squalene hypothesis of Heilbron et al. (14) and Robinson (19) received scant attention because this hydrocarbon was then known to occur in shark liver but not elsewhere, and was regarded as a metabolic aberration rather than a normal metabolite. Even though Channon had furnished some biochemical evidence in favor of a squalene-cholesterol conversion as early as 1926 (5), his experiments, which were of the balance type and therefore inconclusive, aroused little comment.

The experimental phase of the study of steroid biogenesis dates from 1937, and the earlier hypotheses may now be considered in the light of what has since been learned: the steroidal carbon skeleton is synthesized by the condensation of numerous small molecular units (18) and these have been shown to be identical with acetic acid (4). From the distribution pattern of acetate carbon in cholesterol it was suspected that the over-all transformation proceeded in several stages: (1) a condensation of three acetate units to a branched-chain intermediate having five (or possibly six) carbon atoms; (2) multiple condensation or polymerization of these intermediate units to a polyisoprenoid chain of high molecular weight, (3) formation of the steroidal ring system by folding and cyclization of the high-molecular aliphatic intermediate (26). Gradually then the weight of the experimental evidence pointed to a terpene or polyisoprenoid derivative as a key intermediate, bringing back to mind those earlier schemes which had considered compounds of this general type. The squalene hypothesis in particular seemed sufficiently attractive to warrant an experimental check. There has now 
accumulated considerable evidence to show that squalene is converted to cholesterol in the animal body (15), and even though it may turn out that squalene itself is not the acyclic precursor, the principle of the postulated conversion is probably correct. The specific mechanism which Robinson had proposed (19) (Figure 2A) seemed at first a reasonable one to adopt, since it conformed with the distribution pattern which at that

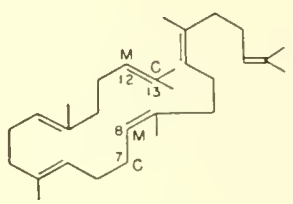

A

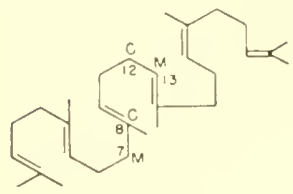

B

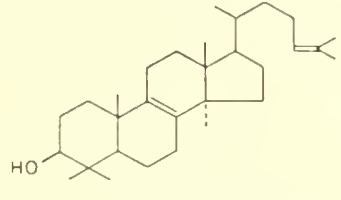

LANOSTEROL

VOSER ET AL. 1952

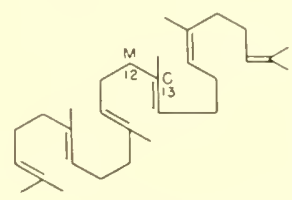

ISOSOUALENE

MONDON 1953

Fig. 2. Structural relation between squalene and lanosterol; A, cyclization of squalene according to Robinson (19); B, cyclization of squalene according to Woodward and Bloch (25); M, C: derived biologically from methyl and carboxyl carbon atoms of acetate, respectively.

time had been established for 17 of the 27 carbon atoms in the steroid molecule $(9,26)$. Nevertheless, it was realized that this analytical information was too incomplete to restrict the number of possible cyclization mechanisms. Robinson's formulation had the virtue of rationalizing the presence of the angular methyl groups in the positions in which they appear in fact in all the steroids. On the other hand, to judge from the chemical behavior of the terpenes a ring closure along the suggested lines did not seem probable. For example, the acid-catalyzed transition of an isoprene derivative to the cyclic isomer proceeds in 
general by addition of a proton to the most strongly basic double bond. In hydrocarbons of the squalene type this addition will occur at the two terminal double bonds. The resulting carbonium ion will be prone to cyclize

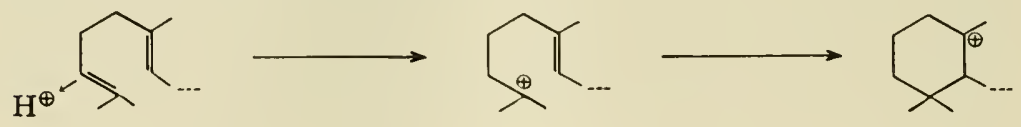

and the cyclization product will be six-membered if the relative position of the double bonds and their orientation are as shown above. These prerequisites for the transformation of an acyclic terpene to a cyclic product are not met by the arrangement of the squalene chain in Robinson's scheme.

For facilitating the more recent advances in the knowledge of steroid biogenesis much credit must be given to Ruzicka and Jeger (24) for their structural work on the steroid alcohol lanosterol, which is a major component of wool fat. Hitherto regarded as a triterpene because, like squalene, it contains 30 carbon atoms, lanosterol was shown to have in fact the same tetracyclic ring system as the steroids and was identified as a 4,4',14 trimethylcholestadienol (Figure 2). Lanosterol therefore constitutes the structural link between the triterpenes and the steroids which had been foreshadowed by biogenetic considerations. What distinguishes lanosterol structurally from other triterpenes is its failure to obey the isoprene rule; it cannot be constructed formally from isoprene residues, though its elementary composition suggests an origin from 5-carbon units. On the other hand, as a sterol, lanosterol is atypical by virtue of the three additional methyl groups which are attached to the ring system. If lanosterol were to be included in a general scheme of steroid biogenesis, then squalene should be its precursor as it is for cholesterol. Yet a ring closure in line with scheme $A$ (Figure 2) is clearly incapable of rationalizing the position of the branched methyl groups in lanosterol. Thus, apart from considerations of the course of acid-catalyzed transformations in the terpene field the structure of lanosterol also called for a revision of Robinson's cyclization scheme. The alternative 
mechanism $B$ shown in Figure 2 was therefore proposed (25). It will be noted that this formulation incorporates some of the features of the scheme suggested by Bryant (Figure 1) many years ago, but it recognizes the fact that it cannot be carotene which is the polyisoprenoid precursor.

From the work of Cornforth and Popjak (10) the isotopic composition of biosynthetic squalene is now known to conform with the pattern expected for isoprene derivatives, and hence one can more safely predict in which way the distribution pattern of acetate carbon in the steroidal transformation product will be altered by substituting one mechanism for the other. If squalene were to cyclize according to $B$ instead of $A$, the derivation of 4 of the 27 carbon atoms of cholesterol will be reversed, methyl carbon atoms of acetate appearing now at $\mathrm{C}_{7}$ and $\mathrm{C}_{13}$ and acetate carboxyl at $\mathrm{C}_{8}$ and $\mathrm{C}_{12}$. Degradation experiments have verified this prediction for $\mathrm{C}_{7}$ and $\mathrm{C}_{13}(3,11,25)$, and hence scheme $A$ is no longer tenable. The same results bear also on the interesting suggestion made by Mondon (16) that the aliphatic precursor of the steroids is not squalene, but an as yet unknown isomeric hydrocarbon "isosqualene" (Fig. 2) in which the irregular junction between two isoprene units is displaced from the center of the molecule towards the end of the chain by one isoprene unit. According to Mondon's hypothesis $\mathrm{C}_{13}$ of cholesterol should be derived from a carboxyl group of acetate, but this has since been ruled out by experiment (25).

The modified cyclization scheme $(B)$ removes some of the objections which have been properly raised against the proposed role of squalene as visualized earlier, but by postulating the shifting of methyl groups, i.e., a rearrangement of the original carbon skeleton, it introduces another feature which is unusual at least as a biochemical reaction.*

* The rearrangement which occurs in the conversion of $p$-hydroxyphenylpyruvic acid to homogentisic acid is the only biochemical example of an intramolecular shift which is well established. A pinacol rearrangenent of acetolactic acid to an isovaleric acid derivative has been suggested as a mechanism for the biosynthesis of the amino acid valine in yeast (21), but it lacks direct experimental support so far. 
Much experimental work along enzymatic lines remains to be done to establish the principle and the details of the cyclization process. Nevertheless it is exceedingly useful to draw on the broad chemical background which already exists in the terpene field and to attempt a rationalization of the squalene-cholesterol conversion. Taking the squalene hypothesis as a basis, Ruzicka and his collaborators have recently
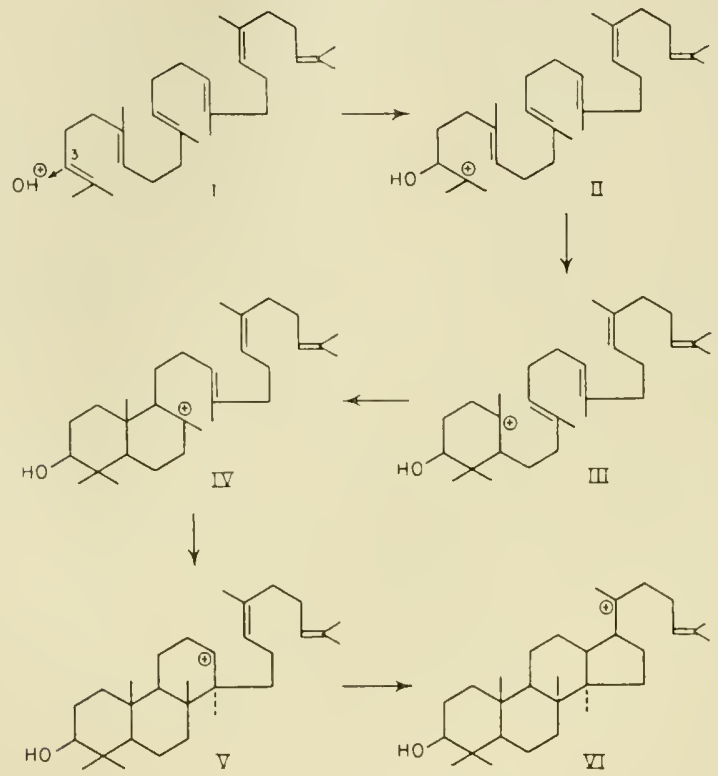

Fig. 3. Ionic mechanism for the cyclization of squalene to steroids according to Ruzicka (20).

presented a comprehensive reaction scheme which connects squalene with the steroids as well as with the various pentacyclic triterpenes, and serves as a highly stimulating working hypothesis for future biochemical work (20). According to Ruzicka the cyclization of squalene is initiated by a cationic attack at the carbon atom which corresponds to $\mathrm{C}_{3}$ of the steroid skeleton, one of the two positions which are most susceptible to attack by electrophilic reagents. Thereafter, the tetracyclic ring system is 


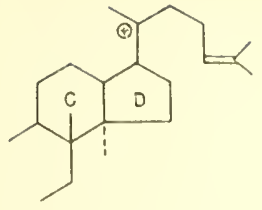

II

$\downarrow-H^{\oplus}$

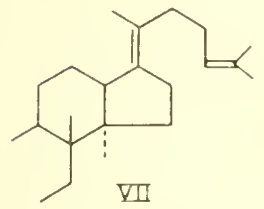

$\mid+H^{\oplus}$

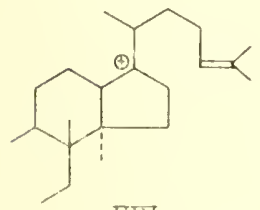

VII

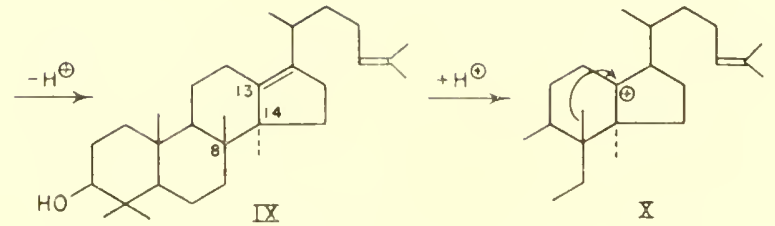

ISOEUPHOL

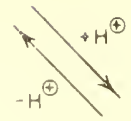

LANOSTEROL

$\uparrow-H^{\oplus}$

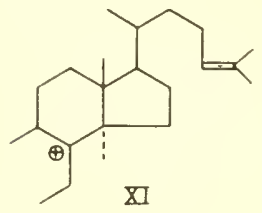

$\uparrow$

Z
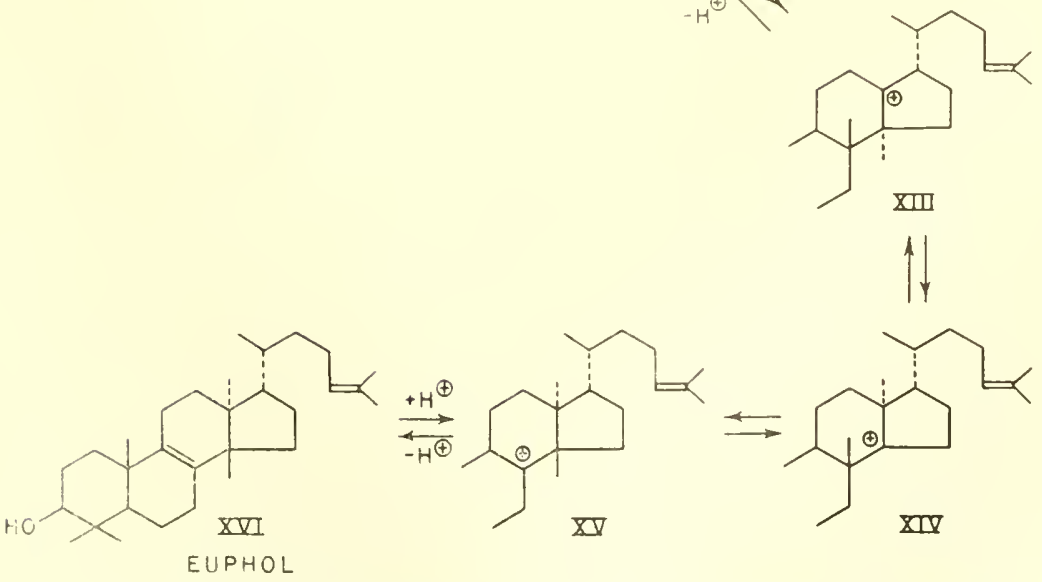

Fig. 4. Postulated mechanism for the formation of Ianosterol and euphol. 
thought to be established by the successive or perhaps synchronous passage through various carbionic forms without stabilization of any of the transition stages. The reaction sequence I through VI (Figure 3) is that proposed by Ruzicka; on the whole it follows the rules established in the acid-catalyzed cyclization of terpenes and assumes lanosterol (XII, Figure 4) to be the first stabilized product. In the slightly modified version of the cyclization process proposed here (Figure 4) the conversion of the intermediate VI to lanosterol is somewhat more detailed and a stabilized intermediate (isoeuphol, IX) is postulated at an earlier stage. This suggestion is based on the constitution of euphol, another triterpenoid alcohol intermediate in structure between the classical sterols and the triterpenes. Euphol (XVI, Figure 4) has only recently been identified as an isomer of lanosterol from which it differs by having the $\mathrm{C}_{13}$ methyl group and the isooctyl side chain in the $\alpha$ configuration and the methyl group at $\mathrm{C}_{14}$ in the $\beta$ configuration $(1,2)$. This configuration imposes a strain on the molecule and explains the facile transformation of euphol into the isomeric isoeuphol (IX) in the presence of mineral acid (23). The isomerization involves a shift of the double bond to ring $\mathrm{D}$ and transposition of the methyl group at $\mathrm{C}_{14}$ to $\mathrm{C}_{8}$ and of the $\mathrm{C}_{13}$ methyl to $\mathrm{C}_{14}$ (reactions IX, XIII to XVI). What is unique for the structure of isoeuphol and of particular interest for the present discussion is the presence of the completed tetracyclic ring system of the steroids, with retention of the two methyl groups (at $\mathrm{C}_{8}$ and $\mathrm{C}_{14}$ ) in vicinal positions. This structural feature has not been encountered among naturally occurring steroids but is typical of the pentacyclic triterpenes. If isoeuphol were a key intermediate in the cyclization of squalene, euphol could be formed biologically by reversal of the steps IX, XIII to XVI. Since the configuration of the methyl group at $\mathrm{C}_{8}$ of isoeuphol is $\beta$ and of the methyl at $\mathrm{C}_{14} \alpha$, the isomerization process would be expected to involve two 1,2 methyl shifts (from $\mathrm{G}_{14}$ to $\mathrm{G}_{13}$ and from $\mathrm{C}_{8}$ to $\mathrm{C}_{14}$ ). Because of the particular stereochemical arrangement, euphol is an unstable molecule and therefore the euphol- 
isoeuphol reaction should be difficult to reverse chemically, but this would not preclude the feasibility of the analogous reaction in an enzymatic system. In the event that isoeuphol is also the precursor of lanosterol (IX to XII, Figure 4), a single 1,3 methyl shift (from $\mathrm{C}_{8}$ to $\mathrm{C}_{13}$ ) would establish the methyl group at $\mathrm{C}_{13}$ in the $\beta$ configuration which is typical of lanosterol and of all the natural steroids except euphol.*

It is perhaps significant that in the pentacyclic triterpenes, the only naturally occurring products which are branched at both the 8 and 14 positions, the configurations of the methyl substituents are without exception $\beta$ and $\alpha$, respectively, as they are in isoeuphol. It is therefore reasonable to expect that the same configuration will be assumed by the first tetracyclic transition state or intermediate (VI, Figure 3). The subsequent course of the reactions will be governed by the stereochemical nature of the end products, the $\beta$ configuration at $\mathrm{C}_{13}$ in lanosterol resulting from a single 1,3 shift of the $\beta$ methyl at $\mathrm{C}_{8}$, whereas the formation of euphol is more readily explained by two consecutive 1,2 methyl shifts.

For the further biochemical exploration of steroid biogenesis it will be crucial to ascertain whether cyclization is initiated by an oxidative attack on squalene, as Ruzicka suggests (20), or whether the hydroxyl group at carbon atom 3 is introduced after the tetracyclic ring system has been completed. The chemical behavior of squalene, i.e., the acid-catalyzed formation of tetracyclo-squalene (Figure 5), merely shows that, as expected,

* Recent evidence obtained by Dr. Tchen in this laboratory suggests that squalene is converted to lanosterol without formation of stabilized intermediates. In the presence of $\mathrm{D}_{2} \mathrm{O}$, squalene cyclizes to lanosterol without incorporating deuterium. This result eliminates any reaction mechanism involving an irreversible attachment of protons to the carbon skeleton, e.g., reactions VI to $\mathrm{X}$ in Figure 4, and thus rules out isoeuphol as an intermediate in lanosterol formation. On the other hand, the experimental results obtained with $\mathrm{D}_{2} \mathrm{O}$ can be rationalized by assuming intramolecular hydride shifts from $\mathrm{C}_{17}$ to $\mathrm{C}_{20}$ and from $\mathrm{C}_{13}$ to $\mathrm{C}_{17}$ accompanied by methyl shifts, events which would yield lanosterol from the carbonium ion VI (Figure 4) without uptake of hydrogen from an external source. 
ring closure need not be preceded by oxidation. Of some interest in this connection is the structure of ambrein (Figure 5), a terpene alcohol which is a pathological excretion product of the sperm whale, and undoubtedly originates from squalene by partial cyclization. The linkage of the hydroxyl group of this interesting compound to the carbon corresponding to $\mathrm{C}_{8}$ of the steroids may be taken as evidence that oxidation occurred after rings $A$ and $B$ had been established. In view of the obscure circumstances which attend the origin of this alcohol, it would be

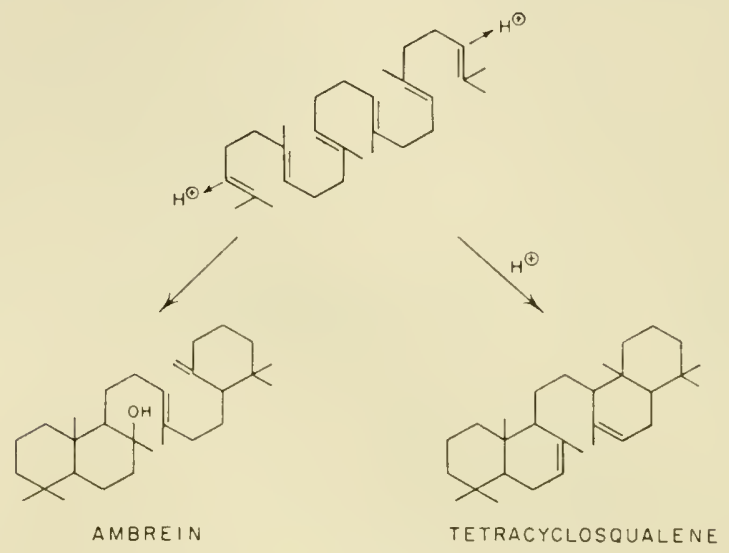

Fig. 5. Transformations of squalene.

hazardous to say that ambrein, though it is derived from a biological source, is actually the product of an enzymatic action. In any event, it is questionable that much can be deduced from the structure of either tetracyclosqualene or ambrein regarding the stage at which the oxidation step occurs in steroid biogenesis. It must also be borne in mind that in the chemical cyclization the reagent attacks the symmetrical squalene chain at two equivalent points. One would suspect, on the other hand, that in the analogous biological reaction one of the two sensitive double bonds must be shielded so that the aliphatic portion which becomes the isooctyl side chain of the sterol can be preserved. Conceivably, the squalene molecule is rendered asym- 
metric by attaclıment of one of the two terminal isopropyliclene groups to a cationic site on the enzyme surface, leaving only one portion of the chain free to cyclize. It is evident that the same purpose would be achieved by the formation of a nonsymmetric derivative of squalene in the course of its synthesis.

From what has been said it is clear that knowledge of the structure of lanosterol has permitted the formulation of a plausible mechanism for the cyclization of squalene. Equally important, it has indicated the direction and the area in which further biochemical experimentation might be pursued. As an example the results of some recent experiments may be cited. Lanosterol has in the past been isolated only from two natural sources, from yeast (kryptosterol) and from wool fat, which contains three other closely related sterols, dihydrolanosterol, agnosterol, and dihydroagnosterol. Once its structure was established it became reasonable to ask whether lanosterol is a metabolic side product or a normal intermediate in the synthesis of the more conventional sterols (cholesterol, ergosterol, or the plant sterols). As the example of squalene had demonstrated earlier, the general significance of a tissue constituent is frequently missed simply because it is primarily associated with somewhat unconventional sources. Evidence has now been obtained that lanosterol also is more widely distributed than had earlier been suspected. With the aid of the carrier technique, it can be shown that mammalian liver synthesizes lanosterol from acetate at a rapid rate, and this establishes lanosterol as a normal constituent of cholesterol-producing tissues (6). Besides, a conversion of lanosterol to cholesterol can be demonstrated in the same liver system. The rate of this reaction is sufficiently fast to suggest that the pathway from squalene to cholesterol goes indeed by way of lanosterol (7). The inclusion of lanosterol in the normal process of cholesterol biogenesis reduces at least partially the complexity of the reaction sequence and thereby greatly improves prospects for an enzymatic characterization of individual steps.

Details of the cyclization of squalene to lanosterol have 
already been discussed at some length. Much less can be said at present about the further transformations of lanosterol, notably the demethylation to the more abundant sterols which have 27 carbon atoms. Compounds with either 1 or 2 fewer methyl groups than lanosterol have to date not been encountered in nature, and hence no clues exist to indicate the order in which the 3 methyl substituents are eliminated. That oxidation precedes the removal of the branched methyl groups appears likely from the structure of the numerous cyclic terpenes in which the $4,4^{\prime}$ or 14 methyl groups are replaced by either $\mathrm{CH}_{2} \mathrm{OH}$ or $\mathrm{COOH}$ :
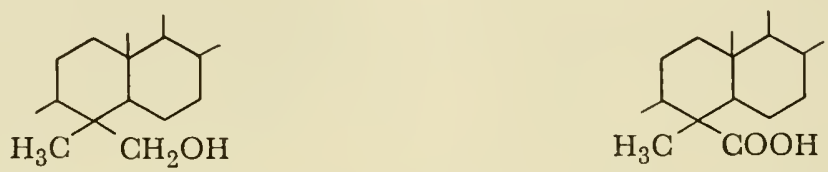

In the event that methyl elimination occurs by way of carboxylic acid derivatives the introduction of keto groups $\beta$ to, or of double bonds $\beta, \gamma$ to the carboxyl group would greatly facilitate decarboxylation reactions. Since in lanosterol the 8,9 double bond is located in $\beta, \gamma$ position with respect to the methyl group at $\mathrm{C}_{14}$, the demethylation process may well be initiated by oxidation of the substituent at $\mathrm{C}_{14}$ (Figure 6). The resulting $\beta, \gamma$ unsaturated acid would then be susceptible to decarboxylation. Alternatively, the 8,9 double bond could first shift to the 7,8 position as it does in the acid-catalyzed isomerization of lanosterol.

There is the second possibility that agnosterol, which is $\Delta 7,9,24$ lanostatrienol and accompanies lanosterol in small amounts in wool fat, is an intermediate in these conversions. The diene system of agnosterol would tend to prevent a migration of the 7,8 double bond to the $\alpha, \beta$ position, a shift which in chemical systems at least accompanies the decarboxylation of $\beta, \gamma$ unsaturated acids. Next, to facilitate the removal of the gem-dimethyl grouping in ring $\mathrm{A}$, a displacement of the double bond to the 5,6 position may be visualized, and for this reaction an enzymatic precedent may exist in the biological conversion 
of $\Delta 7$-cholestenol to cholesterol (8). Oxidation of first one and then of the second methyl group at $\mathrm{C}_{4}$ would once more yield carboxylic acids which are susceptible to decarboxylation because of the double bond in $\beta, \gamma$ position. Admittedly other
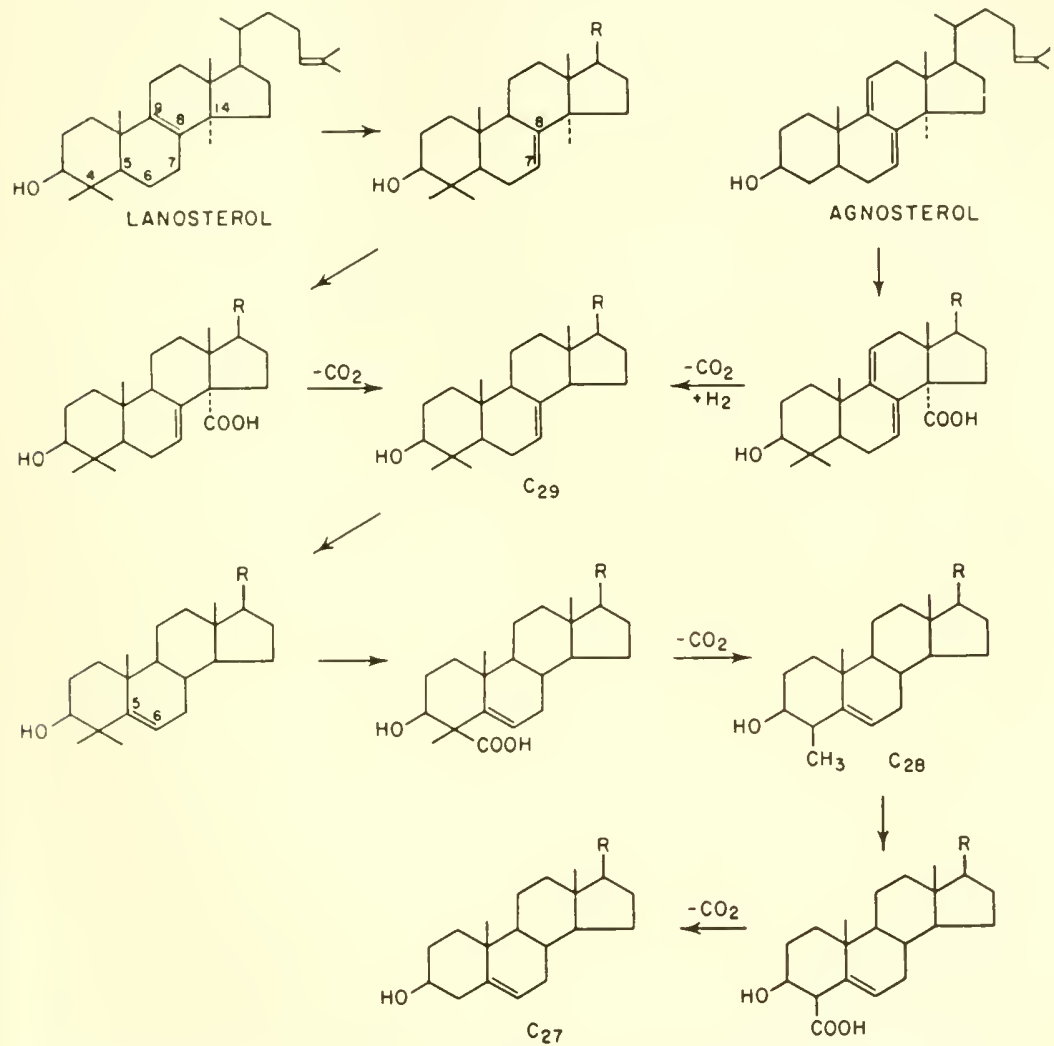

Fig. 6. Scheme for the transformation of lanosterol to cholesterol.

schemes could be and have been devised which are equally plausible (see, for example, Mondon (16)). In fact the structure of zymosterol, a $\Delta 8,24$ cholestadienol present in small amounts in yeast, could well be cited as an argument against the above proposal that the double bond moves from the $\mathrm{B} / \mathrm{C}$ junction to the 5,6 position in the course of the demethylation reac- 
tions. Whether or not the details or even the principle of the suggested scheme are correct, it seems probable nevertheless that in the conversion of lanosterol to cholesterol carboxylic acids will be found as intermediates. If steroid carboxylic acids-other than the bile acids - have not hitherto been detected in animal tissues, it can be pointed out that a thorough search for compounds of this type has not so far been made and that in particular those tissues remain to be examined which are most active in steroid biogenesis.

\section{The Biosynthesis of Myco- and Phytosterols}

It is an obvious defect of current biogenetic schemes that they neglect to provide for the formation of those numerous sterols which carry a methyl or ethyl substituent in the aliphatic side chain, and which are principally found in plants or microorganisms. Experimental work to date has been limited to the demonstration that acetic acid is the carbon source of ergosterol (17), and that the distribution of acetate in a portion of the aliphatic side chain follows the pattern found in cholesterol (12). If the biogenesis of ergosterol and of the plant sterols were to take the same general course as that of the sterols of animal tissues, identical pathways and intermediates up to the lanosterol stage would be anticipated. The occurrence of squalene in plants and microorganisms has been claimed but has not been adequately documented. Lanosterol, on the other hand, is invariably found to be associated with ergosterol in yeast. The structural features of ergosterol and of the typical plant sterols which so far lack a rational biogenetic explanation are the additional branchings at the 24 position of the aliphatic side chain. In this regard the structures of several mold products are of utmost interest. These are the polyporenic and eburicoic acids (Figure 7) from molds such as Polyporus betulinus, identified by the investigations of Jones and co-workers and in the Zurich laboratories as steroid carboxylic acids with 31 carbon atoms (reviewed in ref. 13). Their carbon skeleton and configuration 
is that of lanosterol, but in addition they possess the substituent at $\mathrm{C}_{24}$ which is typical of ergosterol. The acids isolated from the molds probably represent secondary oxidation products rather than metabolic intermediates; yet the disclosure of this structural type strongly suggests that the carbon skeleton of lanosterol can be enlarged by attachment of carbon atoms to the

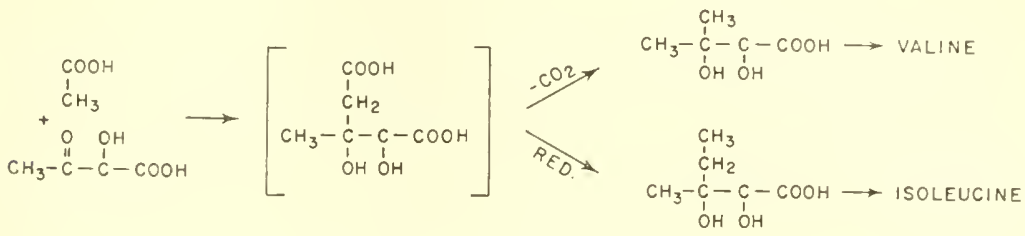

TATUM AND ADELBERG, 195
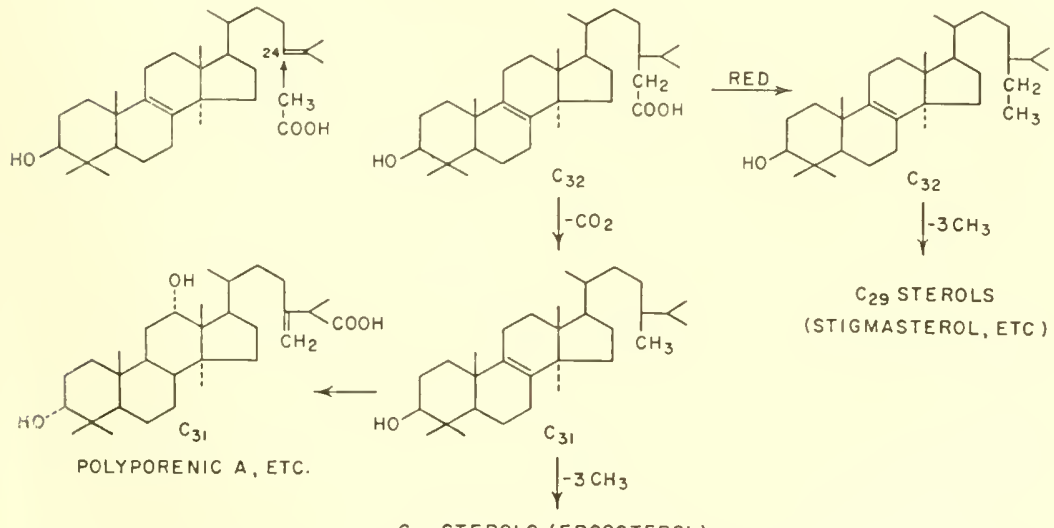

$\mathrm{C}_{29}$ STEROLS

(STIGMASTEROL, ETC)

$C_{28}$ STEROLS (ERGOSTEROL)

Fig. 7. Postulated mechanism for the synthesis of ergosterol and plant sterols from lanosterol.

aliphatic side chain. The carbon source for the alkyl substituents of the plant sterols (stigmasterol or sitosterol) and of ergosterol is obscure, but it is not unreasonable to presume that they are derived from acetate also. There exists one biochemical process for the branching of carbon chains which may have some bearing on the synthesis of the $\mathrm{C}_{28}$ and $\mathrm{C}_{29}$ sterols. The amino acids valine and isoleucine are the $\beta$-methyl and the 
$\beta$-ethyl derivatives, respectively, of $\alpha$-aminobutyric acid. Tatum and Adelberg, working with Neurospora mutants, have obtained evidence that a precursor common to both amino acids is formed by a "methyl" condensation of acetate with a 4-carbon hydroxy-keto acid (22). Their results point to a 6-carbon dicarboxylic acid as a common intermediate which can be metabolized by either one of two pathways: (1) the "acetate" carboxyl is reduced and the reduction product converted further to isoleucine; (2) the same carboxyl group is removed by decarboxylation, leaving the carbon skeleton of valine (Figure 7). By analogy one may visualize a branching of the isooctenyl side chain of lanosterol through attachment of an acetate unit to $\mathrm{C}_{24}$, affording an intermediate with 32 carbon atoms. Either one of two pathways may then be followed: (1) reduction of the branched portion to an ethyl group to yield the $\mathrm{C}_{32}$ precursor of the plant sterols, or (2) decarboxylation to a hypothetical ergosterol precursor containing 31 carbon atoms. Finally, removal of the methyl groups at $\mathrm{C}_{4,4^{\prime}}$ and $\mathrm{C}_{14}$ and introduction of the appropriate double bonds will afford either ergosterol or the $\mathrm{C}_{29}$ sterols (Figure 7).

What is proposed here is admittedly highly speculative, and one would wish for some evidence at least capable of supporting the proposed mechanism for the biogenesis of plant and mycosterols. One single finding may be cited that is relevant, although negative in nature. According to Hanahan and Wakil (12), $\mathrm{C}_{28}$, the branched carbon of ergosterol, is not derived from a carboxyl group of acetate, as one would expect from the scheme postulated in Figure 7.

It has been the object of this discussion to emphasize how critically the progress of biochemical research, in some areas at least, depends upon the discovery and identification of novel structures in natural sources. Had it not been for the isolation of squalene from shark liver or of lanosterol from wool fat, the task of recognizing intermediates in the complex transformation of acetic acid to cholesterol would have been infinitely more difficult. Similarly, with the discovery of the polyporenic acids, 
clues have been provided for the first time to suggest a rational approach to the biosynthesis of ergosterol and the plant sterols. There is little doubt that the organic chemist will continue to chance upon compounds which will aid in the further elucidation of biogenetic pathways. Conversely, the elaboration of biosynthetic mechanisms will provide the background for predicting the existence of novel chemical structures, and thereby serve to intensify the exploration of natural sources. Though the chemist's principal task will continue to be to determine structures and the biochemist's to find out how they are made, their efforts tend increasingly toward the same goal and hence they are bound to benefit by a more intimate acquaintance with their mutual problems.

\section{References}

1. Arigoni, D., R. Viterbo, M. Dunnenberger, O. Jeger, and L. Ruzicka, Helv. Chim. Acta, 37, 2306 (1954).

2. Barton, D. H. R., J. F. McGhie, M. K. Pradhan, and S. A. Knight, Chemistry \& Industry, 1954, 1325.

3. Bloch, K., Helv. Chim. Acta, 36, 1611 (1953).

4. Bloch, K., and Rittenberg, D., J. Biol. Chem., 745, 625 (1942).

5. Channon, H. J., Biochem. J. (London), 20, 400 (1926).

6. Clayton, R. B., and K. Bloch, Federation Proc., 14, 194 (1955).

7. Clayton, R. B., and K. Bloch, J. Biol. Chem., 218, 319 (1956).

8. Cook, R. P., A. Kliman, and L. F. Fieser, Arch. Biochem. and Biophys., 52, 439 (1954).

9. Cornforth, J. W., G. D. Hunter, and G. Popjak, Biochem. J. (London), 5ł, 597 (1953).

10. Cornforth, J. W., and G. Popjak, Biochem. J. (London), 58, 403 (1954).

11. Dauben, W. G., J. Am. Chem. Soc., 75, 6302 (1953).

12. Hanahan, D. J., and S. J. Wakil, J. Am. Chem. Soc., 75, 273 (1953).

13. Halsall, T. G., Ann. Repts. on Progr. Chem. (Chem. Soc. London), 50, 212-214 (1953).

14. Heilbron, I. M., E. D. Kamm, and W. M. Owens, J. Chem. Soc., 1926, 1630.

15. Langdon, R. G., and K. Bloch, J. Biol. Chem., 200, 135 (1953).

16. Mondon, A., Angew. Chem., 65, 333 (1953).

17. Ottke, R. C., E. L. Tatum, I. Zabin, and K. Bloch, J. Biol. Chem., 186, 581 (1951). 
18. Rittenberg, D., and R. Schoenheimer, J. Biol. Chem., 127, 235 (1937).

19. Robinson, R., J. Soc. Chem. Ind. (London), 53, 1062 (1934).

20. Ruzicka, L., Experientia, 9, 357 (1953).

21. Strassman, M., A. J. Thomas, and S. Weinhouse, J. Am. Chem. Soc., 75, 5135 (1953).

22. Tatum, E. L., and E. A. Adelberg, J. Biol. Chem., 190, 843 (1951).

23. Vilkas, M., G. Dupont, and R. Dulou, Bull. soc. chim. France, 1949, 813.

24. Voser, W., M. V. Mijovic, H. Heusser, O. Jeger, and L. Ruzicka, Helv. Chim. Acta 35, 2414 (1952).

25. Woodward, R. B., and K. Bloch, J. Am. Chem. Soc., 75, 2023 (1953).

26. Würsch, J., R. L. Huang, and K. Bloch, J. Biol. Chem., 195, 439 (1952). 


\section{THE ROLE OF NUCLEOTIDES AND COENZYMES IN ENZYMATIC PROCESSES}

FRANK M. HUENNEKENS, Department of Biochemistry, University of II'ashington, Seattle, WTashington

\section{Introduction}

During the past thirty-five years the field of enzyme chemistry has evolved from a tiny island in the realm of biochemistry to such vast and heroic proportions that it now begins to overwhelm the field itself by virtue of its almost universal applicability. Some idea of the accelerated pace in enzymology can be gained from the fact that it required a number of years in the 1930's before Warburg completed his classical studies showing that the oxidation of glucose-6-phosphate was mediated by the concerted action of four highly purified components, two proteins, a dehydrogenase and an electron carrier, and two coenzymes, TPN and FMN.* Today the isolation and description of a new enzyme system, perhaps with its attendant coenzyme, is greeted with far less amazement, simply because such events have become commonplace. This casual attitude is not wholly unexpected, since, as the history of science so eloquently shows, it

* The following abbreviations will be used: DPN, TPN, di- and triphosphopyridine nucleotides; FMN, riboflavin-5'-phosphate; FAD, flavin adenine dinucleotide; CoA, coenzyme A; AMP, ADP, ATP, adenosine mono-, di-, and tri-phosphate; $P$, inorganic phosphate; PP, pyrophosphate; UDPG, uridine diphosphate glucose. 
reflects a certain maturity in the area. Nor is it to be regarded as evidence that the problems have become static, since each plateau of knowledge to which we have ascended becomes, in turn, a stepping stone to the next higher level.

The present treatise has been envisaged by its editor as a collection of essays surveying the past, present, and future of some aspect of biochemistry, inter alia, enzyme chemistry. Since other contributions will undoubtedly emphasize other aspects of enzymatic reactions, the present discussion will be concerned exclusively with the role of nucleotides and coenzymes in enzymatic reactions without, however, any attempt to document extensively the properties of individual members. Rather, attention will be directed toward salient features of our present state of knowledge in this area and will extend to a few speculative thoughts toward future directions.

It is common knowledge that whereas all enzymatic reactions require a protein component (the enzyme), certain ones require, in addition, a cooperative partner (the coenzyme). The coenzyme, sometimes termed cofactor, prosthetic group, or activator, ${ }^{*}$ is usually a heat-stable, dialyzable, organic molecule or metal ion. To omit at present the metal ions, whose function will be considered later, or certain rare cases where a simple anion (e.g., $\mathrm{Cl}^{-}$with amylase) serves as a coenzyme, it may be stated that most, but not all, coenzymes contain a nitrogenous base (often a vitamin), a sugar (ribose), and phosphate groups, therefore placing these substances in the class of compounds commonly called nucleotides. Conversely, certain other nucleotides, the precursors and breakdown products of nucleic acids, exist in tissues without acting as coenzymes. Thus, the 5'nucleotides either may serve as building blocks for nucleic acids, and as such participate in the energy-requiring process of protein synthesis, or they may become portions of coenzymes involved in the breakdown of energy-yielding metabolites. As

* Although each of these terms has been used, on occasion, in a special sense, the distinction between them appears to be vanishing in the literature; herice the term coenzyme will be used synonomously for all. 
Potter (22) has pointed out, it is precisely this competition by alternate pathways for limited amounts of key substances and the proper balance between energy production and utilization that may well determine the precarious balance between normal and uncontrolled growth.

Recognition of a coenzyme as part of an enzymatic process has been accomplished in a variety of ways. Often excellent inferential evidence is gained from experiments in the field of nutrition. Thus, Elvehjem (1) many years ago showed that riboflavin deficiency in rats results in a decreased level of succinic dehydrogenase in the liver. This implication of a flavin coenzyme in succinic dehydrogenase was verified only recently upon isolation of the highly purified enzyme by Green et al. (5) and by Singer and Kearney (38). The connection of a folicacid coenzyme with reactions involving one-carbon groups was likewise obtained initially from vitamin-deficiency experiments. Sometimes preliminary evidence has been elicited from experiments where coenzyme or vitamin analogues are added to crude systems with the resultant inhibition of a specific enzyme.

By far the most convincing, and hence most often used, criterion for coenzyme function is to obtain the enzyme free from its coenzyme (i.e., the apoenzyme), and then to demonstrate the absence of activity until the addition of the coenzyme.* In a dissociated system the coenzyme becomes a second substrate whose kinetic behavior can be analyzed by the conventional Michaelis-Menten treatment or its extensions. The Michaelis constants, or $K_{m}$ values, so obtained are a general, but not exact, measure of the affinity of enzyme for coenzyme.

With certain of the coenzymes, notably the flavin nucleotides and pyridoxal phosphate, it is sometimes difficult to dissociate the tightly bound coenzyme without concomitant destruction of the apoenzyme. In this event, it is usually presumptive evidence if the coenzyme can be shown to be present,

* Resolved systems of this sort, usually with purified apoenzymes, provide an excellent assay for the particular coenzyme even in crude tissue extracts. 
by microbiological assay of its vitamin form or specific enzymatic assay of the denaturated preparation, and to be concentrated in a parallel fashion with the enzyme. In other cases, the highly purified enzyme may exhibit an absorption spectrum indicative of the bound coenzyme, and, as in the case of certain flavoproteins, a change in the absorption spectrum (i.e., corresponding to reduction of the flavin) may occur upon addition of the appropriate substrate.

By one or another of the techniques just described, the series of coenzymes listed in Table I have been established.

\section{TABLE I}

Function of Coenzymes

\begin{tabular}{ll}
\hline \multicolumn{1}{c}{ Coenzyme } & \multicolumn{1}{c}{ Group transferred } \\
\hline Diphosphopyridine nucleotide & Hydrogen ions and electrons \\
Triphosphopyridine nucleotide & Hydrogen ions and electrons \\
Flavin adenine dinucleotide & Hydrogen ions and electrons \\
Riboflavin phosphate & Hydrogen ions and electrons \\
Lipoic acid & Hydrogen ions and electrons; acyl \\
Coenzyme A & Acyl \\
Diphosphothiamin & Aldehyde; carbonyl \\
Pyridoxal phosphate & Amino \\
Tetrahydrofolic acid & Formyl (hydroxymethyl) \\
S-Adenosyl methionine & Methyl \\
Adenosine diphosphate (or & \\
monophosphate) & Acyl \\
Uridine diphosphate & Sugar \\
Cytidine disphosphate & Choline \\
Guanosine diphosphate & Phosphate \\
\hline
\end{tabular}

Inspection of the table reveals several items of interest. First, all of the coenzymes participate in group transfer reactions, which may be represented in the general case by the following equations:

$$
\begin{aligned}
& \mathrm{X}+\mathrm{D}-\mathrm{G} \longrightarrow \mathrm{X}-\mathrm{G}+\mathrm{D} \\
& \mathrm{A}+\mathrm{X}-\mathrm{G} \longrightarrow \mathrm{A}-\mathrm{G}+\mathrm{X}
\end{aligned}
$$

where $\mathrm{X}$ is the coenzyme, $\mathrm{D}-\mathrm{G}$, the donor with group $\mathrm{G}$ to be 
transferred, and A, the acceptor. The net reaction is, of course, the sum of (1) and (2):

$$
\mathrm{D}-\mathrm{G}+\mathrm{A} \longrightarrow \mathrm{A}-\mathrm{G}+\mathrm{D}
$$

so that the coenzyme, like the enzyme, functions in a catalytic role. Reaction (1) involves the activation of the group on the substrate and transfer to the coenzyme, an energy-requiring reaction, whereas (2) represents the transfer of the group from the coenzyme to a suitable acceptor, an isoenergetic process. Where no coenzyme appears to be involved (i.e., dehydration, transpeptidation, transglycosidation reactions), the over-all reaction is usually characterized by a small change in free energy.

The groups which undergo transfer include representatives of most of the major metabolites encountered in enzymatic reactions. As yet, the activation of amino acids for peptide synthesis is not well understood, but by analogy with known reactions, such as the condensation of benzoyl CoA with glycine, one might surmise that the formation of alanyl glycine, for example, would involve the condensation of alanyl-CoA (or perhaps alanyl-AMP, of. equation (5)) with glycine.

It should be noted that the adenosine phosphates are an exception to the rule that the coenzyme acts catalytically. ATP functions irreversibly as a substrate, rather than a coenzyme in the kinase reactions:

$$
\mathrm{ATP}+\text { acceptor } \longrightarrow \mathrm{ADP}+\text { acceptor phosphate }
$$

and in the activation of acyl groups:

$$
\begin{gathered}
\text { acetate }+\mathrm{ATP}+\mathrm{CoA} \longrightarrow \text { Acetyl CoA }+\mathrm{AMP}+\mathrm{PP} \\
\text { succinate }+\mathrm{ATP}+\mathrm{CoA} \longrightarrow \text { Succinyl CoA }+\mathrm{ADP}+\mathrm{P} \\
\text { glutamate }+\mathrm{ATP}+\mathrm{NH}_{3} \longrightarrow \text { Glutamine }+\mathrm{ADP}+\mathrm{P}
\end{gathered}
$$

In reactions (5) and $\left(5^{\prime}\right)$ it is believed that the first step involves the formation of acetyl-AMP or succinyl-ADP (17) followed by an isoenergetic transfer of the acyl group to CoA. Likewise, UDPG is probably formed from a reaction between glucose-1phosphate and uridine triphosphate (27) (cf. equation (12)), 
cytidine diphosphate choline from phosphocholine and cytidine triphosphate (24), and $S$-adenosyl methionine from ATP and methionine (11). However, all of these reactions yield stable intermediates of the coenzyme and the mobile group and have been included, therefore, in Table I. Guanosine disphosphate is, however, a true coenzyme, since it functions catalytically, and is regenerated at the end of the reaction, in the process of oxidative phosphorylation at the substrate level (see equation (7)).

Pyridoxal phosphate participates in reactions where $\alpha$ amino acids are decarboxylated or racemized, and uridine diphosphate is concerned in the structural conversion of galactose-1-phosphate to glucose-1-phosphate. However, each of these coenzymes also is involved in normal reactions of equations (1) and (2), and even in the exceptions cited here, the coenzymes "anchor" the amino acid and sugar residues.

There are several notable omissions in the above table. Ascorbic acid has often been considered to be involved in electron transport, but the high level of this substance in tissues, compared to other vitamins, has made it appear to be an exception to the "vitamin-trace substance" theory. Recently, however, it has been shown to participate at catalytic levels in the oxidative breakdown of tyrosine. Biotin, too, has been implicated in carboxylation reactions, although it has been difficult to demonstrate it as a coenzyme in these processes. It is quite possible that both of these substances, ascorbic acid and biotin, actually exist as more complex coenzymes, perhaps of analogous structure to those listed in Table I. This is not to argue that all coenzymes must contain something in addition to the moiety bearing the "active center" (to which the group being transferred is attached), since lipoic acid is certainly anomalous in this respect, although Reed (36) has suggested that lipoic acid may be conjugated to diphosphothiamin-a suggestion challenged by Gunsalus (18).

Vitamin $B_{12}$ has also been omitted from consideration, since its structure is not completely established, although it does con- 
tain a nitrogenous base (the cyano-complex of a cobalt porphyrin) linked to a nucleotide (the 2'- or 3'-ribotide of benzimidazole). Likewise, its function is obscure, although the possible oxido-reduction of the cobalt porphyrin lends support to the hypothesis that it mediates the reduction of disulfide groups.

Glucose-1,6-diphosphate functions catalytically $\left(K_{m}=\right.$ $\left.4 \times 10^{-7} \mathrm{M}\right)$ in the conversion of glucose-1-phosphate to glucose6-phosphate, but here again we are dealing with a special case, since the catalyst actually "primes the pump" and is itself used up in the reaction, viz.

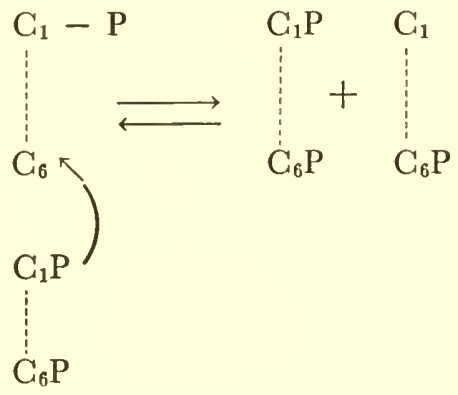

In a like manner, 2,3-diphosphoglycerate participates in the conversion of 2- to 3-phosphoglycerate.

There are also several substances, such as the AMP-ATP complex of Kiessling and Meyerhof (26) (see also Ohlmeyer (34)), the prosthetic group associated with rabbit muscle phosphorylase (10), the second prosthetic group of the crystalline yeast flavoprotein (4), or the factor replacing autoxidizable dyes involved in the enzymatic oxidation of reduced pyridine nucleotides (19), which would appear to be coenzymes because of their structure, function, or association with highly purified enzymes.

It is probably correct to say, however, that most of the major coenzymes or growth factors have been discoveredmajor in the sense of the amounts existing in tissues-but there are still undoubtedly new frontiers to be explored in that area which Lardy (29) has so aptly termed "the micro-catalytic vitamins." 


\section{Structure of Coenzymes}

One may pause at this point and inquire how we have reached our present state of knowledge that enables us to assemble the information in Table I. When a coenzyme has been established definitely as part of an enzymatic process by the criteria mentioned above, there ensues the fascinating game of isolating, if possible in pure form, and of characterizing the coenzyme in question. On occasion the game is played in a spirit of intense rivalry between competing groups. One might also remark parenthetically that of late the pace of the game has been stepped up-as witnessed, for example, by the relatively short time that elapsed between the postulation of lipoic acid as a coenzyme of pyruvic oxidase and its isolation, characterization, and total synthesis.

In part, this acceleration is due to the introduction of revolutionary new methods. In the past, the isolation of a coenzyme was accomplished principally by means of precipitation as heavy metal salts, precipitation by organic solvents, or extraction into organic solvents. These methods were somewhat nonspecific, so that impurities were only slowly removed over many steps with the resultant loss in yield. Now, these methods have not been abandoned and still find a useful place in purification schemes, but the emphasis has shifted toward newer techniques, based mainly upon principles of chromatography. Contemporary workers in the field are presented with a glittering array of new tools: ion-exchange chromatography, adsorption chromatography, partition chromatography, including countercurrent distribution, and even large-scale paper chromatography. The success of the chromatographic method lies in the fact that it induces a change of phase, in turn, of each component in a mixture. Thus, the components are spread out for display, as it were, before the eyes of the investigator. For example, the basic technique introduced by Cohn and Carter (12), i.e., separation of nucleotides on Dowex-1 ion-exchange resins, has been modified by Potter and his colleagues (23) so that the composition of the eluting medium is constantly changing 
(gradient elution). This permits a complete scparation of the acid-soluble nucleotides in tissues, and has revealed a complete spectrum of mono-, di-, and tri-phospho derivatives of the various purine and pyrimidine nucleosides.

The usefulness of such ion-exchange columns cannot be oreremphasized. Not only does this method provide a tool for rapidly determining the constituent nucleotides of a given tissue, but it can be applied with equal success to the large-scale isolation of individual nucleotides. It came as a shock to some, and a pleasant surprise to others, to learn that supposedly pure samples of ATP of commercial origin were, in reality, wonderfully complex mixtures of the other triphosphates, and undoubtedly many ATP-requiring systems will bear re-evaluation. Initially, participation of guanosine diphosphate in the reaction:

succinyl CoA $+\mathrm{P}+\mathrm{ADP} \longrightarrow$ succinate $+\mathrm{CoA}+\mathrm{ATP}(7)$

was overlooked until careful scrutiny of ADP samples by Sanadi and co-workers (37) revealed the presence of the true coenzymea situation somewhat reminiscent of the discovery of glucose1,6-diphosphate as a trace impurity in samples of glucose-1phosphate.

During purification a most serious problem is the lability of the coenzyme to chemical degradation, especially at the pyrophosphate and $N$-glycoside linkages. A corollary of this problem is the facile migration of phosphate groups. Although phosphate groups can be located to some degree on sugar residues by the periodate titration method, the technique does not distinguish between 2 '- and 3 '-isomers. The ready interconversion of adenosine-2-'phosphate to the $3^{\prime}$-isomer through the cyclic $2^{\prime}, 3^{\prime}$-diester caused the expenditure of great effort in several laboratories before it was finally established that pure adenylic acid "a" was the 2'-isomer and "b" the 3'-isomer, rather than vice versa.

One other fact should be emphasized at this point, and that is the ease with which nucleotides form cyclic phosphate derivatives. Thus, glucose-1,2-phosphate, adenosine-2', 3 '-phosphate 
(similarly for other purine and pyrimidine nucleotides), pantothenic-2,4-phosphate, FAD-X (a cyclic derivative of FAD), and riboflavin- $4^{\prime}, 5^{\prime}$-phosphate, all cyclic phosphates, are formed during the degradation of uridine diphosphate glucose, nucleic acids, CoA, and FAD, respectively. Such cyclic phosphates may also appear and play a role during enzymatic processes, for these substances are powerful phosphorylating agents.

With the advent of methods for obtaining coenzymes largely free from impurities, especially of similar structure, it has become possible to achieve final and conclusive purification via crystallization - a finding which has helped to restore nucleotides to their rightful place amongst respectable organic compounds. Occasionally, it has been necessary to employ a crutch for this process by crystallizing the nucleotide as a complex with some organic base such as acridine, quinine, or cyclohexylamine.

Pure coenzymes, if isolated in sufficient quantity, can be subjected to structure determination both by the methods of classical organic chemistry and by certain sensitive, smallscale methods suggested by enzymology itself. The coenzymes usually have no melting points, owing to their saltlike character, and elemental analysis is usually reserved for confirmatory evidence. However, degradation in acid or basic media, and subsequent examination of the liberated fragments, can provide a great deal of information. Small quantities of the fragments can be detected and compared with authentic compounds by means of paper chromatography. Tremendous ingenuity has been exercised in the composition of solvent systems for separating closely related compounds and in devising spray reagents or other means for locating these compounds on paper. A classical example of the potentialities of this technique has been afforded by the work of Baddiley and Thain (3) who obtained decisive evidence on the structure of CoA, although only minute amounts of the partially purified coenzyme were available.

Finally, it is extremely fortunate if the derived fragments can be estimated enzymatically, for example, AMP by a specific 
adenylic deaminase, or FMN by its action as a coenzyme for yeast TPN-cytochrome $c$ reductase. Assays of this sort are sensitive, require minute amounts of material, and are highly specific, so that they may be performed even in the presence of impurities.

Once a coenzyme has been isolated, and its structure determined, a number of ancillary facts about its chemistry begin to accumulate. Necessary documentation for the proper understanding of coenzyme function is provided by physical data such as: (1) absorption spectra, at various $p \mathrm{H}$ values; (2) $p \mathrm{~K}$ values obtained by classical titrimetric methods or by the recently introduced electrophoretic mobility technique of Bock and Alberty (7); (3) crystal structure, bond angles and distances (i.e., the exact three-dimensional shape of the molecule) as revealed by x-ray diffraction patterns; (4) oxidation potentials of appropriate groups; (5) free energies of hydrolysis of phosphate groups; (6) chemical studies such as the behavior and interaction of reactive groupings, and (7) the comparative study of appropriate model compounds. Too often such data have been sorely needed but unavailable, and enzymology is indebted to Meyerhof, Michaelis, R. Kuhn, and Borsook, to name only a few, who have enriched the literature with accurate information in the above categories. A singularly outstanding effort along the above lines has been the complete and extensive documentation of the properties of vitamin $B_{12}$, as described by Lester Smith and his group (14) and by Folkers (15).

Concerning the dynamic state of coenzymes in tissues, there has accumulated considerable evidence on the enzymatic breakdown and synthesis of these substances. As with most other classes of metabolites, knowledge of the former phase has preceded the latter. We know of pyrophosphatases which split the dinucleotides, of nucleotidases, i.e., phosphatases, which are selective for the various isomeric positions of the phosphate group, and nucleosidases which cleave the $N$ glycoside bonds. Other enzymes, which degrade the ring and sugar moieties, are well known but outside the purview of the 
present discussion. Suffice it to say, even the most hardy coenzymes can be rent asunder by the concerted action of various enzymes in the repertoire of the enzymologist.

Enzymatic synthesis of coenzymes is a more complicated affair, but satisfactory and accelerated progress is being made in this area. The biosynthesis of vitamins and various other nitrogen-containing heterocycles has been approached largely with the aid of isotopic tracers. As an example of this, one may cite the tour de force of Buchanan and his colleagues (9), who fed various labeled molecules to pigeons, isolated and degraded the excreted uric acid, and thus established that the purine ring has carbon atoms 2 and 8 derived from formate, carbon atom 6 from $\mathrm{CO}_{2}$, carbon atoms 4 and 5 and nitrogen atom 7 from glycine, nitrogen atoms 3 and 9 from glutamine, and nitrogen atom 1 from glutamic or aspartic acids. Subsequent work, notably by Buchanan and by G. R. Greenberg (16), has provided further information as to the sequence in which these building blocks unite and the nature of the intermediates preceding the intact purine. For example, glycine amide ribotide has been proved to be an intermediate, thus confirming the earlier finding that the purine ribotide, rather than the free purine, was the molecule initially synthesized. Likewise carbon atoms 2 and 8 are known to serve as "ring-closers" under the influence of a folic-acid coenzyme. It is to be hoped that these definitive studies will encourage similar efforts on the biosynthetic pathways of other important ring structures, e.g., riboflavin.

The enzymatic synthesis of nucleosides takes place under the influence of a phosphorylase, e.g. :

nicotinamide + ribose-1-P $\rightleftarrows$ nicotinamide riboside $+\mathrm{P}$ (8)

or by the action of certain DPN-ase enzymes which catalyze the reversible hydrolytic cleavage of DPN:

$\mathrm{DPN}+\mathrm{H}_{2} \mathrm{O} \rightleftarrows$ nicotinamide + adenosine diphosphate ribose 
Nucleotides are then formed by specific ATP-kinases, e.g.,

$$
\text { adenosine }+\mathrm{ATP} \longrightarrow \mathrm{AMP}+\mathrm{ADP}
$$

or, directly from the base by the reaction (28):

adenine +1 -pyrophosphoryl-ribose-5-phosphate $\longrightarrow \mathrm{AMP}+\mathrm{PP}$

Dinucleotides are formed from the corresponding mononucleotides by the representative reaction:

$$
\mathrm{FMN}+\mathrm{ATP} \longrightarrow \mathrm{FAD}+\mathrm{PP}
$$

where the ATP donates its nucleoside portion ( $c f$. also the synthesis of active methionine) instead of a phosphate as in equation (4), and additional phosphates are supplied to the dinucleotide (as in TPN and CoA) by another ATP-kinase reaction:

$$
\mathrm{DPN}+\mathrm{ATP} \longrightarrow \mathrm{TPN}+\mathrm{ADP}
$$

The entire biosynthetic route to CoA has been established by Lipmann and Novelli (33) and by Snell and colleagues (8), and is reproduced in the scheme below, since it illustrates many of the above principles.

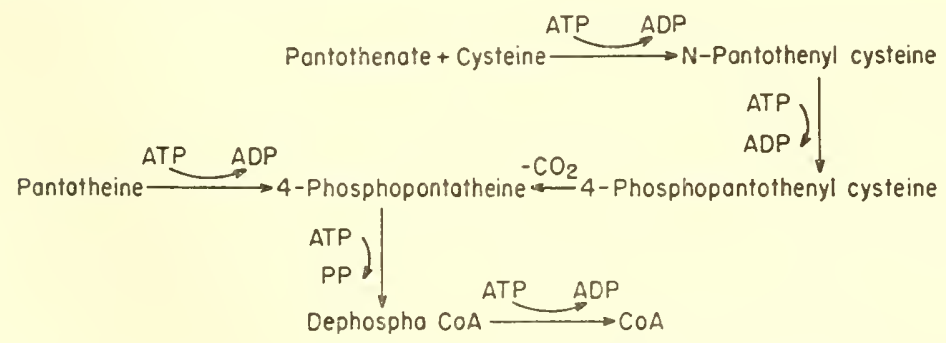

No discussion of coenzymes would be complete without reference to the chemical synthesis of these substances. Early workers, notably Levene, Kuhn, Karrer, and Folkers, had accomplished the total synthesis of several coenzymes of the mononucleotide class. But no member of the dinucleotide group had been prepared, owing to the formidable obstacles in the synthesis of unsymmetrically substituted pyrophosphates. About 10 years ago A. R. Todd (40) and his associates at Cam- 
bridge undertook a systematic and comprehensive program directed toward the chemical synthesis of mono- and dinucleotides and related substances. In addition to their contributions to the fundamental chemistry of nucleotides and phosphate esters, some of their monumental achievements include the total synthesis of: (1) purines and pyrimidines; (2) various ribo- and deoxyribonucleosides (demonstrating, in passing, that the sugar moieties were joined by $\beta$-glycoside linkages at the $3^{\prime}$ and $9^{\prime}$ positions of the pyrimidines and purines, respectively); AMP, ADP and ATP, FMN and nicotinamide riboside; (4) the $2^{\prime}, 3^{\prime}$, and $5^{\prime}$ mononucleotides of the purines and pyrimidines; and finally, (5) the dinucleotides, UDPG and FAD. The latter feat, which represented the first total synthesis of a dinucleotide, deserves a word of additional comment. It was carried out by condensation of the two key substances:

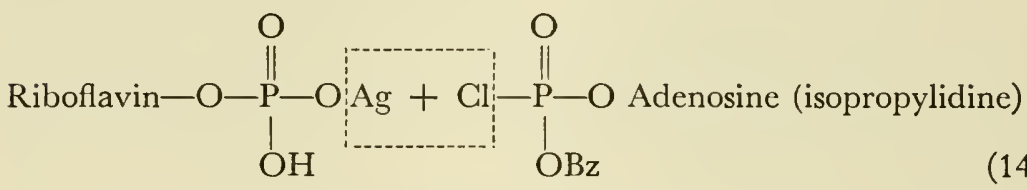

followed by removal of the protective benzyl $(\mathrm{Bz})$ and isopropylidine groups.

Chemical syntheses of nucleotides are costly at present and attended by relatively low yields ( $c f$., however, Khorana's synthesis of adenosine and uridine polyphosphates (25)), and it is doubtful whether they can compete commercially with isolation procedures until these difficulties are overcome. However, the value of this approach lies first in that it provides unambiguous proof of structure (sometimes lacking in the original assignment of structure to a coenzyme), and secondly in that it provides routes to otherwise unavailable coenzyme analogues or even to those coenzymes whose isolation from natural sources is not readily feasible.

Perhaps the successful efforts of Todd in this area and the dramatic demonstration by Lynen (30) that the mystical "active acetate" is, in reality, a straightforward organic substance, 
acetyl CoA, will encourage other investigators to attempt the synthesis of other labile or inaccessible substances such as the transient coenzyme phosphates (e.g., DPN $\sim$ P), often invoked, but never verified experimentally, as the initial carriers of highenergy phosphate in oxidative phosphorylation at the coenzyme level.

\section{Mechanism of Coenzyme Action}

By means of the techniques described in Section II we have now arrived at a seemingly comfortable state of knowledge where quite a respectable number of coenzymes have been characterized fully as to their chemical structure and properties, and have been established unequivocally as functional co-partners in their respective enzymatic systems. But we are still confronted with the important, and largely unanswered, question: "What is the precise mechanism by which these coenzymes exert their specific effects?" To a great extent this question is interwoven with the more fundamental question, namely, the mechanism of action of enzymes in general.

It is known that enzyme-catalyzed reactions proceed with lower activation energies than their counterparts wherein the catalysts are $\mathrm{H}^{+}, \mathrm{OH}^{-}$, other ions, or organic molecules. Of course, a given reaction has a fixed net change of free energy associated with it, and this quantity is simply the net difference of free energies between the products and reactants. A catalyst cannot change the equilibrium position of such a reaction* but merely hastens the rate at which the reaction proceeds by decreasing the activation energy. We are still largely ignorant of how a given molecule looks in its "activated" or transition state, but we may surmise that the molecule has been distorted in such a way that otherwise refractory bonds are weakened or positional barriers reduced, so that impacts due to ordinary ther-

* A contradiction to this general rule is supplied by the work of Theorell and Bonnichsen (39), who found that binding of substrates by high concentrations of alcohol dehydrogenase does, in fact, alter the equilibrium of the reaction. 
mal energy have a much greater probability of completing the reaction. A catalyst produces these favorable distortions by binding the substrate in a strained configuration, and, to be sure, this temporary debt of the binding energy must be repaid out of the net proceeds gained from the reaction.

It follows from the above discussion that in enzymatic reactions we must direct our attention to the binding of substrates by the protein catalysts. Many enzymatic reactions may be divided into three categories.

a. The components of one substrate are used to cleave a bond in the second substrate:

$$
\mathrm{H}_{2} \mathrm{O}+\mathrm{A}-\mathrm{B} \longrightarrow \mathrm{AH}+\mathrm{BOH}
$$

b. Two substrates form a new bond by the loss of a group from each; where $\mathrm{H}_{2} \mathrm{O}$ is removed the reaction is the reverse of equation (15).

c. The transfer of a group from a donor substrate to a similar acceptor substrate, e.g., equations (1) and (2).

All of these categories involve two substrates* so that we may envision a ternary complex where both reactants, or substrates, are bound in close juxtaposition on the enzyme surface and the necessary transfers take place in serial order. It may be argued that a ternary complex is not necessary (and perhaps less likely in a kinetic sense), so that two consecutive binary complexes are formed, i.e., in the case of $(a)$ above, where $\mathrm{E}<_{\mathrm{OH}}^{\mathrm{H}}$ is the enzyme:

$$
\mathrm{A}-\mathrm{B}+\mathrm{E} \mathrm{O}_{\mathrm{OH}}^{\mathrm{H}} \longrightarrow \mathrm{AH}+\mathrm{BOH}+\mathrm{E}
$$

followed by regeneration of the hydrated enzyme:

$$
\mathrm{E}+\mathrm{H}_{2} \mathrm{O} \longrightarrow \mathrm{E}<\mathrm{OH}
$$

* There is some evidence that even reactions involving internal rearrangements of a single substance, i.e., $\mathrm{A} \rightleftarrows \mathrm{A}^{\prime}$, are bimolecular with respect to $\mathrm{A}$. 
In contrast to reactions of type $(a)$, where no coenzyme is required, type $(c)$ reactions often requirc a coenzyme. This is because the enzyme lacks the proper chemical groupings to act as "middleman" carrying, at one stage, the group being transferred. Hence, it must call in a special assistant, the coenzyme, which is bound to the enzyme and which intervenes as shown in equations (1) and (2) where the symbol $\mathrm{X}$ might well be replaced by $\mathrm{X}-\mathrm{E}$. It is unlikely that in this case we can reduce the problem to a series of binary complexes, for this would involve the enzyme, at one stage, holding the transferring group, thereby apparently obviating the need for the coenzyme.

Reactions of type $(b)$ are more complex for they involve the creation of a new bond, usually with the elimination of water (e.g., esters, anhydrides, peptides, glycosides). The energy requirement for this sort of reaction is met by the addition of still another substrate to the reaction, usually ATP, which activates one of the condensing partners. The sequence may be written:

$$
\mathrm{BOH}+\mathrm{ATP} \longrightarrow \mathrm{B}-\mathrm{AMP}+\mathrm{PP}
$$

followed by a transfer to the actual coenzyme

$$
\mathrm{B}-\mathrm{AMP}+\mathrm{CoA} \longrightarrow \mathrm{B}-\mathrm{CoA}+\mathrm{AMP}
$$

and, finally, transfer of the activated group from the coenzyme to the acceptor:

$$
\mathrm{B}-\mathrm{CoA}+\mathrm{AH} \longrightarrow \mathrm{A}-\mathrm{B}+\mathrm{CoA}-\mathrm{H}
$$

Obviously, there are many possible variations of the above schemes, but these illustrations may be considered as representative.

In light of the above discussion one may return to the question of what is so special about the structure of each of the coenzymes in Table I, that makes it unique in being able to transfer particular groups. Each of the coenzymes shown has itself a functional group which seems of suitable structure to carry reversibly the group being transferred. To press further along these lines would undoubtedly be desirable, but at present 
would lead only to the fruitless position of inspecting the structure of biologically active agents in the hope of understanding their mechanism of action. The remainder of the molecule is concerned then, in all probability, with the binding of the coenzyme to the protein. Thus, DPN and TPN have the same oxidation potentials and undoubtedly could replace each other as hydrogen carriers, except for the rigid structural requirements-undoubtedly a reflection of some control mechanismfor binding to the appropriate apoenzyme. Again, one need not invoke any special function of the uridine molecule as a carrier of activated sugar groups or cytidine for choline; probably each could replace the other in an energetic sense were it not for the binding requirement.

These remarks are not meant to imply that the portion of the coenzyme carrying the functional group is not also involved in binding. The inhibition of FAD-protein binding by riboflavin, and the loss of fluorescence when FAD is bound to enzymes, interpreted as a linkage between the $\mathrm{N}-3$ position on the isoalloxazine ring and the protein, are evidence for this point. Since many coenzymes have in common a simple mononucleotide structure, or the components thereof, it would appear instructive to examine, from both a thermodynamic and kinetic view, the binding of a typical mononucleotide, e.g., AMP, by various model peptides. In the event that no binding could be found to approximate that of typical coenzymes and apoenzymes, this, in itself, might be considered as preliminary evidence for "cage" type of binding, where the total binding is more than the sum of individual group effects.

The plain fact is that, at present, very little is known about the manner in which coenzymes are bound to proteins. In order to understand this type of binding we need to have information about the reactive groups and topography of both components. In the latter case this is indeed a formidable undertaking, and progress has been understandably slow. However, with the availability of pure, crystalline nucleotides and the adoption of physical methods we are being presented now with 
accurate, dimensioned pictures of the coenzymes. Actually, some information along this line can sometimes be gained from simple experiments. Thus, Bessey et al. (6) found that FAD at $p \mathrm{H} 7$ has only about $14 \%$ of the fluorescence of FMN but that upon acidification of FAD the fluorescence rises to an equal value. That this is not simply a splitting of the dinucleotide in acid solution is shown by the fact that the fluorescence again falls upon readjusting the $p \mathrm{H}$ to 7 . Since admixture of an equimolal amount of AMP with FMN does not depress the fluorescence to this extent, it may be assumed that in FAD the adenylic portion lies in close juxtaposition to, and interacts with, the isoalloxazine moiety. This may be similar to the resonance interactions observed between the parallel aromatic rings of the para-cyclophanes (13):

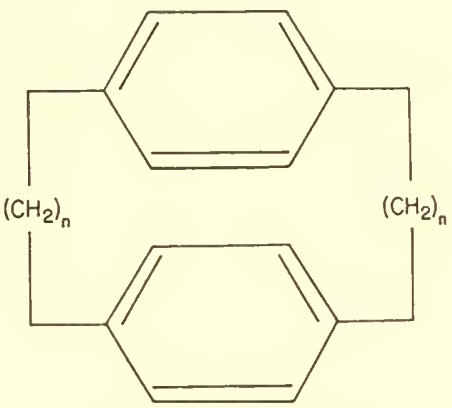

Assuming, then, that we may know the exact structure of a coenzyme and something, at least, of the potential binding groups on the protein (e.g., $-\mathrm{OH},-\mathrm{NH}_{2},-\mathrm{COOH},-\mathrm{SH}$, imidazole groups), can we draw any conclusions as to the type of binding? Are there covalent bonds, electrostatic bonds, hydrogen bonds, van der Waals' forces, cage effects, or combinations of these? We might also raise the question: Since the active centers on the protein for both coenzyme and substrate are probably small relative to the entire protein, what then is the function of the bulk of the protein which is known to be essential for enzyme catalysis? Unfortunately, kinetic studies yield only $K_{m}$ values, whose exact interpretation in terms of 
affinity, or binding, of the two components, remains obscure. Other quantitative data, such as the variation in binding with $p \mathrm{H}$, ionic strength, and temperature, are noticeably lacking. There remain only fragmentary, qualitative observations based upon chemical modification of the coenzyme or protein. These studies are similar in rationale to the search for the active center of certain proteolytic enzymes, where the broad specificity of the enzyme permits a variety of structurally similar substrates to be used and the variation in reaction kinetics noted. Unfortunately, the problem is somewhat analogous to the use of quantum mechanical methods to relate absorption spectra to structure of complex organic molecules; the correlation becomes more exact in proportion to the a priori knowledge of both factors.

Some very interesting observations have been made recently by Racker, Velick, Boyer, and Chance on the binding of DPN by 3-phosphoglyceraldehyde dehydrogenase (35). It has been suggested that the nicotinamide portion of DPN is bound to a glutathione residue of the protein, as shown below

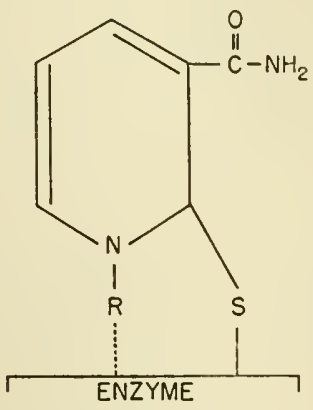

Whether or not this tentative structure is correct, it is known that combination of DPN and the enzyme gives rise to a new absorption band at $360 \mathrm{~m} \mu$. By contrast, reduced DPN forms a spectroscopic complex $\left(\lambda_{\max }\right.$ at $\left.325 \mathrm{~m} \mu\right)$ with horse liver alcohol dehydrogenase or lactic dehydrogenase, but neither complex is observed with glutamic dehydrogenase or yeast alcohol dehydrogenase. The question has been raised repeatedly whether 
pyridine nucleotides are firmly bound in zivo to their apoenzymes, or whether they form only transient complexes enabling them to shuttle between two dehydrogenases of a linked reaction, as in glycolysis, for example. The fact that pyridinoproteins, except 3-phosphoglyceraldehyde dehydrogenase, have been isolated as completely dissociated enzymes, with the corollary finding that most $K_{m}$ values for the reconstituted enzymes are relatively high, argues in favor of the latter hypothesis. However, Huennekens and Green (20) have presented evidence that many of the pyridinoproteins within mitochondria appear to be conjugated with respect to DPN, and upon disorganization of the mitochondrial architecture, destruction or dispersion of the DPN causes a requirement for added DPN to appear. In addition it has been shown recently that the tightly bound DPN of 3-phosphoglyceraldehyde dehydrogenase can be utilized, apparently without being dissociated, in a linked reaction with lactic apodehydrogenase, or as a substrate for DPN-cytochrome $c$ reductase.

In addition, it should be remarked that there is a considerable body of evidence scattered throughout the literature to the effect that in certain cases a coenzyme may be bound originally in a one-to-one ratio with its protein, but after dissociation has once occurred, rebinding of the components may be of a degenerate type where a great excess of coenzyme must be present.

In the field of flavoproteins early work by Kuhn implicated the $\mathrm{N}-3$ position in the isoalloxazine ring and the phosphate group as binding sites. More recently, Mahler (31) has suggested a more detailed picture to explain the respective bindings in metalloflavoproteins. Even the seemingly innocuous sugar moieties may be more than insulating groups and may participate in binding. Huennekens and Snell (21) have found that the lyxoflavin analogues of FMN and FAD have noticeably higher $K_{m}$ values than the parent compounds when compared in enzymatic assay systems. Recent findings have revealed the surprising fact that FAD, bound to succinic dehydrogenase, is not entirely released by trichloracetic acid or heat denaturation 
unless the enzyme is first digested with a proteolytic enzyme $(5,38)$. This would suggest that the coenzyme is either bound by a peptide or ester linkage, or possibly enmeshed in a protein cage. The nature of the protein groups involved in flavin binding has been investigated by Theorell, who suggested that both amino groups and aromatic hydroxyl groups were responsible. Whatever the possible types of binding are for the cases cited above, it seems evident that for certain flavoproteins, notably D-amino acid oxidase, where the conjugated enzyme is resistant to dialysis but readily dissociated upon exposure to acid conditions, the binding cannot be covalent.

From this discussion of various aspects of nucleotide coenzymes, it should be apparent why metal ions, anions, and other cofactors or activators like cysteine, were rather arbitrarily excluded from the present group. Cysteine, and other reducing agents, probably keep labile sulfhydryl groups on the enzyme in the reduced state (although glutathione has a special role in the glyoxalase reaction and ascorbic acid is specific in the enzymatic reduction of folic acid). The anions and the monovalent cations $\left(\mathrm{Na}^{+}, \mathrm{K}^{+}, \mathrm{NH}_{4}{ }^{+}\right.$) play an important, but obscure, role in maintaining the integrity of certain enzymes, possibly by controlling the degree of hydration around the protein. The other metal ions can be divided into two separate categories. The first are those such as copper, molybdenum, and iron which are themselves capable of oxido-reduction, and which have potentials within the range of other oxido-reduction coenzymes. These metals may often participate as regular electron carriers, although in metalloflavoproteins they may be concerned, in addition, with binding of FAD to the protein or to the reactants.

The other metals, such as $\mathrm{Mg}^{++}$and $\mathrm{Mn}^{++}$, probably assist the binding of substrate or coenzyme to the protein. At physiological $p \mathrm{H}$ most substrates and coenzymes are anions and where the "active center" of the protein may be deficient in appropriate cationic groups, the metal can then serve as a bridge between anionic groups of the coenzyme or substrate and the protein. The almost universal requirement for $\mathrm{Mg}^{++}$in 
systems where anionic pyrophosphate groups are involved argues strongly for the above hypothesis, as does the fact that $K_{m}$ values for the metal ion are often of the same order of magnitude as the coenzyme. Also, metal ions are often not very specific, as for example, the replacement of $\mathrm{Mg}^{++}$by $\mathrm{Mn}^{++}$ in many systems, and the finding that aldolases from different sources require $\mathrm{Fe}^{++}, \mathrm{Zn}^{++}$, or $\mathrm{Co}^{++}$, whereas muscle aldolase is inhibited by all of these ions.

No discussion of metal ion effects would be complete without reference to the recent elegant work on metal ion-pyridoxal phosphate complexes. Baddiley (2) and Snell (32) have shown that ternary Schiff-base complexes containing a metal, pyridoxal phosphate, and amino acids can carry out a remarkable series of reactions (transamination, deamination, decarboxylation) in the absence of enzymes. Studies of this sort, where welldefined, simple model systems can duplicate accurately the enzymatic processes, give us valuable insight into the mechanism of enzyme and coenzyme action.

The above material has been presented as a survey of current thinking and trends in the field of coenzymes and nucleotides. At the time of this writing there come to mind five areas in which major discoveries are still to be made. These are: oxidative phosphorylation at the coenzyme level, protein synthesis, biosynthesis of large molecules, hormonal control of enzymes, and genetic control of enzyme synthesis. To this may be added the sixth, and more vast, area: the relation of enzyme chemistry to problems of medicine. Present knowledge of coenzymes, and their function in many metabolic pathways, gives us assurance that such nucleotides of biological importance undoubtedly play a key role as well in these unconquered frontiers.

\section{References}

1. Axelrod, A. E., V. R. Potter, and C. A. Elvehjem, J. Biol. Chem., 142, 85 (1942).

2. Baddiley, J., Nature, 170, 711 (1952). 
3. Baddiley, J., and E. M. Thain, J. Chem. Soc., 1951, 2253.

4. Ball, E. G., J. Gen. Physiol., 29, 413 (1946).

5. Basford, R. E., S. Mii, and D. E. Green, Federation Proc., 14, 178 (1955).

6. Bessey, O. A., O. H. Lowry, and R. H. Love, J. Biol. Chem., 180, 755 (1949).

7. Bock, R. M., and R. A. Alberty, M. Biol. Chem., 193, 435 (1951).

8. Brown, G. M., and E. E. Snell, J. Am. Chem. Suc., 75, 2782 (1953).

9. Buchanan, J. M., J. C. Sonne, and A. M. Delluva, J. Biol. Chem., 173 69, 81 (1948); J. Am. Chem. Soc., 75, 1516 (1953).

10. Buell, M. W., Federation Proc., 17, 192 (1952).

11. Cantoni, G. L., J. Biol. Chem., 189, 745 (1951).

12. Cohn, W., and C. E. Carter, J. Am. Chem. Soc., 72, 4273 (1950).

13. Cram, D. J., and H. Steinberg, J. Am. Chem. Soc., 73, 5091 (1951).

14. Fantes, K. H., J. E. Page, L. F. J. Parker, and E. L. Smith, Proc. Roy. Soc. (London), B136, 492 (1949).

15. Folkers, K., and D. E. Wolf, Vitamins and Hormones, 12, 2 (1954).

16. Goldthwait, D. A., R. A. Peabody, and G. R. Greenberg, J. Am. Chem. Soc., 76, 5258 (1954).

17. Green, D. E., in D. M. Greenberg, ed., Chemical Pathways of Metabolism, Vol. 1. Academic Press, New York, 1954.

18. Gunsalus, I. C., in W. D. McElroy and B. Glass, eds., Mechanism of Enzyme Action, p. 607. Johns Hopkins Press, Baltimore, 1954.

19. Huennekens, F. M., R. E. Basford, and B. W. Gabrio, J. Biol. Chem., 273, 951 (1955).

20. Huennekens, F. M., and D. E. Green, Arch. Biochem., 27, 417, 428 (1950).

21. Huennekens, F. M., and E. E. Snell, unpublished observations.

22. Hurlburt, R. B., and V. R. Potter, J. Biol. Chem., 209, 1 (1954).

23. Hurlburt, R. B., H. Schmitz, A. F. Brumm, and V. R. Potter, J. Biol. Chem., 209, 23 (1954).

24. Kennedy, E. P., and S. B. Weiss, J. Am. Chem. Soc., 77, 250 (1955).

25. Khorana, H. G., J. Am. Chem. Soc., 76, 3517, 5050 (1954).

26. Kiessling, W., and O. Meyerhof, Biochem. Z., 296, 410 (1938).

27. Kornberg, A., Phosphorus Metabolism, 1, 411 (1951).

28. Kornberg, A., I. Lieberman, and E. L. Simms, J. Am. Chem. Soc., 76, 2027 (1954).

29. Lardy, H. A., Record Chem. Progr. (Kresge-Hooker Sci. Lib.), 12, 9 (1951).

30. Lynen, F., and E. Reichert, Z. angew. Chem., 63, 47 (1951).

31. Mahler, H. R., and D. B. Elowe, J. Biol. Chem., 210, 165 (1954).

32. Metzler, D. E., J. B. Longnecker, and E. E. Snell, J. Am. Chem. Soc., 75, 2786 (1953); ibid. 76, 639 (1954).

33. Novelli, G. D., Federation Proc., 12, 675 (1953).

34. Ohlmeyer, P., J. Biol. Chem., 190, 21 (1951). 
35. Racker, E., in IV. D. McElroy and B. Glass, eds., Mechanism of Enzyme Action. Johns Hopkins Press, Baltimore, 1954.

36. Reed, L. J., and B. G. DeBusk, J. Am. Chem. Soc., 71, 3964 (1952).

37. Sanadi, D. R., D. M. Gibson, P. Ayengar, and L. Ouellet, Biochim. et Bisphys. Acta, 13, 146, 309 (1954).

38. Singer, T. P., and E. B. Kearney, Federation Proc., 14, 282 (1955).

39. Theorell, H., and R. Bonnichsen, Acta Chem. Scand., 5, 1105 (1951).

40. Todd, A. R., Proc. Roy. Soc. (London), A226, 70 (1954). 


\section{THE BIOSYNTHESIS OF PORPHYRINS; THE SUCCINATE-GLYCINE CYCLE}

DAVID SHEMIN, The Department of Biochemistry, College of Physicians and Surgeons, Columbia University, New York

There has been tremendous progress in the last twenty years in the elucidation of the biochemical reactions and transformations which occur in living organisms. Some of the general concepts which have emerged, summarized very briefly, are that the basic reactions in the cells are surprisingly simple, that the cell synthesizes its complex molecules from relatively simple and available substances, and that there is a biochemical unity in living matter.

Although these concepts were perhaps not fully appreciated when a study of the biosynthesis of porphyrins was begun ten years ago, the picture which has emerged is rather a good illustration of these basic concepts. The studies have revealed that the complicated looking molecule, protoporphyrin, is synthesized from two simple and available compounds, glycine and succinate, by relatively simple reactions. Furthermore it has been established that the synthesis of this esoteric looking molecule is intimately related to the citric acid cycle, since the "active" succinate utilized in porphyrin synthesis is produced in these cyclic reactions. Although most of the investigations have been concerned with the biosynthesis of protoporphyrin, it 
appears that all the porphyrins in nature, including chlorophyll, in all different types of cells are synthesized by the same basic pathway. The different porphyrins merely arise by modifications occurring in the side chains in the $\beta$-positions of the pyrrole units.

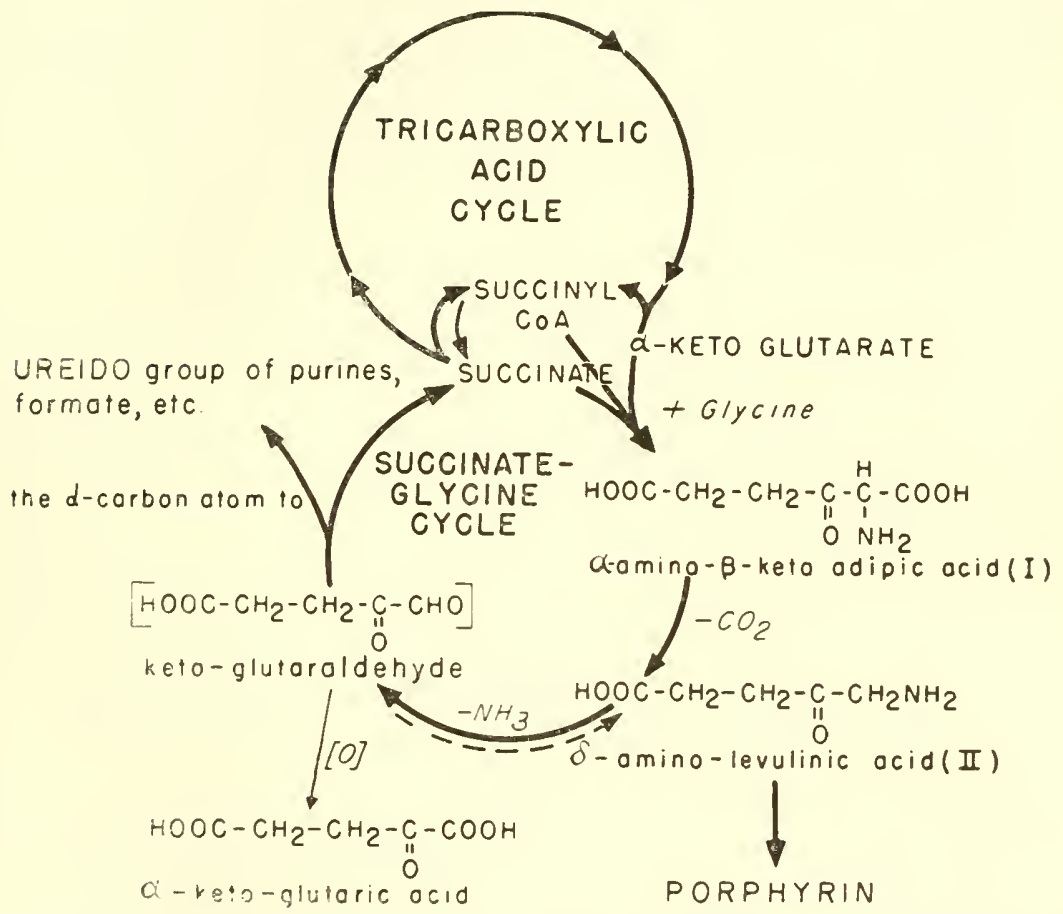

Fig. 1. Succinate-glycine cycle: a pathway for the metabolism of glycine $(28,29)$.

The problem of the biosynthesis of porphyrins, in the formation of the initial intermediates, can be considered, from a more general point of view, to be merely one aspect of the metabolism of glycine. Many over-all aspects of glycine metabolism are now known, and especially the metabolic pattern of the $\alpha$-carbon atom of glycine in its utilization for the synthesis of other compounds has been established. The $\alpha$-carbon atom of 
glycine, no longer attached to the carboxyl group, is utilized for the synthesis of porphyrins, the ureido groups of purines, the $\beta$-carbon atom of serine, and for methyl groups. This metabolic pattern is similar to that of the so-called " $\mathrm{G}_{1}$ " compounds, with the exception that the latter cannot substitute for glycine in porphyrin synthesis. It would seem therefore that these apparently unrelated compounds (porphyrins, purines, serine, and methyl groups) have one common feature, namely, the participation of the $\alpha$-carbon atom of glycine for their synthesis.

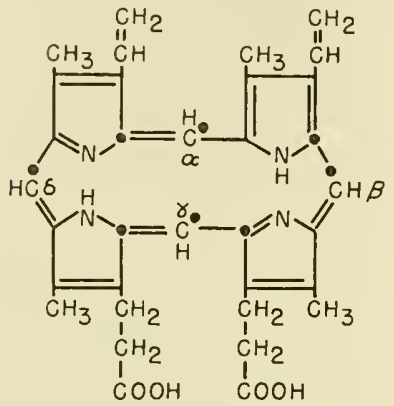

PROTOPORPHYRIN IX

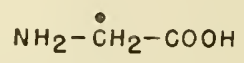

Glycine $-2-\mathrm{C}^{14}$

$\mathrm{NH}_{2}-\dot{\mathrm{CH}}_{2}-\mathrm{CO}-\mathrm{CH}_{2}-\mathrm{CH}_{2}-\mathrm{COOH}$

6-Aminolevulinic Acid-5- $\mathrm{C}^{14}$

Fig. 2. The carbon atoms of protoporphyrin which arise from the $\alpha$-carbon atom of glycine and from the $\delta$-carbon atom of $\delta$-aminolevulinic acid.

It would appear reasonable from a unitarian approach, therefore, to consider the possibility that glycine is metabolized via a pathway in which intermediates are produced which then can be utilized for the synthesis of these different compounds. In an attempt to unify the reactions of glycine we have postulated a series of reactions, called the succinate-glycine cycle (Figure 1). This pathway for glycine metabolism suggested itself from a study of the mechanism of porphyrin synthesis. In this pathway it is postulated that "active" succinate condenses on the $\alpha$-carbon atom of glycine to give rise to $\alpha$ amino- $\beta$-ketoadipic acid. This $\beta$-keto acid decarboxylates to give rise to $\delta$-aminolevulinic acid, which can be utilized for porphyrin synthesis or further metabolized in such a manner 
that its $\delta$-carbon atom (originally the $\alpha$-carbon atom of glycine) is utilized for the synthesis of the ureido groups of purines, for the $\beta$-carbon atom of serine, and for methyl groups, while the remaining four-carbon atom residue is reconverted to succinate. Since this succinate-glycine cycle was arrived at from our study of porphyrin synthesis, it may then be worth while to consider the pertinent facts of porphyrin synthesis which led to the elaboration of this cycle, and the findings which lend their support and broaden the implications of the postulated series of reactions. Furthermore, data will be presented to support the

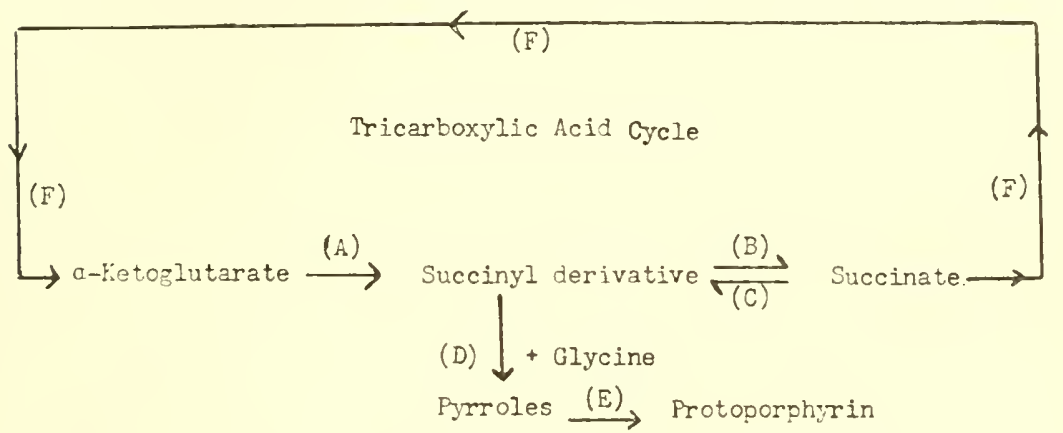

Fig. 3. The relationship of the citric acid cycle and protoporphyrin formation.

statement that $\delta$-aminolevulinic acid, one of the intermediates, is formed from glycine and "active" succinate and is the source of all the atoms of the porphyrin molecule.

It has been previously demonstrated that although the four nitrogen atoms of protoporphyrin are derived from glycine $(15,22,26,27,33)$ eight carbon atoms of the porphyrin molecule are derived from the $\alpha$-carbon atom of glycine $(16,18,34)$; four of these carbon atoms are still attached to the nitrogen atom and four are not. The carbon atoms of protoporphyrin derived from the $\alpha$-carbon atom of glycine are shown in Figure 2 (34). Furthermore, it has been found that the carboxyl group of glycine is not utilized for any of the carbon atoms of the porphyrin $(12,18)$. This latter negative finding was an important 
clue in the elucidation of the mechanism of condensation of the succinate and glycine. It has also been demonstrated that a succinyl intermediate, arising from the tricarboxylic acid cycle, is the source of the remaining twenty-six carbon atoms of protoporphyrin $(24,30)$. The relationship of the tricarboxylic acid
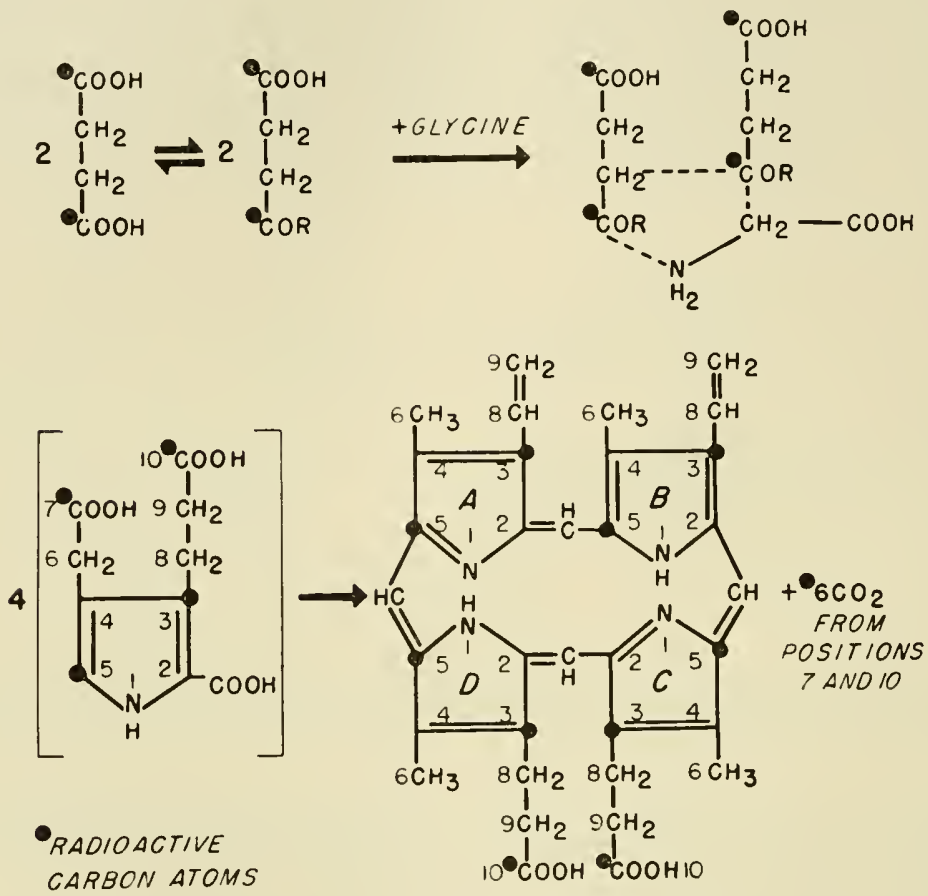

Fig. 4. The position of succinate in protoporphyrin and the labeling pattern obtained in protoporphyrin synthesized from succinate-1,4- $\mathrm{C}^{14}$.

cycle to porphyrin formation was formulated previously as shown in Figure 3. This relationship was further documented by studying the synthesis of protoporphyrin from $\alpha$-ketoglutarate-5- $\mathrm{C}^{14}, \alpha$-ketoglutarate- $1,2-\mathrm{C}^{14}$, and citrate-1,5- $\mathrm{C}^{14}$. In each case the predicted carbon atoms of the porphyrin were labeled from the $\mathrm{C}^{14}$-labeled substrate (35). The experiments in these studies were carried out by incubating either whole (25) or 
hemolyzed red blood cells $(14,24)$ of a duck with the $\mathrm{C}^{14}$ labeled compounds, and subsequently chemically degrading the isolated hemin. The degradation was done in such a manner that individual carbon atoms from a particular position in the porphyrin could be isolated $(30,34)$. The carbon atoms arising from succinate and glycine are shown in Figures 2 and 4 . It would appear merely from the $\mathrm{C}^{14}$-labeling pattern obtained that each pyrrole unit is made up of 2 moles of succinate and 1 mole of glycine. Since the methene bridges also arise from the $\alpha$-carbon atom of glycine, the synthesis of the porphyrin molecule requires 8 moles of both succinate and glycine.

It then became of interest to find the chemical mechanism by which the succinate and glycine combine to form the pyrrole unit of the porphyrin. Our studies on the utilization of the $\alpha$-carbon of glycine for porphyrin formation have led us to the following mechanism. It was difficult for some time to understand the distribution of the $\alpha$-carbon atom of glycine in the porphyrin molecule (four in the pyrrole rings and the four methene bridge carbon atoms, Figure 2). A possible explanation for the observed distribution of the $\alpha$-carbon atom of glycine was that the glycine was utilized for porphyrin synthesis via two pathways.

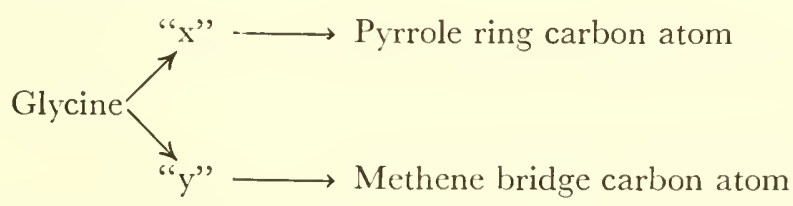

Since the metabolic pattern of the $\alpha$-carbon atom of glycine was similar to that of the " $\mathrm{C}_{1}$ " compounds, and since the methene bridge carbon atoms are no longer attached to the nitrogen atom, it would appear that a " $\mathrm{C}_{1}$ " compound might be substituted for compound "y." Studies were undertaken to test this point. In no case could $\mathrm{CH}_{3} \mathrm{OH}, \mathrm{H}_{2} \mathrm{CO}, \mathrm{HCOOH}$, $\mathrm{CH}_{3} \mathrm{NH}_{2}, \mathrm{CO}_{2}$, or $\mathrm{CHO}-\mathrm{COOH}$ substitute for glycine. These negative findings, coupled with the finding that the $\alpha$-carbon atom of glycine was always equally utilized for both the pyrrole- 
ring carbon atom and the methene-bridge carbon atom, led us to conclude that the same derivative of glycine was utilized for both of these positions.

These findings limit the number of possible ways in which the succinate and glycine combine to form a pyrrole unit. The mechanism of condensation of succinate and glycine becomes a bit more obvious if one considers that the product of this condensation should not only supply a reasonable mechanism of
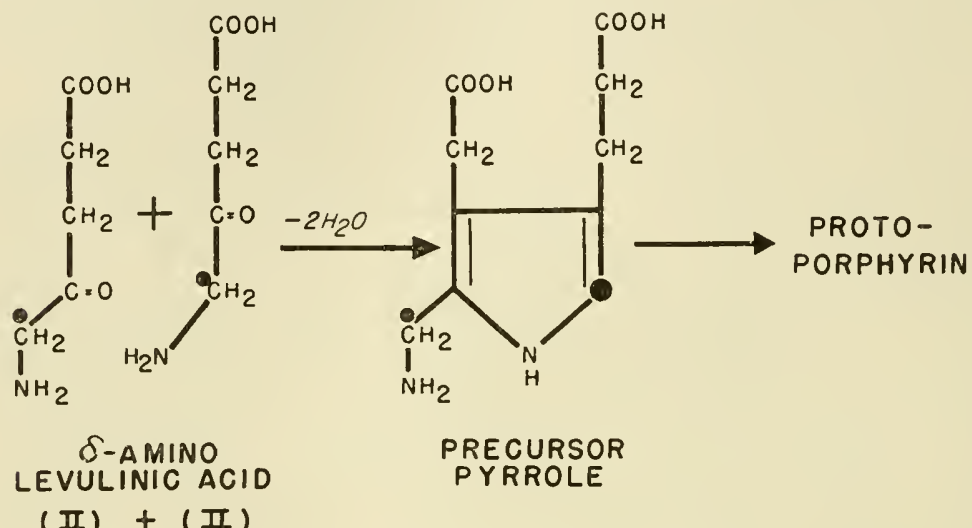

PRECURSOR

PYRROLE

\section{(II) + (III)}

Fig. 5. The mechanism for the formation of the monopyrrole, porphobilinogen, by condensation of 2 moles of $\delta$-aminolevulinic acid. The carbon atoms bearing the closed circles $(\bullet)$ were originally the $\alpha$ carbon atom of glycine.

pyrrole formation but should also suggest a reasonable mechanism by which the $\alpha$-carbon atom of glycine is detached from its carboxyl group and should explain the distribution of the $\alpha$-carbon atom of glycine in the porphyrin molecule. The condensation of succinate on the $\alpha$-carbon atom of glycine to form $\alpha$-amino- $\beta$-ketoadipic acid would appear to agree with the above findings. The compound formed, being a $\beta$-keto acid, could then readily be decarboxylated, and thus provide a mechanism by which the $\alpha$-carbon atom of glycine becomes detached from its carboxyl group. It would also resolve the apparent 
paradox that whereas only the $\alpha$-carbon atom of glycine is utilized for porphyrin synthesis, yet no " $\mathrm{C}_{1}$ " compound can act as a substitute. 'The product of the decarboxylation would then be $\delta$-aminolevulinic acid. Condensation of 2 moles of the latter, by a Knorr type of condensation (Figure 5), would readily give rise to a pyrrole in which the $\alpha$-carbon atom of glycine would be distributed in the positions previously observed. To test this hypothesis $\delta$-aminolevulinic acid was synthesized in three independent ways $(28,29)$. This was done to insure the correctness of the structure. The compound synthesized had the correct analysis and took up 1 mole of periodate. From the periodate reaction the expected products, formaldehyde and succinic acid, were isolated in excellent yields. Prior to our synthesis of $\delta$-aminolevulinic acid, Wynn and Corwin (36) had prepared this compound by another series of reactions.

In the initial experiments, unlabeled $\delta$-aminolevulinic acid was added to duck red blood cell hemolyzates along with either $\mathrm{C}^{14}$-labeled glycine or $\mathrm{C}^{14}$-labeled succinate. The radioactivities of the hemin samples isolated in these experiments were compared with those obtained from controls in which the unlabeled $\delta$-aminolevulinic acid was omitted. The rationale for these dilution-type experiments is as follows: if $\delta$-aminolevulinic acid is an intermediate formed from the condensation of glycine and succinate, any labeled $\delta$-aminolevulinic acid formed from these labeled substrates will be diluted by the added unlabeled compound, and consequently this should be reflected in the lowered radioactivity of the hemin samples synthesized in the presence of unlabeled $\delta$-aminolevulinic acid. It can be seen from Table I that the hemin samples made in the presence of unlabeled $\delta$ aminolevulinic acid contained less $\mathrm{C}^{\mathrm{I} 4}$ than those of the controls made either from $\mathrm{C}^{14}$-labeled glycine or succinate $(28,29)$. These results, which are in full agreement with the hypothesis, can also be explained, however, by the possibility that $\delta$-aminolevulinic acid is acting not as a diluent but as an inhibitor of heme synthesis. To rule out the latter possibility, the $\delta$-aminolevulinic acid added in Experiment 2 (Table I) was labeled with 
$\mathrm{N}^{15}$. It can be seen that whereas the incorporation of $\mathrm{C}^{14}$ from the glycine was lowered, there was a comparatively large incorporation of $\mathrm{N}^{15}$ into the porphyrin, thus demonstrating that the lowered $\mathrm{C}^{14}$ activity of the hemin sample was due to dilution rather than inhibition. Further proof that $\delta$-aminolevulinic acid arises by the condensation of glycine and succinate was obtained by incubating red blood cell hemolyzates with glycine2- $\mathrm{C}^{14}$ and unlabeled $\delta$-aminolevulinic acid, and subsequently isolating the $\delta$-carbon atom. In such an experiment it was

\section{TABLE I}

Comparison of $\mathrm{C}^{14}$-Activities of Hemin Samples Synthesized from

Glycine-2-C ${ }^{14}(0.05 \mathrm{mc} . / \mathrm{mM})$ or Succinic AcId-2-C ${ }^{14}(0.05 \mathrm{mc} . / \mathrm{mM})$ in the Presence and Absence of Nonradioactive $\delta$-Aminolevulinic Acid $(28,29)$

\begin{tabular}{|c|c|c|c|c|c|}
\hline \multirow{3}{*}{$\begin{array}{l}\text { Experi- } \\
\text { ments }\end{array}$} & \multirow{2}{*}{\multicolumn{3}{|c|}{ Substrates }} & \multicolumn{2}{|c|}{$\begin{array}{c}\text { Isotope } \\
\text { concentration } \\
\text { in hemin }\end{array}$} \\
\hline & & & & \multirow{2}{*}{$-\mathrm{C}^{14}$} & \multirow{2}{*}{$\begin{array}{c}\mathrm{N}^{15} \\
\text { (atom } \\
\% \\
\text { excess) }\end{array}$} \\
\hline & $\mathrm{C}^{14}$-labeled & $\mathrm{N}^{16}$-labeled & Unlabeled & & \\
\hline \multirow[t]{2}{*}{1} & $\begin{array}{l}\text { Glycine-2-C } \mathrm{C}^{14} \\
(0.05 \mathrm{mM})\end{array}$ & - & - & 125 & \\
\hline & $\begin{array}{r}\text { Glycine-2-C }{ }^{14} \\
(0.05 \mathrm{mM})\end{array}$ & - & $\begin{array}{l}\delta \text {-Aminolevulinic } \\
\text { acid }(0.05 \\
\mathrm{mM})\end{array}$ & 15 & \\
\hline \multirow[t]{3}{*}{2} & $\begin{array}{r}\text { Glycine-2-C } \mathrm{C}^{14} \\
(0.05 \mathrm{mM})\end{array}$ & - & - & 230 & \\
\hline & $\begin{array}{r}\text { Glycine-2-C } \mathrm{C}^{14} \\
(0.05 \mathrm{mM})\end{array}$ & $\begin{array}{l}\delta \text {-Aminolevulinic } \\
\text { acid }(0.05 \\
\mathrm{mM})^{a}\end{array}$ & - & 48 & 0.21 \\
\hline & - & $\begin{array}{l}\text { Glycine }(0.33 \\
\mathrm{mM})^{a}\end{array}$ & - & & 0.06 \\
\hline \multirow[t]{2}{*}{3} & $\begin{array}{c}\text { Succinate-2-C } \mathrm{C}^{14} \\
(0.1 \mathrm{mM})\end{array}$ & - & - & 660 & \\
\hline & $\begin{array}{c}\text { Succinate-2-C } \mathrm{C}^{14} \\
(0.1 \mathrm{mM})\end{array}$ & - & $\begin{array}{r}\delta \text {-Aminolevulinic } \\
\quad \operatorname{acid}(0.1 \mathrm{mM})\end{array}$ & 180 & \\
\hline
\end{tabular}

${ }_{a}$ The isotopic concentrations of these samples were 34 atom $\%$ excess $\mathrm{N}^{15}$. In each of the experiments the volume of the hemolyzed preparation was $30 \mathrm{ml}$. Unlabeled succinate $(0.1 \mathrm{mM})$ was added to the flasks in which labeled glycine was the substrate, and unlabeled glycine $(0.33 \mathrm{mM})$ was added to the flasks in which labeled succinate was the substrate. Each flask contained $1 \mathrm{mg}$. of iron (ferric). 
found that the formaldehyde liberated upon periodatc oxidation of a crude fraction containing $\delta$-aminolevulinic acid was highly radioactive.

It now remaincd to establish more directly the utilization of $\delta$-aminolevulinic acid for porphyrin formation. The compound was synthesized with $\mathrm{C}^{14}$ in its $\delta$-carbon atom, and its utilization was compared with that of glycine. It can be seen from Table II that the hemin synthesized from an equimolar amount

TABLE II

Comparison of $\mathrm{C}^{14}$ Activities of Hemin Samples

Synthesized from Glycine-2-C ${ }^{14}(0.05 \mathrm{mc} . / \mathrm{mM})$

ANd $\delta$-Aminolevulinic Acid $(0.05 \mathrm{mc} . / \mathrm{mM})(28,29)$

\begin{tabular}{lcc}
\hline \multicolumn{1}{c}{$\mathrm{C}^{14}$ Substrate } & Other Additions & $\begin{array}{c}\mathrm{C}^{14} \\
\text { Activity } \\
\text { of Hemin } \\
\text { (c.p.m.) }\end{array}$ \\
\hline Glycine-2-C $(0.05 \mathrm{mM})$ & Succinate $(0.1 \mathrm{mM})$ & 333 \\
$\delta$-Aminolevulinic acid $(0.05 \mathrm{mM})$ & - & 23,000 \\
$\delta$-Aminolevulinic acid $(0.05 \mathrm{mM})$ & Glycine $(0.33 \mathrm{mM})$ & 21,000 \\
& Succinate $(0.1 \mathrm{mM})$ & \\
\hline
\end{tabular}

of $\delta$-aminolevulinic acid was about 65 times more radioactive than hemin synthesized from glycine. More rigorous proof that $\delta$-aminolevulinic acid is indeed the precursor for porphyrin synthesis was obtained by degrading a hemin sample synthesized from $\delta$-aminolevulinic acid-5- $\mathrm{C}^{14}$ and from $\delta$-aminolevulinic acid$1,4-\mathrm{C}^{14}$. The $\delta$-carbon atom of the former compound should label the same carbon atoms of protoporphyrin as those which we have previously found to arise from the $\alpha$-carbon atom of glycine, since according to the hypothesis the latter carbon atom is the biological source of the $\delta$-carbon atom of $\delta$-aminolevulinic acid. Furthermore, the $\delta$-aminolevulinic acid-1,4-C $\mathrm{C}^{14}$ should label the same carbon atoms of protoporphyrin found to arise from the carboxyl groups of succinate, since from Figures 1 and 4 these carbon atoms arise from succinate.

It can be seen from Table III that the same $\mathrm{C}^{14}$ distribution pattern was found in protoporphyrin synthesized from $\delta$ - 
aminolevulinic acid-5- $\mathrm{C}^{14}$ as from glycine-2- $\mathrm{C}^{14}$; 50 per cent of the $\mathrm{C}^{14}$ activity resides in the pyrrole rings and 50 per cent in the methene bridge carbon atoms (see Figure 2) (29).

\section{TABLE III}

Distribution of $\mathrm{C}^{14}$-Activity in Protoporphyrin Syntilesized from $\delta$-Aminolevulinic ACid-5-C ${ }^{14}$ ANd from Glycine-2-C ${ }^{14}(16,25)$

\begin{tabular}{lcc} 
& \multicolumn{2}{c}{$\begin{array}{c}\text { Molar activity (\%) in fragments } \\
\text { of porphyrin synthesized from }\end{array}$} \\
\cline { 2 - 3 } \multicolumn{1}{c}{ Fragments of porphyrin } & $\begin{array}{c}\delta \text {-Aminolevulinic } \\
\text { acid-5-C }\end{array}$ & Glycine-2-C ${ }^{14}$ \\
\hline Protoporphyrin & 100 & 100 \\
Pyrrole rings A + B (methylethyl- & & 24.6 \\
$\quad$ maleimide) & 24.5 & 25.3 \\
Pyrrole rings C + D (hematinic acid) & 25.2 & 49.9 \\
Pyrrole rings A + B + C + D & 49.7 & 50.1 \\
Methene bridge carbon atoms & 50.3 & \\
\hline
\end{tabular}

Also it can be seen from Table IV that the same $\mathrm{C}^{14}$ distribution pattern was found in protoporphyrin synthesized from $\delta$-aminolevulinic acid-1,4- $\mathrm{C}^{14}$ as from succinate-1,4- $\mathrm{C}^{14}$; ten carbon atoms are equally radioactive, 40 per cent of the $\mathrm{C}^{14}$ activity resides in pyrrole rings $A$ and $B, 60$ per cent of the activity resides in pyrrole rings $\mathrm{C}$ and $\mathrm{D}$, and the carboxyl groups contain 20 per cent of the $\mathrm{C}^{14}$ activity (see Figure 4) (20).

\section{TABLE IV}

Distribution of Cit Activity in Protoporphyrin Syntifesized from $\delta$-Aminolevulinic Acid-1,4-C ${ }^{14}$ ANd from Succinate-1,4-CI4 (20)

\begin{tabular}{|c|c|c|}
\hline \multirow[b]{2}{*}{ Fragments of porphyrin } & \multicolumn{2}{|c|}{$\begin{array}{l}\text { Molar activity }(\%) \text { in fragment } \\
\text { of porphyrin synthesized from }\end{array}$} \\
\hline & $\begin{array}{l}\delta \text {-Aminolevulinic } \\
\text { acid-5- } \mathrm{C}^{14}\end{array}$ & $\begin{array}{l}\text { Succinic } \\
\text { acid-1,4-C }\end{array}$ \\
\hline Protoporphyrin & 100 & 100 \\
\hline $\begin{array}{l}\text { Pyrrole rings } \mathrm{A}+\mathrm{B} \text { (methylethyl- } \\
\text { maleimide) }\end{array}$ & 38.0 & 39.4 \\
\hline Pyrrole rings $\mathrm{C}+\mathrm{D}$ (hematinic acid) & 61.5 & 59.5 \\
\hline Pyrrole rings $\mathrm{A}+\mathrm{B}+\mathrm{C}+\mathrm{D}$ & 99.5 & 98.5 \\
\hline Carboxyl groups & 20.4 & 20.5 \\
\hline
\end{tabular}


Thus all the carbon atoms of protoporphyrin are derived from $\delta$-aminolevulinic acid. The role of $\delta$-aminolevulinic acid in porphyrin synthesis was also actively pursued by Neuberger and Scott (17), and just subsequent to our initial finding they published a confirmatory paper; further confirmation was published by Dresel and Falk (6). Furthermore, it may be well to point out that the theoretical formulation of the structure of the precursor pyrrole (28) is the same structure which was determined by Westall (32) and by Cookson and Rimington (3) for porphobilinogen, a compound excreted in the urine of patients with acute porphyria. The utilization of $\delta$-aminolevulinic acid for porphyrin formation adds to the certainty that porphobilinogen (7) is an intermediate in porphyrin synthesis. These findings make $\alpha$-amino- $\beta$-ketoadipic acid an obligatory intermediate, and we have found experimentally that this $\beta$-keto is indeed an intermediate. Injection of $\delta$-aminolevulinic acid or the diethyl ester of $\alpha$-amino- $\beta$-ketoadipic acid gives rise to the urinary excretion of porphobilinogen (31).

The condensation of "active" succinate and glycine to form $\delta$-aminolevulinic acid subsequently, thus far appears to require the partially intact structure of the red blood cell. It has been found that whereas $\delta$-aminolevulinic acid can be converted to protoporphyrin in either an homogenized preparation or a cell-free extract, the conversion of succinate and glycine to porphyrin takes place only with intact cells or with those cells which have been hemolyzed with water $(23,29)$. Homogenized preparations obtained in a blendor are no longer capable of synthesizing protoporphyrin from succinate and glycine (Table $\mathrm{V})$. It would appear that on homogenization the functional activity of only those enzymes of the system that are involved in the condensation of succinate and glycine is lost. However, the finding that $\delta$-aminolevulinic acid can be converted to protoporphyrin in a cell-free extract opened up the possibility that soluble enzymes, concerned with each of the steps in this conversion, could be isolated.

Indeed, it was subsequently and independently found in 
TABLE V

Comparison of $\mathrm{C}^{14}$ Activities of Hemin Synthesized from $\delta$-Aminolevulinic ACID-5-C $\mathrm{C}^{14}(0.05 \mathrm{mc} . / \mathrm{mM})$, FROM GlyCiNe-2-C'14 $(0.05 \mathrm{mc} . / \mathrm{mM})$ AND FROM SUCCINIC Acid-2-Ci4 $(0.05 \mathrm{mc} . / \mathrm{mM})$ in Different Duck Red Blood Cell Preparations ${ }^{a}$

$(23,29)$

\begin{tabular}{|c|c|c|c|}
\hline $\begin{array}{c}\text { Experi- } \\
\text { ment } \\
\text { no. }\end{array}$ & $\mathrm{C}^{14}$ Substrate & $\begin{array}{c}\text { Red cell } \\
\text { preparation }\end{array}$ & $\begin{array}{c}\mathrm{C}^{14} \\
\text { activity } \\
\text { in } \\
\text { hemin } \\
\text { sample }\end{array}$ \\
\hline \multirow[t]{6}{*}{1} & Glycine $(0.05 \mathrm{mM})^{b}$ & Hemolyzed & 395 \\
\hline & Glycine $(0.05 \mathrm{mM})^{b}$ & Homogenized & 2 \\
\hline & Succinate $(0.05 \mathrm{mM})^{c}$ & Hemolyzed & 565 \\
\hline & Succinate $(0.05 \mathrm{mM})^{c}$ & Homogenized & 1 \\
\hline & $\delta$-Aminolevulinic acid $(0.006 \mathrm{mM})$ & Hemolyzed & 3900 \\
\hline & $\delta$-Aminolevulinic acid $(0.006 \mathrm{mM})$ & Homogenized & 4500 \\
\hline \multirow[t]{4}{*}{2} & $\delta$-Aminolevulinic acid $(0.009 \mathrm{mM})$ & Homogenized & 2200 \\
\hline & $\delta$-Aminolevulinic acid $(0.009 \mathrm{mM})$ & $\begin{array}{l}\text { Supernatant } \\
\quad\left(12 \times 10^{3} \text { g. }\right)\end{array}$ & 1600 \\
\hline & $\delta$-Aminolevulinic acid $(0.009 \mathrm{mM})$ & $\begin{array}{l}\text { Supernatant } \\
\quad\left(47 \times 10^{3} \mathrm{~g} .\right)\end{array}$ & 1600 \\
\hline & $\delta$-Aminolevulinic acid $(0.009 \mathrm{mM})$ & $\begin{array}{l}\text { Supernatant } \\
\quad\left(100 \times 10^{3} \text { g. }\right)\end{array}$ & 1500 \\
\hline \multirow[t]{2}{*}{3} & $\delta$-Aminolevulinic acid $(0.009 \mathrm{mM})$ & $\begin{array}{l}\text { Supernatant } \\
\qquad\left(12 \times 10^{3} \mathrm{~g} .\right)\end{array}$ & 2000 \\
\hline & $\delta$-Aminolevulinic acid $(0.009 \mathrm{mM})$ & $\begin{array}{l}\text { Lyophilized } \\
\text { preparation }\end{array}$ & 1500 \\
\hline
\end{tabular}

a Each preparation represented $25 \mathrm{ml}$. of duck blood prepared as previously described $(14,15)$ and contained $1 \mathrm{mg}$. of iron (ferric).

${ }^{b}$ Plus $0.1 \mathrm{mM}$ of nonradioactive $\alpha$-ketoglutaric acid.

c Plus $0.33 \mathrm{mM}$ of nonradioactive glycine.

three different laboratories that a highly purified protein fraction from ox liver (10), duck erythrocytes (21) and chicken erythrocytes (11) can convert $\delta$-aminolevulinic acid to porphobilinogen. In our laboratory we obtained a highly purified fraction from duck blood, which on incubation with $\delta$-aminolevulinic acid$5-\mathrm{C}^{14}$ produced labeled porphobilinogen. Since the porphobilinogen is presumably synthesized from 2 moles of $\delta$-aminolevulinic acid (Figure 5), its molar radioactivity should be twice that of the $\delta$-aminolevulinic acid used as the substrate. The molar radioactivities of the substrate, $\delta$-aminolevulinic acid, and of the product, porphobilinogen, were found to be $242 \times 10^{3}$ 
c.p.m. and $487 \times 10^{3}$ c.p.m., respectively. This finding demonstrates experimentally the utilization of 2 moles of $\delta$-aminolevulinic acid for porphobilinogen formation. Further evidence that porphobilinogen is an intermediate in protoporphyrin synthesis was obtained by incubating equal volumes of the cellfree extract of duck erythrocytes with equimolar amounts of $\delta$-aminolevulinic acid $(0.018 \mathrm{mc} . / \mathrm{mM})$ and with the enzymatically synthesized radioactive porphobilinogen $(0.036 \mathrm{mc} . / \mathrm{mM})$, and subsequently isolating the hemin and determining its radioactivity. The radioactivities of the hemin samples synthesized from $\delta$-aminolevulinic acid and from the porphobilinogen were 92 c.p.m. and 85 c.p.m., respectively, after a 2-hour incubation, and 350 and 336 c.p.m., respectively, after a 15hour incubation period (21). This latter result is in agreement with the findings of Falk, Dresel, and Rimington (7) and of Bogorad and Granick (2).

Although no evidence has yet been obtained concerning the biological mechanism of conversion of the monopyrrole to the tetrapyrrole structure, several suggestions have been advanced $(2,4)$. We would like to suggest still another possibility which may explain the distribution of the $\alpha$-carbon atom of glycine or the $\delta$-carbon atom of $\delta$-aminolevulinic acid in the porphyrin molecule of the I and III series. This mechanism is based on the synthetic mechanism of dipyrrole and tetrapyrrole formation demonstrated by Corwin and Andrews (5) and by Andrews, Corwin, and Sharp (1).

Condensation of 3 moles of the precursor pyrrole (porphobilinogen) or of a closely related derivative, would lead to a tripyrrylmethane compound, as schematically represented in Figure 6. The tripyrrylmethane then breaks down into a dipyrrylmethane and a monopyrrole. The structure of the dipyrrylmethane is dependent on the place of splitting. An A split would give rise to dipyrrylmethane A, and a B split would give rise to dipyrrylmethane $\mathrm{B}$. Condensation of 2 moles of dipyrrylmethane A would give rise to a porphyrin of the I series, and condensation of a mole of $\mathrm{A}$ and a mole of $\mathrm{B}$ would 
give rise to a prophyrin of the III series. In the formation of the porphyrin of the III series it can be seen from Figure 6 that it is necessary to lose a one-carbon atom compound, since there are three aminomethyl side chains and only two are required to condense the two dipyrroles to the porphyrin structure. If the mechanism similar to that outlined in Figure 6 is concerned with porphyrin synthesis, it would appear that this one-carbon atom compound given off could well be formaldehyde. Consistent with this idea is our finding (29) that on the conversion of por-

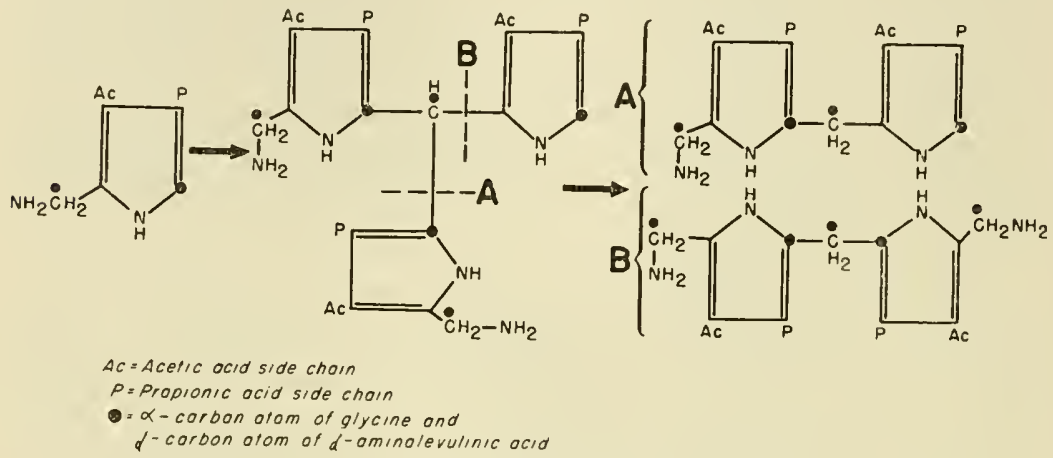

Fig. 6. A mechanism of porphyrin formation from the monopyrrole.

phobilinogen to porphyrins either by heating under acid conditions (32) or by enzymatic conversion in cell-free extracts $(23,29)$ formaldehyde was formed. This was established by heating or incubating porphobilinogen, labeled with $\mathrm{C}^{14}$ in the aminomethyl group, and subsequently isolating radioactive formaldehyde as the dimedon derivative.

It would appear that on conversion of porphobilinogen to porphyrins, formaldehyde from the aminomethyl group is formed and that any postulated mechanism should take this into consideration. It is difficult at present to establish the structure of the intermediate tetrapyrrole compounds which are formed prior to the formation of protoporphyrin. However, we would like to suggest that these intermediate tetrapyrrole compounds may be in a more highly reduced state, containing 
methylene bridge carbon atoms rather than methene bridge carbon atoms, and consequently uroporphyrin and coproporphyrin are oxidized products of the intermediates.

If the proposed succinate-glycine cycle is a pathway of glycine metabolism embracing the reactions known for the $\alpha$-carbon atom of glycine, then the $\delta$-carbon atom of $\delta$-aminolevulinic acid should have the same metabolic spectrum. To test the hypothesis, $\delta$-aminolevulinic acid-5- $\mathrm{C}^{14}$ was injected into a duck, and hemin, guanine, and adenine were isolated from the red blood cells; serine and the methyl group of methionine were obtained from the plasma proteins, and uric acid was isolated from the excreta.

It was found that the ureido groups of the purines (19), the $\beta$-carbon atom of scrine (9), and the methyl group of methionine (8) contained $\mathrm{C}^{14}$. These experimental results, which support the existence of the succinate-glycine cycle, are to be considered as only preliminary in nature, since quantitative aspects have not yet been completely explored. Quantitative evaluation is difficult in whole animals, since cells are not very permeable to $\delta$-aminolevulinic acid and the compound is very unstable as the free amino acid. In an experiment in the bird the $\mathrm{C}^{14}$ activity of both the hemin and the ureido groups of the purines synthesized from $\delta$-aminolevulinic acid-5- $\mathrm{C}^{14}$ was 2.5 times greater than in those samples synthesized in a comparable experiment in which glycine-2- $\mathrm{C}^{14}$ was the substrate. This may be a minimal difference if one considers the experiments in vitro (see Table II) in which the $\mathrm{C}^{14}$ activity of the hemin synthesized from $\delta$-aminolevulinic acid was about 65 times greater than that synthesized from glycine. However, it would appear from the above results that the $\delta$-carbon atom of $\delta$-aminolevulinic acid has the same metabolic pattern as that of the $\alpha$-carbon atom of glycine and likewise as that of the " $\mathrm{C}_{1}$ " compounds beyond the porphyrin synthesis. Further support for this conclusion is our finding that while both glycinc-2- $\mathrm{C}^{14}$ and $\delta$-aminolevulinic acid-5- $\mathrm{C}^{14}$ gave rise to radioactive formate in the urine of a rat, the formate arising from $\delta$-aminolevulinic acid was about 
3 times more radioactive than that made from the $\alpha$-carbon atom of glycine (9).

The cycle also provides a mechanism by which the carbon atoms of glycine can be oxidized to carbon dioxide. The formation of $\alpha$-amino- $\beta$-ketoadipic acid and subsequent decarboxylation of this $\beta$-keto acid would yield 1 mole of carbon dioxide. This mole of carbon dioxide was originally the carboxyl group of glycine. If the postulated ketoglutaraldehyde or some other derivative can be converted to $\alpha$-ketoglutaric acid, a pathway is provided for the conversion of the $\alpha$-carbon atom of glycine to carbon dioxide, for the $\alpha$-carboxyl group of the ketoglutarate was originally the $\alpha$-carbon atom of glycine. In this mechanism, it will be noted, formate is not an obligatory intermediate for the conversion of the $\alpha$-carbon atom of glycine to carbon dioxide. $\quad \alpha$-Ketoglutaric acid isolated from a cell-free extract of duck red blood cells which were incubated with $\delta$-aminolevulinic acid-5- $\mathrm{C}^{14}$ contained radioactivity (29).

Although the evidence for the condensation of succinate and glycine appears to be rather conclusive, it does appear that this reaction may not be unique but rather a prototype of a more general reaction. We observed a few years ago that the utilization of glycine for porphyrin formation is markedly inhibited by the addition of either acetate or pyruvate (13). These compounds appear to inhibit the condensation of succinate and glycine, since the conversion of $\delta$-aminolevulinic acid to porphyrin is not influenced by the addition of these acids. It would appear that "active" acetate may compete with succinate for the glycine, since the inhibition can be overcome by the addition of other members of the citric acid cycle. If acetate condenses with glycine, the product would be $\alpha$-aminoacetoacetic acid. Upon decarboxylation this $\beta$-keto acid would yield aminoacetone, an analogue of $\delta$-aminolevulinic acid. We have carried out some studies with aminoacetone labeled with $\mathrm{C}^{14}$ in the carbon atom bearing the amino group. We have found that this radioactive carbon atom is utilized for the ureido groups of uric acid, that it can be converted both to for- 
mate and carbon dioxide, the radioactivity of which is several times greater than that produced from glycine-2- $\mathrm{C}^{14}(9)$.

The condensation of glycine with either "active" succinate or "active" acetate provides a pathway whereby glycine can be oxidized to carbon dioxide and the intermediates produced in the cycle drawn off for the synthesis of other compounds. This is similar to the citric acid cycle, in which another two-carbon compound is oxidized to carbon dioxide and intermediates are produced which can be drawn off for synthesis. In the succinate-glycine cycle, succinate is the catalyst instead of oxaloacetate.

\section{References}

1. Andrews, J. S., A. H. Corwin, and A. G. Sharp, J. Am. Chem. Soc., 72, 491 (1950).

2. Bogorad. L., and S. Granick, Proc. Natl. Acad. Sci. U. S., 39,1176 (1953).

3. Cookson, G. H., and C. Rimington, Nature, 177, 875 (1953).

4. Cookson, G. H., and C. Rimington, Biochem. J. (London), 57, 476 (1954).

5. Corwin, A. H., and J. S. Andrews, J. Am. Chem. Soc., 59, 1973 (1937).

6. Dresel, E. I. B., and J. E. Falk, Nature, 172, 1185 (1953).

7. Falk, J. E., E. I. B. Dresel, and C. Rimington, Nature, 172, 292 (1953).

8. Foster, G. L., and D. Shemin, unpublished findings.

9. Gatt, S., and D. Shemin, unpublished findings.

10. Gibson, K. D., A. Neuberger, and J. J. Scott, Biochem. J. (London), 58, xli (1954).

11. Granick, S., Science, 120, 1105 (1954).

12. Grinstein, M., M. D. Kamen, and C. V. Moore, J. Biol. Chem., 174, 767 (1948).

13. Labbe, R., and D. Shemin, unpublished results.

14. London, I. M., and M. Yamasaki, Federation Proc., 17, 250 (1952).

15. Muir, H. M., and A. Neuberger, Biochem. J. (London), 45, 163 (1949).

16. Muir, H. M., and A. Neuberger, Biochem. J. (London), 47, 97 (1950).

17. Neuberger, A., and J. J. Scott, Nature, 172, 1093 (1953).

18. Radin, N. S., D. Rittenberg, and D. Shemin, J. Biol. Chem., 184, 745 (1950).

19. Russell, C. S., and D. Shemin, unpublished findings.

20. Schiffman, E., and D. Shemin, unpublished.

21. Schmid, R., and D. Shemin, J. Am. Chem. Soc., 77, 506 (1955).

22. Shemin, D., Cold Spring Harbor Symposia Quant. Biol., 13, 185 (1948). 
23. Shemin, D., T. Abramsky, and C. S. Russell, J. Am. Chem. Soc., 76, 1204 (1954).

24. Shemin, D., and S. Kumin, J. Biol. Chem., 198, 827 (1952).

25. Shemin, D., I. M. London, and D. Rittenberg, J. Biol. Chem., 173, 799 (1948); ibid., 183, 757 (1950).

26. Shemin, D., and D. Rittenberg, J. Biol. Chem., 159, 567 (1945).

27. Shemin, D., and D. Rittenberg, J. Biol. Chem., 166, 621 (1946).

28. Shemin, D., and C. S. Russell, J. Am. Chem. Soc., 75, 4873 (1953).

29. Shemin, D., C. S. Russell, and T. Abramsky, J. Biol. Chem., 215, 613 (1955).

30. Shemin, D., and J. Wittenberg, J. Biol. Chem., 192, 315 (1951).

31. Weliky, I., and D. Shemin, unpublished findings.

32. Westall, R. G., Nature, 770, 614 (1953).

33. Wittenberg, J., and D. Shemin, J. Biol. Chem., 178, 47 (1949).

34. Wittenberg, J., and D. Shemin, J. Biol. Chem., 185, 103 (1950).

35. Wriston, J. C., Jr., L. Lack, and D. Shemin, Federation Proc., 12, 294 (1953); J. Biol. Chem. 215, 603 (1955).

36. Wynn, R. W., and A. H. Corwin, J. Org. Chem., 15, 203 (1950). 


\section{PROBLEMS IN THE STUDY OF MULTIPLE ENZYME SYSTEMS}

G. ROBERT GREENBERG, Department of Biochemistry, School of Medicine, Western Reserve University, Cleveland, Ohio

Many over-all pathways of biosynthesis of cellular constituents have been outlined in recent years. It is obvious that the resolution of these pathways into discrete reaction mechanisms ultimately depends on using in vitro systems and requires all the techniques of biochemistry. Yet in many instances the concepts which help in approaching such problems are quite elementary. In studies of multiple enzyme systems certain factors and ideas have repeatedly entered into our reasoning. Although a good part of the thinking involved is not entirely quantitative, it may nevertheless provide an approach to the problem. Accordingly I should like to bring together some of these ideas at the risk of outlining concepts which belong to the everyday vocabulary of the more sophisticated and with the hope that others may be stimulated to undertake a thorough organization of this subject. Many of the important problems concerned with multi-enzyme systems such as the energetics of coupled reactions, kinetics, and the use of inhibitors have not been included. I hope therefore that these paragraphs will be considered as a reflection of some of our experiences and will be clearly distinguished from a brief do-it-yourself pamphlet. 


\section{LITERATURE}

It is common experience that one of the best training grounds for the investigation of multi-enzyme systems is a thorough study of glycolysis. All of the necessary tools, concepts, and difficulties can be found in these studies which span 50 years. Unfortunately although many of the concepts involved now have emerged in a relatively simple form they tend not to become part of one's armamentarium until some aspects of the scheme have actually been studied.

Dixon (7) has written an excellent little book on the problems of multi-enzyme systems, and this may be recommended as a source of stimulation and of orientation. He has considered the problems arising in the study of glycolysis, such as coupled systems, competing enzyme systems, limiting reactions, equilibria, and the role of $K_{m}$. Green (11) has dealt with enzymes in sequence and has considered many aspects of the organization of enzyme systems. Although many of the newer texts have well-developed chapters on the characteristics of enzymes, little space is devoted to multi-enzyme studies, understandably so because of the qualitative nature of the problem. However, the discussions by Fruton and Simmonds (8) are especially helpful.

\section{Some Approaches to Multi-Enzyme Systems (MES)*}

THE BALANCE

Given a particular pathway to study and knowing the beginning substrates, the student is faced with the problem of obtaining intermediates while fractionating somewhat blindly, assaying for the disappearance of a substrate and the appearance of the product. This approach certainly has yielded important

* Abbreviations: MES, multi-enzyme system; AMP, adenosine-5'phosphate; IMP, inosine-5'-phosphate; IRMP, 5-amino-4-imidazolecarboxamide-5'-phosphoriboside; HX, hypoxanthine; R-5-P, ribose-5-phosphate; R-1-P, ribose-1-phosphate; PRA, 5-phosphoribosylamine; $\mathrm{FAH}_{4}$, tetrahydrofolic acid; $\mathrm{N}^{10}{ }_{-} \mathrm{CHOFAH}_{4}, \mathrm{~N}^{10}$-formyl derivative of $\mathrm{FAH}_{4}$; PRPP, 5-phosphoribosylpyrophosphate. 
information, but it may not always be fruitful at the beginning. One has only to consider the situation in the study of glycolysis. In the carlier work it secms reasonable to assume that fractionation of the soluble extract which converted glucose to ethanol would not have brought about the accumulation of sizable quantities of intermediates, since the over-all reaction is necessary for the regeneration of the proper acceptor systems. To be sure, later when some of the details of the mechanism had become known, individual steps could be isolated and the products studied. This had to await the finding of intermediates in the reaction sequence, however. In this regard then the oldfashioned careful complete balance study is perhaps the most dependable beginning tool. Time and again advances in such studies arise from a consideration of the balance. The work involved in the development of the quantitative methods may be revolting, but the results are never wasted and are always useful. In many cases the balance can differentiate between two possible mechanisms or may provide evidence for the presence of an intermediate compound. Even when the reaction appears to be straightforward, a balance may change one's ideas. The classic findings of Harden and Young depended on a demonstration of net phosphate uptake during glycolysis. It is important to balance as many of the reactants as possible, since a partial balance can lead to an erroneous picture of the reaction.

\section{INTERMEDIATES}

The problem always arises in studying a MES whether to try to isolate possible intermediate compounds in the over-all system or to try to fractionate so as to facilitate the accumulation of such compounds. Naturally no single approach can be applied to all problems. When intermediate compounds accumulate fortuitously, their isolation and characterization becomes an obvious first step. If not, fractionation, the use of inhibitors, or omission of substrates may lead to accumulation of intermediates. In any event it may be necessary to obtain intermediates by special tricks. Once an in vitro system is 
available, every possible source of intermediates should be explored. The correlation by Krebs and Henseleit (19) of citrulline from watermelon seeds with the urea cycle is a classical example. Several of the purine precursors have been shown to accumulate in bacterial systems under various conditions. But one of the most important tools in these studies is the obtaining of information on pathways and intermediates by the use of microbiological mutants. Such studies have been admirably carried out with the tryptophan-synthesizing system (31) and aromatization reactions (6). The use of antimetabolites can give similar results. These tools are not for the confirmed microbiologist alone but are readily available for multi-enzyme studies.

Whether fractionation precedes isolation of intermediate compounds or vice versa, it is clear that progress in the resolution of a MES is greatly dependent on the isolation and identification of intermediate compounds. It seems reasonable to predict that a plot of the activity in the field of glycolysis versus time would show spurts of activity after an intermediate had been isolated and made available. When an intermediate is available, the fractionation of the ensuing reactions in a stepwise manner is greatly facilitated.

Frequently the investigator on the basis of organic-chemical or biochemical precedent or by pure guess resorts to the synthesis of possible intermediate compounds. The author has adopted the principle that (1) only the most straightforward syntheses should be attempted and (2) a compound should be prepared and tested only when some objective data point to it. In the latter category one may include the results of balance studies. Such syntheses can take one far astray. For example, before it was known that purines were synthesized as their ribotide derivatives, the author spent many months synthesizing the free base, 5-amino-4-imidazolecarboxamide, as a likely precursor of hypoxanthine in pigeon liver on the basis of the findings of Shive et al. (28) in bacteria. Actually this compound is not synthesized, per se, although it is utilized (2). Likewise later we prepared and gathered a large number of three-carbon amino- 
malonic acid derivatives to test in this system based on the knowledge that purines could be synthesized this way chemically (26) and guessing that the ribose residue was introduced later. Buchanan and his co-workers (1) made similar excursions.

But synthesis of intermediates can be fruitful. Thus the use of tetrahydrofolate as a one-carbon acceptor was based on the observation that citrovorum factor ( $\mathrm{N}^{5}$-formyltetrahydrofolate) after activation with ATP transferred a one-carbon unit to a purine precursor (13). On the basis of balance studies 5-phosphoribosylamine was synthesized and found to enter into purine nucleotide biosynthesis. On the basis of a number of objective findings Shemin and Russell (27) synthesized aminolevulinic acid and showed it to be an effective precursor of the pyrrole rings and to be synthesized in vitro.

\section{MODEL SYSTEMS}

The organic chemist traditionally studies a complicated mechanism with some model reaction or compound with which he is familiar. This procedure has become increasingly important to the biochemist and is especially useful in the study of multienzyme systems. It may allow him to get at the nature of an intermediate when other approaches have proved more difficult. Acetate is not, per se, an intermediate in metabolism. However, there can be no doubt that the study of the activation of acetate by Lipmann and by Lynen and their co-workers (20, 21) represented a powerful wedge into the problem of pyruvate and fatty acid oxidation. By using $S$-acyl drivatives of $N$ acetyl thioethanolamine as model substrates Lynen (21) was able to conduct a brilliant series of studies on the mechanism of fatty acid synthesis and degradation. Since coenzyme A, the necessary acyl acceptor, was not readily available, he used this model to purify the individual enzyme steps. Williams and Buchanan (30) studied the reaction of hypoxan thine with a ribose derivative to form inosinate to gain information on the nature of the ribose compound ultimately involved in the de novo synthesis of purine ribotides. We have studied the activation of formate, which 
does not appear to be a very important intermediate as such, in order to understand how the purine rings are closed. There can be little doubt that these studies have been concerned with a truly artificial system, for not only formate but also the acceptor compound, tetrahydrofolate, appears to be an unnatural substrate (13). Nevertheless, these studies and those of Jaenicke (17) have given some ideas of the reaction mechanism. It has been clear that $\mathrm{N}^{10}$-formyl rather than $\mathrm{N}^{5}$ derivatives (citrovorum factor is $\mathrm{N}^{5}$-formyltetrahydrofolate) of tetrahydrofolate are involved. In addition ideas about pathways, energy requirements, and oxidation-reduction systems have been obtained from these studies.

This is not to suggest that the results from model systems can be accepted without reservation. At the present time one might find it difficult to introduce coenzyme $\mathrm{A}$ into the problem of protein synthesis merely because it had been found to be involved in forming the peptide linkage in hippuric acid (5). The results of studies on glutathione synthesis (29) may be more to the point.

\section{COUPLED SYSTEMS}

The detailed treatment of coupled systems has been considered by others and is beyond the scope of this paper $(7,11)$. Classical examples are found in the studies of the glycolytic scheme.

Equilibrium constants and $E_{0}^{\prime}$ values soon become a part of the working vocabulary of the investigator entangled in multi-enzyme systems.

The successful coupling of two individual reactions, of course, requires the proper equilibrium conditions. In oxidation-reduction systems the requirements may be expressed in terms of oxidation-reduction potentials. Theoretically we should be able to decide from $E_{0}$ values and from equilibrium data whether a given system will interact with another, but practically this may not always be the case. For example, the 
reaction rates may be very slow, and coupling will not appear to have becn effected.

Usually, however, the coupling system may be chosen on the basis of its thermodynamic characteristics. Where a single reaction is being studied, it is gencrally necessary to couple the system with purified enzymes to prevent an introduction of extraneous reactions. When crude extracts carrying out a multi-enzyme synthesis are being studied, it may be unnecessary to couple the reaction with such a pure enzyme. Frequently the mere addition of the proper substrates and cofactors will make use of the enzymes in the extract, and the proper coupling can be effected with the system being investigated. For example, an ammonium sulfate fraction of muscle extract (24) may be employed to catalyze the regeneration of ATP with 3phosphoglycerate as the substrate, but liver extracts carry out this series of reactions very well. By using ATP at low concentrations and by using a regeneration system, interfering apyrases may be partially circumvented and reasonable phosphate balances effected. As a source of DPNH or of TPNH glucose-6phosphate is frequently added. Ammonium salts and $\alpha$ ketoglutarate in the presence of glutamic acid dehydrogenase may act as a good acceptor of electrons from either TPNH or DPNH providing the $E_{0}^{\prime}$ of the reaction in question is correct. Often, however, the use of pyridine nucleotides as coupled acceptor compounds is beset with difficulties.

High concentrations of extract frequently are required to study complex reactions in crude systems. This appears to be essential because one of the reactions in the chain or one of the coupled reactions is limiting. Therefore it may not be possible to follow the oxidation or reduction of pyridine nucleotides by changes in absorption at $340 \mathrm{~m} \mu$ because the blank may be prohibitive. It may be possible to couple the DPN system to other acceptor systems having absorption maxima at longer wavelengths. However, this procedure can have its difficulties also. Substrate quantities of cytochrome $c$ and DPNH cytochrome $c$ reductase (22) or TPNH cytochrome $c$ reductase (16) provide 
an excellent high positive-potential acceptor from pyridine nucleotides, but cytochrome $c$ is reduced nonenzymatically by various compounds. For example, it is readily reduced by tetrahydrofolic acid. Potassium ferricyanide possesses a high positive potential and its reduction by DPNH can be followed by a drop in its absorption at $420 \mathrm{~m} \mu$. This compound may, however, oxidize substances other than DPNH.

With crude systems one cannot be sure that the reduction of pyridine nucleotides is coupled to the system being studied. Thus in an extract of pigeon liver acetone powder after dialysis there are substrate sources which bring about the reduction of cytochrome $c$. Since these sources appear to possess rather negative $E_{0}^{\prime}$ values, all other conditions being the same, they can play the major role in cytochrome $c$ reduction. Thus reduced pyridine nucleotide derived in a reaction mixture may be associated with the oxidation of only a portion of the hydrogen donor substrate. In the end it may be necessary to make a partial fractionation of the system using other assay methods in order to remove some of these interfering reactions.

Consideration may be given here to the effect that a coupled system has on a multi-enzyme reaction sequence. On first glance a coupled DPN-regenerating reaction may be thought of

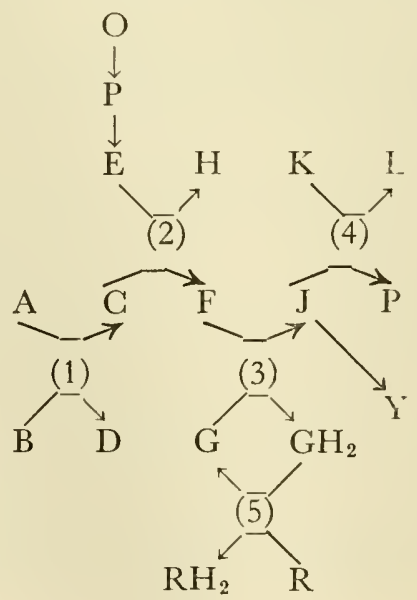


only as a means of providing the electron acceptors. Essentially this is true in the MES, but in addition a failure of the coupled system may result in a failure of the entire reaction chain. More than this is concerned.

In order to pursue the problem further let us look at a MES outlined perhaps in an oversimplified manner in the scheme on the preceding page.

Here $\mathrm{A}, \mathrm{B}, \mathrm{O}, \mathrm{K}$, and $\mathrm{R}$ are beginning substrates or are introduced into the chain along the main synthetic pathway, $\mathrm{A}$, $\mathrm{C}, \mathrm{F}, \mathrm{J}, \mathrm{P}$. The pathway $\mathrm{O} \rightarrow \mathrm{P} \rightarrow \mathrm{E}$ represents the activation of a normal metabolite to a derivative compound. $\mathrm{GH}_{2}-\mathrm{G}$ is an oxidation-reduction carrier system which is reoxidized by substrate R. Y represents a side product. From this figure it will be apparent that reaction (5), which reoxidizes the carrier system $\mathrm{GH}_{2} \rightleftharpoons \mathrm{G}$, could be a key mechanism. If reactions (1), (2), (3),(4) proceed at rates such that none of the intermediate compounds accumulate at more than trace levels, it follows that each of the substrates of the respective enzymes catalyzing these reactions may be present at a different level of saturation (see Dixon (7)). The system is in a steady state and each substrate is present at constant and low concentration. The moment that the rate of reaction (5) decreases below a critical level, the sequence of reactions (1) to (4) is disturbed. F may accumulate and $\mathrm{J}$ and $\mathrm{P}$ decrease. The system reaches a new steady state. This idea has been carefully explored by Chance (4). If the reactions (1) to (4) are reversible, the reactants may reach new levels. The coupled reaction then maintains the MES in a steady state, keeping the substrates below saturation levels.

\section{On the Role of the Michaelis Constant in Multiple Systems}

It is possible that differences in activities of certain enzymes in a MES, i.e., enzyme-limiting steps, may in fact be derived from variations in the dissociation constants of the intermediate compounds with the various enzymes involved. 
A rather interesting example which has not yet been resolved may be cited from the IMP synthesis problem. In studying the de novo synthesis of IMP in pigeon liver extracts it was found that AMP was not being formed at all nor did it appear to be an intermediate as determined by the lack of appearance of a $\mathrm{C}^{\mathbf{1 4}}$ purine precursor in carrier AMP. Yet AMP synthesis does occur in these extracts. 5-Amino-4-imidazolecarboxamide-5'-phosphoriboside (IRMP) is converted to IMP by the reactions:

$\mathrm{HC}^{14} \mathrm{OOH}+\mathrm{ATP}+$ tetrahydrofolate

$\mathrm{N}^{10}-\mathrm{C}^{14} \mathrm{HO}$ tetrahydrofolate $+\mathrm{ADP}+\mathrm{Pi}$

$\mathrm{IRMP}+\mathrm{N}^{10}-\mathrm{C}^{14} \mathrm{HO}$ tetrahydrofolate $\longrightarrow$

$\mathrm{C}^{14}$-IMP + tetrahydrofolate

Under these circumstances it was found that not only IMP but also AMP was labeled. * This situation might be explained by a reference to the following scheme:

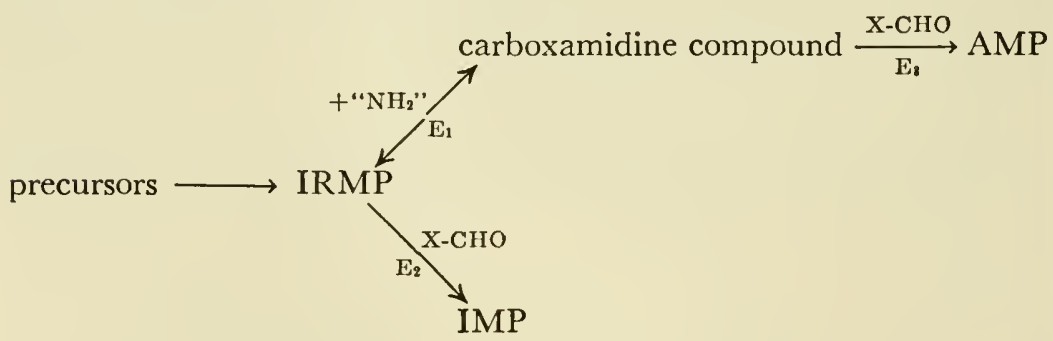

It may be considered that the $K_{m}$ of IRMP with $\mathrm{E}_{2}$ is much lower than it is with $\mathrm{E}_{1}$ and therefore at the low level of IRMP encountered during the steady-state synthesis of IMP not enough IRMP would be present to be carried over route $\mathrm{E}_{1}$ and $\mathrm{E}_{3}$. Thus whereas the actual rate of $\mathrm{E}_{1}$ when saturated with substrate might be greater than that of $\mathrm{E}_{2}$, such a condition may not be satisfied except by the artificial addition of the inter-

* Dr. C. E. Carter has obtained evidence for synthesis of AMP (3) by a pathway other than through IMP and apparently from the carboxamide compound. Private communication. 
mediate IRMP. It is equally probable that X-CHO (active formyl donor compound) has a much lower $K_{m}$ (higher affinity) for enzyme $E_{2}$ than it has for $E_{3}$ and that the equilibrium of $E_{1}$ is toward IRMP. Then it would be necessary to have a large concentration of $\mathrm{X}-\mathrm{CHO}$ to satisfy $\mathrm{E}_{3}$ and to pull IRMP through the carboxamidine compound to AMP.

A consideration of the role of enzyme saturation and the rate of the reaction may be profitable in examining the reason for accumulation of intermediates in a reaction sequence. It is not always clear why such a serendipitous accumulation should occur. Naturally it could be that a compound accumulates because an enzyme is limiting as a result of its being less active or being present in lower concentration than the preceding enzymes in the sequence. In at least one case there is evidence that the accumulation of an intermediate results from the conditions usually present in multi-enzyme systems which are operating in a steady state. That is, under conditions which do not allow saturation of the enzymes the relative rates of the reactions in question may be considerably different from the rates which obtain at saturation. In the synthesis of hypoxanthine by pigeon liver preparations (12) it has been established that inosine-5'-phosphate is an intermediate. Under certain conditions this compound accumulates in the system. The over-all reaction system may be considered diagrammatically:

glycine glutamine ribose-phosphate + other precursors

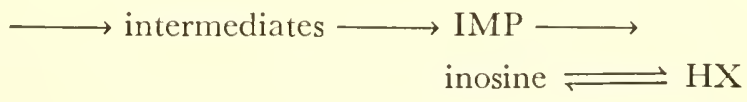

Whereas IMP accumulates in the system it is not because the reaction IMP $\rightarrow$ inosine cannot adequately handle the quantity of IMP formed. This enzyme apparently is not saturated by the IMP and is not able to form inosine rapidly under these circumstances. However, after the IMP reaches a given concentration, the enzyme is able to convert it to inosine at a rate which is equal to the rate of synthesis, and accordingly a constant level of IMP results. A graph describing such results is shown in 
Figure 1. Actually the specific activity (and therefore the $\mathrm{G}^{14}$ activity) of the IMP passes through a rise and fall curve typical of a precursor-product relationship (32). When higher concentrations of IMP were added to the system and the phosphatase was saturated, the rate of conversion of IMP to hypoxanthine could be increased by about 10 times over that observed during de novo synthesis.

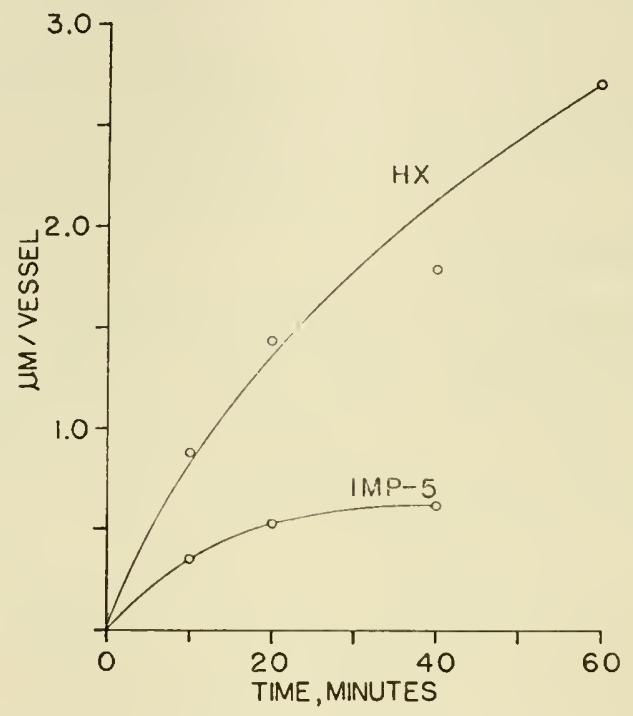

Fig. 1. Constancy of an intermediate compound in a steady-state synthesis.

It is apparent from the discussion above that in a sequence of enzyme reactions the step with the lowest velocity at saturation with its substrate may not be the limiting reaction in the steady state. Under such conditions, another enzyme may become limiting.

SOME ASPECTS OF THE PURIFICATION OF MULTI-ENZYME SYSTEMS

Eventually it becomes necessary to fractionate the crude extract in order to isolate intermediate reactants. To discuss 
some of the problems which arise in approaching such reaction systems it may be useful to consider the previously outlined MES scheme under various conditions.

Consider the situation: the reactions $\mathrm{A} \rightarrow \mathrm{J}$ have a lower rate than $\mathrm{J} \rightarrow \mathrm{P}$. $\mathrm{J}$ will not accumulate under these circumstances. Normal procedure calls for an inhibitor or for fractionation. Inhibitors can be very effective. Hartman, Levenberg, and Buchanan (15) recently employed azaserine, a known broad-spectrum antibiotic, in the system synthesizing inosinic acid and an aliphatic compound, formylglycinamide ribotide, accumulated. Inhibitors can be considered at various points in the reaction. Thus metal-complexing agents, substrate-level inhibitors, cofactor inhibitors, and agents which might interrupt a regeneration mechanism should be considered. Merely treating the extract by dialysis, ion-exchange resin, charcoal, heating, or aging may remove cofactors or an undesired enzyme.

Success of an enzyme fractionation certainly depends on the complexity of the enzyme system, namely, the number of enzymes involved and the integration of the system with another coupled reaction(s). Experience with glycolysis has clearly shown that an interference with one of the carrier systems (adenine or pyridine nucleotides) so that the acceptor or the donor concentration decreases to levels which are too low for optimal rates causes a precipitous fall in the overall rate of glycolysis. Such internally coupled systems generally are very sensitive to changes, and a decrease in activity may begin a vicious cycle which quickly results in a termination of the reaction. Therefore best results might be expected in the fractionation of perhaps a two-step reaction to which any needed coupled system has been added. As usual such fractionations require adequate analytical tools. We have found it useful to analyze both the disappearance of substrate and appearance of the final product, since this may give a measure of the isolation of the system and the accumulation of an intermediate.

A common approach to fractionation has been the separation of the extract into two parts, say A and B. This procedure is 
then followed by the purification of one enzyme from $\mathrm{A}$ in the presence of fraction $\mathrm{B}$, and then $\mathrm{B}$ is purified in the presence of the purified A. Several difficulties arise: (1) the two enzymes may be very similar in physical characteristics and both are not easily separated in good yield; (2) the system may consist of more that two enzymes which increases the difficulties enormously; (3) one of the enzymes is either very labile under the conditions required for fractionating the other enzyme or is limiting because of concentration or activity.

Some of these difficulties may be overcome by looking for another source of one of the enzymes in question. Kornberg and co-workers (18) have used this technique beautifully. Pigeon liver contains an enzyme system which converts orotic acid to uridylic acid at a slow rate.

The reaction mechanism (18) is now known to be

$$
\mathrm{R}-5-\mathrm{P}+\mathrm{ATP} \stackrel{1}{\longrightarrow} \mathrm{PRPP}+\mathrm{AMP}
$$

PRPP + orotic acid $\stackrel{2}{\longrightarrow}$ orotidylic acid + P-P

$$
\text { Orotidylic acid } \stackrel{3}{\longrightarrow} \text { uridylic acid }+\mathrm{CO}_{2}
$$

By adding yeast extracts to the pigeon liver system these authors found very rapid synthesis of UMP. Reaction (1) was found to be present in liver in good activity but (2) and (3) were limiting and were supplied by the yeast system. Therefore enzyme 1 could be purified by using 2 and 3 to analyze for the products and then 2 and 3 could be fractionated.

Purification of one system against another is not always so straightforward. Ratner and co-workers (23) have studied the condensation of aspartic acid with citrulline to form argininosuccinic acid by an ATP-dependent reaction. In an effort to gain insight into the mechanism of this reaction these workers have separated the system into two parts, one from liver and the other from yeast. It was desirable to employ another source such as yeast for one of the enzymes, since the enzyme from liver was unstable. However, whereas both the yeast and the stable liver enzymes could be fractionated to a rather good state of purity, 
a study of the interaction of the resulting two enzymes did not clarify this picture. These workers had considered the possibility that one enzyme might form a high-energy phosphate intermediate and that the other would bring about condensation perhaps as follows:

$$
\begin{gathered}
\text { citrulline }+ \text { ATP } \rightleftharpoons \text { citrulline-P }+ \text { ADP } \\
\text { citrulline-P }+ \text { aspartate } \longrightarrow \text { argininosuccinate }+\mathrm{Pi}
\end{gathered}
$$

However, neither enzyme by itself under any circumstances liberated phosphate from ATP. Both amino acids and both enzymes had to be present. They have correctly pointed out that this does not necessarily eliminate a mechanism such as shown above, for if the equilibrium constant for the first reaction were to favor citrulline, no activity could be demonstrated in the absence of the second reaction, which must necessarily lie far toward the synthesis of argininosuccinate. Since the quantity of Pi liberated was related to the level of ATP and the concentration of yeast enzyme, definitive resolution of the problem was not possible. Thus it could not be ascertained clearly in what sequence the two enzymes acted. However, it appears likely that the over-all reaction is represented by the sum of reactions (1) and (2). The difficulties encountered in this very careful study certainly could not have been foreseen, and it is to be expected that further pursuit of this problem will lead to an elucidation of an important mechanism.

Frequently an intermediate in a reaction sequence is degraded to a by-product.

Consider the situation, $\mathrm{A} \rightarrow \mathrm{J} \rightarrow \mathrm{P}$ and also $\mathrm{J} \rightarrow \mathrm{Y}$ :

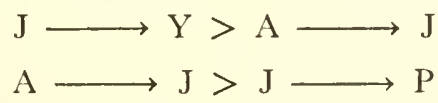

If the $\mathrm{J}$ intermediate is transformed to $\mathrm{Y}$ more rapidly than it is formed, it follows that $J$ will not accumulate but the degradation product $\mathrm{Y}$ and some of the product $\mathrm{P}$ will. The question arises as to how the investigator is to know this since he can show only that two somewhat related compounds occur. In the conver- 
sion of the $\beta$-carbon of serine to the formyl group of $\mathrm{N}^{10}$-formyltetrahydrofolic acid in pig liver extracts, Jaenicke (17) found that he could isolate $\mathrm{N}^{10}$-hydroxymethyl folic acid from the reaction mixture in addition to the product. A reasonable sequence of events to be expected was:

Serine + tetrahydrofolate

$$
\text { glycine }+\mathrm{N}^{10}-\mathrm{CH}_{2} \mathrm{OH} \text { tetrahydrofolate }
$$

$\mathrm{N}^{10}-\mathrm{CH}_{2} \mathrm{OH}$ tetrahydrofolate $+\mathrm{TPN} \longrightarrow$

$$
\mathrm{TPNH}+\mathrm{N}^{10} \text { - } \mathrm{CHO} \text { tetrahydrofolate }
$$

However, in addition an oxidative degradation occurred at such a rate that none of the intermediate accumulated:

$$
\mathrm{N}^{10}-\mathrm{CH}_{2} \mathrm{OH} \text { tetrahydrofolate } \longrightarrow \mathrm{N}^{10}-\mathrm{CH}_{2} \mathrm{OH} \text { folate }+4 \mathrm{H}
$$

By isolating and characterizing the degradation product he gained insight into the nature of the parent compound. It would be expected that the degradation product was derived from the tetrahydro level compounds. Such a system of course is ideal for fractionation. Initial fractionation was carried out using pigeon liver extract. After fractionation $\mathrm{CH}_{2} \mathrm{OH}$-tetrahydrofolate* indeed did accumulate. Pigeon liver has the added advantage that the oxidative degradation reaction is relatively slow in the unfractionated extracts.

Another example of this type may be taken from the mechanism of synthesis of the glycinamide ribotide $(9,10,15)$. The sequence of events which is believed to take place is shown below.

$\mathrm{R}-5-\mathrm{P}+\mathrm{ATP} \longrightarrow \mathrm{PRPP}+\mathrm{AMP}$

$\mathrm{PRPP}+$ glutamine $\longrightarrow$ PRA + glutamate $+\mathrm{P}-\mathrm{P}(\rightarrow 2 \mathrm{Pi})$

$\mathrm{PRA}+$ glycine $+\mathrm{ATP} \longrightarrow$ glycinamide ribotide + ? $\mathrm{ADP}+$ ?Pi

$\mathrm{R}-5-\mathrm{P}+2 \mathrm{ATP}+$ glycine + glutamine glycinamide ribotide + glutamate $+\mathrm{AMP}+\mathrm{ADP}+3 \mathrm{Pi}$

It appears that in this system 5-phosphoribosylamine

* This apparently is one of the compounds found by S. Deodhar and W. Sakami. Private communication. 
(PRA) is deaminated very rapidly to ribose-5-phosphate and $\mathrm{NH}_{4}^{+}$and none accumulates. However, a study of the balance of the second reaction, the glutamine disappearance, and glutamic acid and pyrophosphatc formation called for the presence of phosphoribosylamine. It was apparent that the degradation reaction which might be enzymatic could be removed, and such fractionations are in progress. However, prior to fractionation Goldthwait and co-workers (10) synthesized PRA chemically as it fulfilled the requirements for a simple synthesis (ribose-5-phosphate plus liquid ammonia) and it was tested in the system in place of PRPP and glutamine. PRA was found to be at least 3 times as effective as PRPP and glutamine but still required ATP. These studies also gave some insight into the chemical nature of PRA. It was found to be a rather unstable compound which would require special handling. Hindsight is the simplest branch of science, but it may be useful to backtrack in the development of ideas in a problem. The mechanism as it was visualized after the isolation of glycinamide ribotide went through various revisions. Ribose-1, 5-diphosphate was a logical possibility as the ribosyl donor. It was also possible that ribosc-1-phosphate was involved, forming a riboside which then was phosphorylated. When Kornberg and co-workers (18) first showed the involvement of PRPP in the conversion of orotic acid to uridylic acid, this became a choice possibility. But other problems were involved. Did the ribose derivative react with glutamine or with glycinamide or with a product of glutamine and glycine? Of some importance was the problem of finding a means of activating the amide hydrogen atoms of glutamine which are notably unreactive. Whatever the case, the glutamine does react with PRPP so that fundamentally the problem resolves itself by virtue of the findings without regard to exact mechanism. In retrospect it is clear that an insight into the mechanism could have been gained by a study of the over-all balance. It also is obvious that the key to the development of this problem was the identification of PRPP (18). 
REVERSIBLE SYSTEMS

It may be found that the equilibrium constant of a reaction does not favor synthesis of an intermediate compound. The resulting situation might be presented as:

\section{$\mathrm{C} \leftrightharpoons \mathrm{J} \leftrightharpoons \mathrm{P}$}

Suppose that the equilibrium of $\mathrm{C} \rightleftharpoons \mathrm{J}$ lies far toward $\mathrm{C}$ such that at equilibrium $\mathrm{J}$ is present in approximately $1 / 1000$ the concentration of $\mathrm{G}$, and suppose that the rate of $\mathrm{G} \rightarrow \mathrm{J}<\mathrm{J} \rightarrow \mathrm{P}$. Thus $\mathrm{J}$ would not accumulate. It follows that if the reaction proceeds to $\mathrm{P}$, then $\mathrm{J} \rightarrow \mathrm{P}$ lies to the right and the change in free energy of the over-all reaction is negative. This situation appears to be common in biochemical mechanisms. Consider the activation of formate which has been found to proceed by the following over-all reaction $(13,14)$.

$\mathrm{HCOOH}+\mathrm{FAH}_{4}+\mathrm{ATP} \rightleftharpoons \mathrm{N}^{10} \mathrm{CHOFAH}_{4}+\mathrm{ADP}+\mathrm{Pi}$

Formate appears to be reversibly incorporated, for when a suitable formyl acceptor (5-amino-4-imidazolecarboxamide ribotide) is added the rate of incorporation of formate can be increased several times. This reversibility is also inherent in the studies of Schulman and Buchanan (25). Although it seemed possible that this reaction was more complex (as, for example, enzyme-substrate compounds), the mechanism was first considered as follows:

$$
\begin{gathered}
\mathrm{FAH}_{4}+\mathrm{ATP} \rightleftharpoons \mathrm{FAH}_{4}-\mathrm{P}+\mathrm{ADP} \\
\mathrm{FAH}_{4}-\mathrm{P}+\mathrm{HCOOH} \rightleftharpoons \mathrm{N}^{10}-\mathrm{CHOFAH}_{4}+\mathrm{Pi}
\end{gathered}
$$

Equally possible was the reaction of ATP and formate first.

No labile phosphate was formed unless all of the substrates were present. This was true even when the phosphoenolpyruvate to pyruvate system was added to convert ADP to ATP in order to draw the first reaction in the forward direction. Accordingly the mechanism of the reaction remains obscure. However, if the over-all equilibrium constant is about 1 , then the first reaction, 
whatever its nature, must lie quite far towards the left and the second equally far to the right.

What can be done in this situation? An obvious approach at present is to study the exchange of isotopically labeled compounds, first to aid in establishing the mechanism and, second, to employ the procedure as a measurement of the unfavorable reaction. It is also possible to divide the system into two enzymes (as in the example of argininosuccinic acid synthesis) and to purify one versus the other using the final product as an assay. After purification the problem may yet be unresolved, since the intermediate would not accumulate. Another enzyme system coupled to one of the products or a trapping agent could perhaps be employed.

\section{ISOTOPE STUDIES}

Among the most powerful tools we have at present for detecting intermediate compounds are the radioactive isotopes. Thus if a substrate can be labeled and if even a trace of an intermediate is present, it can be found by chromatographing the reaction filtrate either on paper or on columns, measuring the compound by direct radioactivity determinations of the chromatographic fractions, or by exposing paper chromatograms to $\mathrm{x}$-ray films.

Once a compound is shown to be an intermediate in a complex system, it can be studied by the classical enzyme methods. However, a compound is not necessarily an intermediate on the basis of its becoming labeled by an isotopic substrate. A compound, E, may be labeled under the following circumstances.

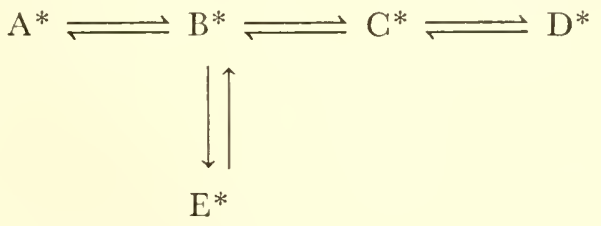

While $\mathrm{E}$ becomes labeled from substrate $\mathrm{A}$, and, in turn, labeled $\mathrm{E}$ is converted to $\mathrm{D}$, it is not an intermediate as such. Frac- 
tionation of the system would eventually show $\mathrm{E}$ to be a side product.

Specific radioactivity measurements do not in themselves prove that a compound is an intermediate. Thus in studying the conversion of hypoxanthine to inosinic acid the author (12) found the following specific activities (counts/ $\mu$ mole) after incubation of $\mathrm{C}^{14}$-hypoxanthine with pigeon liver for 21 minutes: hypoxanthine, 4500; inosine, 2150; and inosinic acid, 1380. Furthermore inosine appeared to be a better precursor of IMP than hypoxanthine was. Accordingly it was considered reasonable to suggest that the reaction mechanism was via inosine perhaps as follows:

hypoxanthine + ribose- 1 -phosphate $\longrightarrow$ inosine $\stackrel{\text { ATP }}{\longrightarrow}$ IMP

However, Buchanan and co-workers (30) have fractionated this system and their results together with the discovery of PRPP* (18) provide evidence that the following holds:

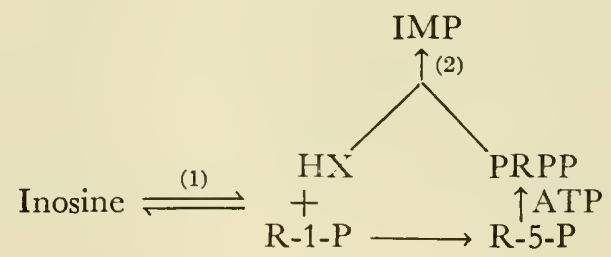

Hypoxanthine was more rapidly equilibrated with inosine (1) than it was converted to IMP (2), and therefore inosine had shown an intermediate specific activity in our experiments.

Specific activity measurements against time may give more positive indication that a compound is an intermediate. But even when the compounds follow the typical precursor-product curves, the results are not unequivocal. More adequate evidence is obtained when in addition the total activity of each fraction is recorded. This allows one to observe that a net quantity of $\mathrm{C}^{14}$ activity has been transferred from one compound to another over a period of time. Since from the specific

* Dr. J. M. Buchanan, private communication. 
activity and the total activity the actual quantity of compound can be calculated, these data are very useful. Such data have been reported in the in vitro system synthesizing IMP (12).

Certain problems arise in employing isotopic compounds to study a MES. For example consider the following:

$$
\mathrm{A}^{*} \rightleftharpoons \mathrm{B} \rightleftharpoons \mathrm{C}
$$

where the equilibria and rates of reaction are such that $\mathrm{B}$ does not accumulate. If we start with labeled $\mathrm{A}$, in the absence of $\mathrm{B}$ or $\mathrm{C}$, then $\mathrm{C}$ will become labeled with time, and the specific activity of $\mathrm{C}$ will approach that of $\mathrm{A}$ at time zero. If, however, a pool of unlabeled $\mathrm{C}$ is added at time zero, the final specific activity of both $\mathrm{A}$ and $\mathrm{C}$ will be much lower. It follows that when a high concentration of $\mathrm{C}$ is present, the rate of incorporation of labeled $\mathrm{A}$ is not a true measure of $\mathrm{G}$ synthesis but represents a minimal value since $\mathrm{A}$ is being diluted by $\mathrm{C}$. This has its practical significance where one attempts to demonstrate exchange reactions. $\mathrm{B}$ can be responsible for a significant dilution even though it does not accumulate in quantity. For example, if $\mathrm{B}$ is synthesized from a source other than A and $\mathrm{C}$ and is in turn converted to another product, i.e., it is turning over rapidly independently of the reaction $\mathrm{ABC}$, a dilution of the radioactive atoms will occur.

If the reaction $\mathrm{B} \rightarrow \mathrm{C}$ is essentially irreversible, a somewhat different situation arises. In the system $\mathrm{A}^{*} \rightleftharpoons \mathrm{B} \rightarrow \mathrm{C}$, where $\mathrm{B} \rightarrow \mathrm{C}$ is limiting because of the absence of a cofactor, $\mathrm{B}$ should accumulate approximately in accord with the equilibrium constant, $\mathrm{B} / \mathrm{A}$. Suppose the rate $\mathrm{B} \rightarrow \mathrm{G}$ is increased by adding the cofactor. Then $\mathrm{A} \rightleftharpoons \mathrm{B}$ is drawn towards $\mathrm{B}$ and more $\mathrm{C}$ will be formed. But the total radioactivity in $\mathrm{B}$ should remain essentially unchanged as long as the concentration of $\mathrm{A}$ is not limiting.

The addition of large levels of a suspected product or intermediate to determine whether a reaction is reversible is a standard practice. If the rate of substrate disappearance is lessened, this is taken as some evidence that the reaction is 
reversible and that the compound is a product, although, of course, the effect could be due to an inhibition. If one observes the utilization of a labeled substrate with and without addition of a suspected product, the results may not be different. Thus, depending on the equilibrium and rate of the reaction $\left(\mathrm{A}^{*} \rightleftharpoons \mathrm{B}\right)$, the labeled substrate $A^{*}$ may be converted quite rapidly into $B$ in short experiments even in the presence of added B. Only after $\mathrm{B}$ dilutes the specific activity of $\mathrm{A}$ does an apparent diminished utilization of $\mathrm{A}$ occur, and this will also depend on the relative concentrations of $\mathrm{A}$ and $\mathrm{C}$.

\section{GONCLUSIONS}

Biosynthetic pathways tend to be relatively slow reactions in comparison with energy-yielding reactions. The enzymes that are involved frequently are present in small quantity. Nevertheless the pathways may manifest their importance as controlling mechanisms, as, for example, the rate of synthesis of DPN. The resolution of the intimate mechanisms of these pathways and of their involvement with other metabolic systems must depend on all the available tools. First a cell-free system carrying out the total synthesis is necessary. Then logical intermediates must be sought wherever possible. With the intermediate a fractionation of the system may be obtained. In many cases a fractionation of the enzyme or the use of special devices may bring about the accumulation of the intermediate.

Finally much of what we have said may be negated by the observation that in these studies the element of intuition and guess plays the largest and most successful role.

\section{References}

1. Buchanan, J. M., B. Levenberg, J. G. Flaks, and J. A. Gladner, in W. D. McElroy and H. B. Glass, eds., Amino Acid Metabolism, p. 743. Johns Hopkins Press, Baltimore, 1955.

2. Buchanan, J. M., and M. P. Schulman, J. Biol. Chem., 196, 513 (1952).

3. Carter, C. E., and L. H. Cohen, J. Am. Chem. Soc., 77, 499 (1955).

4. Chance, B., Harvey Lectures. Ser. 49, 145 (1953-54). 
5. Chantrenne, H., J. Biol. Chem., 789, 227 (1951).

6. Davis, B., in IV. D. McElroy and H. B. Glass, eds., Amino Acid Metabolism, p. 779. Johns Hopkins Press, Baltimore, 1955.

7. Dixon, M., Multi-Enzyme Systems. Cambridge University Press, Cambridge, 1951.

8. Fruton, J. S., and S. Simmonds, General Biochemistry, Ch. 15. Wiley, New York, 1953.

9. Goldthwait, D. A., R. A. Peabody, and G. R. Greenberg, J. Am. Chem. Soc., 76, 5258 (1954).

10. Goldthwait, D. A., R. A. Peabody, and G. R. Greenberg, Biochim. et Biophys. Acta, 18, 148 (1955).

11. Green, D. E., in D. M. Greenberg, ed., Chemical Pathways of Metabolism. Vol. 1, p. 27. Academic Press, New York, 1954.

12. Greenberg, G. R., J. Biol. Chem., 190, 611 (1951).

13. Greenberg, G. R., Federation Proc., 73, 745 (1954).

14. Greenberg, G. R., L. Jaenicke, and S. Silverman, Biochim. et Biophys. Acta, 17, 589 (1955).

15. Hartman, S. C., B. Levenberg, and J. M. Buchanan, J. Am. Chem. Soc., 77, 501 (1955).

16. Horecker, B., J. Biol. Chem., 183, 593 (1950).

17. Jaenicke, L., Biochim. et Biophys. Acta, 17, 588 (1955).

18. Kornberg, A., I. Lieberman, and E. S. Simms, J. Am. Chem. Soc., 76, 2027 (1954).

19. Krebs, H. A., and K. Henseleit, Z. physiol. Chem., 210, 33 (1932).

20. Lipmann, F., Harvey Lectures, Ser. 44, 99 (1950).

21. Lynen, F., Harvey Lectures, Ser. 48, 210 (1953-54).

22. Mahler, H. R., N. K. Sarkar, L. P. Vernon, and R. A. Alberty, J. Biol. Chem., 199, 585 (1952).

23. Ratner, S., in W. D. McElroy and H. B. Glass, eds., Amino Acid Metabolism, p. 231. Johns Hopkins Press, Baltimore, 1955.

24. Ratner, S., and A. Pappas, J. Biol. Chem., 179, 1183 (1949).

25. Schulman, M. P., and J. M. Buchanan, J. Biol. Chem., 202, 241 (1953).

26. Shaw, E., J. Biol. Chem., 185, 439 (1950).

27. Shemin, D., and C. S. Russell, J. Am. Chem. Soc., 75, 4873 (1953).

28. Shive, W., W. W. Ackermann, M. Gordon, M. E. Getzendaner, and R. E. Eakin, J. Am. Chem. Soc., 69, 725 (1947).

29. Snoke, J., and K. Bloch, J. Biol. Chem., 199, 407 (1952).

30. Williams, W. J., and J. M. Buchanan, J. Biol. Chem., 203, 583 (1953).

31. Yanofsky, C., in W. D. McElroy and H. B. Glass, eds., Amino Acid Metabolism, p. 930. Johns Hopkins Press, Baltimore, 1955.

32. Zilversmit, D. B., C. Entenman, and M. C. Fishler, J. Gen. Physiol., 26, 325 (1943). 


\section{ENZYME KINETICS}

ROBERT A. ALBERTY, Chemistry Department, University of Wisconsin, Madison, Wisconsin

In living organisms enzymes determine which of the multitudinous thermodynamically possible reactions occur and at what rates these reactions can proceed. Most of these reactions cannot be duplicated in the laboratory without the use of enzymes, especially in neutral solution and at body temperatures. Thus we are presented with the challenge of probing the secrets of these catalytic mechanisms. Quantitative kinetic studies do not have a monopoly in this field of inquiry, but they play an important role which is discussed in this article.

The role of quantitative studies of enzyme kinetics may perhaps be clarified by consideration of the following analogy. A multicomponent enzyme system may be compared to a watch and the process of discovering the mechanism to the maneuvers of a boy who takes the watch apart. If our young experimentalist is sufficiently careful and observant, he may be able to put it back together again and find that it still works. However, the significant thing about the watch, which is its ability to keep time, does not lie simply in the number of gears or in the fact that it has a spring, but in the quantitative factors that the boy does not measure. The factors which make it possible for the 
watch to keep time can be determined by making quantitative measurements of the dimensions and weight of the balance wheel, the strength of the hair spring, the number of teeth on the gears, etc. Without a properly constructed balance wheel a watch is a machine, but it is not a timepiece. The study of enzyme kinetics is analogous to the quantitative study of a watch and may be required in identifying some of the gears and balance wheels of the enzymatic mechanism.

Thinking in terms of mechanism is useful at all stages in the investigation of enzyme kinetics because a mechanism is a convenient device for the integration of information and a stimulus for further experiments. As more facts are obtained it is possible to build, step by step, a framework into which the final detailed mechanism must fit. In an absolute sense the determination of the mechanism is never complete. Like absolute zero the complete elucidation of the mechanism may be approached, but never reached New types of experiments will require further elaboration of the mechanism, which in this manner becomes more detailed. The mechanism of an enzymatic reaction is necessarily incomplete as long as the chemical nature of the enzyme is unknown.

This article will be concerned only with the information on mechanism which comes directly from kinetic data-that is, the stoichiometry and rate constants of steps which are rate-determining and the nature of the equilibria preceding the ratedetermining step. Another facet of mechanism which lies outside the scope of the present treatment has to do with the manner in which the transfer of electrons and atoms is actually accomplished and with the correlation between structure and kinetic constants. Thus we will refer only in passing to the valuable information concerning enzymatic mechanisms which may be obtained by use of isotopic tracers. Isotopic tracer experiments are useful in interpreting enzyme kinetic experiments because they may be used to determine which bonds are broken, which atoms exchange with the solvent, and which steps are ratedetermining. For example, in the case of the alcohol and lactic 
dehydrogenases it has been possible to exclude all mechanisms which require or allow $\mathrm{H}$ in reduced diphosphopyridine to exchange with the medium during the oxidation-reduction reaction (Vennesland and Westheimer (12)).

It will also not be possible to discuss the theoretical considerations of enzymatic mechanisms which are based on the knowledge of physical organic chemistry. This approach to mechanism is illustrated by the work of Westheimer (12) and of Koshland (12). Enzymatic mechanisms and the mechanisms of other catalytic processes must of necessity have many features in common. Thus acid-catalyzed reactions have their enzymatic counterparts. The enzyme is apparently able to provide a proton at the required position in neutral solution so that it is unnecessary to have a high concentration of hydrogen ions. In addition the enzyme controls the specificity and stereospecificity of the reaction - a feature which is lacking in the nonenzymatic reaction. One advantage of theoretical speculations going hand in hand with experimental work is that important new types of experiment are suggested.

The most important concept which underlies the interpretation of enzyme kinetic data is that one or more intermediates are formed by reaction of the substrate with the enzyme and that the product is produced by the decomposition of such an intermediate. In 1913 Michaelis and Menten (13) derived the rate equation for a simple mechanism based on the concept of an enzyme-substrate complex and showed that this hypothesis made possible a quantitative interpretation of the data for the enzymatic inversion of sucrose. Although the MichaelisMenten equation was found to be widely applicable to enzymatic reactions, the evidence that such an intermediate was actually formed by a second-order reaction was only indirect until Chance (3), some thirty years later, obtained direct evidence. Chance showed that an intermediate was involved in the catalysis by horse-radish peroxidase of the reaction between hydrogen peroxide and malachite green and that the rate of appearance of product was directly proportional to the concentration of this 
intermediate. Actually it turns out to be impossible from kinetic data alone to specify the composition of such an intermediate. However, the fact that the rate of appearance of intermediate is directly proportional to the concentration of substrate and free enzyme proves that the activated complex for the reaction contains onc substrate molecule and one enzyme molecule. The product of this second-order reaction may be something less than a complex containing the whole of both molecules.

One of the themes of this article is that the basic mechanism of Michaelis and Menten can be extended and enlarged upon in many ways to include further phenomena while retaining the essential novel idea of the reversible formation of an intermediate by substrate and enzyme which can decompose or react to yield product and regenerate the enzyme.

\section{Transient-State Studies}

In general, the following three stages may be distinguished in a simple enzymatic reaction. First, immediately after mixing solutions of enzyme and substrate, there is a disappearance of substrate and free enzyme as they react with each other. For most enzymatic reactions this process is so fast that it has been impossible to study it. As the concentration of enzyme-substrate complex increases the rate of appearance of product increases until at a particular concentration of enzyme-substrate complex there is a steady state in which the rate of formation of the complex is balanced by its rate of decomposition. In this second stage of the reaction the rate of appearance of product is constant. If the substrate concentration is high the reaction velocity may remain constant for a sufficiently long time to permit its accurate determination. On the other hand, if the concentration of substrate is low, the reaction rate may drop rapidly as the substrate is used up. In this third and final stage of the reaction, the concentration of the enzyme-substrate complex decreases. Studies of all three phases of the reaction give the most complete information about the mechanism and are referred to as transient-state studies. Chance (3), who in 1940 first studied the 
transient-state kinetics of peroxidase, has pioneered this field of enzyme kinetics. The advantage of transient-state studies over steady-state studies is that the rate constants for individual steps may be determined. Complete analytic solutions have not been obtained for the transient periods of enzymatic reactions, but experimental data may be correlated with the solution of the complete set of differential equations representing the mechanism by use of a differential analyzer.

Since the rate of the initial reaction of enzyme and substrate is so rapid, a special mixing apparatus is required for studies of this phase of the reaction. Two different experimental arrangements have been used. In the "stopped-flow" method the reactants are mixed and quickly brought to rest. Then the concentration of one of the reactants or intermediates is determined as a function of time by a physical method of analysis. A rapidly responding device is required for measuring the rate. In the other method, the "continuous-flow" method, the reactants are mixed and injected through a tube at constant velocity. A device placed at some point along the tube is used to measure the concentration of a reactant or intermediate at that point. This is the concentration which is obtained at a certain time after mixing. Since the composition of the solution at this point in the tube is constant in time, a measuring device with a rather long time constant may be used. By changing the flow rate the composition of the solution may be obtained at some other time after mixing. In essence enzyme is traded for time in this method. Either a large amount of enzyme is required or the reaction must be such that the enzyme may be recycled.

A total of four intermediate compounds of horse-radish peroxidase have been discovered spectrophotometrically by Keilin and Mann, Theorell, and Chance. The strong absorption of peroxidase in the Soret region and the large changes in extinction coefficient upon reaction with substrate have made possible a detailed study of these intermediates. Chance has found that the following mechanism represents the kinetic data of the transient state: 


$$
\begin{gathered}
\text { HRP }+\mathrm{H}_{2} \mathrm{O}_{2} \rightleftharpoons \text { complex I } \\
\text { complex I }+\mathrm{AH} \longrightarrow \text { complex II }+\mathrm{A} \\
\text { complex II }+\mathrm{AH} \longrightarrow \mathrm{HRP}+\mathrm{A}
\end{gathered}
$$

Here horse-radish peroxidase is represented by HRP, the reduced form of the donor by $\mathrm{AH}$, and the oxidized form by $\mathrm{A}$. Complex I reacts with $\mathrm{AH}$ to form complex II at a speed roughly 50 times that of the reaction of complex II with AH. Since the conversion of I to II is so rapid, complex II behaves kinetically like the complex postulated by Michaelis. George's titrations of complex II suggested that in the conversion of complex I to complex II, one of the two oxidation equivalents of complex I is transferred to the donor. This was subsequently proved kinetically by Chance. The transient-state data have been correlated point by point with solutions of the rate equations for the above mechanism obtained with the differential analyzer.

\section{Steady-State Studies}

Most studies of enzyme kinetics have been carried out during the phase of the reaction in which $d(\mathrm{ES}) / d t$ is nearly zero. During this phase the velocity drops off as the substrate concentration decreases. If the initial substrate concentration is used in the rate equation the correct velocity is that obtained by extrapolation to the time of mixing. It should be remembered that the truly initial velocity of disappearance of substrate as determined in a transient-state experiment is something entirely different. An alternative to extrapolation of a slowly changing velocity back to zero time is to use an integrated form of the rate equation. This is the basis of the method of Foster and Niemann (6) for determining the maximum velocity and Michaelis constant without using initial velocities.

In order to determine the effects of independent factors such as the concentrations of substrate, hydrogen ions, metal ions, and inhibitors on the steady-state rate, the effect of changing these variables should be studied over as wide a range of concentration 
as is experimentally possible. The result of a series of steadystate rate measurements at a constant temperature may be expressed as a function of the concentration variables by means of an empirical equation referred to as the rate equation. If the rate equation is simple, that is, if it involves simple powers of the concentrations of the reactants, an interpretation in terms of a simple mechanism may be possible. In discussing the interpretation of steady-state rate equations King (9) points out that the form of the rate equation for mechanisms which consist of one or more independent paths gives information regarding the composition of the activated complex with respect to the components of the reaction mixture whose concentrations have been altered. Thus experiments in dilute aqueous solutions at constant $p \mathrm{H}$ do not yield information as to the number of protons or water molecules in the activated complex. In order to determine the composition of the activated complex it is also necessary to know the predominant forms of the various components in solution, that is, their extent of combination with hydrogen ions, metal ions, etc. The characteristics of rate equations arising from particular types of effects will be seen in the following section. Some types of effects, such as those of ionic strength, per se, or of structural alterations of the protein, may not be expressible in terms of a simple rate equation. The kinetic method of studying interactions of enzymes with protons, metal ions, organic molecules, etc., is of special interest since it provides information about a limited portion of the enzyme molecule.

Mechanisms suggested by steady-state studies may be treated mathematically by assuming that the rate of change in the concentration of intermediate complexes is zero. This assumption leads to a series of simultaneous equations which can be solved algebraically. The coefficients of the terms in the rate equation are related to the rate constants and equilibrium constants for steps in the mechanism, but in general the individual rate constants cannot be calculated from steady-state kinetic data. Much of our information on enzyme kinetics is based on 
steady-state measurements because they are less difficult to perform than transient-state measurements. The fact that a reaction follows a certain rate equation does not prove a certain mechanism is operative, but it does eliminate many mechanisms from consideration. However, if several mechanisms which yield a given rate equation are considered carefully, it may be possible to devise further experiments which will distinguish between the possibilities.

It is interesting to note that rate equations derived for very simple mechanisms may be applicable to reactions that really follow much more complicated mechanisms. This is true since simple rate equations are special cases of the more complicated rate equations which are obtained when certain concentration variables are held constant or experiments are limited to a certain concentration range. For example, at a constant $p \mathrm{H}$ and coenzyme concentration the Michaelis-Menten rate equation is applicable to certain enzymatic reactions. However, the fact that the parameters in this equation are dependent on $p \mathrm{H}$ and coenzyme concentration shows that the mechanism is more complicated than that postulated by Michaelis and Menten.

\section{Experimental Methods}

A good deal has been learned about enzyme kinetics with crude preparations, but purity of all substances involved is basic for accurate quantitative studies. Thus detailed kinetic studies must be preceded by research in which the enzyme is isolated, the products of the reaction are identified, and the possible participation of activators or coenzymes is established.

The success of an enzyme kinetic study is vitally dependent upon the sensitivity, speed, and accuracy of the analytical method. Of the many experimental techniques which have been used to study enzymatic reactions, physical methods are generally more accurate and convenient since they do not require stopping the reaction, and a continuous record of concentration may be 
obtained. In order to determine the initial reaction velocity for use in a steady-state rate equation, it is necessary to use data obtained when the reaction has proceeded only to the extent of a few per cent. Greater sensitivity is thus required than in ordinary kinetic studies in which the reaction is followed to completion. In the transient-state method even greater sensitivity and speed are required since the concentration of any enzyme-substrate complex is so small.

Spectrophotometric methods have been especially useful because of their speed, general applicability, and high sensitivity. Chance (4) has developed spectrophotometric methods extensively and has used them to study reactions in whole cells and particulate suspensions as well as in solutions.

Future progress in the field of enzyme kinetics will be dependent to a large extent upon the development of convenient and sensitive methods for the determination of reaction rates. It is to be hoped that measurements of dielectric constant, magnetic susceptibility, and paramagnetic and nuclear resonance absorption will be developed to the point where they can be used in enzyme kinetic studies to measure small changes in concentration which occur rapidly.

\section{Effects of Independent Variables}

DEPENDENCE OF STEADY-STATE RATE

UPON SUBSTRATE CONCENTRATION

The steady-state rate of an enzymatic reaction is generally found to be directly proportional to the substrate concentration at low substrate concentrations and to approach a limiting value at higher substrate concentrations. This dependence of the rate of the enzymatic inversion of sucrose on the sucrose concentration was interpreted by Michaelis and Menten (13) in terms of the mechanism

$$
\mathrm{E}+\mathrm{S} \underset{k_{2}}{\stackrel{k_{1}}{\rightleftharpoons}} \mathrm{ES} \stackrel{k_{3}}{\longrightarrow} \mathrm{E}+\mathrm{P}
$$


where $\mathrm{E}, \mathrm{S}$, and $\mathrm{P}$ represent enzymatic site, substrate, and product, respectively. The form of the equation for the steadystate velocity $v$ obtained from this mechanism is the same whether it is assumed that ES is in rapid equilibrium with $\mathrm{E}$ and $\mathrm{S}$ or in a steady state.

$$
v=\frac{V}{1+K_{\mathrm{s}} /(\mathrm{S})}
$$

In this equation $V$ is the maximum steady-state velocity which is equal to $k_{3}(\mathrm{E})_{\mathrm{o}}$, and $K_{\mathrm{S}}$ is the Michaelis constant which is equal to $\left(k_{2}+k_{3}\right) / k_{1}$. The mere fact that the rate data for so many enzymatic reactions fit this equation, however, does not prove that they follow mechanism (2). All the rate equation tells us is the composition of the activated complex for the reaction in terms of the predominant forms of the reactants in the solution. Thus for the first step in mechanism (2) the activated complex (not to be confused with the enzyme-substrate compound ES) contains one substrate molecule per enzymatic site. Since this activated complex is built up from a molecule of enzyme and a molecule of substrate, the over-all reaction rate will be proportional to the concentrations of both $\mathrm{E}$ and $\mathrm{S}$ if this is the rate-determining step. At high substrate concentration a complex of composition ES already exists in solution and so the reaction rate will be independent of substrate concentration if the second step is rate-determining.

As pointed out by King (9) the requirements of rate equation (3) that the velocity be first order in (S) at low (S) and zero order at high (S) may be met by several other mechanisms. Two examples are

$$
\begin{gathered}
\mathrm{E}+\mathrm{S} \rightleftharpoons \mathrm{ES} \\
\mathrm{E}+\mathrm{S} \longrightarrow \mathrm{E}+\mathrm{P}
\end{gathered}
$$

in which the ES complex does not yield product and

$$
\begin{aligned}
\mathrm{E} & \rightleftharpoons \mathrm{E}^{\prime} \\
\mathrm{E}+\mathrm{S} & \longrightarrow \mathrm{E}^{\prime}+\mathrm{P}
\end{aligned}
$$


Here the enzyme exists in two isomeric forms which are not rapidly interconvertible, and only $\mathrm{E}$ is catalytically active. Although mechanisms (2), (4), and (5) cannot be distinguished by steady-state kinetic studies at high substrate concentrations, they could be differentiated readily by transient-state studies.

In the derivation of a rate equation for a particular mechanism it is implicitly assumed that the individual rate constants remain constant while the substrate concentration is varied. If the substrate concentration is very low in comparison with that of the buffer which maintains a constant $p \mathrm{H}$ and ionic environment, this ideal condition may be closely approached. However, as the substrate concentration is increased the individual rate constants may be affected because of changes in the ionic environment. Thus deviations from the Michaelis equation at high substrate concentration may result from changes in individual rate constants. Such deviations may also be a consequence of the binding of substrate molecules or ions by the enzyme in such a way as to alter the properties of the enzymatic site. The fact that the rate may be either increased or decreased may be represented by the addition of the following steps to mechanism (2).

$$
\begin{gathered}
\mathrm{E}+\mathrm{S} \rightleftharpoons \mathrm{SE} \\
\mathrm{SE}+\mathrm{S} \rightleftharpoons \mathrm{SES}+\mathrm{P}
\end{gathered}
$$

Here $S$ is written to the left of $E$ to represent its combination at a neighboring site and to the right of $\mathrm{E}$ to represent combination at the enzymatic site. The steady-state treatment of mechanism (2) plus mechanism (6) leads to the following rate equation.

$$
v=\frac{V_{2}+V_{1} K_{2} /\left(1-V_{1} / V_{2}\right)(\mathrm{S})}{1+K_{2} /\left(1-V_{1} / V_{2}\right)(\mathrm{S})+K_{1} K_{2} /\left(1-V_{1} / V_{2}\right)(\mathrm{S})^{2}}
$$

In this equation $K_{1}, K_{2}, V_{1}$, and $V_{2}$ are constants. It may be seen that at low substrate concentrations this equation reduces to the Michaelis equation with maximum initial velocity $V_{1}$ and Michaelis constant $K_{1}$. Equation (7) represents the substrate activation which occurs in the case of fumarase (2). 
The initial rate of hydrolysis of urea by urease has been investigated over a 5000-fold range of urea concentration by Kistiakowsky and co-workers. It was found that a four-parameter equation was required to represent these data, one of the parameters allowing for inhibition at high substrate concentrations. The type of three-parameter rate equation which was found to represent the data at the lower concentrations could be derived by assuming two types of independent active sites, differing in their Michaelis-Menten rate parameters, or pairs of identical sites which interact so that the kinetic parameters of a site are altered when the neighbor site undergoes combination with urea.

\section{MECHANISMS OF COENZYME REACTIONS}

When two substrates are involved in an enzymatic reaction the complexity of the mechanism may be increased considerably. The simplest way in which the Michaelis-Menten mechanism may be elaborated to include a second substrate is

$$
\begin{gathered}
\mathrm{E}+\mathrm{A} \underset{k_{2}}{\stackrel{k_{1}}{\rightleftharpoons}} \mathrm{EA} \\
\mathrm{EA}+\mathrm{B} \stackrel{k_{8}}{\longrightarrow} \mathrm{E}+\mathrm{C}+\mathrm{D}
\end{gathered}
$$

The steady-state treatment of this mechanism yields the rate equation

$$
v=\frac{k_{3}(\mathrm{E})_{\mathrm{o}}(\mathrm{B})}{1+\left[k_{2}+k_{3}(\mathrm{~B})\right] / k_{1}(\mathrm{~A})}
$$

Thus the maximum initial velocity will be directly proportional to (B) and the Michaelis constant of A will be a linear function of $(B)$. Chance has found that this rate equation and mechanism represent the data for peroxidase under conditions where only one enzyme-substrate complex must be considered.

If one of the products is only slowly dissociated from the enzyme as indicated in the following mechanism

$$
\mathrm{E}+\mathrm{A} \underset{k_{2}}{\stackrel{k_{1}}{\rightleftharpoons}} \mathrm{EA}
$$




$$
\begin{gathered}
\mathrm{EA}+\mathrm{B} \underset{k_{4}}{\stackrel{k_{3}}{\rightleftharpoons}} \mathrm{EC}+\mathrm{D} \\
\mathrm{EC} \underset{k_{6}}{\stackrel{k_{6}}{\rightleftharpoons}} \mathrm{E}+\mathrm{C}
\end{gathered}
$$

a different rate equation is obtained. The steady-state treatment of this mechanism yields the following equation for the initial velocity of the forward reaction when terms involving $(\mathrm{C})$ and (D) are omitted.

$$
v=\frac{V}{1+K_{\mathrm{A}} /(\mathrm{A})+K_{\mathrm{B}} /(\mathrm{B})+K_{\mathrm{AB}} /(\mathrm{A})(\mathrm{B})}
$$

The constants in equation (11) are given by $K_{\mathrm{A}}=k_{5} / k_{1}, K_{\mathrm{B}}=$ $k_{5} / k_{3}, K_{\mathrm{AB}}=k_{2} k_{5} / k_{1} k_{3}$, and $V=k_{5}(\mathrm{E})_{0}$. Thus the Michaelis constant for A determined by using a high concentration of $\mathrm{B}$ depends on the rate of dissociation of the EC complex and not at all upon the rate of dissociation of $A$ from $E A$. If the concentration of $\mathrm{B}$ is sufficiently low so that $K_{\mathrm{B}} /(\mathrm{B})$ and $K_{\mathrm{AB}} /(\mathrm{A})(\mathrm{B})$ are much greater than unity, equation (11) reduces to the same form as equation (9), but the two cases may be readily distinguished by experiments at higher concentrations of $B$. If the third step in the above mechanism is rate-determining and the equilibrium in the second step is not far to the right, the rate of the over-all reaction will also depend upon the concentration of $\mathrm{D}$, and it may be extremely difficult to determine the initial velocity if small concentrations of $\mathrm{D}$ have a large effect.

The steady-state rate equations for a number of mechanisms for coenzyme reactions have the form of equation (11), and so it is not possible to distinguish between these mechanisms by use of studies of the steady-state velocities alone. However, for the mechanism

$$
\begin{aligned}
& \mathrm{E}+\mathrm{A} \rightleftharpoons \mathrm{EA} \rightleftharpoons \mathrm{E} \rightleftharpoons \mathrm{E} \rightleftharpoons \mathrm{C} \\
& \mathrm{E}^{\prime}+\mathrm{B} \rightleftharpoons \mathrm{E}+\mathrm{D}
\end{aligned}
$$

where $\mathrm{E}^{\prime}$ might be an oxidized form of the enzyme, rate equation (11) is obtained with $K_{\mathrm{AB}}=0$. 
The most complete studies of any coenzyme reaction are those by Theorell, Chance, and Bonnichsen (15) on horse liver alcohol dehydrogenase. Since reduced diphosphopyridine nucleotide absorbs strongly at $340 \mathrm{~m} \mu$ whereas the oxidized form does not, the over-all kinetics were followed spectrophotometrically. It was found that when DPNH forms a compound with $\mathrm{ADH}$, the absorption maximum of DPNH shifts from 340 to $325 \mathrm{~m} \mu$. This observation was the key which opened the door to a detailed study of the stoichiometry, equilibrium, and kinetics. Since this reaction has been studied by both the steadystate and transient-state methods, it is very reassuring to find agreement between the velocity constants calculated from the different types of experiments. It was found that the transientstate kinetic data could be represented by mechanism (10) where $\mathrm{E}$ is alcohol dehydrogenase, $\mathrm{A}$ is DPNH, $\mathrm{C}$ is DPN, B is aldehyde, and $\mathrm{D}$ is alcohol. The kinetic data may be interpreted without postulating complexes of aldehyde or alcohol with the enzyme or a ternary complex. Michaelis constants for ethyl alcohol and acetaldehyde are obtained, but this does not mean that these substances necessarily form an intermediate complex with the enzyme. However, data obtained by Fisher et al. (5) using deuterium show that a specific hydrogen atom is transferred from one reactant to the other in the enzymatic reaction. It, therefore, appears that this transfer occurs in a ternary complex of the two reactants with the enzyme, the ternary complex having such a short lifetime that it is not detected kinetically.

\section{IONIZATION OF THE ENZYME}

Changing the $p \mathrm{H}$ produces large changes in the ratcs of most enzymatic reactions, even within the range where the enzyme is stable and where the substrate does not change in degree of ionization. Since physiological processes generally occur at a particular $p \mathrm{H}$ value it is perhaps worth inquiring what can be learned from an investigation of kinetics over a range of $p \mathrm{H}$ 
values. Proteins are polybasic acids and because of the large number of ionizable groups the effect of $p \mathrm{H}$ on the kinetics may be extremely complicated. In the neutral $p \mathrm{H}$ range proteins consist of a very large number of isomeric forms. For example, if $h$ of a total of $m$ acidic groups are ionized there are $m ! /(m-h) ! h$ ! different ions corresponding to the number of different ways in which it is possible to choose the $h$ protons from $m$ groups. However, it is to be expected that the ionizable groups in the neighborhood of the active site will exert a preponderant effect. If the gain or loss of a proton by a group in the catalytic site causes a total loss in enzymatic activity, the primary effect of changing the $p \mathrm{H}$ may be represented quite simply. The idea that the bell-shaped plots of activity versus $p \mathrm{H}$ are a consequence of the changing degree of ionization of the enzyme was originally suggested by Michaelis. In order to account for a maximum in such a plot it is necessary to postulate that there are at least two ionizable groups in the enzymatic site. If the enzyme-substrate complex breaks down to yield product only when there is a proton on one group and not on the other, a symmetrical maximum initial velocity versus $p \mathrm{H}$ plot will be obtained and the ionization constants of the two groups may be obtained from the experimental data.

Fumarase has proved to be a very suitable enzyme for a detailed study of the effect of $p \mathrm{H}$. The reaction catalyzed is

$$
\mathrm{F}+\mathrm{H}_{2} \mathrm{O} \rightleftharpoons \mathrm{M}
$$

where the symbols F, fumarate, and $\mathrm{M}, l$-malate, include the various ionized forms of the substrates. The effect of $p \mathrm{H}$ on the reaction rate indicates that the catalytic activity is dependent upon two ionizable groups. The experimental results may be accounted for in terms of the following mechanism, which is the simplest mechanism which will represent the effect of $p \mathrm{H}$ on the maximum initial velocities and Michaelis constants for both the forward and reverse reactions (7). 


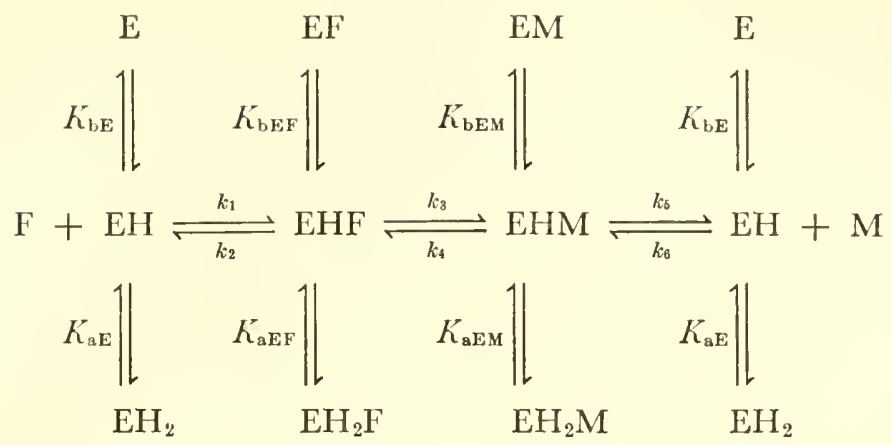

Here the three forms of the catalytic site are represented by $\mathrm{EH}_{2}, \mathrm{EH}$, and $\mathrm{E}$. The acid dissociation constants of the two groups in the enzyme and in the enzyme-substrate complexes are represented by $K_{\mathrm{a}}$ and $K_{\mathrm{b}}$. As indicated by the use of subscripts, it is to be expected that the acid dissociation constants of the groups will be different in the two types of enzyme-substrate complexes and free enzyme.

The steady-state treatment of this mechanism yields the Michaelis-Menten equation for the steady-state velocities of both the forward and reverse reactions, but the maximum initial velocities and Michaelis constants are now functions of the $p \mathrm{H}$. If the steps with rate constants $k_{3}$ and $k_{4}$ are rate-determining, the nature of this dependence is indicated by the equations for the maximum initial velocity $V_{\mathrm{F}}$ and Michaelis constant $K_{\mathrm{F}}$ for the forward reaction.

$$
\begin{gathered}
k_{\mathrm{F}}=\frac{\frac{k_{3} k_{5}(\mathrm{E})_{\mathrm{o}}}{\left(k_{3}+k_{4}+k_{5}\right)}}{1+\left(\mathrm{H}^{+}\right) / K_{\mathrm{aEF}}+K_{\mathrm{bEF}} /\left(\mathrm{H}^{+}\right)} \\
K_{\mathrm{F}}=\frac{\left(k_{2} k_{5}+k_{2} k_{4}+k_{3} k_{5}\right)\left[1+\left(\mathrm{H}^{+}\right) / K_{\mathrm{aE}}+K_{\mathrm{bE}} /\left(\mathrm{H}^{+}\right)\right]}{k_{1}\left(k_{3}+k_{4}+k_{5}\right)\left[1+\left(\mathrm{H}^{+}\right) / K_{\mathrm{aEF}}+K_{\mathrm{bEF}} /\left(\mathrm{H}^{+}\right)\right]}
\end{gathered}
$$

The acid dissociation constants of the enzyme-fumarate complex $K_{\mathrm{aEF}}$ and $K_{\mathrm{bEF}}$ are calculated from the variation of $V_{\mathrm{F}}$ with $p \mathrm{H}$, and the ionization constants of the free enzyme $K_{\mathrm{aE}}$ and $K_{\mathrm{bE}}$ are obtained from the variation of $V_{\mathrm{F}} / K_{\mathrm{F}}$ with $p \mathrm{H}$. As required by 
the mechanism the same values of $p K_{\mathrm{aE}}$ and $p K_{\mathrm{bE}}$ were actually obtained from kinetic data for the forward and reverse reactions.

The ionization constants for the complexes of fumarase with fumarate and $l$-malate are significantly different, and an interesting consequence of the shift in ionization constant induced by substrate is that the enzyme-substrate complex will contain a different number of protons on the average than the free substrate and enzyme molecules at the $p \mathrm{H}$ of the reaction medium. In other words, hydrogen ions will be produced or consumed in the reaction of substrate with the enzyme.

When the reactions of enzyme and substrate and hydrogen ions are interdependent in this way, they are "linked" functions, according to the terminology of Wyman (16). If the binding of substrate at the active site affects the ionization of groups of the enzyme, the ionization of these groups must conversely affect the affinity of the enzyme for the substrate. These reciprocal effects in the oxygen-binding- $p \mathrm{H}$ relation for hemoglobin are well known It is interesting that the effect of $p \mathrm{H}$ on the fumarase reaction is quite similar to the effect on the oxygen binding by hemoglobin and that two imidazole groups appear to be involved in both cases.

The question arises as to why it is that two groups are essential for the catalytic activity and that the intermediate ionized form is the active one. A very attractive explanation is that these two groups per enzymatic site are essential because they are actually involved in the transfer of protons in the enzymatic reaction. In $\mathrm{EHF}$ one ionizable group can donate a proton at one alpha carbon of fumarate and the other can receive a proton from a water molecule to make available a hydroxyl group at the other alpha carbon of fumarate. This mechanism offers an explanation of the fact that the complexes containing two protons or no protons are catalytically inactive. In strongly acidic solutions the catalytic site spends a large fraction of the time with a proton on the group which functions as a proton acceptor in the catalytic process, and during this time the reaction cannot occur. In strongly alkaline solutions, the catalytic 
site spends a large fraction of the time without a proton on the group which functions as a proton donor, and during this time the reaction cannot occur. Protons are transferred very generally in enzymatic reactions, and it may be that the observed $p \mathrm{H}$ dependence of the kinetics may be a consequence largely of the ionization of groups which are involved in providing or accepting hydrogen ions in the reactions.

The dependence of the kinetic constants on $p \mathrm{H}$ for a reaction following mechanism (14) is expected to be somewhat more complicated than indicated by equations (15) and (16) because of the progressive change in ionization constants of individual groups in a protein as the net charge on the molecule changes from large positive values below the isoelectric point to large negative values above the isoelectric point. This effect is well known from studies of acid-base equilibria of proteins of known amino acid composition. From a knowledge of the titration curve of an enzyme and its isoelectric point the electrostatic effect on the free energy of ionization of individual groups may be calculated. Thus it is to be expected that knowledge of classical protein chemistry will be found to be increasingly useful in the interpretation of enzyme kinetic data.

\section{INHIBITION OF ENZYMATIG REACTIONS}

Inhibition studies may provide information about the nature of the enzymatic site. Although this approach is rather indirect, still it is one of the few experimental approaches available. An inhibitor may combine at a site other than the enzymatic site, and this is indeed required in the case of noncompetitive inhibition. An inhibitor which is competitive with the substrate at low concentrations may also combine at other sites at higher concentrations. If the binding of inhibitor at other sites causes a partial inhibition or even an activation, the inhibition may be neither competitive nor noncompetitive. At low substrate concentrations the inhibitory effect may predominate so that there will be a net inhibition while at high substrate concentrations 
activation may predominate. Effects of this type may be represented by adding the following steps to mechanism (2).

$$
\begin{aligned}
\mathrm{E}+\mathrm{I} \rightleftharpoons \mathrm{EI} \\
\mathrm{E}+\mathrm{I} \rightleftharpoons \mathrm{IE} \\
\mathrm{IE}+\mathrm{S} \rightleftharpoons \mathrm{IES} \longrightarrow \mathrm{IE}+\mathrm{P} \\
\mathrm{IE}+\mathrm{I} \longrightarrow \mathrm{IEI}
\end{aligned}
$$

Here combination of $I$ at a neighboring site is indicated by writing $I$ to the left of $\mathrm{E}$. A steady-state treatment of the mechanism including these steps leads to a general equation for inhibition and activation. Activation may occur if IES breaks down to yield product more rapidly than does ES.

Although a large amount of inhibition data has been obtained and the structural features required for good inhibitors of certain enzymes such as chymotrypsin are now well known (14), the quantitative interpretation of inhibition constants has proved to be very difficult. One complication may be that the binding of inhibitor may cause appreciable shifts in ionization constants of groups in the enzyme. When inhibition constants are a function of $p \mathrm{H}$, a comparison of the inhibition constants of a series of compounds at a single $p \mathrm{H}$ may give erroneous indication as to the actual affinities of these inhibitors for a particular ionized form of the enzymatic site. Thus investigation of the effects of $p \mathrm{H}$ on inhibition as well as on the Michaelis constants and maximum velocities would be required.

\section{EFFECT OF BUFFER MEDIUM}

The ideal enzyme kinetic experiment would be carried out in a buffer which has no tendency to interact specifically with the enzyme or substrate but which would serve simply to maintain the $p \mathrm{H}$ and ionic environment constant while the substrate concentration was varied. The need for a constant $p \mathrm{H}$ during a series of velocity determinations is apparent from the preceding section. The need for holding a constant ionic environment is less obvious but equally important. Rate con- 
stants for reactions involving ions may be very dependent upon the nature and concentration of salt, and the rate constants of reactions involving nonelectrolytes are not free from this effect. Unfortunately the ideal buffer does not exist, and so it is necessary to consider specific effects due to the components of the buffer. In order to avoid large alterations in ionic strength, and presumably in the individual rate constants, when the substrate concentration is changed, the buffer concentration is generally maintained at a value considerably higher than the highest substrate concentration.

There are a number of different ways in which electrolytes can affect enzymatic catalysis. The binding of buffer ions by the enzyme may give rise to any of the inhibiting or activating effects discussed in the preceding section. The inclusion of such equilibria in the mechanism alters further the interpretation of the kinetic constants (1).

In addition electrolytes will be expected to exert the same types of effects which are encountered in studies of the kinetics of nonenzymatic reactions. Two types of salt effects were discussed by Brönsted, whose work forms the foundations of this field of physical chemistry. When the concentration of electrolyte is changed, the activity coefficients of the reactants and of the activated complex are altered. Depending upon the signs of the charges on the ions involved in the reaction this effect may result in either a speeding up or slowing down of the reaction. An additional effect of changing the concentration of electrolyte on reactions catalyzed by weak acids or bases is to change the degree of ionization of the catalyst. A similar effect is to be expected in enzymatic reactions if a particular ionized form of the enzyme is a much better catalyst than the other ionized forms. In the case of enzymatic reactions where several of these effects may be of importance, the first problem is to separate them so that the principles discovered in the study of simpler reactions may be applied.

The effect of electrolytes on the activity of urease has been investigated intensively by Kistiakowsky and co-workers (10). 
who find quite different effects at different $p \mathrm{H}$ values. Below $p \mathrm{H} 7$ the concentration of buffer containing sodium maleate and other added salts is without effect on the activity over a 1000fold range. At $p \mathrm{H} 7.0$ and 7.5 the activity decreases slightly with increasing electrolyte concentration, and at $p \mathrm{H} 8.9$ the effect is much greater. The effects are represented quite well by a form of the Debye-Hückel equation.

In the case of fumarase widely different results are obtained in different buffers of the same $p \mathrm{H}$ and ionic strength. Since the Michaelis constants for fumarase determined in phosphate buffer are a linear function of phosphate concentration, phosphate appears to be a competitive inhibitor. However, the situation is more complicated than this, since the maximum initial velocity also depends upon phosphate concentration. Since monovalent and divalent phosphate ions have quite different activating and inhibiting effects and the ratio of the concentrations of these ions changes with $p \mathrm{H}$, the dependence of the kinetic constants upon $p \mathrm{H}$ in phosphate buffers is extremely complicated.

\section{THE HALDANE EQUATION}

If there are appreciable concentrations of both products and reactants at equilibrium so that the kinetics of the catalysis of both the forward and reverse reactions may be studied, it is possible to verify certain conclusions about the mechanism. The kinetics of the forward and reverse reactions are not independent, and Haldane (8) was the first to show that for a reaction like (13) the maximum initial velocities and Michaelis constants for the forward and reverse reactions are related to the equilibrium constant for the over-all reaction in the same medium by

$$
K=\frac{(\mathrm{M})_{\text {eq }}}{(\mathrm{F})_{\text {eq }}}=\frac{V_{\mathrm{F}} K_{\mathrm{M}}}{V_{\mathrm{M}} K_{\mathrm{F}}}
$$

where $V_{\mathrm{F}}$ and $K_{\mathrm{F}}$ are the maximum initial velocity and Michaelis constant for the forward reaction and $V_{\mathrm{M}}$ and $K_{\mathrm{M}}$ for the reverse reaction. If there were activation or inhibition by substrate, 
this relation would not be expected to be obeyed, since the forward reaction would be catalyzed by enzyme activated by $\mathrm{F}$ and the reverse reaction by enzyme activated by $\mathrm{M}$. Although this relation was derived in a very simple way it is applicable even when the mechanism is very complicated and involves $p \mathrm{H}$ and buffer effects, provided only that the steady-state velocities of the forward and reverse reactions are represented by the Michaelis equation. The Haldane equation has been found to be obeyed by the fumarase reaction over a wide range of $p \mathrm{H}$ and buffer concentrations and offers a valuable check on experimental values of the kinetic constants (2).

For reactions involving more than one reactant and one product (other than water and hydrogen ions) there are more complicated relations between kinetic constants for the forward and reverse reactions and the equilibrium constant for the overall reaction (1). Since the form of this relationship depends upon the mechanism of the reaction, it should be possible to differentiate between certain mechanisms for coenzyme reactions by means of steady-state kinetic data on both the forward and reverse reactions.

\section{TEMPERATURE EFFECTS}

The interpretation of apparent activation energies for enzymatic reactions is considerably more difficult than for simpler reactions. This is a consequence of the large number of changes which may occur when the temperature of a protein solution is altered. In addition to the effect of temperature on individual rate constants, there will generally be effects upon the extent of ionization of the protein and its interaction with ions of the buffer solution. The temperature coefficient of a reaction determined without regard to these possibilities remains a hodgepodge of various effects. In order to identify the various participating equilibria, it would be necessary to study the effects of $p \mathrm{H}$, buffer concentration, etc., at a series of temperatures. However, there have been relatively few studies so far in which the effects of temperature on the Michaelis constant and maxi- 
mum initial velocity have been obtained separately, and in some studies, pains have not been taken to differentiate between the reversible and irreversible effects of temperature change.

The effect of temperature on enzymatic reactions has been the subject of controversy. Several authors have claimed that sharp breaks are obtained in plots of logarithm of rate versus reciprocal absolute temperature. To prove that the breaks are quite sharp requires accurate experiments at a large number of temperatures. The interpretation of these data in terms of the shift from one "pace-maker" reaction to another has been criti. cized on physical chemical grounds by Morales (13a) and by Kistiakowsky and Lumry (11). If there is a series of consecutive steps having different activation energies, the experimental activation energy becomes higher the lower the temperature, and the abruptness of the change in slope will be dependent upon the difference in activation energy of the two steps. If this difference is of the order of $10 \mathrm{kcal}$. mole ${ }^{-1}$ the change in slope will occur over approximately an $80^{\circ}$ temperature range. As pointed out by Kistiakowsky and Lumry changes in slope in either direction and sharper changes in slope may be due to a change of the enzyme from one form to another, these two forms catalyzing the reaction with different activation energies. In this case the temperature coefficient for the enzyme transformation will not contribute to the temperature coefficient for the enzymatic reaction but will determine the temperature range over which the transition from one activation energy to the other occurs. In order for the apparent activation energy to change over a small range it is necessary that the energy of isomerization of the protein be very great-of the order of 160 kcal. mole ${ }^{-1}$ if the transition occurs over a temperature range of $5^{\circ}$.

Urease was one of the enzymes for which it had been claimed that joined segments of straight lines are obtained by plotting the logarithm of the rate against the reciprocal absolute temperature with sharp breaks at "critical temperatures." Extensive experimental work by Kistiakowsky and Lumry (11) showed 
that there was a gradual change in slope rather than a sharp break.

\section{Closing}

Much of the enzyme kinetic data in the literature has been obtained under conditions that make a simple quantitative interpretation extremely difficult. As a hypothetical example, a reaction involving a coenzyme and a metal ion activator might be studied under conditions where the concentration of one substrate was so high as to cause inhibition, the metal ion was partially complexed with the anion of the buffer, and the coenzyme was a mixture of ionized forms at the $p \mathrm{H}$ of the medium. It is evident that the details of the mechanism of a reaction of this type would have to be unraveled in the same slow careful way as a snarled fishline. Much is to be gained by isolating effects in their simplest possible form so that experimental results may be expressed mathematically and related to the properties of simpler reactions.

The interpretation of enzyme kinetic data is subject to a number of pitfalls, but these pitfalls are becoming better understood and danger signals are being posted at the dangerous points. Since more than one mechanism may represent a given set of experimental data, anyone who claims that some mechanism is the only one possible for a given reaction is skating on extremely thin ice. However, the fact that the discovery of enzymatic mechanisms is difficult does not diminish interest in them. As we look back on the naiveté of early work in enzyme kinetics surely it is reasonable to assume that the data presently available will be subject to reinterpretation in the future.

When the information presently available on enzyme kinetics is compared with the vast field of potential application, it is true to say that the surface has just been scratched. In the future we may expect that more of the findings of physical chemistry and organic chemistry will find application to the study of enzyme kinetics. There are already indications of the basic 
similarity between enzymatic and nonenzymatic catalysis. As our knowledge of enzyme kinetics becomes more detailed it will no doubt find practical application in the solution of the problems concerned with life.

\section{References}

1. Alberty, R. A., J. Am. Chem. Soc., 75, 1925, 1928 (1953); ibid., 76, 2494 (1954).

2. Alberty, R. A., V. Massey, C. Frieden, and A. R. Fuhlbrigge, J. Am. Chem. Soc., 76, 2485 (1954).

3. Chance, B., in E. S. Guzman Barron, ed., Modern Trends in Physiology and Biochemistry. Academic Press, New York, 1952.

4. Chance, B., F. M. Huennekins, and F. J. W. Roughton, "Investigation of rates and mechanisms of reactions," in S. L. Friess and A. Weissberger, eds., Technique of Organic Chemistry, Vol. 8. Interscience, New YorkLondon, 1953.

5. Fisher, H. F., E. E. Conn, B. Vennesland, and F. H. Westheimer, J. Biol. Chem., 202, 687 (1953).

6. Foster, R. J., and C. Niemann, Proc. Natl. Acad. Sci. (U.S.), 39, 999 (1953); J. Am. Chem. Soc., 77, 1886 (1955).

7. Frieden, C., and R. A. Alberty, J. Biol. Chem., 212, 859 (1955).

8. Haldane, J. B. S., Enzymes. Longmans, Green, London, 1930.

9. King, E. L., personal communication.

10. Kistiakowsky, G. B., and W. H. R. Shaw, J. Am. Chem. Soc., 75, 2751 (1953).

11. Kistiakowsky, G. B., and R. Lumry, J. Am. Chem. Soc., 71, 2006 (1949).

12. McElroy, W. D., and B. Glass, eds., The Mechanism of Enzyme Action. Johns Hopkins Press, Baltimore, 1954.

13. Michaelis, L., and M. L. Menten, Biochem. Z., 49, 333 (1913).

13a. Morales, M. F., J. Cellular Comp. Physiol., 30, 303 (1947).

14. Neurath, H., and G. W. Schwert, Chem. Revs., 46, 69 (1950).

15. Theorell, H., and B. Chance, Acta Chem. Scand., 5, 1127 (1951).

16. Wyman, J., Advances in Protein Chem., 4, 407 (1948). 


\title{
THE INTERCONVERSION OF SUGARS IN NATURE
}

\author{
LUIS F. LELOIR, Instituto de Investigaciones Bioquímicas, Fundación
} Campomar, Buenos Aires

The amazing variety of carbohydrates manufactured by plants and animals has incited the accomplishment of an equally amazing work by the sugar chemists. This work, which gained impetus with Emil Fischer's intelligent research on the structure of sugars, has not slackened, and we are now confronted with an enormous amount of information on the occurrence of different carbohydrates in natural products. But the question which was probably in the minds of all these chemists-how does this organism make this particular sugar?-has only recently fallen within the frontiers of research.

It is fortunate for the biochemist that nature cloes not seem to have "discovered" all the possible isomers of sugars. Thus, of the sixteen possible aldohexoses, only four or five occur naturally. The problem, therefore, becomes more limited, but by no means small, since, including the aldoses, ketoses, amino and deoxy sugars, and uronic acids, the number of monosaccharides known to occur in nature is well over twenty. These substances are distributed in the most varied types of organisms. The biochemist may have to deal with molds or lobsters in order to study glucosamine derivatives, or with umbilical cords or 
pig's noses for amino sugars and uronic acids. Or he might use corn cobs which contain xylose, or perhaps algae and microbes which synthesize complicated polysaccharides. The field of sugar interconversions is a field for the lovers of nature and a fascinating chapter of comparative biochemistry.

Advances in the methodology of enzymes, in analytical procedures, and in the use of tracers have provided us with the means for elucidating many problems of the biogenesis of sugars. Moreover, the first period of enzymology which dealt mostly with catabolic processes has been superseded by the study of synthetic reactions. Following the in vitro synthesis of glycogen, several other polysaccharides have been prepared such as dextrans and levans, but the synthesis of cellulose, xylans, chitin, has not been accomplished yet, nor has that of mixed polymers such as hyaluronic acid, chondroitin sulfate, gums, and mucilages.

As to the disaccharides, trehalose phosphate has been prepared enzymically, and sucrose has been synthesized by three different paths. Although biochemists can be justly proud of the progress achieved, our present knowledge of the interconversion of sugars is limited to several unconnected series of reactions which have been found to occur in many organisms, from bacteria to mammals. We may hope that within a few years the picture will be more complete, and that we may know more about the way in which enzymes work inside the cells. This will require the development of delicate methods to replace the present ones, which usually consist in crushing the cells in order to learn their function.

Organic chemists have developed methods for preparing sugars at will. They can lengthen the chains by cyanohydrin synthesis or with diazomethane. They can shorten them by the Ruff or Wohl degradation. They can oxidize, reduce, epimerize, and invert. Living cells are masters in this technique and can also carry out the most varied reactions. We know that they phosphorylate, oxidize, and shorten the carbon atom chain; transfer two or three carbon atom residues, invert the configura. 
tion, and introduce amino groups. This is what we know, and is probably only a small part of what cells can do.

\section{Transfer of Phosphate Groups}

The study of phosphoric esters and of the corresponding enzymes has been pursued intensely ever since Harden and Young discovered the action of inorganic phosphate in yeast juice fermentation. This discovery was followed by the patient investigations of Robison and co-workers, which led to the isolation of several sugar esters. The mechanism of synthesis of these compounds was partly elucidated by the discovery of hexokinase by Meyerhof. This ubiquitous enzyme is reponsible for the introduction of phosphate in position 6 of glucose, fructose, or mannose using adenosine-triphosphate as donor. More recent work has disclosed the presence of similar enzymes which lead to phosphorylation in position 1 of fructose or galactose.

The specificity of these enzymes is not as stringent as was believed. Thus, the enzyme which acts on glucose also acts on glucosamine, and galactokinase appears to be able to lead to the phosphorylation not only of galactose but also of galactosamine. This fact raises the problem as to whether such reactions are only side effects due to a lack of specificity, or whether they occur normally in cell metabolism. Intriguing reactions of the same type have been observed with phosphoglucomutase. This enzyme catalyzes the transfer of phosphate from position 1 to position 6 of glucose. The reaction is dependent on the presence of catalytic amounts of glucose-1,6-diphosphate and consists in a transfer of phosphate from position 1 of glucose diphosphate to position 6 of glucose-1-phosphate, probably mediated by a phospho enzyme complex. The products are glucose-6-phosphate and glucose-1,6-diphosphate. The latter is, therefore, regenerated at each step. A wider specificity of phosphoglucomutase became apparent when it was found that it acted on mannose-1-phosphate, and that glucose-1,6-diphosphate also acted catalytically in this case (13). The reaction has been formulated in two steps. The first is: 
glucose-1,6-diphosphate + mannose-1-phosphate

$$
\text { mannose-1,6-diphosphate }+ \text { glucose-6-phosphate }
$$

followed by the catalytic action of mannose diphosphate:

mannose-1,6-diphosphate + mannose-1-phosphate $\rightleftharpoons$ mannose-1,6-diphosphate + mannose-6-phosphate

The first step was proved (13) by detecting the formation of mannose diphosphate on incubation of mannose-1-phosphate, glucose diphosphate, and the enzyme. Furthermore, it has been observed (21) that synthetic mannose diphosphate will activate the transformation of either glucose-1-phosphate or mannose-1phosphate. Moreover, similar events take place with the ribose esters. Glucose diphosphate activates the transformation of the 1 - into the 5-phosphate, and the formation of ribose-1,5-diphosphate has been detected (12). With acetylglucosamine-1-phosphate the problem is more complex. Muscle phosphoglucomutase does not act on this ester, but extracts can be obtained from a mold (Neurospora) which catalyze the conversion of the 1 - into the 6-phosphate. These extracts have been separated in different fractions (23) in which the ratio of activity, phosphoglucomutase/phosphoacetylglucosaminomutase, varies from 300 to 0.3 , that is, as if at least two different enzymes were involved. But in either fraction the transformation of the 1-esters is activated by glucose-1,6-diphosphate, and this fact can be taken as an indication that both glucose and acetylglucosamine esters fit the active group of one of the enzymes.

Such an overlapping in the action of these enzymes suggests that there may be a genetic relationship between them, and that their lack of specificity may be the result of a gradual evolutionary change.

If we were to let the imagination loose at the risk of being laughed at by more learned colleagues, we might reason somewhat as follows.

Adaptation experiments suggest that the substrate is in some manner involved in the formation of the corresponding enzyme. It is then possible that if the substrate were changed slightly, the 
enzyme would also be changed. In molds it is likely that there are abundant acetylglucosamine derivatives because the cell wall is made up of the $\beta$-acetylglucosamine polymer, chitin. These derivatives might change the specificity of the enzyme phosphoglucomutase, which in other organisms deals mainly with glucose esters. And if we extend this line of thinking, we can suppose that when an organism is first confronted with a new substrate, it first makes use of the enzymic equipment already available. Thus, an organism which normally deals with glucose could utilize glucosamine by taking advantage of existing enzyme systems. It would phosphorylate it with hexokinase, acetylate it with coenzyme A, change the position of the phosphate group with phosphoglucomutase, etc. The enzymes formed in the new environment might become more efficient. Eventually, if the organism previously made cellulose, it might now make chitin, since the two polymers differ only in the acetyl amino group replacing a hydroxyl.

It is futile to discuss here the possible mechanism of such an imaginary process, but it may be remarked that some sort of adaptation can occur which is independent of the genetic equipment of the cells. A proof is the fact that within the same organism there are cells which contain different enzymes and have different metabolic patterns, as, for instance, liver, brain, and muscle cells.

\section{Ketoses from Aldoses (14)}

Lohmann in 1933 discovered an enzyme in muscle which catalyzes the interconversion of glucose-6-phosphate into fructose-6-phosphate. Since then, several other enzymes of this type have been found. One catalyzes the transformation of ribose-5-phosphate into ribulose-5-phosphate, and another converts 3-glyceraldehyde phosphate into dihydroxyacetone phosphate. Changes of a similar type have been found to take place in some nonphosphorylated sugars. Thus, bacterial enzymes have been prepared which transform D-ribose into D-arabinose, 
and D-xylose to D-xylulose. These investigations have started recently, so that it is impossible to assess their metabolic importance, but it is believed that the enzymes acting on phosphorylated sugars are of more widespread occurrence.

\section{Inversion of Configuration}

The transformation of mannose into glucose requires inversion at position 2, while a similar change in position 4 leads to the formation of glucose from galactose. Both types of reactions occur in nature. Inversion at 2 has been studicd with muscle extracts. Starting with mannose-6-phosphate, the product obtained was a mixture of fructose-6-phosphate and glucose-6phosphate. Since the enzyme acting on mannose phosphate. could not be separated from the isomerase which interconverts glucose phosphate into fructose phosphate, it has not been possible to decide whether the primary product is the glucose or the fructose ester.

Inversion at 4 has been studied with extracts of yeast grown in galactose-containing media. These extracts catalyze the interconversion of $\alpha$-glucose-1-phosphate (II) into $\alpha$-galatose-1phosphate (III); that is, the inversion of the hydroxyl group at

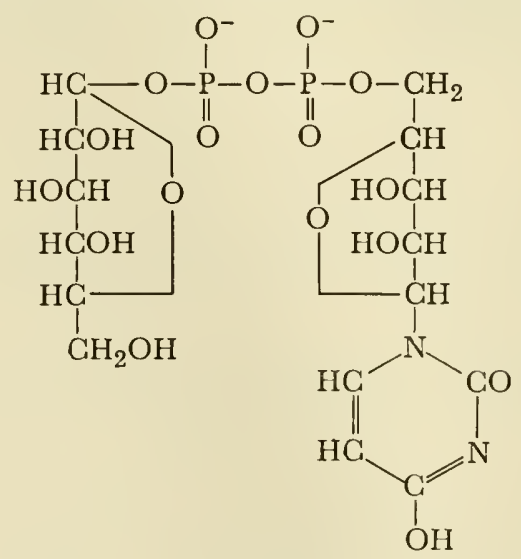

UDPG 


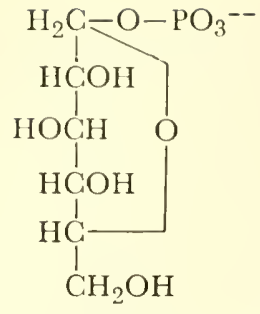

(II)

Glucose-I-phosphate

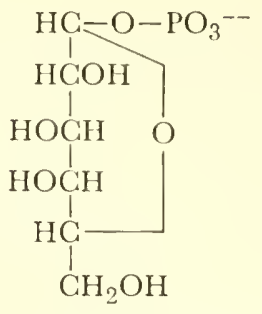

(III)

position 4 of the hexose. The equilibrium mixture contains about $25 \%$ of the galactose ester. Besides the enzyme(s), the reaction requires a coenzyme, uridine-diphosphate-glucose (UDPG) (I), which was first isolated from yeast and has been found to be present in animal and plant tissues. It has also been prepared by synthesis. An indication of how this coenzyme acts was obtained when analyzable amounts of UDPG were incubated with the yeast enzyme. It was found that about one-fourth of the glucose moiety was transformed into galactose, and it was concluded (13) that the yeast extracts contain an enzyme catalyzing the equilibrium

$$
\text { UDP-galactose } \rightleftharpoons \text { UDP-glucose }
$$

In order to relate this fact with the catalytic action of UDPG in the transformation of galactose-1-phosphate, it was assumed that another enzyme would exchange the hexose-1-phosphate residues in UDPG as follows :

galactose-1-phosphate + UDP-glucose $\rightleftharpoons$

$$
\text { glucose-1-phosphate + UDP-galactose }
$$

The sum of these two reactions gives

galactose-1-phosphate $\rightleftharpoons$ glucose-1-phosphate

Information on the mechanism by which UDPG is transformed into the galactose derivative is lacking. Many mechanisms which would lead to such a change in the configuration of carbon 4 can be imagined. Any reaction which produces a loss 
of asymmetry may be suspected, such as a substitution, a breakage of the carbon atom chain, or the formation of a double bond. Further work using labeled compounds would be of the utmost interest.

Another inversion at position 4 has been detected recently. This is the transformation of UDP-acetylglucosamine (UDPAG) (IV) into UDP-acetylgalactosamine (UDPAGA) (V) by liver enzymes. The use of yeast as starting material led to the isola-

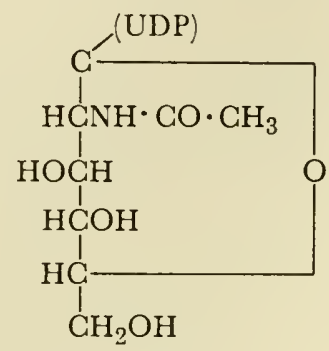

(IV)

UDP-acetylglucosamine

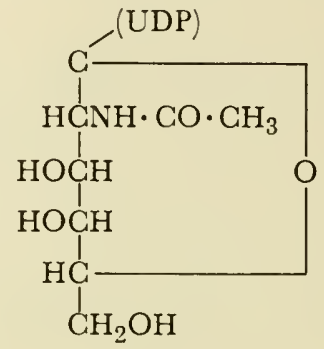

(V)

UDP-acetylgalactosamine

tion of pure UDPG and pure UDPAG (4), but a study of these compounds in liver showed that instead of UDPG, the product obtained was a mixture of UDPG and UDP-galactose. The same occurred with the acetylamino derivatives. Instead of UDP-acetylglucosamine as in yeast, the product obtained from liver contained about $20 \%$ of the acetylgalactosamine derivative (20). Further investigation showed that liver extracts catalyze the transformation of UDPAG into UDPAGA, and this may be the starting point of the study of a practically unexplored field: the enzymology of galactosamine-containing compounds.

Studies on uridine nucleotides have been extended in several directions. The list of sugars which have been found combined with UDP is-glucose, galactose, acetylglucosamine, acetylgalactosamine, glucuronic acid, and three hexosamine amino acid compounds (19). It has also been found that UDPG can act as a glucose donor to fructose, to fructose-6-phosphate, or to glucose-6-phosphate, yielding sucrose, sucrose phosphate, or 
trehalose phosphate. The biosynthesis of UDPG has been observed to be carried out by an enzyme which catalyzes the reaction (12):

UTP + glucose-1-phosphate

UDPG + inorganic pyrophosphate

And UTP (uridine triphosphate), in turn, has been found to be formed by phosphorylation of UDP (uridine diphosphate) by adenosinetriphosphate or phosphopyruvate.

Still one more substance of the nucleotide-sugar family has been isolated from yeast. The structure is like that of UDPG, but instead of uridine it contains guanine, and the glucose residue is replaced by mannose. Its role is unknown at present.

\section{Transfer of Two-Carbon Units}

An enzyme which has been named transketolase (9) has been obtained from yeast, spinach, and liver. When suitably

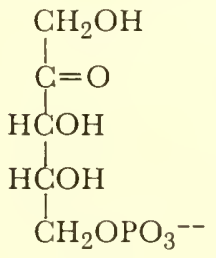

(VI)

D-Ribulose-5-phosphate

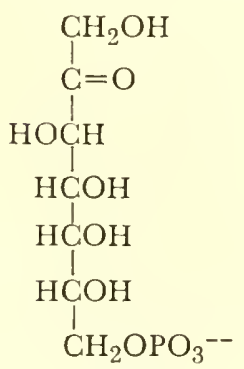

(VIII)

Sedoheptulose-7-phosphate

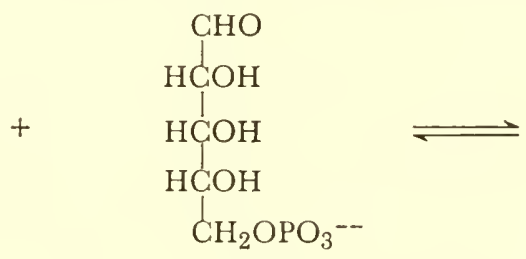

(VII)

D-Ribose-5-phosphate

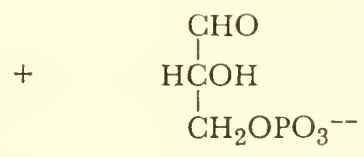

(IX)

D-Glyceraldehyde-3-phosphate 
activated with magnesium ions and diphosphothiamine, it catalyzes the transference of the $\mathrm{CHOH}-\mathrm{CO}-$ group from ketoses to aldoses, as shown in equation (7).

Instead of D-ribulose-5-phosphate as donor of the glycolaldehyde group, the enzyme can use hydroxypyruvic acid, L-erythrulose, or sedoheptulose-7-phosphate. Several substances can serve as acceptors. D-Ribose-5-phosphate and D-glyceraldehyde3-phosphate yield sedoheptulose-7-phosphate and D-ribose-5phosphate, respectively. The products formed with other acceptors have not been identified: L-glyceraldehyde-3-phosphate would probably yield L-ribulose-5-phosphate; D-glyceraldehyde would give D-ribose; and glycolaldehyde would form L-erythrulose.

Thus, transketolase can give rise to the most varied products. When the enzyme is added to the donor in the absence of acceptor, no chemical change can be detected. Diphosphothiamine appears to act as intermediary acceptor and donor of glycolaldehyde.

\section{Transfer of Three-Carbon Units}

It is generally considered that the only function of aldolase is to catalyze the conversion of fructose-1,6-diphosphate into dihydroxyacetone phosphate and glyceraldehyde phosphate, as shown in equation (8).

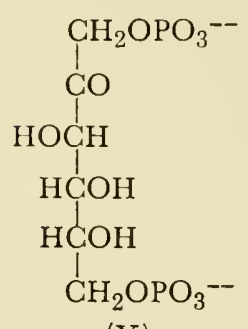

(X)

Fructose-1,6-

diphosphate

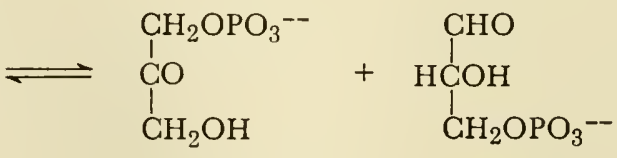

(XI)

Dihydroxyacetone phosphate
(XII)

D-Glyceraldehyde phosphate

Undoubtedly, this reaction is a part of the main metabolic 
channel in most cells, but it is quite likely that the same type of reaction may be used for other purposes. The equilibrium is strongly displaced in favor of fructose diphosphate, so that trapping agents such as cyanide or bisulfite are used in order to detect triose phosphate formation.

Meyerhof and Lohmann found that when aldolase was mixed with fructose diphosphate and different aldehydes, there occurred a transference of the dihydroxyacetone phosphate portion to the added aldehyde. Several of such transference reactions have been observed. Thus, D-fructose-1-phosphate (XIII) is formed on incubating aldolase with fructose-1,6-diphosphate and D-glyceraldehyde. With L-glyceraldehyde the product is L-sorbose-1-phosphate (XIV); with glycolaldehyde it is Dxylulose-1-phosphate (XV); with acetaldehyde it is probably 5-deoxyxylulose-1-phosphate (XVI); and with tetrose phosphate it is sedoheptulose-1,7-diphosphate.

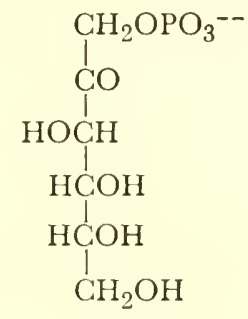

(XIII)

D-Fructose-1-phosphate

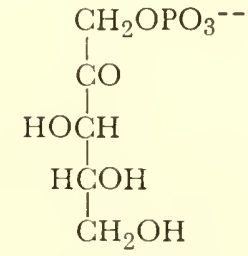

(XV)

D-Xylulose-1-phosphate

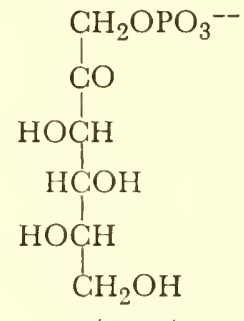

(XIV)

L-Sorbose-1-phosphate

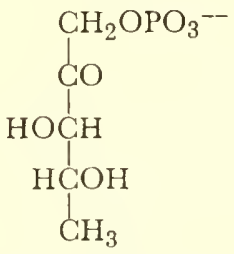

(XVI)

j-Deoxyxylulose-1-phosphate

The synthetic products obtained with aldolase are believed to have the $\mathrm{OH}$ at carbons 3 and 4 always in the trans position 
However, it has been asserted that aldolase will also split Dtagatose-1-phosphate (the ketose corresponding to galactose), in which the 3 and $4 \mathrm{OH}$ groups are cis. If such is the case, it is likely that in the synthetic reactions some of the cis compounds are formed but have not been detected owing to their small amounts.

The action of aldolase on fructose-1-phosphate has been given more attention, since the latter was found to be the product of fructose phosphorylation in liver. The utilization of fructose is considered to occur as follows:

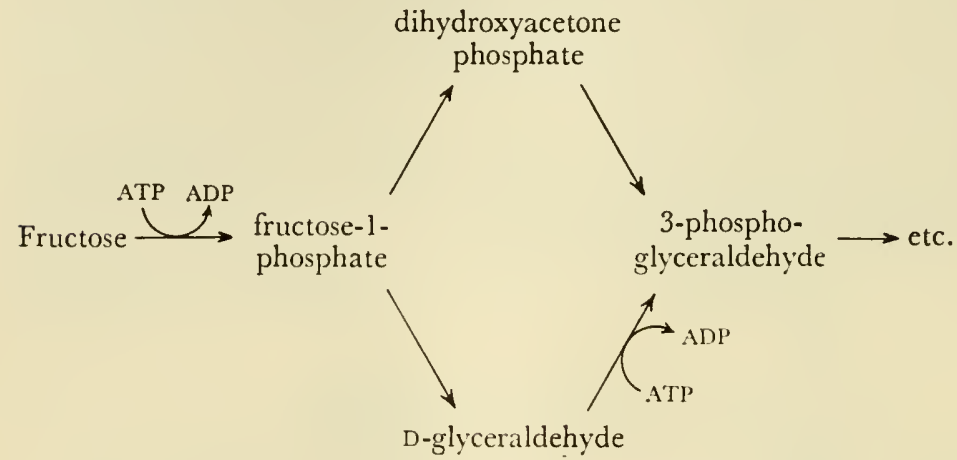

Another enzyme which can transfer three-carbon units is transaldolase (9). It differs from aldolase in that it is the nonphosphorylated dihydroxyacetone residue which is transferred. The following reaction has been used for its detection:

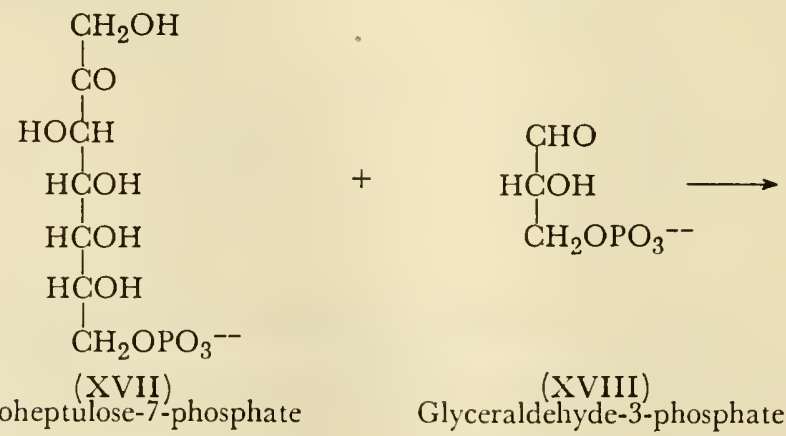




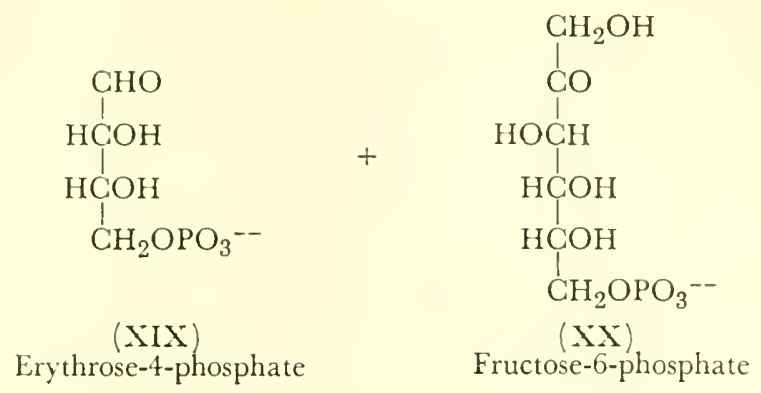

With this new enzyme together with aldolase and transketolase, the number of possible interconversions between ketoses becomes enormous.

\section{Redox Reactions}

The initial studies of Warburg on the oxidation of glucose6-phosphate at position 1 have been followed by several workers, and it has been found that the following sequence of reactions is catalyzed by enzymes present in plant and animals:

glucose-6-phosphate $\stackrel{\text { TPN }}{\rightleftharpoons}$ 6-phosphogluconolactone $\rightleftharpoons$

6-phosphogluconic acid $\underset{\text { TPN }}{\stackrel{-\mathrm{CO}_{2}}{\rightleftharpoons}}$ ribulose-5-phosphate $\rightleftharpoons$

$$
\text { ribose-5-phosphate }
$$

A point of interest is that all the reactions have been proved to be reversible (9), so that starting with ribulose-5-phosphate, $\mathrm{CO}_{2}$, and an appropriate reductant, it is possible to convert ribulose phosphate into glucose phosphate. This set of enzymes, therefore, provides a method for lengthening or shortening the carbon atom chain by one unit.

A similar oxidation of free glucose to gluconic acid has been found to be catalyzed by mold and liver enzymes.

The product of oxidation at position 6 of glucose is glucuronic acid, which is frequently found in nature. It is well known to mammalian physiologists as a "detoxifying substance," 
that is, a substance which combines with toxic products to render them harmless. Glucuronic acid is also present in many polymers such as hyaluronic acid, chondroitin sulfate, heparin, bacterial polysaccharides, and plant gums.

By the use of tracers it has been proved that mammals transform glucose directly into glucuronic acid - that is, without breaking down the carbon atom chain. A signal advance in these studies has been the isolation of UDP-glucuronic acid. It has been found that the formation of glucuronides with liver enzymes requires the presence of a specific donor, which turned out to be a uridine compound (5). The reaction takes place as follows:

UDP-glucuronic acid + phenol $\longrightarrow$ phenol-glucuronide + UDP

The identification of this substance has provided a working hypothesis for the study of the oxidation step which leads from glucose to glucuronic acid. Since studies with tracers prove that free glucuronic acid is not an intermediate in the formation of glucuronides, it may be assumed that the oxidation step takes place either on UDPG or on glucose-1-phosphate, yielding UDPglucuronic acid or glucuronic acid-1-phosphate, respectively.

The knowledge acquired in the formation of glucuronic acid will no doubt be applied to the elucidation of the origin of the other uronic acids such as D-galacturonic acid, which is present in pectins, flaxseed mucilage, and some specific polysaccharides of pneumococci, and D-mannuronic acid, which is the sole constituent of the alginic acid produced by seaweeds.

The oxidation of an uronic acid probably leads to the formation of ascorbic acid. One of the many schemes which have been proposed (10) shown on next page.

An interesting point about this scheme is the mechanism by which the molecule passes from the $\mathrm{D}$ to the $\mathrm{L}$ series. This is accomplished by reducing the aldehyde group of $\mathrm{D}$-galacturonic to a primary alcohol, and thus forming L-galactonic acid. In order to adhere to the usual convention of writing carbon atom 

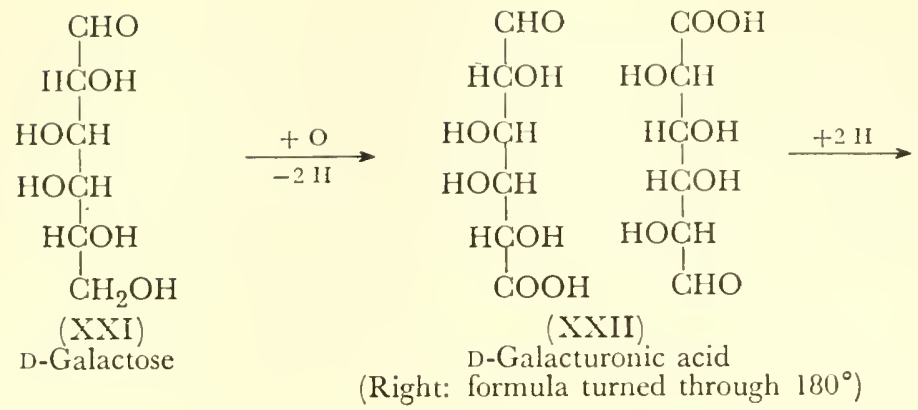<smiles>O=C(O)C(O)C(O)C(O)CO</smiles>

(XXIII)

L-Galactonic acid
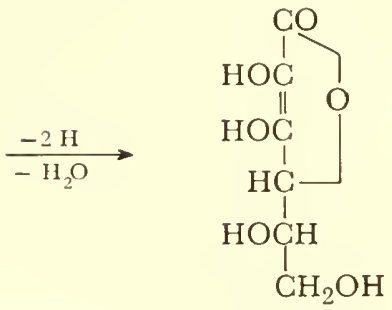

(XXIV)

L-Ascorbic acid

1 at the top, it is necessary to rotate the formula for XXII through $180^{\circ}$.

Some of the experimental facts supporting such a mechanism are the following. Tracer experiments show that D-glucose is transformed into ascorbic acid without breakage of the carbon chain. Glucose labeled at carbon atom 1 is converted into ascorbic acid labeled at carbon atom 6, and similarly, glucose labeled at 6 gives ascorbic acid labeled at 1. Moreover, administration of some intermediates such as D-galacturonic and L-galactonic acids give rise to an increased formation of ascorbic acid in the rat and in cress seedlings.

Another problem which on paper can be solved by the simple expedient of turning the molecule around through $180^{\circ}$ is that of the formation of L-galactose, which is found in agar and flaxseed mucilage. If the primary alcohol group of galactose is oxidized to an aldehyde, a substance would be obtained which on reduction can yield either D- or L-galactose. 
There is still another redox reaction of interest. This is the oxidation at position 2 of a sugar alcohol, D-sorbitol, to the corresponding ketose, D-fructose, which was found to be catalyzed by a liver enzyme with diphosphopyridine nucleotide as oxidant (18). Since the reaction is reversible, it appears likely that polyhydric alcohols are formed in plants by reduction of ketoses.

\section{Amination}

Glucosamine is present in the acetylated and polymerized form as chitin in the cell walls of fungi and in the shells of crustacea. Hyaluronic acid, a mixed polymer of glucosamine and glucuronic acid, is present in vitreous humor, umbilical cord, and synovial fluid, and is formed by some hemolytic streptococci. Tracer evidence has shown that the carbon skeleton of glucosamine is derived directly from glucose without breakage of the chain. Evidence from the enzymic side has shown that extracts can be obtained (16) from Neurospora which will catalyze the reaction

hexose-6-phosphate + glutamine

glucosamine phosphate + glutamic acid

It may be pointed out that Neurospora was selected for this study, not because it is an especially interesting organism, but because it was believed that its glucosamine metabolism should be high in order to supply the organism with the necessary material for the building of its chitin walls. Following this assumption, a fairly active enzyme was obtained from Neurospora, and now that we know something about the reaction we can look for it in other organisms.

The enzyme has not been separated from the isomerase which catalyzes the interconversion of glucose-6-phosphate into fructose-6-phosphate, so that a discussion of the mechanism is premature. It might be formulated as shown in reaction (15) and would involve an exchange of an $\mathrm{OH}$ for an $-\mathrm{NH}_{2}$ group. 
and would be different from the transminase reaction (16), in which there is an exchange of $-\mathrm{NH}_{2}$ for $=\mathrm{O}$. Since one of the reactants is an amide, the name transaminase is not applicable. A suggestion is to call the enzyme transamidase. The mechanism of the reaction is at present obscure. No cofactor has been found

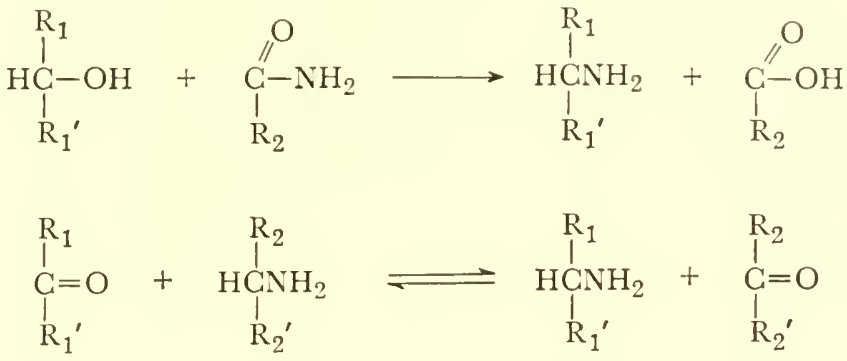

to be necessary, but a further search in this direction should be carried out, especially on recalling the difficulties which attended the recognition of pyridoxal phosphate as the coenzyme of transaminases.

\section{Deoxy Sugars}

Only in one case has an enzyme been found which will synthesize a deoxy sugar (22). It has been obtained from animal tissues and from microorganisms and catalyzes the following reaction:

glyceraldehyde-3-phosphate + acetaldehyde

deoxyribose-5-phosphate

Deoxyribose-5-phosphate $(\mathrm{XXV})$ can be converted by another enzyme of the phosphoglucomutase type into the 1-phosphate, which in turn can react with certain purines to yield deoxyribosides. Other deoxy sugars which occur fairly frequently are L-fucose (XXVI) and L-rhamnose (XXVII). L-Fucose is related (on paper) to $\mathrm{L}$-galactose by reduction of the $\mathrm{CH}_{2} \mathrm{OH}$ to $\mathrm{CH}_{3}$; L-rhamnose is similarly related to L-mannose. Both methyl pentoses are found in plants, and L-fucose is a constituent 
of blood group substances which contain, in addition, glucosamine, galactose, and mannose.

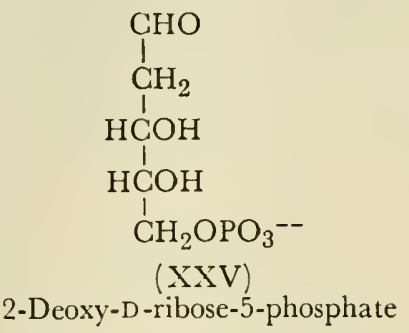

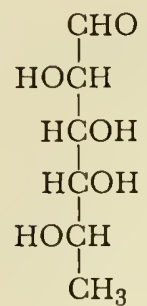

(XXVI)

L-Fucose

(6-Deoxy-L-galactose)

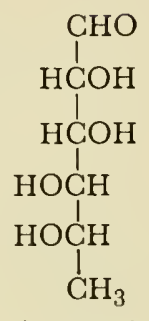

(XXVII)

L-Rhamnose

(6-Deoxy-L-mannose)

\section{Some Unknown Processes}

Whereas ribose phosphate has been obtained in vitro by two different processes, by oxidation of gluconic acid 5-phosphate and by transfer of a two-carbon unit to glyceraldehyde phosphate, information on the origin of other pentoses is lacking. Nothing is known on the biogenesis of $\mathrm{D}$-xylose, present in plants as the polymer xylan, which is second only to cellulose in abundance. Another pentose which is widely distributed in plant products is L-arabinose. It is found in hemicelluloses, pectins, gums, and some bacterial polysaccharides.

The stereochemical relationships of these two pentoses are made apparent by the following scheme.

$$
\begin{gathered}
\text { D-glucose } \stackrel{+\mathrm{O}_{2}}{\longrightarrow} \text { D-glucuronic acid } \stackrel{-\mathrm{CO}_{2}}{\longrightarrow} \text { D-xylose } \\
\text { D-galactose } \stackrel{+\mathrm{O}_{2}}{\longrightarrow} \text { D-galacturonic acid } \stackrel{-\mathrm{CO}_{2}}{\longrightarrow} \text { L-arabinose }
\end{gathered}
$$


Thus, D-xylose and L-arabinose might originate from glucose and galactose by oxidation and decarboxylation of the free sugars or some derivatives such as the 1-phosphate. The vertical relationship in the scheme is of interest because each substance in the lower row might be obtained from that in the upper row by inversion at position 4. Such an inversion has been obtained experimentally in the pair UDP-glucose-UDP-galactose, and UDP-glucuronic acid has been isolated, so that workers should be alert to the possibility of discovering UDP-galacturonic acid, and perhaps UDP-xylose and UDP-L-arabinose, as well as the enzymes catalyzing the interconversions.

\section{The Synthesis of Disaccharides}

When organic chemists wish to prepare a glycoside, they usually start by preparing a derivative in which the $\mathrm{OH}$ at position 1 of the sugar is replaced by a halogen atom. They then react the halogen sugar with the acceptor in the presence of silver or mercury ions. In this manner, the energy of the reactants is stepped up and that of the products is stepped down (by removing the halogen ion as an insoluble salt), so that the equilibrium of the reaction becomes favorable to the synthesis or, in other words, the $\Delta F$ becomes negative. In living cells this stepping up of the reactant may be accomplished by the phosphorylation of glucose to glucose-1-phosphate, or it may be stepped up higher by the introduction of the uridine pyrophosphate residue. Both mechanisms can be used for the synthesis of sucrose.

Doudoroff, Hassid, and co-workers (8) prepared an enzyme from bacteria (Pseudomonas saccharophila) which catalyzes sucrose synthesis according to the following reaction:

glucose-1-phosphate + fructose $\rightleftharpoons$ sucrose + inorganic phosphate

The equilibrium constant is 0.053 at $p \mathrm{H} 6.6$ and $30^{\circ} \mathrm{C}$. $(\Delta F=+1770)$. The original enthusiasm which led to believe that this reaction explained sucrose synthesis in plants was soon 
followed by disappointment when sucrose phosphorylase could not be detected in plant tissues.

More recently, another enzyme has been found (17) which catalyzes the formation of sucrose as follows:

$$
\mathrm{UDPG}+\text { fructose } \rightleftharpoons \mathrm{UDP}+\text { sucrose }
$$

In this case the enzyme was found to be present in the seeds of several plants, and the equilibrium constant is about $5(\Delta F$ about -1000).

Reaction (21) was believed to explain the long-sought mechanism of sucrose synthesis in plants, until another enzyme was found which catalyzes the following reaction:

UDPG + fructose-6-phosphate $\rightleftharpoons$ UDP + sucrose phosphate

Wheat germ was found to contain both enzymes, and since phosphatase is also present, the reaction product may be rapidly dephosphorylated to free sucrose and accumulates only when a purified enzyme is used.

A sucrose phosphate was detected by Buchanan, Calvin, and others (3) as a product of photosynthesis in algae and green leaves. It is not known whether it is the same as that formed in reaction (22), which has the phosphate group in position 6 of the fructose moiety.

A study of the relative activities of the enzymes which synthesize sucrose and sucrose phosphate in different parts of the plant would be of physiological interest, but has not been carried out owing to technical difficulties. Very likely sucrose phosphate follows some metabolic path different from that of free sucrose, so that it is apt to become the subject of interesting investigations.

Another disaccharide has been synthesized enzymically starting with UDPG. This is trehalose phosphate. Yeast extracts have been obtained (15) which catalyze the reaction:

UDPG + glucose-6-phosphate $\longrightarrow$ trehalose phosphate + UDP 
Trehalose phosphate had been previously prepared by Robison and Morgan from the mixture of sugar esters formed during the fermentation of sugars by dried yeast.

Free trehalose is found in some plants, in yeast, and in fungi. In mushrooms it tends to disappear with age and is replaced by mannitol. Trehalose is considered to be some sort of storage form of sugar in those organisms which do not store sucrose.

The discovery of the role of UDPG as glucose donor immediately leads to speculation on the role of the other uridinepyrophosphate-containing substances. The formation of phenol glucuronides from phenol and UDP-glucuronic acid has already been mentioned. As to UDP-galactose, it might transfer galactose to glucose or glucose phosphate to yield lactose or lactose phosphate. In this connection, it should be pointed out that lactose is $\beta$-galactosyl-4-glucose, whereas in sucrose and trehalose, and also in UDPG, the linkage of glucose is $\alpha$. Therefore, the synthesis of sucrose and trehalose takes place without inversion, whereas for lactose it would be accompanied by inversion. That such a type of inversion can take place was proved by Fitting and Doudoroff (7) in a study of a bacterial enzyme which catalyzes the following reaction:

maltose $(\alpha$-glucosyl-4-glucose $)+$ phosphate $\rightleftharpoons$

$$
\beta \text {-glucose-1-phosphate }+ \text { glucose }
$$

Moreover, phenol glucuronides are usually $\beta$, and if UDPglucuronic acid is $\alpha$, like UDPG, an inversion should take place in this reaction also.

Disaccharides and higher saccharides can be formed by enzymes whose main action is hydrolytic (1). To take the case of lactase, the events may be pictured as follows. The substrate, lactose, reacts with lactase to give a galactosyl-enzyme compound which then reacts with an - $\mathrm{OH}$-containing substance which may be water or another molecule. The reaction product will depend on the type of acceptor available, and since water is usually the most abundant, the main reaction will be a hydrolysis of the galactosyl-enzyme compound. However, if galactose con- 
centration is high, a digalactoside will be formed, or with a high lactose concentration the main product will be a trisaccharide containing two galactose molecules and one glucose molecule. In this manner, a number of galactose-containing di- or oligosaccharides can be formed. All these products disappear if the reaction is allowed to proceed to completion, and have therefore $\beta$ linkages. When water is the acceptor, the product is also the $\beta$ anomer, as was shown many years ago by Armstrong.

Similar events have been found to take place with other hydrolytic enzymes such as yeast invertase, which can transfer fructose residues. It seems rather doubtful that these reactions have a role in biosynthetic processes, because they usually require high substrate concentrations.

The fact that hydrolytic enzymes can act as transferring enzymes raises the question of whether enzymes which are considered as purely transferring have some hydrolytic action which is usually attributed to impurities. Perhaps the difference between these two types of enzymes is not quite clear-cut, and all the intermediate types can be found.

\section{The Synthesis of Polysaccharides}

Several polysaccharides have been already synthesized in vitro. Except for the case of phosphorylase, all the other enzymes use a disaccharide as substrate: maltose in some cases, and sucrose in others. The glucose half of sucrose can be used in making dextrans, while other enzymes use the fructose half for the synthesis of levans or fructans (1).

It is interesting to point out how UDPG could be used by cells to step up the energy of fructose in order to make a fructan. Two known reactions could be combined as follows:

$$
\begin{gathered}
\text { UDPG }+ \text { fructose } \rightleftharpoons \text { sucrose }+ \text { UDP } \\
\text { sucrose } \left.+(\text { fructose })_{n} \rightleftharpoons \text { glucose }+ \text { (fructose }\right)_{n+1}
\end{gathered}
$$

The result would be the hydrolysis of UDPG to UDP and free glucose, and the introduction of free fructose into the polymer. It is not known whether UDP-sugar compounds lead to 
the formation of disaccharides which are then transformed into polysaccharides, or whether the transference of the sugar residucs can be direct to the polymer.

With the knowledge available at present, it should not be very long before many other polysaccharides are synthesized in vitro. Cellulose, chitin, xylan, mannan, and galactans containing a single sugar should prove easier to synthesize than mixed polymers like hyaluronic acid or chondroitin sulfate, which, in addition to galactosamine and glucuronic acid, contains sulfate groups. And these in turn, seem to be simple cases compared to blood group substances (2), which contain galactose, glucose, hexosamines, fucose, and amino acids; or to gum arabic (11), where the sugar constituents are galactose, glucuronic acid, Larabinose, and L-rhamnose.

A consideration of these complicated polysaccharides leads to a digression on their role in living cells. Besides the more obvious structural role of substances like cellulose and the role of glucose and fructose polymers as reserve substances, it seems that sometimes polysaccharides form a chemical barrier which protects the organism from attack. The barrier can be overcome only by using the right key, that is, an enzyme which will dissolve the barrier. The more unusual barriers will be those which afford a greater survival value for their possessors. Examples of this are numerous. Hyaluronic acid does not allow the spreading of infectious agents in the dermal tissue, but microbes secreting the corresponding enzyme, hyaluronidase, are not affected by the hyaluronidase barrier. Spreading of the infection occurs also if hyaluronidase is injected together with microbes which do not have the enzyme.

Virulent pneumococci differ from the nonvirulent by the presence of a capsule which surrounds the cells. In this case, the polysaccharide capsules, which are formed by different combinations of sugars, uronic acids, and hexosamines (6), appear to be resistant to the enzyme equipment which forms a part of the host's anti-infectious mechanism.

The plant gums have been thought to represent a protective mechanism by which the injured parts of the plant are sealed 
off and thereby protected from the attack of microorganisms. The complicated structure of these gums should certainly afford a good protection, since very special enzymes would be necessary to break them down.

\section{References}

1. Barker, S. A., and E. J. Bourne, Quart. Revs. (London), 7, 56 (1953).

2. Bray, H. G., and M. Stacey, "Blood group polysaccharides." Advances in Carbohydrate Chem., 4. 37 (1949).

3. Buchanan, J. G., J. A. Bassham, A. A. Benson, D. F. Bradley, M. Calvin, L. L. Daus, M. Goodman, P. M. Hayes, V. H. Lynch, L. T. Norris, and A. T. Wilson, "The path of carbon in photosynthesis. XVII. Phosphorus compounds as intermediates in photosynthesis." Phosphorus Metabolism, 2, 440 (1952).

4. Cabib, E., L. F. Leloir, and C. E. Cardini, J. Biol. Chem., 203, 1055 (1953).

5. Dutton, G., and D. E. Storey, Biochem. J. (London), 57, 275 (1954).

6. Evans, T. H., and H. Hibbert, "Bacterial polysaccharides." Advances in Carbohydrate Chem., 2, 203 (1946).

7. Fitting, C., and M. Doudoroff, J. Biol. Chem., 199, 153 (1952).

8. Hassid, W. Z., and M. Doudoroff, "Synthesis of disaccharides with bacterial enzymes." Advances in Enzymol., 10, 123 (1950).

9. Horecker, B. L., Brewers Dig., 28, 214 (1953).

10. Isherwood, F. A., Y. T. Chen, and L. W. Mapson, Biochem. J. (London), 56,1 (1954).

11. Jones, J. K. N., and F. Smith, "Plant gums and mucilages." Advances in Carbohydrate Chem., 4, 243 (1949).

12. Kalckar, H. M., and H. Klenow, Ann. Rev. Biochem., 23, 527 (1954).

13. Leloir, L. F., "The metabolism of hexosephosphates," in Phosphorus Metabolism, 1, 67 (1951).

14. Leloir, L. F., "Enzymic Isomerization and Related Processes." Advances in Enzymol., 14, 193 (1953).

15. Leloir, L. F., and E. Cabib, J. Am. Chem. Soc., 75, 5445 (1953).

16. Leloir, L. F., and C. E. Cardini, Biochim. et Biophys. Acta, 12, 15 (1953).

17. Leloir, L. F., and C. E. Cardini, J. Am. Chem. Soc., 75, 6084 (1953).

18. McCorkindale, J., and N. L. Edson, Biochem. J. (London), 57, 518 (1954).

19. Park, J. T., J. Biol. Chem., 79.1, 897 (1952).

20. Pontis Videla, H. G., Ciencia e invest. (Buenos Aires), 10, 236 (1954).

21. Posternak, T., and J. P. Rosselet, Helv. Chim. Acta, 36, 1614 (1953).

22. Racker, E., J. Biol. Chem., 196, 347 (1952).

23. Reissig, J. L., unpublished. 


\section{A THEORY OF THE PRIMARY EVENT IN MUSCLE ACTION}

MANUEL F. MORALES and JEAN BOTTS, Division of Physical Biochemistry, U.S. Naval Medical Research Institute, Bethesda, Maryland

The fundamental problem in muscle physiology is not hard to describe: Somewhere in the multitude of reactions going on in the excited fiber there must be a "chemical" reaction which proceeds in such a way that it requires, compels, or achieves an extensive condensation of matter along one axis of space. The problem is to identify the participants of the "reaction" and the matter condensed in this transduction between "chemical" and mechanical energy.

At the time of this writing, knowledge is sufficiently advanced to warrant reasonable speculation about the nature of this primary event, but not so advanced as to discriminate with assurance between two or three hypotheses. For example, it has been shown that two rather pure proteins extractable from muscle, viz., myosin and actin, have an attraction for one another which can be largely abolished by the nucleotide, adenosinetriphosphate (ATP). It is a priori reasonable to think that in muscles myosin and actin are so disposed that the attraction between them causes elements of the two proteins to condense axially whenever ATP is withdrawn from the system, and to separate passively whenever ATP is introduced into the system. 
A theory based on this principle has been proposed collaboratively by A. F. Huxlcy and Niedergerke (39), and H. E. Huxley and Hanson (40), and has been adopted by Bailey (3). Along a different line, it has been shown that actin undergocs a selfreaction in the course of which ATP is hydrolyzed. Spatially, this self-reaction is at least a partial condensation, and although the nature of the interaction between actin condensation and ATP hydrolysis is quite unknown, one can reasonably conceive of muscle actin so disposed that upon introduction of ATP the material condenses axially. Such a theory has been proposed by Straub (67) and has been occasionally advocated by Mommaerts (52). Either of these theories would be greatly strengthened if it could be shown, at least in vitro, that it is possible to construct a contractile, work-performing system operating on either principle. Actually this has never been accomplished, and an examination (57) of present evidence bearing on these theories reveals inconsistencies that, in our opinion, render the theories highly improbable. By contrast, the theory to be elaborated here stems from the brilliant Engelhardt experiments (especially as improved by Weber and Szent-Györgyi), showing that a thread spun from myosin will contract and do work in the presence of $\mathrm{Mg}^{++}$and ATP $(20,41)$. These experiments not only make it probable that myosin, $\mathrm{Mg}^{++}$, and ATP are parts of the muscle "machine" but also suggest that macroscopic contractility results from intrinsic contractility of molecular chain elements, not from reactions in which mechanically discontinuous elements clump together.

Much physicochemical thinking and experimentation of the past fifteen years has sought to explain the basic EngelhardtWeber-Szent-Györgyi experiments; it is only recently that new experiments have placed additional requirements on a theory of the primary event. In the present essay we shall, correspondingly, commence by outlining what seems a plausible theory of why myosin deforms on ATP addition, and then we shall attempt to assimilate complications by elaborating the basic theory. 
The abstract features of our "plausible" theory are these: It is supposed that the basic contractile element is a flexible molecular chain which for certain reasons would-in the absence of the next stipulation-be considerably curled at equilibrium. It is further supposed, however, that fixed along the chain are discrete electrical charges. The mutual repulsion generally existing among these charges introduces an extensile force preventing complete contraction of the chain. Under these circumstances, varying the number of fixed charges will vary the linear dimensions of the chain.* Application of these notions to the myosin-ATP system is unstrained. It is very likely that the basic elements of fibrous myosin, viz., its polypeptide chains, are intrinsically capable of curling. It is also known that even "on the average" myosin is rich in polar residues, and recent indications that myosin is spatially heterogeneous only assure that certain regions of myosin will be especially dense in electrical charge. Therefore, it is not hard to imagine that its electrical charge keeps a myosin system expanded far beyond its uncharged equilibrium shape. To introduce a mechanism of altering the electrical charge on myosin we have to recall two properties of ATP, viz., that it is itself a highly charged anion ( $c a .4-$ charge in neutral solution), and that it is - as Engelhardt showed (20) - a substrate for the enzymatic activity of myosin. If one accepts the Michaelis theory

* The operating principles used in this theoretical model are well known, and in fact underlie the modern theory of polyelectrolytes. In reference to muscle action, Wöhlisch (73), Guth (30), and Bull (18) early pointed out that a "certain reason" why a single molecular chain left to itself in a structureless, inert medium would be curled is that a greater configurational entropy is associated with moderate curling than with full extension. Likewise, Meyer (51) early saw the mechanical implications of electrical fields arising from the abundant ionized groups of myosin. The first conceptual synthesis of these two principles into the model just described was due to Riseman and Kirkwood (64); it was also these authors who first proposed that ATP changed the charge on the myosin, but by a mechanism quite different from that to be proposed here. The practical realization of the model was soon thereafter achieved by Kuhn, Katchalsky, and their associates $(42,45)$. 
of enzyme action in the case of myosin ATPase, then it follows that the adsorption of ATP in the course of the enzymatic act is a mechanism for (algebraically) reducing the local charge on the myosin. To predict the dimensional consequence of ATP adsorption we must introduce the third participant in the reaction-an adsorbable cation, in all probability $\mathrm{Mg}^{2+}$ (vide infra). Quantitative amino acid analyses $(2,43)$ indicate that, except for some possibly very specialized regions of the myosin molecule, its net charge in salt-free neutral solution is negative. Under such conditions the preferential adsorption of ATP should expand or extend the structure. Contraction, on the other hand, should be possible if the deformable regions of myosin are positively charged prior to ATP adsorption. Electrophoretic studies (66) of myosin early indicated that positivity of the protein as a whole (and so presumably of some of its subregions) can be achieved by adsorption of $\mathrm{Mg}^{2+}$; more recent research (vide infra) indicates that at least some magnesium is held by the protein with great tenacity. It has therefore seemed to us reasonable to construct our simplest contractile system by supposing that in such a system, deformable regions of the myosin molecule, made positive and extended through $\mathrm{Mg}^{++}$adsorption, suffer a reduction in charge - hence a mechanical collapsethrough subsequent adsorption of $\mathrm{ATP}^{4-}$.

Although we have mentioned that myosin is an ATPase, we have used the fact only to indicate that ATP must be specifically adsorbable to the myosin. No use has been made of the fact that ATP goes on to be decomposed into adenosinediphosphate (ADP) and orthophosphate (P); indeed in this model the subsequent ATP cleavage is unnecessary to the mechanism of deformation. In experimental models (e.g., dissolved myosin particles), however, the subsequent reaction generally occurs, and ADP and $\mathrm{P}$ accumulate in the solution until virtually all the ATP has been decomposed. In at least some of these situations it can be shown that $\mathrm{ADP}+\mathrm{P}$ are bound to myosin much less than is ATP, and upon exhaustion of ATP the deformation (in these cases an expansion rather than a contraction) of the myosin particles is 
largely reversed. From these observations we judge that whereas the hydrolysis of adsorbed ATP is not what energetically "drives" the deformation, it is necessary for reversing the deformation of this simplest model, and hence for cyclical deformation.

Before testing this theoretical model against experiments or before elaborating it to accommodate other facts, it is well to consider certain theoretical questions. If the model is rigged to do work, it becomes a simple transducer of "chemical" into "mechanical" energy. One may inquire about the maximum efficiency of this transduction or ask, as is often done in physiology, whether chemical energy is supplied for contraction or for relaxation. Thermodynamic analysis (38) of an idealized cyclic isothermal process in which (1) the amount of ATP adsorbed on a loaded myosin fiber is allowed to increase, thereby causing the weight to be lifted; (2) the lifted weight is removed and the bound ATP is (catalytically) decomposed to equilibrium with ADP and P, thereby permitting the fiber to lengthen; (3) the weight is restored on the fiber; and (4) the decomposed ATP is resynthesized, reveals that in steps (1) to (3) work is done at the expense of converting ATP in solution into ADP $+\mathrm{P}$ in solution; ultimately this is "paid for" in the "recovery step" (4). In the efficiency expression the "output" is therefore the net work done in steps (7) to (3), and the "input" is the increment in the number of moles of ATP bound, multiplied by the difference in chemical potential of ATP before and during the binding process. Therefore, although mechanistically the energy transfer step does not involve hydrolysis of ATP, the over-all energetics of the process can nonetheless be calculated from the net changes in the system, i.e., work done, and a certain amount of hydrolysis. The analysis is noncommittal as to "when" (contraction or relaxation) energy is supplied; it merely states that in the cyclic process the system performs work at the expense of its free energy. This result merely confirms what perhaps should be (but sometimes is not) obvious to most of us, viz., that the question of where in the cycle energetic coupling occurs is not a 
proper thermodynamic question. Another instructive by-product is the conclusion that several current molecular models are "thermodynamic homeomorphs." For example, the aforementioned theory of the Huxleys, wherein ATP-binding is said to allow relaxation and ATP removal to permit contraction, is subject to exactly the same analysis except for changes in algebraic sign at obvious places. Likewise the experiments of Pryor (61) on tendons, and the subsequent ones of Laki and Bowen (48) on myosin fibers, wherein adsorption of $\mathrm{I}^{-}$and of $\mathrm{SCN}^{-}$ causes a contraction which can then be abolished by rinsing, are susceptible to the same analysis, the only difference being that in those cases the contracting adsorbate is removed not by chemical conversion into a noncontracting adsorbate, but by dilution.* Foreshadowing a later suggestion, we note that any exergonic reaction which removes ATP (split or unsplit) from the myosin sites, or which cancels the electrostatic effects of bound ATP, can be invoked to construct a contracting-relaxing model of the primary event.

The second theoretical consideration we have in mind has to do not with thermodynamics but with molecular events. We have said above that in our theoretical myosin model the abolition of extensile electrostatic repulsions through ATP adsorption leads, "for certain reasons," to contraction. It has been pointed out that for an idealized molecular chain in a structureless medium one of these reasons is that (configurational) entropy is gained (free energy is lost) on passing isothermally from an extended to a semicurled state. In a more realistic model the factors to be considered are many more, and they cannot, strictly speaking, be separated one from the other, or even resolved into purely energetic or purely entropic contributions. But in a qualitative fashion it is nonetheless valuable to consider what are apt to be first-order effects $(33-35,55)$. To be specific, let us consider one of several theoretical models analyzed by our colleague, Dr. Hill, viz., a cylindrical array

* This was, of course, recognized by Pryor, who developed a thermodynamic treatment appropriate to his experiment. 
of roughly parallel myosin molecular chains, at either end fixed to transversely rigid end plates (this might be crudely thought of as a metameric unit, say $400 \mathrm{~A}$ long, in a muscle filament), and immersed in a solution containing ions, including ATP. Suppose the model to be at its equilibrium* length for a specified tension and chemical potential of ATP, and suppose that the solution concentration of ATP is increased. If the myosin array is initially (+)-charged, it will adsorb ATP and do work because the system as a whole thereby loses free energy. However, this loss is now the net result of several important processes, some already mentioned and others not. There is, by hypothesis, a specific attraction between $\mathrm{ATP}^{4-}$ and any one adsorbing site on the myosin, and since generally there may be more vacant sites than ATP ions which will be adsorbed, the binding process per se has a configurational term. As in the idealized molecular chain the entropy of the individual chains will increase on shortening. Also as before, the electrostatic energy of the system of charges fixed to the chains is altered, both because the average distance between charges is lessened and because the number of net $(+)$-charges is reduced; however, computation of the electrostatic "energy" (now really a free energy) must now take into account the fact that the forces between charges fixed to the myosin chains are attenuated by "atmospheres" of predominantly oppositely charged mobile ions (e.g., $\mathrm{K}^{+}, \mathrm{Cl}^{-}$) which form around each myosin-fixed ion. Recognition that the solvent really consists of molecules which may interact with the material of the chains introduces an entirely new factor. To see this, we may consider what happens when a roughly parallel array of tightly packed "dry" myosin chains is immersed in pure solvent. Clearly, maximization of the entropy is served by intermingling of the solvent molecules with the chain molecules; the resultant swelling would in turn cause linear deformation of the array. This tendency to intermingle may be enhanced or offset, however, depending on the strengths of the

* Here we temporarily assume that adsorbed ATP does not go on to be split. 
relative molecular force interactions: solvent-myosin, solventsolvent, and myosin-myosin. In any event, there is an interplay between length of the array and array-solvent mixingespecially dramatic in passing from dry to immersed array, but of course equally operative on changing the length of an immersed array. From this interplay we can also expect a contribution to the free energy of deformation. Another factor, additional to those encountered in the idealized single molecular chain, is that of $\mathrm{H}$-bonding. There is evidence that $\mathrm{H}$-bonding plays a role in the aggregation of myosin systems (10), and recent theories of regular folding of polypeptide chains have stressed that $\mathrm{H}$-bonding influences the stability* of various chain configurations. More concretely, if one assumes that, as an array of polypeptide chains is unfolded, intra-chain, $\mathrm{H}$-bonds give way to inter-chain bonds, then again any change in the linear dimension of the array is accompanied by a free-energy change due to H-bonding, and this effect too has a purely configurational factor (24), since a given macroscopic stretch can in general be achieved in several microscopically different ways (e.g., undoing any one of many folds along the same molecular chain). Hill's analysis of the foregoing factors has shown that on the basis of realistic values for certain constants of the model (e.g., number of charges per myosin residue, binding constant for ATP, ionic strength of the medium) one predicts mechanical effects of about the magnitude observed in actual experimental models or in muscle. This result attests to the mechanistic plausibility of our model. The analysis has also turned up at least one effect not hitherto realized in elastomeric theory. For certain plausible choices of the constants specifying the (myosin chain)-(solvent) mixing processes, or for certain choices of the energies of intra- and inter-chain $\mathrm{H}$-bonds, the analysis predicts that as the ATP concentration surrounding a free myosin fiber

* Some stability calculations, however, neglect the effect of competitive H-bonding by water molecules or else estimate it very roughly. Nevertheless it is certainly plausible that the H-bonding should have an effect and, as we have said, there is evidence so indicating. 
is increased from zero the fiber will progressively shorten, that at a critical value of the concentration the fiber will shorten discontinuously to a very reduced length, and then that as the concentration is further increased the fiber will again progressively shorten. The discontinuity in the length-concentration curve reflects a phase transition, that is to say, at the critical concentration (and only at this concentration) two separate phases can coexist, and are in equilibrium with one another. In the first case the two phases correspond to two (myosin chain)-(solvent) mixtures of different composition; in the second case, to chains with predominantly intra-chain H-bonding and to chains with predominantly inter-chain H-bonding. The effect of a phase transition is to exaggerate the dimensional changes in the operation of the model, and also to introduce a critical condition (e.g., ATP concentration); this last may be the basis of the "razor-edge" character of muscle contraction.

The foregoing theoretical model of the Engelhardt-WeberSzent-Györgyi experiments is simple enough, and sufficiently understandable in straightforward physicochemical terms to invite diversified experimental test. We shall next consider three general lines of test.

Following Engelhardt's discovery that myosin was an ATPase, the idea that ATP might participate in the primary event of muscle action gained wide acceptance because ATP has long been considered an "energy-rich" compound, i.e., its hydrolysis has been thought to be highly exergonic and exothermic (49). It has not been unreasonable, for example, to suppose that ATP transfers its third phosphate to myosin, and that this phosphorylated myosin is for some reason a contracted structure; indeed much of Webcr's elegant experimentation can, at first sight, be so interpreted $(70,71)$. Such a hypothesis asserts, in essence, that contraction is coupled with hydrolytic cleavage, and since in the absence of enzyme this cleavage is very exergonic, the hypothesis seems further strengthened. By the same token the theory expounded here frequently meets spirited, albeit intuitive, resistance because it implies a major free-energy 
drop temporally prior to, or even without, actual ATP splitting. What seems to us a clarification of this situation has come from three types of research. First the experiments of Bowen $(12,14)$ and others (1) have shown that under various conditions ATP splitting and myosin deformation can be dissociated, thereby disproving any simple coupling between the two processes. Along the same lines, the experiments of Koshland et al. (44) show that, if it exists at all, simple phosphorylated myosin cannot be the contracted form of myosin, for the steady-state concentration of such myosin is negligibly small, whereas the percentage of contracted myosin can exceed $80 \%$ (57). Second, recent investigation (60) has shown that the thermodynamic constants of ATP hydrolysis have probably been hitherto overrated, and that at $20^{\circ} \mathrm{C}$. and $p \mathrm{H} 7,-\Delta F^{\circ}$ is 6 to $8 \mathrm{kcal} . /$ mole (rather than 11 ), and $-\Delta H$ is $5 \mathrm{kcal} . /$ mole (rather than 12). Third, enzyme kinetic methods give for $-\Delta F^{\circ}$ of the reaction

(undeformed myosin) + (nucleotide)

(deformed myosin.nucleotide)

values of the order of 6 to $7 \mathrm{kcal}$./mole when the nucleotide is $\mathrm{ATP}^{4-}$ (59) and, under different conditions, over $5 \mathrm{kcal} . / \mathrm{mole}$ when the nucleotide is $\mathrm{ADP}^{3-}$ (29). The first type of research has upset the idea that ATP splitting per se is coupled with myosin deformation, but it is quite consistent with a coupling between ATP adsorption and deformation; the Koshland result is likewise consistent with the hypothesis that the deformed enzyme holds intact, noncovalently bonded ATP, which in a subsequent step "explodes" into ADP and $\mathrm{P}$ through reaction with water. The second and third types of research suggest that when ATP is, as in our model, hydrolyzed through a route involving an intermediate ATP complex with myosin (this being deformed myosin) the free-energy drop between initial state and complex is a large fraction of the free-energy drop in going from initial to final state, i.e., to hydrolysis equilibrium.*

* It has also seemed to us very significant that the newest values for the $-\Delta F^{\circ}$ of ATP hydrolysis exceed only slightly the difference between the electrostatic free energy of ATP and ADP $(36,37)$. 
This conclusion should allay fears that our model is wasteful of the "energetic wealth" of ATP.

Implicit in the model under consideration is the idea that myosin-more specifically, a myosin network structurebehaves in its deformations like a flexible polyelectrolyte. Amino acid analyses $(2,43)$ have established that myosin is a polyelectrolyte, but whether its mechanical properties reflect this compositional feature is a matter for other investigations. Early experimental confirmation was the result $(9,10,70)$ that for a myosin thread immersed in a solution of low ionic strength $(\partial S / \partial l)_{\mathrm{p}, \mathrm{T}}<0$, and $(\partial E / \partial l)_{\mathrm{p}, \mathrm{T}}<0$, precisely as would be expected from the idealized molecular chain model (vide supra).* The bulk of the remaining evidence consists of observations in which the structural effect of ion-binding on myosin has been observed. Thus variations of parameters such as ionic strength or $p \mathrm{H}$ (i.e., extent of $\mathrm{H}^{+}$binding) have been shown to produce profound structural changes in myosin systems $(5,31)$. Under conditions in which ATP addition would cause contraction, it has been shown by Churney (19) that other anions (e.g., $\mathrm{SO}_{4}{ }^{2-}, \mathrm{Fe}-$ $\left.(\mathrm{CN})_{6}{ }^{3-}, \mathrm{Fe}(\mathrm{CN})_{6}{ }^{4-}\right)$ will also bring about contraction, though at concentrations higher than those of ATP. Similarly, Laki and Bowen (48) have shown that $\mathrm{I}^{-}$and $\mathrm{SCN}$ - produce marked contraction under these circumstances. As might be expected, binding of ATP shows special features, but its effects seem, in general, to be explainable in simple electrostatic terms. Again, Churney (19) has shown that a myosin fiber pretreated with acetic acid contracts upon ATP addition, whereas a fiber pretreated with ammonium hydroxide relaxes upon ATP addition. If one considers that acid treatment induces positive charging of the fiber, and alkali treatment induces negative charging, then the oppositely directed ATP effects are quite comprehensible;

* Here $S$ is entropy, $E$ is internal energy, and $l$ is length. Considering the overenthusiastic application of thermoelastic analysis to nonequilibrium situations, it is wise to state that no ATP was present in these experiments, so that the appropriate thermodynamic expression $(9,10)$ could be expected to apply. 
indeed, the Churney experiments on myosin fibers approach the Katchalsky (42) experiments (on polymethylacrylate threads) in the extent to which they suggest polyelectrolytic behavior. There are other mechanical effects of ATP which seem best explainable in terms of an electrostatic model. Perhaps the most striking of these is that while ATP causes contraction of myosin structures immersed in $0.05 M \mathrm{KCl}$ and $0.001 M \mathrm{MgCl}_{2}$, it causes extension of myosin particles (7) dissolved in $0.60 \mathrm{M} \mathrm{KCl.*}$ The possible significance of this result becomes apparent on noting that the conditions for contraction are thought (65) to favor positive charging of the myosin, whereas under the conditions for extension the myosin is certainly negatively charged; thus again the opposite effects of ATP addition are very simply explainable in elementary terms. Finally it may be added that certain relaxing effects of relatively massive ATP concentrations could conceivably be attributed to "overcharging" initially positive myosin with more than enough ATP anions $(15,63)$; in this case it is possible, however, that interactions of ATP with divalent cations also play a role (57).

Combination of chemical kinetic inferences with mechanical observations provides further support for the model in question. As already mentioned, when certain conditions are met, it is possible to infer from a kinetic analysis the equilibrium constant of the binding-deformation reaction between myosin and an enzymatic substrate $(6,54,59)$. From the present theory it follows that in comparing various substrates the bigger is this equilibrium constant the more effective should be the substrate in deforming myosin. Also, for any one substrate, any "modifier" (11) or other agency which increases the apparent equilibrium constant should also increase the effectiveness of the sub-

* An analogous reversal of mechanical effect can also be shown on one and the same myosin film $(32,58)$. Although the question of whether ATP distends or dissociates myosin particles remains controversial, we feel that the result under well prescribed circumstances is distension. Either process, however, suggests the operation of forces directed in the sense opposite to contraction. 
strate as a deforming agent. Such predictions have been borne out by experiment. Thus in a series of homologous substrates* the equilibrium constants inferred from kinetic analysis are in the order, $K_{\mathrm{ATP}}>K_{\mathrm{UTP}}>K_{\mathrm{ITP}}>K_{\mathrm{TPP}}^{r}(6,26)$, and wherever a corresponding comparison has been carried out on ability to deform a myosin system, just the same order has been observed (57). $\mathrm{Mg}^{2+}$, which is found kinetically either to leave $\mathrm{K}$ unchanged or (more frequently) to increase it, is par excellence an enhancer of deformation; indeed recent experiments $(1,14,69)$ indicate that $\mathrm{Mg}^{++}$(or a substitute such as $\mathrm{Mn}^{++}$) is indispensable for contraction (69). In considering this indispensability of $\mathrm{Mg}^{++}$ it should be recalled that our model assigns to this cation the role of charging positively the sites to which $\mathrm{ATP}^{4-}$ is adsorbed. Finally, there are to be considered the clever but somewhat more complicated experiments of Laidler and his associates $(46,47)$, in which they studied the dependence of $K_{\text {ATP }}$ on bulk dielectric constant, ionic strength, and hydrostatic pressure. The dependence of $K_{\mathrm{ATP}}$ on these "electrical" parameters was in every case strong, confirming in a general way the postulated condensation of charged ATP with charged protein, the release of oriented water, and the consequent increase in volume and entropy of the system. Moreover, the results observed on varying ionic strength were quite consistent with the idea that anionic ATP binds to cationic protein in dilute $\mathrm{KCl}$, and to anionic protein in more concentrated $\mathrm{KCl}$ (compare the results on reversal of ATP deformation cited above). The results observed on varying bulk dielectric constant, however, were unclear, for although the free-energy change of the bindingdeformation process proved to be proportional to inverse dielectric constant, its magnitude increased with decreasing dielectric constant under conditions in which both ATP and protein were anionic.

To this point we have been primarily concerned with attempting to explain the mechanical deformation of $\mathrm{Mg}^{++}$-myosin

* UTP = uridine triphosphate; ITP $=$ inosine triphosphate: TPP $=$ tripolyphosphate. 
on interaction with ATP, in other words, to explain the Engelhardt experiment. The ultimate objective, of course, is beyond this, i.e., it is to explain the mechanical deformation in living muscles. It is to be expected that the situation in muscles will differ from that of the model system in important respects (this is already apparent from recent experimentation); so that one must be prepared to modify or complicate the model correspondingly; however, up to this time the similarities between model and muscles outweigh the apparent differences.

Although we are aware of the fallacies of gauging the eventual importance of recent results, we are nonetheless impressed by the following experimental observations on muscles. (1) Repetitive activity (electrically induced) in muscle fibers does not increase the rate of incorporation of orthophosphate (radioactively labeled) into the ATP of such fibers compared with the rate in resting fibers (8). (2) Under certain well specified conditions unit activity (e.g., single twitches) in muscles is not accompanied by changes in the concentration of ATP or ADP (changes in $\mathrm{P}$ seem to us uncertain), under conditions in which regeneration is very probably ruled out $(22,23,53)$. (3) Microinjection of ATP into muscle fibers does not cause contraction (21). An overly simple conclusion from these findings is that ATP has nothing to do with the action of living muscles, and that the Engelhardt-type of experiments are but confounding coincidences. Such alarm should be tempered, however, by three considerations: first, there exist too many genuine similarities $(70,71)$ between models and muscles; second, even in the model experiments it is reasonable-as we have argued above-for the mechanical deformation (being coupled with the ATP-adsorption process) not to require the hydrolytic cleavage of ATP; and third, there have recently been discovered relations between the models and other substances which may eventually resolve today's "discrepancies."

The new relations to which we have alluded exist between "relaxation factors"* and the ATP-myosin-Mg model systems.

* As we have suggested elsewhere (57), it may be better to term these substances "modifying factors," for in many instances they appear to enhance contraction as well as relaxation. 
These factors are able to inhibit or reverse the mechanical response (e.g., contraction) observed with the model alone, and likewise to inhibit or enhance the ATPase activity of the system.

Five such factors have to date been recognized: myokinase (50), ATP-creatine transphosphorylase + creatine phosphate (28), pyrophosphate (17), "EDTA" or ethylene diamine tetraacetate $(16,69)$, and the arginine transphosphorylase system (68). The third and fourth substances clearly upset what might otherwise seem a good correlation between factor and transphosphorylase activity; moreover, there is seemingly no correlation between conditions promoting factor activity and conditions promoting the usual activity of the transphosphorylases. Two common features which do seem significant, however, are that (as first pointed out to us by Watanabe) these substances all have a strong affinity for $\mathrm{Mg}^{++}$, and that they are all anionic as used. There are also similarities in their behavior. (1) $\mathrm{Mg}^{++}$and $\mathrm{ATP}$ are both required by all factors. (2) In the presence of $\mathrm{Mg}^{++}$and ATP factors are tightly bound to myosin, * most probably in cooperative fashion. (3) $\mathrm{Ca}^{++}$can abolish the action of all factors, and (4) increasing ionic strength and decreasing $p \mathrm{H}$ appear to favor factor action.

Of the five factors listed, at least the myokinase and ATPcreatine transphosphorylase systems exist in mammalian muscles, and the ATP-arginine transphosphorylase system exists in pecten. It is therefore cogent to inquire how the operation of these factors may complicate the experimentally observed behavior of the simple ATP-myosin-Mg system, for we think it is this inquiry which may resolve present discrepancies between models and muscles. Specifically, it seems possible that in

* Students of the relaxation phenomenon reached this conclusion by estimating ease of rinsing factor away. At least in the case of EDTA the same conclusion was reached in another way. The investigations by Friess, Bowen, Kerwin, and one of us $(13,25,27)$ on the activation of dissolved myosin ATPase by EDTA indicate that EDTA exerts its effect by actually binding on the myosin, that this binding occurs only when ATP is present, and that in all probability this binding is mediated by $\mathrm{Mg}$-possibly $\mathrm{Mg}$ which is an integral part of the enzymatic site. 
muscles at rest $\mathrm{ATP}^{4-}$ and anionic factor are both adsorbed to $\mathrm{Mg}$-myosin in such quantitative relations that the anions together "overcharge" negatively appropriate regions of the myosin structure. We may then imagine that excitation makes $\mathrm{Ca}^{2+}$ (or possibly some other metallic cation) available. By any one of several reactions (vide supra, p. 614), e.g., simple electrostatic discharge, spatial removal of ATP, the $\mathrm{Ca}^{2+}$ may temporarily abolish factor, thereby effecting contraction. Subsequent withdrawal of $\mathrm{Ca}^{2+}$ would bring about relaxation. This suggestion sets the energetic problem back one step, i.e., poses the question as to what energy source releases and withdraws $\mathrm{Ca}^{2+}$ from the contractile system. It also leaves it uncertain whether conditions would or would not permit ATP hydrolysis during, say, a very short exposure to $\mathrm{Ca}^{2+}$. At the same time, the suggestion is not without advantage. The ingredients required for a straightforward physicochemical explanation of the operation of in vitro model systems are retained, and with them the fundamental connection between models and muscle. The new ingredients added - "factor" and $\mathrm{Ca}^{2+}$ - are added because experiment has already shown how important they are to the operation of the simple systems. Yet this simple elaboration suggests an explanation of the three in vivo observations cited early in this section, for this more elaborate system (1) could operate in the absence of ATP hydrolysis, and (2) would be insensitive to ATP (inasmuch as ATP would already be there). Curiously enough, such a system would also behave in keeping with two further observations: First (72), microinjection of $\mathrm{Mg}^{2+}$ into a living fiber does not cause contraction $\left(\mathrm{Mg}^{2+}\right.$ is already there and bound), but microinjection of $\mathrm{Ca}^{2+}$ does cause contraction ( $\mathrm{Ca}^{2+}$ abolishes factor action). Second, in a fatiguing fiber (62) it is relaxation that progressively slows (rate of refilling sites falls as ATP concentration falls), while contraction continues unaffected (so long as only $\mathrm{Ca}^{2+}$ needs to be presented).

In closing an essay which, perhaps prematurely, has sought to explain a physiological phenomenon, the authors are moved to philosophical comment. An "explanation" is certainly more 
than a recitation of incontestable experimental observations. On the other hand, in our day, an "explanation" also has to be less than metaphysical, i.e., it must restrict itself to words and concepts which can be found in good textbooks of mathematics, physics, and chemistry. Within these two constraints the diligent invent $a$ theory, and the genius invents the theory; that is why this essay is entitled as it is.

\section{References}

1. Ashley, C. A., A. F. Schick, A. Arasimavicius, and G. M. Hass, "Isolation and characterization of mammalian striated myofibrils," in C. Ragan, ed., Connective Tissues, Transactions of the Fourth Conference; Josiah Macy Jr. Foundation, New York, 1953.

2. Bailey, K., Biochem. J. (London), 43, 271 (1948).

3. Bailey, K., "Structure proteins. II. Muscle," in H. Neurath, and K. Bailey, eds., The Proteins, Vol. 2. Academic Press, New York, 1954.

4. Bendall, J. R., Nature, 170, 1058 (1952).

5. Blum, J. J., Arch. Biochem. and Biophys., 43, 176 (1953).

6. Blum, J. J., Arch. Biochem. and Biophys., 55, 486 (1955).

7. Blum, J. J., and M. F. Morales, Arch. Biochem. and Biophys., 43, 208 (1953).

8. Bollman, J. L., and E. V. Flock. J. Biol. Chem., 152, 371 (1944).

9. Botts, J., F. Johnson, and M. Morales, J. Cellular Comp. Physiol., 37, 247 (1951).

10. Botts, J., and M. Morales, J. Cellular Comp. Physiol., 37, 27 (1951).

11. Botts, J., and M. Morales, Trans. Faraday Soc., 49, 696 (1953).

12. Bowen, W. J., Am. J. Physiol., 169, 218 (1952).

13. Bowen, W. J., and T. D. Kerwin, J. Biol. Chem., 211, 237 (1954).

14. Bowen, W. J., and T. D. Kerwin, Biochim. et Biophys. Acta, 18, 83 (1955).

15. Bozler, E.. Federation Proc., 10, 19 (1951).

16. Bozler, E., J. Gen. Physiol., 38, 149 (1954).

17. Bozler, E., J. Gen. Physiol., 38, 53 (1954).

18. Bull, H., Quart. Bull. Northwestern Univ. Med. School, 20, 175 (1946).

19. Churney, L., Federation Proc., 13, 26 (1954).

20. Engelhardt, W. A., and M. N. Ljubimova, Nature, 744, 669 (1939).

21. Falk, G., and R. W. Gerard, J. Cellular Comp. Physiol., 43, 393 (1954).

22. Fleckenstein, A., J. Janke, R. E. Davies, and H. A. Krebs, Nature, 174, 1081 (1954).

23. Fleckenstein, A., and J. Janke, Pfügers Arch. ges. Physiol., 258, 177 (1953).

24. Frenkel, J., Kinetic Theory of Liquids, Ch. 8. Oxford University Press, London, 1946. 
25. Friess, E. T., Arch. Biochem. and Biophys., 51, 17 (1954).

26. Friess, E. T., and M. Morales, Arch. Biochem. and Biophys., 56, 326 (1955).

27. Friess, E. T., M. F. Morales, and W. J. Bowen, Arch. Biochem. and Biophys., 53, 311 (1954).

28. Goodall, M. G., and A. G. Szent-Györgyi, Nature, 172, 84 (1953).

29. Green, J., and W. F. H. M. Mommaerts, J. Biol. Chem., 210, 695 (1954).

30. Guth, E., "Muscular contraction and rubberlike elasticity," in Muscular Contraction, Annals N. Y. Acad. Sci., 47, Art. 6, 1947.

31. Harkness, M. L. R., and A. Wasserman, J. Chem. Soc., 1954, 1344.

32. Hayashi, T., and R. Rosenbluth, Proc. Natl. Acad. Sci. U.S., 39, 1285 (1953).

33. Hill, T. L., J. Chem. Phys., 20, 1259 (1952).

34. Hill, T. L., Record Chem. Progr. (Kresge-Hooker Sci. Lib.), 13, 101 (1952).

35. Hill, T. L., "Energetics and molecular mechanisms in muscle action, Part II," in The Physical Chemistry of Proteins. Discussions Faraday Soc., 13, 132 (1953).

36. Hill, T. L., Arch. Biochem. and Biophys., 57, 229 (1955).

37. Hill, T. L., and M. F. Morales, J. Am. Chem. Suc., 73, 1656 (1951).

38. Hill, T., and M. F. Morales, Arch. Biochem. and Biophys., 37, 425 (1952).

39. Huxley, A. F., and R. Niedergerke, Nature, 173, 971 (1954).

40. Huxley, H., and J. Hanson, Nature, 773, 979 (1954).

41. Kafiani, K. A., and V. A. Engelhardt, Doklady Akad. Nauk SSSR, 92, 385 (1953).

42. Katchalsky, A., O. Künzle, and W. Kuhn, J. Polymer Sci., 5, 283 (1950).

43. Kominz, D. R., H. Hough, P. Symonds, and K. Laki, Arch. Biochem. and Biophys., 50, 148 (1954).

44. Koshland, D. E., Jr., Z. Budenstein, and A. Kowalsky, J. Biol. Chem., 217, 279 (1954).

45. Kuhn, W., and B. Hargitay, Z. Elektrochem., 55, 490 (1951).

46. Laidler, K. J., and A. J. Beardell, Arch. Biochem. and Biophys., 55, 138 (1955).

47. Laidler, K. J., and M. C. Ethier, Arch. Biochem. and Biophys., 44, 338 (1953).

48. Laki, K., and W. J. Bowen, Biochim. et Biophys. Acta, 16, 301 (1955).

49. Lipmann, F., "Metabolic generation and utilization of phosphate bond energy." Advances in Enzymol., 1, 99 (1941).

50. Marsh, B. B., Biochim. et Biophys. Acta, 9, 247 (1952).

51. Meyer, K. H., Biochem. Z., 214, 253 (1929).

52. Mommaerts, W. F. H. M., Biochim. et Biophys. Acta, 7, 477 (1951).

53. Mommaerts, W. F. H. M., Nature, 174, 1083 (1954).

54. Morales, M. F., J. Am. Chem. Soc., 77, 4169 (1955).

55. Morales, M., and J. Botts, Arch. Biochem. and Biophys., 37, 283 (1952). 
56. Morales, M., and J. Botts, "Energetics and molecular mechanisms in muscle action, Part I, Outline of a theory of muscle action and some of its experimental basis," Discussions Faraday Society, 13, 125 (1953).

57. Morales, M., J. Botts, J. J. Blum, and T. L. Hill, Physiol. Revs., 35, 475 (1955).

58. Munch-Petersen, A., Nature, 162, 537 (1948).

59. Oucllet, L., K. J. Laidler, and M. F. Morales, Arch. Biochem. and Biophys., 39, 37 (1952).

60. Podolsky, R. J., and M. F. Morales, J. Biol. Chem., 218, 945 (1956).

61. Pryor, M. G. M., "Mechanical properties of fibres and muscles." Progr. Biophys. and Biophys. Chem., 7, 216 (1950).

62. Ramsey, R. W., and S. F. Street, Federation Proc., 1, 70 (1942).

63. Ranney, R. E., Am. J. Physiol., in press.

64. Riseman, J., and J. Kirkwood, J. Am. Chem. Soc., 70, 2820 (1948).

65. Sarkhar, N. K., Enzymologia, 7., 237 (1950).

66. Snellman, O., and T. Erdos, Biochim. et Biophys. Acta, 2, 650 (1948).

67. Straub, F. B., and G. Feuer, Biochim. et Biophys. Acta, 4, 445 (1950).

68. Tonomura, Y., K. Yagi, and H. Matsumiya, Arch. Biochem. and Biophys., 59, 76 (1955).

69. Watanabe, S., Arch. Biochem. and Biophys., 54, 559 (1955).

70. Weber, H. H., and H. Portzehl, "Muscle contraction and fibrous muscle proteins." Advances in Protein Chem., 7, 161 (1952).

71. Weber, H. H., and H. Portzehl, "The transference of the muscle energy in the contraction cycle." Progr. Biophys. and Biophys. Chem., 4, 60 (1954).

72. Wiercinski, F. J., Federation Proc., 17, 172 (1952).

73. Wöhlisch, E., Naturwissenschaften, 28, 305 (1940). 


\title{
TRENDS IN THE BIOCHEMISTRY OF NERVE ACTIVITY*
}

\author{
DAVID NACHMANSOHN and IRWIN B. WILSON, Department \\ of Neurology, College of Physicians and Surgeons, \\ Columbia Unizersity, New York
}

\section{Developments in the Biochemical Approach to Cellular Function}

At the turn of this century virtually nothing was known about the chemical reactions associated with cellular function. Information about cells was limited to knowledge of anatomical structure, a number of chemical constituents, and those aspects of function which could be studied and recorded by physical methods. Enzyme chemistry was just in its beginning. The development of chemistry at an ever-increasing rate changed the situation. The progress of organic, physical, and protein chemistry, especially the spectacular advance of enzyme chemistry along with the elaboration of micromethods and isotope techniques, the introduction of physical methods for the study of proteins such as ultracentrifugation and electrophoresis, and various other advances form the basis of what is referred to today as dynamic biochemistry. Discussions of these developments

* The investigations of this Laboratory, upon which a large part of this Discussion is based, have been supported in part by the Medical Research and Development Board, Department of the Army, Office of the Surgeon General, Contract No. DA-49-007-MD-37, and in part by the Division of Research Grants and Fellowships of the National Institutes of Health, Grants No. B-573 and B-400, United States Public Health Service, and by the Atomic Energy Commission. Contract No. AT (30-1)-1503. 
may be found in the classical addresses of Hopkins collected in the Commemoration Volume presented at the First International Congress of Biochemistry (26).

These developments combined with the recognition that molecular events must underlie all displays of biological function, so strongly emphasized by Liebig, have considerably promoted knowledge of the chemical reactions associated with cellular function. If de Broglie (7) speaks of "the revolution in physics" in referring to the progress since the beginning of this century, one may well say that biology underwent a series of revolutions. Research of the living cell changed from a more or less descriptive science to one in which mechanisms are being studied in terms of physics and chemistry, and explanations are being sought at molecular levels. The classical example in this respect is the function of striated muscle. Hopkins, Parnas, Hill and Meyerhof, Embden, Lundsgaard, H. H. Weber, C. F. and G. T. Cori, von Muralt, are just a few of the leading investigators whose names are associated with this field.

A special role in this development must be attributed to Otto Meyerhof (24). Owing to the wide range of his knowledge and interest, his philosophical approach and the depth of his mind, his imagination and originality, he has not only contributed many fundamental facts, but has revolutionized the thinking of this generation of biochemists, as was recognized at the special session of the Third International Congress of Biochemistry dedicated to his memory (6). Among the methods and principles which he so strongly emphasized may be mentioned (1) the importance of thermodynamics in the study of intermediary metabolism; (2) the cyclic character of cellular reactions; (3) the paramount importance of correlating chemical and physical events, i.e., metabolism and function; and (4) the necessity of establishing the sequence of energy transformations for this aim. Today these concepts have become a guide for biochemists and form the basis of any approach to the study of cellular function.

When, in 1937, the Perspectives in Biochemistry (27) appeared, a book which was dedicated to Hopkins' 75 th birthday and may 
be considered to be the precursor of the Currents in Biochemical Research (11), the biochemistry of the intermediary metabolism associated with muscular contraction was far advanced. Most of the enzymatic steps of the glycolytic cycle had been established, with the exception of the so-called "coupling reaction," the mechanism of which was elucidated in 1939 by Otto Warburg (31). The energy released by the hydrolysis of ATP was assumed to be the primary source of energy for the contractile process. Although the study of the pathways of intermediary metabolism was in the forefront of that period, in addition quite a few correlations had been established between chemical reactions and events recorded on living muscle fibers by a variety of physical methods.

At that period, studies of structural muscle proteins, carried out mainly in the laboratories of H. H. Weber and Edsall, were still at an early stage. During the following decade, however, greatly stimulated by the observations of Engelhardt and Lyubimova (8), the investigation of the structural proteins and their interaction with ATP, i.e., the elementary processes of muscular contraction, moved into the center of interest of muscle physiologists. The properties of these proteins and the molecular forces affecting the contractile process and their interdependence with structure is today the most active field in muscle research (32).

Although muscular activity has been the most thoroughly studied problem and offers the most dramatic example of the progress in our understanding of the chemistry of cellular function, advance on similar lines was achieved in respect to other functions, such as vision, renal secretion, and bioluminescence. In this paper some trends of the biochemistry of nerve function will be discussed, but it is obvious that they reflect similar trends in various other fields.

\section{Biochemistry of Nerve Activity}

In spite of all progress achieved in the biochemistry of muscular activity at the time of the appearance of the Perspectives, 
virtually nothing was known about chemical reactions associated with nerve activity, as was stated by Feng in his revicw on heat production in nerve (9); this was in striking contrast to all the great advances in this field obtained with physical methods, especially electrical recordings. During the decade between the appearance of the Perspectives and the first volume of the Currents in Biochemical Research essential progress had been achieved (17). Investigations were carried out following lines of approach similar to those which had been applied to the biochemistry of muscle.

\section{SEQUENGE OF ENERGY TRANSFORMATIONS}

These studies had revealed that the action of acetylcholine is associated with the elementary process of conduction, i.e., acetylcholine is necessary for generating the bioelectric potentials which propagate the impulse in conducting tissues. The sequence of energy transformations was established, the enzyme systems associated with the formation and hydrolysis of acetylcholine were studied, and acetylcholine was integrated into the metabolic pathways of the nerve cell. A variety of chemical and electrical processes were correlated. The use of electric tissue, since 1937, was helpful in overcoming many difficulties. Electric organs are the most powerful bioelectric generators known in nature and the choice of this material proved decisive in many respects, especially, however, in determining the sequence of energy transformations. These organs contain acetylcholinesterase in amazingly high concentrations: $100 \mathrm{~g}$. of organ (fresh weight) may hydrolyze 200 to $400 \mathrm{~g}$. of acetylcholine per hour, although the tissue is poor in protein ( 2 per cent) and its water content is very high (92 per cent). Since the electric organs are highly specilized as to their function, the remarkable concentration of acetylcholinesterase appeared suggestive as to a direct relationship with the process by which bioelectric currents are generated. A direct proportionality exists indeed between voltage per centimeter and acetylcholinesterase concentration. 
This and various other observations seemed to link acetylcholine action with the electric discharge.

Further investigations revealed that the energy released by phosphocreatine breakdown is adequate to account for the total electric energy released by the action potential. It appeared safe to assume that the breakdown of phosphocreatine would not be associated directly with the generation of bioelectric potentials, but would be used as in muscle for immediaterephosphorylation of ADP resulting from the breakdown of ATP. But in contrast to the situation in muscle, where ATP is assumed to be the primary source of energy for the contractile process, it appeared for many reasons unlikely that ATP hydrolysis would provide the energy for the elementary process in conduction. It appeared more likely that acetylcholine hydrolysis would precede that of ATP and that ATP would be used in the recovery process as energy source for the synthesis of acetylcholine. It has indeed been demonstrated, in 1943, that ATP hydrolysis provides the energy for the acetylation of choline (23). This result was of interest for biochemistry in general. It was the first demonstration of the use of ATP energy outside the glycolytic cycle and the first enzymatic acetylation achieved in a soluble system. The observations opened the way for a study of the mechanism of acetylation in general, which played such a role in recent developments. They, moreover, indicated that acetylcholine hydrolysis precedes that of ATP; they made it possible, in combination with many other facts, to attribute to acetylcholine an essential function in the primary events of conduction. A more detailed account of these investigations on the chemical reactions underlying nerve activity may be found in recent summaries $(18-22,50)$. Before discussing more recent trends, the picture which has emerged as to the role of acetylcholine in the elementary process of conduction may be briefly outlined.

ROLE OF ACETYLCHOLINE IN THE ELEMENTARY PROCESS OF CONDUCTION

Nerve impulses are propagated along nerve and muscle fibers by small electric currents. The mechanism of the genera- 
tion of electric currents is generally assumed to be as follows: ions are the carriers of electrical currents in living cells. As is today well established, sodium ions are present at a much higher concentration on the outside of nerve and muscle fibers than inside the cell, and the opposite is true for potassium ions. These concentration gradients are the source of the action potentials and their resulting currents which propagate and are yet part of the impulse. During activity there is an influx of sodium ions $(28,29)$. Apparently owing to a sudden increase in permeability, these ions enter the fibers during the rising phase of the action potential $(12,14)$. The influx of sodium ions is followed by an equivalent outflow of potassium ions coinciding essentially with the falling phase.

Naturally, the question arises, by what mechanism do the ionic concentration gradients, the potential source of EMF, suddenly become effective? What alterations occur in the membrane rendering it more pervious to sodium ions? Knowledge of this process is fundamental for the understanding of the mechanism of generation of the bioelectric currents, that is of conduction. As was postulated by Kurt H. Meyer in 1937, the increased ion permeability must be preceded by a chemical reaction (16). However, the difficulty of identifying this reaction is considerable. The increase in permeability must occur at an extremely high speed, within microseconds, and the barrier must be re-established within a period of time of the order of a millisecond, so that the next impulse may pass again. The studies mentioned above indicate that the acetylcholine system is essential for the nerve impulse and suggest that it is responsible for the alterations of conducting membranes which produce the increased ion permeability. The tentative picture which has emerged as to the role of acetylcholine may be briefly outlined (Figure 1).

Acetylcholine is, in the rcsting cell, present in an inactive bound form which may be tentatively called the storage form. It appears likely that the ester is bound to a protein or a lipopro- 
tein, but no information is at present available about the nature of the storage form. During activity, as is well established experimentally, acetylcholine is released from the bound form. The free acetylcholine acts upon a receptor, the acetylcholine receptor, and this action is responsible for the change of perme-

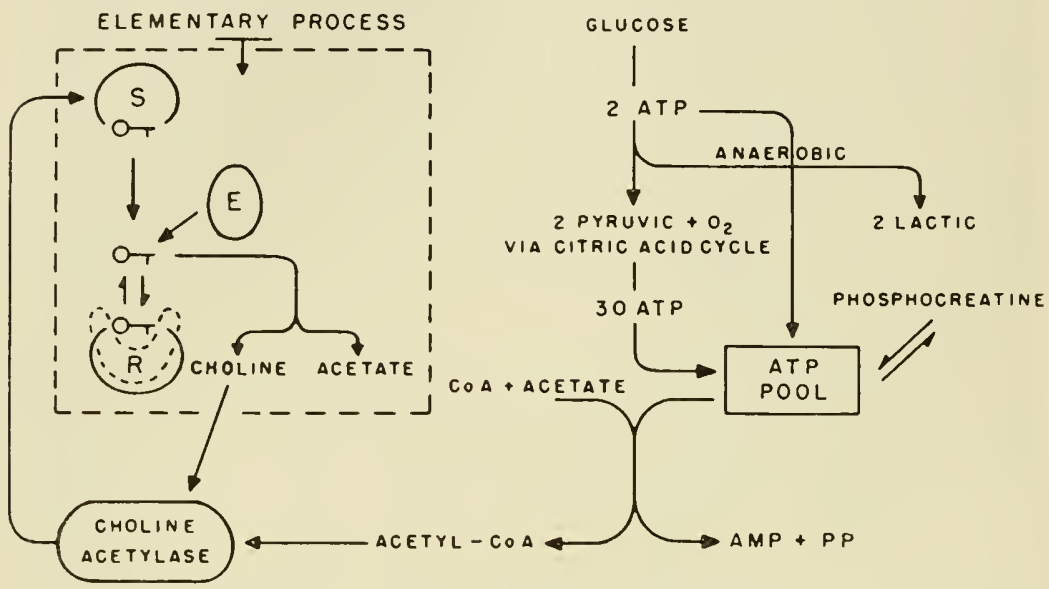

Fig. 1. Sequence of energy transformations associated with conduction, and integration of the acetylcholine system into the metabolic pathways of the nerve cell. The elementary process of conduction may be tentatively pictured as follows:

(7) In resting condition acetylcholine $\left(\mathrm{O}_{-\mathrm{T}}\right)$ is bound, presumably to a storage protein (S). The membrane is polarized.

(2) ACh is released by current flow (possibly hydrogen ion movements) or any other excitatory agent. The free ester combines with the receptor (R), presumably a protein.

(3) The receptor changes its configuration (dotted line). This process increases the $\mathrm{Na}$ ion permeability and permits its rapid influx. This is the trigger action by which the potential primary source of EMF, the ionic concentration gradient, becomes effective and by which the action current is generated.

(4) The ester-receptor complex is in dynamic equilibrium with the free ester and the receptor; the free ester is open to attack by acetylcholinesterase (E).

(5) The hydrolysis of the ester permits the receptor to return to its original shape. The permeability decreases, and the membrane is again in its original polarized condition. 
ability and thus the generation of the electric potential. Although the acetylcholine receptor has not yet been isolated, the most likely assumption appears to be that it is a protein. Some data to be discussed later suggest that by its reaction with acetylcholine the configuration of the protein is changed.

The complex between acetylcholine and its receptor is in dynamic equilibrium with the free ester and the receptor. The free ester is susceptible to attack by the esterase and its hydrolysis permits the receptor to return to its resting condition. The barrier for the rapid ion movements is thus reestablished. This action of the enzyme leads to immediate recovery and ends the cycle of the elementary process. The rapidity of the restoration process is made possible by the high speed of the enzymatic hydrolysis, which may occur in microseconds. Thus the nerve may respond to the next stimulus within a few milliseconds. The further recovery leads to the resynthesis of acetylcholine in its bound form and to the restoration of the concentration gradients.

A sharp distinction must be made between the activity and recovery phases. The flux of sodium and of potassium during activity is in the direction of the concentration gradients. The suddenly increased rate of flow for the extremely short period of time requires very little energy to effect the transient change of permeability. The change in permeability is only a trigger process making the potential energy effective. This view is supported by the extremely small amount of heat released during nerve activity, which is, at most, of the order of magnitude of $10^{-11}$ of one small calorie per square centimeter per impulse. Fundamentally different is the situation in respect to the ion movements during recovery. The restoration of the original steady state requires extrusion of sodium and uptake of potassium against the concentration gradient. These latter processes require a relatively large amount of energy in excess of that necessary for maintaining the resting condition. Most of the heat produced during nerve activity is developed after the electrical changes and is presumably associated with the restoration of the 
electrolyte distribution. The energy for this "active" ion transport must be assumed to be provided by the chemical energy derived from reactions common to most cells.

\section{The Proteins of the Acetylcholine System}

Once the integration of acetylcholine into the metabolic pathways of the neuron had been accomplished, by 1950, the mechanism of the elementary process and the interaction of acetylcholine with the specific proteins of the system became of primary interest. To a certain extent the situation resembled that in muscle physiology, when biochemical studies in the late thirties had resulted in the recognition that ATP is the primary energy source in the elementary process of contraction and the properties of myosin and actin and their reactions with ATP began to dominate muscle research. In the case of nerve the situation is somewhat different: of the four proteins reacting with acetylcholine directly, only the two enzymes, the esterase and the acetylase, are available for studies in vitro. In contrast to the situation in muscle physiology the receptor is not available in solution; its reactions may be tested in intact structures only. Nothing is known about the storage protein.

ACTIVE SURFACE OF ACETYLCHOLINESTERASE;

THE HYDROLYTIC PROCESS

The most suitable protein for studying the molecular forces responsible for interaction with acetylcholine is the esterase. This is due to its stability and great reactivity with a large variety of compounds. However, information obtained with this enzyme might be of considerable value in understanding the reactions with the other proteins of the system. A molecule such as acetylcholine has only a limited number of features which may contribute to its interaction with proteins. All proteins of the system reacting specifically with the ester must do so through the same elementary interactions. We may, therefore, assume that they are similarly constituted at the active site. Small differences might lead to a considerable alteration of function. 
Extensive investigations of the molecular forces involved in the interaction of acetylcholine with the esterase were carried out during the last few years (Wilson (38)). The main results may be only very briefly summarized: The active surface of the enzyme protein contains a negatively charged site, the "anionic site," which reacts by Coulombic forces with the positive electric charge of acctylcholine and contributes hereby to the attraction, fixation, and orientation of the substrate upon the enzyme surface. The existence of such a negative site has been demonstrated in various ways with suitable substrates as well as competitive inhibitors (42). In addition to the Coulombic forces,

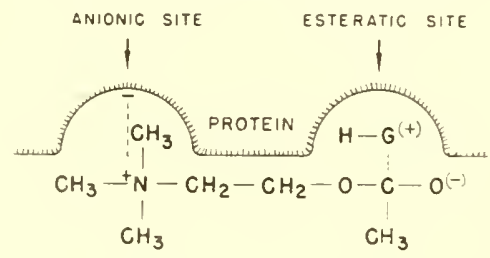

Fig. 2. Schematic presentation of interaction between the active groups of acetylcholinesterase and its substrate.

the methyl or alkyl groups of the nitrogen contribute to the binding by van der Waals forces. This has been demonstrated by the use of methylated competitive inhibitors of the ammonium and hydroxyethyl ammonium series (Wilson (36)). Besides the anionic site attracting the cationic nitrogen there is also a group in the enzyme surface which reacts with the ester group and has been referred to as the "esteratic site." The carbonyl group has a marked polar character. The importance of the electrophilic character of the carbon has been demonstrated experimentally (2). The observations suggest that a covalent bond is formed between the carbon and some basic group in the enzyme. A further clue was obtained from observations on the $p H$ dependence of the catalysis. Since the constitution of acetylcholine does not vary with $p H$, changes in enzyme activity must be attributed to changes in the protein. The changes were interpreted in terms of the dissociation of acidic and basic groups 
(43). Analysis of the postulated equilibria and relations which were borne out by experiment led Wilson to the calculation of the $p \mathrm{~K}$ 's of the basic and acidic groups in the esteratic site of the enzyme surface. Figure 2 represents a scheme, illustrating the interaction between acetylcholinesterase and its substrate.

On the basis of these studies Wilson proposed the following mechanism of hydrolysis:

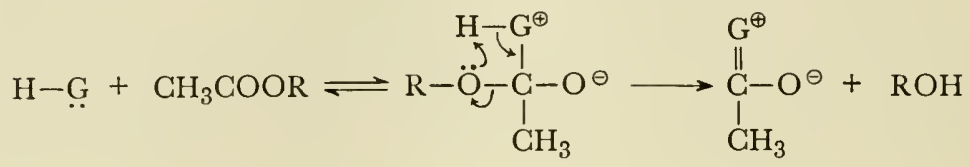

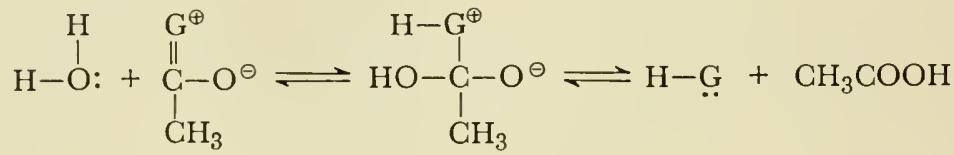

(C)

Here $\mathrm{H}-\mathrm{G}$ represents the esteratic site of the enzyme bearing an acidic group (H) and a basic group (..). Intermediate (B) is a resonance form of acetyl-enzyme. (A) and (C) are MichaelisMenten complexes. The proposed mechanism has been verified in a variety of ways $(33,34,44)$.

In the last few years, the mechanism of enzymatic action and the analysis of molecular forces acting in this process, as was outlined here for acetylcholinesterase, has increasingly preoccupied biochemists, supplementing the study of metabolic pathways. This development parallels in a certain way the studies of structural proteins such as actin and myosin. The results of these investigations are beginning to contribute to our understanding of cellular function on a molecular level, although, as will be discussed below, there are still many gaps between the in vitro studies and the phenomena observed in intact cells; the greatest obstacle is undoubtedly the lack of knowledge of ultrastructure. In the following, two developments may be 
briefly outlined as illustrating how the studies of the molecular forces in the interaction between acetylcholinesterase and its substrate have advanced and stimulated the understanding of the pharmacology and physiology of the nerve cell.

\section{MECHANISM OF NERVE GAS ACTION}

Certain phosphate esters, such as tetraalkyl pyrophosphates, dialkyl p-nitrophenyl phosphates, and dialkyl fluorophosphates, are potent irreversible inhibitors of acetylcholinesterase (and esterases in general). These compounds are of general interest because the most potent chemical warfare gases and some powerful insecticides belong to this class and owe their lethal action to their inactivation of cholinesterase (25).

During the studies of the molecular forces of interaction between acetylcholinesterase and its substrate and the hydrolytic mechanism, the mechanism of nerve gas inhibition became evident $(35,37,42)$. Inhibition by these substances is not removed by dialysis nor by dilution, i.e., it is, according to the customary criteria, irreversible. The structure of these compounds and the fact that the highest inhibition is obtained at those $p H$ 's in which the enzyme is most active, indicated that these compounds enter the hydrolytic mechanism and phosphorylate the same basic group in the esteratic site which-in the physiological processis acetylated. Thus

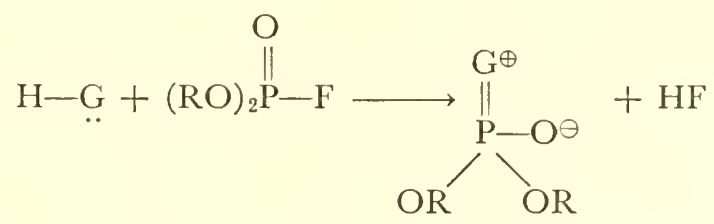

The dialkylphosphoryl enzyme, in contrast to the acetyl enzyme does not readily react with water to regenerate the free and active enzyme, and thus these substances are inhibitors rather than substrates. The reaction with water is, however, rapid enough to be readily demonstrated in a number of cases. Diethylphosphoryl enzyme obtained either by tetraethylpyrophosphate 
or diethylfluorophosphate inhibition hydrolyzes in a few days, as evidenced by the return of enzyme activity (35).

Since it was apparent that reactivation would require the dephosphorylation of the inhibited enzyme, reactivating agents were sought amongst nucleophilic reagents and a large number of compounds such as hydroxylamine, pyridine, imidazole, esters and amides of amino acids, amidines, guanidines, oximes, hydroxamic acids, and choline were found to be active in periods of time varying from one minute to several hours $(35,37,39,47-49)$.

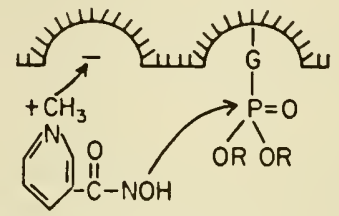

Fig. 3. Promotion of reactivation of alkylphosphate inhibited enzyme by anionic site. Attack of the reactivator (nicotinohydroxamic acid methiodide) upon phosphoryl group is aided by Coulombic attraction.

Since an alcohol group could hardly be considered a strong nucleophilic function, it appeared that choline was active largely because its intrinsic activity could be promoted by interaction of the quaternary ammonium structure with the anionic site. The anionic site, it might be noted, is only indirectly affected by the inhibition. It has been possible in many instances such as illustrated by nicotinohydroxamic acid and its methiodide to increase the activity of a reactivator by introducing a cationic center in the molecule (Figure 3), and in all cases of reactivators containing a cationic center it has been possible to demonstrate a marked promotion by the anionic site (Wilson (40)). What is the nature of this promotion? There is clearly to be expected a general enhancement resulting from the Coulombic attraction of oppositely charged reactants, but is there evidence for a more specific type of interaction? The fact that dimethylaminoethanol ammonium ion is very much poorer than choline would 
appear to indicate that the interaction of choline with diethylphosphoryl enzyme is of the latter type. There is another peculiarity associated with choline reactivation. The reactivation of the inhibited enzyme requires the restoration of a proton. In the case of pyridine reactivation there is no acidic group and the proton must be restored by the solvent, thus:
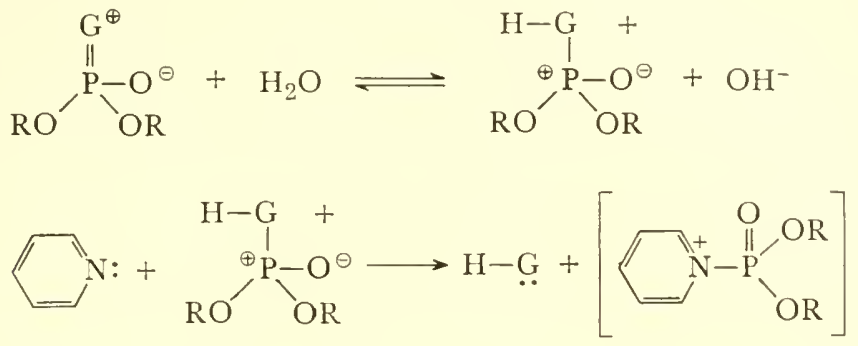

As illustrated above the reaction is acid-catalyzed, and it is in fact found that in general for these reactivators, the rate of reactivation decreases above $p \mathrm{H} 7$. In the case of choline, however, the rate does not decline as the $p \mathrm{H}$ is raised, and it would appear that the alcohol function, which is considerably more acidic than in simple alcohols, makes a combined acid-base attack. Is there a type of specific interaction with the anionic site which promotes the reactivation by facilitating a combined acid-base attack at the phosphorylated esteratic site?

These studies have not only given a satisfactory explanation of the mechanism of nerve gas action, which has puzzled biochemists for more than a decade. They have led also to the development of an antidote (2-pyridine aldoxime methiodide) which has been shown to be effective in vivo (14a,47a); whereas previous antidotes, such as atropin, were essentially based on relief of symptoms, the new type of antidote is capable of repairing the chemical lesion produced by the toxic agent. Finally, a particularly remarkable aspect from the point of view of theory is the demonstration that molecular forces in the protein, once established, may be put to work for purposes other than those for which the protein functions in the biological process. 


\section{REACTION OF TERTIARY AND QUATERNARY NITROGEN}

DERIVATIVES WITH THE ACETYLGHOLINE RECEPTOR

Another development which illustrates the value of the study of molecular forces for a better understanding of cellular function is the remarkable difference between the reaction of the proteins of the acetylcholine system to tertiary and that to quaternary nitrogen derivatives.

In the esterase studies it was in general found that there was not a very pronounced difference in the binding of tertiary and quaternary ammonium ions, but that in the case of substrates and nerve gas reactivators there was a much greater promotion by the anionic site. In other words, the extra methyl group seems to increase greatly the functional activity. Owing to a favorable entropy of activation the formation of acetyl enzyme proceeds about 10 times faster with acetylcholine as substrate than with the tertiary ester (45). The same increase in functional activity is observed with choline acetylase: choline is acetylated about 12 times as fast as dimethylethanolamine (3).

The interaction of cell receptors (probably proteins) with applied substances, receptor activators, and receptor inhibitors, might be expected to obey the law of mass action $(5,15,41)$. Having only a limited number of interaction features, acetylcholine must interact with the receptor in much the same way as with the enzyme and we may, therefore, infer a marked similarity in the action sites of both proteins. The hydrolytic process will, of course, be absent. In its place will be the receptor function. Therefore, much that has been learned about the binding forces and such features of specific interaction as "promotion" may well find their counterpart with the receptor. Receptor inhibitors would be expected to be bound, but the inhibitor lacks those special features which are necesary to bring about the process whereby the receptor functions. As in the case of enzymes, where poorer substrates serve as inhibitors, poorer receptor activators under suitable circumstances may be receptor inhibitors. We are thus in a position to apply 
the (qualitative) consequences of enzyme theory to receptors. We cannot apply the same quantitative techniques because except for actomyosin no receptor has ever been isolated and work must be done for the most part with living cells. Thus we are faced with the problem of permeability. Even if the question of relative permeability were not harassing, we do not at this time know the functional relationship between the activity and the number of receptor-activator complexes (some authors have tacitly assumed a direct proportionality).

In regard to the promotion of activity, the receptor function might be expected, by analogy, to be much more pronounced for acetylcholine as compared to the ammonium ions of dimethylaminoethyl acetate or, since hydrolysis does not appear to be in volved here, to be more pronounced for quaternary as compared to tertiary cations, in general. As is well known, this is in fact the case-suitable quaternary ions depolarize cell membranes whereas tertiary ions do not. Less known is that tertiary ions are receptor inhibitors. They are bound but lack the promotional feature necessary to bring about receptor activity. In work with the electroplax, i.e., the electric cells of electric organs, it was shown that a number of quaternary and tertiary cations block the generation of electrical potential by inhibiting the acetylcholine receptor rather than acetylcholinesterase $(1,30)$. This was the first experimental distinction between acetylcholinesterase and the long suspected receptor. The quaternary ions, by reacting with the receptor, blocked the action potential and also depolarized the cell membrane; the tertiary ions blocked without depolarization. But the tertiary ions antagonized the depolarizing effect of the quaternary ions. For example, carbamylcholine blocks the action potential with depolarization of the membrane, but procaine blocks without depolarization. If a cell is blocked with procaine, subsequent adclition of the same quantity of carbamycholine fails to produce a depolarization. Larger quantities produce depolarization. Similarly, if a cell is blocked with carbamylcholine, the addition of larger quantities of procaine will restore the membrane potential. 
The ammonium ion of dimethylaminoethyl acetate, although somewhat unusual for a tertiary ion in that it causes depolarization, does so with very much less vigor than acetylcholine. How does the "extra" methyl group of acetylcholine lead to such a marked promotion? A clue to this action is to be found in the tetrahedral configuration of these ions. If the fourth group is not to merely project into the surrounding medium, in which case we should be at a loss to explain its activity, there must occur a folding of the protein structure whereby this fourth group is brought into the close contact with the protein necessary for the operation of short-range molecular forces. Such a change in receptor configuration could well be associated with the changes in ionic permeability observed during the propagated action potential. Let us assume, for preciseness, that positively charged amino groups of a protein chain form an obstacle in the pathway of sodium ion permeation through the membrane. By a slight change in configuration of this protein chain, so that a negative carboxyl group takes the place of the amino group, the obstacle may be removed. Of course, a number of other possibilities may be readily conceived. But the fact that quaternary ions are so much more efficient in decreasing the potential difference between inside and outside than tertiary ions, in other words, activate the receptor much more efficiently, indicates the remarkable parallelism with the promoting function of the extra methyl group observed in the case of the esterase and the acetylase.

\section{Gap between Biochemical Data and the Actions of the Living Cell}

The essentiality of the acetylcholine system in the elementary process of conduction appears established beyond any reasonable doubt. Studies of the precise mechanism by which this system generates the electric potentials are in their initial phase. The views presented are an attempt to integrate the vast amount of biochemical and physicochemical data with the 
knowledge derived from physical measurements, especially with that of ion movements. Our knowledge is, however, still very limited. The existence of the receptor has been experimentally demonstrated, but the receptor has not yet been isolated. The difference of its reactions towards certain tertiary and quaternary nitrogen derivatives is one very special feature and has been known from pharmacological experiments for a long time. On the basis of protein studies in vitro an interpretation has been proposed and by a fortunate coincidence of several factors experimental support has been obtained. Otherwise, however, our knowledge of the receptor is extremely scanty: the evidence appears strong that the active site is different from that of acetylcholinesterase. But is it a different protein? There may be even two different receptor proteins as in actomyosin. The number of actual sites is unknown, as is the reactivity with all kinds of compounds and physicochemical factors on which the reaction of a protein depends. In the case of actomyosin intensive investigations over more than a decade have revealed a great number of important properties, and yet even there the interaction between ATP and actomyosin is still full of unsolved problems (32).

In the case of the acetylcholine system the difficulties are incomparably greater. We do not have something comparable to the model, i.e., the glycerinated fiber, in which all the compounds of low molecular weight have been removed and many other complicating elements have been eliminated. In the case of nerve impulse conduction we do not yet even know where the so-called active membrane is located. We know today that there is a barrier which suddenly changes its properties as to ion permeability and that this change is responsible for the generation of the electric potentials. But the greatest obstacle to an understanding of the reactions of the acetylcholine system is the complete lack of knowledge of the structural peculiarities of this barrier. The great complexity of cellular structures has long been known (10). Electron microscope studies have thrown new light upon the intricacies of ultrastructure, which appears 
even more complex than it was suspected. But here too we are in the initial state, and as far as the active membranc of conducting cells is concerned there exists not even yet a beginning, especially as to the detailed properties of this structure.

The generation of bioelectric potentials is one of the most delicate and fastest biological functions known. Obviously, pharmacological methods, i.e., injection into blood vessels of whole animals or the application of chemicals or drugs from the outside, are wholly inadequate as methods of analysis of this mechanism. Such observations may raise interesting problems and occasionally permit conclusions as to certain aspects to an extremely limited extent. A. J. Clark (4) wrote in 1933: "The physical chemist can reasonably hope to simplify his conditions and to reduce the number of variables, until he obtains a system that provides formal proof of the laws which he enunciates, but the pharmacologist is interested in the action of drugs on the living cell and any attempt to simplify this material results in death. Hence he cannot hope to obtain formal proof for his theories and must be content with intelligent guesses. Even in the most favorable cases where quantitative relations have been established for the action of drugs on cells there probably remain dozens of unknown variables, and there is usually a considerable range of possible alternative explanations."

Certain factors, as for instance the existence of permeability barriers surrounding nerve fibers and impervious to certain chemical structures, have been overlooked for a long time and this situation led to the conclusion that acetylcholine has a physiological function only at synaptic junctions. The experimental demonstration of such barriers in a variety of ways has shown that the failure to produce effects with acetylcholine, prostigmine, curare, and related compounds upon the propagation of the spike potential does in no way contradict the assumption of the essentiality of the acetylcholine system for conduction, but is simply due to the fact that the system is protected by permeability barriers. 
The vast amount of biochemical data accumulated, the energetics, and the many correlations between chemical and electrical events indicate the primary role of the acetylcholine system. But it is naïve to believe that we should therefore be able to predict or explain all the electrical phenomena of the intact cell. It would be tedious to enumerate even the known factors involved about which we have little or no information. Let us just discuss one or two as an illustration. What are the molecular changes in the membrane which occur during the action potential and how do they increase the permeability to sodium ion? The picture of acetylcholine action mentioned above is just one possibility. How many obstacles, on a molecular level, have to be changed by chemical reactions in the pathway of sodium ions moving from the outside to the cell interior? How many receptors have to be activated in a single pathway in order to obtain a propagated spike, how many have to be inactivated to block it? Apparently only a small fraction of the neuronal surface is active at any given time. But are these always the same foci or are there many alternatives? We can estimate that one molecule of acetylcholine released permits the passage of at least 400 sodium ions. But is it not possible that three to five molecules located at a certain distance in one single pathway must be released either simultaneously or perhaps in a special sequence to permit 1200 to 2000 sodium ions to pass at any single point?

If we apply our crude methods to the cell, we should not be surprised if the reaction does not correspond to expectations on the basis of the known in vitro properties of the system or, more correctly, of part of the system. The use of microelectrodes inserted into a single fiber is a step forward compared with the techniques of the 19th century. This method made it possible to demonstrate with a suitable preparation and under appropriate experimental conditions that mono- and di-quaternary compounds such as acetylcholine and curare may affect not only synaptic transmission but also the propagated spike (1), as was postulated on the basis of chemical studies. It took a century, 
after Claude Bernard's first observations, to achieve this progress and to explain satisfactorily the exclusive action of curare upon the neuromuscular junction and its failure to affect conduction. But the mysteries and complexities of the living cell will not be revealed even with such methods. Is it really so surprising that by such crude techniques as, e.g., injecting acetylcholine into the cell interior, we just interfere with the physiological progress but cannot reproduce it exactly? that we cannot reproduce in a precise way the effect of acetylcholine released in a few millionths of a second at very special points either simultaneously or in a special sequence and acting upon the receptor at a distance of a few Angströms, probably in a highly peculiar and refined structural organization? The warning of Hippocrates:

\section{$\grave{\eta} \delta \grave{\epsilon} \pi \epsilon \tilde{\imath} \rho \alpha \sigma \varphi \alpha \lambda \epsilon \rho \dot{\eta}, \quad \grave{\eta} \delta \grave{\epsilon} \kappa \rho \grave{l} \sigma \iota s \chi \alpha \lambda \epsilon \pi \dot{\eta}$}

(the experiment is deceptive, the judgment difficult) is certainly true for the study of intact living cells.

Recognition of the complexity of a function such as conduction, or in fact of any biological function, is the prerequisite for any real progress in analyzing its mechanism. Conclusions based upon biochemical studies permit certain tests on the living cell. It was indeed justified to postulate, for instance, that if the view of the primary role of acetylcholine in conduction was to be accepted, it was necessary to demonstrate the impossibility of dissociating conduction and acetylcholinesterase activity. Claims that such a dissociation is possible presented therefore a real challenge. In those cases the experiments were based upon inadequate techniques and the difficulties overlooked have since been amply explained. With a great variety of techniques and many different types of nerves, conclusive evidence has been provided that a dissociation is impossible and that conduction fails if the enzyme concentration falls below 20 per cent of the initial (e.g., 46). The successful demonstration of the necessity of acetylcholinesterase activity for conduction was due to the availability of highly specific inhibitors and the 
application of very special methods. But blocking a complex biological function is always much easier and fundamentally different from an attempt to reproduce it or to modify its various aspects. We have here to deal with observations on two different levels. The existence of a chemical mechanism established in vitro, which satisfies all the characteristics required to be responsible for the primary process and knowledge of some of its properties, is one level. Testing the system organized in a special structure in the intact cell is an entirely different level. Between them is a tremendous gap. This is true not only for the problem of nerve impulse conduction but for all those biological functions where knowledge has advanced to the molecular level. Physical methods are frequently extremely sensitive. They may give valuable clues on the cellular level, they may raise interesting questions. They never can explain mechanisms of living cells, which can be understood only with knowledge of the structure and the underlying molecular processes. Without an integration of the results of various types of approach a satisfactory understanding of conduction is impossible.

In 1949 Otto Warburg closed a lecture on respiration presented in New York with the remark: "100 years ago Claude Bernard made the statement that not a single biological mechanism is really understood. What was true then seems true still today." No claim has been made that the elementary process of conduction is now understood, but a variety of chemical facts have been established which have opened new ways of approach to the understanding and to the investigation of the mechanism of conduction. These basic biochemical facts cannot be ignored. Any new progress must take into account these developments just as well as the results of physical recordings and the facts available concerning structure and ultrastructure.

\section{Perspectives}

The preceding chapter should not be interpreted as a resignation to the belief: "Ignoramus, ignorabimus." In 
1846 Johannes Mueller made the statement that we would never be able to measure the speed with which nerve impulses are conducted. Six years later Helmholtz succeeded in doing it with a most simple tcchnique. History has shown too often that the human mind may cross barriers which appear insurmountable. The ultimate aim of biology to explain phenomena of life in terms of molecular dynamics as postulated by Frederick G. Hopkins (13) in his famous lecture "Some Chemical Aspects of Life" seems today within our reach. Vitalism in its various modifications, still vigorously supported in the early part of this century, has practically vanished and very few scientists will deny the possibility of explaining eventually life processes by physicochemical forces. Progress in all fields proceeds, however, at such an astonishing pace that it is not easy to grasp the implications even in a single field. This may explain some of the difficulties which have to be overcome if piogress in different fields is to be integrated. For the understanding of the living cell advances in the borderline between physics, chemistry, and ultrastructure are essential. Just there the difference in approach and way of thinking makes communication between the various fields of endeavour sometimes difficult. Here the human element, intuition and ingenuity will play a decisive role. Selection of the right material, proper arrangement of experimental conditions, judgment and critical appraisal, and above all imagination and boldness of concepts must be combined in order to overcome the difficulties. In looking back at the amazing changes of the last three decades, the hope seems more than ever justified that we will be able to bridge the gaps between metabolism and function, between molecular dynamics observed in vitro and the same reaction occurring within a special and elaborate structural organization of living cells and tissues.

This article is dedicated to Dr. Hans T. Clarke, Professor of Biochemistry at the College of Physicians and Surgeons, Columbia University, on the occasion of his retirement as Chairman of the Department. The authors want to express their deep affection for him and their gratitude for his invaluable guidance, inspiration, and encouragement. 


\section{References}

1. Altamirano, M., IV. L. Schleyer, G. W. Coates, and D. Nachmansohn, Biochim. et Biophys. Acta, 76, 268 (1955).

2. Bergmann, F., I. B. Wilson, and D. Nachmansohn, J. Biol. Chem., 186, 693 (1950).

3. Berman, R., I. B. Wilson, and D. Nachmansohn, Biochim. et Biophys. Acta, 12, 315 (1953).

4. Clark, A. J.. The Mode of Action of Drugs on Cells. Edward Arnold, London, 1933.

5. Clark, A. J., in WV. Hcubner and J. Schüller, eds., Handb. Experiment. Pharmakol., Vol. 4. Springer, Berlin, 1937.

6. Compte rendu II. Congrès International de Biochimie. Masson, Paris, 1953.

7. de Broglie, L., The Revolution in Physics. Noonday Press, New York, 1953.

8. Engelhardt, V. A., and M. N. Lyubimova, Nature, 14t, 668 (1939).

9. Feng, T. P., Ergeb. Physiol., biol. Chem. u. exptl. Pharmakol., 38, 73 (1936).

10. Frey-Wyssling, A., Submicroscopic Morphology of Protoplasm. Elsevier, Amsterdam, 1953.

11. Green, D. E., ed., Currents in Biochemical Research. Interscience, New York-London, 1946.

12. Hodgkin, A. L., and A. F. Huxley, Cold Spring Harbor Symposia Quant. Biol., 17, 43 (1953).

13. Hopkins, F. G., Smithsonian Repts., 129 (1934).

14. Huxley, A. F., in H. T. Clarke, ed., Ion Transport Acruss Membranes, pp. 23-34. Academic Press, New York, 1954.

14a. Kewitz, H., and I. B. Wilson, Arch. Biochem. and Biophys., 60, 261 (1956).

15. Kirschner, L. B., and W. E. Stone, J. Gen. Physiol., 31, 821 (1951).

16. Meyer, K. H., Helv. Chim. Acta, 20, 634 (1937).

17. Nachmansohn, D., in D. E. Green, ed., Currents in Biochemical Research, pp. 335-356. Interscience, New York, 1946.

18. Nachmansohn, D., Modern Trends in Physiol. and Biochem., 1952, 229-276.

19. Nachmansohn, D., in Harvey Lectures 1953/1954, Academic Press, New York, pp. 57-99.

20. Nachmansohn, D., in J. F. Fulton, ed., A Textbook of Physiolog., pp. 192204. W. B. Saunders, Philadelphia, 1955.

21. Nachmansohn, D., Ergeb. Physiol. biol. Chem. u. exptl. Pharmacol., 48, 575 (1955).

22. Nachmansohn, D., Am. J. Phys. Med., 31, 33 (1955).

23. Nachmansohn, D., and A. L. Machado, J. Neurophysiol., 6, 397 (1943).

24. Nachmansohn, D., S. Ochoa, and F. Lipmann, Science, 115, 365 (1952).

25. Nachmansohn, D., and I. B. IVilson, Advances in Enzymol., 12. 259 (1951). 
26. Needham, J., and E. Baldwin, Hopkins and Biochemistry. W. Heffer and Sons, Cambridge, 1949.

27. Needham, J., and D. E. Green, Perspectives in Biochemistry. Cambridge University Press, Cambridge, 1937.

28. Rothenberg, M. A., Trans. Am. Neurol. Assoc., 230 (1949).

29. Rothenberg, M. A., Biochim. et Biophys. Acta, 4, 96 (1950).

30. Schleyer, W. L., Biochim. et Biophys. Acta, 76, 396 (1955).

31. Warburg, O., and W. Christian, Biochem. Z., 203, 40 (1939).

32. Weber, H. H., and H. Portzehl, Progr. Biophys. and Biophys. Chem., 4. 60 (1954).

33. Wilson, I. B., Biochim. et Biophys. Acta, 7, 466 (1951).

34. Wilson, I. B., Biochim. et Biophys. Acta, 7, 520 (1951).

35. Wilson, I. B., J. Biol. Chem., 190, 111 (1951).

36. Wilson, I. B., J. Biol. Chem., 197, 215 (1952).

37. Wilson, I. B., J. Biol. Chem., 199, 113 (1952).

38. Wilson, I. B., in W. D. McElroy and B. Glass, eds., The Mechanism of Enzyme Action, pp. 642-657. Johns Hopkins Press, Baltimore, 1954.

39. Wilson, I. B., J. Am. Chem. Soc., 77, 2383 (1955).

40. Wilson, I. B., Discussions Farday Soc., in press.

41. Wilson, I. B., and M. Altamirano, Neurochemistry Symposium, in press.

42. Wilson, I. B., and F. Bergmann, J. Biol. Chem., 185, 479 (1950).

43. Wilson, I. B., and F. Bergmann, J. Biol. Chem., 186, 683 (1950).

44. Wilson, I. B., F. Bergmann, and D. Nachmansohn, J. Biol. Chem., 186, 781 (1950).

45. Wilson, I. B., and E. Cabib, J. Am. Chem. Soc., 78, 202 (1956).

46. Wilson, I. B., and M. Cohen, Biochim. et Biophys. Acta, 17, 147 (1953).

47. Wilson, I. B., and S. Ginsburg, Arch. Biochem. and Biophvs., 54, 569 (1955).

47a. Wilson, I. B., and S. Ginsburg, Biochim. et Biophys. Acta, 18, 168 (1955).

48. Wilson, I. B., S. Ginsburg, and E. K. Meislich, J. Am. Chem. Suc., 77, 4286 (1955).

49. Wilson, I. B., and E. K. Meislich, J. Am. Chem. Soc., 75, 4628 (1953).

50. Wilson, I. B., and D. Nachmansohn, in H. T. Clarke, ed., Ion Transport Across Membranes, pp. 35-64. Academic Press, New York, 1954. 


\section{BLOOD: SOME FUNCTIONAL CONSIDERATIONS}

DOUGLAS M. SURGENOR, Department of Biological Chemistry, Harvard Medical School, Boston, Massachusetts

The functions of blood reflect its unique physical and chemical relation to the tissues of the body. In biochemical terms it can be regarded as a continuously circulating tissue phase which is in dynamic equilibrium with the tissues it bathes. It supplies the tissues with the sustenance they require for the performance of their physiological functions; it provides the means whereby important intermediates and specific tissue products such as the hormones are transported to other parts of the organism; and it removes the waste products of metabolism. A striking feature of this transport facility is the efficiency with which the transported substances are delivered to specific destinations throughout the body. The blood in turn depends upon the tissues for a continuous supply of new cells and proteins and for the removal and breakdown of old, since it does not possess regenerative facilities of its own. This dependency is often so direct that the composition of the blood reflects the state of activity of the hematopoietic tissues.

A second major role of blood is the defense of the organism against invading bacteria, viruses, and other foreign substances. Here again distinct and highly specialized systems exist, based on 
the phagocytic action of the leukocytes, on the interactions of specific antibodies, and on more diffuse, but nonetheless important, bactericidal properties of the plasma. Finally, the blood is endowed with facilities for the maintenance of its own integrity, both with respect to its aqueous content and fluidity and also to loss through openings in the blood vessels. The elucidation of the biochemical mechanisms of these complex processes and of the means by which they are controlled and directed to their respective ends presents many intriguing problems. Developments of the last few years have expanded our knowledge of certain of these problems to an extent that a basic framework begins to emerge, and it is now possible to contemplate the patterns which nature has devised to achieve these many specialized and remarkable functions of the blood.

\section{Transport Functions}

The most completely understood transport mechanism is without doubt that by which oxygen is carried from the lungs to the tissues. This familar process is based upon the reversible reaction of hemoglobin and oxygen. Control and direction to meet the tissue demand are accomplished through the mass action principle which governs both the loading and unloading phases of the reaction. Dissociation of oxygen is augmented in the tissues by the effect of carbon dioxide on the affinity of hemoglobin for oxygen. Moreover, since the rates of reaction are rapid, they proceed without the aid of enzymatic mediation. The simplicity and elegance of this mechanism are enhanced by its quasi-reciprocal relation to the transport of carbon dioxide. The latter depends upon the decreased acidity of hemoglobin following dissociation of its oxygen which "makes room" for additional bicarbonate and carbamino anions. Favorable transfer rates are assured by the action of the red cell carbonic anhydrase in catalyzing the hydration of carbon dioxide and the breakdown of carbonic acid when the reaction is reversed in the lungs. The net effect of these reactions is the 
efficient utilization of the red cell compartment in its round trip through the circulatory network. As we shall sec, certain features of these transformations are encountered in other systems.

The transport of iron in the plasma bears many similarities to the oxygen transport mechanism. A specific plasma protein, the $\beta_{1}$-metal combining protein (34), possesses two unique and apparently identical sites which combine with iron in the ferric state; the resultant complex is salmon pink in color, reminiscent of the iron complexes of aspergillic acid and related compounds (13). The affinity of the protein for iron is such that within the range of the binding capacity, the concentration of free, or unbound, iron in the plasma is of the order of $10^{-7}$ molar or less. Clinical studies (8) have confirmed these findings and have established that the metal combining protein provides the means for physiological transport of plasma iron. The protein also reacts with copper, although less tightly than with iron, but despite the fact that the protein is normally only partially saturated with iron in venous blood, it does not appear to contribute to the physiological transport of copper.

Unlike oxygen, it seems improbable that unloading of iron is achieved simply by reversal of the binding reaction. Since the chemical equilibrium is so preponderantly in favor of the metal-protein complex, dissociation of ferric iron would take place only in the presence of an acceptor substance of higher affinity for the metal than that of the plasma protein. This reaction can be operated in vitro, but at a very slow rate. Alternatively, since the affinity of the protein is $p H$-dependent, the iron is released upon acidification of the system, but only at reactions more acid than would be encountered in the tissues. The most likely unloading mechanism involves the reduction of the iron to the ferrous state. This reaction proceeds in vitro at neutral $p \mathrm{H}$ in the presence of a suitable reducing system (10). Recently, Mazur, Baez, and Shorr (24) have found that a small fraction of the iron in ferritin is reduced to the ferrous state under anaerobic conditions or by treatment of ferritin with mild reducing agents. This ferrous iron is then available for binding 
by the metal-combining protein. A similar reaction has been shown to occur in vivo in animals with experimentally produced hypoxia. On the basis of these and other observations, they have suggested that the reverse reaction, transfer of iron from metalcombining protein to ferritin with ferrous iron as the intermediate, may constitute the physiological mechanism for unloading circulating plasma iron in the bone marrow.

Distinctly different mechanisms appear to be responsible for the transport of copper and zinc. In neither case is there an analogy with that of iron. A blue copper-containing protein, caeruloplasmin (17), has been isolated from plasma but the metal appears to be an integral part of the molecule; it is not dissociated by reduction and can be freed only by denaturing the protein. Caeruloplasmin does not appear to be identical with, but may be related to, hemocuprein, the copper protein of red cells. The plasma level of caeruloplasmin is markedly reduced in Wilson's disease, a serious congenital disease characterized by marked neurological changes; however, the protein deficiency does not appear to be the primary etiologic factor.

Plasma contains significant amounts of zinc, of the order of $2 \times 10^{-5}$ molar, which is intimately associated with one or more proteins, as revealed by its behavior during fractionation and by its failure of removal by cation-exchange resins under conditions where added zinc is quantitatively removed. Some preliminary studies with radioactive $\mathrm{Zn}^{65}$-labeled plasma suggested that at acid reactions, near $p \mathrm{H} \mathrm{5}$, the physiological zinc began to be exchangeable. No zinc-containing proteins have been isolated in pure form from plasma; however, since plasma is known to contain trace amounts of certain peptidases, a group of enzymes often found to be zinc-activated, it is likely that at least a part of the plasma zinc is associated with these enzymes. Like copper, zinc is found in the red cells as a constituent of carbonic anhydrase; it is also an important constituent of white cells.

The incompatibility of most free lipids with the aqueous environment of the body demands an unusually specialized vehicle for their transport; the lipoproteins provide this mecha- 
nism. Indeed they are so effective that the characteristic properties of the lipids are completely submerged and are dominated by the hydrophilic properties of the proteins. Although the first lipoprotein was isolated from horse plasma by Macheboeuf in 1929 (22), little attention was paid this highly important class of conjugated proteins until World War II. Since then they have received the intense scrutiny of both chemists and clinicians. The chemical focus has been directed primarily toward the isolation and study of the composition and structure of the lipoproteins. Because of the disparate nature of the chemical moieties involved, the lipoproteins have an inherent fragility; the most extreme example is probably the $\beta$-lipoprotein which has been extensively studied by Oncley and collaborators (26). The composition of this species is predominantly lipid; only $23 \%$ of the anhydrous weight is peptide. Despite this, the substance has characteristic protein-like solubility properties. The lipid constituents show a striking lack of solubility even when the molecule is exposed to organic solvents. Only after the lipoprotein has been denatured do the lipids pass freely in to the organic layer. The forces which hold the molecule together appear to be largely of the van der Waals type. Oncley has calculated that even if spread in a layer one peptide chain thick, on the surface of the spherical molecule, the peptide moiety would cover only about half the surface. If the charged polar groups of the phospholipids were also oriented at the surface, these would contribute further to the amphoteric behavior of the molecule. Such an arrangement at the surface would result in an interior structure composed chiefly of water and nonpolar lipids. The structural importance of the water is suggested by the deep-seated, irreversible, changes which occur when the state of the water is changed, as by freezing or drying.

Clinical interest in the plasma lipoproteins derives from the finding that they may reflect predisposition toward or actual occurrence of certain diseases such as atherosclerosis, of which the characteristic lesion is a fatty deposition in certain areas of the circulatory system. Barr and collaborators in New York (4). 
and Lever and associates in Boston (21), using an analytical fractionation of small amounts of plasma, have shown that in diseases associated with lipemia, the increased lipid is found almost exclusively in the $\beta$-lipoprotein compartment. These increases may represent an acceleration of processes which occur more slowly in normals with increased chronological age. Differences in $\beta$-lipoprotein concentration are observed between young men and young women, suggesting that the lipid composition is under partial hormonal control. Working on this hypothesis, Barr, Russ, and Eder found that administration of estrogen in adult males with high $\beta$-lipoprotein levels resulted in a decrease in both plasma and $\beta$-lipoprotein cholesterol. The levels of $\alpha$-lipoprotein, the other major lipoprotein group, remain strikingly constant with respect to age and sex and during disturbances in lipid metabolism; no physiological function has been suggested for this lipoprotein species. Further proof of a relationship between the $\beta$-lipoproteins and lipid metabolism and transport comes from the findings of Gofman and his collaborators at Berkeley $(14,15)$. In addition to the major $\beta$-lipoprotein isolated by Oncley, they have discovered a related series of trace lipoproteins within the $\beta$ group with increasing amounts of triglycerides, decreasing density, and increasing molecular weight; these are characterized by their rate of flotation $\left(S_{f}\right.$ constant) during ultracentrifugation in media of high density. Originally it was thought that a high degree of correlation existed between the amounts of discrete $S_{f}$ classes of lipoproteins and predisposition to or actual occurrence of atherosclerosis. An "atherosclerogenic index" was derived to express this correlation. However, as the studies have continued, the correlating spectrum has been broadened until it now includes essentially the whole $\beta$-lipoprotein compartment as isolated by chemical fractionation.

Despite these and many other studies which strongly associate the occurrence of atherosclerosis with abnormalities in the plasma lipoproteins and more specifically with the lipoprotein cholesterol, and the diagnostic aid offered by analytical studies 
of the $\beta$-lipoproteins, the etiology of the disease and the singular distribution of atheromata at sites of vascular stress remain completely obscure. Our ignorance of this olsviously abnormal pathway of lipid transport or metabolism is not surprising, however, in view of the fact that we still do not know the normal mechanisms by which the lipids are loaded and unloaded.

Nature has adopted the device of interaction between a specific protein and smaller molecular species to an extraordinary degree. Qualitatively almost all such interactions resemble those already cited; they differ primarily in quantitative aspects such as the number of molecules bound per molecule of protein, and the tightness of binding, or association constant. The frequency of occurrence of binding can be seen from a recent survey by Goldstein (16), where only in the case of sodium and potassium did there appear not to be a bound phase. Evidence of binding has been obtained for almost every other substance normally found in plasma. For certain of these, such as urea and glucose, the evidence is somewhat controversial, owing in large part to the difficulty of demonstrating binding when the degree and tightness of binding are small. Other interactions, of which the binding of calcium by several plasma proteins is a good example, fall in an intermediate category. Here the binding is readily demonstrated $(\log K=2.22)$, as shown by McLean and Hastings (25), but in terms of mass, only about half the calcium ions of plasma are in the bound form under normal circumstances.

One of the most important transport compartments has proved to be the albumin of plasma, which enters into unusually specific combination with a wide range of apparently unrelated groups of small molecules, both physiological and otherwise. The latter include many drugs, dyes, alkyl sulfates, fatty acids, aromatic compounds, chloroform, and long-chain aliphatic alcohols. Theoretical studies on many of these interactions have been greatly fostered by the availability of crystallized human and bovine serum albumin, and much useful information has been derived concerning the physical chemical aspects of the 
reactions, such as the number of groups reacting and the tightness of the binding, and even some suggestions as to the mechanisms involved. Many of the physiologically important interactions of albumin were brought to light by the pioneer investigations of Bennhold and his collaborators at Hamburg (6), who studied the binding of bile and heme pigments and of many drugs and pharmacologic agents. The binding of hematin was suggested by Fairley (12), who showed the presence of abnormal amounts of methemalbumin in Blackwater fever. This reaction has since (31) been shown to involve the very tight combination of two molecules of hematin (Ferriprotoporphyrin IX) with each albumin molecule. Similarly, albumin can bind up to 3 moles of bilirubin (23). Unlike hematin, however, bilirubin is also bound by $\alpha$-globulin, apparently more tightly than by albumin, although both forms occur under physiological conditions.

Only recently has the significance of the interaction of fatty acids with albumin become apparent. Binding was first suggested by Forrest Kendall (18), who obtained crystalline human albumin preparations (from plasma that has been stored for some time prior to fractionation) which contained $1.8 \%$ to $2.9 \%$ fatty acid. This could be extracted only by vigorous treatment, suggestive of a high affinity. Later Hughes (9), working with albumin crystallized from fresh plasma, found only about $0.4 \%$ fatty acid, corresponding to 1 mole per mole of albumin. The difference in the two preparations may have depended on the time which elapsed before the plasma was fractionated, as we now know that fatty acid is one of the products of the "clearing reaction." So termed because of a decrease in turbidity of lipemic plasma, this reaction has been studied in some detail by Anfinsen (3) and others. The optical clearing is the result of an enzymatic reaction in which low density (high molecular weight) lipoproteins are transformed into lower molecular weight products. Analytically this change is accompanied by a decrease in the neutral triglyceride content. The $\beta$-lipoprotein characterized by Oncley contains little or no 
fatty acid triglyceride. Working on this clue, Anfinsen found that the clearing factor possesses lipase activity, the products being free fatty acids, glycerol, mono- and di-glycerides. Albumin, by binding fatty acids, is essential for the reaction to proceed. Without it, or with albumin containing six to ten molecules of sodium oleate, the clearing reaction was inhibited by the accumulation of fatty acid. Fatty acid binding and transport must therefore be considered one of the important physiological functions of albumin. The potential size of this compartment is considerable. The albumin in each liter of plasma can bind approximately $1 \mathrm{~g}$. of fatty acid.

The quantitative relationships between extent of binding and physiological function are perhaps no better illustrated than with the chemotherapeutic agents. Since the site of action of most drugs is not in the blood stream but in a tissue, binding to a plasma protein may seriously interfere with the access of the drug to other areas of the body. Only the unbound species is freely diffusible, and it is the concentration of diffusible drug which corresponds most nearly to the effective concentration. This was clearly shown by Davis (11) in the case of several sulfa drugs. The concentration in cerebrospinal fluid corresponded quite well with the concentration of unbound drug in the blood, despite wide variations in amount bound by blood components. The fraction of drug which is bound acts as a reservoir since it is generally in equilibrium with the diffusible species. It follows that binding does not necessarily interfere with the effectiveness of a drug; the latter is determined by the concentration of the drug at the site of its action. If only a very low concentration is needed for activity, the drug may be effective even though over $90 \%$ of the amount administered is bound. Conversely, where high concentrations are required to produce pharmacological activity, even relatively low binding may result in impaired usefulness.

The hormones present a curious anomaly. The term hormone was introduced in 1904 by Bayliss and Starling (5); its origin was the concept, derived from physiological observations, 
of a chemical messenger released into and transported by the blood stream. However, experimental prcof of the existence of hormones in the blood has been very slow in accumulating. The difficulty has been partly one of technique, for even under optimal conditions the plasma level of a given hormone is extremely low and is variable with time. Assays which have been developed and used during isolation and purification of hormones from endocrine tissue have not generally been sufficiently sensitive to determine plasma levels. This difficulty of assay was circumvented in the case of the thyroid hormone, by direct analysis for protein-bound iodine. It has been found (10) that the iodine in plasma is concentrated in a protein fraction (Fraction IV-4) under conditions where free thyroxin ( $\mathrm{MW}=$ 777) should have been soluble, suggesting that the physical chemical state of the hormone in the plasma is different from that of the free hormone. The plasma protein has not yet been purified, however, and its possible relationship to the glandular tissue protein, thyroglobulin, remains to be established. During World War II, in collaboration with Hisaw, activities ascribable to luteinizing, follicle-stimulating, and thyrotropic hormones were also observed in plasma fractions (10). Later, the estrogenic hormone estriol was identified as a specific constituent of the $\beta$-lipoprotein by Roberts and Szego, who also made some interesting observations on its state of association with the huge lipoprotein molecule (30). Quite recently in our laboratory, insulin-like activity has been reproducibly demonstrated in fractions from small pools of human plasma and, by using selected donors, in whom elevated amounts of certain hormones are known to occur, gonadotropic hormone activity has been partially concentrated from post-menopausal plasma, and growth hormone activity has been demonstrated, although less easily, in the plasma from acromegalic donors. These and other findings strongly support the original concept of transmission of hormonal activity by the blood stream. Many important questions remain, however, for an understanding of the individual mechanisms involved. 
In contemplating these examples of the transport functions of blood, scveral striking features descrve emphasis. The hemoglobin-oxygen interaction and its localization in the red cell represents a signally efficient mechanism for providing relatively large amounts of oxygen to the tissues. The lipoproteins represent the answer to the problem of transporting substances which are physically incompatible with the aqueous environment of the body. The transport of iron typifies the conversion to a state of chemical or physiological inertness of substances which might otherwise be toxic during transport in the blood stream. In this connection, Cartwright and Wintrobe (8) have shown that iron ascorbate could be injected into the blood stream without incident so long as the binding capacity of the circulating metal-combining protein was not exceeded. If more than the stoichiometric amount of iron were introduced, toxic reactions attributable to free iron were immediately observed. Furthermore, the excess iron did not remain in the blood; the plasma iron concentration could not be raised above the iron-binding capacity. Finally, tight chemical binding to specific carrier substances allows for directed transport to specific sites. Selectivity derives from the chemical characteristics of the unloading reaction. Dissociation of the transported substance may result from chemical modification of the bound material (as by reduction in the case of iron), from the action of a specific tissue component with higher affinity for the bound material, from reduction of the concentration of the unbound species as the result of utilization in metabolic reactions, or indeed from any combination of circumstances which displace the mass law equilibrium in the direction of the dissociated components.

Over-all, however, the specificity of many of these interactions remains their most striking and least understood characteristic. Some advances have been made toward elucidating the steric nature of the binding site on the protein and its relation to the small ion. Studies with related series of compounds have shed light on the structural parts of the small ion 
which participate in the binding reaction. Similarly, the effect of $p \mathrm{H}$, temperature, and competing substances can be used to deduce certain of the fcatures of the participating groups of the protein. Klotz (19), for example, has suggested that methyl orange and azosulfathiazole interact with albumin through the $\epsilon$-amino groups of lysine. Obviously this suggestion requires amplification in order to differentiate between binding by albumin and lack of binding by $\gamma$-globulin, since both contain appreciable amounts of lysine. The analogy to the enzymes is of course evident, but particularly in the case of albumin because of the wide range of interacting substances, many of which apparently fail to share any common property, attempts to find a rational theory have thus far been fruitless. The frustrating nature of the problem is epitomized by the interaction of albumin with chloride ions (32). At physiological concentrations approximately six chloride ions are bound per molecule of albumin, but no experimental evidence has been obtained concerning the mechanism of this interaction.

\section{Defense against Infection}

The study of the various mechanisms by which the organism combats infection has long been a separate and specialized area of investigation which is beyond the scope of this discussion. Some recent developments warrant comment, however, because of their bearing on some of the biochemical aspects of the problem. Recently in the course of investigations on the purification of the components of complement, Pillemer (29) discovered a new factor, intimately involved with the functions of complement, but readily separable therefrom by adsorption on zymosan. Termed properdin, this new component apparently acts only in combination with complement and magnesium ions, and is essential for the bactericidal and antiviral properties of normal serum. Of even greater interest is the finding that properdin levels dropped sharply following irradiation, whereas complement levels were unaffected or even increased slightly. 
These data, together with independent observations by Stroud and Brues (33) on the protective action of injected Fraction III, the fraction into which properdin is concentrated during plasma fractionation, led Pillemer to the cautious suggestion that a lack of properdin is connected with the susceptibility to bacteremia following total body irradiation. It seems clear that the chance discovery of this important substance has already made possible a unified rational approach to several basic and hitherto poorly understood phenomena.

The primary mechanism for resistance against bacterial invasion depends upon the phagocytic function of the leukocytes. Consistent with the active nature of this process, white cells are extremely active metabolically. Isolated white cells require a well-balanced biochemical environment for normal activity (36). Citrate ions and other chelating agents commonly used as anticoagulants are toxic, probably because many of the intracellular enzymes require metal activators. Consistent with this, divalent cations such as calcium and magnesium are essential components of any solution which promotes optimal activity. As might be anticipated, preservation of white cells outside the body is successful only when the cells are effectively freed from other formed elements and from plasma, and are placed in an environment where metabolic and physical activity is minimal. The latter conditions are approached when the temperature is reduced to $4^{\circ} \mathrm{C}$. and the cells are immobilized in a buffered gelatin gel. After storage under these conditions for a week, approximately $50 \%$ of the cells exhibit normal viability characteristics when returned to a normal environment and temperature. It was observed, however, that preserved cells would exhibit normal phagocytic activity only when fresh serum was added to the medium. Attempts to substitute pure albumin for the serum protein failed; indeed, albumin inhibited the slight residual phagocytic ability of the cells in protein-free media. Following this lead, two discrete fractions have been obtained (37) from plasma; when either is added to the white cell medium. good phagocytic activity is observed. Tentatively called phago- 
cytosis promoting factors, they are effective at extremely low concentrations. Under the conditions presently being used for their assay it has not been possible to differentiate their effect on the white cells. In solubility, electrophoretic mobility, and other properties, however, they differ markedly. Neither possesses hemolytic complement or $\mathrm{C}^{\prime} 3$ activity, but it remains to be established whether they represent other components of complement. Treatment of serum by the procedure described by Pillemer (29) for removal of properdin resulted in slightly decreased phagocytosis promoting activity, suggesting that properdin may be involved but not as a primary factor.

It may be anticipated that detailed study of the mechanisms by which these purified protein components interact with the white cell, whether by affecting the physical properties of the cell membrane, by enzymatic triggering of a metabolic response, or in some other fashion, will clarify many conflicting views on the nature and action of the opsonins of serum. As with so many other systems, the value of prior purification of components as a prerequisite to determination of physiological roles is well illustrated by properdin and the phagocytosis promoting factors.

\section{Clotting Mechanisms}

The complex series of interactions concerned with blood coagulation represents one of the most active areas of investigation today. Although certain isolated reactions, i.e., the conversion of fibrinogen to fibrin by thrombin, appear to be well characterized, it is not yet possible to draw a picture that would not arouse intense debate by protagonists on various sides. The problem is an intriguing one. Less than 20 years ago the most widely accepted mechanisms involved a relatively small number of reactants; in a recent review, Alexander (2) has proposed a "Modern Scheme of Coagulation" which includes over 20 interacting components. Clcarly, nature has evolved an elaborate and delicately balanced system of enzymes, enzyme precursors, 
inhibitors, and even possibly inhibitor precursors which are triggered by an ill-understood combination of circumstances into a forward reaction of almost explosive velocity. On the other side, equally effective conditions exist for neutralizing the active products at a rapid rate; so efficient are the controls on these reactions that despite the local formation of more than enough thrombin to coagulate the total circulating blood volume, the extent of reaction is effectively restricted.

It is interesting to consider the comparative aspects of blood clotting mechanisms. In lower forms of life hemostasis depends upon the formation of cellular aggregates. Later, rudimentary mechanisms occur which involve both cellular elements and the fluid phase of the blood. In higher forms, the role of plasma components in clotting becomes the dominant one, while the aggregation of blood cells assumes less physiological importance. In man, the platelets have multiple functions; they are involved at the beginning of the coagulation process and also retain many properties reminiscent of those of amoebocytes in lower forms, including the tendency to formation of relatively pure platelet thrombi and conferral of retractility to fibrin clots. BucltzOlsen (7) has suggested that the latter property is an evolutionary holdover which is relatively unimportant in view of the efficiency of the fibrin clot. The formation of thrombi, whether or not an evolutionary holdover of only secondary physiological importance, may nevertheless result in serious pathological disturbances in the circulatory system.

Many of the best clues to the nature of the components involved in blood coagulation have resulted from quirks of nature in which a deficiency occurs of a single component. These deficiencies may be either congenital or acquired; they have a good chance of being detected (in contrast to other deficiency states) because of the gross disturbances in the over-all clotting mechanisms which result. Discrete congenital diseases due to the lack of almost every participating factor in clotting have now been observed by many investigators. In more than one instance the inability to ascribe the abnormality to known patterns 
has led to postulation of new factors. No small part of the present day confusion regarding the mechanisms of blood coagulation is due to such seemingly well-founded postulates which await confirmation by study in isolated systems.

The situation is typified by hemophilia, a hereditary deficiency disease of historical record. The property of normal plasma to compensate for this defect in clotting has been termed antihemophilic globulin. However, this factor has not yet been isolated free of other identifiable clotting factors, largely because of its inherent fragility. As a result the precise biochemical role of the antihemophilic factor still remains to be elucidated, although it is generally believed to be utilized in the first stage of coagulation, the formation of thromboplastin. The most recent chapter in the study of hemophilia has made the picture more complicated by forcing the division of hemophilia into at least two discrete groups. This development stemmed from the observation of Pavlovsky (28) that the blood of certain patients thought to be hemophiliacs repaired the coagulation defect of blood from other hemophiliacs. Subsequent studies have established that approximately one-fifth of those individuals previously thought to suffer from a deficiency of antihemophilic factor were in reality deficient in a new factor, which hemophiliacs possess. The new factor has been purified and characterized by Aggeler (1); the contrast in its properties with those ascribed to antihemophilic factor such as its stability and its apparent failure to be consumed during coagulation, bear out the clinical laboratory findings. In still another respect this new factor epitomizes the confusion felt by the neophyte who delves into the coagulation area-the new component already possesses a number of pseudonyms, including plasma thromboplastin component, plasma thromboplastin factor B, factor IX, and Christmas factor.

From the standpoint of reaction kinetics, the coagulation process presents several interesting features. The forward process is generally considered as consisting of three reactions. The first of these yields a product, thromboplastin, which is an enzyme for the second reaction; this in turn yields a product, 
thrombin, which is the enzyme for the third, the formation of fibrin. The over-all velocity from beginning to end is extremely rapid and resembles an autocatalytic reaction. Closer analysis, however, reveals that an over-all reaction of this character would result if the rate of the first reaction were the limiting rate for all three. This would be the more likely in the present case, since the product of the first reaction is an enzyme for the second. (There is no reason to believe that the rate of thrombin formation is the limiting rate, especially in light of the startling mass of fibrinogen converted to fibrin by minute amounts of thrombin; vide infra.) Despite this rationalization, the pseudo-autocatalytic nature of the clotting process has led to the use of the term autocatalytic in much of the literature on blood coagulation. On the other hand, preliminary studies on the kinetics of the second reaction in the author's laboratory have failed to reveal any behavior attributable to autocatalysis; the reaction appears to be first-order with respect to prothrombin in so far as studicd with purified components.

A second interesting feature emerges. The rather unusual finding of a rapid over-all reaction involving several components including protein enzymes and the platelets, practically demands that, in the blood stream, most, if not all, of the components be present in a preformed, organized complex. It would otherwise be difficult to understand the rapidity of the reaction, since the rate of formation of an enzyme-substrate complex, as for example, that of prothrombin, thromboplastin, accelerator globulin, convertin, and calcium, would in itself be very slow. This can be readily shown in isolated systems. There is, of course, ample precedent for such a complex, but usually, as in the mitochondria, it assumes a three-dimensional form which exists as a separate phase. There is also good presumptive evidence for a preformed coagulation complex. One clue is the affinity of the platelet for calcium. Platelets can be selectively removed from blood by passage over a cation-exchange resin if a very small proportion of the exchange sites have previously been reacted with calcium; magnesium does not share this property 
with calcium. In addition, the affinity of not one, but several, of the soluble coagulation components for insoluble salts of the alkaline earth metals, has already been noted (35). These components include prothrombin, convertin, and proconvertin, and plasma thromboplastin factor B. It is thus not difficult to visualize a complex involving the platelet and the several interacting components, with calcium as the cementing substance, aided by other forces which promote the formation of enzymesubtrate complexes.

These and other implications are perhaps better understood by referring to the data in Table I, where the familiar list of plasma proteins has been used to estimate the number of molecules of the major proteins and the number of blood cells in a unit volume of whole blood, for convenience a volume of $10^{-10} 1$. In these terms there are 120 million molecules of prothrombin for every 25 platelets ( 5 million molecules per platelet). Assum-

TABLE I

Estimated Numerical Ocaurrence of Certain Components in a $10^{-10}$ Liter Volume of Human Blood ${ }^{a}$

\begin{tabular}{lr}
\hline \multicolumn{1}{c}{ Component } & $\begin{array}{c}\text { Number of cells } \\
\text { or molecules }\end{array}$ \\
\hline Leukocytes & 1 \\
Platelets & 25 \\
Erythrocytes & 500 \\
Properdin & 500,000 \\
Cholinesterase & 670,000 \\
Caeruloplasmin & $66,700,000$ \\
$\beta$-Lipoproteins & $89,700,000$ \\
$\alpha_{2}$-Glycoproteins & $93,300,000$ \\
Prothrombin & $120,000,000$ \\
Fibrinogen & $206,000,000$ \\
$\alpha$-Lipoproteins & $350,000,000$ \\
Acid glycoprotein & $500,000,000$ \\
$\beta_{1}$-Metal-combining protein & $770,000,000$ \\
$\gamma$-Globulins & $1,630,000,000$ \\
Albumins & $17,300,000,000$ \\
Hemoglobin (inside erythrocytes) & $133,000,000,000$ \\
\hline
\end{tabular}

a Based in part on estimates from data in references 17, 20, 27, and 29. 
ing the platelet to be a sphere of about 1 micron diameter, the surface area would be of the order of $3 \times 10^{8} \mathrm{~A}^{2}$. This would provide space for approximately 500,000 molecules of a protein the size of prothrombin, if oriented endwise. It is not intended to imply that prothrombin is, in fact, the interacting species; consideration of the clotting system might suggest a more likely component whose frequency of occurrence in plasma might be one or two orders of magnitude less than that of prothrombin. In this connection, the molecular ratio of prothrombin to fibrinogen presents a striking contrast to that normally found for an enzyme and its substrate. With the use of data obtained with relatively impure thrombin, it can be calculated that one molecule of prothrombin is capable of catalyzing the conversion of 100,000 molecules of fibrinogen to fibrin. In terms of the enzymatic reaction for which it is the substrate, the high molar concentration of prothrombin must favor the formation of the enzyme-substrate complex and consequently a rapid rate of thrombin formation.

\section{Summary}

The fragmentary glimpse of blood in its cellular and molecular dimensions lends perspective to the understanding of the biochemical functions of this disperse tissue. The paramount importance of the oxygen transport mechanism is confirmed by the overwhelming molecular superiority of hemoglobin in comparison to plasma proteins. The contribution of albumin in the maintenance of the circulating fluid volume of the blood, and the size of the transport facility which derives from the affinity of albumin for a diversity of small molecules and ions (without significantly interfering with colloid osmotic pressure) need hardly be stressed when viewed in these terms. In contrast to the above, as well as to the $\beta$-metal combining globulin and the $\gamma$-globulins, all of whose physiological interactions can be characterized in terms of classical mass law chemistry, are the hormones, enzymes, and other species which exert their influence 
in catalytically small amounts. Thus the relative numerical inferiority of properdin belies the probable significance of this substance to the basic defense mechanisms of the body. $\alpha$ Lipoprotein molecules occur four times as frequently as $\beta$ lipoproteins, although there is twice as much of the latter by weight. Moreover, despite relatively high molar concentrations, precise functional roles have not yet been ascribed to the $\alpha$-lipoproteins, acid glycoprotein, or $\alpha_{2}$-glycoproteins. Although the plasma components represented in Table I account for approximately $85 \%$ of the protein weight, they comprise considerably less than half of the protein species known to be present in plasma. The latter have not been included in Table I because they have not yet been characterized in the necessary parameters. It is safe to estimate that the majority of components not listed would fall at the low end of the numerical spectrum.

The dissection of blood into its molecular and cellular parts has contributed greatly to the study of the biochemical mechanisms by which the manifold functions of the blood are accomplished within the body. In attempting to assimilate such knowledge into a rational picture, one parameter may be overlooked. Unlike the anatomist, who can extrapolate back from his dissected parts to the gross morphology of the organisms, we must ask ourselves whether the blood we started with, e.g., our unit volume, was a suspension of formed elements in a random solution of plasma proteins, or might there perhaps have been a chemical morphology which was unintentionally disrupted when the blood was removed from the circulation or, more likely still, when the unnatural chemical and physical instruments of dissection were applied? Obviously no categorical answer can be made to this question. On the other hand, there is good evidence for the existence of favored orientations within the blood; the possible role of the platelet in attracting a selected environment of clotting factors has already been noted. The natural occurrence of rouleaux within the circulatory network brings to mind the selective interactions of red cells and certain asymmetric plasma proteins, including fibrinogen and the $\alpha_{2}$-muco- 
protein, which result in rouleaux formation. Beneficial effects of certain plasma proteins upon the red cell during preservation have also been recorded in the literature. In addition to the strong orienting forces at the surface of cells which favor selected protein atmospheres, there are ample forces within the fluid phase itself capable of effecting orientation of proteins with respect to each other. These consist of the same kinds of forces upon which many of the physiological interactions of the blood depend. Tempting though it may be to postulate in this direction, the present status of our knowledge is clearly only sufficient to allow for intelligent surmise.

\section{References}

1. Aggeler, P. M., T. H. Spaet, and B. E. Emery, Science, 119, 806 (1954).

2. Alexander, B., New Engl. J. Med., 252, 432, 484, 526 (1955).

3. Anfinsen, C. B., in Atherosclerosis, Publication 338, National Academy of Sciences, National Research Council, 1955.

4. Barr, D. P., E. M. Russ, and H. A. Eder, in J. L. Tullis, ed., Blood Cells and Plasma Proteins. Academic Press, New York, 1952.

5. Bayliss, W. M., and E. H. Starling, Proc. Roy. Soc. (London), B73, 310 (1904).

6. Bennhold, H., in H. Bennhold, E. Kylin, and S. Russnyak, eds., Die Eiweisskörper des Blutplasmas. Steinkopf, Leipzig, 1938.

7. Budtz-Olsen, O. E., Clot Retraction. Charles C. Thomas, Springfield, Illinois, 1951.

8. Cartwright, G. E., and M. M. Wintrobe, J. Clin. Invest., 28, 86 (1949).

9. Cohn, E. J., W. L. Hughes, Jr., and J. H. Weare, J. Am. Chem. Soc., 69, 1753 (1947).

10. Cohn, E. J., D. M. Surgenor, and M. J. Hunter in J. T. Edsall, ed., Enzymes and Enzyme Systems. Academic Press, New York, 1951.

11. Davis, B. D., in C. M. MacLeod, ed., Evaluation of Chemotherapeutic Agents. Columbia University Press, New York, 1949.

12. Fairley, N. H., Quart. J. Med., 10, 115 (1941).

13. Fiala, S., and D. Burke, Arch. Biochem., 20, 172 (1949).

14. Gofman, J. W., Advances in Biol. and Med. Phys., 2, 269 (1951).

15. Gofman, J. W., H. B. Jones, T. P. Lyon, F. T. Lindgren, B. Strisower, D. Coleman, and V. Herring, Circulation, 5, 119 (1952).

16. Goldstein, A., J. Pharmacol. Exptl. Therap., 95, 102 (1949).

17. Holmberg, C. G., and C. B. Laurcll, Acta Chem. Scand., 7, 944 (1947). 
18. Kendall, F. E., J. Biol. Chem., 138, 97 (1941).

19. Klotz, I. M., and F. M. Walker, J. Am. Chem. Soc., 69, 1609 (1947).

20. Lamy, F., and D. F. Waugh, J. Biol. Chem., 203, 489 (1953).

21. Lever, W. F., and N. A. Hurley, in J. L. Tullis, ed., Blood Cells and Plasma Proteins. Academic Press, New York, 1952.

22. Machebocuf, M., Bull. soc. chim. biol., 11, 268, 483 (1929).

23. Martin, N. H., J. Am. Chem. Soc., 71, 1230 (1949).

24. Mazur, A., S. Baez, and E. Shorr, J. Biol. Chem., 213, 147 (1955).

25. McLean, F. C., and A. B. Hastings, J. Biol. Chem., 108, 285 (1935).

26. Oncley, J. L., and F. R. N. Gurd, in J. L. Tullis, ed., Blood Cells and Plasma Proteins. Academic Press, New York, 1952.

27. Oncley, J. L., G. Scatchard, and A. Brown, J. Phys. E Colloid Chem., 51, 184 (1947).

28. Pavlovsky, A., Blood, 2, 185 (1947).

29. Pillemer, L., L. Blum, I. H. Lepow, O. A. Ross, E. W. Todd, and A. C. Wardlaw, Science, 120, 279 (1954).

30. Roberts, S., and C. M. Szego, Endocrinology, 39, 183 (1946).

31. Rosenfeld, M., and D. M. Surgenor, J. Biol. Chem., 183, 663 (1950).

32. Scatchard, G., I. H. Scheinberg, and S. H. Armstrong, Jr., J. Am. Chem. Soc., 72, 535 (1950).

33. Stroud, A. N., and A. M. Brues, Fed ration Proc., 13, 147 (1954).

34. Surgenor, D. M., B. A. Koechlin, and L. E. Strong, J. Clin. Invest., 28, 73 (1949).

35. Surgenor, D. M., and J. F. Noertker, J. Am. Chem. Soc., 74, 3448 (1952).

36. Tullis, J. L., in J. L. Tullis, ed., Blood Cells and Plasma Proteins. Academic Press, New York, 1952.

37. Tullis, J. L., and D. M. Surgenor, Ann. N. Y. Acad. Sci., in press. 


\section{AN INTEGRATED CONCEPT OF CARCINOGENESIS*}

HAROLD P. RUSCH, The McArdle Memorial Laboratory, Medical School, University of Wisconsin, Madison, Wisconsin

\section{Introduction}

In 1937 the Surgeon General of the United States Public Health Service appointed a committee of leading scientists to "formulate, as far as possible, the fundamental aspects of the cancer problem and to suggest various lines of work which merit investigation." The report of this distinguished committee was published in 1938 (5). Some of their most pertinent conclusions were as follows:

1. The causes of cancer "are multiple and diverse and vary for each type of cancer."

2. "Once malignancy is established in a cell it becomes an automatic process, independent of the presence of a continuously acting agent of outside origin, and the new character of the cell becomes a fixed one which is passed unchanged to the descendants."

* Adapted from an address delivered before the forty-fifth annual meeting of the American Association for Cancer Research, Atlantic City, N. J., April 11, 1954, and published in Cancer Research, 14, 407 (1954).

Financial support for the preparation of this report was provided by the American Cancer Society Institutional Grant No. 71A; by the National Cancer Institute, Public Health Service Grant No. C-828; and by the Alexander and Margaret Stewart Trust Fund. 
3. "The inherited state is an unstable or poorly balanced system confined to an organ or tissue in which the specific cells tend to become malignant either from functional strain or from unfavorable environmental conditions."

4. "Almost all, if not all, classes of cells may be rendered malignant under the influence of one or more agents."

Although no recent discoveries have invalidated the general conclusions reached almost 20 years ago, research during the intervening years has advanced at an ever-increasing tempo, and it is only natural that some of the older beliefs would be clarified, others modified, and that sufficient evidence would be available for additional concepts. Progress during the past few years in areas such as cellular physiology, genetics, and biochemistry has provided the framework for some new conclusions and interpretations, which form the basis of this paper. Some of the mechanisms by which metabolic patterns can be altered will be considered first.

\section{Inherited Cellular Changes}

To keep pace with the rapid advances in cellular inheritance it is necessary to turn to fundamental experiments that were carried out with various forms of life without reference to the cancer problem. The geneticist, for example, has found that metabolic pathways are genetically determined. Although it is impossible to cite all the references that support such conclusions, the line of research on mutations in Neurospora carried out by Beadle (6) and by Beadle and Tatum (7) illustrates this type of study. The finding that the mutant strains have their counterparts in many naturally occurring forms of life suggests the generality of the conclusions. From this work have come stimulating concepts of the origin of life and the idea that life is continually evolving $(32,46,51)$.

It is a tempting leap to the assumption that each type of cell within the body of a higher animal represents a sort of mutant from some predecessor. With such an assumption the 
conversion of a normal cell to a cancer cell might simply be considered but another expression of the same process more clearly typified by the conversion of a streptomycin-sensitive strain of bacteria to a streptomycin-resistant strain. But these hypotheses still remain to be established. They are, however, becoming more amenable to testing in higher animals because of the manifold possibilities for studying the metabolism of tumors and normal tissues by means of isotopic tracers, and such work forms a significant proportion of the present research at the McArdle Memorial Laboratory $(28,40,41,59,60)$. This concept of evolutionary change is applicable not only to the conversion of normal cells to cancer cells or to the individual stages in this process, but also to the conversion of drug-sensitive cancer cells to drug-resistant cancer cells. This process has been studied intensively in leukemic cells at the National Cancer Institute by Law (37), who has emphasized that the conversion is mainly a process of natural selection, in which a few resistant or dependent mutants survive and become the basis of a new line, while the sensitive cells are eliminated.

Ideas on the conversion of normal cells to cancer cells stem from the newer knowledge of how one cell type is transformed to another. These ideas are at present in a state of ferment, and great changes are occurring in some of the concepts of hereditary mechanisms. What was once regarded only in terms of mutations of nuclear genes and the resultant Mendelian heredity can now be considered in the light of at least four clear-cut extraMendelian mechanisms for changing the metabolic pattern of a cell. These are:

1. Hereditary change by cytoplasmic segregation. This change is the result of the continued division of cells at a rate that outpaces the formation of cytoplasmic particles, leading to the loss of such particles by simple dilution. Thus, those enzymes whose formation is controlled by these particles will be lost. Observations of this nature have been made by Sonneborn (62), Ephrussi (17,18), Spiegelman (64), and others (34,77).

2. Partial hereditary transfer (genetic transduction) by deoxyri- 
bonucleic acid $(D N A)$. It is now widely accepted that the pneumococcus transforming principle is a specific type of DNA and that the introduction of this material into a receptor strain of bacteria induces one or more new heritable traits, presumably owing to the production of more of the same type of DNA (3). The phenomenon has been extended to other transformations (33).

3. Partial hereditary transfer (genetic transduction) by viruses. The most recent of the extra-Mendelian mechanisms has startling implications if the phenomenon can be extended to mammalian cells. It has been found that a bacteriophage grown in one strain of bacteria can infect another strain without killing it and, in so doing, can carry with it (or transduce) a new metabolic property into the new host (38). In all experiments thus far, only one property could be transduced at a time, but by successive transductions as many as five new metabolic characters or reactions could be introduced into and retained by a strain that originally lacked all five of them, thus converting it into five strains. This mechanism may be intrinsically similar to the one described under point (2) if the genetic fragments carried by the bacteriophage are considered to be DNA.

4. Hereditary change by the establishment of lysogeny. Freeman and Morse (20) demonstrated that an avirulent culture of a diphtheria bacillus could be changed to a virulent one from a symbiotic infection with a bacteriophage. In lysogeny the phage per se produces the heritable alteration in contrast to transduction, where the phage merely acts as the carrier of genetic material from one cell to another.

\section{Carcinogenesis}

With all these possibilities of cellular change, it is clear that studies on the problem of carcinogenesis have ample inspiration. Any or all of the above mechanisms could be concerned in the conversion of normal cells to cancer cells, and cancer investigators are deeply indebted to the biologists 
who discovered these interesting mechanisms. Before examining the evidence which indicates that similar mechanisms are involved in carcinogenesis, it is necessary to emphasize that the development of cancer is far more complex than the transformations observed in the unicellular organisms. A pure strain of cells living under controlled conditions is not subject to the action of antibodies, fibroblasts, leukocytes, hormones, and others factors that help to regulate life in multicellular organisms. Thus, it is to be expected that the changes leading to cancer will be complicated and extended because of the moderating influence of the host in combatting deviations from the norm. Despite these complications, it is not premature to examine the biological and biochemical evidence which indicates a similarity in the processes that result in heritable cellular changes in unicellular organisms and in cancer.

First, there is some indirect evidence that the cause of some cancers is associated with mutations of nuclear genes. Such evidence is based on the findings that nuclear changes are associated with cancer and that carcinogens cause chromosomal aberrations (26), and on the corollary finding that some carcinogens are mutagenic and some mutagens are carcinogenic $(2,29,30,36)$.

Second, carcinogenesis may result from deletions of cytoplasmic factors in a manner analogous to that observed by Ephrussi in yeast transformations $(17,18)$. In the McArdle Memorial Laboratory, James and Elizabeth Miller $(44,45)$ have obtained evidence compatible with this view. They have demonstrated that the carcinogenic amino azo dyes form firm complexes with normally occurring proteins of the liver; but no similar binding was found in hepatic neoplasms. Their results suggest that the carcinogen causes a gradual deletion of certain proteins that are necessary for specialized features of the cell but not for the growth pattern. Similar complexes between proteins of the skin of mice and carcinogenic hydrocarbons have also been demonstrated by E. C. Miller (43) and by Heidelberger (72-74). Of special significance is the fact 
that the carcinogenic activity of the compounds tested is correlated with the amount or rate of binding $(44,45)$. Collaborative experiments by Potter, Price, Miller, and Miller (50) have demonstrated pronounced progressive decreases in certain cytoplasmic enzymes during carcinogenesis in the rat liver.

Third, if nucleic acids or nucleoproteins as pure chemical entities or in a form carried by viruses can be passed from one strain of cells to another and thereby induce or transduce heritable alterations in the metabolic pattern in bacteria, one must inquire whether similar phenomena occur in some types of carcinogenesis. At present there are only the cases in which viruses have been shown to convert normal cells to cancer cells (10, $53,54,61)$, and it is not clear whether the process is analogous to transduction or lysogeny or whether the introduction of the virus leads to a cytoplasmic deletion by competitive interaction. It appears probable that the viruses act at the cytoplasmic level and that their action is not strictly analogous to the DNA transformations, which evidently act at the nuclear level.

It is of interest to recall at this time that a phenomenon similar to transduction or lysogeny was foreshadowed and illustrated by the pioneer work of Peyton Rous and his associates (55) when they demonstrated that a cell-free extract of an osteochondrosarcoma could "transduce" connective tissues in voluntary muscle into osteochondrosarcomas. The implication of this finding was not lost to them, since in 1912 they stated (55), "It is very remarkable that such an agent should bring about a differentiation ordinarily foreign to the tissue."

The solution of carcinogenesis would be complicated enough if a thorough understanding of the mechanisms of heritable cellular changes were the only aspect of the problem requiring clarification. It is now recognized that the formation and growth of some tumors are prolonged processes, in some types involving three or more separate periods: (1) the stage in which a heritable cellular change occurs (called the period of initiation), (2) the period called promotion, in which the altered cells proliferate and become an autonomous cluster, and (3) the period 
during which the autonomous mass invades and metastasizes. Usually it is not until this last stage that cancer is clinically recognizable. The three periods are illustrated in the experiment in which tumors are initiated in the skin of mice by a single application of a carcinogenic hydrocarbon and promoted to an autonomous cluster of cells by the frequent applications of croton oil $(9,56,57)$. The autonomous mass then grows and invades the adjacent tissues in the absence of inciting agents. Other examples of the prolonged process of cancer formation are presented in papers by Blum (11), Furth (22), and Gardner (24).

On the basis of hundreds of experiments on the subject of cancer and ancillary fields, it is now possible to present a simplified diagrammatic picture of the factors which control the reduplication and specialization of cells and to indicate how this normal pattern can be altered during the formation and subsequent growth of neoplasms. Although the concept to be presented takes root from such facts, it must be admitted that it is partly speculative, and one should not be deluded with the belief that the picture is complete or perfect. One reason for presenting this concept is the hope that it may suggest further experimentation. Normal growth and differentiation will be considered first.

\section{Normal Growth}

For the purposes of the present paper all cellular functions are divided into two chief categories: one, the function of reduplication, and two, all other special functions not primarily concerned with cellular multiplication. The following presentation is based on the concept that the process of cell reduplication and the special functions interact in two distinct ways. In addition to being mutually dependent, these functions must also be recognized as mutually competitive with respect to the available cellular nutrients. Thus, the development of cells is a resultant of the outcome of the dynamic balance between cell reduplication and special function. 
Figure 1 is a diagrammatic representation of an unfertilized ovum containing symbols which represent these two chief cellular functions together with the cell food necessary for such activity. This representation has been reduced to the very simplest terms. Potential mechanisms for cellular reduplication are represented by a hollow square, and potential mechanisms for specialized cellular functions are shown by hollow circles. The numerous specialized functions of the cell have been reduced to four, each represented by a circle, which may represent a single enzymatic reaction or a series of integrated metabolic processes; but, since cellular reduplication is basically similar in

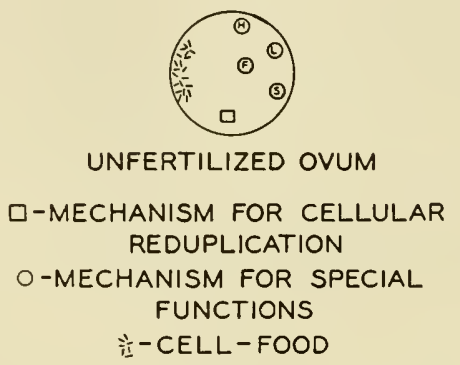

Figure 1.

all cells, only one square is used. The short straight bars arranged at random at one side of the cell represent the cell food or nutrients, also frequently referred to in the literature as building blocks, precursors, or substrates. Although it is tempting to indicate whether the various mechanisms are nuclear or cytoplasmic, no implications are here intended.

The term cellular reduplication is preferred, because there can be no mistake about its meaning; but the terms cellular division, proliferation, and "growth" are also used. Growth is the least desirable term because of its broad meaning, but it has the advantage of brevity.

Figure 2 illustrates the sequence of events associated with the "growth" and differentiation of normal cells. Cells with the most specialized functions are shown at the right, and the 
immature ones are at the left. The arrows within the cells represent the concepts of dependence and of competition. The arrows between the cells represent many stages and cells in transition.

In this diagram, cell $A$ represents the toti-potential fertilized ovum. Immediately following fertilization, the mechanisms for reduplication and for special functions become activated, and this is indicated by the arrows from the cell food to the functions. Both mechanisms have increased in size.

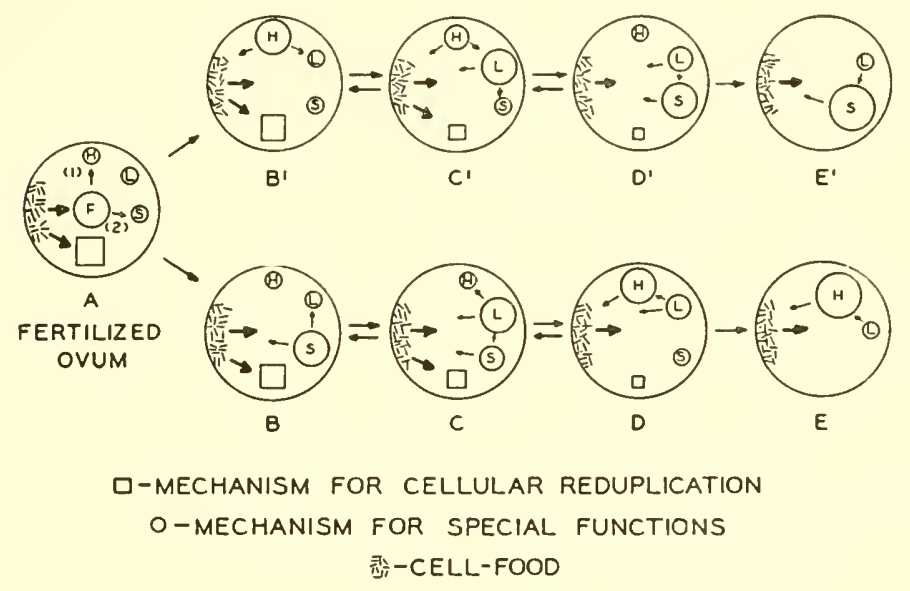

Figure 2.

The first special system to become functional is the one represented by the circle labeled $F$. As the products of its reactions become quantitatively significant, some of these metabolites are used to catalyze and initiate the next sequence of reactions, as depicted by the arrows labeled 7 and 2 . The ultimate characteristics of the differentiated cells are predetermined by the particular system of reactions which is nurtured in the first few generations of cells. If, in a given cell, the $H$ function is the first one to be stimulated, as indicated by arrow 7 , the subsequent course of events in descendants of this cell will follow the upper 
sequence of events culminating in cell $E^{\prime}$. If the special function $S$ is the first to be activated, as shown by arrow 2 , the end results are represented by cell $E$. The course of events associated with the normal growth and differentiation of the mature cell $E$ will be described first.

Cell $B$ represents the primordial cell which is destined to develop into specialized mature cell $E$. The first specialized function, marked by $F$ in the fertilized ovum, has disappeared during the many stages between cells $A$ and $B$. The products of the reactions of the special function $S$ have by this time attained sufficient proportions to trigger and sustain the next sequence of events, function $L$, and to provide energy and metabolites for other intra- and extracellular functions. This flow of metabolites from circle $S$ is indicated by arrows. The utilization of cell food from the general pool by the mechanisms of reduplication and specialized functions at this stage is shown by the heavier arrows.

Cell $C$ represents a further development, as indicated by the increased size of the special function $L$. Besides contributing its own distinctive attributes to the cell, this function in turn stimulates the third function, $H$, and may feed metabolites back into the general pool of cell food. This stage clearly represents how one reaction activates a second, and the second starts a third, and so on; thus, a series of mutually dependent, endogenously induced enzyme formations leads to an orderly, sequential development of differentiation. Without such provision, growth and maturation would be chaotic. This mechanism for sequential development is not idle speculation but is based on the fact that a number of interlocking metabolic reactions have already been shown to initiate and sustain secondary chemical reactions in this manner $(42,49,52,75)$. It is also based on the observation that some enzyme-forming systems in unicellular organisms can be stimulated to produce the enzyme for a substrate by the introduction or accumulation of suitable levels of the given substrate, thereby producing profound changes in the metabolic patterns of a cell without altering its hereditary makeup $(16,66)$. This 
phenomenon was formerly referred to as adaptive enzyme formation but is now known as induced enzyme formation (14).

Only meager evidence exists for the occurrence of induced enzyme formation in higher organisms. For example, Knox (35) has found that the tryptophan peroxidase activity of mammalian liver increases many-fold in response to injections of large amounts of tryptophan. For a variety of reasons he considers this effect due to an increase in the actual quantity of this enzyme. It might be anticipated that the loss of any given enzyme at a particular stage of differentiation or carcinogenesis would lead to the accumulation of the substrate for that enzyme. By the mechanisms of enzyme induction, such an accumulation could then facilitate the increase of enzymes for alternative pathways.

This schema may give some clues concerning the late manifestations of certain inherited defects. The explanation for some of these imperfections appears intelligible if we consider that the responsible defective function is one of the last induced systems to unfold in the developmental process.

The next stage (cell $D$ ) illustrates several interesting changes; the activity of the last special function, labeled $H$, has reached significant proportions, while the reactions of function $S$ have decreased to an extent where they no longer influence the metabolic pattern of the cell, as shown by the absence of arrows. This cell is a functional one, and the mechanism for reduplication, although still present, is no longer effectively competing for cell food, as indicated by the absence of an arrow from the precursors to the small square. At this stage the cell may still retrace its steps during the process of reduplication or it may continue on its path to final irreversible maturation and death. The path to the irreversible stage is shown by an arrow leading to cell $E$. This cell has lost its potentiality to reduplicate (note the absence of the square), and all the precursors are funneled into the special functions of the cell. Any mature cell which is no longer capable of multiplication is representative of this stage. Thus, the course of events leading to differentiation is 
pictured as the sequential deletion or segregation of some metabolic patterns and the concomitant activation or induction of other systems. Similar suggestions have been made by several investigators $(18,65,70,71,76)$. Such deletions are represented by the absence from cell $E$ of function $S$. The concurrent increase in the remaining special functions is depicted by the enormous size of function $H$.

If the path to be followed is reduplication, the course of events is in the opposite direction from that depicted for irreversible maturation. Cell $D$ represents a mature cell with a potential capacity for reduplication, but, in order to divide, it must first retrace some of the processes that led to its maturation. The mechanism for reduplication might be described as follows: When a cell divides, the mechanisms for special functions and reduplication are diluted in the daughter cells and must be gradually rebuilt. For some unknown reason the patterns for specialization recover most rapidly, but the mechanism for growth also recovers, although at a slower pace. As the growth mechanism slowly gains ascendancy, the special functions decrease in the reverse order in which they increased; function $H$ becomes inactive before function $L$, and so on. Eventually the processes for reduplication become dominant and utilize an ever-increasing share of the cell food until a time is reached when the specialized systems no longer play a dominant role in the over-all metabolism of the cell. At this time mitosis begins.

Cell $E^{\prime}$ represents an irreversibly mature cell of a type different from cell $E$. The sequence of events controlling its growth and differentiation is similar to that just described. The chief difference is that it acquires some special characteristic metabolic patterns which differ from those in cell $E$ and retains some features identical with those in cell $E$. This is shown by the dominance of function $S$ in cell $E^{\prime}$ and function $H$ in cell $E$. The $L$ system is common to both cells. During the earliest stages in embryonic development some cells along the path $A-B$ ' "induce" differentiation in cells along the path $A-B$ $(31,63)$. The mechanism of induction might be similar to the 
orderly and synchronous development of the metabolic patterns just described, except that the inducing substances originate in neighboring cells.

It should be stressed that the competitive pathways leading to reduplication or differentiation are in delicate equilibrium; only slight shifts are required to divert them one way or the other. Cell reduplication could be stimulated by any process that diverts the cell food to the growth pattern. One way to accomplish this is to inhibit one or more of the special functions. Perhaps estrogenic hormones and croton oil stimulate mitosis in this manner. In the presence of excessive demands on the special functions, cell division would be retarded and hypertrophy result, whereas factors harmful to special functions would stimulate growth and result in hyperplasia.

On the basis of the evidence which engendered the diagrammatic scheme just presented, the following statements are offered as a concept of normal growth:

1. Normal primordial cells contain many potential mechanisms which ultimately determine differentiation. These mechanisms become functionally active when the constituents attain certain quantitative levels.

2. During the process of growth and differentiation, all living cells acquire or maintain certain biochemical processes or metabolic patterns in common, but in addition each cell type has special characteristic deviations from the general pattern, resulting from variations in enzyme make-up.

3. The specific cell pattern results from the deletion of some of the mechanisms constituting the original make-up of the cell and from the concomitant increase in other functions. These losses and increases occur in a sequence which serves a useful function and is beneficial to the general economy of the organism.

4. In normal cells the competing processes for reduplication and for special function alternate in dominance until the final stage of maturation is reached. Then the pattern for reduplication is irrevocably lost in some cells. 


\section{Theory of Cancer Formation}

Some aspects of carcinogenesis will now be considered. Two of the conclusions concerning the nature of cancer made by the committee in 1938 were that the causes of cancer "are multiple and diverse" and that "once malignancy is established in a cell it becomes an automatic process. . . which is passed unchanged to the descendants." Research during the intervening

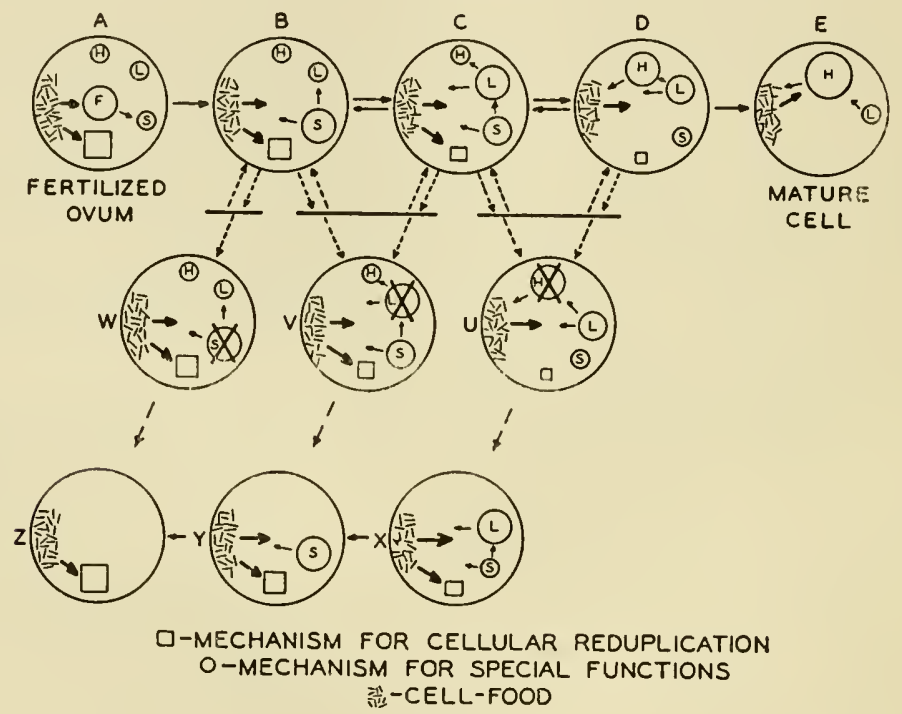

Figure 3.

years has sustained this report, and, in addition, sufficient information has been accumulated to indicate that all carcinogens induce heritable cellular changes.

This change is depicted in the alteration of the normal cells $B, C$, and $D$ to the neoplastic cells $U, V$, and $W$ as shown in Figure 3. At first glance little difference is noted between the top two rows as to the patterns in the various cells; both have the same quantity of cell food, and both possess considerable specialized function. But one crucial difference exists. A key reaction in one of the special metabolic patterns has been 
irrevocably lost in the cells of row 2-a defect which is heritable. This is indicated by the large cross covering the system involved. As will be described later, some ancillary reactions of the altered pattern may persist temporarily, as indicated by the short arrows pointing away from the crossed circles. The initial alteration may be either nuclear or cytoplasmic in origin.

Although the heritable alterations in cells $U, V$, and $W$ affect some specialized function, it will be noted that a different function is altered in each cell depicted. On closer scrutiny it is evident that the future course of the cells is determined by the particular system which has been altered. If the special function is one of the last to develop, as depicted by function $H$ in cell $U$, most of the remaining specialized functions are unaltered, the general cellular economy experiences little change, and the cell represents the type destined to give rise to benign tumor cells. However, if the altered special function occupies a more dominant position in the economy of the cell, as depicted by the circle labeled $L$ in cell $V$, some other functions of the cell will be secondarily involved, and the result is a cell capable of producing cells of intermediate malignancy. When the altered function occupies a key position in relation to other special functions, as represented by function $S$ in cell $W$, many of the other specialized functions will be secondarily disturbed. Such a cell is potentially capable of forming a highly anaplastic neoplasm. Since each cell has an abundance of many special functions, it becomes obvious that almost any variation of cell type is possible. Indeed, it is fortuitous for any two tumors to be exactly alike when one considers the variety of combinations that can result when one or more functions are altered simultaneously or sequentially. Defects in special functions may be induced by carcinogens in any stage of normal development such as cells $B, C, D$, or $E$, but neoplastic cells can never result from cell $E$ because there is no mechanism for transmitting the change.

It is also obvious that carcinogens produce a variety of biochemical lesions which are not heritable. The cell may recover 
from some of these lesions and succumb to others. Some of the initial changes produced by carcinogens may not differ from lesions resulting from other toxic agents; the chief difference is that carcinogens can also induce heritable alterations.

While it appears possible that the altered cells $U, V$, and $W$ may arise from any one of the stages of normal cells $B, C$, and $D$, it is probable that cell $U$ is more likely to arise from cell $D$ than from cell $B$, and cell $W$ is more apt to arise from cell $B$ than from cell $D$. Some, but not all, of the pathways from the normal to the defective cells are indicated by arrows made of dash lines. Each set of arrows depicts a series of many cells and stages rather than a single change. The period of transition from the normal cells $B, C$, and $D$ to the altered ones $U, V$, and $W$ corresponds to the period of initiation as defined by Rous (21) and others (8). The horizontal lines which break the dash lines indicate the time when the reversible condition passes into an irreversible one. That the process of initiation may be reversible for a period is demonstrated by the fact that certain carcinogens must be administered for a considerable time before neoplasms result $(13,58)$.

Although each cell represented by $U, V$, and $W$ has a heritable loss of one specialized function and is therefore capable of giving rise to a neoplasm, the conversion into an autonomous cluster of cells does not always proceed rapidly. The cell still retains some competitive cellular constituents which were a part of the once active special system. Such constituents must disappear before the final conversion is complete. The removal of such substances is by a gradual deletion, usually occurring with each cell division and culminating in the change of cells $U, V$, and $W$ to $X, Y$, and $Z$, respectively. This change is depicted by the absence from cells $X, Y$, and $Z$ of the specialized functions marked with the crosses in cells $U, V$, and $W$. The final conversion just described corresponds to the period of promotion as described by Rous (21) and others (8), and the series of changes during this period is shown by the arrows made of the long dash lines. The specialized systems, $H$ in cell $V$ and $L$ and $H$ 
in ccll $W$, which were directly dependent on the products of the inactivated precursor reactions, are not shown in cells $Y$ and $Z$. It must be remembered, however, that some specialized systems may be functioning at very low levels. It is obvious that many variations are possible in this schema, but that cell $Z$ represents the ultimate degree of anaplasia. In the benign tumor (cell $X$ ) the arrows indicate a reactivation of function $S$ at a level comparable to that seen in normal cell $C$. Such activation is possible because of the reoriented equilibrium of the cellular systems following the loss of one or more of the other functions.

Some neoplastic cells may lie relatively quiescent for long periods without losing their capacity to be promoted. This is illustrated by epidermal and testicular tumors, which may be promoted with croton oil $(9,56,57)$ and estrogen (23), respectively, one year after the initiation of the tumor. Similarly, the hormonally dependent tumors, as studied by Furth (22) and Gardner (24), also require a considerable period for initiation and promotion to the neoplastic state.

Cells intermediate in malignancy (e.g., cell $Y$ ) may suffer a further loss of specialized function and pass to the next lower level. The continued deletion of special characteristics from neoplastic cells through successive cell generations has been clearly demonstrated by various investigators who have studied the genetic factors required for successful transplantation of neoplasms $(4,25,27)$. Such studies demonstrate that tumor cells, once incapable of growth in homologous strains, may after prolonged growth be successfully transplanted to previously resistant hosts. This indicates that the number of histocompatibility genes required for successful transplantation may be progressively reduced in the growing cancer cell (25). This process is probably to be identified with the term progression as used by Foulds for describing the changes of neoplastic cells to the more malignant state (19). Thus, whereas normal cells are genetically stable, instability is common once a permanent, heritable change has occurred in a cell.

Cell $Z$ represents the most advanced stage of anaplasia. 
Such a cell has lost its major potential for specialized function and is doomed to a continuous process of reduplication. It represents a reversion to a unicellular type of life. This advanced stage seldom if ever occurs in an animal bearing a spontaneous or induced cancer, because the host does not live sufficiently long to permit a complete regression, but such neoplastic cells are illustrated by certain types of ascites tumor cells derived from tumors which have undergone repeated transfer. The tendency for unicellularity is evident to some degree in all neoplasms which tend to form abnormal architectural patterns and which give rise to cells which metastasize.

On the basis of the discussion just presented, the conclusions reached by the committee in 1938 may be extended with the following ideas :

1. Carcinogens induce a change in one or more of the special functions of the cell. The resulting change is heritable.

2. An untimely alteration of function disrupts the normal sequence of differentiation and the resulting metabolic patterns. In such cells, the pattern for reduplication is retained and predominates to varying degrees.

3. Cells which have suffered from such heritable change may require the additional loss of accessory factors before becoming completely autonomous neoplasms.

4. The formation and growth of cancer cells are affected by conditions within the host-the genetic constitution, the hormone balance, the diet, the presence of irritation, chemotherapeutic agents - any factor that affects the internal environment of the host.

It appears clear from the above points that the causes of cancer may be varied and multiple and that the point of initiation may be nuclear or cytoplasmic. It is also clear that the metabolic patterns of neoplasms may include a diversity of features which they have in common with normal cells.

\section{Course of Action}

The concepts just presented are far from complete; at 
best, they can only emphasize weaknesses in our present knowledge and help to provide the framework for the design of further experiments. At this point, it may be appropriate to emphasize a few of the approaches that are worthy of further consideration.

First, in searching for the causes of cancer and their eventual elimination, it is well to consider the dual roles of initiation and promotion as causal mechanisms in the formation of some types of cancer. Not only must the understanding of the mechanisms by which carcinogens produce heritable changes be clarified, but the methods by which these processes may be modified must also be investigated. For example, hormones $(22,24)$, caloric restriction $(12,67)$, and irritation influence tumor formation, yet too few investigators are scarching for new agents to block hormones, for methods to neutralize the favorable influence of caloric excesses on carcinogenesis, and for means to combat the more subtle types of irritation.

Second, further information is needed about the metabolic patterns which characterize cellular reduplication and specialization. The step-by-step mapping of the individual reactions of many alternative pathways forms a large part of the research program at the McArdle Memorial Laboratory (28, $39,41,47,48,59,60,68,69)$. In this work the eventual localization of the sites of action of antigrowth agents is one of the important goals. This knowledge is required as a firm foundation for the chemotherapy of neoplastic diseases and helps to orient our thoughts about the various approaches to therapy One possibility would be to divert cell food from the growth pattern by increasing the level of some quiescent special function. Another would be to supply artificial substrates to the malignant growth process in order to obtain a defective growth mechanism in the cancer cells.

Third, investigations of the phenomena of invasion and metastasis deserve greater emphasis as another approach to therapy. Additional information is nceded concerning the role of fibroblasts, leukocytes, and enzymes in limiting or enhancing 
invasion and metastasis. The experiments of Algire (1), Coman (15), and others provide stimulating leads. Further work in this direction may lead to definitive experiments that will permit us to understand how the invading cell evokes a favorable vascular response and overcomes the forces that prevent normal, growing tissues from overstepping the boundaries that are compatible with the best interest of the host.

Finally, since neoplasms in human beings usually retain certain specialized features of the mature cell, the response of such tumors to therapy must depend to a considerable extent on the proportion of the retained characteristics. Therefore, no chemotherapy screening program should depend entirely on animal tumors which have suffered a considerable loss of special functions through repeated transplantation.

\section{Conclusion}

It should be emphasized that the attack on the cancer problem is a fine example of a research effort in which the various branches of biological science cooperate in a common cause and in which the old departmental classifications no longer apply in a strict sense. The modern investigator of malignant growth frequently brings to bear not only the disciplines of physiology and biochemistry but also the specialities of genetics, embryology, bacteriology, pathology, virology, and many others, which stimulate and nurture one another.

The concept of carcinogenesis presented in this report was based on facts which have emerged from numerous seemingly unrelated investigations in these various fields. Such concepts are worth while only if they engender additional advances, since each advance is dependent on some prior gain made elsewhere, and each advance, from the preliminary spadework to the completed harvest, stimulates and catalyzes new advances.

The report published in 1938 ended with a statement which is still applicable today, "In any program for cancer research, patience and the adoption of a long-time point of view are absolutely essential." 


\section{References}

1. Algire, G. H., F. Y. Legallais, and B. F. Anderson, J. Natl. Cancer Inst., 14, 879 (1954).

2. Auerbach, C., Biol. Revs. Cambridge Phil. Soc., 24, 355 (1949).

3. Avery, O. T., C. M. MacLeod, and M. McCarty, J. Exptl. Med., 79, 137 (1944).

4. Barrett, M. K., Cancer Research, 12, 535 (1952).

5. Bayne-Jones, S., R. G. Harrison, C. C. Little, J. Northrop, and J. B. Murphy, Public Health Repts. (U. S.), 53, 2121 (1938).

6. Beadle, G. W., "Chemical genetics," in L. C. Dunn, ed., Genetics in the 20th Century, pp. 221-239. Macmillan, New York, 1951.

7. Beadle, G. W., and E. L. Tatum, Am. J. Botany, 32, 678 (1945).

8. Berenblum, I., Cancer Research, 14, 471 (1954).

9. Berenblum, I., and P. Shubik, Brit. J. Cancer, 1, 379 (1947).

10. Bittner, J. J., Ann. N. Y. Acad. Sci., 49, 69 (1947).

11. Blum, H. F., J. Natl. Cancer Inst., 17, 463 (1950).

12. Boutwell, R. K., M. K. Brush, and H. P. Rusch, Am. J. Physiol., 154, 517 (1948).

13. Clayton, C. C., and C. A. Baumann, Cancer Research, 9, 575 (1949).

14. Cohn, M., J. Monod, M. R. Pollock, S. Spiegelman, and R. Y. Stanier, Nature, 172, 1096 (1953).

15. Coman, D. R., Cancer Research, 13, 397 (1953).

16. Davies, R., and E. F. Gale, Adaptation in Micro-Organisms, Third Symposium of the Society for General Microbiology. Cambridge University Press, Cambridge, 1953.

17. Ephrussi, B., "Remarks on cell heredity," in L. C. Dunn, ed., Genetics in the 20th Century, pp. 241-262. Macmillan, New York, 1951.

18. Ephrussi, B., Nucleo-cytoplasmic Relations in Micro-organisms: Their Bearing on Cell Heredity and Differentiation. Oxford University Press, London, 1953.

19. Foulds, L., Cancer Research, 14, 327 (1954).

20. Freeman, V. J., and I. U. Morse, J. Bacteriol., 63, 407 (1952).

21. Friedewald, W. F., and P. Rous, J. Exptl. Med., 80, 101 (1944).

22. Furth, J., Cancer Research, 13, 477 (1953).

23. Gardner, W. U., Cancer Research, 5, 497 (1945).

24. Gardner, W. U., Advances in Cancer Research, 1, 173 (1953).

25. Gorer, P. A., Brit. J. Cancer, 2, 103 (1948).

26. Haddow, A., Proc. Roy. Soc. Med., 41, 263 (1951).

27. Hauschka, T. S., Cancer Research, 12, 615 (1952).

28. Heidelberger, C., Advances in Cancer Research, 1, 274 (1953).

29. Heston, W. E., J. Natl. Cancer Inst., 10, 125 (1949).

30. Heston, W. E., J. Natl. Cancer Inst., 14, 131 (1953). 
31. Holtfreter, J., Growth, 15. 117 (1951).

32. Horowitz, N. H., Proc. Natl. Acad. Sci. U. S., 31, 153 (1945).

33. Hotchkiss, R. D., "The biological nature of the bacterial transforming factors," in The Chemistry and Physiology of the Nucleus, Exptl. Cell Research Suppl. 2, 383-390 (1952).

34. Hutner, S. H., and L. Provasoli, "The phytoflagellates," in A. Lwoff, ed., Biochemistry and Physiology of Protozoa, pp. 79-85. Academic Press, New York, 1951.

35. Knox, W. E., and A. H. Mehler, Science, 113, 237 (1951).

36. Koller, P. C., "The experimental modification of nucleic acid systems in the cell," in Nucleic Acid, Symposia Soc. Exptl. Biol., 1, 270-290 (1947).

37. Law, L. W., Cancer Research, 14, 695 (1954).

38. Lederberg, J., Physiol. Revs., 32, 403 (1952).

39. LePage, G. A., Cancer Research, 13, 178 (1953).

40. LePage, G. A., and C. Heidelberger, J. Biol. Chem., 188, 593 (1951).

41. LePage, G. A., V. R. Potter, H. Busch, C. Heidelberger, and R. B. Hurlbert, Cancer Research, 12, 153 (1952).

42. Lwoff, A., Cold Spring Harbor Symposia Quant. Biol., 11, 139 (1946).

43. Miller, E. C., Cancer Research, 11, 100 (1951).

44. Miller, E. C., and J. A. Miller, Cancer Research, 12, 547 (1952).

45. Miller, J. A., and E. C. Miller, Advances in Cancer Research, 1, 339 (1953).

46. Miller, S. L., Science, 117, 528 (1953).

47. Moldave, K., and C. Heidelberger, J. Am. Chem. Soc., 76, 679 (1954).

48. Mueller, G. C., J. Biol. Chem., 204, 77 (1953).

49. Potter, V. R., and C. Heidelberger, Physiol. Revs., 30, 487 (1950).

50. Potter, V. R., J. M. Price, E. C. Miller, and J. A. Miller, Cancer Research, 10, 28 (1950).

51. Pringle, J. W. S., "The origin of life," in Evolution, Symposia Soc. Exptl. Biol., 7, 1-21 (1953).

52. Recknagel, R. O., and V. R. Potter, J. Biol. Chem., 192, 263 (1951).

53. Rous, P., Am. J. Cancer, 28, 233 (1936).

54. Rous, P., J. Am. Med. Assoc., 122, 573 (1943).

55. Rous, P., J. B. Murphy, and W. H. Tytler, J. Am. Med. Assoc., 59, 1793 (1912).

56. Rusch, H. P., and B. E. Kline, Arch. Pathol., 42, 445 (1946).

57. Rusch, H. P., Proc. Soc. Exptl. Biol. Med., 69, 90 (1948).

58. Rusch, H. P., B. E. Kline, and C. A. Baumann, Arch. Pathol., 37, 135 (1941).

59. Schmitz, H., V. R. Potter, and R. B. Hurlbert, Cancer Research, 14, 58 (1954).

60. Schmitz, H., V. R. Potter, R. B. Hurlbert, and D. M. White, Cancer Research, 1., 66 (1954). 
61. Shope, R. E., J. Exptl. Mcd., 58, 607 (1933).

62. Sonncborn, T. M., "The role of the genes in cytoplasmic inheritance," in L. C. Dunn, ed., Genetics in the 20th Century, pp. 291-314. Macmillan, New York, 1951.

63. Spemann, H., Embryonic Dcoclopment and Induction. Yale University Press, New Haven, 1938.

64. Spiegelman, S., Cold Spring Harbor Symposia Quant. Biol., 16, 87 (1951).

65. Spratt, N. T., Jr., Physiol. Revs., 34, 1 (1954).

66. Stanier, R. Y., Ann. Rev. Microbiol., 5, 35 (1951).

67. Tannenbaum, A., and H. Silverstone, Cancer Rescarch, 9, 724 (1949).

68. Tyner, E. P., C. Heidelberger, and G. A. LePage, Cancer Research, 12, 158 (1952).

69. Tyner, E. P., C. Heidelberger, and G. A. LePage, Cancer Research, 13, 186 (1953).

70. Waddington, C. H., Organiscrs and Genes. Cambridge University Press, Cambridge, 1940.

71. Weiss, P., J. Embryol. EO Exptl. Morphol., 1, 181 (1953).

72. Wiest, W. G., and C. Heidelberger, Cancer Research, 13, 246 (1953).

73. Wiest, W. G., and C. Heidelberger, Cancer Research, 13, 250 (1953).

74. Wiest, W. G., and C. Heidelberger, Cancer Research, 13, 255 (1953).

75. Williams, C. M., Federation Proc., 10, 546 (1951).

76. Wilson, E. B., The Cell in Development and Heredity, 3rd ed. Macmillan, New York, 1925.

77. Woods, M. W., and H. G. duBuy, J. Natl. Cancer Inst., 17, 1105 (1951). 








